\title{
Schweiz - Europäische Union: Grundlagen, Bilaterale Abkommen, Autonomer Nachvollzug
}

Oesch, Matthias

\begin{abstract}
Die schweizerische Europapolitik steht auf dem Prüfstand. Nach der Ablehnung der Volksinitiative «Für eine massvolle Zuwanderung (Begrenzungsinitiative)» rückt das geplante Institutionelle Abkommen in den Fokus der Öffentlichkeit. Die EU erwartet von der Schweiz, sich zum vorliegenden Text zu bekennen. Der Bundesrat beurteilt den Entwurf zwar positiv, hat bislang aber darauf verzichtet, ihn zu unterzeichnen. Er verlangt Klärungen und Präzisierungen in Bezug auf den Lohnschutz, die Unionsbürgerrichtlinie und die Beihilferegeln. Die nächsten Monate werden zeigen, ob der Bundesrat und die Europäische Kommission einen tragfähigen Kompromiss finden und bereit sind, das Abkommen den Vertragsgebern in der Schweiz und der EU zur Genehmigung vorzulegen, oder ob wir auf eine Phase der Unsicherheit und Erosion der bilateralen Beziehungen zusteuern. Letzteres wäre keine verlockende Perspektive - weder für die Schweiz noch für die EU.
\end{abstract}

DOI: https://doi.org/10.36862/eiz-297

Posted at the Zurich Open Repository and Archive, University of Zurich

ZORA URL: https://doi.org/10.5167/uzh-193359

Monograph

Published Version

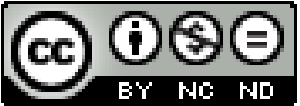

The following work is licensed under a Creative Commons: Attribution-NonCommercial-NoDerivatives 4.0 International (CC BY-NC-ND 4.0) License.

Originally published at:

Oesch, Matthias (2020). Schweiz - Europäische Union: Grundlagen, Bilaterale Abkommen, Autonomer Nachvollzug. Zürich: EIZ Publishing.

DOI: https://doi.org/10.36862/eiz-297 
Matthias Oesch

Schweiz Europäische Union

Grundlagen Bilaterale Abkommen Autonomer Nachoollzug 
Schweiz - Europäische Union 

Matthias Oesch

\section{Schweiz - Europäische Union}

Grundlagen

Bilaterale Abkommen

Autonomer Nachvollzug 


\section{(ㄷ) (1) $\circledast \Theta$}

Schweiz - Europäische Union von Matthias Oesch wird unter Creative Commons Namensnennung-Nicht kommerziell-Keine Bearbeitung 4.0 International lizenziert, sofern nichts anderes angegeben ist.

(C) 2020 - CC BY-NC-ND (Werk), CC BY-SA (Text)

Autor: Prof. Dr. Matthias Oesch

Verlag: EIZ Publishing

Produktion, Satz \& Vertrieb: buch \& netz, buchundnetz.com

Cover: Dominik Huber, Scientific Visualisation and Visual Communication, Universität Zürich

ISBN:

978-3-03805-297-5 (Print - Hardcover)

978-3-03805-336-1 (PDF)

978-3-03805-337-8 (ePub)

978-3-03805-338-5 (mobi/Kindle)

DOI: https://doi.org/10.36862/eiz-297

Version: $1.04-20201002$

Dieses Werk ist als buch \& netz Online-Buch und als eBook in verschiedenen Formaten sowie als gedrucktes Buch verfügbar. Weitere Informationen finden Sie unter der URL:

https://buchundnetz.com/werke/schweiz-europaeische-union/. 


\section{Vorwort}

Die schweizerische Europapolitik steht auf dem Prüfstand. Nach der Ablehnung der Volksinitiative «Für eine massvolle Zuwanderung (Begrenzungsinitiative)» rückt das geplante Institutionelle Abkommen in den Fokus der Öffentlichkeit. Die EU erwartet von der Schweiz, sich zum vorliegenden Text zu bekennen. Der Bundesrat beurteilt den Entwurf zwar positiv, hat bislang aber darauf verzichtet, ihn zu unterzeichnen. Er verlangt Klärungen und Präzisierungen in Bezug auf den Lohnschutz, die Unionsbürgerrichtlinie und die Beihilferegeln. Die nächsten Monate werden zeigen, ob der Bundesrat und die Europäische Kommission einen tragfähigen Kompromiss finden und bereit sind, das Abkommen den Vertragsgebern in der Schweiz und der EU zur Genehmigung vorzulegen, oder ob wir auf eine Phase der Unsicherheit und Erosion der bilateralen Beziehungen zusteuern. Letzteres wäre keine verlockende Perspektive - weder für die Schweiz noch für die EU.

Das Buch bezweckt, den Boden zu legen für eine informierte Diskussion über den bilateralen Weg der Schweiz und die nächsten Integrationsschritte. Es stellt die Entstehung der bilateralen Abkommen vor, ihre Grundzüge, ihre institutionelle Ausgestaltung und die mit dem Institutionellen Abkommen avisierten Novellierungen. Es skizziert den Inhalt und die Funktionsweise der einzelnen Abkommen. Es beleuchtet die Politik des autonomen Nachvollzugs von EU-Recht in der Schweiz. Insgesamt zeigt sich, dass die Doppelstrategie der völkerrechtlichen Anbindung und der selbstgewählten Anpassung zu einer weitgehenden Integration der Schweiz in das Recht der EU geführt hat deutlich weitgehender als dies einer breiten, auch juristisch fachkundigen Öffentlichkeit bewusst ist. Die Schweiz ist längst zu einem «zugewandten Ort» der EU mutiert. Sie tut gut daran, sich der mannigfaltigen Gemeinsamkeiten, Interdependenzen und Abhängigkeiten bewusst zu sein, wenn die nächsten europapolitischen Weichen gestellt werden.

Nina Burghartz, Susanne Gutzwiller, Stefan Höfler, Vanessa König, Veena Manikulam, Tobias Naef, Thomas Pfisterer und Gabriel Speck haben mich auf Unzulänglichkeiten hingewiesen und mich mit $\mathrm{Zu}$ - und Widersprüchen herausgefordert. Dominik Huber hat den Umschlag gestaltet. Titus Nemeth hat typographische Hinweise gegeben. Tobias Baumgartner, Petra Bitterli und Michael Mayer haben mich verlagsseitig begleitet. Ihnen allen sei herzlich gedankt. Das Buch baut in Teilen auf Vorarbeiten von mir auf: Switzerland and the European Union: General Framework, Bilateral Agreements, Autono- 
mous Adaptation, 2018; EU-Schweiz, in: Krenzler et al. (Hrsg.), EU-Aussenwirtschafts- und Zollrecht, 2018 (zusammen mit Nina Burghartz); Europarecht, 2. Aufl., 2019. Das Manuskript wurde im Wesentlichen Ende August 2020 abgeschlossen.

Das Buch erscheint beim Verlag EIZ Publishing, einem juristischen Fachverlag, der vom Europa Institut an der Universität Zürich (EIZ) getragen wird. Es wird als Printexemplar und als kostenfreies E-Book vertrieben (open access; www.eizpublishing.ch; www.rwi.uzh.ch/oesch).

Matthias Oesch 


\section{Inhaltsübersicht}

\section{Erster Teil - Grundlagen}

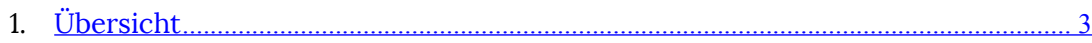

2. Die Schweiz als «zugewandter Ort» der EU ……...................................................... 7

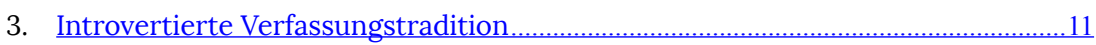

\section{Zweiter Teil - Bilaterale Abkommen}

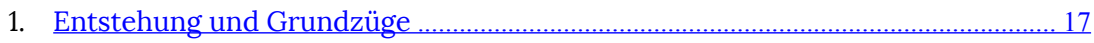

2. Institutionelle Ausgestaltung ………...................................................................... 43

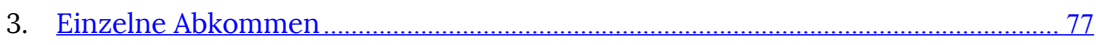

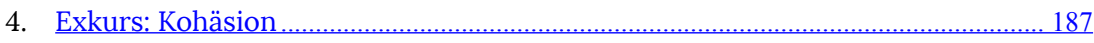

\section{Dritter Teil - Autonomer Nachvollzug}

1. Politik der Europaverträglichkeit ………………………….................................. 193

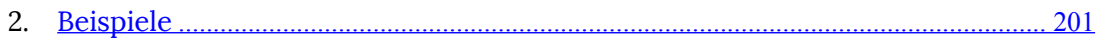

3. Europaverträgliche Auslegung ............................................................................. 209

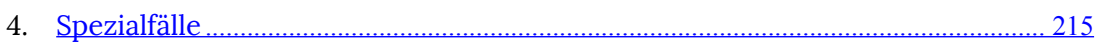

$\underline{\text { Vierter Teil - Epilog } \ldots \ldots \ldots \ldots \ldots \ldots \ldots \ldots \ldots \ldots \ldots \ldots \ldots \ldots \ldots \ldots \ldots \ldots \ldots \ldots \ldots \ldots \ldots \ldots \ldots \ldots \ldots \ldots \ldots \ldots \ldots \ldots \ldots \ldots . .27}$ 



\section{Inhaltsverzeichnis}

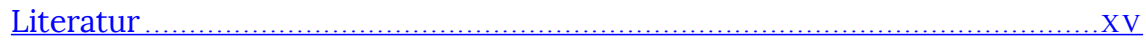

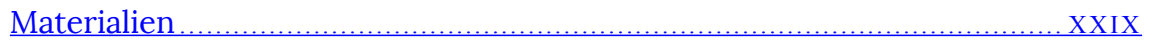

Berichte und weitere Informationen ............................................... XXXIII

Abkürzungsverzeichnis..........................................................................XXV

\section{Erster Teil - Grundlagen}

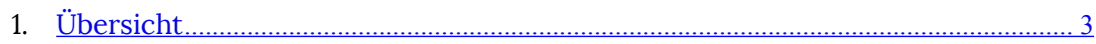

2. Die Schweiz als «zugewandter Ort» der EU ………………………………………….. 7

3. Introvertierte Verfassungstradition …………………………………………….....11

\section{Zweiter Teil - Bilaterale Abkommen}

1. Entstehung und Grundzüge ………………………………………………………….. 17

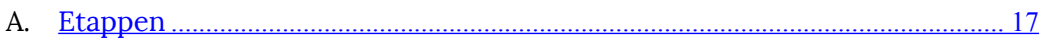

1. Erste Annäherung (1956-1972) ……………………………………………... 17

2. Abkommen im Waren-, Versicherungs- und Transitbereich (1972-1992) 18

3. Ablehnung des EWR-Beitritts (1992) ……………………………………... 19

4. Bilaterale I (1999) ………………………………………………………….... 20

5. Bilaterale II (2004) ………………………………………………....... 22

6. Punktuelle Ergänzungen (2005-heute) ……………………………………...24

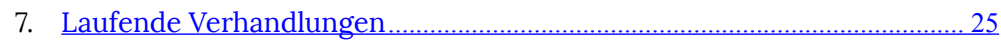

B. Parteien ………………………………………………………………… 27

1. «EU only»-Abkommen ……………………………………………..... 27

2. Gemischte Abkommen …………………………………………………...2 29

C. Typologie …………………………………………………………….... 30 
D. Verhältnis zum unionalen und schweizerischen Recht .................................. 32

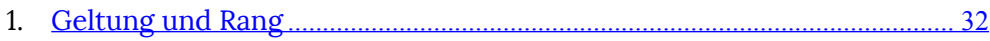

2. Völkerrechtskonforme Auslegung ........................................................... 34

3. Unmittelbare Anwendbarkeit .......................................................................... 35

E. Seitenblick: Bilaterale Abkommen und direkte Demokratie ........................... 36

1. Hohe demokratische Legitimation …………............................................. 36

2. Aushöhlung der Substanz der demokratischen Rechte ............................. 39

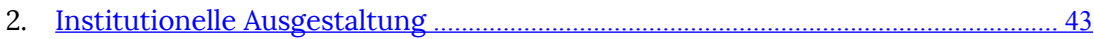

A. Durchführung, Überwachung, Rechtsschutz ……………………………......... 43

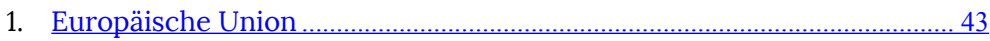

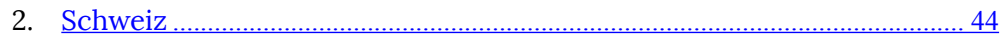

3. Spezielle Regelungen ………............................................................... 45

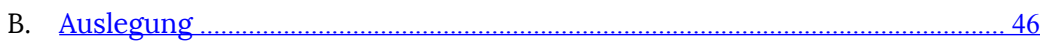

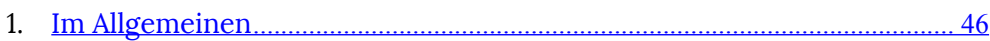

2. Sinngemäss oder wörtlich übernommenes EU-Recht ................................ 47

3. Positivrechtliche Regelungen................................................................... 50

4. Einseitige Erklärungen ................................................................................. 53

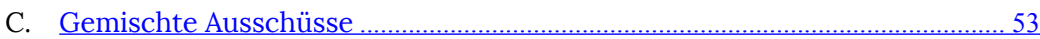

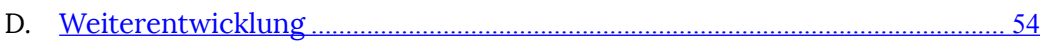

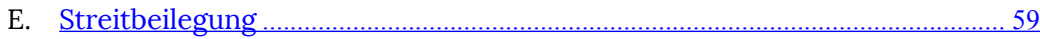

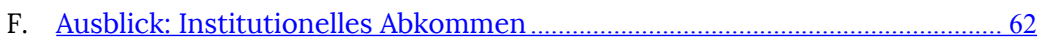

1. Ausgangslage und Verhandlungen ............................................................... 62

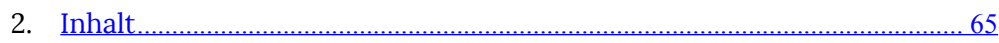

3. Weiteres Vorgehen ………................................................................. 73

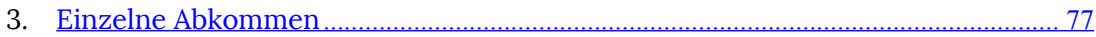

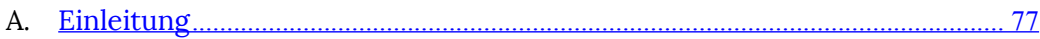

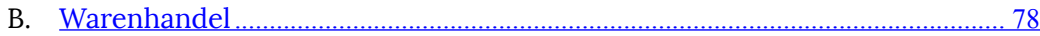

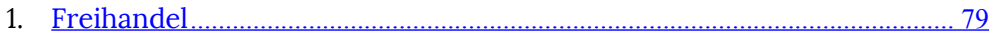

2. Technische Handelshemmnisse …………….............................................. 83 


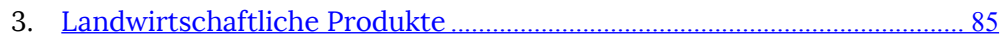

4. Verarbeitete landwirtschaftliche Produkte ……………................................ 87

5. Zollerleichterungen und Zollsicherheit ................................................... 89

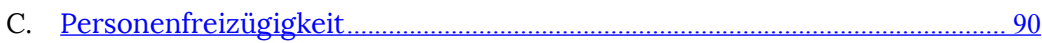

1. Freizügigkeitsabkommen: Im Allgemeinen ................................................ 91

2. Einreise, Aufenthalt, Familiennachzug ………........................................ 94

3. Nichtdiskriminierung, Stillhalteklausel....................................................... 98

4. Rechtfertigung von Einschränkungen ................................................... 100

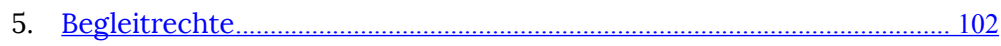

(a) Koordinierung der Systeme der sozialen Sicherheit....................... 103

(b) Anerkennung beruflicher Qualifikationen.......................................... 104

D. Dienstleistungshandel ..................................................................................... 107

1. Direktversicherungen .................................................................................. 109

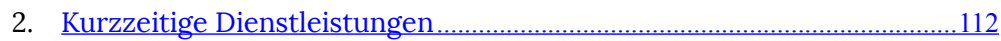

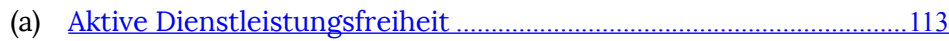

(b) Flankierende Massnahmen...................................................................115

(c) Passive Dienstleistungsfreiheit …………….....................................117

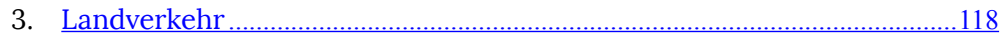

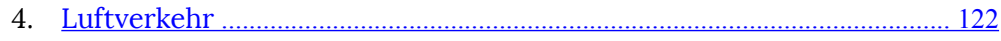

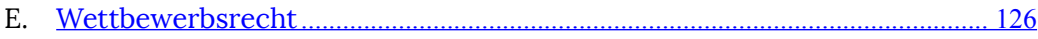

1. Kartellrechtliche Bestimmungen .................................................................... 127

2. Beihilferechtliche Bestimmungen …………............................................... 128

3. Öffentliches Beschaffungswesen ........................................................... 131

4. Zusammenarbeit der Wettbewerbsbehörden …………............................ 133

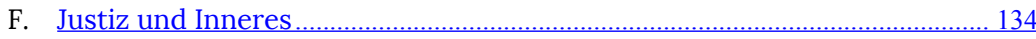

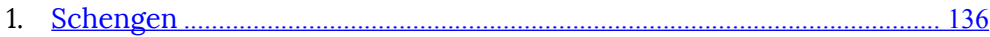

(a) Schengen-Assoziierungsabkommen: Im Allgemeinen ….................. 138

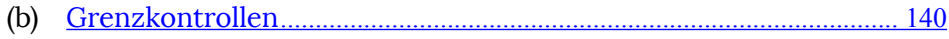

(c) Schengen-Visum............................................................................ 143 
(d) Rückführung von Drittstaatsangehörigen ...................................... 145

(e) Polizei, Amts- und Rechtshilfe, Sicherheit ....................................... 147

(f) Frontex, ISF - Grenzen und Visa....................................................... 151

2. Betrugsbekämpfung, Europol, Eurojust.................................................... 154

(a) Betrugsbekämpfung ………………………....................................... 154

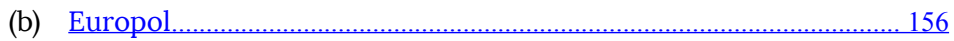

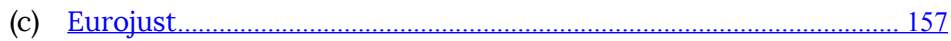

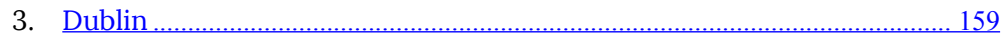

(a) Dublin-Assoziierungsabkommen: Im Allgemeinen ........................... 161

(b) Für die Bearbeitung eines Asylantrags verantwortlicher Staat ...... 163

(c) Eurodac ............................................................................................. 166

(d) Europäisches Unterstützungsbüro für Asylfragen .......................... 167

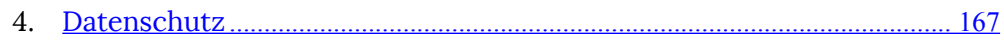

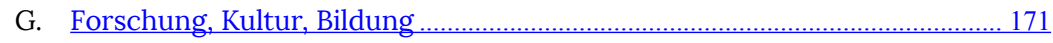

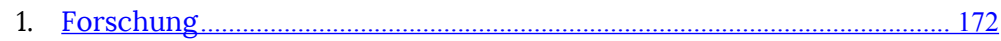

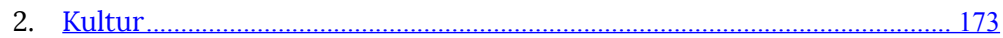

3. Bildung, Berufsbildung, Jugend ……………......................................... 175

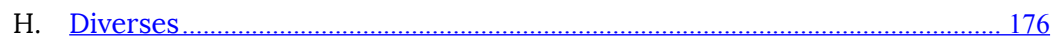

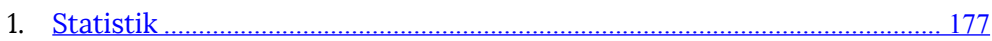

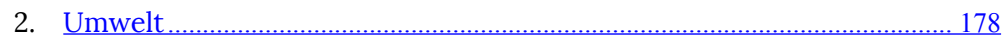

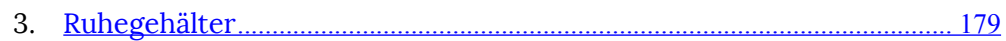

4. Zinsbesteuerung / Automatischer Informationsaustausch .................... 180

5. Verteidigung.......................................................................................... 182

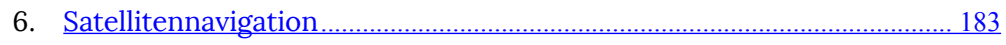

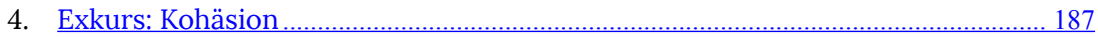




\section{Dritter Teil - Autonomer Nachvollzug}

1. Politik der Europaverträglichkeit ........................................................................ 193

A. Begriff und Zweck ................................................................................................ 193

B. Verhältnis zur Rechtsvergleichung …………............................................... 196

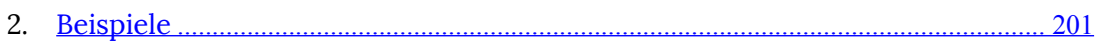

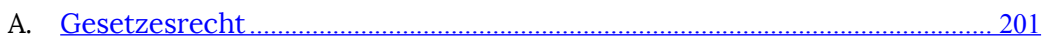

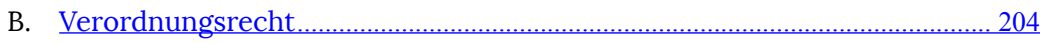

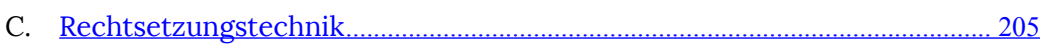

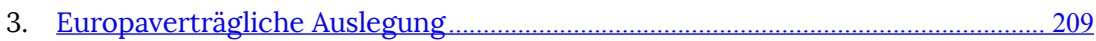

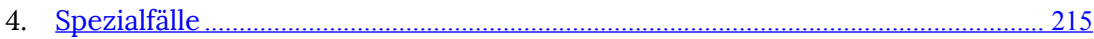

A. Faktische Alternativlosigkeit ........................................................................ 215

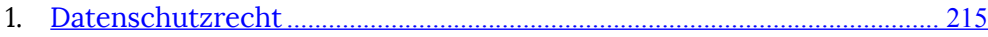

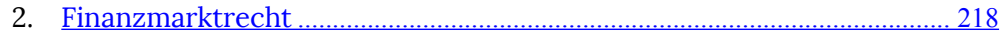

3. COVID-19: Ausfuhr persönlicher Schutzausrüstung ……………….......... 221

B. Cassis de Dijon-Prinzip ………………………............................................ 222 



\section{Literatur}

Diese Liste enthält die einschlägige Literatur sowie eine Auswahl der Publikationen zu den rechtlichen Beziehungen der Schweiz zur EU. Bei mehreren Publikationen einer Autorin oder eines Autors wird bei der Referenzierung im Text das in Klammern gesetzte Kurzzitat beigestellt.

Altermatt UrS, Die Schweiz in Europa: Antithese, Modell oder Biotop?, 2011

AMBÜHL MiCHAEL/SCHERER DANIELA, Ein Interimsabkommen könnte den Rahmenvertrag mit der EU retten, NZZ vom 25. November 2019 (zit. Interimsabkommen)

AMBÜHL MichaEL/SCHERER DANIELA, Zum Entwurf des Institutionellen Abkommens: Auf der Suche nach einem Interessenausgleich, Jusletter 4. Februar 2019 (zit. Entwurf)

AMbÜHL Michael/Scherer Daniela, Personenfreizügigkeit und der Schatten des Rahmenabkommens, NZZ vom 29. Juni 2020 (zit. Personenfreizgüigkeit)

AMSTUTZ MARC, Normative Kompatibilitäten: Zum Begriff der Europakompatibilität und seiner Funktion im Schweizer Privatrecht, SJER 2004/2005, 235

ARBIA ALI, The Road not taken: Europeanisation of Laws in Austria and Switzerland (1996-2005), 2006

ARIOLI SiLviO, Der Abbau von technischen Handelshemmnissen, in: Cottier/Oesch (Hrsg.), Die sektoriellen Abkommen Schweiz-EG, 2002, 123 (zit. Abbau)

ARIOLI SiLVIO, Replik: Zur Tragweite des Freihandelsabkommens im Beihilfenbereich aus rechtlicher Sicht, Jusletter 7. Mai 2007 (zit. Tragweite)

ARIOLI SILVIO, Übernahme des EU-Rechts in den Marktzugangsabkommen: Kein EU-Diktat sondern Sachzwang, www.suisse-en-europe.ch (zit. Übernahme)

AUER ANDREAS et al., Stellungnahme zur Volksinitiative «Schweizer Recht statt fremde Richter (Selbstbestimmungsinitiative)», Jusletter vom 20. Februar 2017

AuER ANDREAS/MALINVERni Giorgio/HotTelier Michel, Droit constitutionnel suisse, Vol. I: L'Etat, 3 éd., 2013

BALDI MARINO, Zu den Auswirkungen des geplanten Rahmenabkommens mit der EU: Auswirkungen auf das geltende Freihandelsabkommen (FHA 1972) und dessen allfällige «Modernisierung», insbesondere bezüglich Beihilfenkontrolle, Jusletter 18. März 2019

BAUDENBACHER CARL, Zum Nachvollzug europäischen Rechts in der Schweiz, EuR 1992, 309

BAUDENBACHER CARL, Wie sollen Konflikte im Verhältnis Schweiz - EU gelöst werden?, in: Sethe et al. (Hrsg.), Kommunikation, Festschrift für Rolf H. Weber zum 60. Geburtstag, 2011, 821

BAUDENBACHER CARL, Swiss Economic Law Facing the Challenges of International and European Law, ZSR 2012 II, 419

BAUDENBACHER CARL, Judicial Independence: Memoirs of a European Judge, 2018

BAUDENBACHER CARL, Das Schweizer EU-Komplott, 2019 (zit. Komplott)

Baudenbacher Laura Melusine, Das Personenfreizügigkeitsabkommen EU-Schweiz ist doch kein Integrationsvertrag, ELR 2010, 34

BAUdENBACHER LAURA MELUSINE, EuGH überträgt die Polydor-Rechtsprechung auch auf das Luftverkehrsabkommen, ELR 2013, 173 (zit. Luftverkehrsabkommen) 
BAUR GEORGES, Die EWR/EFTA-Staaten im Schengen/Dublin-System, in: Breitenmoser/Gless/ Lagodny (Hrsg.), Schengen und Dublin in der Praxis: Aktuelle Fragen, 2015, 27 (zit. Schengen)

BAUR GEORGES, Datenschutz-Grundverordnung Schengen-relevant? Vielleicht nicht, aber ..., in: Breitenmoser/Lagodny/Uebersax (Hrsg.), Schengen und Dublin in der Praxis: Aktuelle Herausforderungen, 2018, 165 (zit. Datenschutz)

BAUR GEORGES, The European Free Trade Association: An Intergovernmental Platform for Trade Relations, 2020

BECK BETtinA, Die Regelung von Slots (Zeitnischen) im Luftverkehrsrecht, 2008

BERTHOUD FRÉDÉRIC, Etudier dans une université étrangère: L'équivalence académique des diplômes en application de la Convention de reconnaissance de Lisbonne et des conventions bilatérales conclues entre la Suisse et ses pays limitrophes, 2012 (zit. Etudier)

BERTHOUD FRÉDÉRIC, La reconnaissance des qualifications professionelles: Union européenne et Suisse - Union européenne, 2016 (zit. reconnaissance)

BesSON SAMANTHA, Droit constitutionnel européen, 2019

BIAGGinI GiovANNI, Die Öffnung des Verfassungsstaates als Herausforderung für Verfassungsrecht und Verfassungslehre, in: Ehrenzeller et al. (Hrsg.), Der Verfassungsstaat vor neuen Herausforderungen, Festschrift für Yvo Hangartner, 1998, 957 (zit. Öffnung)

BiaGgini GiovanNI, Das Verhältnis der Schweiz zur internationalen Gemeinschaft, AJP 1999, 722 (zit. Verhältnis)

BiAGGini GiovanNI, Zur Umsetzung von Art. 121a BV durch die Bundesversammlung: Wo liegt und welcher Art ist das Problem (verfassungsrechtlich gesehen)?, ZBl 2016, 588 (zit. Umsetzung)

BiAGGini GiovanNi, BV-Kommentar, 2. Aufl., 2017 (zit. BV-Kommentar)

BOILLET VÉRONIQUE, L'interdiction de discrimination en raison de la nationalité au sens de l'Accord sur la libre circulation des personnes, 2010

BORER JÜRG, Massnahmen gleicher Wirkung wie mengenmässige Einfuhrbeschränkungen im Freihandelsabkommen Schweiz-EWG: eine vergleichende Darstellung von Art. 13 Freihandelsabkommen mit Art. 30 EWG-Vertrag, 1988

BORGHI ALVARO, La libre circulation des personnes entre la Suisse et l'UE: Commentaire article par article de l'accord du 21 juin 1999, 2010

BRADFORD ANU, The Brussels Effect, Northwestern University Law Review 2012, 1

BRADFORD ANU, The Brussels Effect: How the European Union Rules the World, 2020

BREITENMOSER STEPHAN, Sectoral Agreements between the EC and Switzerland: Contents and Contexts, CMLRev 2003, 1137

BREITENMOSER STEPHAN, Welche Ergänzungen für das Rahmenabkommen notwendig sind - und keine Neuverhandlungen benötigen, NZZ vom 31. Januar 2019 (zit. Ergänzungen)

BREITENMOSER STEPHAN/Weyeneth ROBERT, Europarecht: Unter Einbezug des Verhältnisses Schweiz-EU, 3. Aufl., 2017

Breuss Fritz/Cottier Thomas/Müller-Graff Peter-Christian (Hrsg.), Die Schweiz im europäischen Integrationsprozess, 2008

BUCHER OLIVER, Open Access im Schienenverkehr: eine Auslegeordnung der rechtlichen Rahmenbedingungen der Schweiz mit einem Überblick über die Regelung in der EU, 2006

BURRI NINA, Landesverweisung und Freizügigkeitsabkommen - Bundesgericht vertagt Entscheid über Normenkonflikt, sui-generis 2019, 64

BURRI THOMAS/PIRKER BENEDIKT, Stromschnellen im Freizügigkeitsfluss: Von der Bedeutung von Urteilen des Europäischen Gerichtshofes im Rahmen des Freizügigkeitsabkommens, SZIER 2010, 165 
CEyssens JAN/TARDE ANDREAS, Äquivalenz im Finanzdienstleistungsrecht der Europäischen Union, EuZW 2019, 805

Church Clive H. (ed.), Switzerland and the European Union: a close, contradictory and misunderstood relationship, 2012

Classen Claus Dieter, Nationales Verfassungsrecht in der Europäischen Union, 2013

COlella StÉPHANIE U., Protection des données: l'influence de l'UE sur le processus normatif suisse, LeGes 2020, Heft 2

COTTIER THOMAS, Recht und Macht im Europäischen Wirtschaftsraum, AJP 1992, 1204

COTTIER THOMAS, Die Bundesverfassung im postnationalen Europa: Vorbild oder Hemmschuh?, in: Kreis (Hrsg.), Erprobt und entwicklungsfähig: Zehn Jahre neue Bundesverfassung, 2009, 177 (zit. Bundesverfassung)

COTTIER THOMAS (Hrsg.), Die Europakompatibilität des schweizerischen Wirtschaftsrechts: Konvergenz und Divergenz, ZSR-Beiheft 50, 2012

CotTier THOMAS, Die Schweiz und Europa: Herausforderungen im Vierten Kreis der Integration, in: Neuhaus (Hrsg.), Europa zu Beginn des 21. Jahrhunderts, 2014, 141

COTTier THOMAS, Ein Europa-Artikel für die Bundesverfassung, 2014, www.sga-aspe.ch (zit. Europa-Artikel)

COTTIER ThOMAS, Der Rechtsschutz im Rahmenabkommen Schweiz-EU, 2019, www.suisse-eneurope.ch (zit. Rechtsschutz)

COTTIER THOMAS, Der Strukturwandel des Aussenwirtschaftsrechts, SRIEL 2019, 203 (zit. Strukturwandel)

COTTIER THOmAS, Die Souveränität und das institutionelle Rahmenabkommen, SJZ 2019, 345 (zit. Souveränität)

COTTIER THOMAS/DiEBold NiColas, Warenverkehr und Freizügigkeit in der Rechtsprechung des Bundesgerichts zu den Bilateralen Abkommen, Jusletter 2. Februar 2009

CotTIER ThOMAS/DZAmKo DANIEL/EvTimov ERIK, Die europakompatible Auslegung des schweizerischen Rechts, SJER 2003, 357

CotTiER ThOmas et al., Die Rechtsbeziehungen der Schweiz und der Europäischen Union, 2014

COTTIER ThOmas/Evtimov ERIK, Probleme des Rechtsschutzes bei der Anwendung der sektoriellen Abkommen mit der EG, in: Cottier/Oesch (Hrsg.), Die sektoriellen Abkommen SchweizEG, 2002, 179

COTTIER ThOMAS/HERREN DAVID, Das Äquivalenzprinzip im schweizerischen Aussenwirtschaftsrecht: von Cassis de Dijon zu Cassis de Berne, SJER 2009/2010, 249

CotTIER ThomAS/Liechti RACHEL (Hrsg.), Die Schweiz und Europa: Wirtschaftliche Integration und institutionelle Abstinenz, 2009

COTTIER ThOMAS/Liechti RACHEL, Zwischen Skylla und Charybdis: Die Rezeption des europäischen Wirtschaftsrechts in der Schweiz, EuZW 2012, 849

COTTIER THOMAS/LIECHTI RACHEL, KVG-Teilrevision: Zur Vereinbarkeit mit dem bilateralen Freizügigkeitsabkommen Schweiz-EU, Jusletter 10. Juni 2013 (zit. KVG)

CotTIER ThOMAS/MatTEOTTI RENÉ, Der Steuerstreit Schweiz-EG: Rechtslage und Perspektiven, SJER 2006/2007, 221

CotTier Thomas/Oesch MatThias (Hrsg.), Die sektoriellen Abkommen EG-Schweiz: Ausgewählte Fragen zur Rezeption und Umsetzung der Verträge vom 21. Juni 1999 im schweizerischen Recht, 2002

COTTIER THOMAS/WÜGER DANIEL, Auswirkungen der Globalisierung auf das Verfassungsrecht: Eine Diskussionsgrundlage, in: Sitter-Liver (Hrsg.), Herausgeforderte Verfassung: Die Schweiz im globalen Kontext, 1999, 241 
DELLI CHANTAL, Verbotene Beschränkungen für Arbeitnehmende?, Überlegungen zur Tragweite des Personenfreizügigkeitsabkommen zwischen der Schweiz sowie der EG und ihren Mitgliedstaaten, 2009

DELORS JACQUES, Déclaration sur les orientations de la Commission faite devant le Parlement européen, Strasbourg, 17 et 18 janvier 1989, Bulletin des Communautés européennes Supplément $1 / 89$

De SÉPIBUS JoËLLE, Ein institutionelles Dach für die Beziehungen zwischen der Schweiz und der Europäischen Union - Wie weiter?, Jusletter 14. Juli 2014

Dettling-Ott Regula, Das bilaterale Luftverkehrsabkommen der Schweiz und der EG, in: Thürer et al. (Hrsg.), Bilaterale Verträge I \& II Schweiz - EU, 2007, 491

DieBold NiCOLAS, Gerichtliche Sachverständige als hoheitlich tätige Organe?, AJP 2012, 1162 (zit. Sachverständige)

DiebolD Nicolas, Freizügigkeit im Mehrebenensystem: Eine Rechtsvergleichung der Liberalisierungsprinzipien im Binnenmarkt-, Aussenwirtschafts- und Europarecht, 2016 (zit. Freizügigkeit)

Diebold Nicolas/Ludin Martin, Das Cassis de Dijon-Prinzip in Praxis und Politik, SJER 2016/ 2017, 371

Diebold Nicolas/Oesch MatTHias, Die Durchsetzung von WTO-Recht durch Schweizer Unternehmen, AJP 2008, 1525

Diggelmann Oliver, Der liberale Verfassungsstaat und die Internationalisierung der Politik: Veränderungen von Staat und Demokratie in der Schweiz, 2005 (zit. Internationalisierung)

DigGelmann Oliver, Das Phänomen der «Scherbenhaufen»-Referenden, Jusletter 13. Oktober 2008 (zit. Scherbenhaufen)

DiMITRIJEWITSCH JOVAN, Scheinselbständigkeit im grenzüberschreitenden Dienstleistungsverkehr: Eine Analyse des Unionsrechts und des bilateralen Rechts Schweiz-EU, 2020

Du Bois Pierre, La Suisse et le défit européen 1945-1992, 1989

DUCREY PATRIK, Das Abkommen zwischen der Schweiz und der EU über die Zusammenarbeit bei der Anwendung ihrer Wettbewerbsrechte, Jusletter 30. September 2013

Dumont JANine/KelLerhals ANDREAS, Kanada-Abkommen als Vorbild für Ausnahmen vom Beihilfeverbot im Rahmenabkommen Schweiz-EU?, EuZ 2020, 4

DuRIĆ HANS-PETER, Die Freihandelsabkommen EG-Schweiz: die rechtliche Problematik, 3. Aufl., 1998

EHRENZELLER BERnhard/GerTSCH Gabriel, Art. 139, in: Ehrenzeller et al. (Hrsg.), St. Galler BVKommentar, 3. Aufl., 2014

EMMENEGGER SUSAN/Bigler ANDREA, Fortress Europe: Die Äquivalenzverfahren der EU im Bereich der Finanzdienstleistungen, SZW 2019, 155

EMmENEGGER SusAn/TSCHENTSCHER AXeL, Art. 1 ZGB, in: Berner Kommentar: Einleitung und Personenrecht, 2012

EPINEY ASTRID, Zur Bedeutung der Rechtsprechung des EuGH für Anwendung und Auslegung des Personenfreizügigkeitsabkommens, ZBJV 2005, 1

EPINEY ASTRID, Steuern, Europa und die Schweiz - Ausgewählte Aspekte der «Europakompatibilität» kantonaler Steuerregime aus rechtlicher Sicht, in: Jäger (Hrsg.), Steuerwettbewerb: Die Schweiz im Visier der EU, 2008, 75 (zit. Steuern)

EPINEY ASTRID, Das Verbot der Diskriminierung aus Gründen der Staatsangehörigkeit im Personenfreizügigkeitsabkommen, SJZ 2009, 25

EPINEY ASTRID, How Does European Union Law Influence Swiss Law and Policies?, in: Narath/ Varone (Hrsg.), Rediscovering Public Law and Public Administration in Comparative Policy Analysis: a Tribute to Peter Knoepfel, 2009, 179 
EPINEY ASTRID, Zur Verbindlichkeit der EU-Grundrechte in der und für die Schweiz, in: Altermatt/Casasus (Hrsg.), 50 Jahre Engagement der Schweiz im Europarat 1963-2013, 2013, 141 (zit. EU-Grundrechte)

EPINEY ASTRID, Vorübergehende Wiedereinführung der bedarfsabhängigen Zulassung frei praktizierender Ärzte, Jusletter 22. April 2013 (zit. Wiedereinführung)

EPINEY ASTRID, Quadratur des Kreises in der Europapolitik gelungen, NZZ vom 23. August 2013 (zit. Quadratur)

EPINEY ASTRID, Das Verhältnis von Völkerrecht und Landesrecht aus der Sicht des Bundesgerichts, Jusletter vom 18. März 2013 (zit. Völkerrecht)

EPINEY ASTRID, § 26 Europäische Freihandelszone (EFTA), in: Hatje/Müller-Graff (Hrsg.), EnzEur Bd. 1, 2014, 1105

EPINEY ASTRID, Art. 84, in: Waldmann/Belser/Epiney (Hrsg.), Bundesverfassung, Basler Kommentar, 2015 (zit. Art. 84)

EPINEY ASTRID, Auslegung und Verhältnis des Freizügigkeitsabkommens zum nationalen Recht. Zum Urteil des Bundesgerichts 2C_716/2014 vom 26. November 2015, Jusletter 14. März 2016 (zit. Verhältnis)

EPINEY ASTRID, «Brexit» und FZA: Zu den Perspektiven der Freizügigkeit zwischen der Schweiz und der EU im Gefolge des «Brexit», Jusletter 20. März 2017 (zit. Brexit)

EPINEY ASTRID, Der Entwurf des Institutionellen Abkommens Schweiz-EU, Jusletter 17. Dezember 2018 (zit. Entwurf)

EPINEY ASTRID, Brexit und die Schweiz: «Mind the Gap», SRIEL 2019, 231 (zit. Mind the Gap)

EPINEY ASTRID, Strafrechtliche Landesverweisung und FZA. Anmerkung zum Urteil des Bundesgerichts 6B_378/2018 vom 22. Mai 2019, Jusletter 19. August 2019 (zit. Landesverweisung)

EPINEY ASTRID/AFFolter SinA, Das Institutionelle Abkommen und die Unionsbürgerrichtlinie, Jusletter 11. März 2019

EPINEY ASTRID/FREI NULA, Zur Übernahme weiterentwickelten EU-Rechts durch den Bundesrat, Jusletter 12. August 2019

EPINEY ASTRID/Frei NULA, Sicherheitsnormen im alpenquerenden Schwerverkehr und Völkerrecht, Jusletter 24. August 2020 (zit. Schwerverkehr)

EPiney Astrid/Hehemann Lena, Die Tragweite des Institutionellen Abkommens im Bereich der Arbeitnehmerentsendung, Jusletter 8. April 2019

EPINEY ASTRID/HEUCK JENNIFER/KeRN MARKUS, Unionsrechtliche Vorgaben für die Beschränkungen des alpenquerenden Strassengütertransports, 2013

EPINEy Astrid/METz BEATE/MOsters RoBert (Hrsg.), Das Personenfreizügigkeitsabkommen Schweiz - EU, 2011

EPiney AStrid/Metz BEATE/PirKer BEnEdiKT, Die Vereinbarkeit eines gesetzlichen Mindestpreises für Alkoholika und ausgewählter Modelle von Lenkungsabgaben auf Alkoholika mit dem Freihandelsabkommen Schweiz-EU und der Wirtschaftsfreiheit, Jusletter 24. Januar 2011 (zit. Vereinbarkeit)

Epiney Astrid/Metz BEATe/PIRKer BEnEdikT, Zur Parallelität der Rechtsentwicklung in der EU und in der Schweiz, 2012 (zit. Parallelität)

EPINEY Astrid/SchleIsS YvonNe, § 4, in: Belser/Epiney/Waldmann (Hrsg.), Datenschutzrecht, 2011

ERNST WOLFGANG, Vollharmonisierung und autonomer Nachvollzug, EuZ 2010, 2

ERNST WOLFGANG, Fremde Richter - damals und heute, AJP 2013, 1374 (zit. Richter)

ETTER CHRISTIAN, Das Dienstleistungsabkommen der Uruguay-Runde und seine Bedeutung für die Schweiz, in: Cottier (Hrsg.), GATT-Uruguay Round: Neun Beiträge, 1995, 91 
FARGAHI BABAK/PRiUli VALERIO/DE WECK FAnNy, Die ersten Urteile des Bundesgerichts zur Landesverweisung (Art. 66a ff. StGB), Jusletter vom 15. April 2019

FreibuRGHAUS Dieter, Königsweg oder Sackgasse? Schweizerische Europapolitik von 1945 bis heute, 2. Aufl., 2015

FREIBURGHAUs Dieter/EPINEY ASTRID (Hrsg.), Beziehungen Schweiz - EU: Standortbestimmung und Perspektiven, 2010

FreibuRghaus Dieter/Kreis Georg (Hrsg.), Der EWR - verpasste oder noch bestehende Chance?, 2012

Frommelt Christian, In Search of Effective Differentiated Integration: Lessons from the European Economic Area (EEA), 2017

GAMMENTHALER NiNA, Diplomanerkennung und Freizügigkeit: Unter besonderer Berücksichtigung der Richtlinie über die Anerkennung von Berufsqualifikationen 2005/36/EG und ihrer möglichen Umsetzung in der Schweiz, 2010

GaVA Roy/Sciarini PASCAL/VARONE FrÉDÉRIC, Twenty Years After the EEA Vote: The Europeanization of Swiss Policy-Making, SPSR 2014, 197

GENTINETTA KATJA/KoHLER GEORG (Hrsg.), Souveränität im Härtetest. Selbstbestimmung unter neuen Vorzeichen, 2010

GLASER ANDREAS, Umsetzung und Durchführung des Rechts der Bilateralen Verträge in der Schweiz, in: Glaser/Langer (Hrsg.), Die Verfassungsdynamik der europäischen Integration und demokratische Partizipation, 2015, 133

GLASER ANDREAS, Wandel der Volksrechte, in: Diggelmann/Hertig Randall/Schindler (Hrsg.), Verfassungsrecht der Schweiz, Band I Grundlagen, 2020, 461 (zit. Wandel)

GLASER ANDREAS, Das Institutionelle Abkommen Schweiz-EU - ein Fall für das obligatorische Staatsvertragsreferendum?, sui-generis 2020, 210 (zit. Staatsvertragsreferendum)

GLASER ANDREAS/BrunNer ARThuR, Politik in der Defensive: Zwischen Vorrang des FZA und dynamischer Rezeption der EuGH-Rechtsprechung, Anmerkung zum Urteil des Bundesgerichts 2C_716/2014 vom 26. November 2015, Jusletter 18. April 2016

GLASER ANDREAS/DÖRIG HeIDI, Die Streitbeilegung in den Bilateralen Abkommen Schweiz - EU, SJER 2017/2018, 451

GLASER ANDREAS/LANGER LORENZ, Die Institutionalisierung der Bilateralen Verträge: Eine Herausforderung für die schweizerische Demokratie, SZIER 2013, 563

Gless Sabine/Petrig AnNa/Tobler Christa, Ein fachübergreifendes Prüfprogramm für die obligatorische Landesverweisung nach Art. 66a StGB. forumpoenale 2018, 97

Good PAUL-LuKaS, Die Schengen-Assoziierung der Schweiz, 2010

GRÄDEL MARKUS, Vereint marschieren - getrennt schlagen! Die Schweiz, Österreich, Norwegen und Schweden zwischen EWR und Beitritt zur Europäischen Union, 2007

GRÜTER BARBARA/NUSSBAUMER MARKUS, Redaktionelle Aspekte bei der Übernahme von EU-Recht, in: Uhlmann/Höfler (Hrsg.), Rechtsetzung im Mehrebenensystem: Gemeinden, Kantone, Bund, EU, 2020, 103

GUNDEL JÖRG, Die Öffnung des Vorabentscheidungsverfahrens zum EuGH für nichtmitgliedstaatliche Gerichte, EuZW 2019, 934

HAHN MiCHAEL, Die Kündigung des FZA als Voraussetzung für die Einführung von Kontingenten in Umsetzung des Art. 121a BV - Zugleich Anmerkung zum Entscheid des Bundesgerichts, II. öffentlich-rechtliche Abteilung, vom 26. November 2015, 2C_716/2014 (BGE 142 II 35), SJER 2015/2016, 457

HAilBronner KAI/Thym DANiel (eds.), EU Immigration and Asylum Law: A Commentary, $2^{\text {nd }}$ ed., 2016 
HEINEMANN ANDREAS, Rechtliche Transplantate zwischen Europäischer Union und der Schweiz, in: Fahrländer/Heizmann (Hrsg.), Europäisierung der schweizerischen Rechtsordnung, 2013, 3

HirSBRUNNER SimON, Könnte die Schweiz ein Verbot staatlicher Beihilfen verkraften?, EuZ 2017, 60

HÖFLER STEFAn, Gute Gesetzessprache aus dem Blickwinkel der Verwaltung: Die Redaktionskommission der schweizerischen Bundesverwaltung, in: Uhlmann/Höfler (Hrsg.), Gute Gesetzessprache als Herausforderung für die Rechtsetzung, 2018, 65

HöFler Stefan/Nussbaumer Markus/Uhlmamnn Felix, Legislation in Switzerland, in: Karpen/ Xanthaki (eds.), Legislation in Europe: A Country by Country Guide, to be published in 2020

Human Rights Watch, Pushed Back, Pushed Around. Italy's Forced Return of Boat Migrants and Asylum Seekers, Libya's Mistreatment of Migrants and Asylum Seekers, 2009

HUMMER WALDEMAR, Integrationspolitische Alternativen der Schweiz, EuZ 2012, 128

IMSTEPF RALF, Der Einfluss des EU-Rechts auf das schweizerische Mehrwertsteuerrecht, unter besonderer Berücksichtigung der Europakompatibilität des Leistungsortes, der ausgenommenen Leistungen und der Steuersätze, 2011

Institut für Europarecht der Universität Freiburg (Hrsg.), Die Schweiz und die europäische Integration: 20 Jahre Institut für Europarecht, 2015

JAAG TOBIAS/HÄNNI JULIA, Europarecht: Die europäischen Institutionen aus schweizerischer Sicht, 4. Aufl., 2015

JAAG TOBIAS/ZiHLMANN MAGDA, Institutionen und Verfahren, in: Thürer et al. (Hrsg.), Bilaterale Verträge I \& II Schweiz - EU, 2007, 65

JACOT-GuILlARMOD OliviER (éd.), Accord EEE: commentaires et réflexions, 1992

JENNI SABINE, Direkte und indirekte Europäisierung der schweizerischen Bundesgesetzgebung, LeGes 2013, 489

Jenni SABINE, Switzerland's Regulatory European Integration: Between Tacit Consensus and Noisy Dissensus, SPSR 2015, 508

JENNI SABInE, Switzerland's Differentiated European Integration, 2016 (zit. Differentiated)

Jutzi Thomas/Wess KSEnia/SiERADZKi DAmiAn, Die neue Finanzmarktarchitektur im europäischen Regulierungskontext, AJP 2020, 572

KADDOUS CHRISTINE, L'Accord CH-UE sur la libre circulation des personnes et l'ordre juridique suisse, in: Breuss/Cottier/Müller-Graff (Hrsg.), Die Schweiz im Europäischen Integrationsprozess, 2008, 153

KADDOUs Christine, Stamm et Hauser, Grimme, Fokus Invest AG, Hengartner et Gasser ou les accords bilatéraux ne créent pas un marché intérieur, SZIER 2010, 129

KADDOUS Christine, § 20 Die Zusammenarbeit zwischen der EU und der Schweiz, in: Hatje/Müller-Graff (Hrsg.), EnzEuR Bd. 1, 2014 (zit. Zusammenarbeit)

Kaddous Christine/JAmetTi-Greiner Monique (éds.), Accord bilatéraux II Suisse-UE, 2006

KÄLIN WALTER, Die Bedeutung des Freizügigkeitsabkommens für das Ausländerrecht, in: Cottier/ Oesch (Hrsg.), Die sektoriellen Abkommen Schweiz-EG, 2002, 11

KAUFMANN MARIANNE, Anerkennung der Berufsqualifikationen von Primarlehrpersonen, 2015

KELLENBERGER JAKOB, Wo liegt die Schweiz? Gedanken zum Verhältnis CH-EU, 2014

KELLER HeLEN, Rezeption des Völkerrechts, 2003

KELLER HELEN/LANTER MARKUS/FISCHER ANDREAS, Volksinitiativen und Völkerrecht: die Zeit ist reif für eine Verfassungsänderung, ZBl 2008, 121

Kellerhals Andreas/Baumgartner TOBIAS, Das «Cassis-de-Dijon»-Prinzip und die Schweiz, SJZ 2006, 321 
KIENER REGINA/KRÜSI MELANIE, Bedeutungswandel des Rechtsstaats und Folgen für die (direkte) Demokratie am Beispiel völkerrechtswidriger Volksinitiativen, ZBl 2009, 237

KLÄSER JULIAN, The Swiss Confederation and the European Union: their constitutional systems, bilateral agreement-based constitutional cooperation and a Swiss company tax regime facing challenges of constitutional and legal feasibility, 2017

KLEY ANDREAS, Verfassungsgeschichte der Neuzeit, 4. Aufl., 2020

KOHLER EMILIE, Influence du droit européen sur la législation suisse: analyse des années 2004 à 2007, Jusletter 31. August 2009 (zit. Influence)

KOHLER EMILIE, Le rôle du droit de l'Union européenne dans l'interprétation du droit suisse, 2015 (zit. rôle)

KOLLER HEINRICH, Der schweizerische Gesetzgeber vor der internationalen Herausforderung: Erfahrungen mit «EUROLEX» - «SWISSLEX» - «GATTLEX», ZBl 1994, 241

KÖLZ ALFRED/MÜLLER JÖRG PAUL, Entwurf für eine Bundesverfassung vom 16.5.1984, 3. Aufl., 1995

KRAMER ERnSt A., Juristische Methodenlehre, 6. Aufl., 2019

KREIS GEORG, Fremde Richter: Karriere eines politischen Begriffs, 2018

KROPF CATHERINE, Multi-Level-Gesetzgebung: europäisches, bilaterales, nationales Recht und wieder zurück, in: Uhlmann/Höfler (Hrsg.), Rechtsetzung im Mehrebenensystem: Gemeinden, Kantone, Bund, EU, 2020, 85

KunZ PeTER V., Instrumente der Rechtsvergleichung in der Schweiz bei der Rechtssetzung und bei der Rechtsanwendung, ZVglRWiss 2009, 31 (zit. Rechtsvergleichung)

Kunz Peter V., Wirtschaftsrecht: Grundlagen und Beobachtungen, 2019 (zit. Wirtschaftsrecht)

LANGER LORENZ, Staatsvertragsreferendum und Bilaterale Verträge, in: Glaser/Langer (Hrsg.), Die Verfassungsdynamik der europäischen Integration und demokratische Partizipation, 2015, 21

LAVENEX SANDRA/SCHwok RENÉ, The Swiss way: The nature of Switzerland's relationship with the EU, in: Eriksen/Fossum (eds.), The European Union's non-members: independence under hegemony?, 2015, 36

MADER LUZIUS/Kropf CATHERINE, Verweisungen auf das Recht der Europäischen Union in der Bundesgesetzgebung: Vom Fotografieren und Filmen, in: Institut für Europarecht (Hrsg.), Die Schweiz und die europäische Integration, 2015, 69

MAIANI FRANCESCO, Legal Europeanization as Legal Transformation: Some Insights from Swiss «Outer Europe», in: Maiani et al. (eds.), European Integration Without EU Membership: Models, Experiences, Perspectives, EUI Working Paper MWP 2009/2010, 111 (zit. Europeanization)

MAIANI FRANCESCO, La «saga Metock», ou des inconvénients du pragmatisme helvétique dans la question des rapports entre droit européen, droit bilatéral et droit interne, ZSR 2011, 27

MAIANI FRANCESCO, Lost in translation: euro compatibility, legal security, and the autonomous implementation of EU law in Switzerland, ELRev 2013, 29

MARTENET Vincent, Pour un Pacte européen, 2017

MARTENET VinCENT/Boillet VÉRONIQUe, L'égalité dans les relations entre particuliers et l'Accord sur la libre ciruclation des personnes, SJER 2007/2008, 311

MARTI SimON, Schweizer Europapolitik am Wendepunkt: Interessen, Konzepte und Entscheidungsprozesse in den Verhandlungen über den Europäischen Wirtschaftsraum, 2013

MAURHOFER ROLAND, Die schweizerische Europapolitik vom Marshallplan zur EFTA 1947 bis 1960: Zwischen Kooperation und Integration, 2001

MaYer Franz C./Wendel MatTias, $§ 4$ Die verfassungsrechtlichen Grundlagen des Europarechts, in: Hatje/Müller-Graff (Hrsg.), EnzEur Bd. 1, 2014 
MaYoraz JEAN-François, Staatliche Beihilfen in den Rechtsbeziehungen der Schweiz und der Europäischen Union, unter besonderer Berücksichtigung von ausgewählten Aspekten aus der schweizerischen Elektrizitätswirtschaft, 2018

MAZILLE ClémENTINE, L'institutionnalisation de la relation entre l'Union européenne et la Suisse: recherche sur une construction européenne, 2018

MERKT BENOÎT, Harmonisation internationale et entraide administrative internationale en droit de la concurrence, 2000

MuHEIM FRANZ, Der dornenvolle Weg der Schweiz nach Europa, 1999

MÜLler FeliX E., Kleine Geschichte des Rahmenabkommens, 2020

MÜLLER GEORG/UhLmann FElix, Elemente einer Rechtsetzungslehre, 3. Aufl., 2013

Muser MARCo/TOBler CHRISTA, Schiedsgerichte in den Aussenverträgen der EU: Neue Entwicklungen unter Einbezug der institutionellen Verhandlungen Schweiz-EU, Jusletter 28. Mai 2018

MUSLIU NAGIHAN, Umsetzung eidgenössischer Volksinitiativen, 2019

NAEF TOBIAS, Popular Initiatives Contrary to International Law - A Swiss Dilemma, in: Kellerhals/ Baumgartner (Hrsg.), Rule of Law in Europe - Current Challenges, 2017, 241

NAJY Cenni Michelangelo, The EU in the negotiations of the Bilateral Agreements I with Switzerland (1992-99): Assessing the role of the Commission and the Council, 2019

NeIER Christina, Der Kernbestandsschutz der Unionsbürgerschaft, 2019

NEUMANN Simon, Die Europäische Grenzschutzagentur Frontex: Integrierter Aussengrenzschutz und humanitäre Standards, 2014

NOTTER MARKUS, Institutionelles Rahmenabkommen - ein europapolitischer Zwischenruf, EuZ 2019, 4

NYFFELER FRANZ, Die Anwendung autonom nachvollzogener Normen des EU-Rechts, in: Aargauischer Anwaltsverband (Hrsg.), Festschrift 100 Jahre Aargauischer Anwaltsverband, 2005, 35

Oesch MatTHiAS, Regionale Integration Schweiz - Europäische Union und die Welthandelsorganisation (WTO), in: Breuss/Cottier/Müller-Graff (Hrsg.), Die Schweiz im Integrationsprozess, 2008, 207 (zit. Integration)

OESCH MatthiAS, Die einseitige Einführung des Cassis-de-Dijon-Prinzips, Anwaltsrevue 2009, 519

Oesch Matthias, Brüssel und Luxemburg bald wichtiger als Bern und Lausanne, Plädoyer 5/ 2011, 33

OESCH MATTHIAS, Die Europäisierung des schweizerischen Rechts, ZSR Beiheft 50, 2012, 13

OESCh MATTHIAS, Staatliche Subventionen und auswärtige Wirtschaftsteilnehmer, ZSR 2012, 255 (zit. Subventionen)

Oesch MatTHiAS, Grundrechte als Elemente der Wertegemeinschaft Schweiz-EU: Zur Auslegung der bilateralen Verträge, ZBl 2014, 171

OEsch MatTHias, Wirtschaft, in: Tschudi et al. (Hrsg.), Die Grenzüberschreitende Zusammenarbeit der Schweiz, 2014, 307 (zit. Wirtschaft)

Oesch MATTHiAS, Aussenwirtschaftsrecht: Grundlagen, in: Biaggini et al. (Hrsg.), Fachhandbuch Verwaltungsrecht, 2015, 1261 (zit. Aussenwirtschaft)

OESCH MatTHIAS, Ein Europa-Artikel für die schweizerische Bundesverfassung, in: Glaser/Langer (Hrsg.), Die Verfassungsdynamik der europäischen Integration und demokratische Partizipation, 2015, 163 (zit. Europa-Artikel)

Oesch MatTHIAS, Der Einfluss des EU-Rechts auf die Schweiz - von Gerichtsdolmetschern, Gerichtsgutachtern und Notaren, SJZ 2016, 53 (zit. Einfluss) 
OESCH MATTHIAS, Urteilsbesprechung: Bundesgericht (II. öffentlich-rechtliche Abteilung), Urteil vom 26. November 2015, 2C_716/2014, ZBl 2016, 208 (zit. Urteilsbesprechung)

OEsch MatThIAS, Die bilateralen Abkommen Schweiz-EU und die Übernahme von EU-Recht, AJP 2017, 638

Oesch Matthias, The Swiss Model of European Integration, in: Biondi et al. (eds.), Brexit: The Legal Implications, 2018, 35

OESCh MatThias, Das geplante institutionelle Abkommen Schweiz-EU und der Rechtsschutz für Private, in: Notter et al. (Hrsg.), Europäische Idee und Integration - mittendrin und nicht dabei? Liber amicorum für Andreas Kellerhals, 2018, 57

OesCh Matthias, Switzerland and the European Union: General Framework, Bilateral Agreements, Autonomous Adaptation, 2018 (zit. Switzerland)

OEsch MATTHIAS, Europarecht, Band I: Grundlagen, Institutionen, Verhältnis Schweiz-EU, 2. Aufl., 2019 (zit. Europarecht)

OEsCh MATTHIAS, Switzerland-EU Bilateral Agreements, The Incorporation of EU Law and the Continuous Erosion of Democratic Rights, Oxford Yearbook of European Law 2020, www.academic.oup.com/yel (zit. Incorporation)

OESCH MATTHiAS, Verfassungswandel durch Globalisierung und Europäisierung, in: Diggelmann/ Hertig Randall/Schindler (Hrsg.), Verfassungsrecht der Schweiz, Band I Grundlagen, 2020, 137 (zit. Verfassungswandel)

Oesch MatThiAS, Wird die handelspolitische Autonomie der EFTA-Staaten überschätzt?, EuR Behiheft 1, 2020, 329 (zit. Autonomie)

Oesch MatThias/BurgharTZ Nina, EU-Schweiz, in: Krenzler et al. (Hrsg.), EU-Aussenwirtschafts- und Zollrecht: Kommentar, 2018

Oesch Matthias/BurghartZ AliÉnor Nina/Manikulam VeEna, The Jurisprudence of WTO Dispute Resolution (2019), SRIEL 2020, 265

Oesch Matthias/Lang AnOuK, EU-Agenturen und die Schweiz, EuZ 2014, 132

Oesch Matthias/Naef Tobias, EU-Grundrechte, der EuGH und die Schweiz, ZSR 2017, 117

Oesch Matthias/Neier Christina, Die Komitologie im Unionsrecht und die Schweiz, in: Belser/ Waldmann (Hrsg.), Mehr oder weniger Staat? Festschrift für Peter Hänni zum 65. Geburtstag, 2015, 59

Oesch Matthias/Renfer Stefan, BGBM, in: Oesch/Weber/Zäch (Hrsg.), Wettbewerbsrecht II: Kommentar, 2. Aufl., erscheint 2021

Oesch Matthias/Speck Gabriel, Das geplante institutionelle Abkommen Schweiz-EU und der EuGH, SJER 2016/2017, 257

Oetiker Christian/Weibel Thomas, Protokoll 2, in: Oetiker/Weibel (Hrsg.), Lugano-Übereinkommen, Basler Kommentar, 2. Aufl., 2016

Oppermann Thomas/Classen Claus Dieter/NetTesheim Martin, Europarecht, 8. Aufl., 2018

Pahud de Mortanges René, Schweizerische Rechtsgeschichte: Ein Grundriss, 2. Aufl., 2017

PÄRLI KURT, Entsendegesetz (EntsG): Stämpflis Handkommentar, 2018 (zit. Entsendegesetz)

PÄRLI KURT, Eurolohn: Berufung auf das Diskriminierungsverbot ist rechtsmissbräuchlich. Kritische Bemerkungen zum Urteil des Bundesgerichts 4A_230/2018 vom 15. Januar 2019, Jusletter 20. Mai 2019 (zit. Eurolohn)

PETERSMANN ERNST-UlRICH/ZIEGLER ANDREAS R., Verfassungsgebung in der Europäischen Union: Lehren für die Nachführung der schweizerischen Bundesverfassung, in: Sitter-Liver (Hrsg.), Herausgeforderte Verfassung, Die Schweiz im globalen Kontext, 1999, 479

PFÄFFLI ROLAND/LIECHTI FABRIZIO ANDREA, Bemerkungen zu den rechtlichen Einschätzungen der eidgenössischen Wettbewerbskommission (WEKO) zur Freizügigkeit der Notare, Jusletter 16. Dezember 2013 
PFisterer Thomas, Die Kantone mit dem Bund in der EU-Zusammenarbeit, 2014

PIRKER BENEDIKT, Zu den für die Auslegung der Bilateralen Abkommen massgeblichen Grundsätzen - Gedanken zu BGE 140 II 112 (Gerichtsdolmetscher), ZBl 2015, 295 (zit. Auslegung)

PIRKER BENEDIKT, Zum Schiedsgericht im Institutionellen Abkommen, Jusletter 3. Juni 2019 (zit. Schiedsgericht)

PROBST ThOmAS, Die Rechte des Flugzeugpassagiers - oder - «Wenn einer im verspäteten Flugzeug sitzt, dann kann er was erleben...», in: Emmenegger et al. (Hrsg.), Brücken bauen, Festschrift für Thomas Koller, 2018, 763

Progin-Theuerkauf Sarah, Expulsion pénale et ALCP, ZSR 2020, 35

Progin-Theuerkauf SARAH/ZufFerey VinCENT, Umsiedelung von Asylsuchenden innerhalb der EU - Eine unendliche Geschichte?, EuZ 2020, 92

REICH JoHANNES, Direkte Demokratie und völkerrechtliche Verpflichtungen im Konflikt, ZaöRV 2008, 979 (zit. Demokratie)

Reich Johannes, Switzerland [The «Globalization Paradox» in Constitutional Practice], in: Albert et al. (eds.), 2018 Global Review of Constitutional Law, 2019, 298 (zit. Switzerland)

Rhinow RenÉ/Schefer Markus/Uebersax PETER, Schweizerisches Verfassungsrecht, 3. Aufl., 2016

RODRIK DANI, The Globalization Paradox, 2011

RuSCH ARNOLD F., Methoden und Ziele der Rechtsvergleichung, Jusletter 13. Februar 2006

RÜTSCHE BERNHARD, Rechtsvergleichung im öffentlichen Recht: Auslegungsmethode oder blosse Inspirationsquelle?, in: Schmid et al. (Hrsg.), Die Rechtsvergleichung in der Rechtsprechung: Praxis, Legitimität und Methodik, 2014, 121

SAURER JOHANNES, Die rechtliche Beziehung der Schweiz zu den Europäischen Agenturen, ZBl 2013, 415

SCHLEGEl StePHAN, in: Wohlers/Godenzi/Schlegel (Hrsg.), Schweizerisches Strafgesetzbuch: Handkommentar, 4. Aufl., 2020

SCHMidT-ASSMANN EBERHARD, Einleitung, in: Schmidt-Assmann/Schöndorf-Haubold (Hrsg.), Der Europäische Verwaltungsverbund, 2005, 1

SCHNELl CHRISTINA, Arbeitnehmerfreizügigkeit in der Schweiz: Ausgewählte rechtliche Aspekte zum Personenfreizügigkeitsabkommen, 2010

Schotт MARкus, Die Bedeutung der direkten Demokratie im Verhältnis Schweiz-EU, in: Rüssli/ Hänni/Häggi Furrer (Hrsg.), Staats- und Verwaltungsrecht auf vier Ebenen, Festschrift für Tobias Jaag, 2012, 693

SCHUHMACHER FloRian, Art. 101, in: Grabitz/Hilf/Nettesheim (Hrsg.), Das Recht der Europäischen Union: Kommentar, EL Mai 2018

SChweIZer MaX/UrSPRUnG Dominique (Hrsg.), Integration am Ende? Die Schweiz im Diskurs über ihre Europapolitik: Ein Lesebuch, 2015

SCHWEIZER RAINER J., Wie das europäische Recht die schweizerische Rechtsordnung fundamental beeinflusst und wie die Schweiz darauf keine systematische Antwort findet, in: Epiney/ Rivière (Hrsg.), Auslegung und Anwendung von «Integrationsverträgen», 2006, 23 (zit. Antwort)

SCHWEIZER RAINER J., Datenschutzrechte der betroffenen Personen in Schengen- und DublinVerfahren, Jusletter 3. September 2018 (zit. Datenschutzrechte)

SchwoK RenÉ, Suisse-Union européenne, l'adhésion impossible?, 3e éd., 2015

SCHWOK RENÉ, Switzerland-EU Relations: The Bilateral Way in a Fragilized Position, EFAR 2020, 159

SEeHASE Juliane, Die Grenzschutzagentur Frontex: Chance oder Bedrohung für den Europäischen Flüchtlingsschutz, 2013 
SEILER HANSJÖRG, Einfluss des europäischen Rechts und der europäischen Rechtsprechung auf die schweizerische Rechtspflege, ZBJV 2014, 265

SEILER HANSJÖRG, Dynamik oder Statik in der Rechtsprechung des Bundesgerichts zum EURecht?, in: Glaser/Langer (Hrsg.), Die Verfassungsdynamik der europäischen Integration und demokratische Partizipation, 2015, 77

SEITz Claudia/Berne AndRÉ S., Der Entwurf des institutionellen Abkommens Schweiz-EU: Eine erste Analyse der beihilferechtlichen Bestimmungen, EuZW 2019, 594

SETHE RolF, MiFIR/MiFID II - Drittstaatenzugang für die Schweiz und Äquivalenz von FINIG/ FIDLEG, in: Gericke (Hrsg.), Private Equity VI, 2018, 135

SOLLBERGER KASPAR, Konvergenzen und Divergenzen im Landverkehrsrecht der Europäischen Gemeinschaft und der Schweiz: Unter besonderer Berücksichtigung des bilateralen Landverkehrsabkommens, 2003

SOLLBERGER KASPAR/EPINEY ASTRID, Verkehrspolitische Gestaltungsspielräume der Schweiz auf der Grundlage des Landverkehrsabkommens, 2001

SPINNER BRUNO/MARITZ DANIEL, EG-Kompatibilität des schweizerischen Wirtschaftsrechts: Vom autonomen zum systematischen Nachvollzug, in: Forstmoser et al. (Hrsg.), Der Einfluss des europäischen Rechts auf die Schweiz. Festschrift für Professor Roger Zäch zum 60. Geburtstag, 1999, 127

StadelHofer Julie-Antoinette/Zirlick BeAt, THG, in: Oesch/Weber/Zäch (Hrsg.), Wettbewerbsrecht II: Kommentar, 2. Aufl., erscheint 2021

STERN JOACHIM/TOHIDIPUR TIMO, § 14 Migration von Drittstaatsangehörigen, in: von Arnauld (Hrsg.), EnzEur Bd. 10, 2014

STURNY MONIQUE, Der Einfluss des EU-Rechts auf das schweizerische Kartellrecht: Historische Entwicklung und Analyse, 2014

SUNDE MARTINA, Freizügigkeitsabkommen und Steuerrecht: Auslegung im Spannungsfeld von nationalem Recht, Unionsrecht und Völkerrecht, 2018

THÜRER DANIEL, Europaverträglichkeit als Rechtsargument, in: Haller et al. (Hrsg.), Im Dienst an der Gesellschaft. Festschrift für Dietrich Schindler zum 65. Geburtstag, 1989, 561 (zit. Europaverträglichkeit)

THÜRER DANIEL, Verfassungsfragen rund um einen möglichen Beitritt der Schweiz zur Europäischen Union, SZIER 2005, 21 (zit. Verfassungsfragen)

THÜRER DANIEL, Gutachten über mögliche Formen der Umsetzung und Anwendung der Bilateralen Abkommen, 7. Juni 2011, www.eda.admin.ch/dea und Link zu Studien (zit. Gutachten)

THÜRER DANIEL, Europa und die Schweiz: Status quo und Potenziale einer Partnerschaft, SJZ 2012, 477

THÜRER DANIEL, Europa als Erfahrung und Experiment: Grundidee Gerechtigkeit, Bd. 3, 2015

THÜRER DANIEL et al. (Hrsg.), Bilaterale Verträge I \& II Schweiz - EU, 2007

ThÜRER DANiEl/Hillemanns CAROLIN, Allgemeine Prinzipien, in: Thürer et al. (Hrsg.), Bilaterale Verträge I \& II Schweiz - EU, 2007, 39

TIEFENTHAL JÜRG MARCEL, Flankierende Massnahmen zum Schutz des schweizerischen Arbeitsmarktes, 2008

TOBLER CHRISTA, Der Acquis der rechtlichen Verbindung der Schweiz zur EG und EU - Eine unsichere Grösse?, in: Breuss/Cottier/Müller-Graff (Hrsg.), Die Schweiz im Europäischen Integrationsprozess, 2008, 11

TOBLER Christa, Die EuGH-Entscheidung Grimme - Die Wiederkehr von Polydor und die Grenze des bilateralen Rechts, SJER 2009/2010, 369

TOBLER CHRISTA, Schiedsgerichte im bilateralen Recht?, SZIER 2012, 1 (zit. Schiedsgerichte) 
TOBLER CHRISTA, Zur Beteiligung der Schweiz an der Europäischen IT-Agentur, Jusletter 1. Oktober 2012 (zit. IT-Agentur)

TOBLER CHRISTA, Luftverkehrsrecht: Auslegung des Luftverkehrsabkommens EU-Schweiz - Keine Anwendbarkeit der Dienstleistungsfreiheit, Anmerkung zum EuGH Urteil Rs. C-547/10, EuZW 2013, 429 (zit. Luftverkehrsrecht)

TOBLER CHRISTA, Die Erneuerung des bilateralen Wegs: Eine wachsende Annäherung an den EWR in den zur Diskussion gestellten Modellen, Jusletter 3. Juni 2013

TOBLER CHRISTA, Bilaterales Wirtschaftsrecht Schweiz-EU: Uneinheitlich, ineffizient und irrelevant?, ZSR 2013, 3 (zit. Wirtschaftsrecht)

TOBler CHRISTA, One of Many Challenges After «Brexit»: The Institutional Framework of an Alternative Agreement - Lessons from Switzerland and Elsewhere?, MJ 2016, 575

TOBLER CHRISTA, Fluggastrechte nach bilateralem Recht bei grossen Verspätungen: Parallel zum EU-Recht, und wenn ja, inwieweit?, SJER 2016/2017, 505 (zit. Fluggastrechte)

TOBler CHRISTA, Homogenität im Rechtsbestand der Schengen- und Dublin-Abkommen: Übernimmt die Schweiz im Assoziationsrahmen nicht notifiziertes Asyl- und Datenschutzrecht der EU?, SRIEL 2017, 211 (zit. Homogenität)

TOBLER Christa, Personenfreizügigkeit mit und ohne Unionsbürgerrichtlinie, SJER 2017/2018, 433 (zit. Personenfreizügigkeit)

TOBLER CHRISTA, Wie weiter mit dem Institutionellen Abkommen? Varianten zum Umgang mit den drei heiklen Punkten, Jusletter 20. Januar 2020 (zit. Varianten)

TOBler CHrista/Beglinger JACQues, Grundzüge des bilateralen (Wirtschafts-) Rechts Schweiz-EU. Systematische Darstellung in Text und Tafeln, 2 Bände, 2013 (zit. Grundzüge)

TOBler Christa/Beglinger JACQues, Brevier zum institutionellen Abkommen Schweiz-EU, Ausgabe 2020-02.1, www.brevier.eur-charts.eu (zit. Brevier)

VAHL MARIUS/GROLIMUND NinA, Integration ohne Mitgliedschaft: die bilateralen Verträge der Schweiz mit der Europäischen Gemeinschaft, 2007

VON BÜREN ROLAND, Auswirkungen des Luftverkehrsabkommens auf das Wettbewerbsrecht, in: Cottier/Oesch (Hrsg.), Die sektoriellen Abkommen Schweiz-EG, 2002, 71

WALTER HANS PETER, Das rechtsvergleichende Element - Zur Auslegung vereinheitlichten, harmonisierten und rezipierten Rechts, ZSR 2007, 259

WIEGAND WOLFGANG, Zur Anwendung von autonom nachvollzogenem EU-Privatrecht, in: Forstmoser et al. (Hrsg.), Der Einfluss des europäischen Rechts auf die Schweiz. Festschrift für Professor Roger Zäch zum 60. Geburtstag, 1999, 172

WÜGER DANIEL, Anwendbarkeit und Justiziabilität völkerrechtlicher Normen im schweizerischen Recht: Grundlagen, Methoden und Kriterien, 2005 (zit. Anwendbarkeit)

WÜGER DANIEL, Bundesgericht wendet Freihandelsabkommen erstmals unmittelbar an - ein Schritt vorwärts, ein Schritt zurück, Jusletter 4. April 2005 (zit. Freihandelsabkommen)

WÜGER DANIEL/SCARPELLI SAMUELE, Die vernachlässigten institutionellen Aspekte der Bilateralen Verträge und die Aushandlung eines Rahmenabkommens, SJER 2005/2006, 287

WydER Rudolf, Die Schweiz und der Europarat 1949-1971: Annäherung und zehn Jahre Mitarbeit in der Parlamentarischen Versammlung, 1984

WYSS MARTIN P., Europakompatibilität und Gesetzgebungsverfahren im Bund, AJP 2007, 717

ZÄCH ROGER, Cassis de Dijon - Meilenstein des schweizerischen Wirtschaftsrechts?, in: Rechtliche Rahmenbedingungen des Wirtschaftsstandortes Schweiz, hrsg. von der Rechtswissenschaftlichen Fakultät der Universität St. Gallen (HSG), 2007, 295

ZÄCH ROGER, Die Übernahme von EU-Recht - ein Vorschlag zur Verbesserung der institutionellen Mechanismen, SJZ 2011, 405 
Zäch Roger/Heizmann Roger, Die Anwendung des Cassis de Dijon-Prinzips durch die Schweiz im Verhältnis zum EWR, EuZW 2012, 876

ZBINDEN MARTIN, Der Assoziationsversuch der Schweiz mit der EWG 1961-1963: ein Lehrstück schweizerischer Europapolitik, 2006

ZIEGLER ANDREAS R., Die Schweiz und die Europäische Wirtschaftsintegration (EG und EFTA), in: Senti/Ziegler (Hrsg.), Die Schweiz und die internationalen Wirtschaftsorganisationen, 2005,1

ZiEgLER ANDREAS R., Die De-facto Mitgliedschaft der Schweiz in der EU: Binnen- und Aussenbeziehungen, ZEuS 2007, 247 (zit. De-facto-Mitgliedschaft)

ZIEGLER ANDREAS R., Internationales Wirtschaftsrecht: Eine Einführung (unter Einschluss des Aussenwirtschaftsrechts der Schweiz), 2. Aufl., 2017

Ziegler ANDREAS R./OdendAHL KeRSTIN, Bundesverfassung und Völkerrecht, in: Ehrenzeller et al. (Hrsg.), St. Galler BV-Kommentar, 3. Aufl., 2014

ZÜND ANDREAS, Grundrechtsverwirklichung ohne Verfassungsgerichtsbarkeit, AJP 2013, 1349

ZuRbrÜGG MatThias/HruschKa Constantin, Art. 66a, in: Niggli/Wiprächtiger (Hrsg.), Strafrecht I, Basler Kommentar, 4. Aufl., 2019

ZURKINDEN PHILIPP, Institutionelles Abkommen - Rechtsgutachten zuhanden der WAK-N; Fragen zu Staatsbeihilfen und zum Freihandelsabkommen CH-EU sowie zu weiteren Fragen,

21. Februar 2019, www.parlament.ch und Link zu Services 


\section{Materialien}

Diese Liste enthält Botschaften und weitere Berichte und Erläuterungen des Bundesrates und weiterer Behörden. Sie sind in chronologischer Reihenfolge aufgeführt. Bei der Referenzierung im Text wird das in Klammern gesetzte Kurzzitat verwendet.

Botschaft betreffend den Beitritt der Schweiz zu dem am 16. April 1948 in Paris unterzeichneten Abkommen über die europäische wirtschaftliche Zusammenarbeit vom 20. August 1948, BBl 1948 II 1177 (zit. Botschaft OEEC)

Botschaft des Bundesrates an die Bundesversammlung über die Beteiligung der Schweiz an der Europäischen Freihandels-Assoziation vom 5. Februar 1960, BBl 1960 I 841

Botschaft des Bundesrates an die Bundesversammlung über die Genehmigung der Abkommen zwischen der Schweiz und den Europäischen Gemeinschaften vom 16. August 1972, BBl 1972 II 653 (zit. Botschaft FHA)

Botschaft über die Volksinitiative «Gleiche Rechte für Mann und Frau» vom 14. November 1979, BBl 1980 I 69 (zit. Botschaft Gleiche Rechte für Mann und Frau)

Bericht des Bundesrates über die Stellung der Schweiz im europäischen Integrationsprozess vom 24. August 1988, BBl 1988 III 249 (zit. Integrationsbericht 1988)

Bericht des Bundesrates über einen Beitritt der Schweiz zur Europäischen Gemeinschaft vom 18. Mai 1992, BBl 1992 III 1185 (zit. Bericht EG-Beitritt)

Botschaft zur Genehmigung des Abkommens über den Europäischen Wirtschaftsraum vom 18. Mai 1992, BBl 1992 IV 1 (zit. Botschaft EWR)

Botschaft über das Folgeprogramm nach der Ablehnung des EWR-Abkommens vom 24. Februar 1993, BBl 1993 I 805 (zit. Botschaft Folgeprogramm 1993)

Botschaft zum Bundesgesetz über die Gleichstellung von Frau und Mann (Gleichstellungsgesetz) und zum Bundesbeschluss über die Genehmigung einer Änderung der Verordnung über die Zuweisung der Ämter an die Departemente und der Dienste an die Bundeskanzlei vom 24. Februar 1993, BBl 1993 I 1248 (zit. Botschaft Gleichstellungsgesetz)

Bericht über die Aussenpolitik der Schweiz in den 90er Jahren vom 29. November 1993, BBl 1994 I 153

Botschaft zu einem Bundesgesetz über den Binnenmarkt (Binnenmarktgesetz, BGBM) vom 23. November 1994, BBl 1995 I 1213 (zit. Botschaft BGBM)

Botschaft über eine Bundesverfassung vom 20. November 1996, BBl 1997 I 1 (zit. Botschaft Bundesverfassung)

Integrationsbericht 1999 vom 3. Februar 1999, BBl 1999 IV 3935 (zit. Integrationsbericht 1999)

Botschaft zur Genehmigung der sektoriellen Abkommen zwischen der Schweiz und der EG vom 23. Juni 1999, BBl 19996128 (zit. Botschaft Bilaterale I)

Aussenpolitischer Bericht 2000. Präsenz und Kooperation: Interessenwahrung in einer zusammenwachsenden Welt vom 15. November 2000, BBl 2001261

Bericht der Aussenpolitischen Kommission des Ständerates über die Optionen der schweizerischen Integrationspolitik vom 18. März 2002, BBl 20026326 (zit. Bericht Integrationspolitik Ständerat)

Botschaft zum Bundesgesetz über genetische Untersuchungen beim Menschen vom 11. September 2002, BBl 20027361 (zit. Botschaft genetische Untersuchungen) 
Botschaft zur Genehmigung der bilateralen Abkommen zwischen der Schweiz und der Europäischen Union, einschliesslich der Erlasse zur Umsetzung der Abkommen («Bilaterale II») vom 1. Oktober 2004, BBl 20045965 (zit. Botschaft Bilaterale II)

Botschaft über die Änderung des Binnenmarktgesetzes vom 24. November 2004, BBI 2005465 (zit. Botschaft revBGBM)

Botschaft zur Änderung des Patentgesetzes und zum Bundesbeschluss über die Genehmigung des Patentrechtsvertrags und der Ausführungsordnung vom 23. November 2005, BBl 20061 (zit. Botschaft Änderung Patentgesetz)

Botschaft zur Vereinheitlichung des Strafprozessrechts vom 21. Dezember 2005, BBl 20061085 (zit. Botschaft Strafprozessrecht)

Europabericht 2006 vom 28. Juni 2006, BBl 20066815 (zit. Europabericht 2006)

Bericht des Bundesrates zu den Auswirkungen verschiedener europapolitischer Instrumente auf den Föderalismus in der Schweiz vom 15. Juni 2007, BBl 20075907 (zit. Föderalismusbericht)

Botschaft zur formellen Bereinigung des Bundesrechts vom 22. August 2007, BBl 20076121 (zit. Botschaft Bereinigung Bundesrecht)

Botschaft zur Weiterführung des Freizügigkeitsabkommens sowie zu dessen Ausdehnung auf Bulgarien und Rumänien vom 14. März 2008, BBl 20082135 (zit. Botschaft Weiterführung FZA)

Botschaft über die Genehmigung und die Umsetzung des Notenaustauschs zwischen der Schweiz und der EU betreffend die Übernahme des Rahmenbeschlusses 2008/977/JI vom 27. November 2008 über den Schutz von Personendaten im Rahmen der polizeilichen und justiziellen Zusammenarbeit in Strafsachen vom 11. September 2009, BBl 20096749 (zit. Botschaft Rahmenbeschluss Personendaten)

Bericht des Bundesrates über das Verhältnis von Völkerrecht und Landesrecht vom 5. März 2010, BBl 20102263 (zit. Bericht Völkerrecht-Landesrecht)

Bericht des Bundesrates über die Evaluation der schweizerischen Europapolitik (in Beantwortung des Postulats Markwalder [09.3560] «Europapolitik. Evaluation, Prioritäten, Sofortmassnahmen und nächste Integrationsschritte») vom 17. September 2010, BBl 20107239 (zit. Europabericht 2010)

Bericht des Bundesrates zum Verhältnis der Schweiz zu den europäischen Agenturen (in Erfüllung des Postulates David [08.3141]) vom September 2010, www.eda.admin.ch/dea und Link zu Berichte (zit. Agenturbericht)

Zusatzbericht des Bundesrates zu seinem Bericht vom 5. März 2010 über das Verhältnis von Völkerrecht und Landesrecht vom 30. März 2011, BBl 20113613 (zit. Zusatzbericht VölkerrechtLandesrecht)

Bericht des Bundesrates in Erfüllung des Postulats Hans Fehr 10.3857 vom 1. Oktober 2010: Konsequenzen des Schengen-Anpassungszwangs vom 7. Juni 2013, BBl 20136319 (zit. Bericht Schengen 2013)

Botschaft zur Änderung des Publikationsgesetzes (Primatwechsel von der gedruckten zur elektronischen Version der amtlichen Veröffentlichungen) vom 28. August 2013, BBl 20137057 (zit. Botschaft Änderung Publikationsgesetz)

Botschaft über die Genehmigung und die Umsetzung der Notenaustausche zwischen der Schweiz und der EU betreffend die Übernahme der Verordnungen (EU) Nr. 603/2013 und (EU) Nr. 604/2013 (Weiterentwicklungen des Dublin/Eurodac-Besitzstands) vom 7. März 2014, BBl 20142675 (zit. Botschaft Dublin 2014)

Botschaft zum Finanzmarktinfrastrukturgesetz (FinfraG) vom 3. September 2014, BBl 20147483 (zit. Botschaft FinfraG)

Bericht des Bundesrates in Beantwortung des Postulats Keller-Sutter (13.4022) «Freihandelsabkommen mit der EU statt bilaterale Abkommen» vom Juni 2015, www.eda.admin.ch/dea und Link zu Berichte (zit. Bericht Freihandelsabkommen statt bilaterale Abkommen) 
Botschaft zum Finanzdienstleistungsgesetz (FIDLEG) und zum Finanzinstitutsgesetz (FINIG) vom 4. November 2015, BBl 20158901 (zit. Botschaft FIDLEG und FINIG)

Botschaft zur Genehmigung des Notenaustauschs zwischen der Schweiz und der EU betreffend die Übernahme der Verordnung (EU) Nr. 515/2014 zur Schaffung des Fonds für die innere Sicherheit im Bereich Aussengrenzen und Visa (Weiterentwicklung des Schengen-Besitzstands) vom 3. Juni 2016, BBl 20165083 (zit. Botschaft Fonds für die innere Sicherheit)

Bericht zur Aussenwirtschaftspolitik 2016 und Botschaften zu Wirtschaftsvereinbarungen sowie Bericht über zolltarifarische Massnahmen im Jahr 2016 vom 11. Januar 2017, BBl 2017813 (zit. Bericht Aussenwirtschaftspolitik 2016)

Aussenpolitischer Bericht 2016 vom 11. Januar 2017, BBl 20171233 (zit. Aussenpolitischer Bericht 2016)

Botschaft zur Förderung der internationalen Mobilität in der Bildung in den Jahren 2018-2020 vom 26. April 2017, BBl 20173885 (zit. Botschaft Mobilität in der Bildung)

Botschaft zur Aufhebung der Ausfuhrbeiträge für landwirtschaftliche Verarbeitungsprodukte vom 17. Mai 2017, BBl 20174351 (zit. Botschaft Ausfuhrbeiträge)

Botschaft zum Bundesgesetz über die Totalrevision des Bundesgesetzes über den Datenschutz und die Änderung weiterer Erlasse zum Datenschutz vom 15. September 2017, BBl 20176941 (zit. Botschaft Totalrevision Datenschutzgesetz)

Bericht zur Aussenwirtschaftspolitik 2017 und Bericht über zolltarifarische Massnahmen im Jahr 2017 vom 10. Januar 2018, BBl 2018821 (zit. Bericht Aussenwirtschaftspolitik 2017)

Weisungen VEP: Weisungen und Erläuterungen zur Verordnung über die Einführung des freien Personenverkehrs des Staatssekretariats für Migration (SEM) vom Juli 2018, www.sem.admin.ch und Link zu Publikationen \& Service (zit. Weisungen VEP)

Botschaft zum zweiten Schweizer Beitrag an ausgewählte EU-Mitgliedstaaten zur Verringerung der wirtschaftlichen und sozialen Ungleichheiten in der erweiterten EU sowie zur Unterstützung von Massnahmen im Bereich der Migration vom 28. September 2018, BBl 2018 6665 (zit. Botschaft zweiter Schweizer Beitrag)

Erläuterungen des EDA zum Institutionellen Abkommen Schweiz-EU, 16. Januar 2019 , www.eda.admin.ch/dea und Link zu Verhandlungen (zit. Erläuterungen des EDA)

Botschaft zur Volksinitiative «Für eine massvolle Zuwanderung (Begrenzungsinitiative)» vom 7. Juni 2019, BBl 20195027 (zit. Botschaft Begrenzungsinitiative)

Aussenpolitischer Bericht 2019 vom 29. Januar 2020, BBl 20201565 (zit. Aussenpolitischer Bericht 2019)

Botschaft zur Finanzierung der Schweizer Beteiligung an den Massnahmen der Europäischen Union im Bereich Forschung und Innovation in den Jahren 2021-2027 (Horizon-Paket 2021-2027) vom 20. Mai 2020, BBl 20204845 (zit. Botschaft Horizon Paket)

Staatssekretariat für Wirtschaft (SECO), FlaM Bericht 2019: Umsetzung der flankierenden Massnahmen zum freien Personenverkehr Schweiz-Europäische Union, 11. Juni 2020, www.seco.admin.ch und Link zu Publikationen \& Dienstleistungen (zit. FlaM Bericht 2019)

Botschaft zur Übernahme und Umsetzung der Verordnung (EU) 2019/1896 über die Europäische Grenz- und Küstenwache und zur Aufhebung der Verordnungen (EU) Nr. 1052/2013 und (EU) 2016/1624 (Weiterentwicklung des Schengen-Besitzstands) und zu einer Änderung des Asylgesetzes, noch nicht im BBl veröffentlicht (Pressemitteilung des Bundesrates vom 26. August 2020, Bundesrat verabschiedet die Botschaft zur erweiterten Europäischen Grenz- und Küstenwache; zit. Botschaft Frontex) 
Der Rat der EU veröffentlicht periodisch Schlussfolgerungen zu den Beziehungen der EU zur Schweiz. Sie sind auf www.consilium.europa.eu/de einsehbar.

Schlussfolgerungen des Rates zu den Beziehungen zwischen der EU und den EFTA-Ländern vom 8. Dezember 2008

Schlussfolgerungen des Rates zu den Beziehungen zwischen der EU und den EFTA-Ländern vom 14. Dezember 2010

Schlussfolgerungen des Rates zu den Beziehungen zwischen der EU und den EFTA-Ländern vom 20. Dezember 2012

Schlussfolgerungen des Rates zu einem homogenen erweiterten Binnenmarkt und den Beziehungen der EU zu nicht der EU angehörenden westeuropäischen Ländern vom 16. Dezember 2014

Schlussfolgerungen des Rates zu den Beziehungen der EU zur Schweizerischen Eidgenossenschaft vom 28. Februar 2017

Schlussfolgerungen des Rates zu den Beziehungen der EU zur Schweizerischen Eidgenossenschaft vom 19. Februar 2019 


\section{Berichte und weitere Informationen}

Das SJER und die SZIER/SRIEL informieren einmal pro Jahr umfassend über die Entwicklungen in den bilateralen Beziehungen Schweiz-EU. Die EuZ berichtet regelmässig über Initiativen, Gesetzgebungsprojekte und Judikatur in der EU und ihre Bedeutung für die Schweiz. Das EIZ verfasst viermal jährlich einen kostenlosen Newsletter (www.eiz.uzh.ch). Das Plädoyer enthält in jeder Ausgabe die Rubrik «Luxemburg aktuell» mit Zusammenfassungen und Würdigungen von EuGH-Urteilen, welche auch für die Schweiz bedeutsam sind. 



\section{Abkürzungsverzeichnis}

$\mathrm{ABl}$.

Abs.

ACER

AETR

AEUV

AG

AgrarA

AHV

AIAA

AIG

AJP

Art.

AS

AsylG

Aufl.

BAZL

$\mathrm{BBl}$

BBV

betr.

BGBM

BGE

BGer

BGFA

BGG

BGMD
Amtsblatt der Europäischen Union

Absatz / Absätze

Agentur für die Zusammenarbeit der Energieregulierungsbehörden Accord Européen sur les Transports Routiers vom 31. März 1971

Vertrag über die Arbeitsweise der Europäischen Union

Aktiengesellschaft

Abkommen zwischen der Schweizerischen Eidgenossenschaft und der Europäischen Gemeinschaft über den Handel mit landwirtschaftlichen Erzeugnissen vom 21. Juni 1999 (Landwirtschaftsabkommen, SR 0.916.026.81)

Alters- und Hinterlassenenversicherung

Abkommen zwischen der Schweizerischen Eidgenossenschaft und der Europäischen Union über den automatischen Informationsaustausch über Finanzkonten zur Förderung der Steuerehrlichkeit bei internationalen Sachverhalten vom 26. Oktober 2004

(SR 0.641.926.81)

Bundesgesetz über die Ausländerinnen und Ausländer und über die Integration (Ausländer- und Integrationsgesetz) vom 16. Dezember 2005 (SR 142.20)

Allgemeine Juristische Praxis

Artikel

Amtliche Sammlung des schweizerischen Bundesrechts

Asylgesetz vom 26. Juni 1998 (SR 142.31)

Auflage

Bundesamt für Zivilluftfahrt

Bundesblatt

Verordnung über die Berufsbildung vom 19. November 2003 (Berufsbildungsverordnung, SR 412.101)

betreffend

Bundesgesetz über den Binnenmarkt vom 6. Oktober 1995 (Binnenmarktgesetz, SR 943.02)

Bundesgerichtsentscheid

Bundesgericht

Bundesgesetz über die Freizügigkeit der Anwältinnen und Anwälte vom 23. Juni 2000 (Anwaltsgesetz, SR 935.61)

Bundesgesetz über das Bundesgericht vom 17. Juni 2005 (Bundesgerichtsgesetz, SR 173.110)

Bundesgesetz über die Meldepflicht und die Nachprüfung der Berufsqualifikationen von Dienstleistungserbringerinnen und -erbringern in reglementierten Berufen vom 14. Dezember 2012 (SR 935.01) 
BGMK

BJ

BNE

BPI

Bsp.

Bst.

BSV

Bull.

BV

BVerfG

BVerfGE

BVerwG

BVGE

bzw.

Cedefop

CETA

cf.

$\mathrm{CHF}$

CML Rev

COVID-19

DAA

DEA

DEZA

d.h.

Doc.

DSG

E.

EACEA

EAD

EAG

EAGV

EAPC

EASA

EASO
Bundesgesetz über die Mitwirkung der Kantone an der Aussenpolitik des Bundes vom 22. Dezember 1999 (SR 138.1)

Bundesamt für Justiz

Bruttonationaleinkommen

Bundesgesetz über die polizeilichen Informationssysteme des Bundes vom 13. Juni 2008 (SR 361)

Beispiel

Bestimmung(en)

Verordnung über die Schifffahrt auf schweizerischen Gewässern vom 8. November 1978 (Binnenschifffahrtsverordnung, SR 747.201.1)

Bulletin

Bundesverfassung der Schweizerischen Eidgenossenschaft vom 18. April 1999 (SR 101)

deutsches Bundesverfassungsgericht

Entscheid des deutschen Bundesverfassungsgerichts

Bundesverwaltungsgericht

Entscheid des Bundesverwaltungsgerichts

beziehungsweise

Europäisches Zentrum für die Förderung der Berufsbildung

Umfassendes Wirtschafts- und Handelsabkommen (EU-Kanada)

confer (vgl.)

Schweizer Franken

Common Market Law Review

Coronavirus Disease 2019

Abkommen zwischen der Schweizerischen Eidgenossenschaft und der Europäischen Gemeinschaft über die Kriterien und Verfahren zur Bestimmung des zuständigen Staates für die Prüfung eines in einem Mitgliedstaat oder in der Schweiz gestellten Asylantrags vom 26. Oktober 2004 (Dublin-Assoziierungsabkommen, SR 0.142.392.68)

Direktion für Europäische Angelegenheiten

Direktion für Entwicklung und Zusammenarbeit

das heisst

Document

Bundesgesetz über den Datenschutz vom 18. Juni 1992 (Datenschutzgesetz, SR 235.1)

Erwägung(en)

Exekutivagentur Bildung, Audiovisuelles und Kultur

Europäischer Auswärtiger Dienst

Europäische Atomgemeinschaft

Vertrag zur Gründung der Europäischen Atomgemeinschaft

Euro-Atlantischer Partnerschaftsrat

Europäische Agentur für Flugsicherheit

Europäisches Unterstützungsbüro für Asylfragen 


\begin{tabular}{|c|c|}
\hline ECDC & $\begin{array}{l}\text { Europäisches Zentrum für die Prävention und die Kontrolle von } \\
\text { Krankheiten }\end{array}$ \\
\hline ECHA & Europäische Chemikalienagentur \\
\hline ECTS & European Credit Transfer and Accumulation System \\
\hline ed. & editor / edition \\
\hline éd. & éditeur / édition \\
\hline EDA & Eidgenössisches Departement für auswärtige Angelegenheiten \\
\hline EDI & Eidgenössisches Departement des Innern \\
\hline EDÖB & $\begin{array}{l}\text { Eidgenössische Datenschutz- und Öffentlichkeitsbeauftragte / Eid- } \\
\text { genössischer Datenschutz- und Öffentlichkeitsbeauftragter }\end{array}$ \\
\hline eds. & editors / editions \\
\hline éds. & éditeurs / éditions \\
\hline EEA & Einheitliche Europäische Akte \\
\hline EES & Einreise-/Ausreisesystem \\
\hline EFAR & European Foreign Affairs Review \\
\hline EFSA & Europäische Behörde für Lebensmittelsicherheit \\
\hline EFTA & Europäische Freihandelsassoziation \\
\hline EG & Europäische Gemeinschaft(en) \\
\hline EGKS & Europäische Gemeinschaft für Kohle und Stahl \\
\hline EGNOS & European Geostationary Navigation Overlay System \\
\hline EGV & Vertrag zur Gründung der Europäischen Gemeinschaft \\
\hline EHR & Europäischer Hochschulraum \\
\hline eidg. & eidgenössische/r/s \\
\hline Eionet & Europäisches Umweltinformations- und Umweltbeobachtungsnetz \\
\hline EIOPA & $\begin{array}{l}\text { Europäische Aufsichtsbehörde für das Versicherungswesen und die } \\
\text { betriebliche Altersversorgung }\end{array}$ \\
\hline EIZ & Europa Institut an der Universität Zürich \\
\hline EL & Ergänzungslieferung \\
\hline ELR & European Law Reporter \\
\hline ELRev & European Law Review \\
\hline EMIR & $\begin{array}{l}\text { Verordnung (EU) Nr. 648/2012 über OTC-Derivate, zentrale Gegen- } \\
\text { parteien und Transaktionsregister }\end{array}$ \\
\hline EMRK & $\begin{array}{l}\text { Konvention zum Schutze der Menschenrechte und Grundfreiheiten } \\
\text { vom 4. November } 1950 \text { (Europäische Menschenrechtskonvention, } \\
\text { SR 0.101) }\end{array}$ \\
\hline endg. & endgültig \\
\hline EntsG & $\begin{array}{l}\text { Bundesgesetz über die flankierenden Massnahmen bei entsandten } \\
\text { Arbeitnehmerinnen und Arbeitnehmern und über die Kontrolle der } \\
\text { in Normalarbeitsverträgen vorgesehenen Mindestlöhne vom } \\
\text { 8. Oktober } 1999 \text { (Entsendegesetz, SR 823.20) }\end{array}$ \\
\hline EnzEur & Enzyklopädie Europarecht \\
\hline $\mathrm{ERC}$ & European Research Council \\
\hline ESA & Europäische Weltraumorganisation \\
\hline
\end{tabular}




\begin{tabular}{|c|c|}
\hline ESMA & Europäische Wertpapier- und Marktaufsichtsbehörde \\
\hline ESP & Europäisches Statistisches Programm \\
\hline ESS & Europäisches Statistisches System \\
\hline et al. & und andere \\
\hline ETIAS & Europäisches Reiseinformations- und -genehmigungssystem \\
\hline $\mathrm{EU}$ & Europäische Union \\
\hline EUA & Europäische Umweltagentur \\
\hline EU-EHS & EU-Emissionshandelssystem \\
\hline $\mathrm{EuG}$ & Gericht (erster Instanz) \\
\hline EuGH & Gerichtshof der EU \\
\hline eu-LISA & $\begin{array}{l}\text { Agentur der EU für das Betriebsmanagement von IT-Grosssystemen } \\
\text { im Raum der Freiheit, der Sicherheit und des Rechts }\end{array}$ \\
\hline EuR & Europarecht (Zeitschrift) \\
\hline EUR & Euro \\
\hline Euratom & Europäische Atomgemeinschaft \\
\hline Eurojust & Europäische Einheit für justizielle Zusammenarbeit \\
\hline EUROSUR & Europäisches Grenzüberwachungssystem \\
\hline EUStA & Europäische Staatsanwaltschaft \\
\hline EUV & Vertrag über die Europäische Union \\
\hline EuZ & Zeitschrift für Europarecht \\
\hline EuZW & Europäische Zeitschrift für Wirtschaftsrecht \\
\hline EVA & Europäische Verteidigungsagentur \\
\hline EWG & Europäische Wirtschaftsgemeinschaft \\
\hline EWGV & Vertrag zur Gründung der Europäischen Wirtschaftsgemeinschaft \\
\hline EWR & Europäischer Wirtschaftsraum \\
\hline EWRA & Abkommen über den Europäischen Wirtschaftsraum \\
\hline EWRS & Frühwarn- und Reaktionssystem \\
\hline $\mathrm{EZB}$ & Europäische Zentralbank \\
\hline FHA & $\begin{array}{l}\text { Freihandelsabkommen / Abkommen zwischen der Schweizerischen } \\
\text { Eidgenossenschaft und der Europäischen Wirtschaftsgemeinschaft } \\
\text { vom 22. Juli } 1972 \text { (SR 0.632.401) }\end{array}$ \\
\hline FIDLEG & $\begin{array}{l}\text { Bundesgesetz über die Finanzdienstleistungen vom 15. Juni } 2018 \\
\text { (Finanzdienstleistungsgesetz, SR 950.1) }\end{array}$ \\
\hline FinfraG & $\begin{array}{l}\text { Bundesgesetz über die Finanzmarktinfrastrukturen und das Markt- } \\
\text { verhalten im Effekten- und Derivatehandel vom 19. Juni } 2015 \\
\text { (Finanzmarktinfrastrukturgesetz, SR 958.1) }\end{array}$ \\
\hline FINIG & $\begin{array}{l}\text { Bundesgesetz über die Finanzinstitute vom 15. Juni } 2018 \text { (Finanzinsti- } \\
\text { tutsgesetz, SR 954.1) }\end{array}$ \\
\hline FINMA & Eidgenössische Finanzmarktaufsicht \\
\hline FlaM & flankierende Massnahmen \\
\hline FMG & Fernmeldegesetz vom 30. April 1997 (SR 784.10) \\
\hline ForschA & $\begin{array}{l}\text { Abkommen über die wissenschaftliche und technologische Zusam- } \\
\text { menarbeit (Forschungsabkommen, AS } 2002 \text { 1998) }\end{array}$ \\
\hline
\end{tabular}


Frontex

FusG

FZA

GASP

GATS

GATT 1947/1994

GEAS

GGA

GlG

GLONASS

GNSS

GPA

GPS

GRC

GRG

GSVP

GTG

GTR

GUB

HABM

HMG

Hrsg.

HS

HSG

ibid.

ICN

i.d.F.

inkl.

InstA
Europäische Agentur für die Grenz- und Küstenwache

Bundesgesetz über Fusion, Spaltung, Umwandlung und Vermögensübertragung vom 3. Oktober 2003 (Fusionsgesetz, SR 221.301)

Abkommen zwischen der Schweizerischen Eidgenossenschaft einerseits und der Europäischen Gemeinschaft und ihren Mitgliedstaaten andererseits über die Freizügigkeit vom 21. Juni 1999 (Freizügigkeitsabkommen, SR 0.142.112.681)

Gemeinsame Aussen- und Sicherheitspolitik

Allgemeines Abkommen über den Dienstleistungshandel vom 15. April 1994 (SR 0.632.20)

Allgemeines Zoll- und Handelsabkommen vom 30. Oktober 1947 / 15. April 1994 (SR 0.632.20)

Gemeinsames Europäisches Asylsystem

geografische Angaben

Bundesgesetz über die Gleichstellung von Frau und Mann vom 24. März 1995 (Gleichstellungsgesetz, SR 151.1)

Global Navigation Satellite System

European Global Navigation Satellite System

Übereinkommen über das öffentliche Beschaffungswesen vom 15. April 1994 (SR 0.632.231.422)

Global Positioning System

Charta der Grundrechte

Grossratsgesetz des Kantons Freiburg vom 6. September 2006 (SGF 121.1)

Gemeinsame Sicherheits- und Verteidigungspolitik

Bundesgesetz über die Gentechnik im Ausserhumanbereich vom 21. März 2003 (Gentechnikgesetz, SR 814.91)

Gesetzestechnische Richtlinien des Bundes

geschützte Ursprungsbezeichnungen

Harmonisierungsamt für den Binnenmarkt

Bundesgesetz über Arzneimittel und Medizinprodukte vom 15. Dezember 2000 (SR 812.21)

Herausgeberin / Herausgeber

Internationales Übereinkommen über das Harmonisierte System zur Bezeichnung und Codierung der Waren vom 14. Juni 1983

(SR 0.632.11)

Hochschule (Universität) St. Gallen

ibidem (daselbst, am gleichen Ort, an gleicher Stelle)

International Competition Network

in der Fassung

inklusive

Abkommen zur Erleichterung der bilateralen Beziehungen zwischen der Europäischen Union und der Schweizerischen Eidgenossenschaft in den Bereichen des Binnenmarkts, an denen die Schweiz teilnimmt (Institutionelles Abkommen, Entwurf) 
i.S.

ISF

i.V.m.

KAG

KBBK

KG

$\mathrm{km}$

KonfBA

Kt.

KVG

LandVA

LFG

LGV

lit.

LMG

LSVA

LugÜ

LVA

MessG

Mia.

MiFID II

MiFIR

Mio.

MJ

MRA

MSchG in Sachen

Fonds für die innere Sicherheit

in Verbindung mit

Bundesgesetz über die kollektiven Kapitalanlagen vom 23. Juni 2006 (Kollektivanlagengesetz, SR 951.31)

Kommission für das Beschaffungswesen Bund-Kantone

Bundesgesetz über Kartelle und andere Wettbewerbsbeschränkungen vom 6. Oktober 1995 (Kartellgesetz, SR 251)

Kilometer

Abkommen zwischen der Schweizerischen Eidgenossenschaft und der Europäischen Gemeinschaft über die gegenseitige Anerkennung von Konformitätsbewertungen vom 21. Juni 1999 (Abkommen über technische Handelshemmnisse, MRA, SR 0.946.526.81)

\section{Kanton}

Bundesgesetz über die Krankenversicherung vom 18. März 1994 (SR 832.10)

Abkommen zwischen der Schweizerischen Eidgenossenschaft und der Europäischen Gemeinschaft über den Güter- und Personenverkehr auf Schiene und Strasse (Landverkehrsabkommen, SR 0.740.72)

Bundesgesetz über die Luftfahrt vom 21. Dezember 1948 (Luftfahrtgesetz, SR 748.0)

Lebensmittel- und Gebrauchsgegenständeverordnung vom 16. Dezember 2016 (SR 817.02)

litera(e)

Bundesgesetz über Lebensmittel und Gebrauchsgegenstände vom 20. Juni 2014 (Lebensmittelgesetz, SR 817.0)

leistungsabhängige Schwerverkehrsabgabe

Übereinkommen über die gerichtliche Zuständigkeit und die Anerkennung und Vollstreckung von Entscheidungen in Zivil- und Handelssachen vom 30. Oktober 2007 (Lugano-Übereinkommen, SR 0.275.12)

Abkommen zwischen der Schweizerischen Eidgenossenschaft und der Europäischen Gemeinschaft über den Luftverkehr vom 21. Juni 1999 (Luftverkehrsabkommen, SR 0.748.127.192.68)

Bundesgesetz über das Messwesen vom 17. Juni 2011 (Messgesetz, SR 941.20)

Milliarde(n)

Richtlinie 2014/65/EU über Märkte für Finanzinstrumente

Verordnung (EU) Nr. 600/2014 über Märkte für Finanzinstrumente

Million(en)

Maastricht Journal of European and Comparative Law

Abkommen über die gegenseitige Anerkennung von Konformitätsbewertungen (Abkommen über technische Handelshemmnisse, Mutual Recognition Agreement, KonfBA, SR 0.946.526.81)

Bundesgesetz über den Schutz von Marken und Herkunftsangaben vom 28. August 1992 (Markenschutzgesetz, SR 232.11) 
m.w.H.

\section{MWSTG}

N.

NATO

NBG

NEAT

NR

$\mathrm{Nr}$.

N-SIS-Verordnung

NZZ

OECD

OEEC

ÖffBA

OLAF

OR

ParlG

PASEI

PatG

PEM-Übereinkommen

PESCO

PfP

PJZS

PKK

PNR

PrHG

PrSG

Rn.

RPW

Rs.

Rz.

S. mit weiteren Hinweisen

Bundesgesetz über die Mehrwertsteuer vom 12. Juni 2009 (Mehrwertsteuergesetz, SR 641.20)

Note(n)

Organisation des Nordatlantikvertrags / Nordatlantikpakt-Organisation

Bundesgesetz über die Schweizerische Nationalbank vom 3. Oktober 2003 (SR 951.11)

Neue Eisenbahn-Alpentransversale

Nationalrätin / Nationalrat

Nummer

Verordnung über den nationalen Teil des Schengener Informationssystems (N-SIS) und das SIRENE-Büro vom 8. März 2013 (SR 362.0)

Neue Zürcher Zeitung

Organisation für wirtschaftliche Zusammenarbeit und Entwicklung

Organisation für europäische wirtschaftliche Zusammenarbeit

Abkommen zwischen der Schweizerischen Eidgenossenschaft und der Europäischen Gemeinschaft über bestimmte Aspekte des öffentlichen Beschaffungswesens vom 21. Juni 1999 (SR 0.172.052.68)

Europäisches Amt für Betrugsbekämpfung

Bundesgesetz betreffend die Ergänzung des Schweizerischen Zivilgesetzbuches vom 30. März 1911 (Fünfter Teil: Obligationenrecht, SR 220)

Bundesgesetz über die Bundesversammlung vom 13. Dezember 2002 (Parlamentsgesetz, SR 171.10)

Protection of Autonomous Systems against Enemy Interference

Bundesgesetz über die Erfindungspatente vom 25. Juni 1954 (Patentgesetz, SR 232.14)

Regionales Übereinkommen über Pan-Europa-Mittelmeer-Präferenzursprungsregeln vom 15. Juni 2011 (SR 0.946.31)

Permanent Structured Cooperation

Partnerschaft für den Frieden

Polizeiliche und Justizielle Zusammenarbeit in Strafsachen

Arbeiterpartei Kurdistans

Passenger Name Records

Bundesgesetz über die Produktehaftpflicht vom 18. Juni 1993 (Produktehaftpflichtgesetz, SR 221.112.944)

Bundesgesetz über die Produktesicherheit vom 12. Juni 2009 (SR 930.11)

Randnummer(n)

Recht und Politik des Wettbewerbs

Rechtssache

Randziffer(n)

Seite(n) 
S.

SAA

SBFI

SDG

SDSG

SDÜ

SECO

SEM

SEMP

SES

SGF

SGK

sGS

SIR

SIRENE

SIS

SJER

SJZ

SNF

sog.

SPSR

SR

SRIEL

STAF

StGB

$\mathrm{StGH}$

SÜ

SZIER siehe

Abkommen zwischen der Schweizerischen Eidgenossenschaft, der Europäischen Union und der Europäischen Gemeinschaft über die Assoziierung dieses Staates bei der Umsetzung, Anwendung und Entwicklung des Schengen-Besitzstands vom 26. Oktober 2004 (Schengen-Assoziierungsabkommen, SR 0.362.31)

Staatssekretariat für Bildung, Forschung und Innovation

Sustainable Development Goals

Bundesgesetz über den Datenschutz im Rahmen der Anwendung des Schengen-Besitzstands in Strafsachen vom 28. September 2018 (Schengen-Datenschutzgesetz, SR 235.3)

Übereinkommen zur Durchführung des Übereinkommens von Schengen vom 14. Juni 1985 zwischen den Regierungen der Staaten der Benelux-Wirtschaftsunion, der Bundesrepublik Deutschland und der Französischen Republik betreffend den schrittweisen Abbau der Kontrollen an den gemeinsamen Grenzen vom 19. Juni 1990 (Schengener Durchführungsübereinkommen)

Staatssekretariat für Wirtschaft

Staatssekretariat für Migration

Swiss-European Mobility Programme

Single European Sky

Systematische Gesetzessammlung des Kantons Freiburg

Schengener Grenzkodex

systematische Gesetzessammlung des Kantons St. Gallen

Schweizerisches Institut für Rechtsvergleichung

Supplementary Information Request at the National Entry

Schengener Informationssystem

Schweizerisches Jahrbuch für Europarecht

Schweizerische Juristen-Zeitung

Schweizerischer Nationalfonds

sogenannt(e)

Swiss Political Science Review

Systematische Sammlung des schweizerischen Bundesrechts

Swiss Review of International and European Law

Bundesgesetz über die Steuerreform und die AHV-Finanzierung vom 28. September 2018 (noch nicht in der SR veröffentlicht)

Schweizerisches Strafgesetzbuch vom 21. Dezember 1937 (SR 311.0)

Staatsgerichtshof des Fürstentums Liechtenstein

Übereinkommen zwischen den Regierungen der Staaten der Benelux-Wirtschaftsunion, der Bundesrepublik Deutschland und der Französischen Republik betreffend den schrittweisen Abbau der Kontrollen an den gemeinsamen Grenzen vom 14. Juni 1985 (Schengen Übereinkommen)

Schweizerische Zeitschrift für Internationales und Europäisches Recht 
SZW

THG

TRIMs-Abkommen

TRIPs-Abkommen

TTIP

u.a.

UNEP

UNFCCC

UNO

URG

US

U.S.

USA

u.U.

v.a.

VEP

VersA

VGG

vgl.

VIPaV

VIS

VMD

VöB

VPB

VPiB

VVDStRL
Schweizerische Zeitschrift für Wirtschafts- und Finanzmarktrecht

Bundesgesetz über die technischen Handelshemmnisse vom 6. Oktober 1995 (SR 946.51)

Abkommen über handelsrelevante Investitionsmassnahmen vom 15. April 1994 (SR 0.632.20)

Abkommen über handelsbezogene Aspekte der Rechte an Geistigem Eigentum vom 15. April 1994 (SR 0.632.20)

Transatlantische Handels- und Investitionspartnerschaft (EU-US)

unter anderem

UN Environment Programme

United Nations Framework Convention on Climate Change

Vereinte Nationen

Bundesgesetz über das Urheberrecht und verwandte Schutzrechte vom 9. Oktober 1992 (Urheberrechtsgesetz, SR 231.1)

United States

United States

Vereinigte Staaten von Amerika

unter Umständen

vor allem

Verordnung über die schrittweise Einführung des freien Personenverkehrs zwischen der Schweizerischen Eidgenossenschaft und der Europäischen Union und deren Mitgliedstaaten sowie unter den Mitgliedstaaten der Europäischen Freihandelsassoziation vom 22. Mai 2002 (Verordnung über die Einführung des freien Personenverkehrs, SR 142.203)

Abkommen betreffend die Direktversicherung mit Ausnahme der Lebensversicherung (Versicherungsabkommen, SR 0.961.1)

Bundesgesetz über das Bundesverwaltungsgericht vom 17. Juni 2005 (Verwaltungsgerichtsgesetz, SR 173.32)

vergleiche

Verordnung über das Inverkehrbringen von nach ausländischen technischen Vorschriften hergestellten Produkten und über deren Überwachung auf dem Markt vom 19. Mai 2010 (Verordnung über das Inverkehrbringen von Produkten nach ausländischen Vorschriften, SR 946.513.8)

Visa-Informationssystem

Verordnung über die Meldepflicht und die Nachprüfung der Berufsqualifikationen von Dienstleistungserbringerinnen und -erbringern in reglementierten Berufen vom 26. Juni 2013 (SR 935.011)

Verordnung über das öffentliche Beschaffungswesen vom 11. Dezember 1995 (SR 172.056.11)

Verwaltungspraxis des Bundes

Verordnung der Bundesversammlung über die Pflege der internationalen Beziehungen des Parlamentes vom 28. September 2012 (SR 171.117)

Veröffentlichungen der Vereinigung der Deutschen Staatsrechtslehrer 
WEF

WEKO

WettbA

WEU

WTO

WVRK

z.B.

ZBJI

ZBJV

ZBl

ZEuS

ZGB

Ziff.

ZinsbestA

zit.

ZollA

ZRK

ZSR

ZVglRWiss

\section{World Economic Forum}

Wettbewerbskommission

Abkommen zwischen der Schweiz und der EU über die Zusammenarbeit bei der Anwendung ihres Wettbewerbsrechts von 2013 (Wettbewerbsabkommen, SR 0.251.268.1)

Westeuropäische Union

Welthandelsorganisation

Wiener Übereinkommen über das Recht der Verträge vom 23. Mai 1969 (Wiener Vertragsrechtskonvention, SR 0.111)

zum Beispiel

Zusammenarbeit in den Bereichen Justiz und Inneres

Zeitschrift des Berner Juristenvereins

Schweizerisches Zentralblatt für Staats- und Verwaltungsrecht

Zeitschrift für Europarechtliche Studien

Schweizerisches Zivilgesetzbuch vom 10. Dezember 1907 (SR 210)

Ziffer

Abkommen zwischen der Schweizerischen Eidgenossenschaft und der Europäischen Gemeinschaft über Regelungen, die den in der Richtlinie 2003/48/EG des Rates im Bereich der Besteuerung von Zinserträgen festgelegten Regelungen gleichwertig sind (Zinsbesteuerungsabkommen, AS 2005 2571)

zitiert

Abkommen zwischen der Schweizerischen Eidgenossenschaft und der Europäischen Gemeinschaft über die Erleichterung der Kontrollen und Formalitäten im Güterverkehr und über zollrechtliche Sicherheitsmassnahmen vom 25. Juni 2009 (Zollabkommen, SR 0.631.242.05)

Zollrekurskommission

Zeitschrift für Schweizerisches Recht

Zeitschrift für Vergleichende Rechtswissenschaft 
Erster Teil

Grundlagen 



\section{1. Übersicht}

Die Schweiz und die EU sind durch eine Vielzahl von bilateralen Abkommen unterschiedlicher Generationen miteinander verbunden. Dazu gehören rund zwanzig Hauptabkommen und über hundert weniger bekannte gegenseitige Abmachungen in der Form von Sekundärabkommen. Die Grundlage dieses dichten Vertragsnetzes bildet das Freihandelsabkommen von 1972. 1999 wurde das Paket der Bilateralen I abgeschlossen; dabei handelt es sich um sieben Abkommen, welche vornehmlich den Marktzugang und die Personenfreizügigkeit zum Gegenstand haben. 2004 wurde das Paket der Bilateralen II geschnürt; die davon betroffenen neun Abkommen gehen über den hauptsächlich wirtschaftlichen Rahmen der Bilateralen I hinaus und betreffen namentlich auch Justiz und Inneres, Visa und Asyl, Umwelt, Kultur und Bildung. Diese Abkommen sind als Antwort auf konkrete Bedürfnisse und im Rahmen von windows of opportunities entstanden und bilden kein umfassendes, kohärentes System. Gleichwohl bestehen zwischen den Abkommen Querbezüge und Abhängigkeiten. Zeitweise teilen einzelne Abkommen ein gemeinsames rechtliches Schicksal (Guillotine-Klausel). Zeitweise macht die EU die Anpassung eines Abkommens an neues EU-Recht oder den Abschluss neuer Abkommen von einem Entgegenkommen der Schweiz in anderen Dossiers abhängig.

Die EU blockierte im Nachgang zur Annahme der Volksinitiative «Gegen Masseneinwanderung» von 2014 die Übernahme neuer EU-Rechtsakte in das KonfBA, um den Druck auf die Schweiz zu erhöhen, eine mit dem FZA vereinbare Umsetzung der Initiative zu wählen. Aktuell ist die EU nicht bereit, neue Binnenmarktabkommen - wie das Stromabkommen abzuschliessen, solange die Schweiz dem InstA nicht zustimmt. Sie ist ebenso wenig bereit, bestehende Binnenmarktabkommen zu aktualisieren, sofern keine Fortschritte beim Abschluss des InstA erzielt werden (Aussenpolitischer Bericht 2019, S. 1582); dies betrifft etwa die Übernahme der Novellierung der Richtlinie zur Medizinaltechnik. Ähnliches gilt in Bezug auf die Assoziierung an Horizon Europe oder den Abschluss des Gesundheitsabkommens (Antwort des Bundesrates vom 13. Februar 2019 auf die Anfrage 18.1079 «Erpressungen durch die EU im Gesundheitsbereich?»; Aussenpolitischer Bericht 2019 S. 1583); auch hier verlangt die EU ein Entgegenkommen der Schweiz beim InstA, obwohl weder die Assoziierung an Horizon Europe noch das Gesundheitsabkommen (dessen Inhalt öffentlich allerdings nicht bekannt ist) typische Binnenmarktabkommen sind.

Die EU hat wiederholt bekräftigt, dass sie den baldigen Abschluss des Institutionellen Abkommens erwartet. Der seit Dezember 2018 vorliegende Entwurf eines solchen Abkommens bietet eine Gelegenheit, sich grundsätzlich mit der Gestaltung der Beziehungen zur EU auseinanderzusetzen. Der bilaterale Weg in seiner jetzigen Form steht auf dem Prüfstand. 
In Ergänzung zu den bilateralen Abkommen wird das Verhältnis der Schweiz zur EU und zu ihren Mitgliedstaaten durch eine Vielzahl weiterer staatsvertraglicher Regelwerke und internationaler Organisationen geprägt. Dazu gehören zuvörderst die UNO-Pakte I und II, die OECD, der Europarat, die EMRK und die weiteren Konventionen unter der Ägide des Europarates (Minderheitenschutz, Soziales, Bildung, Kultur, Privat- und Strafrecht), wobei die EU bei diesen Organisationen und Regelwerken nicht Vertragspartei ist, wohl aber ihre Mitgliedstaaten. Im Handelsrecht stellt das WTO-Recht auch für das bilaterale Verhältnis zwischen der Schweiz und der EU die Grundordnung dar; hier ist die EU parallel zu ihren Mitgliedstaaten formell Vertragspartei. Im Bereich der gerichtlichen Zuständigkeit und der Vollstreckung gerichtlicher Entscheidungen in Zivil- und Handelssachen ist das revidierte Lugano-Übereinkommen (LugÜ) bedeutsam; dieses Übereinkommen gilt zwischen der EU, Dänemark sowie den EFTA-Mitgliedstaaten Island, Liechtenstein, Norwegen und der Schweiz. Im Klimaschutz besteht eine Vielzahl von Verträgen und Protokollen, welche häufig unter dem Dach der UNO und ihres Umweltprogramms UNEP ausgehandelt wurden. Dazu gehören die Klimaschutzrahmenkonvention (UNFCCC) von 1992, das Kyoto-Protokoll von 1997 und das Pariser Abkommen von 2015; auch bei diesen Verträgen ist die EU parallel zu ihren Mitgliedstaaten Partei. Unzählige Verträge zwischen der Schweiz und einzelnen europäischen Staaten - insbesondere den Nachbarstaaten Deutschland, Österreich, Liechtenstein, Italien und Frankreich - komplementieren diese Regelwerke. In Ergänzung zu klassischen völkerrechtlichen Bindungen bedienen sich Staaten und Entitäten zunehmend auch internationaler Foren und transnationaler Netzwerke, deren Berichte, Empfehlungen und Verlautbarungen zwar rechtlich nicht verbindlich sind, faktisch aber gleichwohl weitreichende Wirkung entfalten; dazu gehört etwa der Basler Ausschuss für Bankenaufsicht, dessen Standards für die Regulierung und Beaufsichtigung von Banken (z.B. Eigenmittelanforderungen) weltweit und damit auch für die EU und die Schweiz normgebend wirken und Eingang in die unionalen und nationalen Rechtsordnungen finden.

Zeitweise werden Verträge direkt zwischen den Gemeinwesen in den Grenzregionen abgeschlossen (Art. 56 BV; kleine Aussenpolitik). Zurzeit sind deutlich mehr als 100 Verträge zwischen Kantonen und anderen Staaten oder grenznahen Regionen in Kraft (R. RHINOW/ M. SCHEFER/P. UEBERSAX, Rz. 3730), typischerweise in den Bereichen Verkehr, Bildung, Kultur, Raumplanung, Umweltschutz und Wirtschaft. Beispiele sind die Gegenrechtsvereinbarungen zwischen dem Kanton St. Gallen und Österreich bzw. dem österreichischen Bundesland Niederösterreich über die Anerkennung von Jagdkarten (1976, sGS 853.155) bzw. der Jägerprüfung (1999, sGS 853.163). 
Diese völkerrechtlichen Regelwerke - auf die bei der Darstellung der bilateralen Abkommen bereichsspezifisch Bezug genommen wird - sind beim Studium der bilateralen Abkommen allesamt zu berücksichtigen. Dasselbe gilt für allgemeine Rechtsgrundsätze und Völkergewohnheitsrecht. Bei Kollisionen sind spezielle Vorgaben (s. etwa Art. 21 FZA betr. Doppelbesteuerungsabkommen; Art. 16 LVA betr. Vereinbarungen über Verkehrsrechte für Luftfahrtunternehmen) und die allgemeinen Regeln über die Einhaltung, Anwendung und Auslegung von völkerrechtlichen Verträgen anwendbar (Art. 26-33 WVRK), zuvörderst die Grundsätze lex specialis derogat legi generali und lex posterior derogat legi priori.

Parallel zum völkervertragsrechtlich abgesicherten Bilateralismus verfolgt die Schweiz seit 1988 eine Politik des autonomen Nachvollzugs bzw. - synonym verstanden - der Europaverträglichkeit. Neue Gesetze und Verordnungen wie auch die Änderung von bestehenden Erlassen werden auf Bundesebene im Vorbereitungsstadium systematisch auf ihre Europakompatibilität hin überprüft (Art. 141 Abs. 1 lit. a ParlG). Bereits 1998 hielt der Bundesrat fest, dass mit der Einführung der Europaverträglichkeitsprüfung ein «Europareflex» geschaffen wurde; neue schweizerische Erlasse «sind im allgemeinen eurokompatibel, ausnahmsweise nicht» (Antwort des Bundesrates vom 13. Mai 1998 auf die Einfache Anfrage 98.1032 «Swisslex II»). Damit wird das schweizerische Recht fortlaufend europäisiert, ohne dass staatsvertragliche Verpflichtungen den Anpassungsprozess auslösen. Die Politik des autonomen Nachvollzugs gehört mittlerweile zum Standardrepertoire des Gesetz- und Verordnungsgebers in Bern. Es gibt in der Schweiz kaum mehr ein Rechtsgebiet, welches nicht direkt oder indirekt vom EU-Recht beeinflusst wird. Dieses wird auch in der Schweiz zum gelebten ius commune.

Dieses Buch behandelt die rechtlichen Beziehungen zwischen der Schweiz und der EU. Der erste Teil skizziert die sektoriell weit fortgeschrittene Integration der Schweiz in den unionalen Rechtsraum und reflektiert die fehlende Abbildung dieser Integration in der Bundesverfassung. Der zweite Teil stellt die bilateralen Abkommen vor, ihre Entstehung und Grundzüge, ihre institutionelle Ausgestaltung - inkl. Seitenblick auf das geplante Institutionelle Abkommen - sowie den Inhalt der einzelnen Abkommen. Der dritte Teil widmet sich dem autonomen Nachvollzug von EU-Recht in der Schweiz. Ein Epilog rundet die Ausführungen ab. 



\section{Die Schweiz als «zugewandter Ort» der EU}

Die Doppelstrategie der völkerrechtlichen Anbindung und selbstgewählten Anpassung führt zu einer sektoriell weitreichenden Integration der Schweiz in das Recht der EU, weshalb die Schweiz als «zugewandter Ort» der EU (D. Freiburghaus, S. 391; M. Oesch/A. LanG, S. 135) oder als «de facto Mitglied der EU» (T. COTTIER et al., Rz. 998; A.R. ZIEGLER, De-facto Mitgliedschaft, S. 270) qualifiziert wird oder von einer «Passivmitgliedschaft» (U. ALTERMATT, S. 155) oder einer «Integration ohne Mitgliedschaft» (M. VAHL/N. GROLIMUND, passim) die Rede ist.

Die Schweiz ist mit der EU in der Tat ausserordentlich eng verbunden. Politisch, gesellschaftlich, kulturell und wissenschaftlich teilen die Schweiz und die EU gemeinsame Grundwerte. Die geographische Lage inmitten Europas verschafft der Schweiz in Verkehrsfragen eine zentrale Stellung. Auch die wirtschaftliche Vernetzung ist gross (s. für diese Zahlen www.eda.admin.ch/ dea und Link zu Europapolitik der Schweiz). Knapp $52 \%$ aller Exporte aus der Schweiz werden in den EU-Raum geliefert. Rund $70 \%$ aller Importe in die Schweiz stammen aus dem EU-Raum. Das Hauptaugenmerk liegt auf dem deutschen Markt, der knapp 20 \% aller Exporte aus der Schweiz abnimmt und für $27 \%$ aller Importe in die Schweiz verantwortlich zeichnet (wobei die Handelsbeziehungen mit den Nachbarbundesländern Baden-Württemberg und Bayern ganz im Vordergrund stehen). Mehr als die Hälfte der schweizerischen Auslandinvestitionen fliesst in den EU-Raum. Damit ist die EU bei weitem die wichtigste Handelspartnerin der Schweiz. Eine Studie schätzt, dass die Schweiz - als Drittstaat! - pro Kopf von allen europäischen Staaten am meisten von der Errichtung des Binnenmarktes profitiert hat (Bertelsmann Stiftung, Ökonomische Effekte des EU-Binnenmarktes in Europas Ländern und Regionen, 2019). Umgekehrt ist aber auch die Schweiz für die EU wichtiger, als es ihre Stellung als Kleinstaat vermuten lässt. In absoluten Zahlen ist die Schweiz nach den Vereinigten Staaten und China die drittwichtigste Handelspartnerin der EU. Auch bei den Personen, welche sich regelmässig in der EU bzw. Schweiz aufhalten - sei es als Arbeitskräfte, sei es als Pensionierte, Privatiers oder Studierende, sei es als Familienangehörige von freizügigkeitsberechtigten Personen -, ist die Verflechtung gross: Aktuell sind mehr als 450000 Schweizerinnen und Schweizer in der EU niedergelassen (wobei rund $3 / 4$ dieser Personen Mehrfachbürgerinnen und Mehrfachbürger sind). Umgekehrt wohnen mehr als 1400000 EU/EFTA-Bürgerinnen und Bürger in der Schweiz; damit kommen rund zwei Drittel der ständigen ausländischen 
Wohnbevölkerung in der Schweiz aus der EU/EFTA. 320000 Grenzgängerinnen und Grenzgänger aus der EU arbeiten in der Schweiz und überqueren die Grenzen in der Regel täglich oder mindestens einmal pro Woche.

Die Schweiz beteiligt sich an diversen Agenturen, Programmen, Einrichtungen und sonstigen Stellen der EU. Dabei sticht insbesondere die Teilnahme an Agenturen ins Auge, welche als selbständige Verwaltungseinheiten dezentralisiert Verwaltungsaufgaben erledigen und ein wesentliches Rückgrat der Eigenverwaltung der EU bilden. Sie sind Teil des europäischen Verwaltungsverbunds (E. SCHMIDT-ASSMANN, S. 1; s. zu diesem Label auch BVerfGE 140, 317 [338] - Identitätskontrolle), wobei dieser Verbund durch die Beteiligung von Drittstaaten über die EU hinaus erweitert wird und paneuropäische Dimensionen annimmt. Aktuell beteiligt sich die Schweiz an der Europäischen Agentur für Flugsicherheit (EASA), an der Europäischen Umweltagentur (EUA) und am Europäischen Umweltinformations- und Umweltbeobachtungsnetz (Eionet), an der Europäischen Agentur für die Grenz- und Küstenwache (Frontex), am Europäischen Unterstützungsbüro für Asylfragen (EASO), an Europol und Eurojust, an den Satellitennavigationsprogrammen Galileo und EGNOS und an Forschungsprogrammen (s. zum Ganzen Agenturbericht, passim; M. OESCH/A. LANG, S. 135-139; C. TOBLER, IT-Agentur, Rz. 53-72). Ergänzend pflegt die Schweiz mit weiteren Agenturen und Einrichtungen lose, unterschiedlich weitgehende Formen der Zusammenarbeit (fachlicher Austausch, Beobachterstatus); dazu gehören das Harmonisierungsamt für den Binnenmarkt (HABM), die Europäische Verteidigungsagentur (EVA), die Europäische Wertpapierund Marktaufsichtsbehörde (ESMA), die Europäische Aufsichtsbehörde für das Versicherungswesen und die betriebliche Altersversorgung (EIOPA) und die Europäische Behörde für Lebensmittelsicherheit (EFSA). Auf diese Weise erhält die Schweiz Zugang zu einschlägigen Gremien und Netzwerken. Sie profitiert von Vorarbeiten und kann mit eigenen Beiträgen Einfluss auf die Politikgestaltung nehmen. Zeitweise besitzen diese Agenturen die Befugnis, für die Schweiz verbindliche Entscheide zu treffen; dies gilt etwa für die Europäische Agentur für Flugsicherheit (EASA).

Die Beteiligung der Schweiz an unionalen Einrichtungen ist auch eine Folge davon, dass sich das Umfeld für die intergouvernementale Zusammenarbeit in Europa in den letzten zwei Jahrzehnten verändert hat. Die Tätigkeiten und Aufgaben von internationalen Organisationen und informellen Foren wurden vermehrt auf Einrichtungen der EU übertragen und damit gleichsam «vergemeinschaftet» bzw. «unionalisiert». Beispiele dafür sind die Joint Aviation Authorities (JAA), welche zur Harmonisierung der in Europa geltenden Vorschriften im Bereich der technischen und operationellen Flugsicherheit beitrugen, und der informelle und ad hoc orchestrierte Informationsaustausch zwischen europäischen Staaten zur Bekämpfung übertragbarer Krankheiten. Diese Aufgaben werden heute im Wesent- 
lichen durch die Europäische Agentur für Flugsicherheit (EASA) und das Europäische Zentrum für Prävention und Kontrolle von Krankheiten (ECDC; s. zu den Verhandlungen über eine Beteiligung der Schweiz N. 35) wahrgenommen; die EASA und das ECDC sind Agenturen der EU. Die Integration von europäischen Organisationen und informellen Netzwerken in den EU-Rechtsrahmen und die damit einhergehende Formalisierung und Verrechtlichung führen dazu, dass die Beteiligung von Drittstaaten grundsätzlich in Frage gestellt wird und nurmehr unter klar definierten Vorgaben - und ohne Stimmrecht - im Angebot ist (Agenturbericht, S. 2-3; S. LAVENEX/R. SCHWOK, S. 44).

Die EU ermöglicht der Schweiz, sich bei der Vorbereitung neuer EU-Rechtsakte im Anwendungsbereich der bilateralen Abkommen zu beteiligen und den Inhalt mitzugestalten (N. 94). Expertinnen und Experten aus der Schweiz tauschen sich in Arbeitsgruppen, Ausschüssen und weiteren - auch informellen Netzwerken mit den Kolleginnen und Kollegen der EU-Eigenverwaltung und der Verwaltungen der EU-Mitgliedstaaten aus (proposal shaping), verfügen in aller Regel aber über kein Stimmrecht (decision making).

Der Einfluss der Schweiz hängt wesentlich von der Qualität und Überzeugungskraft der Argumente ab (power of the pen). Diese fallen umso eher auf fruchtbaren Boden, je technischer eine Materie ist. Umgekehrt verfügen Äusserungen eines «Passivmitglieds» zwangsläufig über weniger Gewicht als Einwürfe von «Aktivmitgliedern», wenn politisch umstrittene Grundsatzfragen debattiert werden. Das ist für die Schweiz nachteilig, auch wenn die meisten Entscheidungen in solchen unionalen Gremien im Konsens getroffen werden (Agenturbericht, S. 46, 51, 106; Bericht Schengen 2013, S. 6334). Entsprechend essentiell ist, dass die Schweiz ihre Mittel möglichst effektiv einsetzt und sich mit anderen Staaten, deren Interessen gleichlaufen, verbündet.

Insgesamt wirkt die Schweiz damit punktuell in mitgliedstaatsähnlicher Weise im europäischen Staatenverbund (s. zu diesem Label BVerfGE 89, 155 [181] Maastricht) mit. Politisch bleibt die Schweiz eine Aussenseiterin und ist für Mitwirkungsrechte auf das Entgegenkommen der EU angewiesen; funktional ist sie eng eingebunden - deutlich enger als dies prima vista und allein mit Blick auf die klassisch völkerrechtlich ausgestalteten Vertragsbeziehungen aussehen mag (S. LAVENEX/R. SCHWOK, S. 49). Dabei verändert die Mitwirkung in den unionalen Agentur- und Programmverwaltungen sowie bei der Vorbereitung neuer EU-Rechtsakte den tradierten Integrationsmodus der Schweiz (J. SAURER, S. 428). Die traditionelle Politik des Bilateralismus trägt reaktive Züge. Die EU bestimmt Rhythmus und Themen. Die Schweiz folgt hintenan nach. Sie übernimmt passiv «fertiges» EU-Recht, sei es im Rahmen des Abschlusses und der Weiterentwicklung bilateraler Abkommen, sei es im Rahmen des autonomen Nachvollzugs. Demgegenüber verlangt die Mitarbeit in unionalen Gremien eine proaktive und konstruktive Mitwirkung - verbunden mit der Verpflichtung, (Mit-) Verantwortung für die Politikgestaltung im paneuropäischen Kontext zu übernehmen. 



\section{Introvertierte Verfassungstradition}

Die Bundesverfassung atmet einen weltoffenen und völkerrechtsfreundlichen Geist (vgl. auch A. Auer/G. MALINVERni/M. HotTELIER, Rz. 31; G. BiAGginI, Öffnung, S. 965). Sie verpflichtet den Bund, «in Solidarität und Offenheit gegenüber der Welt» zu handeln (Präambel), sich «für eine friedliche und gerechte internationale Ordnung» einzusetzen (Art. $2 \mathrm{BV}$ ), «zur Linderung von Not und Armut in der Welt, zur Achtung der Menschenrechte und zur Förderung der Demokratie, zu einem friedlichen Zusammenleben der Völker sowie zur Erhaltung der natürlichen Lebensgrundlagen» beizutragen (Art. $54 \mathrm{BV}$ ) und «die Interessen der schweizerischen Wirtschaft im Ausland» zu wahren (Art. 101 BV). Diese Zielbestimmungen geben der Aussenpolitik - verbindlich, wenngleich offen formuliert und konkretisierungsbedürftig - inhaltlich die Richtung vor. Der Grundrechtekatalog beruht wesentlich auf der EMRK, welche auf diese Weise «konstitutionalisiert» wurde (G. BIAGGINI, Verhältnis, S. 727). Die Aussenverfassung regelt die kompetenziellen Fragen (Art. 54-56, Art. 140-141, Art. 166 und Art. 184 BV). Das Staatsvertragsreferendum - 1921 eingeführt, 1977 erstmals erweitert - wurde 2003 als Reaktion auf den fortschreitenden Prozess der Globalisierung und Europäisierung des Rechts weiter ausgebaut mit dem Ziel, die direkt-demokratische Legitimierung völkerrechtlicher Verträge sicherzustellen (s. zur demokratischen Legitimation der bilateralen Abkommen N. 59-68). Die Völkerrechtsfreundlichkeit wird durch die Verpflichtung zur Beachtung des Völkerrechts durch Bund und Kantone betont (Art. 5 BV). Diese Vorgaben gelten allesamt auch bei der Gestaltung der Beziehungen der Schweiz zur EU. Beachtenswert ist, dass der Beitritt zu supranationalen Gemeinschaften dem obligatorischen Referendum untersteht, womit die Tür auch für einen EU-Beitritt verfassungsrechtlich offensteht (Art. $140 \mathrm{BV}$ ).

Das BGer hat das Verhältnis von Völker- und Landesrecht weiter ausdifferenziert. Eine reichhaltige Praxis besteht dabei auch zur Geltung und zum Rang der bilateralen Abkommen im schweizerischen Recht, zur Auslegung des schweizerischen Rechts im Licht der bilateralen Abkommen sowie zu ihrer unmittelbaren Anwendbarkeit (N. 50-58).

Gleichzeitig schweigt sich die Bundesverfassung über die Bedeutung der Einbindung der Schweiz in globale und regionale Organisationen und Vertragswerke und die damit einhergehenden Interdependenzen und Abhängigkeiten weitgehend aus. Die einzige internationale Organisation, welche namentlich erwähnt wird, ist die UNO. Der durch eine Volksinitiative angestossene Beitritt der Schweiz zur UNO von 2002 hat es immerhin in die Übergangsbestimmungen geschafft (Art. 197 Ziff. 1 BV). Dieses verzerrte Bild ist bedauerlich und der 
Orientierungsfunktion der Bundesverfassung abträglich (s. dazu auch A.R. ZieGLER/K. OdENDAHL, Rz. 39; G. BIAGGINI, Öffnung, S. 969). Gleichzeitig ändert dieser blinde Fleck der Verfassung nichts an der grundlegend konstitutiven Bedeutung, welche Organisationen und Regelwerke wie die WTO, die OECD, die UNO-Pakte I und II und andere Menschenrechtskonventionen für die jeweiligen Politikbereiche in der Schweiz haben.

Der Befund der Verfassungsblindheit gilt ebenso für die Beteiligung der Schweiz am europäischen Integrationsprozess. Die Leserin und der Leser suchen die Begriffe Europa, EU, EFTA, bilaterale Abkommen, Europarat und EMRK in der Bundesverfassung vergeblich. Es besteht ein Widerspruch zur gelebten (Verfassungs-) Wirklichkeit, der sich mit jedem weiteren Integrationsschritt der Schweiz akzentuiert. Weder die enge Vernetzung der Schweiz mit der EU noch die überragende Bedeutung der EMRK für den Grundrechtsschutz in der Schweiz werden abgebildet. Es finden sich keine Aussagen über die Grundsätze, Ziele und Grenzen der schweizerischen Europapolitik. Die Bundesverfassung von 1999 kommt bewusst «europaneutral» daher (Botschaft Bundesverfassung S. 119). Die Öffentlichkeit war nach der Ablehnung des Beitritts zum EWR verunsichert und in Bezug auf die Europafrage gespalten. Der Verfassungsgeber scheute das Risiko, die Konsensfähigkeit des neuen Verfassungstexts durch konkrete Aussagen zum Verhältnis der Schweiz zur EU aufs Spiel zu setzen, und nahm die «Tabuisierung des Themas Europa» bewusst in Kauf (T. CotTIER, Bundesverfassung, S. 183).

Während es der Schweiz in der Folge gelang, die Beziehungen zur EU qua bilateraler Abkommen auf eine tragfähige Basis zu stellen, wurde die Frage des Verhältnisses der Schweiz zu Europa verfassungsrechtlich ausdrücklich nur noch bei zwei Volksinitiativen aufgeworfen. Sowohl die Volksinitiative «EU-Beitrittsverhandlungen vors Volk!» von 1997 als auch die Volksinitiative «Ja zu Europa!» von 2001 wurden wuchtig abgelehnt (s. dazu und zu weiteren europapolitisch bedeutsamen Volksabstimmungen N. 60-64).

Rechtsgelehrte haben vorgeschlagen, zumindest die weitreichende Verflechtung mit Europa verfassungsrechtlich abzubilden und auf diese Weise Verfassungstext und gelebte Praxis in Einklang zu bringen (s. A. KÖLZ/J.P. MÜLLER, Art.113; im Hinblick auf einen EU-Beitritt D. THÜRER, Verfassungsfragen, S. 30-33; zum Ganzen auch E.-U. PeterSmann/A.R. ZIEGLER, passim). Der Zweck eines solchen Europa-Artikels oder - systematisch wohl kluger - eines Europa-Absatzes, der Art. 54 BV ergänzte, bestünde darin, über die Grundsätze, Ziele und Grenzen der schweizerischen Europapolitik Auskunft zu geben. Ein Vorschlag lautet wie folgt (s. für diesen Vorschlag M. OESCH, Verfassungswandel, S. 177-181; ein ähnlicher Vorschlag stammt von T. CотTIER, Europa-Artikel, passim): 
Art. 54 Auswärtige Angelegenheiten

$1 \ldots$

$2 \ldots$

$2^{\text {bis }}$ Er [der Bund] wirkt am Prozess der europäischen Integration mit. Die Schweiz ist Mitglied des Europarates und strebt eine aktive und enge Zusammenarbeit mit der Europäischen Union an. Sie achtet die gemeineuropäisch anerkannten Werte und setzt sich für ihre Förderung ein; dazu gehören zuvörderst Frieden, Freiheit, Wohlergehen, Demokratie, Rechtsstaatlichkeit und die Achtung der Menschenrechte.

$2^{\text {ter }}$ Er berücksichtigt bei der Aushandlung und Umsetzung völkerrechtlicher Verträge insbesondere die gesamtwirtschaftlichen Interessen der Schweiz, die Bedürfnisse der Wohnbevölkerung und die natürlichen Lebensgrundlagen in der Schweiz. Er erlässt im Rahmen seiner Zuständigkeiten flankierende Massnahmen.

$3 \ldots$

Der vorgeschlagene Abs. $2^{\text {bis }}$ reflektiert eine betont europafreundliche Grundhaltung und schliesst einen Alleingang der Schweiz in Europa aus. Namentlich werden der Europarat und die EU genannt, ohne dass damit eine Gering(er)schätzung weiterer Organisationen und Vertragswerke impliziert wird. Abs. $2^{\text {bis }}$ verzichtet darauf, die Art und Weise der Zusammenarbeit und das Instrumentarium festzulegen. Er überlässt den Entscheid über weitere Integrationsschritte den Behörden, wobei zumindest bei einem EU-Beitritt ohnehin Volk und Stände das letzte Wort haben und sich eine Neufassung dieser Bestimmung aufdrängt. Umgekehrt scheint es sinnvoll, im Zug der Einführung eines solchen Europa-Artikels Art. 121a und Art. 197 Ziff. 11 BV aufzuheben; diese Bestimmungen zielen auf den Alleingang der Schweiz und sind mit der aktuellen Integrationspolitik der Schweiz nicht vereinbar. Gute Gründe sprechen dafür, die Sorgen und Ängste, welche zur Annahme der Volksinitiative «Gegen Masseneinwanderung» geführt haben, verfassungsrechtlich weiterhin zu reflektieren. Abs. $2^{\text {ter }}$ verpflichtet den Bund, die mitunter als negativ gewerteten Folgen der europäischen Integration zu bedenken und flankierende Massnahmen zu erlassen. Auch dieser vorgeschlagene Absatz ist programmatischer Natur. Er schafft keine neuen Bundeskompetenzen.

Ein solcher Vorschlag mag auf den ersten Blick blutleer und unspektakulär 17 daherkommen. Gleichwohl sollte seine rechtspolitische Bedeutung nicht unterschätzt werden. Abs. $2^{\text {bis }}$ nimmt auf die Grundwerte des Europarates, der EMRK und der EU Bezug, wie sie auch in der Bundesverfassung zum Ausdruck kommen. Er hält den Bund an, sich für die Förderung dieser Werte einzusetzen. Auch bilden diese Werte Mindeststandards bzw. Schranken, welche es bei der Mitwirkung am europäischen Integrationsprozess stets zu beachten gilt. 


\begin{abstract}
Abs. $2^{\text {bis }}$ unterstreicht die Wichtigkeit enger und stabiler Beziehungen zwischen der Schweiz und der EU und nimmt damit ein Anliegen auf, über das in Politik und Gesellschaft ein breiter Konsens besteht.
\end{abstract}

Dieser Vorschlag orientiert sich an der Rechtslage diverser EU-Mitgliedstaaten, deren Verfassungstexte sich spezifisch zum Verhältnis zur EU und zur Übertragung von Hoheitsrechten äussern (s. dazu die Übersichten bei C.D. CLASSEN, S. 285-306; F.C. MAYER/ M. WENDEL, passim). Wenngleich solche Öffnungs- oder Integrationsklauseln für die Schweiz nur beschränkt fruchtbar gemacht werden können, zielen sie im Kern hier wie da doch aufs Gleiche: Sie übernehmen eine wichtige Scharnierfunktion zwischen der nationalstaatlichen und der europäischen Ebene. Sie spiegeln die überstaatlichen Bedingtheiten des Staates und die (nurmehr) begrenzte Steuerungs- und Gestaltungsfunktion der nationalstaatlichen Verfassung insbesondere mit Blick auf die fortschreitende Europäisierung. Sie tragen zur Entwicklung eines gesamtheitlichen Systems im Sinne eines europäischen Verfassungsverbunds (s. zu diesem Label BVerfGE 140, 317 [338] - Identitätskontrolle) bei, indem sie nicht nur einseitig kompetenzielle Fragen regeln, sondern der Europäisierung die Richtung vorgeben und Schranken setzen. 


\section{Zweiter Teil}

\section{Bilaterale Abkommen}





\section{Entstehung und Grundzüge}

\section{A. Etappen}

Das Verhältnis der Schweiz zur europäischen Integration unter dem Dach der

EGKS/EWG/EG/EU ist gekennzeichnet durch das Bestreben, sektoriell bilaterale Abkommen abzuschliessen, ohne sich institutionell (allzu) weitreichend zu integrieren. Die zentralen Wegmarken - von den ersten Annäherungen in den 1950er Jahren über den Abschluss des Freihandelsabkommens, die Ablehnung des Beitritts zum EWR und den Abschluss der Bilateralen I und II bis hin zu den aktuell laufenden Verhandlungen - lassen sich wie folgt zusammenfassen.

\section{Erste Annäherung (1956-1972)}

Die Aussenpolitik der Schweiz beruhte nach dem Zweiten Weltkrieg auf den Pfeilern der Souveränität, der Unabhängigkeit, der Neutralität und der handelspolitischen Autonomie. Die schweizerische Exportwirtschaft profitierte von bilateralen Handelsabkommen mit einer Vielzahl von Staaten und von einer starken Auslandnachfrage im Rahmen des europäischen Wiederaufbaus. Die Schweiz war gut aufgestellt, ihren Wohlstand zu mehren. Das Landwirtschaftsrecht war auf die Unterstützung des Bauernstandes ausgerichtet und bot massiven Grenzschutz. Vor diesem Hintergrund erstaunt es nicht, dass die Schweiz bemüht war, sich nur selektiv völkerrechtlich $\mathrm{zu}$ binden. Diese Zurückhaltung galt zuvörderst mit Blick auf globale Organisationen und Regelwerke sowie (verteidigungs-) politische Bündnisse.

Die Schweiz trat erst 1966 definitiv dem GATT bei. Der Beitritt zu den Bretton Woods-Institutionen und zu den Vereinten Nationen erfolgte 1992 und 2002. 1995 war die Schweiz Gründungsmitglied der WTO. Der Westeuropäischen Union (WEU) blieb die Schweiz zeitlebens fern. Sie ist ebenso wenig Mitglied der NATO; seit 1996 beteiligt sie sich an der Partnerschaft für den Frieden (PfP) und am Euro-Atlantischen Partnerschaftsrat (EAPC).

Ähnlich defensiv agierte die Schweiz auch auf dem europäischen Kontinent. Sie fungierte 1948 zwar als Gründungsmitglied der Organisation für europäische wirtschaftliche Zusammenarbeit (OEEC, 1961 umgetauft in OECD), machte ihre Beteiligung aber von der Einhaltung gewisser Vorbehalte abhängig; dazu gehört die Beachtung der Neutralität, die Weitergeltung der handelspolitischen Autonomie und die Nichtverbindlichkeit von Entscheiden, denen die Schweiz nicht zugestimmt hat (Botschaft OEEC, S. 1177). Eine Teilnahme am europäischen Integrationsprozess im Rahmen der EGKS/EWG/Euratom kam 
für die Schweiz nicht in Frage. Die kriegsverschonte Schweiz blieb gegenüber dem zentralen Projekt der europäischen Friedenssicherung distanziert. Stattdessen engagierte sie sich im Verbund mit den anderen Staaten der «Äusseren Sieben» 1960 bei der Gründung der Europäischen Freihandelsassoziation (EFTA). Sie erblickte darin eine willkommene Gelegenheit, Freihandel, Agrarprotektionismus und nationale Souveränität zu vereinen. Dem Europarat trat die Schweiz 1963 bei; seit 1974 ist sie Vertragspartei der EMRK.

Die Anfänge des vertraglichen Bilateralismus mit den Europäischen Gemeinschaften bildeten ein Konsultationsabkommen zwischen der Schweiz und der Hohen Behörde der EGKS von 1956 sowie ein Abkommen über die Einführung direkter internationaler Eisenbahntarife im Verkehr mit Kohle und Stahl im Durchgang durch das schweizerische Gebiet zwischen dem Schweizerischen Bundesrat einerseits und den Regierungen der EGKS-Mitgliedstaaten und der Hohen Behörde der EGKS andererseits von 1956 (AS 1957 71, AS 1957 381; C. TOBLER/J. BEGLINGER, Grundzüge, Rz. 12); diese Abkommen sind heute nicht mehr in Kraft. Weitere Abkommen mit der EWG folgten. Dazu gehört das Abkommen betreffend die Erzeugnisse der Uhrenindustrie zwischen der Schweiz und der EWG und ihren Mitgliedstaaten von 1967, das bis heute in Kraft ist (SR 0.632.290.13).

\section{Abkommen im Waren-, Versicherungs- und Transitbereich (1972-1992)}

Die EFTA beabsichtigte ursprünglich, «die baldige Schaffung einer multilateralen Assoziation zur Beseitigung der Handelsschranken und zur Förderung einer engeren wirtschaftlichen Zusammenarbeit zwischen den Mitgliedern der Organisation für europäische wirtschaftliche Zusammenarbeit, einschliesslich der Mitglieder der Europäischen Wirtschaftsgemeinschaft, zu erleichtern» (Präambel des Übereinkommens zur Errichtung der EFTA von 1960, AS 1960 589). Bestrebungen zur Gründung einer vor allem auch von Grossbritannien favorisierten grossen europäischen Freihandelszone scheiterten allerdings. Stattdessen handelten die EFTA-Staaten mit der EWG anfangs der 1970er Jahre gemeinsam bilaterale Freihandelsabkommen aus. Die einzelnen, inhaltlich weitgehend identischen Abkommen wurden von der EWG und jedem EFTA-Mitgliedstaat separat unterzeichnet und in Kraft gesetzt. Das Freihandelsabkommen zwischen der EWG und der Schweiz datiert von 1972 (FHA, SR 0.632.401). Es trat 1973 in Kraft, nachdem es in der Schweiz im Rahmen eines obligatorischen Referendums von Volk (72.5\%) und Ständen (alle) gutgeheissen worden war; das obligatorische Referendum wurde ange- 
ordnet, weil das Abkommen «in seinem Gehalt derart bedeutsam [ist] und einen Teil der öffentlichen Meinung in so starkem Masse [beschäftigt], dass die Diskrepanz zu den sonst üblichen Mitwirkungsrechten des Volkes bei der Bildung des Landesrechts [andernfalls] als zu gross erscheinen müsste» (Botschaft FHA, S. 737). Das Freihandelsabkommen bildet seither zusammen mit den unzähligen Zusatzvereinbarungen, welche in der Folge ausgehandelt wurden, die Grundlage - quasi «der erste Stein» (Europabericht 2010, S. 6846) für die präferenziellen Wirtschaftsbeziehungen zwischen der Schweiz und der EWG/EG/EU. 1991 wurde zudem das Abkommen über die Erleichterung der Kontrollen und Formalitäten im Güterverkehr in Kraft gesetzt (AS 1991 1490); es wurde 2011 vom Zollabkommen (ZollA) abgelöst.

Im Bereich des wirtschaftlich stetig wichtiger werdenden Dienstleistungssektors führten langwierige Verhandlungen 1989 zur Unterzeichnung des Abkommens betreffend die Direktversicherung mit Ausnahme der Lebensversicherung (Versicherungsabkommen, VersA, SR 0.961.1). Dieses Abkommen trat 1993 in Kraft und bildet bis heute das einzige Abkommen, welches Aspekte des Marktzugangs für die Versicherungsbranche regelt.

1993 trat das Abkommen über den Güterverkehr auf Strasse und Schiene von 1992 in Kraft (Transitabkommen, AS 1993 1198). Die Geltung dieses Abkommens war auf zwölf Jahre befristet; es blieb in Teilen bis 2005 in Kraft.

\section{Ablehnung des EWR-Beitritts (1992)}

In den 1980er-Jahren einigten sich die EWG-Mitgliedstaaten darauf, die Vollendung des Binnenmarktes voranzutreiben. Die Verabschiedung der Einheitlichen Europäischen Akte (EEA) und des damit verbundenen Binnenmarktprogramms weckte unter den EFTA-Mitgliedstaaten die Befürchtung, wirtschaftlich (weiter) ins Hintertreffen zu geraten und Diskriminierungen ausgesetzt zu sein. Der damalige Kommissionspräsident Jacques Delors baute den EFTAMitgliedstaaten eine Brücke. Er schlug 1989 die Gründung eines Europäischen Wirtschaftsraums (EWR) vor mit dem Ziel, «binnenmarktähnliche Verhältnisse» auch für die EFTA-Mitgliedstaaten zu schaffen und zu diesem Zweck eine neuartige Form der Assoziierung einzurichten (J. Delors, passim). Verhandlungen zwischen der EWG-Kommission und den Vertreterinnen und Vertretern der damals sieben EFTA-Mitgliedstaaten führten am 2. Mai 1992 zur Unterzeichnung des EWR-Abkommens. Auch die Schweiz unterzeichnete das Abkommen. Am 26. Mai 1992 reichte der schweizerische Bundesrat - im Gleichschritt mit den meisten anderen EFTA-Staaten - ein Gesuch um Aufnahme von EG-Beitrittsverhandlungen ein. Dabei definierte er den EG-Beitritt 
ausdrücklich als Ziel der schweizerischen Europapolitik und betrachtete «den EWR als eine besonders auch innenpolitisch wichtige Etappe in Richtung auf dieses Ziel» (Bericht EG-Beitritt, S. 1186, 1194; s. auch J. KELLENBERGER, S. 30-32).

Bundesrat und Parlament entschieden mit Blick auf die überragende politische und wirtschaftliche Bedeutung des EWR-Abkommens, die Frage des EWR-Beitritts dem obligatorischen Referendum zu unterstellen (Botschaft EWR, S. 541, 542). Die Vorlage war in hohem Masse umstritten. Im Vordergrund stand die Kritik an den institutionellen Mängeln und an der Verpflichtung, EU-Recht ohne Mitentscheidung faktisch zwingend übernehmen zu müssen. Die Personenfreizügigkeit wurde in weiten Kreisen als problematisch erachtet. Das Engagement der Wirtschaft und ihrer Verbände war bis kurz vor der Abstimmung gering. Erstmals wurde «Europa» als Bedrohung thematisiert und diente einer aufstrebenden nationalkonservativen Strömung als Instrument der politischen Mobilisierung. Die EWR-Mitgliedschaft wurde als antizipierter EUBeitritt dargestellt. Umgekehrt begannen auch EU-Beitrittsbefürworter an der Notwendigkeit eines Zwischenschritts auf dem Weg zur Vollmitgliedschaft zu zweifeln. Am 6. Dezember 1992 lehnten Volk (50.3 \%) und Stände (14 Kantone, 4 Halbkantone) den Beitritt zum EWR ab.

Das Beitrittsgesuch von 1992 blieb auch nach der Ablehnung des EWR-Beitritts in Brüssel liegen, hatte aber keine praktische Bedeutung mehr. 2016 wurde es formell zurückgezogen. Der Bundesrat degradierte das strategische Ziel des EU-Beitritts bereits 2005 zu einer längerfristigen Option unter mehreren (N. 378).

\section{Bilaterale I (1999)}

Nach der Ablehnung des EWR-Beitritts - dem defining moment der jüngeren schweizerischen Europapolitik - nahm die Schweiz die Tradition pragmatischbilateraler Beziehungen wieder auf und konzentrierte sich gleichsam faute de mieux auf kurzfristig realisierbare, sektorielle Verhandlungen mit der EU. Die EU bot dafür Hand; ein wesentlicher Grund für dieses Entgegenkommen war die Erwartung, der Schweiz mit einer schrittweisen und massgeschneiderten Annäherung den Weg zur Vollmitgliedschaft zu ebnen. Gleichzeitig liess die Europäische Kommission verlauten, es müsse verhindert werden, dass die Schweiz nur von den Vorteilen des abgelehnten EWR-Arrangements profitieren würde; auch würde der Acquis communautaire die Basis für die Verhandlungen bilden (Mitteilung vom 1. Oktober 1993, COM (93) 486 endg., Rz. 10, 13). 1994 führte die Annahme der Alpenschutzinitiative (N. 200) zu einer Blockade, welche erst aufgelöst werden konnte, als die Schweiz eine nichtdiskriminierende Umsetzung des verfassungsrechtlichen Auftrags zum Schutz der Alpen in Aussicht stellte (Botschaft Bilaterale I, S. 6129). 1999 wurden die Bilateralen I 
unterzeichnet. Dazu gehören die folgenden sieben Abkommen, welche - mit Ausnahme des Forschungsabkommens - das Freihandelsabkommen von 1972 und das Versicherungsabkommen von 1989 durch eine schrittweise und kontrollierte gegenseitige Marktöffnung ergänzen:

- Abkommen über die Freizügigkeit (Freizügigkeitsabkommen, FZA, SR 0.142.112.681);

- $\quad$ Abkommen über die gegenseitige Anerkennung von Konformitätsbewertungen (KonfBA; auch: Abkommen über technische Handelshemmnisse, Mutual Recognition Agreement, MRA, SR 0.946.526.81);

- Abkommen über bestimmte Aspekte des öffentlichen Beschaffungswesens (ÖffBA, SR 0.172.052.68);

- Abkommen über den Handel mit landwirtschaftlichen Erzeugnissen (Landwirtschaftsabkommen, AgrarA, SR 0.916.026.81);

- Abkommen über den Luftverkehr (Luftverkehrsabkommen, LVA, SR 0.748.127.192.68);

- Abkommen über den Güter- und Personenverkehr auf Schiene und Strasse (Landverkehrsabkommen, LandVA, SR 0.740.72);

- Abkommen über die wissenschaftliche und technologische Zusammenarbeit (Forschungsabkommen, ForschA, AS 2002 1998); dieses Abkommen bezog sich auf die Teilnahme der Schweiz am Fünften Rahmenprogramm im Bereich der Forschung (1998-2002); die Teilnahme der Schweiz an den Nachfolgeprogrammen wurde in separaten Abkommen geregelt.

Die Bilateralen I sind durch eine Guillotine-Klausel verbunden (s. etwa Art. 25 Abs. 4 FZA). Sofern ein Abkommen durch eine Vertragspartei gekündigt wird, treten sechs Monate danach auch alle anderen automatisch ausser Kraft. Damit wurde dem Begehren der EU entsprochen, ein ausgewogenes Gesamtergebnis zu erzielen und zu verhindern, dass einzelne Abkommen - im Sinne eines «Rosinenpickens» (Botschaft Bilaterale I, S. 6436) - in der Schweiz in einer Referendumsabstimmung abgelehnt werden. Dieses Schicksal hätte insbesondere dem Freizügigkeitsabkommen gedroht, bei dem die EU als demanderesse auftrat und die Schweiz nur widerwillig einlenkte; auf die Forderung der Schweiz ging die Befristung dieses Abkommens auf sieben Jahre zurück (Art. 25 Abs. 2 FZA; Botschaft Bilaterale I, S. 6313; vgl. NZZ vom 16. September 2020, S. 13). Die Bilateralen I wurden am 1. Juni 2002 in Kraft gesetzt, nachdem das Schweizer Stimmvolk dem Paket mit $67 \%$ der abgegebenen Stimmen zugestimmt hatte. Auf Seiten der EU wurde das Paket gestützt auf Art. 310 i.V.m. Art. 300 EGV (Art. 217 i.V.m. Art. 218 AEUV) durch den Rat und das Europäische Parlament (bzw. die Kommission betr. des ForschA) genehmigt 
(Beschluss 2002/309/EG/Euratom; s. zur Bestimmung der Rechtsgrundlage C. KADDOus, Zusammenarbeit, Rz.69). Das Freizügigkeitsabkommen ist ein gemischtes Abkommen und musste folglich auch durch die Mitgliedstaaten ratifiziert werden (N. 44-45). Anlässlich der Osterweiterungen der EU von 2004 und 2007 wurde das Freizügigkeitsabkommen formell auf die zwölf neuen Mitgliedstaaten ausgedehnt. Beide Zusatzprotokolle wurden in Referendumsabstimmungen genehmigt (2005 mit 56 \%, 2009 mit $59 \%$ der abgegebenen Stimmen), wobei in der zweiten Abstimmung auch über die Weiterführung des Freizügigkeitsabkommens entschieden (vgl. Art. 25 Abs. 2 FZA) und damit der bilaterale Weg in der bisherigen Form insgesamt bestätigt wurde. Die Ausdehnung auf Kroatien - EU-Mitglied seit 2013 - verzögerte sich nach der Annahme der Volksinitiative «Gegen Masseneinwanderung»; sie erfolgte 2017, nachdem sich der schweizerische Gesetzgeber für eine FZA-konforme Umsetzung dieser Initiative entschieden hatte (N. 63).

\section{Bilaterale II (2004)}

Weitere Verhandlungen primär über left overs, über welche im Rahmen der Bilateralen I keine Einigung erzielt werden konnte, führten 2004 zur Unterzeichnung der Bilateralen II. Dieses Paket umfasst die folgenden neun Abkommen, welche über den hauptsächlich wirtschaftlichen Rahmen der Bilateralen I hinausgehen und namentlich auch Justiz und Inneres, Visa und Asyl, Umwelt, Kultur und Bildung betreffen:

- Abkommen über die Assoziierung der Schweiz bei der Umsetzung, Anwendung und Entwicklung des Schengen-Besitzstands (SchengenAssoziierungsabkommen, SAA, SR 0.362.31);

- Abkommen über die Kriterien und Verfahren zur Bestimmung des zuständigen Staates für die Prüfung eines in einem Mitgliedstaat oder in der Schweiz gestellten Asylantrags (Dublin-Assoziierungsabkommen, DAA, SR 0.142.392.68);

- $\quad$ Abkommen über Regelungen, die den in der Richtlinie 2003/48/EG im Bereich der Besteuerung von Zinserträgen festgelegten Regelungen gleichwertig sind (Zinsbesteuerungsabkommen, ZinsbestA, AS 2005 2571); dieses Abkommen wurde durch das Abkommen über den automatischen Informationsaustausch über Finanzkonten zur Förderung der Steuerehrlichkeit bei internationalen Sachverhalten von 2015 abgelöst, N. 31); 
- $\quad$ Abkommen über die Zusammenarbeit zur Bekämpfung von Betrug und sonstigen rechtswidrigen Handlungen, die die finanziellen Interessen der Vertragsparteien beeinträchtigen (Betrugsbekämpfungsabkommen, SR 0.351.926.81);

- $\quad$ Abkommen zur Änderung des Freihandelsabkommens von 1972 in Bezug auf die Bestimmungen über landwirtschaftliche Verarbeitungserzeugnisse (Abkommen über landwirtschaftliche Verarbeitungserzeugnisse, SR 0.632.401.23);

- Abkommen über die Beteiligung der Schweiz an der Europäischen Umweltagentur (EUA) und dem Europäischen Umweltinformations- und Umweltbeobachtungsnetz (Eionet) (Umweltabkommen, SR 0.814.092.681);

- Abkommen im Bereich audiovisuelle Medien über die Festlegung der Voraussetzungen und Bedingungen für die Beteiligung der Schweiz an den Gemeinschaftsprogrammen MEDIA Plus und MEDIA-Fortbildung (AS 2006 1039); dieses Abkommen bezog sich auf die Teilnahme der Schweiz an den genannten Programmen (2001-2006); die Teilnahme der Schweiz an nachfolgenden Programmen wurde in separaten Abkommen geregelt;

- Abkommen über die Zusammenarbeit im Bereich der Statistik (Statistikabkommen, SR 0.431.026.81);

- $\quad$ Abkommen zur Vermeidung der Doppelbesteuerung von in der Schweiz ansässigen ehemaligen Beamten der Organe und Agenturen der Europäischen Gemeinschaften (SR 0.672.926.81).

Die Verhandlungen beruhten - diesmal vor allem auf Drängen der Schweiz auf dem Prinzip des angemessenen Parallelismus. Gleichwohl sind die Abkommen nicht durch eine Guillotine-Klausel verbunden. Nur das Schicksal der Schengen- und Dublin-Assoziierungsabkommen ist verknüpft (kleine Guillotine; Art. 15 Abs. 4 SAA, Art. 14 Abs. 2 DAA). In der Schweiz wurden diese beiden Abkommen - die einzig umstrittenen - in einer Referendumsabstimmung mit $54 \%$ der abgegebenen Stimmen angenommen. Auf Seiten der EU wurden die neun Abkommen gestützt auf die jeweils einschlägigen vertraglichen Bestimmungen verabschiedet (s. dazu C. KADDous, Zusammenarbeit, Rz. 69). Die Inkraftsetzung erfolgte für die Abkommen zeitlich gestaffelt. Das Betrugsbekämpfungsabkommen stellt ein gemischtes Abkommen dar (N. 44-45), wobei auf Seiten der EU noch die Ratifizierung von Irland fehlt (www.eda.admin.ch/ dea und Link zu Bilaterale Abkommen II (2004)/Betrugsbekämpfung). Aus die- 
sem Grund wird es seit 2009 von der Schweiz nur gegenüber denjenigen Mitgliedstaaten der EU angewendet, welche das Abkommen ebenfalls ratifiziert und eine Erklärung über die vorzeitige Anwendung abgegeben haben.

\section{Punktuelle Ergänzungen (2005-heute)}

31 Nach dem erfolgreichen Abschluss und der schrittweisen Inkraftsetzung der Bilateralen II haben die Schweiz und die EU nur noch punktuell weitere Abkommen abgeschlossen und Memoranda of Understanding unterzeichnet. Dazu gehören die folgenden:

- Memorandum of Understanding von 2006, in dem die Schweiz ihre Bereitschaft bekräftigte, sich während zehn Jahren finanziell an der Kohäsionspolitik der EU zu beteiligen; in der Folge unterstützte die Schweiz Projekte in den neuen mittel- und osteuropäischen EU-Mitgliedstaaten autonom mit rund 1,3 Mia. CHF («Kohäsionsmilliarde»); 2017 wurde der Boden gelegt für einen weiteren Beitrag (N. 329);

- Abkommen über die Erleichterung der Kontrollen und Formalitäten im Güterverkehr und über zollrechtliche Sicherheitsmassnahmen von 2009 (Zollabkommen, ZollA), in Kraft seit 2011 (SR 0.631.242.05); dieses Abkommen löste das Abkommen über die Erleichterung der Kontrollen und Formalitäten im Güterverkehr von 1990 ab (N. 22);

- Abkommen zum Schutz von Ursprungsbezeichnungen (GUB) und geografischen Angaben (GGA) für Agrarerzeugnisse und Lebensmittel von 2011, in Kraft seit 2011 (AS 2011 5149); dieses Abkommen stellt einen integralen Bestandteil des Landwirtschaftsabkommens von 1999 dar;

- $\quad$ Abkommen zwischen der Schweiz und der EU über die Zusammenarbeit bei der Anwendung ihres Wettbewerbsrechts von 2013 (Wettbewerbsabkommen, WettbA), in Kraft seit 2014 (SR 0.251.268.1);

- Memorandum of Understanding von 2014 zur gütlichen Beilegung des Steuerstreits, d.h. der Auseinandersetzung um die Vereinbarkeit von kantonalen Steuerprivilegien für im Ausland tätige Unternehmen mit Sitz in der Schweiz mit dem Freihandelsabkommen (N. 223);

- $\quad$ Abkommen über den automatischen Informationsaustausch über Finanzkonten zur Förderung der Steuerehrlichkeit bei internationalen Sachverhalten von 2015 (AIAA), in Kraft seit 2017 (SR 0.641.926.81); dieses Abkommen - formell als Änderungsprotokoll ausgestaltet (AS 2016 5003) - löste das Zinsbesteuerungsabkommen von $2004 \mathrm{ab}$; 
- $\quad$ Abkommen zur Verknüpfung der jeweiligen Systeme für den Handel mit Treibhausgasemissionen von 2017, in Kraft seit 2020 (SR 0.814.011.268);

- diverse Abkommen über die Teilnahme der Schweiz an unionalen Agenturen, Einrichtungen und Programmen: Europol, 2004, in Kraft seit 2006 (SR 0.362.2); Eurojust, 2008, in Kraft seit 2011 (SR 0.351.6); Europäische Verteidigungsagentur, 2012 (s. für das Framework for Cooperation www.eda.admin.ch/dea und Link zu Bilaterale Abkommen und Kooperationen ab 2004/Zusammenarbeit EVA); Satellitennavigationsprogramme Galileo und EGNOS, 2013, vorläufig angewendet seit 2014 (SR 0.741.826.8); Horizon 2020, 2014, in Kraft seit 2015 (SR 0.424.11); Europäisches Unterstützungsbüro für Asylfragen (EASO), 2014, in Kraft seit 2016 (SR 0.142.392.681).

2019 wurde das Abkommen zur Vertiefung der grenzüberschreitenden Zusammenarbeit unterzeichnet (Prümer Zusammenarbeit). Es regelt die Zusammenarbeit der Polizeibehörden im Bereich des automatisierten Informationsaustauschs und befindet sich im Zustimmungsprozess in der EU und der Schweiz.

\section{Laufende Verhandlungen}

Die Schweiz und die EU diskutieren und verhandeln über den Abschluss von weiteren Abkommen. Im Vordergrund steht der geplante Abschluss des Institutionellen Abkommens. Die EU hat mehrmals klargestellt, dass sie ein solches Abkommen als conditio sine qua non betrachtet für den Abschluss weiterer Marktzugangsabkommen, welche auf EU-Recht beruhen (N. 104).

Die EU und die Schweiz verhandeln seit 2007 über den Abschluss eines Stromabkommens. Damit soll die Teilnahme der Schweiz am europäischen Strommarkt (vgl. Art. 194 AEUV) geregelt und der gegenseitige Marktzugang zwischen der Schweiz und der EU vertraglich abgesichert werden. Die Strominfrastruktur in der Schweiz, insbesondere das Übertragungsnetz und dessen Ausbau, soll weiterhin in das europäische System eingebunden sein. Die bestehenden Netze sollen koordiniert und effizient genutzt werden, womit die Netzstabilität und die Versorgungssicherheit verbessert werden. Die Schweiz soll die unionalen Vorgaben zur Förderung von Energie aus erneuerbaren Quellen übernehmen. Das Stromabkommen soll die Teilnahme der Schweiz in der Agentur für die Zusammenarbeit der Energieregulierungsbehörden (ACER) regeln. Die EU macht den Abschluss dieses Abkommens vom Abschluss des Institutionellen Abkommens abhängig (www.eda.admin.ch/dea und Link zu Verhandlungen und offene Themen). 
Seit 2008 verhandeln die Schweiz und die EU über die Schaffung von Abkommen im Bereich der Landwirtschaft, der Lebensmittelsicherheit, der Produktsicherheit und der öffentlichen Gesundheit. Damit wird bezweckt, die Akteure der Wertschöpfungskette im Agrar- und Ernährungssektor stärker zu vernetzen und die Zusammenarbeit in den Bereichen der Lebensmittel- und Produktsicherheit sowie der öffentlichen Gesundheit $\mathrm{zu}$ intensivieren (www.eda.admin.ch/dea und Link zu Verhandlungen und offene Themen). Die Verhandlungen über den Agrarfreihandel, die Lebensmittelsicherheit und die Produktsicherheit sind seit längerem sistiert. Die Verhandlungen im Bereich der Gesundheit sind demgegenüber weit fortgeschritten. Hier soll hauptsächlich die Teilnahme der Schweiz am Dispositiv der EU über grenzüberschreitende Gesundheitsgefahren, am mehrjährigen EU-Gesundheitsprogramm und am Europäischen Zentrum für die Prävention und die Kontrolle von Krankheiten (ECDC) geregelt werden (www.eda.admin.ch/dea und Link zu Verhandlungen und offene Themen). Die EU macht die Unterzeichnung des Gesundheitsabkommens von einer Lösung im Bereich der institutionellen Fragen abhängig (Antwort des Bundesrates vom 13. Februar 2019 auf die Anfrage 18.1079 «Erpressungen durch die EU im Gesundheitsbereich?»).

Die Schweiz war während der Bekämpfung der durch das Coronavirus (COVID-19) ausgelösten epidemiologischen Krise im Frühjahr 2020 bei diversen Gesprächskreisen und Koordinationsgruppen beteiligt, zu denen sie zuvor keinen Zugang hatte (N. 380).

Die Schweiz beteiligt sich seit längerem an zivilen Missionen zur Krisenbewältigung und Friedensförderung der EU im Rahmen ihrer Gemeinsamen Sicherheits- und Verteidigungspolitik (GSVP, vgl. Art. 42-43 EUV). Armeeangehörige und zivile Expertinnen und Experten aus der Schweiz nehmen an solchen Missionen teil. Bis anhin hat die Schweiz mit der EU jeweils separate Abkommen über die Modalitäten der Schweizer Einsätze in EU-Missionen abgeschlossen (vgl. z.B. für die Beteiligung der Schweiz an der Operation ALTHEA in Bosnien und Herzegowina SR 0.510.268.1). Nun ist vorgesehen, ein «Framework for Participation Agreement» (FPA) mit der EU auszuhandeln, in dem der allgemeine rechtliche und organisatorische Rahmen für künftige Schweizer Einsätze in zivilen EU-Missionen geregelt werden soll (www.eda.admin.ch/dea und Link $\mathrm{zu}$ Verhandlungen und offene Themen). Zu diesem Zweck hat der Bundesrat im Juni 2020 vorbehältlich der Konsultation der Aussenpolitischen Kommissionen des National- und Ständerates ein Verhandlungsmandat verabschiedet (Pressemitteilung des Bundesrates vom 12. Juni 2020, Frieden und Stabilität: Bundesrat will rechtlichen Rahmen für die Zusammenarbeit mit der EU bei der zivilen Friedensförderung regeln). 
Zwei Bereiche, in denen bis heute keine umfassenden Abkommen zwischen der Schweiz und der EU bestehen, betreffen den Dienstleistungsverkehr und den Kapitalverkehr. Nur vereinzelt regeln die bilateralen Abkommen Elemente der Kapitalverkehrsfreiheit (N. 121) und des freien Dienstleistungsverkehrs (N. 180-181). In diesen Bereichen laufen keine exploratorischen Gespräche oder Verhandlungen.

\section{B. Parteien}

Die bilateralen Abkommen werden zwischen der Schweiz und der EU abgeschlossen («EU only»-Abkommen). Ein Spezialfall sind gemischte Abkommen, bei denen auf der Seite der EU nicht nur die EU, sondern auch die Mitgliedstaaten als Vertragsparteien auftreten (gemischte Abkommen).

\section{1. «EU only»-Abkommen}

Die EU ist in denjenigen Sachbereichen, in denen sie über eine umfassende Vertragsschlusskompetenz verfügt, allein befugt, mit der Schweiz bilaterale Abkommen abzuschliessen (Art. 2 und Art. 3 i.V.m. Art. 216 AEUV; «EU only»Abkommen). Den Mitgliedstaaten ist es untersagt, sich in denjenigen Sachbereichen, in denen die EU ausschliesslich zuständig ist, völkerrechtlich zu binden (Sperrwirkung), wobei die Mitgliedstaaten von der EU ermächtigt werden können, vorläufig an ihrer statt tätig zu werden (Art. 2 Abs. 1 AEUV; s. Urteil Donckerwolke, 41/76, EU:C:1976:182, Rn. 31/37).

Ein Beispiel ist der Beschluss (EU) 2020/853 zur Ermächtigung Deutschlands, seine bilaterale Vereinbarung über den Strassenverkehr mit der Schweiz zur Genehmigung von Kabotagebeförderungen bei der Bereitstellung von grenzüberschreitenden Personenkraftverkehrsleistungen mit Kraftomnibussen in der Grenzregion der beiden Länder zu ändern (Erwägungsgrund 8).

Die allermeisten bilateralen Abkommen zwischen der Schweiz und der EU betreffen Sachbereiche, in denen die EU eine umfassende treaty making power besitzt. Folglich fungieren die EU und die Schweiz als Vertragsparteien; auf Seiten der EU bedürfen solche «EU only»-Abkommen der Zustimmung der zuständigen Organe der EU nach Massgabe von Art. 218 AEUV bzw. Art. 207 AEUV (gemeinsame Handelspolitik).

In der Schweiz verfügt der Bund über eine umfassende Kompetenz für die gesamten auswärtigen Angelegenheiten; dies gilt auch für Sachbereiche, welche innerstaatlich in die Zuständigkeit der Kantone fallen (G. BiAGGinI, BV-Kommentar, Art. 54 Rz. 5; s. zur Stellung der Kantone im Integrationsprozess T. COTTIER et al., Rz. 277-309; T. PFISTERER, passim). 
Bis 2009 besassen allein die E(W)G (Art. 210 EWGV, Art. 281 EGV) und die Euratom (Art. 101 Abs. 1 und Art. 184 EAGV) Rechtspersönlichkeit (Urteil AETR, C-22/70, EU:C:1971:32, Rn. 13-14). Aus diesem Grund wurden völkerrechtliche Verträge bis zum Inkrafttreten des Vertrags von Lissabon 2009 zwischen der $\mathrm{E}(\mathrm{W}) \mathrm{G}$ und/oder Euratom auf der einen Seite und Drittstaaten auf der anderen Seite abgeschlossen. Seit 2009 besitzt die EU Rechtspersönlichkeit und damit die Fähigkeit, Trägerin von völkerrechtlichen Rechten und Pflichten zu sein (Art. 47 EUV; s. auch Art. 37 EUV, Art. 3 Abs. 2 und Art. 216 AEUV). Die EU trat 2009 an die Stelle der EG, deren Rechtsnachfolgerin sie ist (Art. 1 Abs. 3 EUV). Die Abkommen zwischen der Schweiz und der E(W)G, welche vor 2009 abgeschlossen wurden, gelten automatisch weiter, ohne dass sie terminologisch - EU statt $\mathrm{E}(\mathrm{W}) \mathrm{G}$ - angepasst worden wären.

Der räumliche Geltungsbereich der bilateralen Abkommen erstreckt sich vorbehältlich abweichender Regelungen - auf die Gebiete, in denen der E(W)GV bzw. der EUV/AEUV nach Massgabe dieser Verträge angewendet werden (Art. 52 Abs. 2 EUV, Art. 355 AEUV), einerseits und auf das Hoheitsgebiet der Schweiz andererseits (s. etwa Art. 35 FHA, Art. 17 KonfBA). Im Fall des Beitritts eines Staates zur EU wird der Geltungsbereich von «EU only»-Abkommen automatisch auf das Verhältnis des neuen Mitgliedstaats zur Schweiz ausgedehnt. Im Fall des Austritts eines Staates aus der EU verlieren «EU only»Abkommen ihre Gültigkeit zwischen diesem Staat und der Schweiz. Dies ist der Fall beim Austritt des Vereinigten Königreichs aus der EU.

\footnotetext{
Die Schweiz ist bestrebt sicherzustellen, dass die bestehenden Rechte und Pflichten zwischen der Schweiz und dem Vereinigten Königreich auch nach dem Austritt des Vereinigten Königreichs aus der EU und nach Ablauf der Übergangsphase (während der die bilateralen Abkommen Schweiz-EU auch auf das Vereinigte Königreich anwendbar bleiben) weitergelten; zu diesem Zweck wurden diverse Verträge mit dem Vereinigten Königreich abgeschlossen (sog. «Mind the gap»-Strategie, www.eda.admin.ch/dea und Link zu Verhandlungen und offene Themen/Brexit; A. EPINEY, Mind the Gap, passim). Auch eine engere Zusammenarbeit im Bereich der Finanzdienstleistungen wird angestrebt (Pressemitteilung des EFD vom 30. Juni 2020).
}

43 Die bilateralen Abkommen werden in aller Regel zwischen der EU (bzw. früher der E[W]G, N. 41) und der Schweiz (terminologisch korrekt: der Schweizerischen Eidgenossenschaft, vgl. Art. $1 \mathrm{BV}$ ) abgeschlossen. Auf Seiten der EU sind ausnahmsweise die Europäische Kommission (z.B. Abkommen zur Vermeidung der Doppelbesteuerung von in der Schweiz ansässigen ehemaligen Beamten der Organe und Agenturen der EG), eine Agentur oder sonstige Einrichtung (z.B. Abkommen zwischen der Schweiz und Europol) Vertragspartnerin; vereinzelt fungiert zudem nicht nur die EU, sondern auch Euratom als Vertragspartei (z.B. Abkommen zur Assoziierung der Schweiz an Horizon 2020). Auf 
Seiten der Schweiz tritt ausnahmsweise der Bundesrat als Vertragspartner auf (z.B. Abkommen zur Vermeidung der Doppelbesteuerung von in der Schweiz ansässigen ehemaligen Beamten der Organe und Agenturen der EG).

\section{Gemischte Abkommen}

Sofern die EU nicht über eine umfassende Zuständigkeit verfügt, um einen Vertrag mit einem Drittstaat oder einer internationalen Organisation in eigener Regie abzuschliessen, bedarf es notwendigerweise der Beteiligung der Mitgliedstaaten. Diesfalls treten die EU und die Mitgliedstaaten gemeinsam als Vertragsparteien auf und machen konzertiert von ihren jeweiligen Vertragsschlusskompetenzen Gebrauch. Bei solchen Verträgen handelt es sich um gemischte Abkommen (s. zum Ganzen M. OEsch, Europarecht, Rz. 786-790). Sie bedürfen der Genehmigung und Ratifikation durch die EU und ihre Mitgliedstaaten. Völkerrechtlich binden gemischte Abkommen ohne gegenteilige Vereinbarung sowohl die EU als auch die Mitgliedstaaten (s. Art. 27 und Art. 46 WVRK).

Die folgenden Abkommen zwischen der Schweiz und der EU wurden als gemischte Abkommen abgeschlossen. Umgangssprachlich werden auch sie hinlänglich zu den «bilateralen» Abkommen gezählt, wenngleich formell zurzeit bis zu 29 Vertragsparteien beteiligt sind (wobei nur das FZA bereits von allen Mitgliedstaaten der EU ratifiziert worden ist):

- Freizügigkeitsabkommen;

- Betrugsbekämpfungsabkommen;

- $\quad$ Abkommen über die Teilnahme der Schweiz an den Europäischen Satellitennavigationsprogrammen (Galileo, EGNOS).

Im Fall des Beitritts eines Staates zur EU muss ein gemischtes Abkommen formell geändert werden, damit es auch im Verhältnis zwischen dem neuen EUMitgliedstaat und der Schweiz Geltung erlangt. Dies geschieht in der Regel in Form eines Änderungsprotokolls, das gemäss den üblichen Vorgaben im EURecht und im schweizerischen Recht genehmigt und ratifiziert werden muss. Das Freizügigkeitsabkommen wurde anlässlich der Osterweiterungen der EU von 2004, 2007 und 2013 formell auf die 13 neuen Mitgliedstaaten ausgedehnt (2005, 2009 und 2017; N. 28).

Im Fall des Austritts eines Staates aus der EU verlieren gemischte Abkommen ihre Gültigkeit zwischen diesem Staat und der Schweiz, sofern das fragliche Abkommen im Wesentlichen bilateraler Natur ist, d.h. sofern es im Wesentlichen darauf ausgerichtet ist, das Verhältnis der Schweiz zur EU insgesamt zu 
regeln (und nicht - unabhängig davon - auch vis-à-vis der einzelnen EU-Mitgliedstaaten). Dies trifft insbesondere auf das Freizügigkeitsabkommen zu: Das FZA wirkt «zu Gunsten der Staatsangehörigen der Mitgliedstaaten der Europäischen Gemeinschaft und der Schweiz» (Art. 1); es gilt «für das Hoheitsgebiet der Schweiz einerseits und die Gebiete, in denen der Vertrag zur Gründung der Europäischen Gemeinschaft Anwendung findet, und nach Massgabe jenes Vertrags andererseits» (Art. 24; s. zur «Mind the gap»-Strategie der Schweiz N. 42). In anderen Fällen kann es durchaus Konstellationen geben, in denen ein gemischtes Abkommen oder zumindest einzelne Teile davon mit Blick auf den Wortlaut und den Sinn und Zweck des Abkommens oder einzelner Bestimmungen auch nach dem Austritt eines Staates aus der EU im Verhältnis zu diesem Staat weitergelten mögen. Dies ist bei keinem der zwischen der EU und der Schweiz aktuell geltenden gemischten Abkommen der Fall.

\section{Typologie}

Die bilateralen Abkommen folgen keinem gemeinsamen Grundmuster. Sie unterscheiden sich insbesondere in Bezug auf die jeweiligen Ziele, welche sie verfolgen, und damit auch den Grad der Integration der Schweiz in den unionalen Rechtsraum, der damit einhergeht:

- Das Freihandelsabkommen stellt ein klassisches Handelsabkommen dar. Es regelt den gegenseitigen Marktzugang und beabsichtigt nicht, die Schweiz in den unionalen Binnenmarkt zu integrieren. Es ist statisch ausgestaltet und bedarf für das ordnungsgemässe Funktionieren keiner periodischen Weiterentwicklung.

- Diverse Abkommen - wie das Abkommen über die gegenseitige Anerkennung von Konformitätsbewertungen und das Landwirtschaftsabkommen - ergänzen das Freihandelsabkommen und bezwecken die weitere Erleichterung und Intensivierung des Warenhandels, ohne eine sektorielle Integration der Schweiz in den unionalen Rechtsraum anzustreben. Solche Liberalisierungsabkommen beruhen zumindest partiell auf EURecht.

- $\quad$ Einige Abkommen - wie das Freizügigkeitsabkommen, das Luftverkehrsabkommen und die Schengen/Dublin-Assoziierungsabkommen - bezwecken die sektorielle Integration der Schweiz in den unionalen Rechtsraum durch die weitgehende Angleichung des schweizerischen Rechts an dasjenige der EU. Sie räumen der Schweiz in Bezug auf die vereinbarten Rechte und Pflichten eine mitgliedstaatsähnliche Stellung ein. Der EuGH hat ausgeführt, dass mit dem Freizügigkeitsabkommen die Rechte auf die 
Personen und Unternehmen in der Schweiz «ausgedehnt» (Urteil Vereinigtes Königreich/Rat, C-656/11, EU:C:2014:97, Rn. 59 und 63) werden, womit die Schweiz punktuell «einem Mitgliedstaat gleichzustellen» (Urteil Kik, C-266/13, EU:C:2015:188, Rn. 44) ist (s. zur Lesart, wonach auch das Bundesgericht dem FZA eine neuartige Rechtsqualität zubilligt, M. OESCH, Urteilsbesprechung, S. 210-211). Der Bundesrat billigt dem Luftverkehrsabkommen die Qualität eines «partiellen Integrationsvertrags» $\mathrm{zu}$, auch unter Verweis auf die abkommensspezifischen institutionellen Vorgaben (Botschaft Bilaterale I, S. 6156; s. zu den institutionellen Vorgaben N. 75). Diese Abkommen beruhen wesentlich auf EU-Recht und verweisen extensiv auf EU-Sekundärrecht. Die schweizerischen Behörden sind gehalten, die Praxis des EuGH zur Auslegung von Parallelbestimmungen im EU-Recht zu berücksichtigen.

- Diverse Abkommen - wie das Forschungsabkommen, das Umweltabkommen, das Wettbewerbsabkommen und das Abkommen über die Teilnahme der Schweiz an den Europäischen Satellitennavigationsprogrammen (Galileo, EGNOS) - regeln die Zusammenarbeit der EU und der Schweiz in ausgewählten Politikbereichen oder ermöglichen der Schweiz, sich an unionalen Agenturen, sonstigen Einrichtungen und Programmen zu beteiligen. Dabei werden typischerweise die Rahmenbedingungen der Teilnahme der Schweiz und allfällige finanzielle Verpflichtungen vereinbart.

- Das Abkommen über bestimmte Aspekte des öffentlichen Beschaffungswesens baut im Wesentlichen auf dem WTO-Recht auf. Es erweitert den Anwendungsbereich des WTO-Übereinkommens über das öffentliche Beschaffungswesen zwischen der EU und der Schweiz.

Bei denjenigen Abkommen, welche auf EU-Recht beruhen und auf EU-Sekundärrecht verweisen, ist die Schweiz verpflichtet, die referenzierten EURechtsakte oder - zumindest auf dem Papier - gleichwertige Rechte und Pflichten anzuwenden (s. etwa Art. 1 Anhang II und Ziff. 1 Anhang III FZA; Art. 7 Abs. 1, Art. 9 Abs. 4 und Anhang 1 LandVA). Diese Abkommen werden im Einklang mit der Rechtsentwicklung in der EU periodisch aufdatiert, um das ordnungsgemässe Funktionieren nicht zu gefährden. 


\section{Verhältnis zum unionalen und schweizerischen Recht}

50 Ausgangspunkt für die Bestimmung des Verhältnisses der bilateralen Abkommen zum unionalen bzw. schweizerischen Recht ist der Grundsatz der Vertragstreue (pacta sunt servanda, Art. 26 WVRK). Sofern die EU oder die Schweiz gegen eine bilateralrechtliche Verpflichtung verstösst, treten die Rechtsfolgen der völkerrechtlichen Verantwortlichkeit ein. Die andere Vertragspartei kann gestützt auf allgemeine völkerrechtliche Grundsätze oder abkommensspezifische Vorgaben geeignete Massnahmen ergreifen (N. 98). Von der völkerrechtlichen Bindung sind die Fragen der Geltung und des Rangs der bilateralen Abkommen im unionalen bzw. schweizerischen Recht, der völkerrechtskonformen Auslegung des unionalen bzw. schweizerischen Rechts und der unmittelbaren Anwendbarkeit der bilateralen Abkommen zu unterscheiden. Dabei handelt es sich um Fragen, welche im Wesentlichen durch das jeweilige Verfassungsrecht zu beantworten sind.

Die Bestimmung des Verhältnisses zwischen den bilateralen Abkommen und unionalem bzw. schweizerischem Recht ist verknüpft mit der Frage der demokratischen Legitimation des bilateralen Wegs (s. dazu N. 59-68).

\section{Geltung und Rang}

Das EU-Recht und das schweizerische Recht beruhen auf dem Grundsatz der automatischen Geltung des Völkerrechts (Monismus; vgl. zum EU-Recht Art. 216 Abs. 2 AEUV, Urteil Haegeman, C-181/73, EU:C:1974:41, Rn. 26; zum schweizerischen Recht Art. 5 Abs. 4 BV; BGE 105 II 49; Bericht VölkerrechtLandesrecht, S. 2302-2303). Auch die bilateralen Abkommen bilden einen integralen Bestandteil der unionalen und der schweizerischen Rechtsordnung; Transformationsakte sind nicht notwendig (s. zum Spezialfall gemischter Abkommen C. KADDOUs, Zusammenarbeit, Rz. 104-111).

Die Feststellung, dass die Abkommen einen integralen Bestandteil der unionalen Rechtsordnung darstellen, hat für das EU-Recht zwei gewichtige Konsequenzen: Die bilateralen Abkommen beanspruchen als Bestandteil des EU-Rechts Vorrang vor mitgliedstaatlichem Recht. Des Weiteren ist der EuGH letztinstanzlich zuständig für die Auslegung der bilateralen Abkommen; er stellt auf diese Weise ihre einheitliche Anwendung in der gesamten EU sicher (Art. 218 Abs. 11, Art. 263 und Art. 267 AEUV; zum Rechtsschutz N. 72).

In der EU stehen völkerrechtliche Verträge gemäss ständiger Praxis des EuGH im Rang zwischen Primär- und Sekundärrecht (Urteil Kadi, C-402/05 und C-415/05, EU:C:2008:461). Es ist davon auszugehen, dass diese Rangordnung auch in Bezug auf das Verhältnis zwischen EU-Recht und bilateralen Abkommen gilt. Primärrecht inkl. der Grundrechtecharta geht im Kollisionsfall dem 
bilateralen Vertragsrecht folglich vor (zurzeit soweit ersichtlich eine hypothetische Konstellation; s. zur grundrechtskonformen Auslegung N. 78); letzteres bindet demgegenüber den EU-Gesetzgeber und die Mitgliedstaaten und beansprucht im Fall einer Kollision mit Verordnungen und Richtlinien sowie mitgliedstaatlichem Recht Vorrang (wobei für die praktische Wirksamkeit einer bilateralrechtlichen Norm die Bereitschaft der Behörden entscheidend ist, die Norm unmittelbar anzuwenden, N. 57).

In der Schweiz gilt grundsätzlich der Vorrang von Völkerrecht (vgl. Art. 5 Abs. 4 BV; zum Ganzen G. BIAGGINI, BV-Kommentar, Art. 5 Rz. 29-35). Dem Bundesgesetzgeber ist es allerdings nicht verwehrt, bewusst von einer völkerrechtlichen Verpflichtung abzuweichen; die rechtsanwendenden Behörden sind diesfalls gehalten, eine solche Entscheidung des Gesetzgebers zu akzeptieren und der völkerrechtswidrigen innerstaatlichen Norm Vorrang einzuräumen (SchubertPraxis; BGE 99 Ib 39, E. 3/4). Diese Option besteht in zwei Konstellationen nicht: Völkerrechtliche Menschenrechtsgewährleistungen, d.h. insbesondere die EMRK, gehen Bundesgesetzen in jedem Fall vor (PKK-Gegenausnahme; BGE 125 II 417, E. 4d). Weiter gilt die Schubert-Praxis «im Verhältnis zur Europäischen Union und den von der Schweiz im Freizügigkeitsrecht staatsvertraglich eingegangenen Pflichten nicht» (FZA-Gegenausnahme); daran ändert auch eine bewusste Missachtung der vertraglichen Verpflichtungen durch den Gesetzgeber nichts, wie das Bundesgericht in einem Urteil von 2015 beiläufig - d.h. als obiter dictum - ausführte (BGE 142 II 35, E. 3.2; s. G. BIAGGINI, BVKommentar, Art. 5 Rz. 32; A. EPINEY, Verhältnis, passim; A. GLASER/A. BRUNNER, passim; M. OESCH, Urteilsbesprechung, passim). Das Urteil erging im November 2015, also nach der Annahme der Masseneinwanderungsinitiative durch Volk und Stände im Februar 2014, und erteilte den Vorschlägen eine Absage, die Initiative unter Verletzung des Freizügigkeitsabkommens umzusetzen (s. zu dieser «Umsetzung» N. 63). Argumentativ beruht das Urteil auf einer nachvollziehbaren Logik: Die Schweiz beteiligt sich in den vom Abkommen erfassten Bereichen mitgliedstaatsähnlich an der unionalen Personenfreizügigkeit. Vor diesem Hintergrund ist es ein kleiner Schritt, in Analogie zum EU-Recht von einem umfassenden Vorrang des Abkommens auszugehen und auf diese Weise sein ordnungsgemässes Funktionieren zu sichern. Allerdings äusserte sich das Bundesgericht nicht zur besonderen Stellung der Schweiz im europäischen Integrationsprozess. Die Schweiz ist ein Drittstaat ohne institutionelle Einbindung und direkte Einflussmöglichkeit auf die Weiterentwicklung des EU-Rechts. Auch die Tatsache, dass in der EU mit dem Vorabentschei- 
dungsverfahren ein Mechanismus zur Verfügung steht, mit dem die Gerichte den EuGH mit Gültigkeits- und Auslegungsfragen befassen können, zu dem schweizerische Gerichte aber keinen Zugang haben, wurde nicht thematisiert.

Die bundesgerichtliche Begründung für den Vorrang des FZA löst eine Fülle von Fragen aus, welche vorderhand unbeantwortet bleiben. Es ist ungeklärt, ob der Vorrang auch gegenüber direkt anwendbarem Verfassungsrecht wirkt. Die Ausführungen des BGer enthalten jedenfalls keine Anhaltspunkte, weshalb dies nicht der Fall sein sollte. Es ist ungeklärt, ob sich der Vorrang auch auf andere bilaterale Abkommen erstreckt. Offensichtliche Kandidaten sind das Luftverkehrsabkommen und die Schengen/Dublin-Assoziierungsabkommen, welche ähnlich wie das FZA eine Integration in den unionalen Rechtsraum vorsehen, im Unterschied zu letzterem aber keine Rechtsangleichung qua Grundfreiheiten avisieren. Es ist ungeklärt, ob der Vorrang - wie vom BGer suggeriert - unbedingt wirkt, oder ob das BGer sich vorbehält, in Ausnahmefällen den Vorrang zu verneinen und die schweizerische Norm anzuwenden. Es wäre analog zu den vom deutschen Bundesverfassungsgericht entwickelten Vorbehalten zum Vorrang des EU-Rechts (M. OESCH, Europarecht, Rz. 134-146, 335-344; s. zuletzt das Urteil des BVerfG vom 5. Mai 2020, 2 BvR 859/15 u.a., in dem der Beschluss der EZB zum Staatsanleiheprogramm - trotz des anderslautenden Urteils des EuGH in der Rs. Weiss, C-493/17, EU:C:2018:1000 - als Ultra-vires-Massnahme qualifiziert wurde) oder auch zu den vom Liechtensteinischen Staatsgerichtshof entwickelten Vorbehalten zum Vorrang des EWR-Rechts (StGH 2013/196) denkbar, dass das BGer im Kollisionsfall einen EU-Rechtsakt nicht anwendet, weil er gegen elementare Grundrechtsgewährleistungen verstösst, oder ein EuGH-Urteil nicht befolgt, weil der EuGH die Grenzen der zulässigen Auslegung offensichtlich überschritten hat.

\section{Völkerrechtskonforme Auslegung}

Ein wirkmächtiges Instrument zur Vermeidung von Kollisionen zwischen Völkerrecht und innerstaatlichem Recht - zu dem in diesem Kontext auch EURecht gehört - ist die völkerrechtskonforme Auslegung. Die rechtsanwendenden Behörden sind gehalten, unionales bzw. schweizerisches Recht soweit als möglich, d.h. im Rahmen der anerkannten Auslegungsmethoden, unter Berücksichtigung des einschlägigen Völkerrechts auszulegen und anzuwenden (s. allg. zur völkerrechtskonformen Auslegung im EU-Recht Urteil Bosphorus, C-84/95, EU:C:1996:312, Rn. 11-18; im schweizerischen Recht BGE 94 I 669, 678). Die völkerrechtskonforme Auslegung findet ihre normative Grundlage im völkerrechtlichen Grundsatz der Vertragstreue und im völkerrechtlichen Anspruch auf Vorrang vor innerstaatlichem Recht (Art. 26 und Art. 27 WVRK). Diese Auslegungsmaxime ist auch im Kontext der bilateralen Abkommen anwendbar (T. CotTier/E. Evtimov, S. 188-189; C. Tobler/J. BEglinger, Grundzüge, Rz. 100). völkerrechtskonforme Auslegung belassen, besteht eine Kollision. Den rechts- 
anwendenden Behörden bleibt diesfalls nichts anderes übrig, als die einschlägigen Kollisionsregeln fruchtbar zu machen, d.h. primär die Regeln zum Vorrang und zur unmittelbaren Anwendbarkeit.

\section{Unmittelbare Anwendbarkeit}

Verwaltungsbehörden und Gerichte in der Schweiz und in der EU bejahen die unmittelbare Anwendbarkeit eines völkerrechtlichen Vertrags, sofern der Vertrag mit Blick auf seine Art und Struktur einer unmittelbaren Anwendbarkeit nicht entgegensteht und die fragliche Bestimmung inhaltlich hinreichend bestimmt und unbedingt formuliert - kurzum: justiziabel - ist (s. allg. zur unmittelbaren Anwendbarkeit im EU-Recht M. OESCH, Europarecht, Rz. 809-817; im schweizerischen Recht BGE 136 I 297, 308, E. 8.1; H. KELLER, passim; D. WÜGER, Anwendbarkeit, passim). Diese Voraussetzungen gelten auch bei der Bestimmung der unmittelbaren Anwendbarkeit der bilateralen Abkommen.

Der EuGH bejaht die unmittelbare Anwendbarkeit der bilateralen Abkommen grosszügig. Dies gilt unbestrittenermassen für die justiziellen Bestimmungen der Bilateralen I und II. Der EuGH hat sich bis heute nicht zur unmittelbaren Anwendbarkeit des Freihandelsabkommens geäussert. Mit Blick auf seine langjährige Praxis, die Eignung von Freihandelsabkommen zur unmittelbaren Anwendbarkeit mit Blick auf ihre Rechtsnatur und Systematik grundsätzlich zu bejahen (Urteil Kupferberg, C-104/81, EU:C:1982:362, Rn. 17-26), ist davon auszugehen, dass er auch das Freihandelsabkommen mit der Schweiz unmittelbar anwenden würde.

Das Bundesgericht bejaht die unmittelbare Anwendbarkeit des Versicherungsabkommens (BGE 138 I 378, E. 10; N. 185) sowie der Bilateralen I und II (T. JAAG/ J. HÄNNI， Rz. 4020-4022; D. ThÜRER/C. HILlEMANNS， Rz.12-15; s. betr. FZA N. 155; betr. LVA N. 212). Dabei sind auch EU-Richtlinien, auf die in einem Abkommen verwiesen wird, unmittelbar anwendbar; die Schweiz verzichtet in pragmatischer Weise häufig darauf, solche Richtlinien ins schweizerische Recht umzusetzen (s. betr. FZA N. 170; C. KROPF, S. 93). In Bezug auf das Freihandelsabkommen zeigt sich das Bundesgericht zurückhaltender (T. COTTIER/ E. EvTimOV, S. 190-198). Es hat dem Abkommen die unmittelbare Anwendbarkeit wiederholt abgesprochen, wobei hier vor allem auf den Sinn und Zweck des Abkommens als «reines Handelsabkommen» abgestellt wurde (BGE 104 IV 175, E. 2c; BGE 105 II 49, E. 3b; s. auch BGE 118 Ib 367, E. 6b; einzig die Ursprungsregeln hat das BGer unmittelbar angewendet, s. BGE 112 IV 53, E. 2/ 3; BGE 114 Ib 168). In jüngeren Urteilen hat das Bundesgericht die unmittelbare 
Anwendung zumindest für gewisse Bereiche nicht mehr kategorisch ausgeschlossen (Urteil 2A.593/2005 vom 6. September 2006, E. 5.4; s. auch Urteil des BVerwG A-8382/2007 vom 29. September 2008, E. 11; die Eidg. Zollrekurskommission hielt bereits 2001 fest, dass das Verbot diskriminierender Steuern gemäss Art. 18 FHA unmittelbar anwendbar ist, VPB 66.44, E. 5a/ bb; dazu N. 127). Eine Kehrtwende scheint das Bundesgericht in einem Urteil von 2005 vollzogen zu haben, in dem es eine Abgabe umfassend auf ihre Vereinbarkeit mit dem Freihandelsabkommen überprüfte (ohne die unmittelbare Anwendbarkeit allerdings ausdrücklich zu bejahen, BGE 131 II 271, E. 10; dazu D. WÜGER, Freihandelsabkommen, passim). Die Praxis in der Schweiz präsentiert sich insgesamt uneinheitlich und wenig gefestigt. Sie ist aus individualrechtlicher Sicht problematisch. Unbefriedigend ist die zögerliche Anerkennung der unmittelbaren Anwendbarkeit staatsvertraglich garantierter Marktzugangsrechte umso mehr, als die verfassungsrechtlich gewährleistete Aussenwirtschaftsfreiheit in der Praxis nur über beschränkte Wirkkraft verfügt (M. OEsCH, Aussenwirtschaftsrecht, Rz. 30.31-30.33).

\section{E. Seitenblick: Bilaterale Abkommen und direkte Demokratie}

Die Bestimmung des Verhältnisses zwischen den bilateralen Abkommen und unionalem bzw. schweizerischem Recht ist verknüpft mit der Frage der demokratischen Legitimation des bilateralen Wegs. Diese Frage wird in der Schweiz mit Verve diskutiert. Verkompliziert wird die Sachlage durch das helvetische Spezifikum, wonach das Volk und mitunter auch die Stände nicht nur über die grundlegenden europapolitischen Weichenstellungen befinden (Volksinitiative, Staatsvertragsreferendum), sondern auch das letzte Wort haben über einzelne Weiterentwicklungen der bilateralen Abkommen (Staatsvertragsreferendum). Ein Blick auf die Praxis der letzten fünf Jahrzehnte offenbart zwei Tendenzen: Zum einen wird der bilaterale Weg an der Urne regelmässig bestätigt. Zum anderen wird die Substanz der demokratischen Rechte bei Vorlagen, welche einen direkten Bezug zu den bilateralen Abkommen haben, eingeschränkt, weil sich die Rahmenbedingungen für ihre Ausübung verändert haben.

\section{Hohe demokratische Legitimation}

60 Die direkt-demokratische Mitsprache, d.h. das Recht, Initiativen und Referenden zu initiieren und zu unterzeichnen und darüber abzustimmen, zählt zu den tragenden Strukturelementen der schweizerischen Verfassungsordnung (Art. 139-141 BV). Sie spielt auch in den Aussenbeziehungen eine bedeutsame 
Rolle. Volk und Stände können mittels einer Volksinitiative aussenpolitische Entwicklungen anstossen. Der Beitritt der Schweiz zur UNO 2002 wurde auf diese Weise in die Wege geleitet (heute Art. 197 Ziff. 1 BV). Das Staatsvertragsreferendum - 1921 eingeführt, 1977 und 2003 ausgebaut (L. LANGER, S. 28-39) - erlaubt dem Volk, einem völkerrechtlichen Vertrag die Zustimmung zu versagen und damit korrigierend in die Gestaltung der Aussenbeziehungen einzugreifen. Auf diese Weise geniesst das aussenpolitische Handeln der Schweiz innerstaatlich eine hohe demokratische Legitimation.

Die Volksrechte sind auch für die schweizerische Europapolitik prägend. 1972 stimmten Volk und Stände dem Abschluss des Freihandelsabkommens zu (N. 22). 1992 lehnten Volk und Stände den von der Bundesversammlung sowie von allen grossen Parteien ausser der SVP und weiten Teilen der wirtschaftlichen Elite befürwortete Beitritt zum EWR ab (N. 26). Seither bestätigten die Stimmbürgerinnen und Stimmbürger den bilateralen Weg wiederholt in Referendumsabstimmungen (N. 28, N. 30, N. 93 und N. 328). Verfassungsrechtlich wurde die Frage nach dem Verhältnis zwischen der Schweiz und Europa im Rahmen von zwei Volksinitiativen von 1997 und 2001 explizit aufgeworfen. Sowohl die Volksinitiative «EU-Beitrittsverhandlungen vors Volk!», welche den Abbruch bisher eingeleiteter Beitrittsverhandlungen mit der EU verlangt und die Aufnahme von neuen Verhandlungen von der Zustimmung von Volk und Ständen abhängig gemacht hätte, als auch die Volksinitiative «Ja zu Europa!», welche den Bund zur Aufnahme von Beitrittsverhandlungen verpflichtet hätte, wurden abgelehnt. Einen direkten Bezug zur Europapolitik hatte auch die 2018 verworfene Volksinitiative «Schweizer Recht statt fremde Richter (Selbstbestimmungsinitiative)». Sie sah den Vorrang der Bundesverfassung gegenüber dem Völkerrecht (mit Ausnahme von ius cogens) vor; im Falle eines Widerspruchs hätten die völkerrechtlichen Verpflichtungen an die Bundesverfassung angepasst werden müssen, nötigenfalls durch Kündigung der betreffenden völkerrechtlichen Verträge (A. AUER et al., passim). Die Volksinitiative «Für eine massvolle Zuwanderung (Begrenzungsinitiative)», welche die Kündigung der Personenfreizügigkeit mit der EU verlangte, wurde 2020 von Volk und Ständen verworfen.

Die Tatsache, dass der Abschluss bilateraler Abkommen unter dem Vorbehalt der Zustimmung durch das Volk steht, wirkt sich auch auf die Verhandlungen aus. Die Unterhändlerinnen und Unterhändler der Schweiz und der EU wissen, dass das Vereinbarte nicht nur im Bundesrat und im Parlament, sondern auch im Volk mehrheitsfähig sein muss. Vor diesem Hintergrund ist das Beharren der EU, die Bilateralen I durch eine Guillotine-Klausel zu verbinden, verständlich; es beruhte auf der berechtigten Befürchtung, dass andernfalls allein das Freizügigkeitsabkommen eine Referendumsabstimmung hätte überstehen müssen (N. 28). 
In den letzten Jahren nahmen Volk und Stände mehrere Volksinitiativen an, welche punktuell nicht mit den bilateralen Abkommen - insbesondere mit dem Freizügigkeitsabkommen - vereinbar waren. 2010 wurde die Volksinitiative «für die Ausschaffung krimineller Ausländer (Ausschaffungsinitiative)» angenommen, welche auf eine Verschärfung der Wegweisungspraxis ohne Berücksichtigung des Einzelfalls abzielte (Art. 121 Abs. 3-6 und Art. 197 Ziff. 8 BV). Sofern Ausländerinnen und Ausländer wegen eines namentlich aufgeführten Straftatbestands rechtskräftig verurteilt worden sind oder missbräuchlich Leistungen der Sozialversicherungen oder der Sozialhilfe bezogen haben, verlieren sie automatisch ihr Aufenthaltsrecht und müssen aus der Schweiz ausgewiesen werden. Der Gesetzgeber führte im Rahmen der Umsetzung dieser neuen Verfassungsbestimmungen zwar eine Härtefallklausel ein (Art. 66a Abs. 2 StGB), womit eine einzelfallgerechte Beurteilung zwingend geboten ist. Darüber hinaus ist es aber den Gerichten überantwortet, tatsächlich für eine EMRK- und FZA-konforme Anwendung zu sorgen (s. BGE 139 I 16; zum Ganzen G. BIAGGinI, BV-Kommentar, Art. 121 Rz.16-22; A. EPINEY, Völkerrecht, passim; J. REICH, Demokratie, passim; zur Praxis des BGer zum Verhältnis von Art. 66a und Art. $66 a^{\text {bis }}$ StGB zum FZA N. 168).

2014 nahmen Volk und Stände die Volksinitiative «Gegen Masseneinwanderung» an, welche den bilateralen Weg in seiner jetzigen Form ernsthaft in Frage stellte. Die neu eingeführten Art. 121a und Art. 197 Ziff. 11 BV sind offenkundig gegen die Weiterführung des Freizügigkeitsabkommens gerichtet, auch wenn sie dieses Abkommen nicht ausdrücklich benennen. Sie verlangen, die Zuwanderung von Ausländerinnen und Ausländern eigenständig zu steuern und zu diesem Zweck jährliche Höchstzahlen und Kontingente sowie einen Schweizervorrang bei der Stellensuche einzuführen; völkerrechtliche Verträge, welche diesen Vorgaben widersprechen, sind innert drei Jahren neu zu verhandeln und anzupassen (s. zum Ganzen G. BIAGGINI, Umsetzung, passim; N. MusLIU, S. 255-306; M. OESCH, Europa-Artikel, S. 170-174). Nachdem die EU verlautete, zwar Hand zu bieten für einen Austausch über «practical problems related to the implementation of the Agreement» (Brief der Hohen Vertreterin der EU für die GASP, Catherine Ashton, vom 24. Juli 2014, www.eda.admin.ch/ dea und Link zu Personenfreizügigkeit), nicht aber für eine formelle Neuverhandlung des Abkommens, achtete die Bundesversammlung bei der «Umsetzung» der Verfassungsbestimmungen darauf, das Freizügigkeitsabkommen nicht zu verletzen; sie führte den Inländervorrang light ein (Art. 21a AIG). Damit gewichtete die Bundesversammlung den Grundsatz pacta sunt ser- 
vanda höher als den verfassungsrechtlichen Auftrag zur eigenständigen Steuerung der Zuwanderung; ein europapolitisch begrüssenswertes, verfassungsrechtlich aber problematisches Vorgehen.

Für zusätzliche Spannung sorgte während der Debatte über die Umsetzung der Masseneinwanderungsinitiative der Zwischenruf aus Lausanne, wonach die Schubert-Praxis «im Verhältnis zur Europäischen Union und den von der Schweiz im Freizügigkeitsrecht staatsvertraglich eingegangenen Pflichten nicht» gelte (BGE 142 II 35, E. 3.2; N. 53). Dieses Diktum erteilte den Vorschlägen, die Initiative unter Verletzung des FZA umzusetzen, apodiktisch eine Absage.

Initiativkomitees sind gut beraten, bei der Formulierung von Initiativtexten in der Form des ausgearbeiteten Entwurfs (Art. 139 Abs. 2 BV) die Folgen für die bilateralen Abkommen mit der EU klar zu benennen und den Behörden entsprechende Handlungsanweisungen zu geben (vgl. T. NAEF, S. 264-270, wonach ein völkerrechtlicher Vertrag nur gekündigt werden soll, wenn der Initiativtext dies ausdrücklich vorsieht). Die Stimmbürgerinnen und Stimmbürger haben ein Recht darauf, transparent über die konkreten Anliegen einer Initiative und die rechtlichen Konsequenzen einer Annahme auch in Bezug auf den europapolitischen Kurs der Schweiz informiert zu werden. In diesem Sinne war die 2020 abgelehnte Begrenzungsinitiative (N. 61) konsequent.

\section{Aushöhlung der Substanz der demokratischen Rechte}

Die fortschreitende Globalisierung und Europäisierung des Rechts führt zu Spannungen im schweizerischen Verfassungsgefüge. Die Substanz der demokratischen Rechte wird eingeschränkt, weil sich die Rahmenbedingungen für ihre Ausübung verändern (s. zum Ganzen T. COTTIER/D. WÜGER, passim; O. DigGelmanN, Internationalisierung, S. 67-121; M. OESCH, Verfassungswandel, passim). Das von Dani Rodrik beschriebene «Globalisierungsparadox» wonach Demokratie, nationale Selbstbestimmung und wirtschaftliche Globalisierung nicht gleichzeitig verwirklicht werden können (D. RODRIK. S. 184-206) - trifft auch auf die Schweiz zu (J. REICH, Switzerland, S. 298).

Dies zeigt sich paradigmatisch bei Vorlagen an die Stimmbürgerinnen und Stimmbürger, welche einen direkten Bezug zu den bilateralen Abkommen mit der EU haben (ähnlich M. ScHOTT, S. 702-704):

- Beim Referendum steht das Volk mitunter unter Druck, ein neues Abkommen oder eine abkommensrelevante Weiterentwicklung des EU-Rechts «durchzuwinken», weil ihm die Opportunitätskosten einer Ablehnung welche mit jeder Bestätigung des eingeschlagenen Kurses steigen 
(L. LANGER, S. 48) - schlicht zu hoch erscheinen. Formell bleibt das Staatsvertragsreferendum und damit das Recht des Volkes, auch in europapolitischen Belangen das letzte Wort zu haben, bestehen; praktisch existiert eine tatsächliche Wahlfreiheit zeitweise nur auf dem Papier. So stellte der Bundesrat 2008 mit Blick auf die Abstimmung über die Weiterführung des Freizügigkeitsabkommens und dessen Ausdehnung auf Bulgarien und Rumänien fest: «Von der Zustimmung zu den beiden Vorlagen hängt für die Schweiz letztlich die Weiterführung des bilateralen Wegs ab.» (Botschaft Weiterführung FZA, S. 2136). In Bezug auf das Freizügigkeitsabkommen besteht gleichsam ein «faktisches Kündigungsverbot» (A. GLASER, Wandel, S. 479). Ähnliches gilt auch für die Weiterentwicklung des Schengen/Dublin-Besitzstandes: Im Vorfeld der Abstimmung von 2009 über die Schengen-relevante Einführung biometrischer Pässe führte der Bundesrat aus: «Die Einführung von E-Pässen schafft (...) die Voraussetzung dafür, dass (...) die Schweiz nicht wieder aus dem Schengen-Raum ausgeschlossen wird.» (Volksabstimmung vom 17. Mai 2009, Erläuterungen des Bundesrates [«Bundesbüchlein»], S. 15; s. zum Übernahmemechanismus beim SAA/DAA N. 93 und N. 95; zu dieser Abstimmung N. 250). 2013 stellte der Bundesrat fest, dass bei Schengen-Weiterentwicklungen zwar kein «Demokratiedefizit» bestehe, sich aber gleichwohl «eine gewisse Relativierung der Entscheidungsautonomie der Schweiz und damit auch der Volksrechte de facto aus dem Umstand ergibt, dass eine allfällige Nichtübernahme einer Weiterentwicklung mitunter mit erheblichen Nachteilen verbunden sein kann, würde doch im äussersten Fall die Zusammenarbeit von Schengen und Dublin als Ganzes beendet» (Bericht Schengen 2013, S. 6394-6395).

- Auch das Initiativrecht verliert an Wirkung. In bilateralrechtlich überdachten Bereichen reduziert sich seine Wirkmacht faktisch darauf, ein Zeichen zu setzen und den Bund zu verpflichten, in den von den bilateralen Abkommen gesetzten Grenzen tätig zu werden. Das Initiativrecht kommt im Bereich der Europapolitik nurmehr dort uneingeschränkt zur Geltung, wo keine bilateralrechtlichen Vorgaben bestehen, sowie als Instrument dafür, im Grundsätzlichen über die Teilnahme der Schweiz am europäischen Integrationsprozess zu befinden.

67 Der Bedeutungsverlust der Volksrechte in bestimmten Bereichen löst mit Blick auf das in der Schweiz vorherrschende Demokratieverständnis Unbehagen aus. Patentlösungen, dieses Unbehagen zu beseitigen, gibt es nicht: 
- Dies gilt insbesondere für das Referendumsrecht. Das naheliegende Rezept, nämlich die Einschränkung des Referendumsrechts in Konstellationen, in denen heikle europapolitische Weichenstellungen zur Debatte stehen - etwa durch die Ausnahme der Beschlüsse zur Übernahme von EU-Recht vom Referendum - ist kaum ratsam. Die Stimmbürgerinnen und Stimmbürger würden entmündigt; sie würden gleichsam vor der eigenen Unverfrorenheit, eine Übernahme abzulehnen, geschützt, was in breiten Kreisen kaum goutiert würde (vgl. auch O. DigGelmanN, Scherbenhaufen, Rz. 13).

- $\quad$ Mit Blick auf das Initiativrecht wäre es zwar denkbar, die Ungültigkeitsgründe zu erweitern und etwa Initiativtexte, welche gegen die bilateralen Abkommen verstossen, Volk und Ständen nicht vorzulegen (s. für prüfwürdige Ungültigkeitsgründe, v.a. auch im Licht der EMRK, Bericht Völkerrecht-Landesrecht, S. 2328-2337; Zusatzbericht Völkerrecht-Landesrecht, S. 3632-3653; B. EhrENZELler/G. GERTSCH, Art. 139 Rz. 53; H. Keller/M. Lanter/A. Fischer, S. 149; R. Kiener/M. KRÜSI, S. 252). Allerdings wäre eine derart grundlegende Änderung des etablierten Systems des Initiativrechts mit diversen Fragezeichen behaftet (Praktikabilität, Akzeptanz, Auswirkungen auf das politische System insgesamt).

Letztlich bleibt auf absehbare Zeit nichts anderes übrig, als sich im aktuellen System der direkten Demokratie zurechtzufinden. Urnengänge, bei denen europapolitische Sachzwänge eine vorbestimmte Stimmabgabe nahelegen, sind die unvermeidliche Folge davon, dass sich die Schweiz - zu Recht - für die sektorielle Beteiligung am unionalen Binnenmarkt und an weiteren Politiken der EU entschieden hat und in mitgliedstaatsähnlicher Weise in das EURecht eingebunden ist. Das demokratische Unbehagen, welches sich bei solchen Urnengängen einstellen mag, gehört zum Preis, den die Schweiz dafür bezahlt, von den Segnungen der europäischen Integration zu profitieren. Dabei gehört es zu den Daueraufgaben der Behörden, der Parteien und der Verbände, den Stimmbürgerinnen und Stimmbürgern die Vorteile der engen Anbindung an die EU wie auch ihre Nachteile aufzuzeigen und auf diese Weise den Boden für informierte Sachentscheidungen an der Urne zu legen. Das System der direkten Demokratie in der Schweiz muss in der Lage sein, die aufgezeigten Spannungen auszuhalten. 



\section{Institutionelle Ausgestaltung}

\section{A. Durchführung, Überwachung, Rechtsschutz}

Der bilaterale Acquis Schweiz-EU kennt keinen allgemeingültigen institutionellen Rahmen. Jedes Abkommen enthält ein eigenes Set an Regeln für die Durchführung, die Überwachung und den Rechtsschutz. Die Parteien sind grundsätzlich selbst dafür verantwortlich, das ordnungsgemässe Funktionieren der Abkommen sicherzustellen (Zweisäulenprinzip). Sie treffen sich in Gemischten Ausschüssen, um sich auszutauschen und - im Idealfall - Unstimmigkeiten auszuräumen. Eine Unterstellung unter gemeinsame Instanzen gibt es - etwa im Gegensatz zum EWR-Recht, wo der EWR/EFTA-Pfeiler gemeinsame Behörden kennt (EFTA-Überwachungsbehörde, EFTA-Gerichtshof) nicht. Es werden keine supranationalen Elemente eingeführt. Mit Ausnahme der Schengen/Dublin-Assoziierungsabkommen und des Zollabkommens enthalten die Abkommen zudem keine Vorgaben für die Übernahme von EURecht (N. 93).

Die ordnungsgemässe Durchführung der Abkommen, ihre Überwachung und die Gewährung von Rechtsschutz obliegen im jeweiligen Hoheitsgebiet den Vertragsparteien. Sie sind verpflichtet, alle geeigneten Massnahmen zu treffen, um die Erfüllung der sich aus den Abkommen ergebenden Verpflichtungen zu gewährleisten, und sich aller Massnahmen zu enthalten, welche die Erreichung der mit den Abkommen verfolgten Ziele gefährden (s. etwa Art. 22 FHA, Art. 17 LVA).

\section{Europäische Union}

In der EU obliegt die Durchführung der Abkommen primär den Mitgliedstaaten (s. zur unmittelbaren Anwendbarkeit der Abkommen N. 57). Die Europäische Kommission überwacht die ordnungsgemässe Umsetzung und Anwendung der Abkommen durch die Mitgliedstaaten (Art. 17 Abs. 1 EUV, Art. 291 Abs. 1 AEUV). Zu diesem Zweck steht ihr die Vertragsverletzungsklage zur Verfügung (Art. $258 \mathrm{AEUV}$ ).

Griechenland erhob in den Jahren vor 2010 auf Dividenden, welche von griechischen Tochtergesellschaften an ihre Schweizer Muttergesellschaften bezahlt wurden, eine Quellensteuer von $10 \%$. Die Europäische Kommission erachtete diese Praxis als nicht vereinbar mit dem Zinsbesteuerungsabkommen von 2004. Sie übermittelte Griechenland eine Stellungnahme gemäss Art. 258 AEUV mit der Aufforderung, dieses Abkommen zu respektieren (Pressemitteilung der Kommission vom 30. September 2010, IP/10/1253). - 2011 ordnete 
die Europäische Kommission eine Untersuchung gegen Italien an, weil sie Anhaltspunkte dafür hatte, dass Italien gegen das bilaterale Abkommen über bestimmte Aspekte des öffentlichen Beschaffungswesens verstiess. Daraufhin beschloss der italienische Wirtschafts- und Finanzminister, die umstrittenen Massnahmen gegen Schweizer Unternehmen aufzuheben (Stellungnahme des Bundesrates vom 18. Mai 2011 zur Motion 11.3157 «Beziehungen zwischen der Schweiz und Italien. Wogen glätten»). In beiden Fällen wurden die Unstimmigkeiten beigelegt, ohne dass der EuGH involviert werden musste.

Rechtsschutz wird durch die mitgliedstaatlichen Gerichte und den Gerichtshof der EU gewährt (Art. 19 Abs. 1 EUV, Art. 267 AEUV). Bis heute drehten sich die meisten Urteile des Gerichtshofs der EU - rund 30 - zu den bilateralen Abkommen um die Auslegung des Freizügigkeitsabkommens; der EuGH wurde dabei qua Vorabentscheidungsverfahren mit der Auslegung dieses Abkommens befasst (s. zu den Urteilen bis 2016 M. OESCH/G. SPECK, S. 265-268). Ausnahmsweise ist die Kommission oder eine sonstige Einrichtung der EU (z.B. Agenturen) für die Durchführung zuständig; dies ist etwa der Fall beim Luftverkehrsabkommen und beim Wettbewerbsabkommen. Diesfalls wird der Rechtsschutz direkt durch den Gerichtshof der EU gewährt (Art. 263 AEUV).

\section{Schweiz}

In der Schweiz fällt die Durchführung der bilateralen Abkommen je nach der betroffenen Materie in die Zuständigkeit der Kantone oder des Bundes (T. JAAG/M. ZIHLMANN, Rz. 25; s. zur unmittelbaren Anwendbarkeit der Abkommen N. 58).

Referenzierte EU-Rechtsakte, welche nicht unmittelbar anwendbar sind, werden in der Schweiz stufengerecht (Art. 164 BV; s. zur Umsetzungspflicht der Kantone Art. 7 BGMK) und unter Berücksichtigung der Grundsätze der hiesigen Rechtsetzungstechnik (www.bk.admin.ch und Link zu Übernahme von EU-Recht/Faustregeln) eingepasst. Dabei gelten mutatis mutandis - bei der Durchführung der bilateralen Abkommen ist die Schweiz verpflichtet, die unionalen Rechtsakte oder (zumindest auf dem Papier) gleichwertige Vorschriften anzuwenden - die gleichen Grundsätze wie beim autonomen Nachvollzug (N. 351-353).

Auch die Gewährung von Rechtsschutz erfolgt entweder auf der Stufe der Kantone oder auf der Stufe des Bundes. In jedem Fall steht die Beschwerde an das Bundesverwaltungsgericht oder das Bundesgericht offen (Art. 31f. VGG, Art. 82ff. und Art. 113ff. BGG). Die schweizerischen Gerichte verfügen über keine Möglichkeit, den EuGH im Wege der Vorabentscheidung mit Auslegungsfragen zu befassen, was etwa mit Blick auf den unbedingten Vorrang des Freizügigkeitsabkommens nicht ideal ist (N. 53). Gleichwohl haben die schweizerischen Gerichte Strategien entwickelt, um die Praxis des EuGH zum EURecht einfliessen zu lassen und auf diese Weise sicherzustellen, dass die 
Homogenität der Rechtsanwendung im Regelfall sichergestellt ist. Sie legen die bilateralen Abkommen, die auf EU-Recht beruhen und deren ratio legis dahin geht, die Schweiz sektoriell in den unionalen Binnenmarkt zu integrieren und eine parallele Rechtslage zu schaffen, gestützt auf die spezifisch EUrechtlichen Auslegungsmethoden und mit Blick auf die Präjudizien des EuGH aus (N. 83). Des Weiteren warten die schweizerischen Gerichte mit der Klärung einer Rechtsfrage im schweizerischen Kontext mitunter bewusst zu, wenn der EuGH sich zu dieser Frage demnächst im Kontext des EU-Rechts äussern wird (s. für ein Beispiel einer solchen Strategie - wenngleich im Rahmen des autonomen Nachvollzugs ergangen - das Urteil des BVerwG vom 13. September 2010, B-3064/2008; N. 362). Dieses Vorgehen illustriert den typisch schweizerischen Pragmatismus, mit dem die Gerichte in der Schweiz den Rechtsschutz im Rahmen der bilateralen Abkommen handhaben.

\section{Spezielle Regelungen}

Abweichend vom Grundsatz, wonach die Vertragsparteien für die Durchführung der Abkommen zuständig sind, obliegt die Durchführung des Luftverkehrsabkommens partiell auch vis-à-vis der Schweiz den zuständigen Organen der EU, insbesondere der Europäischen Kommission und der Europäischen Agentur für Flugsicherheit (Art. 11 und Art. 18 Abs. 2 LVA, Verordnung 216/2008 zur Festlegung gemeinsamer Vorschriften für die Zivilluftfahrt und zur Errichtung einer Europäischen Agentur für Flugsicherheit; s. Art. $42 a$ KG zur Zuständigkeit bei Untersuchungen in Verfahren in der Schweiz gestützt auf das LVA). Der Gerichtshof der EU ist diesfalls für die Gewährung des Rechtsschutzes zuständig (Art. 20 LVA; zum Fluglärmstreit N. 214).

Eine spezielle Klausel enthält auch das Abkommen zwischen der Schweiz und der EU über zusätzliche Regeln in Bezug auf das Instrument für die finanzielle Unterstützung für Aussengrenzen und Visa im Rahmen des Fonds für die innere Sicherheit für den Zeitraum von 2014 bis 2020 von 2018 (SR 0.362.314, dazu N. 266). Demnach ist allein der EuGH befugt, die Vollstreckung einer Entscheidung der Kommission, welche andere Rechtspersonen als Staaten zu einer Zahlung verpflichten, auszusetzen (Art. 5). 


\section{B. Auslegung}

\section{Im Allgemeinen}

Die bilateralen Abkommen werden gestützt auf die Auslegungsmethoden gemäss Art. 31-33 WVRK ausgelegt; diese Bestimmungen kodifizieren geltendes Völkergewohnheitsrecht (s. etwa BGE 133 V 329, E. 8.4; BGE 132 V 53, E. 6.3; Urteil Hengartner und Gasser, C-70/09, EU:C:2010:430, Rn. 36; Urteil Wächtler, C-581/17, EU:C:2019:138, Rn. 35; zum Ganzen T. BURRI/B. PIRKER, passim; T. CotTier/N. Diebold, passim; A. EPiney/B. Metz/B. PirKer, Parallelität, passim). Ausgehend vom Wortlaut und gestützt auf Treu und Glauben steht die gewöhnliche Bedeutung einer Norm im Licht ihres Zusammenhangs sowie ihres Ziels und Zwecks im Vordergrund. Der für die Sinnermittlung erforderliche Zusammenhang kann sich aus weiteren Übereinkünften ergeben; gleichermassen zu berücksichtigen ist die Praxis zur Anwendung der Verträge. Dieser Methode liegt ein vornehmlich objektiver Ansatz zugrunde; es gilt der im Völkerrecht etablierte Grundsatz der vertragsautonomen Auslegung, womit die einheitliche Auslegung und Anwendung in den Vertragsstaaten gewährleistet werden soll.

Die bilateralen Abkommen enthalten keine Grundrechtskataloge. Ebenso wenig verweisen sie direkt auf die EMRK, die Grundrechtecharta der EU oder andere Grundrechtsinstrumente (s. für die Nennung der EMRK in den Präambeln der Schengen/Dublin-Assoziierungsabkommen N. 246 und N. 281). Mitunter verpflichten EU-Rechtsakte, welche qua Verweis im bilateralen Verhältnis anwendbar sind, ausdrücklich zur Beachtung der Grundrechtecharta, womit sich die Frage stellt, ob und, sofern dies der Fall ist, inwieweit solche Kaskadenverweisungen dazu führen, dass das grundrechtliche Schutzsystem der EU auch im bilateralen Verhältnis direkt wirkt:

- $\quad$ Der EuGH dürfte nicht zögern, die Abkommen bzw. ihre Durchführung im EU-Recht auf die Vereinbarkeit mit den unionalen Grundrechten zu überprüfen und eine grundrechtskonforme Anwendung einzufordern (vgl. zur Grundrechtsrelevanz im Allgemeinen Urteil Kadi, C-402/05 P und C-415/ 05 P, EU:C:2008:461; Gutachten Abkommen PNR EU-Kanada, 1/15, EU:C:2017:592; zum Vorrang des unionalen Primärrechts vor Völkerrecht N. 52).

- $\quad$ Die Gerichte in der Schweiz legen die bilateralen Abkommen durchwegs im Licht völkerrechtlicher Grundrechtsgewährleistungen aus; im Vordergrund steht die EMRK (s. BGE 136 II 177, E. 3.2.2; BVGE 2012/27; im erstge- 
nannten Entscheid wird zudem auch auf die Kinderrechtskonvention von 1989 [SR 0.107] Bezug genommen). Darüber hinaus ist durchaus vorstellbar, dass die schweizerischen Behörden bei Bedarf auch auf die Grundrechtecharta rekurrieren; dies gilt bei denjenigen Abkommen, welche auf EU-Recht beruhen und die sektorielle Integration der Schweiz in den unionalen Rechtsraum bezwecken, grundsätzlich unabhängig davon, ob referenzierte Rechtsakte ausdrücklich auf die Grundrechtecharta verweisen oder nicht (A. EPINEY, EU-Grundrechte, passim; M. OESCH/T. NAEF, passim). Ansätze in diese Richtung zeigen sich vor allem in der Praxis des Bundesverwaltungsgerichts zur Auslegung der Dublin III-Verordnung, welche im Dublin-Assoziierungsabkommen referenziert wird; hier verweist das Bundesverwaltungsgericht regelmässig auf die Grundrechtecharta (N. 282).

Spezielle Fragen stellen sich bei der Auslegung von Bestimmungen, welche EU-rechtlichen Normen nachgebildet sind oder solche Normen wörtlich übernehmen (2.). Vereinzelt enthalten Abkommen spezielle Regeln zur Berücksichtigung der Praxis in der EU (3.).

\section{Sinngemäss oder wörtlich übernommenes EU-Recht}

Diverse Abkommensbestimmungen sind ähnlich oder wortgleich formuliert wie die Parallelbestimmungen im EU-Recht. Dies gilt offenkundig für unionale Rechtsakte, welche tel quel in den bilateralen Acquis übernommen werden. Diesfalls stellt sich die Frage, ob dergestalt parallele Bestimmungen in gleicher Weise ausgelegt werden sollen oder ob ihr allenfalls unterschiedlicher Normgehalt nahelegt, eine autonome Lesart zu wählen und die Praxis des EuGH zum Gemeinschafts- bzw. Unionsrecht nicht auf das bilaterale Verhältnis zu übertragen.

Der EuGH hat bei der Auslegung von Freihandels- und Assoziierungsabkommen mit Drittstaaten wiederholt betont, dass eine parallele Auslegung nur dann in Betracht kommt, wenn der Zweck und der Kontext der Vertragsbestimmung, insbesondere auch mit Blick auf die Integrationstiefe des Vertrags, vergleichbar sind mit dem Zweck und dem Kontext der gemeinschafts- bzw. unionsrechtlichen Bestimmung. Diese mittlerweile jahrzehntealte Praxis geht auf das Urteil Polydor, 270/80, EU:C:1982:43, zurück (und wird entsprechend als Polydor-Prinzip bezeichnet; C. TOBLER, Polydor, S. 369). Sie ist gemäss EuGH auch bei der Auslegung der bilateralen Abkommen mit der Schweiz einschlägig. Der EuGH führte 2009 aus, dass «die den gemeinschaftsrechtlichen 
Bestimmungen über den Binnenmarkt gegebene Auslegung nicht automatisch auf die Auslegung des [Freizügigkeitsabkommens] übertragen werden [kann], sofern dies nicht im Abkommen selbst ausdrücklich vorgesehen ist» (Urteil Grimme, C-351/08, EU:C:2009:697, Rn. 29; s. auch Urteil Hengartner und Gasser, C-70/09, EU:C:2010:430, Rn. 41-42; Urteil Picart, C-355/16, EU:C:2018:184, Rn. 29).

Im Fluglärmstreit zwischen der Schweiz und Deutschland entschied der EuGH, dass die unionsrechtlich gewährleistete Dienstleistungsfreiheit (Art. 56 AEUV) nicht unbesehen auf das LVA übertragen werden kann. Er wies darauf hin, dass die Schweiz nach der Ablehnung des EWR-Beitritts «nicht dem Binnenmarkt der Union beigetreten [ist], mit dem alle Hindernisse beseitigt werden sollen, um einen Raum vollständiger Freizügigkeit entsprechend einem nationalen Markt zu schaffen, der u. a. die Dienstleistungsfreiheit umfasst (...). Deshalb kann die den unionsrechtlichen Vorschriften über den Binnenmarkt gegebene Auslegung nicht automatisch auf die Auslegung des Luftverkehrsabkommens EG-Schweiz übertragen werden, sofern dies nicht im Abkommen selbst ausdrücklich vorgesehen ist (...).» Der EuGH stellte fest, dass das LVA keine dem Unionsrecht nachgebildete Dienstleistungsfreiheit enthält, womit die Praxis des EuGH zu Art. 56 AEUV nicht auf das LVA übertragen werden kann (Urteil Fluglärmstreit, C-547/10 P, EU:C:2013:139, Rn. 78-81; s. dazu auch N. 214).

Sofern eine bilaterale Norm ähnlich oder wortgleich formuliert ist wie die Parallelnorm im EU-Recht und Zweck und Kontext der Normen vergleichbar sind, scheint der EuGH - zumindest mit Blick auf seine neuere Praxis - von der Vermutung einer parallelen Auslegung auszugehen. Die meisten Fälle, mit denen sich der EuGH dabei bis anhin befasste, betreffen das Freizügigkeitsabkommen. Er zögert nicht, die bilateralen Abkommen zugunsten einer beschwerdeführenden Partei auszulegen und Rechte daraus abzuleiten, wenn sich eine solche Lesart im Licht der anerkannten Auslegungsmethoden als sachgerecht erweist; dabei greift er auch auf seine Praxis zum EU-Recht zurück (s. Urteil Stamm und Hauser, C-13/08, EU:C:2008:774, Rn. 30-49; Urteil Graf und Engel, C-506/10, EU:C:2011:643, Rn. 22-36; Urteil Ettwein, C-425/11, EU:C:2013:121， Rn. 41-49; A. EPINEY, Brexit, Rz. 5; M. OESCH/G. SPECK, S. 265-268).

Dasselbe Muster zeigt sich in der Praxis des Bundesgerichts. Es legt die bilateralen Abkommen, die auf EU-Recht beruhen und deren ratio legis dahin geht, die Schweiz sektoriell in den unionalen Rechtsraum zu integrieren und eine parallele Rechtslage zu schaffen, gestützt auf die spezifisch EU-rechtlichen Auslegungsmethoden und mit Blick auf die Präjudizien des EuGH zu unionalen Parallelbestimmungen aus. In der Praxis des Bundesgerichts stehen ebenfalls Fälle zum Freizügigkeitsabkommen im Vordergrund. Auch bei der Auslegung der Schengen/Dublin-Assoziierungsabkommen bzw. der darin referenzierten 
Rechtsakte weichen die schweizerischen Behörden «nicht ohne stichhaltige Gründe» von der EuGH-Rechtsprechung ab (Urteil des BVerwG vom 2. Juli 2015, E-594/2015, E. 6.4, betr. DAA). Gleichzeitig betont das Bundesgericht, dass gewisse bilaterale Abkommen je nach Ausgestaltung und Zielsetzung einen im Vergleich zum EU-Recht weniger weitgehenden Integrationsgrad anstreben und folglich nicht automatisch analog zu unionsrechtlichen Parallelbestimmungen auszulegen sind. Dies gilt mithin für das Freihandelsabkommen von 1972. In einem Urteil von 2005 bestätigte das Bundesgericht, dass das Freihandelsabkommen «grundsätzlich autonom auszulegen und anzuwenden» ist; gleichzeitig wies es darauf hin, dass die Praxis des EuGH zu EU-rechtlichen Parallelbestimmungen «nicht unbeachtlich» ist (BGE 131 II 271, E. 10.3; s. auch BGE 118 Ib 367, E. 6; zur Frage, ob gestützt auf Art. 13 FHA das Cassis de Dijon-Prinzip gilt, N. 130).

Der EuGH hat eine parallele Auslegung von Art. 30 und Art. 36 EWGV (Art. 34 und Art. 36 AEUV) und den identisch formulierten Bestimmungen in Freihandelsabkommen (vgl. Art. 13 und Art. 20 FHA) in Bezug auf das Immaterialgüterrecht (Verbot von Parallelimporten) unter Rekurs auf den im Vergleich zum EWG-Recht unterschiedlichen Integrationsgrad von Freihandelsabkommen wiederholt verneint (Urteil Polydor, C-270/80, EU:C:1982:43, betr. FHA EWG-Portugal). Diese Entscheidung gilt als Präjudiz auch für die Schweiz: Das BGer lehnte eine analoge Behandlung von Parallelexporten unter dem EWGV und dem FHA ab (BGE 105 II 49, E. 3; s. dazu auch N. 130). Es führte aus, das FHA sei «ein reines Handelsabkommen (...), das nicht wie der EWG-Vertrag einen einheitlichen Binnenmarkt mit überstaatlicher Wettbewerbsordnung, sondern bloss eine Freihandelszone schaffen will. Es beschränkt sich zudem im Wesentlichen auf den industriellen Freihandel. Bei seiner Aushandlung wurde nicht nur eine Pflicht zur gegenseitigen Angleichung der gemeinschaftlichen und schweizerischen Rechtsnormen bewusst ausgeschlossen; die bestehenden Rechtsordnungen und deren uneingeschränkte autonome Durchsetzung wurden vielmehr gegenseitig vorbehalten (...). Das Abkommen sieht auch kein Organ vor, das wie der Europäische Gerichtshof als Institution der EWG die unmittelbare Anwendbarkeit einzelner Normen für die Vertragsparteien verbindlich festlegen könnte. (...) Diese Unterschiede sind auch bei der Auslegung einzelner Bestimmungen zu beachten, weshalb es entgegen den Einwänden der Beklagten nicht angeht, die Rechtsprechung des Europäischen Gerichtshofes zu analogen Bestimmungen des EWG-Vertrages unbesehen zu übernehmen. Die Schweiz wird durch das Abkommen nicht gezwungen, ihre Wirtschaftspolitik und innere Gesetzgebung mit derjenigen der EWG zu harmonisieren, mag es auch nahe liegen, in konkreten Fällen für gleichartige Probleme ähnliche Lösungen wie die Nachbarstaaten anzustreben (...); dies ändert jedoch nichts daran, dass der schweizerische Richter das Abkommen seinem handelspolitischen Charakter und Zweck entsprechend autonom auszulegen und anzuwenden hat.» 


\section{Positivrechtliche Regelungen}

Das Freizügigkeitsabkommen und das Luftverkehrsabkommen verpflichten die Behörden in der Schweiz und der EU, die Praxis des EuGH zur Auslegung von Parallelbestimmungen im EU-Recht zu beachten. Gemäss Art. 16 Abs. 2 FZA ist die Rechtsprechung des EuGH vor dem Zeitpunkt der Unterzeichnung zu berücksichtigen, «[s]oweit für die Anwendung dieses Abkommens Begriffe des Gemeinschaftsrechts herangezogen werden». Art. 1 Abs. 2 LVA verlangt, die Bestimmungen, welche im Wesentlichen mit dem EU-Recht übereinstimmen, «in Übereinstimmung mit den vor der Unterzeichnung dieses Abkommens erlassenen Urteilen, Beschlüssen und Entscheidungen des Gerichtshofs und der Kommission der Europäischen Gemeinschaften auszulegen.» Beide Bestimmungen begründen in Bezug auf Urteile, welche nach dem Stichtag des 21. Juni 1999 erlassen wurden, eine Informationspflicht; gegebenenfalls stellt der Gemischte Ausschuss die Folgen fest. Die Schengen/Dublin-Assoziierungsabkommen thematisieren die Auslegung des Sekundärrechts, ohne eine Bindungswirkung zu stipulieren (Art. 8 und Art. 9 SAA, Art. 5 und Art. 6 DAA). All diese Bestimmungen sind im Verhältnis zu Art. 31-33 WVRK leges speciales.

Das Bundesgericht hat sich wiederholt zur Tragweite von Art. 16 Abs. 2 FZA geäussert (grundlegend BGE 136 II 5, E. 3.4, dazu auch N. 163; s. auch BGE 136 II 65, E. 3/4; BGE 136 II 177, E. 3.2; BGE 139 II 393, E. 4; BGE 142 II 35, E. 3). Demnach ist die Alt-Rechtsprechung des EuGH, d.h. die Rechtsprechung vor dem Zeitpunkt der Unterzeichnung des Abkommens, zu Parallelbestimmungen im EU-Recht «massgebend» und damit als verbindlich zu betrachten; es besteht eine «Befolgungspflicht» (BGE 139 II 393, E. 4.1.1). In Bezug auf die NeuRechtsprechung des EuGH, d.h. die Rechtsprechung nach dem Zeitpunkt der Unterzeichnung des Abkommens, besteht keine Berücksichtigungspflicht, sondern «höchstens ein Beachtungsgebot in dem Sinn, dass diese [Urteile] nicht ohne sachliche Gründe unbeachtet bleiben sollen, aber aus der Sicht der Vertragspartner auch nicht zu einer nachträglichen Änderung des Vertragsinhalts führen dürfen» (BGE 139 II 393, E. 4.1.1). In der Praxis des Bundesgerichts spielt die Datumsgrenze, also die Unterscheidung zwischen Alt- und NeuRechtsprechung des EuGH, eine untergeordnete Rolle. Das Bundesgericht berücksichtigt regelmässig nicht nur die Präjudizien des EuGH, welche vor dem Zeitpunkt der Unterzeichnung des Abkommens ergangen sind, sondern ganz grundsätzlich das Fallrecht des EuGH zu Fragen, welche sich im bilateralen Verhältnis in analoger Weise stellen. Im Ergebnis findet eine «dynamische Rechtsprechungsübernahme» (A. ZÜND, S. 1357) statt. Bilaterale Freizügigkeitsrechte, die wörtlich oder sinngemäss Unionsrecht nachgebildet sind und die 
den Zweck verfolgen, eine parallele Rechtslage zu schaffen, sind im Regelfall in Anlehnung an die Präjudizien des EuGH auszulegen. Davon abweichende Auslegungsresultate bleiben in Ausnahmefällen zwar möglich und rechtlich zulässig. Sie bedürfen in Kenntnis der Sachlage aber einer triftigen Begründung.

Das BGer führte im leading case zur Tragweite von Art. 16 Abs. 2 FZA aus, es würde auch die nach dem Stichtag vom 21. Juni 1999 ergangene Rechtsprechung des EuGH «in seine Beurteilung einbeziehen und ihnen Rechnung tragen» (BGE 136 II 5, E. 3.4). Dabei sei es möglich, «aus triftigen Gründen» zu einer anderen Rechtsauffassung zu gelangen, wenngleich das BGer «dies aber mit Blick auf die angestrebte parallele Rechtslage nicht leichthin tun» würde (E. 3.4). In casu folgte das BGer einem neuen Urteil des EuGH (Urteil Metock, C-127/08, EU:C:2008:449), in dem der EuGH in ausdrücklicher Abkehr von einem früheren Urteil (Urteil Akrich, C-109/01, EU:C:2003:491) entschied, dass sich ein Drittstaatsangehöriger, welcher der Ehegatte einer Unionsbürgerin ist, die sich in einem Mitgliedstaat aufhält, dessen Staatsangehörigkeit sie nicht besitzt, vor seiner Einreise in den Aufnahmemitgliedstaat nicht bereits rechtmässig in einem anderen Mitgliedstaat aufgehalten haben muss, um sich auf die Unionsbürgerrichtlinie berufen zu können. Das BGer führte aus, dass es sich dem Urteil Akrich «trotz einer gewissen Skepsis vor allem aus Gründen der Rechtsharmonisierung» angeschlossen hatte, und fuhr fort: «Analoge Überlegungen sprechen für eine Übernahme der angepassten Rechtsprechung. Es sind keine triftigen Gründe erkennbar, weshalb es innerhalb der Europäischen Gemeinschaft und in deren Verhältnis mit der Schweiz zwei unterschiedliche Freizügigkeitsregelungen geben sollte. Das Interesse an einer parallelen Rechtslage und mithin an einem möglichst einheitlichen Freizügigkeitsraum geht vielmehr vor.» (E. 3.6.2). Dies galt umso mehr, wie sich «[d]en Gründen für die Änderung der Rechtsprechung durch den EuGH die Überzeugungskraft nicht absprechen» lässt (E. 3.7).

Das Bundesgericht ist bis heute noch nie ausdrücklich von einem EuGH-Urteil abgewichen, weil sich eine davon abweichende, autonome Auslegung als die sachlich naheliegendere erwiesen hätte. Abweichungen drängen sich gemäss Bundesgericht allerdings immer dann auf, wenn eine bilaterale Norm einen anderen Zweck verfolgt als eine ähnlich oder wortgleich formulierte Bestimmung des Unionsrechts, d.h. wenn die Zielsetzung nicht darin liegt, das in der Union geltende Recht auf das Verhältnis zur Schweiz auszuweiten. Dies ist zumindest gemäss Bundesgericht - der Fall bei den Voraussetzungen, welche erfüllt sein müssen, damit eine strafrechtliche Landesverweisung mit dem Freizügigkeitsabkommen vereinbar ist: Das Bundesgericht legt Art. 5 Anhang I FZA «strafrechtlich» nicht analog zur Praxis im EU-Recht aus, weil es sich bei diesem Abkommen «um ein im Wesentlichen wirtschaftsrechtliches Abkommen handelt» (Urteil 6B_378/2018 vom 22. Mai 2019, E. 3.4.4.); bei dieser Argumentation bleibt allerdings manches unklar (N. 168). Eine Abweichung drängt sich gemäss Bundesgericht ebenfalls auf, wenn Ansprüche nicht (primär) gestützt auf die klassischen Freizügigkeitsrechte (i.V.m. dem allgemeinen Diskriminierungsverbot) weiterentwickelt werden, sondern gestützt auf «die 
Bestimmungen über die Unionsbürgerschaft und deren Kernbereich»; «dasselbe gilt für mit der Richtlinie 2004/38/EG neu eingeführte Rechte für die Unionsbürger wie etwa den bedingungslosen Anspruch auf Daueranwesenheit nach ununterbrochenem fünfjährigem (rechtmässigem) Aufenthalt (...) oder das voraussetzungslose Aufenthaltsrecht von bis zu drei Monaten» (BGE 139 II 393, E. 4.1.2; s. auch BGE 136 II 5, E. 3.6.3; BGE 136 II 65, E. 4.2). Die Unionsbürgerschaft ist ein Institut zur «Schaffung einer immer engeren Union der Völker Europas» (Präambel zum EUV), welches im Verhältnis Schweiz-EU nicht greift.

Zur Tragweite von Art. 1 Abs. 2 LVA (N. 84) hat sich das Bundesgericht noch nicht geäussert. Es ist davon auszugehen, dass die Grundzüge der bundesgerichtlichen Praxis zur Auslegung des Freizügigkeitsabkommens in Bezug auf Urteile des EuGH, welche nach dem Stichtag vom 21. Juni 1999 ergangen sind, auch hier greifen: Es gilt ein Beachtungsgebot; von einschlägigen EuGH-Urteilen soll nur bei Vorliegen triftiger Gründe abgewichen werden (C. TOBLER, Fluggastrechte, S. 519).

Das Bezirksgericht Bülach wählte in einem Urteil von 2016 bei der Auslegung des LVA und der darin referenzierten Fluggastrechteverordnung (EG) Nr. 261/2004 (N. 212) einen anderen Weg: Es folgte einem Urteil des EuGH (Rs. Sturgeon, C-402/07, EU:C:2009:716) bewusst nicht, weil «die vom EuGH eingeführte Rechtsprechung faktisch eine Gesetzesänderung zur Folge hat» (E. 4.2.14). Dabei äusserte sich das Bezirksgericht weder zu den Spezifika bei der Auslegung von EU-Recht (es verwies bei der Darlegung der Auslegungsmethoden vielmehr auf den Methodenpluralismus bei der Auslegung von schweizerischem Gesetzesrecht, E. 4.2.7) noch zur systemischen Bedeutung einer parallelen Auslegung für das gute Funktionieren des LVA; auch setzte es sich nicht mit der bundesgerichtlichen Praxis zur Auslegungsregel gemäss Art. 16 FZA auseinander (Urteil vom 2. Februar 2016, FV150044-C/ U AB/ad; s. auch Stellungnahme des Bundesrates vom 29. August 2018 zur Motion 18.3568 «Rechtssicherheit in den Flugpassagierrechten sicherstellen»; kritisch zu diesem Urteil T. PROBST, S. 783-793).

Ein Seitenblick auf das Lugano-Übereinkommen - kein bilaterales Abkommen zwischen der EU und der Schweiz, sondern ein plurilaterales Abkommen zwischen der EU, Dänemark und den EFTA-Staaten, das sich als Parallelabkommen eng an das in der EU geltende Recht zur gerichtlichen Zuständigkeit und zur Anerkennung und Vollstreckung von Entscheidungen in Zivil- und Handelssachen anlehnt - offenbart in Bezug auf die Bedeutung der EuGH-Rechtsprechung für die Auslegung ein ähnliches Schema. Um eine möglichst einheitliche Auslegung des LugÜ und des EU-Rechts sicherzustellen, wurde eine spezielle Auslegungsregel vereinbart: Art. 1 des Protokolls Nr. 2 zum LugÜ verpflichtet die Gerichte der Vertragsstaaten, bei der Anwendung und Auslegung des LugÜ den Grundsätzen gebührend Rechnung zu tragen, die in massgebenden Entscheidungen von Gerichten der anderen Vertragsstaaten entwickelt 
worden sind; in der Praxis stehen Urteile des EuGH dabei ganz im Vordergrund. Gemäss Bundesgericht ist es «unerheblich, ob die europäische Rechtsprechung vor oder nach dem Inkrafttreten des Lugano-Übereinkommens (...) ergangen ist. Eine Differenzierung verbietet sich schon deshalb, weil sonst das mit dem Lugano-Übereinkommen angestrebte Ziel, die Schweiz in einen europäischen Raum vereinheitlichter Gerichtszuständigkeiten in Zivil- und Handelssachen einzubinden, untergraben würde. Der Rechtsprechung des EuGH ist daher bei der Auslegung des Lugano-Übereinkommens grundsätzlich zu folgen. Eine abweichende Auslegung bleibt nur dann vorbehalten, wenn die europäische Rechtsprechung eindeutig an den Zielen der Europäischen Union orientiert ist, welche die Schweiz nicht mitträgt» (BGE 135 III 185, E. 3.2; s. zum Ganzen C. OETIKER/T. WeIBEL, passim).

Ganz vereinzelt wirkt die Bezugnahme auch in umgekehrter Richtung: Der EuGH orientierte sich in einem Urteil von 2009 an einem Urteil des BGer vom 9. November 2004 und erklärte, ihm nach Massgabe von Art. 1 des Protokolls Nr. 2 zum LugÜ gebührend Rechnung zu tragen (Urteil Gambazzi, C-394/07, EU:C:2009:219, Rn. 35).

\section{Einseitige Erklärungen}

Vereinzelt enthalten Abkommen einseitige Erklärungen zur Auslegung. So erklärte die EWG beim Abschluss des Freihandelsabkommens im Anhang zur Schlussakte, sie würde Art. 23 Abs. 1 FHA (Kartell- und Beihilferecht) auf der Grundlage der Kriterien beurteilen, welche sich aus der Anwendung der Bestimmungen des EWGV (Art. 85, Art. 86, Art. 90 und Art. 92 EWGV; Art. 101, Art. 102, Art. 106 und Art. 107 AEUV) ergeben. Die EU rekurrierte im Steuerstreit mit der Schweiz auf diese Erklärung (N. 223).

\section{Gemischte Ausschüsse}

Die meisten Abkommen sehen die Einsetzung von Gemischten Ausschüssen vor, welche für die Verwaltung und das ordnungsgemässe Funktionieren der Abkommen verantwortlich sind. Zurzeit existieren 24 Gemischte Ausschüsse. Sie setzen sich aus einer Delegation der Schweiz und einer Delegation der EU (sowie im Fall von gemischten Abkommen zusätzlich aus Vertreterinnen und Vertretern der Mitgliedstaaten) zusammen. Sie treten bei Bedarf, mindestens einmal jährlich, zusammen und beschliessen in traditionell diplomatischer Manier im gegenseitigen Einvernehmen, d.h. einstimmig (s. etwa Art. 30 Abs. 2 FHA, Art. 14 Abs. 1 FZA, Art. 21 Abs. 1 LVA). Die Aufgaben der Gemischten Ausschüsse bestimmen sich nach den jeweiligen vertraglichen Vorgaben: 
- Information und Konsultation: In den Gemischten Ausschüssen werden Informationen bezüglich Anpassungen und Änderungen von internen Rechtsvorschriften, welche Auswirkungen auf das ordnungsgemässe Funktionieren eines Abkommens haben können, ausgetauscht (s. etwa Art. 14 Abs. 3 und Art. 17 FZA, Art. 7 Abs. 1 ÖffBA);

- Überwachung: Eine zentrale Rolle spielt die Überwachung der Praxis der Vertragsparteien im Anwendungsbereich der Abkommen, um die einheitliche Anwendung zu sichern (s. etwa Art. 1 Abs. 2 LVA, Art. 16 Abs. 2 FZA);

- Abhilfemassnahmen: Gemischte Ausschüsse können ausserordentliche Massnahmen beschliessen, sofern sich solche aufgrund schwerwiegender Probleme oder Störungen aufdrängen (s. etwa Art. 14 Abs. 2 FZA, Art. 47 LandVA);

- Diplomatische Streitbeilegung: Die Vertragsparteien diskutieren Fälle, in denen eine Vertragspartei der anderen vorwirft, gegen ein Abkommen zu verstossen, und suchen nach Lösungen (s. etwa Art. 19 FZA, Art. 54 LandVA; zu den Folgen für den Fall, dass keine gütliche Lösung gefunden wird, N. 98);

- Weiterentwicklung: Gewisse Abkommen übertragen dem Gemischten Ausschuss die Kompetenz, Regelungen in den Anhängen zu ändern (N. 92).

\section{Weiterentwicklung}

Diverse bilaterale Abkommen reichen über Fragen des gegenseitigen Marktzugangs und der zwischenstaatlichen Zusammenarbeit hinaus und bezwecken die sektorielle Integration der Schweiz in den unionalen Rechtsraum. Das Freizügigkeitsabkommen, das Luftverkehrsabkommen und die Schengen/DublinAssoziierungsabkommen ermöglichen der Schweiz, sektoriell an das EU-Recht anzudocken, und stellen Bindeglieder zum sich dynamisch weiterentwickelnden EU-Recht dar (s. zur Typologie N. 48). Diverse weitere Abkommen verweisen ebenfalls auf EU-Rechtsakte, welche dergestalt tel quel Eingang in das bilaterale Recht finden; ihre Anwendbarkeit wird auf die Schweiz «ausgedehnt» (Urteil Vereinigtes Königreich/Rat, C-656/11, EU:C:2014:97, Rn. 59 und 63). Es ist offenkundig, dass Änderungen von solchen Rechtsakten im EU-Recht auch im bilateralen Verhältnis ihren Niederschlag finden sollten, damit das gute Funktionieren der Abkommen nicht gefährdet wird. Entsprechend stellt die unkomplizierte und zügige Anpassung der Abkommen an das EU-Recht ein zentrales institutionelles Postulat dar. 
Zeitweise ist es bei der Ausarbeitung und beim Erlass eines neuen Rechtsaktes in der EU unklar, ob der Rechtsakt auch für den Acquis Schweiz-EU bedeutsam ist (s. zur Kontroverse, ob die Unionsbürgerrichtlinie 2004/38/EG eine Weiterentwicklung des FZA darstellt, N. 96 und N. 109). Ganz vereinzelt wird im Titel eines Rechtsaktes oder in den Erwägungsgründen darauf hingewiesen, dass es sich um einen «Text von Bedeutung für den EWR und die Schweiz» handelt (wobei dieser - ausschliesslich informative - Zusatz in Bezug auf die EWR-Relevanz seit jeher beigefügt wird); Beispiele hierfür sind die Verordnung (EG) Nr. 883/2004 zur Koordinierung der Systeme der sozialen Sicherheit und die Verordnung (EU) 2019/1149 zur Errichtung einer Europäischen Arbeitsbehörde (s. dazu auch N. 194). Allein bei Rechtsakten, welche für die Assoziierung der Schweiz am Schengen/Dublin-Besitzstand bedeutsam sind, wird in den Erwägungsgründen seit jeher und konsequent ein entsprechender Hinweis angebracht.

Die gewandelte Natur staatsvertraglicher Beziehungen zwischen der Schweiz und der EU findet in den Abkommen nur ansatzweise eine institutionelle Entsprechung. Die Abkommen folgen einem tradierten Verständnis nationalstaatlicher Souveränität und stecken formell in klassisch völkerrechtlichen Kleidern. Sie verpflichten die Vertragsparteien zur Beachtung der Rechte und Pflichten, wie sie sich zum Zeitpunkt eines gewissen Stichtags - in der Regel zum Zeitpunkt der Unterzeichnung des Abkommens - präsentieren. Die Abkommen sind statisch ausgestaltet. Es besteht - von wenigen Ausnahmen abgesehen (N. 93) - keine Verpflichtung der Schweiz, neue EU-Rechtsakte dynamisch zu übernehmen. Änderungen der Abkommen bedürfen der Zustimmung der Parteien:

- $\quad$ Änderungen der Abkommen unterliegen in der Schweiz und der EU dem ordentlichen Verfahren der Vertragsgebung. Dies gilt insbesondere in Bezug auf die Änderung der eigentlichen Vertragstexte (im Gegensatz zur Übernahme von EU-Rechtsakten in die Anhänge). Bei gemischten Abkommen müssen auch die EU-Mitgliedstaaten nach Massgabe ihrer verfassungsrechtlichen Vorgaben zustimmen (N. 44), was mitunter zu Verzögerungen und zur Vermischung mit sachfremden Begehrlichkeiten führt.

- Einzelne Abkommen übertragen dem Gemischten Ausschuss die Kompetenz, in eigener Regie Regelungen in den Anhängen (Art. 23 Abs. 4 LVA, Art. 18 FZA, Art. 21 Abs. 2 ZollA) wie auch - ausnahmsweise - der Abkommen selbst (Art. 21 Abs. 2 ZollA) zu ändern. Auf Seiten der Schweiz fasst ein Aide-mémoire der Bundesverwaltung von 2007 die Vorgehensweise zur internen Vorbereitung von Beschlüssen der Gemischten Ausschüsse und die Kompetenzen zur Genehmigung von Beschlüssen zur Weiterentwicklung der Abkommen zusammen (abgedruckt in: T. COTTIER et al., Rz. 176-177). Vermutungsweise ist von einer Zuständigkeit des Bundesrates auszugehen. Beschlüsse, welche Materien betreffen, die in die 
Kompetenz der Bundesversammlung fallen, oder Anpassungen von Bundesgesetzen erfordern, müssen dem Parlament vorgelegt werden und unterstehen dem fakultativen Referendum. Dasselbe gilt für Beschlüsse, welche weitreichende institutionelle Vorkehren enthalten oder Kontrollund Sanktionsaufgaben auf EU-Organe übertragen. Auf Seiten der EU regelt der Beschluss 2002/309/EG/Euratom des Rates (N. 28) die Verfahren der internen Entscheidfindung im Vorfeld eines Beschlusses durch einen Gemischten Ausschuss im Rahmen der Bilateralen I.

Die Schengen/Dublin-Assoziierungsabkommen und das Zollabkommen enthalten spezifische Regelungen zur dynamischen Übernahme von EU-Rechtsakten durch die Schweiz. Die Schweiz ist verpflichtet, die notwendigen Schritte zur Anpassung des betroffenen Abkommens zügig an die Hand zu nehmen:

- Sofern die Schweiz der Übernahme neuer Rechtsvorschriften, welche zum Schengen/Dublin-Besitzstand gehören und der Schweiz von der EU notifiziert werden, nicht zustimmt und sich der Gemischte Ausschuss innert 90 Tagen nicht auf eine einvernehmliche Lösung einigt, gilt das betroffene Abkommen - und kraft Verknüpfung der beiden Abkommen auch das andere (Art. 15 Abs. 4 SAA, Art. 14 Abs. 2 DAA) - als beendet (Art. 7 SAA, Art. 4 DAA). Diese Frist ist so berechnet, dass die in der Schweiz verfassungsrechtlich vorgesehenen Kompetenzen - u.U. inklusive eines Referendums - respektiert werden. Bis heute wurde in zwei Fällen das fakultative Referendum ergriffen: 2009 wurde über die Schengen-relevante Einführung der biometrischen Pässe und Reisedokumente und die Übernahme der Verordnung (EG) Nr. 2252/2004 abgestimmt; das Stimmvolk nahm die Vorlage hauchdünn mit $50.1 \%$ der abgegebenen Stimmen an (N. 250). 2019 wurde das Referendum gegen die Übernahme der Richtlinie (EU) 2017/853 zur Änderung der Waffenrichtlinie 91/477/EWG in das Schengen-Assoziierungsabkommen ergriffen; 63.7 \% der Stimmberechtigten votierten für die Übernahme und die Umsetzungsgesetzgebung (N. 262). Eine Ausnahme zur strikten Folge der Beendigung des Schengen-Assoziierungsabkommens besteht für den Fall einer Änderung des Prinzips der doppelten Strafbarkeit im Bereich der direkten Steuern (Art. 7 Abs. 5 SAA i.V.m. Art. 51 SDÜ).

- Sofern die Schweiz der Übernahme neuer Rechtsvorschriften, welche für das Zollabkommen einschlägig sind, nicht zustimmt, kann die EU angemessene Ausgleichsmassnahmen einschliesslich der Aussetzung der 
Anwendung gewisser Bestimmungen ergreifen (Art. 29 ZollA). Es besteht die Möglichkeit, die Verhältnismässigkeit solcher Massnahmen durch ein Schiedsgericht überprüfen zu lassen (N. 99).

Vereinzelt regeln die bilateralen Abkommen - gleichsam als «institutionelle[s] Gegengewicht» (Bericht Schengen 2013, S. 6332) zur Übernahme von EURecht - den Einbezug von Sachverständigen aus der Schweiz bei der Vorbereitung neuer Rechtsakte in der EU (proposal shaping). Einige Abkommen enthalten allgemeine Vorgaben über die Beteiligung von Expertinnen und Experten aus der Schweiz bei der Erarbeitung von neuen Rechtsakten; andere Abkommen regeln ihre Teilnahme in Ausschüssen und bei der Vorbereitung von Durchführungsmassnahmen der Kommission (s. etwa Art. 23 LVA, Art. 22 ZollA, Art. 52 Abs. 2 LandVA, Erklärung des Rates der EU in den Schlussakten der bilateralen Abkommen von 1999, Art. 6 SAA, Art. 2 DAA). Eine Vereinbarung legt die Modalitäten der Beteiligung der am Schengen-Besitzstand assoziierten Staaten an Komitologieausschüssen fest (Vereinbarung zwischen der EU sowie Island, Liechtenstein, Norwegen und der Schweiz vom 22. September 2011, SR 0.362.11). In der Praxis hat sich zudem eingebürgert, dass die assoziierten Schengen-Staaten an den Beratungen in den Ratsarbeitsgruppen bei Schengen-relevanten Geschäften teilnehmen; auch vor der formellen Beschlussfassung auf Ministerstufe - im Normalfall im JI-Rat (Justiz- und Innenminister) - können sie Stellung beziehen (G. BAUR, Schengen, S. 30). Das decision making obliegt naturgemäss allein den Vertreterinnen und Vertretern der EU-Mitgliedstaaten; Drittstaaten verfügen über keine Stimmrechte.

Ein Beispiel erfolgreicher Einflussnahme der Schweiz betrifft Art. 6 Abs. 6 lit. c in fine der Richtlinie (EU) 2017/853 zur Änderung der Waffenrichtlinie 91/477/EWG; hier gelang es der Schweiz, einen Swiss finish zu installieren (N. 262).

Die Mitwirkung der Schweiz erfolgt primär bei Vorbereitungsarbeiten der Europäischen Kommission und des Rates sowie bei Arbeiten in Agenturen und weiteren Einrichtungen, bei denen die Schweiz beteiligt ist. Demgegenüber fehlt eine Mitwirkung in Arbeitsgruppen des Europäischen Parlaments, welches in der EU mittlerweile in den allermeisten Fällen als Co-Gesetzgeber fungiert (Art. 14 EUV, Art. 289 AEUV). Hier ist die Schweiz auf informelle Lobbykanäle angewiesen. - Immerhin tauschen sich eine Delegation des Europäischen Parlaments (DEEA) und eine Delegation des Schweizerischen Parlaments (EFTA/EUDelegation) regelmässig über aktuelle Themen aus (www.europarl.europa.eu/delegations und Link zu DEEA; Verordnung der Bundesversammlung über die Pflege der internationalen Beziehungen des Parlamentes, SR 171.117).

Im Ergebnis wird das Kardinalsprinzip, wonach die Abkommen rein statischer Natur sind und für die Schweiz keine formalisierte Teilnahme am dynamischen Integrationsprozess der EU begründen, in der Praxis relativiert. Das Ziel, auch 
im bilateralen Verhältnis binnenmarktähnliche Verhältnisse zu schaffen, wird faktisch nur durch eine systematische Anpassung an das massgebliche EURecht erreicht - zeitverzögert und allein formell unter «Wahrung der Souveränität der Schweiz und ihrer innerstaatlichen Entscheidverfahren» (Europabericht 2010, S. 7298). Für gewisse EU-Rechtsakte besteht mit Blick auf das gute Funktionieren der Abkommen ein faktischer Übernahmezwang; dies gilt typischerweise für die Übernahme von Rechtsakten des Schengen/Dublin-Besitzstands. Es erstaunt nicht, dass die Schweiz alle seit 2004 von der EU notifizierten Rechtsakte zu Schengen/Dublin - mehr als 250 an der Zahl (www.eda.admin.ch/dea und Link zu Bilaterale Abkommen II (2004)/Schengen/Dublin) - akzeptiert hat; europapolitisch konsequent, demokratietheoretisch nicht unproblematisch, weil Bundesrat, Parlament und/oder Stimmvolk in solchen Übernahmeverfahren allein die «frustrierende Wahl zwischen Akklamation und destruktiver Opposition» (O. DigGELMANN, Internationalisierung, S. 117) haben und die Substanz der demokratischen Rechte ausgehöhlt wird (N.66). Über Schengen/Dublin hinaus werden im Regelfall auch die anderen Abkommen routinemässig angepasst, ohne dass ein vergleichbarer Anpassungsdruck besteht; dies trifft typischerweise auf die im Abkommen über die gegenseitige Anerkennung von Konformitätsbewertungen, im Landwirtschaftsabkommen und im Luftverkehrsabkommen geregelten technischen Vorschriften zu.

Die Übernahme der Verordnung (EU) 2020/696 in den Anhang des LVA bereits wenige Wochen nach der Inkraftsetzung in der EU manifestiert, wie die EU und die Schweiz bei ausserordentlichen Umständen wie einer epidemiologischen Krise (COVID-19) und den damit einhergehenden existentiellen Schwierigkeiten der Fluggesellschaften in der Lage sind, den Übernahmeprozess eindrücklich speditiv durchzustrophen. Mit dieser Verordnung werden die finanziellen Anforderungen für gewisse Akteure der Luftfahrtbranche vorübergehend gelockert; offenkundig ein zeitlich dringlicher Entscheid.

Demgegenüber verzögerte sich die Übernahme der Richtlinie 2005/36/EG über die Anerkennung von Berufsqualifikationen in Anhang III FZA ungebührlich lange (s. zu dieser Richtlinie N. 173-175). Diese Richtlinie kodifizierte 2005 das bisher geltende Recht - eine Vielzahl von Richtlinien - in einem Rechtsakt. Den EU-Mitgliedstaaten wurde eine Frist von zwei Jahren eingeräumt, um die Richtlinie umzusetzen. Nach Ablauf dieser Frist, d.h. im Oktober 2007, wurden die alten Richtlinien aufgehoben - vorderhand selbstredend nur mit Wirkung für das EU-Recht, während sie im Verhältnis zwischen der Schweiz und der EU weiterhin gültig waren. Obwohl es absehbar war, dass die Richtlinie 2005/36/EG auch auf die Schweiz ausgedehnt werden sollte, um das ordnungsgemässe Funktionieren des FZA nicht zu gefährden, dauerten die Vorbereitungsarbeiten unerwartet lange. Die Schweiz stimmte der Übernahme der Richtlinie in Anhang III FZA erst 2013 zu. Zwischenzeitlich galten bilateral die in der EU überholten Rechtsakte, für die in den EU-Mitgliedstaaten keine Umsetzung mehr existierte. Ein solches «Interregnum» gefährdet das gute Funktionieren des Abkommens und ist der Rechtssicherheit abträglich. Seit 2013 steht die Übernahme der nächsten Weiterentwicklung der Diplomanerkennungsrichtlinie (Richtlinie 2013/55/ 
EU) auf der Agenda. Der Prozess geht aber auch hier nur schleppend voran. Die Vorbereitungsarbeiten zur Übernahme (Entwurf der Umsetzungsgesetzgebung) sind abgeschlossen. Gleichwohl blockiert die EU die Übernahme. Dem Vernehmen nach verknüpft sie auch dieses Dossier mit Fortschritten beim InstA.

Ausnahmsweise nutzt die Schweiz den bestehenden Verhandlungsspielraum, um eine Übernahme abzulehnen. So hat sie wiederholt betont, auf Verhandlungen zur Übernahme der Unionsbürgerrichtlinie 2004/38/EG in das Freizügigkeitsabkommen nicht einzutreten, weil damit zusätzliche Ansprüche im Bereich der Sozialhilfe und des Daueraufenthaltsrechts begründet würden (Pressemitteilung des SEM vom 14. Juni 2011; Pressemitteilung des EDA vom 18. Dezember 2013). Umgekehrt ist es problematisch, dass die EU ihrerseits zeitweise darauf bedacht ist, den Übernahmeprozess zu verzögern. So stellte der Bundesrat anfangs 2017 fest, «im aktuellen Umfeld der Beziehungen Schweiz-EU» würden Anpassungen etwa des Abkommens über die gegenseitige Anerkennung von Konformitätsbewertungen «mehr Zeit als üblich» benötigen, und betonte: «Der Bundesrat hat sein Missfallen über diese Haltung der EU klargemacht und erwartet, dass sie 2017 zu einer Normalisierung Hand bietet» (Bericht Aussenwirtschaftspolitik 2016, S. 867; Aussenpolitischer Bericht 2016, S. 1305). Die EU dürfte damit bezweckt haben, Druck aufzubauen für eine FZA-kompatible Umsetzung der Masseneinwanderungsinitiative (N. 63) sowie mutmasslich auch für ein Entgegenkommen der Schweiz in den Verhandlungen über ein institutionelles Abkommen.

\section{E. Streitbeilegung}

Die Durchführung der Abkommen und die Gewährung von Rechtsschutz 97 obliegen den Vertragsparteien (Zweisäulenprinzip, N. 69). Der bilaterale Acquis Schweiz-EU kennt kein unabhängiges, gerichtsförmiges Organ, welches Streitigkeiten autoritativ entscheiden könnte. Auch der EuGH ist nicht befugt, den schweizerischen Behörden Vorgaben für die Auslegung und Anwendung der Abkommen zu machen. Das Bundesverwaltungsgericht oder das Bundesgericht entscheiden auf Beschwerden hin letztinstanzlich über die Auslegung und Anwendung der Abkommen im Hoheitsgebiet der Schweiz (N. 74). Damit besteht zwar die Gefahr divergierender Auslegungen, was dem guten Funktionieren der Abkommen abträglich sein kann. Solche Divergenzen sind in der Praxis allerdings selten. Das ist insbesondere ein Verdienst des Bundesverwaltungsgerichts und des Bundesgerichts, welche auf die Parallelität der Rechtsentwicklung bedacht sind (N. 83 und N. 85). 
Ein Beispiel für divergierende Auslegungen betrifft die unterschiedlichen Auffassungen des EuGH und des BGer zur Tragweite der im EU-Recht und im FZA praktisch wortgleich formulierten Bereichsausnahmen bei Tätigkeiten, welche mit der Ausübung hoheitlicher Befugnisse verbunden sind. Das BGer wird sich in künftigen Fällen dazu äussern müssen, ob es an der autonomen, von der EuGH-Praxis abweichenden Lesart festzuhalten gedenkt oder nicht (N. 160).

Beim 2014 gütlich beigelegten Steuerstreit (N. 223) und beim ungelösten Disput über die Vereinbarkeit der Acht-Tage-Regel mit dem FZA (N. 195) divergieren die Rechtsauffassungen der Schweiz und der EU, wobei in diesen Fällen die Gerichte in der Schweiz noch (?) nicht befasst wurden. Die causa wurde (Steuerstreit) bzw. wird (Acht-Tage-Regel) regelmässig im Gemischten Ausschuss thematisiert.

Sofern eine Vertragspartei der Meinung ist, die andere Vertragspartei würde gegen vertragliche Verpflichtungen verstossen, kann sie den Gemischten Ausschuss mit der Angelegenheit befassen; dieser versucht, eine einvernehmliche Lösung zu finden (N. 90). Gelingt dies nicht, kann die betroffene Vertragspartei gestützt auf abkommensspezifische Vorgaben oder allgemeine völkerrechtliche Grundsätze einseitig geeignete Massnahmen ergreifen (Gegenbzw. Schutzmassnahmen; s. etwa Art. 50 LandVA, Art. 22 und Art. 31 LVA, Art. 27 FHA); gewisse Abkommen erlauben ausdrücklich die Aussetzung von Vertragsbestimmungen (s. etwa Art. 19 KonfBA, Art. 29 ZollA). Die Schengen/ Dublin-Assoziierungsabkommen sehen die automatische Beendigung der Abkommen vor, sofern ein Streit über die Anwendung der Abkommen innert nützlicher Frist nicht gütlich beigelegt werden kann (Art. 10 SAA, Art. 7 DAA).

2019 wies der Gerichtshof der EU eine Klage eines französischen Arztes ab, der vorgebracht hatte, die Kommission hätte es rechtswidrig unterlassen, Massnahmen gegen die Schweiz zu ergreifen, weil die Beschränkung des Zugangs von Ärztinnen und Ärzten im Kanton Genf (Bedürfnisklausel) mit dem FZA nicht vereinbar war (Beschluss Magnan/ Kommission, T-99/19, EU:T:2019:693; Beschluss Magnan/Kommission, C-860/19, EU:C:2020:227). Das EuG und der EuGH bestätigten hierbei die Praxis, wonach Einzelpersonen keinen Anspruch haben, dass die Kommission in solchen Konstellationen tätig wird; dies gilt auch dann, wenn die Kommission der Auffassung ist, dass ein bilaterales Abkommen verletzt wird. Es ist nämlich unerlässlich, «dass die Unionsorgane und die Mitgliedstaaten über ein freies Ermessen oder zumindest über einen Wertungsspielraum verfügen, der so weit ist, dass die erforderliche Abwägung der sich aus den Beziehungen mit der Schweizerischen Eidgenossenschaft ergebenden divergierenden Interessen vorgenommen und die geeignetste Strategie zum Schutz der Interessen der Union und der Interessen ihrer Bürger und Wirtschaftsteilnehmer festgelegt werden kann» (Beschluss des EuG, Rz. 54).

99 Ausnahmsweise enthalten Abkommen Bestimmungen zur Streitbeilegung über die Befassung der Gemischten Ausschüsse hinaus: 
- Das Luftverkehrsabkommen erklärt den Gerichtshof der EU zuständig zur Streitschlichtung in gewissen im Abkommen geregelten Bereichen (Art. 20 LVA; zum Fluglärmstreit N. 214).

- Das Versicherungsabkommen sieht vor, zur Lösung einer Streitigkeit über die Auslegung und Anwendung des Abkommens ein Schiedsgericht einzusetzen, dessen Entscheidungen für die Parteien bindend sind (Art. 38). Die Vertragsparteien haben von dieser Möglichkeit bis dato noch nie Gebrauch gemacht.

Fraglich ist, ob die Einsetzung eines Schiedsgerichts, welches auch zur verbindlichen Auslegung von «vervölkerrechtlichtem» EU-Recht befugt ist, im Licht der EuGH-Praxis zur Wahrung der Autonomie des Unionsrechts und der vertraglich vorgesehenen Zuständigkeiten des EuGH zulässig ist (s. Gutachten EWR I, 1/91, EU:C:1991:490; Gutachten EMRK II, 2/13, EU:C:2014:2454; Gutachten CETA EU-Kanada, 1/17, EU:C:2019:341, Rn. 109-111; ebenso kritisch C. TOBLER, Schiedsgerichte, S. 4).

- Das Zollabkommen sieht ein spezielles Schiedsverfahren vor für den Fall, dass eine Vertragspartei Ausgleichsmassnahmen ergreift. Das Mandat der für die Durchführung eines solchen Verfahrens eingesetzten drei Schiedsrichter umfasst allerdings nur die Prüfung der Verhältnismässigkeit solcher Massnahmen (Art. 29 Abs. 3 ZollA).

Das WTO-Recht bildet für den Marktzugang für Waren und Dienstleistungen die rechtliche Grundordnung auch für das bilaterale Verhältnis EU-Schweiz (N. 122 und N. 123; N. 178 und N. 181). Es ist denkbar, Streitigkeiten zwischen der EU und der Schweiz, welche auch in den Anwendungsbereich des WTORechts fallen, durch das WTO-Streitbeilegungssystem klären zu lassen. Die bilateralen Abkommen schliessen ein solches Vorgehen nicht aus. Einzig für den Bereich der Bekämpfung der Rinderseuche BSE findet sich im Anhang zum Landwirtschaftsabkommen eine gemeinsame Erklärung, worin sich die Schweiz einseitig (sic!) verpflichtet, im Rahmen der WTO keine Verfahren gegen die EG oder ihre Mitgliedstaaten einzuleiten (Gemeinsame Erklärung zum Anhang 11 AgrarA; kritisch zu dieser Ausnahme T. CotTiER/E. EvTimov, S. 188). An anderer Stelle wird das WTO-Recht hingegen ausdrücklich vorbehalten (s. etwa Art. 8 Anhang 11 AgrarA; Art. 15 ÖffBA). Voraussetzung für die aktive Nutzung des WTO-Streitbeilegungsverfahrens ist auf Seiten der Schweiz selbstredend, dass die Schweiz ihre traditionelle Zurückhaltung gegenüber der Konfliktlösung durch unabhängige (Schieds-) Gerichte im Wirtschaftsvölkerrecht überdenkt und ihren Kompass diesbezüglich neu justiert (s. dazu N. DiebOLD/M. OESCH, S. 1550).

Die Schweiz ist bis anhin einmal aktiv als klägerische Partei vor einem WTO-Panel und dem Appellate Body aufgetreten: Im Streitfall United States - Definitive Safeguard Measures 
on Imports of Certain Steel Products obsiegte sie zusammen mit sieben weiteren klägerischen Parteien - darunter der EG - gegen die Vereinigten Staaten (WT/DS253/AB/R, am 10. Dezember 2003 angenommen). Zurzeit ist sie - erneut gegen die Vereinigten Staaten wegen Zollerhöhungen im Stahlbereich und wiederum gemeinsam u.a. mit der EU in einem zweiten Verfahren vor einem Panel als klägerische Partei involviert (WT/DS556, United States - Certain Measures on Steel and Aluminum Products). Problematisch ist, dass die Vereinigten Staaten die Ernennung von neuen Mitgliedern des Appellate Body blockiert, womit der Appellate Body seit Dezember 2019 nicht mehr funktionsfähig ist; zurzeit arbeiten 21 WTO-Mitglieder - darunter auch die EU und die Schweiz - daran, ein vorläufiges Berufungsverfahren mittels Schiedsgericht einzurichten (vgl. Pressemitteilung des WBF vom 30. April 2020, Die Schweiz und 18 weitere WTO-Mitglieder führen ein vorläufiges Berufungsverfahren für Handelsstreitigkeiten ein; M. OESCH/A.N. BURGHARTZ/ V. MANIKULAM, S. 266-270).

\section{F. Ausblick: Institutionelles Abkommen}

\section{Ausgangslage und Verhandlungen}

Die bilateralen Abkommen funktionieren grundsätzlich gut (Bericht Aussenwirtschaftspolitik 2017, S. 855). Die EU, ihre Mitgliedstaaten und die Schweiz sind bestrebt, die Abkommen ordnungsgemäss durchzuführen. Unstimmigkeiten werden in den Gemischten Ausschüssen gelöst. Ausnahmen - wie die Auseinandersetzung über die Vereinbarkeit der Acht-Tage-Regel mit dem Freizügigkeitsabkommen (N. 195) - bestätigen die Regel. Das Bundesgericht legt die Abkommen konsequent im Licht der EuGH-Praxis aus (N. 83 und N. 85). Die Aufdatierung der Abkommen, welche auf EU-Recht beruhen, erfolgt in aller Regel routinemässig; auch hier sind Ausreisser - wie die harzige Übernahme der Richtlinie 2005/36/EG in das Freizügigkeitsabkommen (N. 95) - selten. Gleichwohl gibt es Anzeichen dafür, dass das geltende System an seine Grenzen stösst. Die gewandelte Natur der staatsvertraglichen Beziehungen, die Notwendigkeit, EU-Recht nach Massgabe eines formalisierten Prozesses zeitnah und unkompliziert in den bilateralen Acquis zu übernehmen, und das Bedürfnis, Streitfälle bei Bedarf zu entpolitisieren und einem unabhängigen Streitbeilegungsmechanismus zu überantworten, finden keine institutionelle Entsprechung. Es handelt sich um eine «fair-weather construction» (M. OESCH, Incorporation, S. 11). Die institutionellen Vorgaben sind nicht in der Lage, Rechtssicherheit, Transparenz und Effizienz sicherzustellen. Sie reflektieren die sektoriell weitgehende Integration der Schweiz in den Binnenmarkt bzw. ihre Beteiligung an einem «multilateralen Projekt» (Schlussfolgerungen des Rates vom 16. Dezember 2014, Rz. 44) und an einem «gemeinsamen Wirtschaftsraum» (Schlussfolgerungen des Rates vom 19. Februar 2019, Rz. 8) nicht adäquat. 
Die EU kritisiert seit 2008, dass «das derzeitige System der bilateralen Abkommen (...) komplex und schwer zu handhaben ist und eindeutig an seine Grenzen stösst» und daher einer Modernisierung bedarf (Schlussfolgerungen des Rates vom 14. Dezember 2010, Rz. 48). Sie verlangt von der Schweiz, Hand zur Schaffung eines institutionellen Abkommens zu bieten, um die nötige Homogenität in der Rechtsanwendung und Weiterentwicklung sicherzustellen. Dabei geht es um die folgenden vier Bereiche: (i) Rechtsanpassung (Verfahren zur dynamischen Weiterentwicklung der Abkommen); (ii) Auslegung (Sicherstellung einer homogenen Auslegung der Abkommen); (iii) Überwachung (Sicherstellung der ordnungsgemässen Anwendung der Abkommen); und (iv) Streitbeilegung (Verfahren zur Lösung von Streitigkeiten zwischen den Vertragsparteien). Der Rat verlangte 2012, für die Überwachung und gerichtliche Kontrolle einen «internationalen Mechanismus» zu schaffen, welcher einen Grad an Rechtssicherheit und Unabhängigkeit aufweist, wie er bei dem im Rahmen des EWR-Abkommens geschaffenen System existiert (Schlussfolgerungen des Rates vom 20. Dezember 2012, Rz. 33). Ein gemeinsamer institutioneller Rahmen soll auf «bestehende und künftige Abkommen über die Beteiligung der Schweiz am Binnenmarkt der EU» anwendbar sein, wobei der Abschluss weiterer Abkommen über die Beteiligung der Schweiz am Binnenmarkt vom Abschluss eines institutionellen Abkommens abhängig gemacht wird (Schlussfolgerungen des Rates vom 28. Februar 2017, Rz. 5). Das Europäische Parlament teilt diese Erwartungshaltung des Rates (Entschliessung vom 9. September 2015, 2015/2061 (INI), Rz. 16).

Die Schweiz wehrte sich anfänglich gegen die Forderung der EU zur Schaffung eines Rahmenabkommens. Es gab hierzulande zwar seit Längerem Bestrebungen, verfahrensrechtliche Fragen, die sich in allen Abkommen in ähnlicher Weise stellen, zu vereinheitlichen und den bilateralen Acquis auf eine solide einheitliche Basis zu stellen (F.E. MÜLLER, S. 29-38). 2002 schlug die Groupe de Réflexion Suisse-Europe vor, die bilateralen Abkommen in einem einzigen Abkommen zu bündeln und einen einheitlichen institutionellen Rahmen vorzusehen (NZZ vom 6. April 2017). Ebenfalls 2002 erwähnte die Aussenpolitische Kommission des Ständerates in einem Bericht über die Optionen der schweizerischen Integrationspolitik eine «Assoziation» mit der EU, «welche es erlaubt, alle bilateralen Abkommen zwischen der Schweiz und der EU unter dem Dach eines Rahmenabkommens zu bündeln» (Bericht Integrationspolitik Ständerat, S. 6332). 2005 wurde der Bundesrat in einem Postulat aufgefordert, «einen Bericht zu erstellen über den Stellenwert eines Rahmenvertrages zwischen der Schweiz und der EU», wobei in der Begründung ausgeführt wurde: «Inhaltliche Anliegen zwischen der Schweiz und der EU würden wie bisher 
diskutiert und wären in diesem Rahmenvertrag nicht enthalten.» (Postulat 05.3564 «Rahmenvertrag zwischen der Schweiz und der EU»). 2010 kam der Bundesrat zum Schluss, dass eine Neugestaltung und Vereinheitlichung der institutionellen Regeln Vorteile bieten mag: «Eine solche Lösung (Rahmenabkommen oder ähnliches Arrangement) würde durch einheitliche Mechanismen die Transparenz und Effizienz der Entscheidfassung in den Gemischten Ausschüssen fördern und letztlich die Rechtssicherheit des bilateralen Wegs erhöhen» (Europabericht 2010, S. 7243). Die umfassende Regelung der von der EU als klärungsbedürftig identifizierten vier Bereiche (N. 102) wurde allerdings nicht offensiv avisiert. Man sträubte sich lange gegen eine allzu weitreichende Verrechtlichung und Dynamisierung der bilateralen Beziehungen. Die Gründe dafür liegen im weitum vorherrschenden nationalstaatlich ausgerichteten Souveränitätsverständnis, welches Selbstbestimmung und direkte Demokratie traditionell in den Mittelpunkt stellt, und im Bestreben, die Beziehungen zur EU primär unter klassisch handelspolitischen Aspekten zu regeln (T. COTTIER, Souveränität, passim). Auch diejenigen Abkommen, welche auf EURecht beruhen und deren Zweck darin liegt, die Schweiz sektoriell in den unionalen Rechtsraum zu integrieren, werden hinlänglich nicht als Beteiligung an einem paneuropäischen Projekt verstanden, bei dem sich zuvor bereits 27 bzw. - wenn die EWR-EFTA-Staaten mitgezählt werden - 30 Staaten unter dem institutionellen Dach der EU bzw. des EWR auf gemeinsame Regeln einigen, sondern als Resultat «bilateraler» Verhandlungen zwischen zwei grundsätzlich gleichberechtigten Partnerinnen; eine Qualifikation der Abkommen, welche ihrer inhaltlichen Substanz nicht entspricht.

2014 einigten sich die Schweiz und die EU, Verhandlungen über ein institutionelles Abkommen aufzunehmen. Ursprünglich ging vor allem die EU davon aus, institutionelle Elemente vorzusehen, welche mit denjenigen des EWRAbkommens vergleichbar sind (C. TOBLER/J. BEGLINGER, Brevier, 15. Frage; F.E. MÜLLER, S. 58-59). Demgegenüber präferierte die Schweiz zumindest im Bereich der Überwachung und Streitbeilegung Mechanismen, welche sich nicht am EWR orientieren. Die Annahme der Masseneinwanderungsinitiative im Februar 2014 führte zu gewissen Verzögerungen. Im März 2018 präzisierte der Bundesrat zudem das Verhandlungsmandat von 2013; die Anpassungen betrafen namentlich einen neuen Ansatz für die Streitbeilegung auf der Basis eines unabhängigen Schiedsgerichts (während ursprünglich angedacht war, den EuGH direkt - d.h. ohne Befassung eines Schiedsgerichts - anrufen zu können). Im Dezember 2018 erklärte die EU den vorliegenden Entwurf als endgültig und die Verhandlungen als beendet. Sie wiederholte, dass der Abschluss weiterer Abkommen über die Beteiligung der Schweiz am Binnenmarkt vom 
Abschluss eines solchen Abkommens abhängt (Schlussfolgerungen des Rates vom 19. Februar 2019, Rz. 9). Sie verlautete, dass sie nicht bereit ist, die geltenden Abkommen weiterhin aufzudatieren (z.B. im Bereich der Medizinaltechnik; vorbehalten bleiben Aufdatierungen, an denen die EU ein überwiegendes Interesse hat), und Hand zu bieten für Entgegenkommen und Kooperationen in weiteren Bereichen (z.B. Anerkennung der Börsenäquivalenz, Assoziierung an Horizon Europe, Zusammenarbeit in den Bereichen Kultur und Gesundheit), sollte die Schweiz dem institutionellen Abkommen nicht zustimmen. Die EU sendet damit die klare Botschaft aus, wonach sie den Status quo nicht mehr als valable Option betrachtet. Der Bundesrat verzichtete im Dezember 2018 allerdings darauf, das Abkommen zu unterzeichnen. Stattdessen veröffentlichte er den Text und führte Konsultationen mit betroffenen Kreisen durch; gleichsam als Vernehmlassungsverfahren sui generis, nachdem die Öffentlichkeit zuvor keinen Zugang zu den Verhandlungsmandaten, Vertragsentwürfen und Konzeptpapieren hatte und in dieser Hinsicht vom Meinungsbildungsprozess ausgeschlossen war.

\section{Inhalt}

Der Entwurf des Institutionellen Abkommens (InstA) regelt hauptsächlich die folgenden Elemente (s. für den Text www.eda.admin.ch/dea und Link zu Verhandlungen).

Vertragsparteien des InstA sind gemäss Titel und Präambel die Schweiz und die EU. Letztere scheint davon auszugehen, dass sie umfassend für den Abschluss des Abkommens zuständig ist (A. EPINEY, Entwurf, Rz. 4-6; zu «EU-only»-Abkommen N. 39). Mit Blick auf die Tatsache, dass das InstA auch auf das FZA - ein gemischtes Abkommen (N. 28) - anwendbar ist, kann mit guten Gründen argumentiert werden, dass es sich recte um ein gemischtes Abkommen handelt. Hier dürften auf Seiten der EU noch vertiefte rechtliche Abklärungen (und politische Überlegungen) anstehen.

Ziel (Art. 1): Das InstA bezweckt, den Vertragsparteien, Wirtschaftsakteuren und Privatpersonen grössere Rechtssicherheit und Gleichbehandlung zu garantieren sowie einheitliche Bedingungen zu gewährleisten; dies gilt für diejenigen Bereiche des Binnenmarkts, an denen die Schweiz teilnimmt. Das InstA schafft einen neuen Rahmen, der einen kontinuierlichen und ausgewogenen Ausbau der Wirtschafts- und Handelsbeziehungen zwischen den Vertragsparteien ermöglicht.

Geltungsbereich (Art. 2): Das InstA ist anwendbar auf das Freizügigkeitsabkommen (FZA), das Luftverkehrsabkommen (LVA), das Landverkehrsabkommen (LandVA), das Landwirtschaftsabkommen (AgrarA) und das Abkommen über 
technische Handelshemmnisse (KonfBA) sowie auf künftige Marktzugangsabkommen, mit denen die Schweiz am Binnenmarkt teilnimmt. Das Freihandelsabkommen von 1972 ist nicht betroffen, wobei die Vertragsparteien gemeinsam ihre Absicht erklären, Verhandlungen über eine Modernisierung dieses Abkommens aufzunehmen und dabei auch die Regelung weiterer Bereiche - wie Dienstleistungshandel, Bekämpfung des Klimawandels und Schutz des geistigen Eigentums - ins Auge zu fassen (M. BALDI, Rz. 18-34). Die Vertragsparteien werden «gemäss dem Umfang der Beteiligung der Schweiz am Binnenmarkt der Union» (Ziff. 9 der Gemeinsamen Erklärung EU-Schweiz zu den Handelsabkommen) bei jedem weiteren Modernisierungsschritt separat entscheiden, ob und, sofern dies der Fall ist, in welchem Umfang das InstA anwendbar sein soll.

Gemäss einem dem InstA beigefügten Entwurf für einen Beschluss des Gemischten Ausschusses nach Art. 29 FHA können die Parteien konsensual entscheiden, Streitfälle unter dem FHA dem Schiedsgericht zu überantworten. In der Präambel dieses Beschlusses wird zudem ausgeführt, «dass die Schweiz und die Europäische Union übereingekommen sind, dass die Bestimmungen von Teil II des institutionellen Abkommens eine spätere Übereinkunft zwischen den Parteien im Sinne von Artikel 31 [WVRK] darstellen, die für die Auslegung von Artikel 23 Absatz 1 Ziffer iii des Abkommens massgebend ist, und dass dessen Anwendung fortan von dieser Auslegung geleitet werden soll.» Die rechtliche Wirkung dieser Erwägung ist unklar. Es ist nicht ausgeschlossen, dass damit suggeriert werden soll, dass die Beihilferegeln des FHA ab Inkrafttreten des Beschlusses auch in der Schweiz (s. zu einer entsprechenden Erklärung der EWG beim Abschluss des FHA 1972 N. 89) im Lichte der in Art. 8A InstA stipulierten materiellen Grundsätze des EU-Beihilferechts inkl. der Praxis des EuGH ausgelegt und angewendet werden sollen, und zwar unabhängig davon, ob die Vertragsparteien eine Streitigkeit einem Schiedsgericht zur Beurteilung zuweisen oder nicht (vgl. Erläuterungen des EDA, S. 16; M. BALDI, Rz. 6-17); ein Auslegungsresultat, welches die Schweiz nicht zu akzeptieren bereit ist. Aus diesem Grund fordert die Schweiz eine Klarstellung, dass die Beihilferegeln gemäss FHA durch die Schweiz weiterhin autonom ausgelegt werden (N. 117).

Auslegung (Art. 4): Die betroffenen Abkommen und die darin referenzierten EU-Rechtsakte werden einheitlich und unter Wahrung der Grundsätze des Völkerrechts ausgelegt und angewendet. Unionsrechtliche Begriffe («notions de droit de l'Union européenne», N. 111) werden gemäss der Rechtsprechung des Gerichtshofs der EU ausgelegt und angewendet. Dies gilt für die vor und eine wichtige Novellierung! - nach der Unterzeichnung des betreffenden Abkommens ergangene Rechtsprechung des EuGH. Dabei bleibt das PolydorPrinzip einschlägig (N. 81-82): Die «Übersetzungsarbeit», d.h. die Bestimmung der Bedeutung der EuGH-Praxis zum EU-Recht für das bilaterale Verhältnis, bleibt eine zentrale Aufgabe der schweizerischen Gerichte.

Dem BGer wird kein Recht eingeräumt, den EuGH auf dem Wege der Vorabentscheidung mit einer Auslegungsfrage zu befassen. Das ist ein Konstruktionsfehler. Es wäre v.a. mit 
Blick auf die Verpflichtung, auch jüngere EuGH-Urteile zu befolgen, sowie das Diktum aus Lausanne, wonach das FZA Bundesgesetzen ausnahmslos vorgeht (N. 53), systemisch naheliegend, das BGer qua Vorabentscheidungsverfahren in den europäischen Rechtsprechungsverbund (s. zu diesem Label BVerfGE 140, 317 [338] - Identitätskontrolle) zu integrieren und ihm das Recht einzuräumen, den EuGH zu befassen. Dadurch würde auch die Streitschlichtung durch Schiedsgerichte (inkl. Befassung des EuGH) entlastet. Immerhin werden das BGer und der EuGH auf einen Dialog verpflichtet, um eine einheitliche Auslegung zu fördern (Art. 11).

Weiterentwicklung (Art.12-14): Die EU ist verpflichtet, der Schweiz abkommensrelevante Rechtsakte zu notifizieren. Im Gemischten Ausschuss werden die notwendigen Anpassungen vorgenommen. Die Schweiz ist sodann verpflichtet, der Übernahme zuzustimmen; diese Übernahmeverpflichtung besteht unabhängig davon, ob ein Abkommen als solches oder nur ein Anhang geändert werden muss (a.M. wohl A. EPINEY, Entwurf, Rz. 28). Die Frist für die Übernahme durch die Schweiz beträgt maximal drei Jahre ab dem Zeitpunkt der Notifikation durch die EU, sofern ein Referendum ergriffen wird. Sofern ein Schiedsgericht feststellt, dass die EU (Notifikation) oder die Schweiz (Übernahme) diese Vorgaben verletzt, kann die andere Partei Ausgleichsmassnahmen ergreifen, deren Verhältnismässigkeit durch ein Schiedsgericht überprüft werden kann (N. 111). Die Schweiz kann die Vorbereitung von EU-Rechtsakten mitgestalten (proposal shaping); diese Mitwirkungsrechte entsprechen denjenigen der EWR-EFTA-Staaten im Rahmen des EWR (vgl. Art. 99-101 EWRA). Damit wird der Übernahmeprozess dynamisiert und formalisiert; das ist der Rechtssicherheit förderlich. Es wird für die EU nicht mehr möglich sein, die Aufdatierung eines Abkommens vom Entgegenkommen der Schweiz in anderen Dossiers abhängig zu machen. Umgekehrt nimmt der Druck auf die Schweiz zu, auch unliebsame Weiterentwicklungen zu akzeptieren, weil andernfalls Ausgleichsmassnahmen drohen (s. zum Ganzen M. OESCH, Incorporation, passim, auch betr. Rechtslage im EWR). Einzelne Massnahmen der Schweiz, welche in den vom InstA betroffenen Abkommen geregelt werden und Schweizer Besonderheiten reflektieren, werden von der dynamischen Rechtsübernahme ausdrücklich ausgenommen (Protokoll 2); dazu gehören etwa die LSVA und das Nacht- und Sonntagsfahrverbot (LandVA) sowie das Verbot internationaler Tiertransporte auf der Strasse (AgrarA).

Die Unionsbürgerrichtlinie 2004/38/EG wird im InstA nicht erwähnt. Weder die EU (ausdrückliche Übernahmeverpflichtung der Schweiz) noch die Schweiz (ausdrücklicher Ausschluss einer Übernahmeverpflichtung) setzten sich mit ihren Ansinnen durch. Vor diesem Hintergrund muss bei einem Inkrafttreten des InstA damit gerechnet werden, dass die EU diese Richtlinie der Schweiz als abkommensrelevant notifizieren wird. Sofern sich die Schweiz und die EU im Gemischten Ausschuss weiterhin nicht auf eine - allenfalls auch nur teilweise (z.B. unter Ausklammerung zusätzlicher Sozialhilfeansprüche von nichter- 
werbstätigen Personen, der verschärften Ausweisungsregeln und des Daueraufenthaltsrechts nach fünf Jahren rechtmässigen Aufenthalts; allesamt Ansprüche, welche sich nach schweizerischen Lesart aus der Unionsbürgerschaft ergeben und bilateralrechtlich nicht greifen; A. EpInEY/S. AfFolter, passim; C. TOBleR, Varianten, Rz. 50-58; C. TOBLER, Personenfreizügigkeit, passim) - Übernahme der Richtlinie in das FZA einigen können, besteht für die EU die Möglichkeit, ein Schiedsgericht anzurufen. Das Schiedsgericht müsste sodann - wohl unter Befassung des EuGH, da der Umfang der Übernahmeverpflichtung auch von der Auslegung von Begriffen des EU-Rechts abhängt - die Frage der Abkommensrelevanz entscheiden (s. zur Rechtslage im EWR N. 117). - Ein ähnliches Bild zeigt sich bei der laufenden Revision der Verordnung (EG) Nr. 883/2004 zur Koordinierung der Systeme der sozialen Sicherheit (N. 172). Der Schweiz ist es im Rahmen der Verhandlungen zum InstA nicht gelungen, diese absehbare Novellierung von der Verpflichtung zur Übernahme in das FZA auszunehmen.

Diese Fälle umstrittener Weiterentwicklungen dürften Ausnahmefälle sein. Die Übernahme von neuen EU-Rechtsakten wird in aller Regel wohl auch in Zukunft unproblematisch sein und kaum zu kontroversen Debatten Anlass geben. Dabei wird die Schweiz gut beraten sein, sich im Rahmen des proposal shaping aktiv zu engagieren und den Inhalt der EUGesetzgebung mitzugestalten (s. dazu auch Motion 19.3170 «Gesetzliche Grundlage zur Wahrung des Mitsprache- und Entscheidungsrechts von Parlament, Volk und Kantonen bei der Umsetzung des Rahmenabkommens»).

110 Überwachung (Art. 6-8C): Die Parteien sind - mit Ausnahme des Beihilferechts (N. 112) - weiterhin für die Durchführung, die Überwachung und den Rechtsschutz zuständig (Zweisäulenprinzip). Sie können Informationen über ihre Tätigkeiten zur Überwachung der Anwendung der Abkommen austauschen und Anliegen von gegenseitigem Interesse besprechen. Im Rahmen der zuständigen sektoriellen Ausschüsse sorgen die Parteien gemeinsam für die Überwachung der Anwendung der Abkommen.

111 Streitbeilegung (Art. 10, Protokoll 3): Jede Partei kann die Einsetzung eines Schiedsgerichts - bestehend aus drei (allenfalls fünf) Personen - verlangen, sofern eine Streitigkeit im Gemischten Ausschuss nicht beigelegt werden kann. Wirft der Streitfall eine Frage betreffend die Auslegung von «Begriffen des EU-Rechts» («notions de droit de l'Union européenne») auf und ist deren Auslegung für die Entscheidfällung notwendig, ruft das Schiedsgericht den EuGH an, dessen Urteil für das Schiedsgericht verbindlich ist (Art. 4 Abs. 2 i.V.m. Art. 10 Abs. 3). Unionsrechtliche Begriffe liegen naturgemäss bei der Auslegung von Bestimmungen referenzierter Verordnungen und Richtlinien vor. Auch bei der Auslegung von Bestimmungen in den Abkommen selbst dürfte dies häufig der Fall sein; gemäss Bundesrat beruhen die betroffenen Abkommen «weitestgehend» auf EU-Recht (Erläuterungen des EDA, S. 7; vgl. auch Art. 16 Abs. 2 FZA, wo Praxis und Lehre diesen Begriff grosszügig auslegen; B. PIRKER, Schiedsgericht, Rz. 12, wonach ein Vorgehen nach Treu und Glauben gebietet, den EuGH im Zweifel zu involvieren). Die Frage, ob ein unionsrecht- 
licher Begriff vorliegt, ist methodisch zu trennen von der (Folge-) Frage, ob die bilateralrechtliche Bestimmung gleich ausgelegt werden soll wie die unionsrechtliche Parallelbestimmung oder ob sich eine autonome Lesart aufdrängt (s. zum Polydor-Prinzip N. 81-82; s. demgegenüber T. COTTIER, Rechtsschutz, S. 6-7, wonach eine Vorlagepflicht nur dort besteht, wo sich auf Grund von Wortlaut, Sinn und Zweck eine identische Anwendung wie im EU-Recht aufdrängt). Beispiele für Bestimmungen, welche keine unionsrechtlichen Begriffe darstellen, sind etwa die Ventilklausel im FZA (Art. 10 FZA, N. 154) und die Ermächtigung, die LSVA einzuführen (Art. 38 und Art. 40 LandVA, N. 205). Beim Einbezug des EuGH ähnelt das Verfahren dem Vorabentscheidungsverfahren im EU-Recht (Art. 267 AEUV); folgerichtig wendet der Gerichtshof die entsprechenden internen Verfahrensvorschriften sinngemäss an (Art. III.9 Abs. 5 Protokoll 3). Sofern die unterlegene Partei einen Schiedsspruch nicht umsetzt, kann die obsiegende Partei Ausgleichsmassnahmen ergreifen, deren Verhältnismässigkeit durch ein Schiedsgericht überprüft werden kann. Ausgleichsmassnahmen könnten z.B. darin bestehen, die Zölle auf Agrarprodukte zu erhöhen (AgrarA), freizügigkeitsrechtliche Ansprüche nicht zu gewähren (FZA, wobei bereits erworbene Rechte von Personen und Unternehmen unberührt bleiben, Art. 10 Abs. 8), Konformitätsbewertungen nicht anzuerkennen (KonfBA) oder die LSVA zu erhöhen (LandVA). Dabei geht es primär darum, das Gleichgewicht von Rechten und Pflichten wiederherzustellen (S. ARIOLI, Übernahme, Kap. 1.3); gleichzeitig kann damit auch Druck gegenüber der anderen Vertragspartei aufgebaut werden mit dem Ziel, dass diese die ursprüngliche Rechtsverletzung beseitigt (NZZ vom 17. Januar 2019, S. 17). Es ist unklar, ob und - sofern dies der Fall ist - unter welchen Voraussetzungen Ausgleichsmassnahmen im Anwendungsbereich eines Abkommens zulässig sind, welches zwar dem InstA unterstellt ist, aber nicht am Ursprung der eigentlichen Streitigkeit steht (cross-retaliation). Unangenehm ist, dass es - wenn überhaupt nur begrenzt möglich sein wird, die Kosten und die weiteren Nachteile der Nichtbefolgung eines Urteils ex ante abzuschätzen.

Der Einbezug des EuGH wird kontrovers diskutiert, wobei zeitweise verkannt wird, dass der EuGH unionsrechtlich - d.h. aus Gründen der Autonomie des EU-Rechts und der in den EU-Verträgen festgelegten Zuständigkeitsordnung - zwingend das letzte Wort über die Auslegung von EU-Recht in der EU haben muss; dazu gehört auch EU-Recht, das «vervölkerrechtlicht» auf einen Drittstaat ausgedehnt wird (Gutachten EWR I, 1/91, EU:C:1991:490; Gutachten EMRK II, 2/13, EU:C:2014:2454; Gutachten CETA EU-Kanada, 1/ 17, EU:C:2019:341; tendenziell kritisch zur Vereinbarkeit der Lösung im InstA mit dem EURecht, weil ein Schiedsgericht abschliessend über eine Vorlage an den EuGH entscheidet, B. PIRKER, Schiedsgericht, Rz. 22-34). Als Alternative käme in Frage, die Andockung der Schweiz an den EFTA-Gerichtshof inklusive der damit verbundenen Vorstellung, diesen Gerichtshof mit einem schweizerischen Richter anzureichern, zu prüfen (Überwachung, 
Rechtsschutz) und die Streitbeilegung analog zum EWR-Modell, wo die an einem Streit beteiligten Vertragsparteien den EuGH gemeinsam involvieren können, auszugestalten (Art. 111 EWRA; C. BAUDENBACHER, Komplott, S. 106-115, 220-222; C. TOBLER, Varianten, Rz. 12; C. TOBLER/J. BEGLINGER, Brevier, 93.-109. Frage; vgl. auch M. AMBÜHL/D. SCHERER, Entwurf, Rz. 24-29, welche vorschlagen, auf eine Beurteilung der Rechtmässigkeit einer Massnahme durch ein Schiedsgericht zu verzichten und der EU stattdessen das Recht einzuräumen, Ausgleichsmassnahmen zu erlassen, deren Verhältnismässigkeit durch ein Schiedsgericht überprüft werden könnte; zum Ganzen auch D. THÜRER, Gutachten, passim). Mit Blick auf denkbare Anwendungsfälle dürfte es allerdings kaum einen Unterschied machen, ob der EuGH oder der EFTA-Gerichtshof mit einer Streitsache betraut wird. Zudem deutet nichts darauf hin, dass der EuGH tendenziell «gegen die Schweiz» entscheiden würde; seine Praxis zur Auslegung von völkerrechtlichen Abkommen ist von einer «Integrationslogik geprägt, welche unabhängig von Partikularinteressen operiert» (A. EPINEY, Quadratur, S. 21; vgl. auch M. OESCH/G. SPECK, passim, für weitere Gründe gegen eine EFTA-Lösung). Dabei hat der Einbezug des EuGH «nichts, aber auch gar nichts» mit der mittelalterlichen Konsolidierung einer heimischen Gerichtsbarkeit durch die blockierte Anrufung kirchlicher oder ausländischer Gerichte zu tun («fremde Richter», W. ERNST, Richter, S. 1375).

Die praktische Bedeutung der avisierten Streitschlichtung qua Schiedsgerichtsbarkeit dürfte sich auf einige wenige - dann allerdings publikumswirksame und politisch aufgeladene - Fälle beschränken. Für die Schweiz ist vor allem der Schutz vor einseitigen Massnahmen der EU bedeutsam. Umgekehrt besteht die Möglichkeit, bilateralrechtliche Ansprüche schiedsgerichtlich einzufordern, was selbstredend voraussetzt, dass die Schweiz bei Bedarf tatsächlich davon Gebrauch macht (N.100). Im Alltag erfolgt der Rechtsschutz bei der Auslegung und Anwendung der bilateralen Abkommen weiterhin zuvörderst durch die Gerichte in der EU und der Schweiz. sätze, welche bei der Gewährung von staatlichen Beihilfen zu beachten sind. Sie entsprechen denjenigen im Binnenmarktrecht (Art. 107 AEUV; s. zum Ganzen C. SEITZ/A.S. BERNE, passim) und gelten vorderhand allein für das LVA. Zusätzlich wird das LVA durch eine Reihe von unionalen Rechtsakten und Mitteilungen - eine merklich umfangreiche Liste mit Elementen, welche den Luftverkehr mitunter nur indirekt betreffen - ergänzt (Anhang X). Die Parteien führen ein Notifizierungsverfahren für staatliche Beihilfen ein. In der EU wird die Europäische Kommission dafür zuständig sein. Auch die Schweiz ist verpflichtet, eine unabhängige Überwachungsbehörde einzurichten, welche über die Zulässigkeit von Beihilfen entscheidet. Unklar bleibt, über welche Kompetenzen diese Behörde verfügen soll: Die Beihilferegeln sollen zwar gemäss der jeweiligen «verfassungsmässigen Kompetenzordnung» angewendet werden. Gleichzeitig ist mit Blick auf die Vorgabe, wonach die «Überwachungsbehörden die ausschliessliche Befugnis [haben], Beihilferegelungen oder einzelne Beihilfevorhaben zu bewilligen, und [sicherstellen], dass entgegen der Bestimmungen über staatliche Beihilfen gewährte Beihilfen zurückgefordert werden» (Art. 8B Abs.4), die Lesart durchaus naheliegend, dass die schweizerische 
Überwachungsbehörde auch gegenüber dem Bundesgesetzgeber und den Kantonen verbindlich über die Zulässigkeit von Beihilfen entscheiden soll. Das wäre ein staatspolitisch grundlegender Systemwechsel; umso bedauerlicher ist, dass der Normtext vage bleibt. Zurzeit ist die WEKO für die Überwachung der Beihilfenregeln im LVA zuständig. Ihre Zuständigkeit und Aufgaben werden im Lichte der zusätzlichen Vorgaben des InstA zu überprüfen sein.

Die EU und die Schweiz verhandeln seit 2007 über den Abschluss eines Stromabkommens, mit dem die Teilnahme der Schweiz am europäischen Strommarkt geregelt und der grenzüberschreitende Stromhandel weiterentwickelt werden soll; dabei werden die im InstA stipulierten Beihilferegeln zur Anwendung kommen, ergänzt um die einschlägigen unionalen Rechtsakte und Mitteilungen im Bereich des Energierechts (und allenfalls damit verwandter Bereiche). Bei der Modernisierung des FHA von 1972 wird die EU bestrebt sein, die Beihilferegeln umfassend anwendbar zu erklären und das Abkommen ebenfalls dem InstA zu unterstellen (s. für mögliche Auswirkungen M. BALDI, Rz. 25-29; P. ZURKINDEN, passim). Dies wird zu einem Paradigmawechsel in Bezug auf die Disziplinierung der Gewährung von staatlichen Beihilfen in der Schweiz führen (M. OESCH, Subventionen, passim; merklich kritisch S. HIRSBRUNNER, passim). Ein erster positiver Aspekt eines solchen Paradigmawechsels wäre die im InstA angelegte Verpflichtung zur Transparenz (Art. 8C; vgl. auch das Postulat 15.3387 «Für einen funktionierenden Wettbewerb. Gegen wettbewerbsverzerrende staatliche Beihilfen», welches den Bundesrat auffordert, Formen und Umfang staatlicher Beihilfen sowie allfällige Handlungsoptionen aufzuzeigen).

Flankierende Massnahmen (Protokoll 1): Die EU und die Schweiz verfolgen gleichermassen das Ziel, ihren Staatsangehörigen sowie Marktteilnehmern gerechte Bedingungen für die freie Erbringung von Dienstleistungen während bis zu 90 Arbeitstagen pro Kalenderjahr (inkl. Entsendung von Arbeitnehmenden) zu gewährleisten und gleichzeitig die Arbeitnehmerrechte zu garantieren («gleicher Lohn für gleiche Arbeit»). Die EU anerkennt, dass dafür nichtdiskriminierende und verhältnismässige Kontrollen sowie weitere Massnahmen notwendig sind (s. zum Ganzen A. EPINEY/L. HEHEMANN, passim). Sie schlägt vor, dass die Schweiz die Revision der Entsenderichtlinie (EU) 2018/957 und die Durchsetzungsrichtlinie 2014/67/EU in ihre nationale Gesetzgebung überführt. Gleichzeitig soll es der Schweiz erlaubt sein, gestützt auf eine branchenspezifische Risikoanalyse eine Voranmeldefrist von vier Arbeitstagen und eine Kautionspflicht für Firmen, welche bei einer früheren Dienstleistungserbringung ihren finanziellen Verpflichtungen nicht nachgekommen sind, vorzusehen. Zur Bekämpfung der Scheinselbständigkeit soll es der Schweiz erlaubt sein, von den selbständigen Dienstleistungserbringerinnen und Dienstleistungserbringern im Rahmen nachträglicher Kontrollen die Vorlage von Dokumenten zu verlangen. Diese Massnahmen werden quasi «immunisiert»: Die Schweiz ist nicht verpflichtet, Weiterentwicklungen des EU-Rechts in diesen Bereichen zu übernehmen; auch sind diese Massnahmen nicht über- 
prüfbar durch ein Schiedsgericht. Weiter soll die Schweiz in das BinnenmarktInformationssystem (IMI, vgl. Verordnung (EU) Nr. 1024/2012) aufgenommen werden; damit wird die Verwaltungszusammenarbeit erleichtert und die Wirksamkeit der Kontrollen von Dienstleistungserbringern verbessert.

\begin{abstract}
Voranmeldefristen, Kautions- und Dokumentationspflichten, welche die Dienstleistungsfreiheit weiter einschränken als die von der EU vorgeschlagenen «immunisierten» Massnahmen, dürften $e$ contrario zumindest in aller Regel mit dem FZA und den darin referenzierten Richtlinien nicht vereinbar sein (A. EPINEY, Entwurf, Rz. 37). Weitere flankierende Massnahmen (FlaM; N. 194-195), deren Rechtmässigkeit nicht restlos geklärt ist, betreffen das System der sozialpartnerschaftlichen Kontrollen durch paritätische und tripartite Kommissionen (inkl. Kompetenzen zur Sanktionierung und Durchsetzung), die Dienstleistungssperren, die Häufigkeit der Kontrollen ausländischer Firmen und die Kumulation von Konventionalstrafen, Verwaltungssanktionen und Strafen; diese Massnahmen werden sich auch in Zukunft am geltenden unionalen Entsenderecht messen lassen müssen (A. EPINEY/ L. HEHEMANN, Rz. 41-42).
\end{abstract}

Horizontaler Gemischter Ausschuss und gemischter parlamentarischer Ausschuss (Art. 15-16): Mit dem InstA werden ein horizontaler Gemischter Ausschuss und ein gemischter parlamentarischer Ausschuss eingesetzt. Ersterer soll die Gesamtübersicht über die Beziehungen zwischen der Schweiz und der EU garantieren, die Koordination zwischen den sektoriellen Ausschüssen sicherstellen und das Funktionieren des InstA überwachen. Letzterer soll über Dialog und Debatten zum besseren Verständnis zwischen der Schweiz und der EU in denjenigen Sachbereichen beitragen, die unter die betroffenen Abkommen fallen; damit wird der Boden gelegt für einen formalisierten Austausch der Volksvertreterinnen und -vertreter der Schweiz und der EU.

115 Kündigung (Art. 22): Bei einer Kündigung des InstA treten auch die von ihm erfassten fünf Abkommen der Bilateralen I ausser Kraft, sofern sich die Vertragsparteien innert drei Monaten nicht auf eine andere Lösung einigen. Die künftigen Abkommen, mit denen die Schweiz am Binnenmarkt teilnimmt und die explizit auf das InstA verweisen, treten gemeinsam mit dem InstA ausser Kraft. Damit wird das Schicksal der fünf betroffenen Abkommen der Bilateralen I und der künftigen Marktzugangsabkommen mit demjenigen des InstA verknüpft.

Diese Verknüpfung ist vor dem Hintergrund, dass die EU das aktuell geltende System nicht mehr als valable Option betrachtet, nachvollziehbar. Keineswegs zwingend scheint allerdings, dass die Abkommen der Bilateralen I bei einer Kündigung des InstA ohne Einigung der Vertragsparteien automatisch ausser Kraft treten. Weniger einschneidend - und ebenso gut begründbar - wäre die Regelung, wonach diese Abkommen bei einer Kündigung des InstA ohne Tätigwerden der Vertragsparteien fortgeführt werden; also Weiterbestand, nicht Kahlschlag by default. Es ist klar, dass mit der vorgeschlagenen Lösung nurmehr ganz überwiegende Gründe eine Kündigung des gesamten Pakets zu rechtfer- 
tigen vermögen (kritisch zu diesem «Ausserkraftsetzungs-Automatismus» und zur faktischen Ausweitung der Guillotine-Klausel der Bilateralen I M. AMBÜHL/D. SCHERER, Entwurf, passim).

Gewisse Themen, welche eine horizontale Regelung verdienen würden, werden im InstA nicht aufgegriffen. Dazu gehört die Klärung der Rechtslage in Bezug auf den Grundrechtsschutz bei der Auslegung und Anwendung der bilateralen Abkommen (N. 78). Dazu gehört die Vereinheitlichung und Formalisierung der Beteiligungen der Schweiz an unionalen Agenturen und Programmen, soweit es um immer wiederkehrende Regelungsgegenstände geht (vgl. Agenturbericht, S. 110; Antwort des Bundesrates vom 20. Mai 2009 auf die Interpellation 09.3249 «Rahmenabkommen mit der EU zur Stärkung des Automatismus?»).

\section{Weiteres Vorgehen}

Der Bundesrat veröffentlichte im Juni 2019 einen Bericht über die Ergebnisse der Konsultationen (www.eda.admin.ch/dea und Link zu Institutionelles Abkommen). Er bekräftigte seine positive Einschätzung des Entwurfs. Gleichzeitig verlangte er von der EU Klärungen und Präzisierungen in drei Bereichen: (i) Rechtssicherheit in Bezug auf das in der Schweiz geltende Lohnschutzniveau (FlaM); (ii) Nicht-Übernahme der Unionsbürgerrichtlinie; (iii) NichtAnwendbarkeit der Beihilferegeln auf das Freihandelsabkommen von 1972 (Schreiben von Bundespräsident Ueli Maurer an Kommissionspräsident JeanClaude Juncker vom 7. Juni 2019, www.eda.admin.ch/dea und Link zu Institutionelles Abkommen; s. für Lösungsvorschläge S. BREITENMOSER, Ergänzungen, passim; M. AMBÜHL/D. SCHERER, Entwurf, passim; C. TOBLER, Varianten, passim). Der National- und Ständerat nahmen Motionen an, welche den Bundesrat ebenfalls zu diesen Nachbesserungen auffordern (Motionen 19.3416 und 19.3420 «Zusatzverhandlungen zum institutionellen Abkommen mit der EU»). Während eine Klärung in Bezug auf den letzten Punkt der genannten Bereiche möglich scheint, sind die Fronten beim Lohnschutz und bei der Unionsbürgerrichtlinie verhärtet. Der Abkommenstext muss zwingend geändert werden, um diesen beiden Anliegen der Schweiz Rechnung zu tragen. Der Rat der EU verlautete demgegenüber im Februar 2019, dass er den baldigen Abschluss «auf der Grundlage des vorliegenden Texts» erwartet (Schlussfolgerungen des Rates vom 19. Februar 2019, Rz. 9). Das Europäische Parlament betonte, «dass sich die EU bei den Verhandlungen über das Abkommen über einen institutionellen Rahmen sehr flexibel gezeigt hat und dass dies von allen beteiligten Parteien anerkannt werden muss»; es gab seiner Hoffnung Ausdruck, dass die Schweiz das institutionelle Abkommen «schnellstmöglich» 
abschliesst (Empfehlung vom 26. März 2019, 2018/2262[INI], lit.d und e). Erschwerend für die Schweiz kommt hinzu, dass die EWR-EFTA-Staaten die Übernahme der Unionsbürgerrichtlinie 2004/38/EG in das EWR-Abkommen längst akzeptiert und damit ein für die Schweiz nachteiliges Präjudiz geschaffen haben (Beschluss des Gemeinsamen EWR-Ausschusses Nr. 158/2007 inkl. gemeinsamer Erklärung der Vertragsparteien, wonach mit dieser Übernahme auch künftig keine Rechte einhergehen, welche sich aus dem Konzept der Unionsbürgerschaft ergeben, und wonach die Einwanderungspolitik nicht unter das EWRA fällt; M. OESCH, Incorporation, S. 21-23; C. TOBLER/J. BEGLINGER, Brevier, 51. Frage). Weiter führen die komplizierten Verhandlungen über das künftige Verhältnis der EU zum Vereinigten Königreich dazu, dass die EU gegenüber Drittstaaten noch konsequenter auftritt und ihnen nur privilegierten Marktzugang gewährt, wenn die von ihr definierten Rahmenbedingungen respektiert werden. Es dürfte für die Schweiz schwierig werden, ihre Forderungen durchzusetzen. Klar ist, dass der Ball bei der Schweiz liegt: Sie ist gefordert, der EU Vorschläge für Klärungen und Präzisierungen zu unterbreiten.

Sofern der Bundesrat dem Abkommen - gegebenenfalls in geänderter Form zustimmt, muss er es dem Parlament zur Genehmigung unterbreiten. Das Parlament entscheidet anlässlich seines Genehmigungsbeschlusses, ob das Abkommen dem obligatorischen Referendum - sofern es einem Beitritt zu einer supranationalen Organisation gleichkommt (Art. 140 Abs. 1 lit. b BV) oder verfassungsmässigen Charakter hat (obligatorisches Staatsvertragsreferendum sui generis) - oder dem fakultativen Referendum (Art. 141 Abs. 1 lit. d BV) zu unterstellen ist. Es ist fraglich, ob das Abkommen die Schwelle für ein obligatorisches Referendum erreicht (a.M. A. GLASER, Staatsvertragsreferendum, passim). Nicht ausgeschlossen ist, dass das Parlament ein obligatorisches Referendum aus primär politischen Gründen anordnet; ein Vorgehen, welches Bundesrat und Parlament bereits beim Abschluss des Freihandelsabkommens von 1972 und beim geplanten EWR-Beitritt 1992 wählten (N. 22 und N. 26).

In der Lehre wurde vorgeschlagen, ein Interimsabkommen mit der EU auszuhandeln, z.B. «in Form eines niederschwelligen Memorandum of Understanding». Auf diese Weise liessen sich negative Entwicklungen abfedern, und die Verhandlungen über das InstA könnten später wieder aufgenommen werden, auch mit einem Seitenblick auf den Gang der Verhandlungen zwischen der EU und dem Vereinigten Königreich (M. AMBÜHL/D. SCHERER, Interimsabkommen, passim; M. AMBÜHL/D. SCHERER, Personenfreizügigkeit, passim). Es ist allerdings nicht leichthin ersichtlich, weshalb die EU in einigen Jahren zu Konzessionen bereit sein sollte, welche sie heute der Schweiz (und auch dem Vereinigten Königreich) nicht gewähren mag. Auch ist mit Blick auf die Erwartungshaltung der EU, das InstA nach 
langen Jahren der Debatte und des Feilschens abzuschliessen, fraglich, ob die EU einem weiteren Aufschub geneigt ist; es scheint, dass sie den Status quo nicht mehr als valable Option betrachtet.

Das Institutionelle Abkommen stellt die Beziehungen mit der EU auf eine solide Grundlage und ermöglicht der Schweiz, ihre sektoriell mitgliedstaatsähnliche Stellung zu konsolidieren und weiterzuentwickeln. Es trägt zur weiteren Verrechtlichung des bilateralen Acquis bei, indem es die Beziehungen in den vom Institutionellen Abkommen abgedeckten Bereichen dynamisiert, die Beteiligung der Schweiz bei der Vorbereitung neuer Rechtsakte formalisiert und die Streitbeilegung entpolitisiert und einer gerichtlichen Instanz überantwortet, was der Schweiz als politisch und wirtschaftlich weniger mächtigen Vertragspartnerin in die Hände spielt. Die Rechtssicherheit wird erhöht. Das Institutionelle Abkommen bereitet den Weg, nach ersten Erfahrungen auch das Freihandelsabkommen sowie neue Abkommen unter diesen Rahmen zu stellen und weitere pragmatische Integrationsschritte der Schweiz an die Hand zu nehmen (vgl. auch Stellungnahme der Vereinigung La Suisse en Europe zum Rahmenabkommen, 9. Februar 2019, www.suisse-en-europe.ch). Gleichzeitig fordert das Institutionelle Abkommen das vorherrschende Staatsund Demokratieverständnis der Schweiz heraus. Die dynamische Rechtsübernahme führt dazu, dass die Schweiz in den vom Institutionellen Abkommen erfassten Bereichen in mitgliedstaatsähnlicher Weise in den unionalen Rechtsraum eingebunden wird und zukünftige Rechtsentwicklungen mitträgt. Schon heute besteht zwischen der fortlaufenden Rechtsübernahme und der (direkten) Demokratie ein Spannungsverhältnis; die Substanz der demokratischen Rechte wird ausgehöhlt (N. 65-68). Diese Entwicklung wird sich mit der Dynamisierung der Beziehungen noch verstärken. Ein neuer institutioneller Rahmen gehört letztlich zum Preis, den die Schweiz dafür bezahlt, sektoriell am unionalen Binnenmarkt teilzuhaben, ohne die gemeinsamen Regeln des Clubs vollumfänglich akzeptieren zu wollen. Sollte die Schweiz diesen weiteren Integrationsschritt ablehnen, droht eine Phase der Unsicherheit und der schleichenden Erosion der bilateralen Beziehungen; das wäre keine verlockende Perspektive - weder für die Schweiz noch für die EU. 



\section{Einzelne Abkommen}

\section{A. Einleitung}

Die Grundlage des Acquis Schweiz-EU bilden rund 20 bilaterale Hauptabkommen. Sie werden in diesem Teil vorgestellt, thematisch gebündelt nach ihrem primären Regelungsinhalt: Warenhandel, Personenfreizügigkeit, Dienstleistungshandel, Wettbewerbsrecht, Justiz und Inneres, Forschung, Kultur und Bildung sowie Diverses. Im Rahmen eines Exkurses wird der Beitrag der Schweiz an die Kohäsionspolitik der EU behandelt, wenngleich dieser Beitrag autonom durch die Schweiz geleistet wird und nicht auf einer vertraglichen Abmachung beruht.

Der bilaterale Acquis enthält keine allgemeinen Regeln zur Kapitalverkehrsfreiheit. Nur ganz vereinzelt finden sich in den bilateralen Abkommen Bestimmungen, welche für Investitionen relevant sind. Dazu gehören die Vorgaben zu Steuern und zum Erwerb von Liegenschaften im Freihandelsabkommen und im Freizügigkeitsabkommen (T. COTTIER et al., Rz. 655). Auf der multilateralen Ebene enthält das WTO-Recht im GATS (N. 178), im TRIPs-Abkommen und im TRIMs-Abkommen Regeln für den Investitionsschutz. Die Schweiz hat mit diversen Mitgliedstaaten der EU zudem bilaterale Investitionsschutzabkommen abgeschlossen (s. für eine Übersicht www.seco.admin.ch und Link zu Aussenwirtschaft/Internationale Investitionen; zur Übergangsregelung für Investitionsschutzabkommen zwischen EU-Mitgliedstaaten und Drittländern Verordnung (EU) Nr. 1219/2012). Schliesslich haben sich die Mitgliedstaaten der OECD auf rechtlich bindende Grundsätze zur Unterstützung der Liberalisierung des Kapital- und Zahlungsverkehrs verpflichtet (Codes of Liberalisation of Capital Movements and of Current Invisible Operations; Bericht in Beantwortung des Postulats 13.4022 «Freihandelsabkommen mit der EU statt bilaterale Abkommen» vom Juni 2015, S. 46; M. OESCH, Aussenwirtschaftsrecht, Rz. 30.26).

Gemäss Art. 63 AEUV sind Beschränkungen des Kapitalverkehrs zwischen den Mitgliedstaaten der EU und dritten Ländern verboten. Diese Regelung gilt allerdings nur vorbehältlich spezifischer Ausnahmen (Art. 64-66 AEUV; s. zu bereits vor 1993 bestehenden Beschränkungen gegenüber dritten Ländern Urteil Fokus Invest, C-541/08, EU:C:2010:74). Aus diesem Grund ist die Abgrenzung der Kapitalverkehrsfreiheit von den anderen Grundfreiheiten für Drittstaaten besonders bedeutsam (s. zur Abgrenzung der Kapitalverkehrsfreiheit von der Dienstleistungsfreiheit Urteil Fidium Finanz, C-452/04, EU:C:2006:631; zum Ganzen T. CotTIER et al., Rz. 626-667; C. TOBLER/J. BEGLINGER, Grundzüge, Rz. 119-120; T. Oppermann/C.D. Classen/M. NetTesheim, § 30 Rz. 12). Aktuell gibt es in der EU Bestre- 
bungen, Investitionen aus Drittstaaten einer verstärkten Kontrolle zu unterwerfen: Die Verordnung (EU) 2019/452 ermöglicht den Mitgliedstaaten, ausländische Direktinvestitionen aus Gründen der Sicherheit oder der öffentlichen Ordnung zu überprüfen. Ein Kooperationsmechanismus stellt sicher, dass sich die Europäische Kommission bei Projekten von Unionsinteresse beteiligen kann. 2020 hat die Kommission Leitlinien zur Anwendung dieser Verordnung erlassen; damit verfolgt sie auch das Ziel zu verhindern, dass die durch die COVID-19-Pandemie ausgelöste Krise zu einem Verlust kritischer Anlagen und Technologien führt (Mitteilung der Kommission 2020/C 99 I/01).

\section{B. Warenhandel}

Die WTO stellt die rechtliche Grundordnung für den Warenhandel zwischen der EU und der Schweiz dar. Das GATT 1994 enthält die Vorgaben in Bezug auf den Marktzugang, die Nichtdiskriminierung und die Transparenz. Es belässt Raum für die Verfolgung öffentlicher Interessen wie den Gesundheits- und Umweltschutz und wird durch eine Vielzahl von Zusatzabkommen und Vereinbarungen ergänzt. Den Vertragsparteien gelang es im Rahmen des GATT 1947 in acht Handelsrunden seit dem Ende des Zweiten Weltkriegs, die durchschnittlichen Zölle auf den Handel mit Industrieprodukten erheblich zu senken. Demgegenüber geniessen landwirtschaftliche Güter in diversen WTOMitgliedern - dazu gehören auch die Schweiz und die EU - seit jeher eine Sonderbehandlung. Dafür wird hinlänglich auf die multifunktionalen Aufgaben der Landwirtschaft verwiesen (vgl. Art. 104 Abs. 3 BV). Mit Inkrafttreten der WTO 1995 wurde der Handel mit landwirtschaftlichen Gütern zwar ansatzweise liberalisiert; das WTO-Abkommen über die Landwirtschaft erlaubt aber weiterhin den Erlass von internen Stützungsmassnahmen (Direktzahlungen) und von - mitunter prohibitiv hohen - Zöllen und Zollkontingenten, um die einheimische Produktion zu schützen.

Der durchschnittliche Zollsatz auf die Einfuhr von Industrieprodukten in die Schweiz liegt bei 2,3\%; bei landwirtschaftlichen Produkten liegt er bei $30.8 \%$ (WTO Trade Policy Review Report, Switzerland and Liechtenstein, 11 April 2017, WT/TPR/S/355, Rz. 3.22). Der durchschnittliche Zollsatz auf die Einfuhr von Industrieprodukten in die EU liegt bei 4,2\%; bei landwirtschaftlichen Produkten liegt er bei $14.2 \%$ (WTO Trade Policy Review Report, European Union, 10 December 2019, WT/TPR/S/395, Rz. 3.1.3.2).

Die nachfolgend dargestellten bilateralen Abkommen bauen auf dem WTORecht auf. Sie begründen in ihrer Gesamtheit eine Freihandelszone gemäss Art. XXIV GATT (Botschaft Bilaterale I, S. 6430 und S. 6433; kritisch dazu M. OESCH, Integration, S. 233-234, v.a. mit Blick auf die nur punktuell vereinbarten Liberalisierungen im Agrarbereich). An der Grenze zwischen der Schweiz und der EU finden im Unterschied zu einer Zollunion weiterhin Zollabfertigungen und stichprobenweise Kontrollen statt, etwa um den Ursprung 
von Waren festzustellen. Dies gilt unabhängig davon, dass die Schweiz ein assoziierter Schengen-Staat ist und an den Binnengrenzen des SchengenRaums keine systematischen Personenkontrollen durchgeführt werden ( $\mathrm{N}$. 247-248).

\section{Freihandel}

Die EWG und die Schweiz unterzeichneten 1972 das Freihandelsabkommen (FHA; zur Entstehungsgeschichte N. 22). Es trat 1973 in Kraft und bezweckt, in Übereinstimmung mit dem GATT «die Hemmnisse annähernd für ihren gesamten Handel schrittweise zu beseitigen» (Präambel). Das Abkommen stellt ein klassisches Handelsabkommen dar, mit dem der gegenseitige Marktzugang geregelt wird, ohne die Schweiz in den Binnenmarkt zu integrieren (N. 48). Gleichwohl sind diverse Bestimmungen ähnlich oder gar wortgleich formuliert wie die Parallelbestimmungen des EWGV bzw. AEUV (Zoll- und Abgaberecht, Verbot mengenmässiger Beschränkungen und Massnahmen gleicher Wirkung, Kartell- und Beihilferecht), womit sich bei der Auslegung dieser Bestimmungen die Frage stellt, welche Präjudizwirkung die Praxis des EuGH zu den Parallelbestimmungen im EU-Recht entfalten soll (s. zum Polydor-Prinzip N. 81-82; zur Erklärung der EU zur Auslegung von Art. 23 Abs. 1 FHA N. 89). Das Abkommen wird durch rund 130 zusätzliche Protokolle, Vereinbarungen und Briefwechsel ergänzt, welche allesamt integrale Bestandteile des Abkommens sind (s. zum Zusatzprotokoll betr. Amtshilfe bei der Sicherstellung der ordnungsgemässen Anwendung des Zollrechts N. 269).

Das Freihandelsabkommen bildet die Grundlage der Handelsbeziehungen zwischen der Schweiz und der EU. 2018 exportierte die Schweiz Waren im Wert von 121 Mia. CHF in die EU. Umgekehrt importierte sie Waren aus der EU im Wert von 142 Mia. CHF (ohne Goldhandel). 2018 war die Schweiz nach den Vereinigten Staaten und China der drittgrösste Absatzmarkt für Waren aus der EU. Ein Grossteil dieser Warenflüsse fällt in den Anwendungsbereich des Freihandelsabkommens.

Institutionell beruht das Abkommen auf dem traditionellen Zweisäulenprinzip (N. 69; zum Steuerstreit N. 223). Es ist davon auszugehen, dass der EuGH das Abkommen unmittelbar anwenden würde (N. 57); die bundesgerichtliche Praxis ist diesbezüglich zurückhaltender (N. 58). Sofern eine Vertragspartei der Auffassung ist, die andere Vertragspartei verstosse gegen das Abkommen, kann sie gestützt auf abkommensspezifische Vorgaben oder allgemeine völkerrechtliche Grundsätze einseitig geeignete Massnahmen ergreifen (Gegenbzw. Schutzmassnahmen, Art. 27 FHA). 
Das geplante InstA enthält eine Gemeinsame Erklärung, worin die Vertragsparteien ihre Absicht bekunden, Verhandlungen über die Modernisierung des Freihandelsabkommens einzuleiten. Weiter ist dem InstA ein Entwurf für einen Beschluss des Gemeinsamen Ausschusses nach Art. 29 FHA beigefügt; demnach können die Parteien konsensual entscheiden, Streitfälle unter dem FHA dem Schiedsgericht zu überantworten (N. 107).

Das Abkommen ist auf Industriegüter und - vor allem seit Inkrafttreten des Abkommens über landwirtschaftliche Verarbeitungserzeugnisse (N. 142-145) ausgewählte verarbeitete landwirtschaftliche Produkte anwendbar, welche in der EU und in der Schweiz ihren Ursprung haben. Dabei handelt es sich um Erzeugnisse, welche unter die Kapitel 25-97 des Harmonisierten Systems zur Bezeichnung und Codierung der Waren (HS, SR 0.632.11) fallen (mit Ausnahme der in Anhang I aufgeführten Waren), und Erzeugnisse, welche in Anhang II und Protokoll Nr. 2 (unter den dort getroffenen Sonderregelungen) genannt werden (Art. 2). Das Protokoll Nr. 3 regelt die Bestimmung des Ursprungs (Art. 11); diese Vorgaben beruhen mittlerweile auf dem Regionalen Übereinkommen über Pan-Europa-Mittelmeer-Präferenzursprungsregeln von 2011 (PEM-Übereinkommen, SR 0.946.31; Beschluss Nr.2/2015 des Gemischten Ausschusses EU-Schweiz vom 3. Dezember 2015; s. zur nachträglichen Prüfung eines Ursprungsnachweises Urteil Les Rapides Savoyards, C-218/83, EU:C:1984:275; BGE 112 IV 53; BGE 114 Ib 168).

In einem Urteil von 1992 äusserte sich das BGer zur Frage, ob Mineralwasser in Getränkeflaschen aus PVC in den Geltungsbereich des Abkommens fiel. Es kam zum Schluss, dass in casu Mineralwasser das relevante Produkt war und nicht die Verpackung. Da Mineralwasser zu jenem Zeitpunkt - anders als heute (vgl. Protokoll Nr. 2, Tabelle II, HS Nr. 2201: «Wasser, einschliesslich natürliches oder künstliches Mineralwasser und kohlensäurehaltiges Wasser, ohne Zusatz von Zucker, anderen Süssmitteln oder Aromastoffen; Eis und Schnee») - nicht vom FHA erfasst wurde, war letzteres nicht anwendbar (BGE 118 Ib 367, E. 6; kritisch dazu T. COTTIER et al., Rz. 317-318).

Das Abkommen verbietet Ein- und Ausfuhrzölle, Abgaben gleicher Wirkung (Art. 3-7) und diskriminierende Steuern (Art. 18). Die Vertragsparteien waren verpflichtet, innert der vereinbarten Übergangsfristen Zölle und Abgaben gleicher Wirkung zu beseitigen. Seither gilt im Warenverkehr zwischen der EU und der Schweiz ein absolutes Verbot von Ein- und Ausfuhrzöllen, Abgaben gleicher Wirkung und diskriminierenden Steuern. Diese Bestimmungen weisen einen ähnlichen Wortlaut auf wie die Parallelbestimmungen im EU-Recht (Art. 12 und Art. 95 EWGV, Art. 30 und Art. 110 AEUV). Sie werden indessen teilweise unterschiedlich ausgelegt und angewendet. Der EuGH tendiert zu einer parallelen Auslegung der Bestimmungen zu Zöllen und Abgaben gleicher Wirkung im Gemeinschafts- bzw. Unionsrecht und in Freihandelsabkommen (s. etwa Urteil Aprile, C-125/94, EU:C:1995:309; Urteil Legros, C-163/90, 
EU:C:1992:326, betr. FHA EWG-Schweden; T. COTTIER et al., Rz. 334-335). Bei der Auslegung der steuerrechtlichen Bestimmungen geht der EuGH differenzierter vor (s. etwa Urteil Metalsa, C-312/91, EU:C:1993:279, betr. FHA EWGÖsterreich; Urteil Kupferberg, C-104/81, EU:C:1982:362, betr. FHA EWG-Portugal; A. EPINEy/B. Metz/B. PIRKER, Vereinbarkeit, Rz. 17). Es ist davon auszugehen, dass diese Praxis des EuGH auch bei der Auslegung des Freihandelsabkommens mit der Schweiz massgebend ist. Die schweizerischen Behörden haben sich demgegenüber nur selten zu diesen Bestimmungen geäussert (s. etwa BGE 131 II 271, E. 10, zur Auslegung von Art. 7 und Art. 18 FHA; kritisch dazu D. WÜGER, Freihandelsabkommen, passim).

Die schweizerische Zollrekurskommission verneinte in einem Entscheid von 2001, dass die schweizerische Automobilsteuer einer Abgabe gleicher Wirkung wie einem Einfuhrzoll gemäss Art. 6 FHA gleichkommt, weil die interne Abgabe nicht diskriminierend i.S.v. Art. 18 FHA ist. Eine Privatperson hatte vorgebracht, diese Steuer würde faktisch allein auf importierte Automobile erhoben, weil die Inlandproduktion von Automobilen in der Schweiz bedeutungslos sei. Damit drang sie nicht durch. Die Zollrekurskommission hielt fest: «Die Steuer ist als Verbrauchssteuer ganz klar Teil des allgemeinen schweizerischen Abgabensystems und sie dient ausschliesslich der Erzielung von Einnahmen für den allgemeinen Staatshaushalt und nicht protektionistischen Zwecken. Von einer Abschottung des nationalen Marktes durch die Erhebung dieser Steuer kann keine Rede sein.» (Entscheid vom 29. August 2001, ZRK 2000-020, E. 6.b, VPB 66.44).

Das Abkommen verbietet mengenmässige Beschränkungen und Massnahmen gleicher Wirkung (Art. 13-14). Die Vertragsparteien waren verpflichtet, die bei Unterzeichnung des Abkommens bestehenden Beschränkungen innerhalb der vereinbarten Übergangsfrist aufzuheben. Dieses Verbot gilt sowohl für Einfuhren als auch für Ausfuhren.

Die Eidgenössische Rekurskommission für Infrastruktur und Umwelt qualifizierte in einem Entscheid von 2005 einen Zuschlag auf den Vorzugstarif für die Beförderung von Presseerzeugnissen, welche im Ausland gedruckt wurden, als Massnahme gleicher Wirkung gemäss Art. 13 FHA und bestätigte gestützt auf eine völkerrechtskonforme Auslegung von Art. 15 des Postgesetzes (in der damaligen Fassung) die Unrechtmässigkeit des Zuschlags (Entscheid vom 20. Oktober 2005, H-2004-174, E. 13.5.4).

Die Europäische Kommission unterstellte bei der Bekämpfung der durch das Coronavirus (COVID-19) ausgelösten epidemiologischen Krise die Ausfuhr von bestimmten Gegenständen im Bereich der medizinischen Schutzausrüstung (Atemmasken, Handschuhe, Schutzbrillen, Schutzkleidung) einer speziellen Ausfuhrgenehmigung (Durchführungsverordnung (EU) 2020/402). Es handelte sich dabei um Produkte, welche notwendig waren, «um eine weitere Ausbreitung der Krankheit zu verhindern und die Gesundheit des medizinischen Personals zu schützen, das infizierte Patientinnen und Patienten behandelt» (ibid., Erwägungsgrund 2). Dieses faktische Ausfuhrverbot war für die Schweiz problematisch, weil sie dringend auf diese Güter angewiesen war. Eine Woche später wurden Ausfuhren in die Schweiz von der Genehmigungspflicht ausgenommen (N. 372-373). Offen bleibt, ob die Ausfuhrbeschränkung unter Rekurs auf Art. 20 FHA gerechtfertigt werden konnte. 
Das Verbot mengenmässiger Beschränkungen und Massnahmen gleicher Wirkung gilt - im Gegensatz zum Verbot von Ein- und Ausfuhrzöllen, Abgaben gleicher Wirkung und diskriminierenden Steuern - nicht absolut. Das Abkommen lässt Ein-, Aus- und Durchfuhrverbote oder -beschränkungen zu, die aus Gründen der öffentlichen Sittlichkeit, Ordnung und Sicherheit, zum Schutz der Gesundheit und des Lebens von Menschen und Tieren oder von Pflanzen, des nationalen Kulturguts von künstlerischem, geschichtlichem oder archäologischem Wert oder des gewerblichen und kommerziellen Eigentums gerechtfertigt sind und weder ein Mittel zur willkürlichen Diskriminierung noch eine verschleierte Beschränkung des Handels darstellen (Art. 20; Ordre public-Vorbehalt). Eine solche Massnahme muss zudem die Verhältnismässigkeit wahren (Urteil Kommission/Italien, C-228/91, EU:C:1993:206, betr. die identische Ausnahmebestimmung im FHA EWG-Norwegen).

\begin{abstract}
Das BGer war 2006 aufgerufen, die Vereinbarkeit eines Werbeverbots für Kosmetika unter Bezugnahme auf Krankheiten mit Art. 13 und Art. 20 FHA zu überprüfen. Dabei handelte es sich um eine mengenmässige Beschränkung. Diese Beschränkung konnte unter Rekurs auf den Gesundheits- und Verbraucherschutz gerechtfertigt werden; auch war sie verhältnismässig und stellte keine verschleierte Beschränkung des Handels dar (Urteil vom 6. September 2006, 2A.593/2005, E. 5).
\end{abstract}

Der Wortlaut von Art. 13 Abs. 1 und Art. 20 FHA ist praktisch identisch mit demjenigen von Art. 30 und Art. 36 EWGV bzw. Art. 34 und Art. 36 AEUV (s. dazu A. EPINEY/B. MeTZ/B. PIRKER, Vereinbarkeit, Rz. 11-24). Zeitweise legt der EuGH diese Bestimmungen in Freihandelsabkommen mit EFTA-Staaten gleich aus wie diejenigen im Gemeinschafts- bzw. Unionsrecht (Urteil Eurim-Pharm GmbH, C-207/91, EU:C:1993:278, betr. FHA EWG-Österreich). Demgegenüber verneint der EuGH eine parallele Auslegung etwa in Bezug auf das Immaterialgüterrecht (Verbot von Parallelimporten) unter Rekurs auf den im Vergleich zum EWG-Recht unterschiedlichen Integrationsgrad von Freihandelsabkommen (Urteil Polydor, C-270/80, EU:C:1982:43, betr. FHA EWG-Portugal). Diese Entscheidung gilt als Präjudiz für die Schweiz: Auch das Bundesgericht lehnte eine analoge Behandlung von Parallelexporten unter dem EWGV und dem Freihandelsabkommen ab (BGE 105 II 49, E. 3, dazu N. 83; BGE 126 III 129, E. 7; T. COTTIER et al., Rz. 368).

Das Verbot von Massnahmen gleicher Wirkung gemäss Art. 13 FHA wird hinlänglich dahingehend verstanden, dass es das im EU-Binnenmarkt entwickelte Herkunftsortsprinzip (Cassis de Dijon-Prinzip) nicht umfasst (vgl. Urteil des BGer vom 6. September 2006, 2A.593/2005, E. 6; C. TOBLER/J. BEGLINGER, Grundzüge, Rz. 146; zum autonom eingeführten Cassis de Dijon-Prinzip in der Schweiz N. 374-376). 
Das Abkommen enthält kartell- und beihilferechtliche Bestimmungen (Art. 23

Abs. 1; N. 219-220 und N. 222-223). Ist eine Vertragspartei der Auffassung, dass eine Praktik mit diesen Bestimmungen unvereinbar ist, so kann sie geeignete Massnahmen treffen (Art. 23 Abs. 2). Auch bei Dumping-Praktiken erlaubt das Abkommen den Erlass geeigneter Massnahmen im Einklang mit dem GATT (Art. 25).

\section{Technische Handelshemmnisse}

Die EG und die Schweiz unterzeichneten 1999 das Abkommen über die gegenseitige Anerkennung von Konformitätsbewertungen (KonfBA, auch: Abkommen über technische Handelshemmnisse, Mutual Recognition Agreement, MRA). Es trat 2002 in Kraft und bezweckt, «die gegenseitige Anerkennung der Ergebnisse der für den Zugang zu den Märkten der Vertragsparteien verbindlich vorgeschriebenen Konformitätsbewertungsverfahren [zu ermöglichen]» (Präambel).

Wertmässig deckt das Abkommen heute mehr als einen Viertel aller Exporte in die EU und mehr als einen Drittel aller Warenimporte aus der EU ab (www.seco.admin.ch und Link zu Aussenwirtschaft/Technische Handelshemmnisse/MRA Schweiz-EU).

Das Abkommen gilt für technische Vorschriften der EU und der Schweiz. In der EU geht es dabei allein um den harmonisierten Bereich. Der nicht-harmonisierte Bereich, d.h. der Bereich, in dem die EU-Mitgliedstaaten weiterhin zum Erlass technischer Vorschriften zuständig sind (wobei der EuGH in diesem Bereich gestützt auf die Warenverkehrsfreiheit das Herkunftsortsprinzip bzw. Cassis de Dijon-Prinzip eingeführt hat, s. Urteil Rewe/Bundesmonopolverwaltung für Branntwein, 120/78, EU:C:1979:42), wird vom Abkommen nicht erfasst (s. zur autonomen Einführung des Cassis de Dijon-Prinzips durch die Schweiz N. 374-376). Das Abkommen beruht auf dem Grundsatz der gegenseitigen Anerkennung von Konformitätsbewertungen.

Gemäss BGer können die Behörden im Rahmen der Marktbeobachtung überprüfen, ob die für das Inverkehrbringen eines in der EU im Verkehr befindlichen Produkts erforderliche Konformitätserklärung zu Recht erfolgt ist (Urteil 2C_754/2010 vom 1. Februar 2011, E. 3.7).

Das Abkommen unterscheidet zwei Konstellationen (Art. 1):

- $\quad$ Keine Anerkennung der Gleichwertigkeit der technischen Vorschriften: Die EU und die Schweiz anerkennen gegenseitig die von den gemäss den im Abkommen festgelegten Verfahren anerkannten Konformitätsbewertungsstellen ausgestellten Berichte, Bescheinigungen, Zulassungen und Konformitätskennzeichen sowie die Konformitätserklärungen der Her- 
stellerin oder des Herstellers, mit denen die Übereinstimmung mit den Anforderungen der anderen Vertragspartei in den vom Abkommen erfassten Bereichen bescheinigt wird.

In den meisten vom Abkommen erfassten Produktsektoren werden die technischen Vorschriften der Schweiz und der EU als gleichwertig anerkannt. Nur in den Produktbereichen Gasverbrauchseinrichtungen und Heizkessel sowie Messmittel und Fertigpackungen wird das jeweilige Recht nicht als gleichwertig anerkannt, womit Exportprodukte in die EU nach den Vorschriften der EU hergestellt werden müssen, die erforderlichen Konformitätsbewertungen jedoch von einer im Rahmen des Abkommens anerkannten schweizerischen Konformitätsbewertungsstelle ausgestellt werden kann (www.seco.admin.ch und Link zu Aussenwirtschaft/Technische Handelshemmnisse/MRA Schweiz-EU).

- Anerkennung der Gleichwertigkeit der technischen Vorschriften: Zur Vermeidung doppelter Verfahren in den Fällen, in denen die schweizerischen Anforderungen mit denen der EU als gleichwertig beurteilt werden, anerkennen die EU und die Schweiz gegenseitig die von den anerkannten Konformitätsbewertungsstellen ausgestellten Berichte, Bescheinigungen und Zulassungen sowie die Konformitätserklärungen der Herstellerin oder des Herstellers, mit denen die Übereinstimmung mit ihren jeweiligen Anforderungen in den vom Abkommen erfassten Bereichen bescheinigt wird. In den Berichten, Bescheinigungen, Zulassungen und Konformitätserklärungen der Herstellerin oder des Herstellers wird insbesondere angegeben, dass die betreffenden Produkte mit den Rechtsvorschriften der EU übereinstimmen. Die in den Rechtsvorschriften einer Vertragspartei vorgeschriebenen Konformitätskennzeichen - in der EU die CE-Kennzeichnung - sind auf den Waren anzubringen, die im Gebiet dieser Vertragspartei in Verkehr gebracht werden. Obwohl das Abkommen von «gleichwertigen» Anforderungen spricht, akzeptiert die EU diese Art der Anerkennung faktisch nur dort, wo das schweizerische Recht qua Anpassung oder Verweisung praktisch identisch ist (S. ARIOLI, Abbau, S. 142).

Anhang 1 legt fest, welche Produktesektoren unter das Abkommen fallen (Art. 3 Abs. 1). Heute werden 20 Produktebereiche vom Abkommen erfasst. Dazu gehören etwa Maschinen, persönliche Schutzausrüstungen, Spielzeuge, Medizinprodukte (aber nicht Medikamente), Druckgeräte, Funkanlagen und Telekommunikationsendgeräte, Baugeräte und Baumaschinen, Kraftfahrzeuge und Bauprodukte. Anhang 1 ist in sektorale Kapitel gegliedert (Rechts- und Verwaltungsvorschriften, Konformitätsbewertungsstellen, benennende Behörden, besondere Grundsätze für die Benennung der Konformitätsbewertungsstellen, gegebenenfalls zusätzliche Bestimmungen). Anhang 1 muss bei 
Weiterentwicklungen des EU-Rechts aufdatiert werden. Dabei geht es regelmässig darum, die Gleichwertigkeit der Gesetzgebung in der Schweiz mit derjenigen in der EU anzuerkennen. Auf diese Weise wird der erreichte Liberalisierungsgrad aufrechterhalten und das ordnungsgemässe Funktionieren des Abkommens sichergestellt. Entsprechende Änderungen erfolgen in aller Regel routinemässig (N. 95); die EU hat in Ausnahmefällen allerdings nicht davor zurückgeschreckt, Weiterentwicklungen zu blockieren, um die Schweiz zu einem Entgegenkommen in anderen Dossiers zu veranlassen (N. 1 und N. 104).

Art. 4 Abs. 2 THG verpflichtet die Bundesbehörden in der Schweiz, die technischen Vorschriften autonom auf die technischen Vorschriften der wichtigsten Handelspartner abzustimmen; damit ist zuvörderst die EU gemeint. Auf diese Weise erledigt die Schweiz ihre Hausaufgaben für die Anerkennung der Gleichwertigkeit durch die EU.

Unterschiedliche Vorschriften existieren etwa weiterhin für das Inverkehrbringen von Alcopops, Reinigungsmittel, welche Phosphat enthalten, und Eier aus in der Schweiz nicht zugelassener Käfighaltung von Hühnern ohne entsprechende Deklaration. Diese Produkte profitieren auch nicht vom einseitig eingeführten Cassis de Dijon-Prinzip (vgl. Art. 2 VIPaV, N. 375).

Das geplante InstA wäre auf das Abkommen über die gegenseitige Anerkennung von Konformitätsbewertungen anwendbar (s. für den Textentwurf des InstA vom Dezember 2018 N. 105-116).

\section{Landwirtschaftliche Produkte}

Das Abkommen über den Handel mit landwirtschaftlichen Erzeugnissen (Landwirtschaftsabkommen) wurde 1999 unterzeichnet. Es trat 2002 in Kraft und bezweckt, «die Freihandelsbeziehungen zwischen den Parteien durch Verbesserung des Marktzugangs für landwirtschaftliche Erzeugnisse der jeweils anderen Partei zu stärken»(Art.1 Abs.1). Als landwirtschaftliche Erzeugnisse gelten die Erzeugnisse der Kapitel 1-24 des Harmonisierten Systems zur Bezeichnung und Codierung der Waren (HS), wobei für die Anwendung der Anhänge 1-3 gewisse Ausnahmen gemacht werden (Art. 1 Abs. 2). Verarbeitete landwirtschaftliche Erzeugnisse werden - von wenigen Ausnahmen abgesehen - nicht erfasst (Art. 1 Abs. 3; s. zum Abkommen über landwirtschaftliche Verarbeitungserzeugnisse N. 142-145).

Die EU ist auch im Bereich des Handels mit landwirtschaftlichen Produkten die wichtigste Handelspartnerin der Schweiz: 2018 gingen rund 58 \% der Ausfuhren aus der Schweiz in die EU; rund $75 \%$ der Einfuhren stammten aus der EU (www.eda.admin.ch/dea und Link zu Bilaterale Abkommen I (1999)/Landwirtschaft). 
Das Abkommen sieht die folgenden Liberalisierungsmassnahmen vor:

- Die Vertragsparteien gewähren sich gegenseitig Zollzugeständnisse (Anhänge 1 und 2). Dies gilt für namentlich aufgeführte Produkte vorwiegend aus den Sektoren Früchte, Gemüse und Gartenbau (einschliesslich Schnittblumen) sowie in beschränktem Umfang auch für Trockenfleischund Weinspezialitäten. Der Käsehandel wurde 2007 vollständig liberalisiert (Anhang 3).

- Die Vertragsparteien treffen Massnahmen zur Beseitigung nichttarifärer Handelshemmnisse im Bereich der Produktevorschriften und Zulassungsbestimmungen. Dies geschieht primär durch die Anerkennung der Gleichwertigkeit der Vorschriften in der Schweiz und in der EU für Produkte im Bereich des Pflanzenschutzes, der Futtermittel, des Saatguts, des Weinbaus und der ökologischen Landwirtschaft (Anhänge 4-11). Im Veterinärbereich, d.h. im Bereich der Gesundheits-, Tierzucht- und Tierschutzmassnahmen für den Handel mit Tieren und Erzeugnissen tierischer Herkunft, wird die Gleichwertigkeit der Vorschriften umfassend anerkannt. Davon profitieren tierische Lebensmittel wie Käse, Fleischspezialitäten und Honig. 2009 wurde ein gemeinsamer europäischer Veterinärraum geschaffen, mit welchem die gegenseitigen tierärztlichen Kontrollen an der Grenze abgeschafft wurden (www.eda.admin.ch/dea und Link zu Bilaterale Abkommen I (1999)/Landwirtschaft).

- 2011 trat das Abkommen zum Schutz von Ursprungsbezeichnungen (GUB) und geografischen Angaben (GGA) für Agrarerzeugnisse und Lebensmittel in Kraft. Dieses Abkommen stellt einen integralen Bestandteil des Landwirtschaftsabkommens dar (Anhang 12). Die namentlich aufgeführten Ursprungsbezeichnungen und geografischen Angaben werden gegenseitig anerkannt und geschützt. Dazu gehören auf Seiten der EU etwa «Tiroler Speck», «Bayerisches Bier», «Lübecker Marzipan» und «Prosciutto di Parma», auf Seiten der Schweiz etwa «St. Galler Bratwurst»/«St. Galler Kalbsbratwurst», «Bündnerfleisch» und «Raclette du Valais»/«Walliser Raclette».

Im Urteil Commune de Champagne, T-212/02, EU:T:2007:194, wies das Gericht (EuG) eine Nichtigkeitsklage u.a. der waadtländischen Gemeinde Champagne gegen die Regelung, wonach Wein aus dieser Gemeinde wegen Verwechslungsgefahr mit dem gleichnamigen französischen Produkt nicht mehr die Bezeichnung «Champagne» tragen kann (Art. 5 Abs. 8 des Titels II in Anhang 7 AgrarA in der Fassung von 1999; sog. Champagnerklausel), als unzulässig ab. Das Gericht kam zum Schluss, dass die Gemeinde Champagne nach Massgabe von Art. 230 Abs. 4 EGV (Art. 263 Abs. 4 AEUV) nicht aktivlegitimiert war, sich 
gegen den Genehmigungsbeschluss des Rates über den Abschluss der bilateralen Abkommen von 1999 (im Hinblick auf die behauptete Unrechtmässigkeit der Champagnerklausel im AgrarA) zu wehren.

Die Anhänge des Abkommens - mehrere hundert Seiten lang - müssen im Zuge der Weiterentwicklungen des EU-Rechts periodisch aufdatiert werden. Dabei geht es regelmässig auch darum, die Gleichwertigkeit der Gesetzgebung in der Schweiz mit derjenigen in der EU anzuerkennen. Auf diese Weise wird der erreichte Liberalisierungsgrad aufrechterhalten und das ordnungsgemässe Funktionieren des Abkommens sichergestellt. Solche Änderungen erfolgen in aller Regel routinemässig (N. 95).

Das geplante InstA wäre auf das Landwirtschaftsabkommen anwendbar (s. für den Textentwurf des InstA vom Dezember 2018 N. 105-116). Demgegenüber sind die 2008 initiierten Verhandlungen über ein Freihandelsabkommen im Agrar- und Lebensmittelbereich (FHAL) seit längerem sistiert; mit einem solchen Abkommen würde ein verbesserter gegenseitiger Zugang zum Agrarund Lebensmittelmarkt entlang der gesamten Produktionskette - vorgelagerte Stufe, welche Produktionsmittel und Investitionsgüter liefert; Landwirtschaft als solche, welche die Rohstoffe herstellt; nachgelagerte Stufe, welche die landwirtschaftlichen Produkte verarbeitet, inkl. erster und zweiter Verarbeitungsstufe - avisiert (www.eda.admin.ch/dea und Link zu Verhandlungen und offene Themen).

\section{Verarbeitete landwirtschaftliche Produkte}

Die EG und die Schweiz unterzeichneten 2004 das Abkommen zur Änderung des Freihandelsabkommens von 1972 in Bezug auf die Bestimmungen über landwirtschaftliche Verarbeitungserzeugnisse (Abkommen über landwirtschaftliche Verarbeitungserzeugnisse). Es wurde 2005 in Kraft gesetzt «in dem Wunsch, den gegenseitigen Zugang zu den Märkten für landwirtschaftliche Verarbeitungserzeugnisse zu verbessern» (Präambel). Mit dem Abkommen wurden Anhang 1 und Protokoll Nr. 2 FHA ersetzt.

Die Schweiz exportierte 2018 in die EU vom Abkommen erfasste verarbeitete landwirtschaftliche Produkte im Wert von rund 7.5 Mia. CHF (www.eda.admin.ch/dea und Link zu Bilaterale Abkommen I (1999)/Landwirtschaftliche Verarbeitungsprodukte). Die Importe aus der EU in die Schweiz erreichten ein Volumen von rund 3.1 Mia. CHF.

Die vom Abkommen erfassten verarbeiteten landwirtschaftlichen Produkte werden in der Tabelle I zum Protokoll Nr. 2 aufgeführt. Dazu gehören etwa Joghurt, Schokolade, Biskuits, Teigwaren, Suppen, andere Nahrungsmittelzubereitungen sowie Mineralwasser (s. zu letzterem auch N. 126). 
Verarbeitete landwirtschaftliche Produkte nehmen eine Sonderstellung zwischen industriellen Produkten (für die mit dem Freihandelsabkommen Zölle und Abgaben gleicher Wirkung abgeschafft wurden) und landwirtschaftlichen Produkten (die vom Freihandelsabkommen grundsätzlich ausgenommen werden) ein (www.eda.admin.ch/dea und Link zu Bilaterale Abkommen I (1999)/Landwirtschaftliche Verarbeitungsprodukte). Sie bestehen aus einem industriellen Verarbeitungsteil und einem landwirtschaftlichen Rohstoffteil. Das Abkommen erlaubt für gewisse Produkte, im Handel zwischen der EU und der Schweiz die Differenz zwischen den Rohstoffpreisen in der Schweiz und der EU auszugleichen (Nettopreiskompensation); in Bezug auf Zucker wurde vereinbart, den Ausgleich vollständig aufzuheben, da die Zuckerpreise in der EU und der Schweiz im langjährigen Mittel vergleichbar sind (T. JAAG/J. HäNNI, Rz. 4107). In der Praxis hat dieser Mechanismus dazu geführt, dass die EU Einfuhrzölle und Abgaben gleicher Wirkung auf verarbeitete landwirtschaftliche Produkte vollständig abgebaut hat und auch auf die Leistung von Exportbeiträgen verzichtet. Die Schweiz hat die Einfuhrzölle und Exportbeiträge auf die Höhe der Differenz der Rohstoffpreise zwischen der Schweiz und der EU gesenkt. Auf diese Weise wird der Wettbewerbsnachteil ausgeglichen, der durch die höheren Beschaffungskosten der landwirtschaftlichen Rohstoffe in der Schweiz entsteht («Rohstoffpreis-Handicap»).

Die WTO-Ministerkonferenz beschloss 2015 in Nairobi, Exportsubventionen für landwirtschaftliche Produkte per Ende 2020 abzuschaffen (Ministerial Decision of 19 December 2015, WT/MIN(15)/45). Die EU und die Schweiz bestätigten diesen Beschluss nach Massgabe der jeweiligen internen Genehmigungsverfahren 2016 und 2017. In der Schweiz wurde zu diesem Zweck das Bundesgesetz über die Ein- und Ausfuhr von Erzeugnissen aus Landwirtschaftsprodukten von 1974 (sog. «Schoggigesetz») totalrevidiert und in Bundesgesetz über die Einfuhr von Erzeugnissen aus Landwirtschaftsprodukten umgetauft; das neue Gesetz trat 2019 in Kraft (SR 632.111.72). Die für verarbeitete landwirtschaftliche Produkte gewährten Ausfuhrbeiträge werden aufgehoben; dies gilt auch im Handel mit der EU. Kompensatorisch werden Begleitmassnahmen erlassen, namentlich Stützungsmassnahmen für Milch und Brotgetreide sowie eine Vereinfachung des Bewilligungsverfahrens des aktiven Veredelungsverkehrs mit gewissen Milch- und Getreidegrundstoffen (Botschaft Ausfuhrbeiträge, S. 4352). 


\section{Zollerleichterungen und Zollsicherheit}

Die EWG und die EFTA-Mitgliedstaaten schlossen 1987 das Übereinkommen zur Vereinfachung der Förmlichkeiten im Warenverkehr ab (SR 0.631.242.03). Damit wurde ein einheitliches Verwaltungspapier (Einheitspapier) eingeführt, das seither für alle Aus- und Einfuhrverfahren sowie für ein im Warenverkehr zwischen den Vertragsparteien geltendes gemeinsames Versandverfahren verwendet wird. 1990 unterzeichneten die EWG und die Schweiz das Abkommen über die Erleichterung der Kontrollen und Formalitäten im Güterverkehr (AS 1991 1490). Es trat 1991 in Kraft und bezweckte, die Kontrollen und Formalitäten im Güterverkehr zwischen der Schweiz und der EWG weiter zu vereinfachen und die Zusammenarbeit an den Grenzstellen zu koordinieren. Die Öffnungszeiten der Zollstellen wurden koordiniert. Dokumente und Kontrollen wurden gegenseitig anerkannt. Bei Waren wurde nurmehr stichprobenweise kontrolliert. Gemeinsame Transitschnellspuren wurden eingerichtet.

2011 wurde das Abkommen von 1990 durch das 2009 unterzeichnete Abkommen über die Erleichterung der Kontrollen und Formalitäten im Güterverkehr und über zollrechtliche Sicherheitsmassnahmen (Zollabkommen, ZollA) ersetzt.

Das Zollabkommen führt dazu, dass der Verkehr an den Grenzübergängen zwischen der Schweiz und der EU weiterhin zügig fliesst. Täglich passieren mehr als 24000 Lastwagen die Schweizer Grenze; jährlich nutzen insgesamt 940000 Lastwagen die Transitachsen in der Schweiz, um Waren von einem EU-Mitgliedstaat in einen anderen zu transportieren (www.eda.admin.ch/dea und Link zu Bilaterale Abkommen bis 1999/Zollerleichterungen und Zollsicherheit).

Das Zollabkommen baut auf den vorbestehenden zollrechtlichen Erleichterungen auf. Es regelt die Zusammenarbeit der Zollverwaltungen in der EU und der Schweiz im Zollsicherheitsbereich und sieht für die Schweiz Ausnahmen in Bezug auf die Voranmeldepflicht für Wareneinfuhren aus Drittstaaten in die EU vor. Schweizerische Unternehmen sind nicht verpflichtet, Importe vorgängig zu melden, wie dies gemäss EU-Recht für Einfuhren aus Drittstaaten der Fall ist (entry summary declaration). Folgerichtig wendet die Schweiz bei Wareneinfuhren aus Drittstaaten in die Schweiz (Luftfracht) ihrerseits die unionalen zollrechtlichen Sicherheitsvorschriften bzw. gleichwertige Vorschriften an.

Die Massnahmen der EU zur Erhöhung der Sicherheit im Warenverkehr mit Drittstaaten waren im Wesentlichen eine Reaktion auf die weltweit veränderte Lage im Bereich der Sicherheit im Nachgang zu den Terroranschlägen vom 11. September 2001 in den Vereinigten Staaten. Mittlerweile enthalten die Verordnung (EU) 952/2013 zur Festlegung des Zollkodex der Union und die delegierten Verordnungen (EU) 2015/2446 und (EU) 2015/2447 
mit Einzelheiten zur Präzisierung und Umsetzung von Bestimmungen des Zollkodex die einschlägigen Vorgaben. Je nach Transportart muss die Anmeldung zwischen einer Stunde (Strassenverkehr) und 24 Stunden (Schiffcontainerfracht) vor dem Grenzübertritt erfolgen; wegen der maximalen Frist in der Hochseeschifffahrt wird landläufig von der «24 StundenRegel» gesprochen. cherheitsraum mit gleichwertigen Sicherheitsstandards (www.eda.admin.ch/ dea und Link zu Bilaterale Abkommen bis 1999/Zollerleichterungen und Zollsicherheit). Das Abkommen beruht auf der Prämisse, dass die EU und die Schweiz gleichwertige zollrechtliche Sicherheitsmassnahmen ergreifen, wobei die EU das angemessene Schutzniveau und die notwendigen Kontrollinstrumente inkl. Risikoanalyse vorgibt. Aus diesem Grund ist es für das ordnungsgemässe Funktionieren des Abkommens unerlässlich, dass Änderungen des EU-Rechts zeitnah auch von der Schweiz übernommen und angewendet werden. Für den Fall, dass die Gleichwertigkeit der zollrechtlichen Sicherheitsmassnahmen nicht mehr gewährleistet ist, sieht das Abkommen vor, dass die Vertragsparteien angemessene Ausgleichsmassnahmen einschliesslich der Aussetzung der betreffenden Bestimmungen des Kapitels III ergreifen können (Art. 29; N. 98 und N. 99).

\section{Personenfreizügigkeit}

Diverse völkerrechtliche Verträge zwischen der Schweiz und europäischen Staaten gewähren Rechte auf Einreise und Aufenthalt im Hoheitsgebiet der anderen Vertragspartei und auf Ausübung einer Erwerbstätigkeit. Solche Verträge gehen mitunter ins 19. Jahrhundert zurück; Beispiele sind der Freundschafts-, Handels- und Niederlassungsvertrag zwischen der Schweiz und dem Vereinigten Königreich von Grossbritannien und Irland von 1855 (SR 0.142.113.671), der Niederlassungs- und Konsularvertrag zwischen der Schweiz und Italien von 1868 (SR 0.142.114.541) und der Niederlassungsvertrag zwischen der Schweiz und Frankreich von 1882 (SR 0.142.113.491). Sie werden zeitweise durch sog. Niederlassungsvereinbarungen ergänzt, die einen Anspruch auf eine Niederlassungsbewilligung beinhalten (T. CотTIER et al., Rz. 437). Sie sind teils immer noch in Kraft, werden seit dem Ersten Weltkrieg in stillschweigendem Einvernehmen aber restriktiv ausgelegt (s. BGE $106 \mathrm{Ib}$ 125, E. 2b, betr. des Niederlassungs- und Konsularvertrags zwischen der Schweiz und Serbien von 1888, SR 0.142.118.181). Darüber hinaus bestehen Abkommen mit den Nachbarstaaten über den (kleinen) Grenzverkehr (s. etwa Abkommen zwischen dem Schweizerischen Bundesrat und der Regierung der Bundesrepublik Deutschland über den Grenzübertritt von Personen im klei- 
nen Grenzverkehr von 1970, SR 0.631.256.913.63). Im Bereich der Anerkennung von Berufsqualifikationen besteht etwa eine Vereinbarung zwischen der Schweiz und Deutschland von 1937, wonach «der deutsche Gesellenbrief dem schweizerischen Fähigkeitszeugnis und das deutsche Meisterdiplom dem schweizerischen Diplom gleichgestellt» sind (BBl 1937 III 491; s. zum Verhältnis dieser Vereinbarung zum FZA N. 174). Das Allgemeine Abkommen über den Dienstleistungshandel (GATS) der WTO gewährt unter gewissen Voraussetzungen ein Recht, natürliche Personen in das Hoheitsgebiet einer anderen Vertragspartei zu entsenden, um eine Niederlassung zu gründen oder Dienstleistungen zu erbringen (Führungskräfte und Spezialisten, intra-corporate transfer and other essential persons); die Rechtslage hängt im Wesentlichen von den Verpflichtungslisten der EU und der Schweiz ab (WTO Doc. GATS/ SC/31, WTO Doc. GATS/SC/83).

Die Kantone haben von ihrem Recht, gestützt auf Art. 56 BV in ihren Zuständigkeitsbereichen Staatsverträge abzuschliessen, durchaus Gebrauch gemacht (s. auch N. 3). Beispiele aus dem Bereich der Personenfreizügigkeit betreffen spezielle Regeln über die Anerkennung von Fähigkeitsausweisen: So hat der Kanton St. Gallen mit Österreich bzw. mit dem österreichischen Bundesland Niederösterreich Gegenrechtsvereinbarungen über die Anerkennung von Jagdkarten (1976) bzw. der Jägerprüfung (1999) abgeschlossen (sGS 853.155 und 853.163). Weiter hat der Kanton St. Gallen eine Gegenrechtserklärung gegenüber dem Fürstentum Liechtenstein über die Anerkennung von Fähigkeitsausweisen für Wirte (1974) abgegeben (sGS 553.153).

Im bilateralen Verhältnis steht das Freizügigkeitsabkommen ganz im Vordergrund. Das Versicherungsabkommen und das Luftverkehrsabkommen enthalten punktuell Bestimmungen über die Niederlassungsfreiheit; dazu gehört in Bezug auf das letztgenannte Abkommen auch das Recht natürlicher Personen, sich im Hoheitsgebiet der anderen Vertragspartei niederzulassen (N. 184 und N. 210). Das Schengen-Assoziierungsabkommen sieht die Abschaffung von Personenkontrollen an den Binnengrenzen vor, enthält - mit Ausnahme der Vorgaben zur Einreise für maximal 90 Tage (Schengen-Visum) - selbst aber keine Regeln über die Einreise und den Aufenthalt.

\section{Freizügigkeitsabkommen: Im Allgemeinen}

Das Abkommen zwischen der Schweiz einerseits und der EG und ihren Mitgliedstaaten andererseits über die Freizügigkeit wurde 1999 unterzeichnet (Freizügigkeitsabkommen, FZA) und 2002 in Kraft gesetzt. Die EU insistierte in den Verhandlungen zu den Bilateralen I, dass das rechtliche Schicksal des Freizügigkeitsabkommens mit demjenigen der weiteren sechs Abkommen von 1999 verbunden wird (Guillotine-Klausel, Art. 25 FZA; N. 28). Das Freizügig- 
keitsabkommen ist ein gemischtes Abkommen und musste folglich auch durch die Mitgliedstaaten ratifiziert werden. Es wurde im Nachgang der Ost-Erweiterungen der EU 2006, 2009 und 2017 formell auf die 13 neuen EU-Mitgliedstaaten ausgedehnt (N. 28). Ziel des Abkommens zu Gunsten der Staatsangehörigen der EU-Mitgliedstaaten und der Schweiz ist, ein Recht auf Einreise, Aufenthalt und Zugang zu einer Erwerbstätigkeit einzuräumen, die Erbringung von kurzzeitigen Dienstleistungen zu erleichtern (N. 187-199), ein Recht auf Einreise und Aufenthalt für Personen, die keine Erwerbstätigkeit ausüben, einzuräumen, und dabei die gleichen Lebens-, Beschäftigungs- und Arbeitsbedingungen wie für Inländerinnen und Inländer zu gewährleisten (Art. 1).

Das SECO veröffentlicht regelmässig Berichte und Statistiken über die wichtigsten Aspekte des freien Personenverkehrs. Im 16. Bericht des Observatoriums zum FZA Schweiz-EU vom 29. Juni 2020 wurden u.a. die folgenden Kernaussagen gemacht (www.seco.admin.ch und Link zu Publikationen): Die Zuwanderung von Arbeitskräften aus der EU/EFTA in die Schweiz ist auf die Bedürfnisse der Wirtschaft fokussiert und wird durch den Arbeitsmarkt und die Wirtschaftsentwicklung in der Schweiz und in den Herkunftsländern bestimmt; dies zeigt die hohe Erwerbsbeteiligung der zugewanderten Personen. Die Arbeitslosenquote von Personen aus der EU/EFTA bleibt gleichwohl leicht über dem nationalen Durchschnitt; Grund dafür ist hauptsächlich die Konzentration von Zugewanderten aus dem EU/ EFTA-Raum auf Branchen, die stärker als andere Branchen von Arbeitslosigkeit betroffen sind. Das FZA begünstigt die Zuwanderung von hochqualifizierten Arbeitskräften, was der Wirtschaft insgesamt zugute kommt. Während für Personen aus der EU/EFTA meist der Antritt einer neuen Stelle den Grund für die Zuwanderung darstellt, ist es bei Personen aus Drittstaaten vor allem der Familiennachzug. Die Erwerbsintegration und der Bezug von Sozialhilfe stehen in engem Zusammenhang; nur 3,4 \% der 2009 aus der EU/EFTA zugewanderten Familienangehörigen haben in den acht Jahren seit ihrer Ankunft Sozialhilfe bezogen. Im Familiennachzug zugewanderte Personen aus der EU/EFTA, die Sozialhilfe beziehen, können sich meist schnell wieder davon lösen und erlangen rasch wieder ihre wirtschaftliche Unabhängigkeit. Je höher das Bildungsniveau ist, desto geringer ist auch das Sozialhilferisiko.

Die Vertragsparteien sind «entschlossen, diese Freizügigkeit zwischen ihnen auf der Grundlage der in der Europäischen Gemeinschaft geltenden Bestimmungen zu verwirklichen» (Präambel). Die zentralen Bestimmungen des Abkommens sind weitgehend dem EU-Recht nachgebildet; verschiedentlich verweist das Abkommen bzw. die Anhänge II (Koordinierung der Systeme der sozialen Sicherheit) und III (gegenseitige Anerkennung beruflicher Qualifikationen) auf das EU-Sekundärrecht. Diese Anhänge müssen regelmässig aufdatiert werden, um den aktuell geltenden EU-Besitzstand zu reflektieren (s. zur schleppenden Übernahme der Richtlinie 2005/36/EG N. 95). Die Rechte werden - in den Worten des EuGH - auf die Personen in der Schweiz «ausgedehnt» (Urteil Vereinigtes Königreich/Rat, C-656/11, EU:C:2014:97, Rn. 59 und 63), womit die Schweiz punktuell «einem Mitgliedstaat gleichzustellen» (Urteil 
Kik, C-266/13, EU:C:2015:188, Rn. 44) ist. Es ist für das gute Funktionieren des Abkommens unabdingbar, dass die Bestimmungen, welche auf EU-Recht beruhen, gleich ausgelegt und angewendet werden wie die Parallelbestimmungen im EU-Recht (N. 84-86). Die Unionsbürgerrichtlinie 2004/38/EG ist im bilateralen Verhältnis nicht einschlägig. Die Schweiz hat wiederholt betont, auf Verhandlungen zur Übernahme dieser Richtlinie nicht einzutreten (N. 96; zu den Verhandlungen über das InstA N. 109). Des Weiteren umfasst das Abkommen nicht alle wesentlichen Elemente der Personenfreizügigkeit und der Dienstleistungsfreiheit, wie sie den Unionsbürgerinnen und Unionsbürgern im EURecht zustehen (Art. 21, Art. 45, Art. 49 und Art. 56 AEUV). So schützt die bilateralrechtliche Niederlassungsfreiheit etwa nur natürliche Personen, und die Dienstleistungsfreiheit ist auf 90 Arbeitstage pro Kalenderjahr beschränkt.

Das Abkommen sieht eine stufenweise und kontrollierte Einführung der Personenfreizügigkeit vor. Die Schweiz ist berechtigt, während einer Übergangsfrist Beschränkungen vorzusehen (Inländervorrang und vorgängige Kontrolle der Lohn- und Arbeitsbedingungen). Unter gewissen Voraussetzungen ist es zulässig, wieder Kontingente einzuführen (Ventilklausel, Art. 10 und Protokolle I-III). Zurzeit sind solche Beschränkungen und Kontingente noch gegenüber Kroatien (Beschränkungen bis 2023, Ventilklausel bis 2026) möglich. Darüber hinaus entscheidet der Gemischte Ausschuss bei schwerwiegenden wirtschaftlichen oder sozialen Problemen über geeignete Abhilfemassnahmen; er tut dies in traditionell diplomatischer Natur einvernehmlich (Art. 14 Abs. 2).

Per Mai 2012 rief der Bundesrat die Ventilklausel an und kontingentierte die Aufenthaltsbewilligungen der Kategorie B für die Angehörigen der EU-8-Staaten (Estland, Lettland, Litauen, Polen, Slowakei, Slowenien, Tschechische Republik und Ungarn). Er verlängerte diese Massnahme per Mai 2013 um ein Jahr und beschloss, sie per Juni 2013 auch gegenüber den Angehörigen der EU-17-Staaten anzuwenden (Belgien, Dänemark, Deutschland, Finnland, Frankreich, Griechenland, Irland, Italien, Luxemburg, Malta, Niederlande, Österreich, Portugal, Schweden, Spanien, das Vereinigte Königreich und Zypern).

Institutionell beruht das Abkommen auf dem klassischen Zweisäulenprinzip (N. 69). Der Gemischte Ausschuss ist für die Verwaltung und die ordnungsgemässe Anwendung des Abkommens verantwortlich (Art. 14). Das Abkommen und seine Anhänge sind unmittelbar anwendbar, d.h. die einschlägigen Rechte können direkt vor den Behörden der Vertragsparteien geltend gemacht werden (s. etwa BGE 129 II 249, E. 3.3; BGE 130 II 49, E. 4.2; BGE 132 II 135; Urteil des BVerwG C-89/2007 vom 2. Juli 2007, E. 3; Botschaft Bilaterale I, S. 6358-6359; N. 57-58); dies gilt unter Umständen auch bei Ansprüchen gegenüber Privaten (Urteil des BGer 4A_593/2009 vom 5. März 2010 betr. GAV; V. MARTENET/V. BoIlLET, passim) und gegenüber dem eigenen Staat 
(Urteil Ettwein, C-425/11, EU:C:2013:121, Rn. 33), sofern der Anwendungsbereich des Abkommens eröffnet ist. Art. 16 FZA sieht eine spezielle Auslegungsregel vor: Die Behörden sind mitunter verpflichtet, die Praxis des EuGH zu berücksichtigen (N. 84-86). Das Abkommen enthält eine ausdrückliche Bestimmung für den Fall, dass es von einer Vertragspartei gekündigt wird. Diesfalls bleiben die erworbenen Ansprüche von Einzelnen unberührt; die Vertragsparteien treffen im gegenseitigen Einvernehmen eine Regelung für die Anwartschaften (Art. 23). Das geplante InstA wäre auf das Freizügigkeitsabkommen anwendbar (s. für den Textentwurf des InstA vom Dezember 2018 N. 105-116).

2010 nahmen Volk und Stände die Volksinitiative «für die Ausschaffung krimineller Ausländer (Ausschaffungsinitiative)» an. Die neuen Verfassungsbestimmungen wurden in problematischer Weise nicht FZA-konform umgesetzt, womit eine völkerrechtskonforme Anwendung den Gerichten überantwortet wurde (N. 62). 2014 nahmen Volk und Stände die Volksinitiative «Gegen Masseneinwanderung» an. Hier achtete die Bundesversammlung darauf, die Umsetzungsgesetzgebung FZA-konform auszugestalten (N. 63). Im September 2020 lehnten Volk und Stände die Initiative «Für eine massvolle Zuwanderung (Begrenzungsinitiative)» ab, welche die Kündigung des Freizügigkeitsabkommens verlangt hätte (N. 61).

\section{Einreise, Aufenthalt, Familiennachzug}

Das Abkommen räumt den Staatsangehörigen einer Vertragspartei weitreichende Freizügigkeitsrechte ein. Im Wesentlichen geht es um Arbeitnehmende, Selbständigerwerbende, nicht erwerbstätige Personen (wie Rentnerinnen und Rentner, Studierende und Privatiers) und Familienangehörige; letztere verfügen über abgeleitete Rechte (N. 163). Diese Rechte können unter gewissen Voraussetzungen eingeschränkt werden (N. 168-169).

Vor diesem Hintergrund ist das bundesgerichtliche Diktum, «dass es sich beim FZA um ein im Wesentlichen wirtschaftsrechtliches Abkommen handelt» und es «lediglich zu einem doppelt bedingten Aufenthalt in der Schweiz [berechtigt], nämlich einerseits nach Massgabe der spezifischen Vertragsvereinbarungen als Voraussetzung eines rechtmässigen Aufenthalts und andererseits nach Massgabe des rechtskonformen Verhaltens im Sinne von Art. 5 Abs. 1 Anhang I FZA», merkwürdig (Urteil vom 22. Mai 2019, 6B_378/2018, E. 3.4.4). Das Abkommen geht erheblich über rein wirtschaftliche Sachverhalte hinaus; der Zweckartikel gemäss Art. 1 FZA reflektiert dies anschaulich (N. 152-153). Auch wird die Gewährleistung der Aufenthalts-, Familiennachzugs- und Verbleiberechte nicht an «Bedingungen» geknüpft; die Staatsangehörigen der Vertragsparteien können sich darauf berufen, sofern sie die einschlägigen Voraussetzungen erfüllen (ebenso kritisch A. EPINEY, Landesverweisung, passim; S. PROGIN-THEUERKAUF, passim). 
Arbeitnehmende, welche Staatsangehörige einer Vertragspartei sind und mit einer Arbeitgeberin oder einem Arbeitgeber des Aufnahmestaates ein Arbeitsverhältnis mit einer Dauer von mindestens einem Jahr eingegangen sind, enthalten eine Aufenthaltserlaubnis mit einer Gültigkeitsdauer von mindestens fünf Jahren. Diese wird bei Fortdauer des Arbeitsverhältnisses automatisch um mindestens fünf Jahre verlängert. Sofern Arbeitnehmende ein Arbeitsverhältnis mit einer Dauer von mehr als drei Monaten und weniger als einem Jahr eingegangen sind, enthalten sie eine Aufenthaltserlaubnis mit einer Gültigkeitsdauer, die der Dauer des Arbeitsvertrags entspricht (Art. 6 Anhang I). Grenzgängerinnen und Grenzgänger, d.h. Personen, die in der Regel täglich oder mindestens einmal in der Woche an ihren Wohnort zurückkehren, benötigen keine Aufenthaltserlaubnis (Art. 7 Anhang I). Die Staatsangehörigen der Vertragsparteien haben das Recht, sich in das Hoheitsgebiet einer anderen Vertragspartei zu begeben, um sich eine Beschäftigung zu suchen, und sich während eines angemessenen Zeitraums von bis zu sechs Monaten dort aufzuhalten; die Arbeitsuchenden haben Anspruch auf die gleiche Hilfe, wie sie die Arbeitsämter dieses Staates eigenen Staatsangehörigen leisten, aber nicht auf Sozialhilfe (Art. 2 Anhang I). Das Freizügigkeitsabkommen knüpft im Grundsatz an die Arbeitnehmerfreizügigkeit, wie sie im Unionsrecht gewährleistet wird (Art. 45 AEUV), an (J. DimitRIJEWITSCH, S. 158-168; A. EPINEY, Verhältnis, Rz. 20; A. EPINEY/B. MeTZ/B. PIRKER, Parallelität, S. 205).

Der EuGH und das BGer nehmen bei der Definition von Arbeitnehmerinnen und Arbeitnehmern - in Abgrenzung von selbständig erwerbstätigen Personen - Bezug auf die Praxis des EuGH zu dieser Abgrenzung im EU-Binnenmarktrecht; entsprechend steht auch bilateralrechtlich das Kriterium der Weisungsgebundenheit bzw. des Unterordnungsverhältnisses im Zentrum (Urteil Wächtler, C-581/17, EU:C:2019:138, Rn. 45; BGE 131 II 339, E. 3.2; BGE 140 II 460, E. 4.1.1). Gestützt auf dieses Kriterium und das Urteil Jany des EuGH, in dem der EuGH die Tätigkeit einer Prostituierten als selbständige Erwerbstätigkeit einstufte (C-268/99, EU:C:2001:616), qualifizierte das BGer in einem Urteil von 2014 die Erwerbstätigkeit einer Prostituierten in einem sog. Club als unselbständige Erwerbstätigkeit; die Beschwerdeführerin, eine rumänische Staatsangehörige, konnte sich in der Folge nicht auf Art. 4 i.V.m. Art. 6 Anhang I FZA berufen, um einen Anspruch auf Erteilung einer Aufenthaltserlaubnis geltend zu machen, weil in jenem Zeitpunkt der Zugang zum schweizerischen Arbeitsmarkt für unselbständig erwerbstätige Staatsangehörige aus Rumänien und Bulgarien gemäss der «Ventilklausel» (N. 154) beschränkt war (BGE 140 II 460).

Staatsangehörige einer Vertragspartei, die sich zwecks Ausübung einer selbstständigen Erwerbstätigkeit im Hoheitsgebiet einer anderen Vertragspartei niederlassen, erhalten eine Aufenthaltserlaubnis mit einer Gültigkeitsdauer von mindestens fünf Jahren, sofern sie den Behörden nachweisen, dass sie tatsächlich selbständig tätig sind, etwa durch den Nachweis, dass ihre Einzelfirma im Handelsregister eingetragen ist, dass sie über das notwendige Start- 
kapital und eine Betriebsstätte verfügen dass sie die gesetzlich vorgeschriebenen sozialversicherungsrechtlichen Abgaben leisten und dass sie über einen eigenen Auftritt am Markt, einen Businessplan und Buchhaltungstools verfügen (Art. 12 Anhang I; s. zum Nachweis der selbständigen Erwerbstätigkeit Weisungen VEP, S. 51-52). Dabei muss eine Person ihre selbständige Tätigkeit im Hoheitsgebiet einer anderen Vertragspartei als derjenigen, deren Staatsangehörigkeit sie besitzt, tatsächlich ausüben; andernfalls kann sie sich nicht auf das Niederlassungsrecht gemäss Art. 12 Anhang I berufen (Urteil Picart, C-355/16, EU:C:2018:184, Rn. 22-24). Selbständige Grenzgängerinnen und Grenzgänger benötigen keine Aufenthaltserlaubnis (Art. 13 Anhang I). Die bilateralrechtliche Niederlassungsfreiheit wird nur natürlichen Personen gewährt; juristische Personen können sich nicht darauf berufen (Urteil Grimme, C-351/ 08, EU:C:2009:697, Rn. 37-39). Abgesehen davon knüpft das Freizügigkeitsabkommen im Grundsatz an die Niederlassungsfreiheit, wie sie im Unionsrecht gewährleistet wird (Art. 49 AEUV), an (A. EPINEY, Verhältnis, Rz. 20).

Eine Vertragspartei kann Staatsangehörigen der anderen Vertragspartei das Recht auf eine Beschäftigung in der öffentlichen Verwaltung oder das Recht auf Ausübung einer selbständigen Erwerbstätigkeit verweigern, sofern diese Tätigkeit die Ausübung hoheitlicher Befugnisse umfasst (Art. 10 Anhang I) bzw. mit der Ausübung öffentlicher Gewalt (Art. 16 Anhang I) verbunden ist. Diese Bereichsausnahmen sind - analog zur Praxis des EuGH zur Auslegung der Bereichsausnahmen im EU-Recht (Art. 45 Abs. 4 und Art. 51 AEUV; vgl. etwa Urteil Kommission/Deutschland, C-54/08, EU:C:2011:339) - restriktiv auszulegen.

Das BGer kam in einem Urteil von 2014 zum Schluss, dass die Tätigkeit von Gerichtsdolmetscherinnen und Gerichtsdolmetschern nicht als hoheitliche Tätigkeit im Sinne von Art. 10 und Art. 16 Anhang I FZA zu qualifizieren ist. Es stützte sich dabei wesentlich auf die Rechtsprechung des EuGH zur Tragweite der Bereichsausnahme der «Ausübung öffentlicher Gewalt» gemäss Art. 51 AEUV, wonach hoheitlichen Tätigkeiten «Entscheidungsautonomie und eine gewisse Letztverantwortung inhärent sein müssen»; dies ist bei der Dolmetschertätigkeit nicht der Fall, weil sie «darin besteht, eine neutrale und hochwertige Übersetzung zuhanden der Gerichte zu erbringen, ohne dass die Meinung des Dolmetschers in die Entscheidungsfindung der Behörden mit einfliesst», und «eine Hilfsfunktion für die entsprechende Bereichsausnahme darstellt» (BGE 140 II 112; s. zum Ganzen N. DieBold, Sachverständige, passim; M. OEsCH, Einfluss, passim; B. PIRKER, Auslegung, passim). - Mit Blick auf die restriktive Praxis des EuGH zur Auslegung der Bereichsausnahme der «Ausübung öffentlicher Gewalt» scheint eine Neubeurteilung der bundesgerichtlichen Praxis, wonach Gerichtsgutachterinnen und Gerichtsgutachter (BGer vom 9. August 2011, 2C_121/2011, E. 3.3) und freiberufliche Notarinnen und Notare (BGE 128 I 280, E. 3) in der Schweiz hoheitlich tätig sind, angezeigt (ebenso in Bezug auf Notare Empfehlung der WEKO vom 23. September 2013 betreffend Freizügigkeit für Notare und öffentliche Urkunden, RPW 2013/3, S. 399; S. Breitenmoser/R. Weyeneth, Rz. 708; a.M. R. PFÄFFLI/F. LieCHTI, 
passim; s. auch Stellungnahme des Bundesrates vom 28. August 2015 zur Motion 15.3728 «Schweiz und EU. Gleich lange Spiesse, keine einseitige Freizügigkeit»; Antwort des Bundesrates vom 17. Februar 2016 zur Anfrage 15.1088 «Europakompatible Auslegung und Ausübung hoheitlicher Befugnisse durch EU-Bürger in der Schweiz»).

Arbeitnehmende und selbständig erwerbstätige Personen haben das Recht auf berufliche und geographische Mobilität im gesamten Hoheitsgebiet des Aufnahmestaates. Die berufliche Mobilität umfasst den Wechsel des Berufs und den Übergang von einer selbstständigen zu einer unselbstständigen Erwerbstätigkeit und umgekehrt. Die geographische Mobilität umfasst den Wechsel des Arbeits- und Aufenthaltsortes (Art. 8 und Art. 14 Anhang I).

Eine Person, welche die Staatsangehörigkeit einer Vertragspartei besitzt und keine Erwerbstätigkeit im Aufenthaltsstaat ausübt, erhält eine Aufenthaltserlaubnis mit einer Gültigkeitsdauer von mindestens fünf Jahren, sofern sie den Behörden den Nachweis dafür erbringt, dass sie für sich selbst und ihre Familienangehörigen über (i) ausreichende finanzielle Mittel verfügt, so dass sie während ihres Aufenthalts keine Sozialhilfe in Anspruch nehmen muss, und (ii) einen Krankenversicherungsschutz verfügt, der sämtliche Risiken abdeckt (Art. 24 Anhang I; s. dazu BGE 135 II 265, E. 2 und 3.3). Die Personen, die ein Arbeitsverhältnis mit einer Dauer von weniger als einem Jahr im Hoheitsgebiet einer Vertragspartei innehatten, müssen für den weiteren Verbleib ebenfalls diese Voraussetzungen erfüllen (Art. 24 Abs. 3 Anhang I). Studierende müssen zusätzlich zu den genannten Voraussetzungen in einer anerkannten Lehranstalt zum Erwerb einer beruflichen Bildung eingeschrieben sein (wobei das Abkommen weder den Zugang zur Ausbildung noch die Unterhaltsbeihilfen für Studierende regelt, Art. 24 Abs. 4 Anhang I).

Die Familienangehörigen einer Person, die Staatsangehörige einer Vertragspartei ist und ein Aufenthaltsrecht hat, haben das (abgeleitete) Recht, bei dieser Person Wohnung zu nehmen (Art. 3 Anhang I; s. zum Anwendungsbereich des FZA in Bezug auf den Familiennachzug bei Doppelbürgerschaften BGE 143 II 57; zum rechtsmissbräuchlichen Nachzug von Kindern Urteil des BVerwG vom 2. April 2019, F-4854/2017, E. 7-9). Abhängig von ihrem Alter haben Familienangehörige das Recht auf Zugang zu einer Erwerbstätigkeit oder zur Teilnahme am allgemeinen Unterricht sowie an der Lehrlings- und Berufsausbildung (Art. 3 Abs. 5 und Abs. 6 Anhang I; s. BGE 139 II 393 betr. Aufenthaltsrecht von Kindern zu Ausbildungszwecken). Staatsangehörige einer Vertragspartei, die ein Aufenthaltsrecht haben und ihren Hauptwohnsitz im Aufnahmestaat nehmen, haben hinsichtlich des Erwerbs von Immobilien die gleichen Rechte wie die Inländerinnen und Inländer (Art. 25 Anhang I). Die Staatsangehörigen einer Vertragspartei und ihre Familienangehörigen haben nach Beendigung 
ihrer Erwerbstätigkeit ein Recht auf Verbleib im Hoheitsgebiet der anderen Vertragspartei; die Verordnung (EWG) Nr. 1251/70 und die Richtlinie 75/34/ EWG, auf die im Abkommen verwiesen wird (Art. 4 Anhang I), sind einschlägig (s. zum Verbleiberecht bei Arbeitsunfähigkeit BGE 141 II 1; Urteil des BGer 2C_134/2019 vom 12. November 2019).

Das BGer entschied in einem leading case von 2009, dass der Nachzug eines Familienmitglieds mit Drittstaatsangehörigkeit nicht voraussetzt, dass sich dieser Familienangehörige bereits rechtmässig in der Schweiz oder einem anderen FZA-Vertragsstaat aufgehalten hat. Das BGer änderte dabei seine bisherige restriktivere Praxis und folgte einem neuen Urteil des EuGH zum Familiennachzug gestützt auf die unionalen Freizügigkeitsrechte (BGE 136 II 5, m.V.a. das EuGH-Urteil Metock, C-127/08, EU:C:2008:449; s. dazu N. 85).

\section{Nichtdiskriminierung, Stillhalteklausel}

Die zentrale Bestimmung des Abkommens ist der Grundsatz der Nichtdiskriminierung. Die Staatsangehörigen einer Vertragspartei, die sich rechtmässig im Hoheitsgebiet einer anderen Vertragspartei aufhalten, werden bei der Anwendung des Abkommens gemäss den Anhängen I, II und III nicht auf Grund ihrer Staatsangehörigkeit diskriminiert (Art. 2; s. zum Ganzen V. Boillet, passim; N. Diebold, Freizügigkeit, Rz. 615-632; A. EPINEY/B. METZ/ B. PIRKER, Parallelität, S. 221-226; W. KÄLIN, S. 31-37; zur Anwendung des Abkommens Urteil Hengartner und Gasser, C-70/09, EU:C:2010:430, Rn. 39; Urteil des BGer 2A.114/2003 vom 23. April 2004, E. 4.2; C. TOBLER/J. BEGLINGER, Grundzüge, Rz. 185-186). Dieser Grundsatz wird in den Anhängen spezifisch für den jeweiligen Anwendungsbereich konkretisiert (Art. 7 lit. a, Art. 8 lit. a, Art. 9, Art. 15 und Art. 19 Anhang I).

\footnotetext{
Der EuGH entschied im Urteil Stamm und Hauser von 2008 (C-13/08, EU:C:2008:774), dass sich auch selbständige Grenzgängerinnen und Grenzgänger auf das Diskriminierungsverbot berufen können. Entsprechend war es den deutschen Behörden untersagt, Landwirtinnen und Landwirte mit Betriebssitz in der Schweiz beim Abschluss von Landpachtverträgen über in Deutschland gelegenes Ackerland schlechter zu behandeln als Landwirtinnen und Landwirte mit Betriebssitz in Deutschland.
}

Der Grundsatz der Nichtdiskriminierung verbietet nicht nur direkte (formelle, unmittelbare, de iure), sondern auch indirekte (verdeckte, mittelbare, de facto) Diskriminierungen (s. etwa BGE 136 II 241, E. 15.2; BGE 137 II 242, E. 3.2; BGE 131 V 209, E. 6.2 und 6.3; BGE 130 I 26, E. 3; Urteil Ettwein, C-425/11, EU:C:2013:121, Rn. 26; Urteil Graf und Engel, C-506/10, EU:C:2011:643, Rn. 26; Urteil Bergström, C-257/10, EU:C:2011:839, Rn. 30; Urteil Stamm und Hauser, C-13/08, EU:C:2008:774, Rn. 39-49): 
- Eine direkte Diskriminierung beruht auf einer rechtlichen Vorgabe, wonach Personen in einer vergleichbaren Situation nach Massgabe ihrer Herkunft unterschiedlich behandelt werden, selbstredend - zumindest in aller Regel - zum Nachteil der auswärtigen Personen. Solche Vorgaben können unter Bezugnahme auf einen ausdrücklich im Abkommen vorgesehenen Ausnahmetatbestand gerechtfertigt werden (N. 168; vgl. Urteil des BGer 2C_150/2016, E. 4.2.3, m.V.a. BGE 140 II; a.M. T. CotTIER et al., Rz. 509; W. KäLIN, S. 32, wonach Ungleichbehandlungen, welche direkt an der Staatsangehörigkeit anknüpfen, unter keinen Umständen zu rechtfertigen sind; gemäss dieser Ansicht gilt das Verbot der unmittelbaren Diskriminierung absolut).

- Eine indirekte Diskriminierung beruht auf einer rechtlichen Vorgabe, welche prima vista zwar nichtdiskriminierend ausgestaltet ist (weil sie auf einheimische und auswärtige Personen unterschiedslos anwendbar ist), deren praktische Wirkung aber gleichwohl zu einer Schlechterbehandlung von auswärtigen Personen führt. Solche Vorgaben - wie etwa die Voraussetzung, für die Ausübung einer Tätigkeit über spezifische Sprachkenntnisse zu verfügen, die Ausbildung an einem spezifischen Ausbildungsort absolviert zu haben oder eine Wohnsitzpflicht zu beachten können auch unter Bezugnahme auf weitere im Abkommen nicht ausdrücklich genannte Gemeinwohlanliegen gerechtfertigt werden (N. 168-169).

Es liegt eine unzulässige indirekte Diskriminierung vor, wenn eine Arbeitgeberin oder ein Arbeitgeber den Grenzgängerinnen und Grenzgängern mit Wohnsitz in Deutschland oder Frankreich aufgrund der Eurokrise den Lohn in Schweizer Franken um 6 \% kürzt, nicht aber den Arbeitnehmenden mit Wohnsitz in der Schweiz (Urteil 40012152 des Kantonsgerichts BL vom 17. Dezember 2012, E. 3.7; s. aber auch Urteile des BGer 4A 230/2018 und 4A_215/2017 vom 15. Januar 2019, wobei eine Berufung auf das FZA in casu rechtsmissbräuchlich war; kritisch dazu K. PÄRLI, Eurolohn, passim). - Ebenso führt die Besteuerung an der Quelle zu einer unzulässigen indirekten Diskriminierung, wenn eine quellenbesteuerte Person schlechter behandelt wird als wenn sie ordentlich besteuert würde, ohne dass dies für die Sicherung des Steuerbezugs gemäss Art. 21 Abs. 3 FZA gerechtfertigt wäre (BGE 136 II 214; BGE 140 II 167; N. DiEBolD, Freizügigkeit, Rz. 594). - Demgegenüber liegt keine indirekte Diskriminierung aufgrund der Staatsangehörigkeit vor, wenn der jährliche Beitrag für die Säule 3a nur für diejenigen Personen steuerlich abzugsfähig ist, welche der AHV-Pflicht unterstellt sind; Staatsangehörige eines EU-Mitgliedstaates in der Schweiz sind diesfalls nicht stärker benachteiligt als Schweizer Bürgerinnen und Bürger (BGE 140 II 364, E. 6; N. DiebOLD, Freizügigkeit, Rz. 529).

Die Vertragsparteien verpflichten sich des Weiteren, in den unter das Abkom166 men fallenden Bereichen keine neuen Beschränkungen für Staatsangehörige der anderen Vertragspartei einzuführen (Art.13). Das Bundesgericht geht 
davon aus, dass die Verpflichtung der Stillhalteklausel nicht weitergeht als das Verbot der mittelbaren Diskriminierung (BGE 130 I 26, E. 3.4; s. auch Urteil Graf und Engel, C-506/10, EU:C:2011:643, Rn. 35).

Es ist fraglich, ob eine derart restriktive Auslegung der Stillhalteklausel sachgerecht ist. Dies gilt insbesondere auch mit Blick auf die Praxis des EuGH zur Auslegung von Stillhalteklauseln in anderen Abkommen, welche die EU mit Drittstaaten abgeschlossen hat (vgl., jeweils betr. Assoziierungsabkommen EWG-Türkei, Urteil Genc, C-561/14, EU:C:2016:247; Urteil CX, C-629/16, EU:C:2018:556; Urteil A, C-70/18, EU:C:2019:823; C. TOBLER/ J. BEGLINGER, Grundzüge, Rz. 191; T. COTTIER/R. LIECHTI, KVG, Rz. 34).

Der EuGH entnimmt der Arbeitnehmerfreizügigkeit und der Niederlassungsfreiheit im EU-Binnenmarktrecht (Art. 45 und Art. 49 AEUV) über das Diskriminierungsverbot hinaus ein Behinderungs- bzw. Beschränkungsverbot. Es ist ungeklärt, ob die Rechte auf Einreise, Aufenthalt und Ausübung einer Erwerbstätigkeit i.V.m. dem Grundsatz der Nichtdiskriminierung nach Massgabe des Freizügigkeitsabkommens ebenfalls ein Behinderungs- bzw. Beschränkungsverbot enthalten oder ob diese Ansprüche mit dem Grundsatz der Nichtdiskriminierung zusammenfallen (s. dazu C. Deldi, S. 291-331; N. Diebold, Freizügigkeit, Rz. 781-792; A. EPINEY, Wiedereinführung, Rz. 13; C. TOBLER/J. BEGLINGER, Grundzüge, Rz. 199; Generalanwalt P. Mengozzi, der in der Rs. Picart, C-355/ 16, EU.C:2017:610, Rn. 72-76, ausführte, dass die bilateralrechtliche Niederlassungsfreiheit nur Diskriminierungen, nicht aber auch Beschränkungen verbietet).

\section{Rechtfertigung von Einschränkungen}

Die Rechte, auf die sich die Staatsangehörigen einer Vertragspartei berufen können, werden nicht absolut gewährt. Dies gilt in Bezug auf die Einreise, den Aufenthalt und die Ausübung einer Erwerbstätigkeit wie auch in Bezug auf die Nichtdiskriminierung. Diese Rechte können aus Gründen der öffentlichen Ordnung, Sicherheit und Gesundheit eingeschränkt werden (Art. 5 Anhang I; s. Urteil Graf und Engel, C-506/10, EU:C:2011:643, Rn. 29-34). Dabei wird auf die Richtlinien 64/221/EWG, 72/194/EWG und 75/35/EWG verwiesen, welche dergestalt im bilateralen Verhältnis anwendbar sind (während diese Richtlinien im EU-Recht im Zug der Neufassung durch die Unionsbürgerrichtlinie 2004/ 38/EG aufgehoben wurden). Diese Gründe sind restriktiv auszulegen. Des Weiteren muss die Verhältnismässigkeit gewahrt sein (BGE 131 V 215, E. 6.2 und 6.3; Urteil des BVerwG vom 18. Dezember 2018, B-2701/2016, E. 4.2). Dies erfordert zwingend eine auf den Einzelfall fokussierte Beurteilung; ausschlaggebend ist das persönliche Verhalten der betroffenen Person (Art. 3 der Richtlinie 64/221/EWG). So setzen etwa Entfernungs- oder Fernhaltemassnahmen 
eine hinreichend schwere und gegenwärtige Gefährdung der öffentlichen Ordnung durch die betroffene Person voraus. Eine strafrechtliche Verurteilung darf nur insofern zum Anlass für eine derartige Massnahme genommen werden, als die ihr zugrunde liegenden Umstände ein persönliches Verhalten erkennen lassen, das eine gegenwärtige Gefährdung der öffentlichen Ordnung darstellt; wesentlich ist das Rückfallrisiko (BGE 129 II 215; BGE 130 II 176, E. 4.2; BGE 136 II 5, E. 4.2).

Das BGer hat die Voraussetzungen, welche erfüllt sein müssen, damit eine Person, die sich gestützt auf das FZA rechtmässig in der Schweiz aufhält, des Landes verwiesen werden kann, in den letzten Jahren zunehmend gelockert. In einem Urteil von 2018 führte es aus, die Landesverweisung gemäss Art. $66 a^{\text {bis }}$ StGB sei «eine eigenständige strafrechtliche Massnahme»; damit einher ginge «keine Beendigung des Aufenthaltsrechts und keine Wegweisung im Sinne des Ausländergesetzes $(\mathrm{AuG} ;$...)»; aus diesem Grund erscheine die vor dem Inkrafttreten der strafrechtlich relevanten Ausführungsgesetzgebung zu Art. 121 BV (Ausschaffungsinitiative; N. 62) ergangene ausländerrechtliche Rechtsprechung, wonach eine behördliche Berufung auf Art. 5 Anhang I FZA nur unter restriktiven Voraussetzungen zulässig sei und sich an der Praxis des EuGH zum unionalen Freizügigkeitsrecht zu orientieren habe, insoweit nicht als einschlägig (BGE 145 IV 55, E. 4.2). In einem Urteil von 2019 hielt das BGer dafür, dass Art. 5 Anhang I FZA «strafrechtlich» nicht analog zur Praxis im EU-Recht auszulegen sei, weil es sich bei diesem Abkommen «um ein im Wesentlichen wirtschaftsrechtliches Abkommen handelt» (Urteil vom 22. Mai 2019, 6B_378/2018, E. 3.4.4). Diese Urteile des BGer lassen Fragen offen; die Erwägungen zur Natur und zur Wirkungsweise des FZA und zu seinem Verhältnis zum innerstaatlichen (Straf-) Recht sind inkohärent und widersprüchlich (ganz mehrheitlich ebenfalls kritisch N. BURRI, passim; A. EPIneY, Landesverweisung, passim; B. Fargahi/V. PriUli/F. DE WeCK, passim; S. GlesS/ A. Petrig/C. Tobler, passim; S. Progin-Theuerkauf, passim; s. auch S. SChlegel, Art. 66a Rz. 15-17; M. ZURBRÜGG/C. HRUSCHKA, Art. 66a Rz. 38-51, 58-81). In der Lehre wird mehrheitlich vorgebracht, dass die sich aus dem FZA ergebenden Aufenthaltsrechte Vorrang vor den innerstaatlichen Regeln zur Landesverweisung haben; diese Lesart steht im Einklang mit dem obiter dictum in BGE 142 II 35, E. 3.2 (s. dazu N. 53).

In einem Urteil von 2017 beurteilte das BGer den Fall eines österreichischen Staatsbürgers, der bei den schweizerischen Behörden die Anerkennung der Gleichwertigkeit seines österreichischen Meisterprüfungszeugnisses für das Handwerk Augenoptik mit dem - in der Schweiz nicht mehr vergebenen, aber weiterhin berufsbefähigenden - eidgenössischen Diplom des Augenoptikers beantragt hatte. Das BGer bestätigte, dass die Behörden bei Tätigkeiten, deren Ausübung vom Nachweis einer Qualifikation, Weiterbildung oder Berufserfahrung abhängig gemacht wird, gestützt auf das FZA verpflichtet sind, die in einer anderen Vertragspartei absolvierten Aus- und Weiterbildungen sowie Erfahrungen zu prüfen und anzuerkennen, sofern sie mit den in der Schweiz einschlägigen Anforderungen gleichwertig sind; dabei muss auch eine nur teilweise gleichwertige Qualifikation, Weiterbildung oder Berufserfahrung unter Beachtung der zwischen den nationalen Regelungen festgestellten Unterschiede nach Massgabe ihrer Gleichwertigkeit berücksichtigt werden. Die Weigerung der schweizerischen Behörden, eine Gleichwertigkeitsprüfung vorzunehmen, war in casu nicht vereinbar mit Art. 2 FZA und Art. 15 Abs. 1 Anhang I FZA (Urteil 2C_472/2017 vom 7. Dezember 2017, E. 3.4; s. zur Richtlinie 2005/36/EG über die Anerkennung von Berufsqualifikationen - welche in casu nicht anwendbar war - N. 173-175). 
Das BVerwG erachtete in einem Grundsatzurteil von 2018 die in Art. 55a Abs. 2 KVG normierte Einschränkung der Zulassung von Ärztinnen und Ärzten, welche nicht mindestens drei Jahre an einer anerkannten schweizerischen Weiterbildungsstätte tätig waren, zur Tätigkeit zulasten der Krankenversicherung als zulässig. Diese Massnahme behandelt inländische und ausländische Ärztinnen und Ärzte zwar indirekt ungleich, kann unter Rekurs auf den Schutz der öffentlichen Gesundheit aber gerechtfertigt werden (BVGE 2018 V/1, E. 9).

Gemäss Bundesgericht können auch ungeschriebene Gemeinwohlanliegen bzw. - in den Worten des EuGH zu Einschränkungen im EU-Recht - zwingende Erfordernisse des Allgemeininteresses, welche über die in Art. 5 Anhang I erwähnten Gründe hinausgehen, Einschränkungen rechtfertigen (BGE 140 II 112, E. 3.6.2; Urteil Radgen, C-478/15, EU:C:2016:705, Rn. 49; dies gilt nicht für direkte Diskriminierungen, N. 165). Des Weiteren enthält das Abkommen spezifische Ausnahmetatbestände, welche punktuell Beschränkungen zulassen (s. etwa Art. 21 Abs. 2 zum Recht der Vertragsparteien, Massnahmen zur Gewährleistung der Besteuerung sowie der Zahlung und der tatsächlichen Erhebung der Steuern und zur Verhinderung der Steuerflucht anzuwenden).

Das BGer qualifiziert die Voraussetzung, eine Landessprache zu beherrschen, in ständiger Praxis und unter Rekurs auf die Rechtsprechung des EuGH als zulässig, sofern solche Kenntnisse für die Wahrnehmung der betreffenden Aufgabe unabdingbar sind. In einem Urteil von 2003 stellte es - wenngleich etwas gar pauschal und wenig differenziert - fest: «Dies ist für den Arztberuf mit seinen Kontakten zu Patienten und Versicherungen ohne weiteres der Fall. Etwaige hieraus resultierende Benachteiligungen von Ausländerinnen und Ausländern aus der EU sind hinzunehmen und nicht diskriminierend im Sinn von Art. 2 FZA.» (BGE 130 I 26, E. 3.3.3.5). Gemäss Art. 53 der Richtlinie 2005/36/EG über die Anerkennung von Berufsqualifikationen (N. 173-175) müssen Personen, deren Berufsqualifikation anerkannt wird, über Sprachkenntnisse verfügen, die für die Ausübung der Berufstätigkeit im Aufnahmemitgliedstaat «erforderlich» sind (wobei die Richtlinie 2013/55/EU, welche noch nicht in den Anhang III FZA übernommen worden ist [N. 95], diese Bestimmung weiter konkretisiert).

\section{Begleitrechte}

Die Vertragsparteien haben vereinbart, die erforderlichen Begleitmassnahmen zu treffen, um die Ausübung der Freizügigkeit zu erleichtern. Dazu gehören die Koordinierung der Systeme der sozialen Sicherheit gemäss Anhang II und die gegenseitige Anerkennung beruflicher Qualifikationen gemäss Anhang III. Die Vertragsparteien wenden untereinander die EU-Rechtsakte an, auf die Bezug genommen wird, oder - zumindest auf dem Papier - gleichwertige Vorschriften. In pragmatischer Weise wird in der Schweiz häufig darauf verzichtet, diese Rechtsakte - inkl. Richtlinien - umzusetzen; die schweizerischen Behörden wenden sie unmittelbar an (s. etwa BGE 132 II 135, E. 6; BGE 134 II 
341, E. 2.1/2.2; Urteil des BVerwG C-89/2007 vom 2. Juli 2007, E. 3.4). Dabei verlieren diese Rechtsakte ihren europarechtlichen Charakter nicht, nur weil sie kraft Verweis formell in ein staatsvertragliches Kleid gehüllt werden; im Rahmen der Koordinierung der Systeme der sozialen Sicherheit und der Anerkennung beruflicher Fähigkeitsausweise findet eine weitgehende Integration in das Unionsrecht statt.

Gemäss Art. 4 Abs. $3^{\text {bis }}$ BGBM erfolgt die Anerkennung von Fähigkeitsausweisen für Erwerbstätigkeiten, die unter das FZA fallen, nach Massgabe dieses Abkommens. Kraft dieser Bestimmung gelten im Binnen- wie im Aussenverhältnis dieselben Anerkennungsregeln. Damit wird eine allfällige Schlechterstellung von Schweizerinnen und Schweizern gegenüber Staatsangehörigen eines EU-Mitgliedstaates verhindert (Inländerdiskriminierung; Botschaft revBGBM 2004, S. 487; s. zum Ganzen M. OESCH/S. RENFER, Art. 4 Rz. 5-9).

\section{(a) Koordinierung der Systeme der sozialen Sicherheit}

Die Vorgaben zur Koordinierung der Systeme der sozialen Sicherheit finden sich massgeblich in der Verordnung (EG) Nr. 883/2004 und der Verordnung (EG) Nr. 987/2009. Auf beide Verordnungen wird in Anhang II verwiesen. Diese Verordnungen bezwecken, die sozialversicherungsrechtlichen Systeme der EU-Mitgliedstaaten und der Schweiz zu koordinieren, ohne eine materielle Vereinheitlichung anzustreben.

Die Verordnung (EG) Nr. 883/2004 gilt für alle klassischen Bereiche der sozialen Sicherheit. Dazu gehören Krankheit, Mutter- und Vaterschaft, Altersrenten, Vorruhestandsregelungen und Leistungen bei Invalidität, Hinterbliebenenrenten, Arbeitslosigkeit, Familienleistungen, Arbeitsunfälle und Berufskrankheiten (s. zum Ganzen www.eda.admin.ch/dea und Link zu Bilaterale Abkommen I (1999)/Personenfreizügigkeit). Es handelt sich um einen komplexen Sachbereich mit unterschiedlichen Regelungen je nach Sozialversicherungsbereich. Die zentralen Eckpunkte lassen sich wie folgt zusammenfassen: Die Verordnung (EG) Nr. 883/2004 garantiert den Berechtigten, d.h. den Staatsangehörigen der Vertragsparteien und ihren Familienmitgliedern, dass die Rechtsvorschriften eines einzigen Landes anwendbar sind und sie dort Beiträge leisten; in der Regel ist dies der Arbeitsort. Die Berechtigten verfügen über dieselben Rechte und Pflichten wie die Staatsangehörigen des Staates, in dem sie sich aufhalten; sie profitieren vom Grundsatz der Nichtdiskriminierung. Frühere Versicherungszeiten in einem anderen Staat werden bei der Berechnung von Leistungen berücksichtigt. Die Berechtigten haben das Recht, Leistungen einzufordern, auch wenn sie sich nicht mehr im dafür zuständigen Staat aufhalten; Leistungen - wie etwa Ansprüche aus der beruflichen Vorsorge - werden diesfalls exportiert. Kranken- und Unfallversicherungsbei- 
träge sind grundsätzlich am Arbeitsort zu entrichten. Dasselbe gilt für die Beiträge der Alters-, Hinterlassenen -und Invalidenversicherung. Die Behörden der Vertragsparteien sind zur Zusammenarbeit verpflichtet.

Derzeit wird in der EU über eine Revision der Verordnung (EG) Nr. 883/2004 zur Koordinierung der Systeme der sozialen Sicherheit debattiert. Ein zentraler Punkt betrifft den Wechsel der Zuständigkeit vom Wohn- zum Arbeitsort bei der Gewährung von Arbeitslosenleistungen an Grenzgängerinnen und Grenzgänger. Je nach Ausgestaltung wäre ein solcher Systemwechsel mit erheblichen Mehrkosten für die Schweiz verbunden (s. Erläuterungen des EDA, S. 14). Der Schweiz ist es im Rahmen der Verhandlungen zum Institutionellen Abkommen nicht gelungen, diese absehbare Novellierung explizit von der Verpflichtung zur Übernahme in das FZA auszunehmen (N. 109). Dessen ungeachtet ist auch ohne InstA davon auszugehen, dass die EU von der Schweiz erwarten wird, diese Rechtsentwicklung mitzutragen (Erläuterungen des EDA, S. 15).

\section{(b) Anerkennung beruflicher Qualifikationen}

Im Vordergrund der in Anhang III referenzierten EU-Rechtsakte steht die Richtlinie 2005/36/EG über die Anerkennung von Berufsqualifikationen (wobei die 2013 im EU-Recht erfolgte Revision dieser Richtlinie durch die Richtlinie 2013/55/EU bis anhin nicht in Anhang III FZA übernommen wurde [was im Urteil des Verwaltungsgerichts des Kantons Aargau vom 21. August 2018, WBE.2018.36, E. 3, verkannt wird]; s. zu dieser Übernahme N. 95). Die Richtlinie gilt für die Staatsangehörigen der Vertragsparteien, welche im Hoheitsgebiet einer anderen Vertragspartei einen reglementierten Beruf ausüben möchten, d.h. eine berufliche Tätigkeit, bei der die Aufnahme oder Ausübung direkt oder indirekt durch Rechts- und Verwaltungsvorschriften an den Besitz bestimmter Berufsqualifikationen gebunden ist (Art. 3; s. für die in der Schweiz reglementierten Berufe www.sbfi.admin.ch/diploma; zum Ganzen F. BERTHOUD, reconaissance, passim; N. GAMMENTHALER, passim; zur Anerkennung von Berufsqualifikationen von Primarlehrpersonen M. KAUfMANN, passim).

Sofern eine Erwerbstätigkeit in der Schweiz weder im Bundesrecht noch im kantonalen Recht reglementiert ist (wobei es durchaus möglich ist, dass eine Tätigkeit in einem Kanton reglementiert wird und in anderen Kantonen nicht, wie dies z.B. der Fall ist für «Bauunternehmer/in / Baumeister/in und Bauspezialistbetreiber/in»), erfolgt der Marktzugang vorbehaltlich der Anforderungen hinsichtlich persönlicher Eigenschaften, Räumlichkeiten, Versicherungen etc. frei. Die Richtlinie 2005/36/EG ist diesfalls nicht anwendbar (Urteil des BVerwG B-1129/2013 und B-4335/2013 vom 25. Februar 2014, E. 6.3.1.1; N. DiEBOLD, Freizügigkeit, Rz. 1155). auch Vorschriften zum Anerkennungsverfahren. Sie regelt die Anerkennung 
von Berufsqualifikationen für Personen, welche sich bloss vorübergehend zur Erbringung einer Dienstleistung in einen anderen Staat begeben (N. 192), und für Personen, welche sich dauerhaft niederlassen wollen. In Bezug auf den letzteren Fall wird zwischen den folgenden Anerkennungsregeln unterschieden:

- $\quad$ automatische Anerkennung von Berufen, für die harmonisierte Ausbildungsstandards bestehen (Ärztinnen und Ärzte, Zahnärztinnen und Zahnärzte, Tierärztinnen und Tierärzte, Pflegepersonal in allgemeiner Pflege, Hebammen, Apothekerinnen und Apotheker, Architektinnen und Architekten; s. etwa Urteil des BGer 2C.668/2012 vom 1. Januar 2013, E. 3; Urteil des BGer 2C.622/2012 vom 17. Juni 2013, E. 3.2.2, beide betr. Berufsausübung von Ärzten);

- Anerkennung von Berufen aufgrund der Berufserfahrung, wobei die Dauer und der Inhalt der Berufserfahrung umschrieben werden (z.B. Fachkräfte im Bereich Handwerk, Handel und Industrie; s. etwa Urteil des BVerwG B-3503/2016 vom 19. März 2018, E. 3.5, betr. Berufserfahrung eines Elektroinstallateurs);

- $\quad$ allgemeines System, bei dem die Ausbildung und Berufserfahrung mit den eigenen Anforderungen verglichen werden; bei erheblichen Unterschieden können Ausgleichsmassnahmen verlangt werden (Anpassungslehrgang oder Eignungsprüfung); das allgemeine System kommt zur Anwendung, wenn eine automatische Anerkennung und eine Anerkennung aufgrund der Berufserfahrung nicht greifen (s. etwa Urteil des BVerwG B-3738/2011 vom 28. Februar 2013, betr. Optiker; Urteil des BVerwG B-6467/2012 vom 27. Juni 2013 betr. Fahrlehrer; BVGE 2015/14 betr. Meisterprüfungszeugnis als Optiker und Verhältnis des FZA zur Vereinbarung vom 1. Dezember 1937 zwischen der Schweiz und dem Deutschen Reich, wonach deutsche Meisterprüfungszeugnisse oder Meisterbriefe «automatisch» anerkannt werden; zu dieser Vereinbarung auch oben N. 150).

Staatsangehörige einer Vertragspartei können sich auch direkt auf das Freizügigkeitsabkommen berufen, um eine Erwerbstätigkeit auszuüben und ihre beruflichen Qualifikationen anerkennen zu lassen (BGE 136 II 470, E. 4.1; BGE 133 V 33, E. 9.4; Gutachten der WEKO vom 16. Juli 2012, RPW 2012/3, S. 708, 713; s. auch N. 168). Diese Anerkennungsregeln kommen als Mindeststandards zur Anwendung, wenn ein Sachverhalt nicht vom sekundärrechtlichen Anerkennungsregime erfasst wird. Das FZA reflektiert in dieser Hinsicht die Rechtslage im Binnenmarktrecht, wo der EuGH Ansprüche auf Berücksichtigung von im Herkunftsstaat erlangten Qualifikationen ebenfalls direkt gestützt auf die Grundfreiheiten ableitet (grundlegend Urteil Hocsman, C-238/98, EU:C:2000:440, Rn. 23).

Die Richtlinie 2005/36/EG verpflichtet die Mitgliedstaaten (inkl. der Schweiz) zur Benennung eines nationalen Koordinators, welcher die Behörden der Auf- 
nahme- und Herkunftsmitgliedstaaten bei der Zusammenarbeit inkl. Amtshilfe unterstützt (Art. 56), und zur Benennung einer nationalen Kontaktstelle, welche die Information der Bürgerinnen und Bürger und der Kontaktstellen der anderen Mitgliedstaaten sicherstellt (Art. 57). In der Schweiz übernimmt das Staatssekretariat für Bildung, Forschung und Innovation (SBFI) diese Funktionen (Art. 71 der Berufsbildungsverordnung, BBV, SR 412.101).

Im EU-Recht legt die Richtlinie (EU) 2018/958 über eine Verhältnismässigkeitsprüfung vor Erlass neuer Berufsreglementierungen zudem einen gemeinsamen Rechtsrahmen zur Durchführung von Verhältnismässigkeitsprüfungen vor der Einführung neuer oder der Änderung bestehender Rechts- und Verwaltungsvorschriften fest, mit denen der Zugang zu reglementierten Berufen oder deren Ausübung in den Mitgliedstaaten beschränkt wird. Diese Richtlinie ist nicht Teil des bilateralen Acquis. Es ist dem Vernehmen nach nicht geplant, sie in den Anhang III FZA aufzunehmen.

Die Anerkennung von gewissen Berufsqualifikationen wird speziell geregelt. Dies trifft etwa auf Rechtsanwältinnen und Rechtsanwälte zu: Die Richtlinie 77/249/EWG regelt die vorübergehende Erbringung anwaltlicher Dienste in einem anderen EU-Mitgliedstaat; das ist unter Verwendung der ursprünglichen Berufsbezeichnung in der Sprache des Herkunftsstaats zulässig. Die Richtlinie 98/5/EG regelt die ständige Ausübung des Rechtsanwaltsberufs in einem anderen EU-Mitgliedstaat als in dem, in dem die Qualifikation erworben wurde; dies ist zulässig unter der Berufsbezeichnung, welche die Anwältin oder der Anwalt im Herkunftsstaat erworben hat. Nach dreijähriger Tätigkeit im Aufnahmestaat besteht die Möglichkeit der Integration in den Berufsstand des Aufnahmestaats; beim fehlenden Nachweis ausreichender Praxiserfahrung im Aufnahmestaat besteht die Verpflichtung, eine Eignungsprüfung abzulegen. Diese Richtlinien gelten qua Verweis in Anhang III FZA auch zwischen der Schweiz und der EU. Die Schweiz hat sie im Anwaltsgesetz von 2000 (SR 935.61) umgesetzt (s. dazu etwa Urteil des BGer vom 9. August 2004, 2A.536/2003, betr. Eintragung in ein kantonales Anwaltsregister; Beschluss der Aufsichtskommission über die Anwältinnen und Anwälte des Kantons Zürich vom 6. September 2012, KF110428, ZR 2012, 221, betr. nicht notwendiger Kenntnisse der Amtssprache als Eintragungsvoraussetzung).

Weitere EU-Rechtsakte, welche Bestandteil des bilateralen Acquis sind, regeln bereichsspezifisch den Zugang zu Berufen und deren Ausübung, typischerweise nicht durch gegenseitige Anerkennung, sondern durch Harmonisierung. Dazu gehören etwa die Verordnung (EG) Nr. 1071/2009 zur Festlegung gemeinsamer Regeln für die Zulassung zum Beruf des Kraftverkehrsunternehmers (LandVA) und die Verordnung (EWG) Nr. 3922/91 zur Harmonisierung der technischen Vorschriften und der Verwaltungsverfahren in der Zivilluftfahrt (LVA; s. dazu N. 212). 
Die Anerkennung von universitären Abschlüssen (akademische Anerkennung) wird in Anhang III FZA und den darin referenzierten Rechtsakten nicht erfasst. In diesem Bereich gilt primär das Übereinkommen über die Anerkennung von Qualifikationen im Hochschulbereich in der europäischen Region von 1997 (Lissaboner Übereinkommen, SR 0.414.8; F. BERTHOUD, Etudier, passim); auch die Schweiz und alle EU-Mitgliedstaaten sind Vertragsparteien dieses Übereinkommens (s. BGE 140 II 185, wonach der im Lissabonner Übereinkommen verankerte Grundsatz der gegenseitigen Anerkennung der im Ausland erworbenen Qualifikationen für den Hochschulzugang unmittelbar anwendbar ist). Des Weiteren hat die Schweiz mit ausgewählten europäischen Staaten bilaterale Verträge abgeschlossen; dazu gehört etwa das Abkommen mit Österreich über die gegenseitige Anerkennung von Gleichwertigkeiten im Hochschulbereich von 1993 (SR 0.414.991.631).

\begin{abstract}
29 europäische Staaten, darunter auch die Schweiz, beschlossen in der Bologna-Erklärung von 1999, einen europäischen Hochschulraum (EHR) zu schaffen. Das übergeordnete Ziel dieses Prozesses ist die Förderung der Mobilität und die Stärkung der Wettbewerbsfähigkeit des Bildungsstandorts Europa. Die zentralen Elemente sind das dreistufige Studiensystem mit Bachelor, Master und PhD, das Leistungspunktesystem ECTS, die Zusammenarbeit bei der Qualitätssicherung und die Einführung von nationalen Qualifikationsrahmen. 2010 wurde der EHR symbolisch eröffnet. Seither haben sich die beteiligten Staaten auf weitere Massnahmen geeinigt. Der Bologna-Prozess ist formell zwar nicht mit dem Lissaboner Übereinkommen verknüpft; gleichwohl bestehen Gemeinsamkeiten und Interdependenzen (s. zum Bologna-System und zur Umsetzung in der Schweiz www.sbfi.admin.ch und Link zu Bologna-Prozess).
\end{abstract}

\title{
D. Dienstleistungshandel
}

Das Allgemeine Abkommen über den Dienstleistungshandel (GATS) der WTO stellt die Grundordnung für den Dienstleistungshandel zwischen der EU und der Schweiz dar. Es legt die wesentlichen Grundsätze für eine schrittweise Liberalisierung fest. Länderspezifische Listen enthalten Verpflichtungen in Bezug auf den Marktzugang und die Gewährung der Meistbegünstigung und Inländerbehandlung (s. für die Verpflichtungsliste der EU WTO Doc. GATS/ SC/31; für die Liste der Schweiz WTO Doc. GATS/SC/83). Die Marktzugangsliste der Schweiz beruht - analog denjenigen vieler anderer WTO-Mitglieder - auf dem Grundsatz des Status quo: Die Schweiz hat sich im Rahmen der Uruguay Runde verpflichtet, den damals bestehenden Marktzugang für auswärtige Dienstleistungen künftig nicht mehr einzuschränken (C. ETTER, S. 101-102). Mit den beiden Separatabkommen über die Finanzdienstleistungen und die Telekommunikation wurden bereichsspezifisch weitergehende Liberalisierungsschritte eingeleitet. 

Verträgen zwischen der Schweiz und einem oder mehreren EU-Mitgliedstaaten Teilaspekte des Dienstleistungshandels. Voraussetzung dafür ist selbstredend, dass die EU-Mitgliedstaaten unionsrechtlich in einem solchen Bereich überhaupt noch über eigene Vertragsschlusskompetenzen verfügen (vgl. Art. 207 AEUV).

Solche Verträge betreffen etwa den grenzüberschreitenden Verkehr auf der Strasse, auf dem Wasser und in der Luft. Beispiele sind die Vereinbarung zwischen dem Eidgenössischen Post- und Eisenbahndepartement und dem Bundesminister für Verkehr der Bundesrepublik Deutschland über den gewerblichen Strassenpersonen- und -güterverkehr vom 17. Dezember 1953 (SR 0.741.619.136; s. dazu Beschluss (EU) 2020/853 zur Ermächtigung Deutschlands, seine bilaterale Vereinbarung über den Strassenverkehr mit der Schweiz zur Genehmigung von Kabotagebeförderungen bei der Bereitstellung von grenzüberschreitenden Personenkraftverkehrsleistungen mit Kraftomnibussen in der Grenzregion der beiden Länder zu ändern; dazu auch N. 39), das Abkommen zwischen der Schweizerischen Eidgenossenschaft und der Republik Österreich über den grenzüberschreitenden Verkehr mit Motorfahrzeugen auf öffentlichen Strassen vom 22. Oktober 1958 (SR 0.741.619.163; s. zum Verhältnis dieser Verträge zum FZA N. 189), das Schweizerisch-deutsche Abkommen über den Grenz- und Durchgangsverkehr vom 5. Februar 1958 (SR 0.631.256.913.61), die Rheinschifffahrts-Akte vom 17. Oktober 1868 (Mannheim-Übereinkommen, SR 0.747.224.101) und das Übereinkommen über die Personenbeförderung im grenzüberschreitenden Gelegenheitsverkehr mit Kraftomnibussen (ASOR) vom 26. Mai 1982 (SR 0.741.618).

Ein weiteres Beispiel ist der Briefwechsel vom 3. Juli 2013/15. August 2013 zwischen der Schweiz und Deutschland zur Verbesserung der grenzüberschreitenden Tätigkeiten im Finanzbereich (SR 0.956.113.61). Hiermit wird Banken in der Schweiz ermöglicht, auch ohne Vermittlung eines in Deutschland bewilligten Kreditinstituts bzw. eines EWR-Kreditinstituts grenzüberschreitende Geschäftsbeziehungen in Deutschland anzubahnen. Banken, welche diese Möglichkeit nutzen wollen, müssen die relevanten deutschen Vorgaben zum Verbraucherschutz und zur Geldwäschereibekämpfung einhalten (FINMA-Mitteilung 54/ 2014 vom 6. Januar 2014; www.finma.ch und Link zu Dokumente).

Der bilaterale Acquis Schweiz-EU kennt kein umfassendes Dienstleistungsabkommen. Bestrebungen, den Dienstleistungsverkehr zwischen der Schweiz und der EU umfassend zu liberalisieren, scheiterten regelmässig. Protektionistische Interessen in zahlreichen Sektoren, das lange Zeit als «unverhandelbar» betrachtete Bankkundengeheimnis und offene Fragen in Bezug auf die Auswirkungen der diesfalls von der EU erwarteten Übernahme des einschlägigen Acquis communautaire (z.B. Konsumentenschutz, Wettbewerbs- und Beihilferecht) verhinderten ernsthafte Verhandlungen (s. zu diesen Gründen T. COTTIER et al., Rz. 541-547). Gleichwohl wird vor dem Hintergrund geänderter Rahmenbedingungen und tendenziell höherer Marktzugangshürden für schweizerische Unternehmen in der EU wieder vermehrt über die Wünsch- 
und Machbarkeit zumindest eines Finanzdienstleistungsabkommens diskutiert (vgl. Schweizerische Bankiervereinigung, Marktzugang für Schweizer Banken, Bedeutung und Perspektiven, Hintergrundpapier vom Juni 2019, S. 4).

Die bilateralen Abkommen enthalten punktuell Bestimmungen über den Dienstleistungshandel. Dazu gehören das Versicherungsabkommen, das Freizügigkeitsabkommen, das Landverkehrsabkommen, das Luftverkehrsabkommen und das Abkommen über bestimmte Aspekte des öffentlichen Beschaffungswesens. Diese Abkommen werden im folgenden vorgestellt (s. zum letztgenannten Abkommen N. 228-234).

Es ist mit Blick auf diesen Flickenteppich gegenseitiger Liberalisierungen (und die Ausnahme von Finanzdienstleistungen vom Geltungsbereich der Dienstleistungsfreiheit im FZA, N. 191) nicht davon auszugehen, dass der Acquis Schweiz-EU die Voraussetzungen von Art. V GATS erfüllt. Gemäss dieser Bestimmung können Ausnahmen von der Meistbegünstigung gemäss Art. II GATS gerechtfertigt werden, sofern eine bilaterale Übereinkunft zur Liberalisierung des Dienstleistungshandels «einen beträchtlichen sektoralen Geltungsbereich» aufweist und «praktisch jede Diskriminierung» beseitigt wird. Damit entfällt für die Schweiz wie auch für die EU die Möglichkeit, allfällige Ausnahmen von der Meistbegünstigung durch Rekurs auf Art. V GATS zu rechtfertigen (M. OESCH, Integration, S. 235-238). Denkbar wäre eine Rechtfertigung auch unter Rekurs auf die von der Schweiz beim Abschluss des GATS verankerten Ausnahmen zur Meistbegünstigung gemäss Art. II Abs. 2 GATS. Dabei wird allerdings nur auf natürliche Personen Bezug genommen (und nicht auf juristische Personen); auch ist umstritten, ob diese Ausnahmelisten auf zehn Jahre befristet waren oder nicht (N. DiEBOLD, Freizügigkeit, Rz. 335-337).

Die Schweiz verfolgt - gleichsam als Ausgleich zum Fehlen eines umfassenden Dienstleistungsabkommens - auch im Dienstleistungsbereich konsequent die Politik des autonomen Nachvollzugs. Auf diese Weise wird zumindest punktuell die Grundlage dafür gelegt, dass die EU schweizerischen Unternehmen auch ohne staatsvertragliche Verpflichtung Marktzugang gewährt (s. zum autonomen Nachvollzug im Finanzmarktrecht N. 369-371).

\section{Direktversicherungen}

Die EWG liberalisierte den Markt für Direktversicherungen mit Ausnahme der Lebensversicherung 1973 durch die Koordinierung der Vorschriften betr. Aufnahme und Ausübung der Tätigkeiten der Direktversicherung und durch die Aufhebung der Beschränkungen der Niederlassungsfreiheit in den Mitgliedstaaten (Richtlinien 73/239/EWG und 73/240/EWG). In der Folge war die Schweiz bestrebt, mit der EWG ein Abkommen abzuschliessen, um die Niederlassungsfreiheit auf schweizerische Unternehmen auszudehnen. Langwierige Verhandlungen führten 1989 zur Unterzeichnung des Abkommens betreffend die Direktversicherung mit Ausnahme der Lebensversicherung (Versiche- 
rungsabkommen, VersA). Es wurde 1993 in Kraft gesetzt (s. für die Anwendung des Abkommens in der EU Richtlinie 91/371/EWG; Urteil Kommission/Spanien, C-360/95, EU:C:1997:624).

Der Versicherungssektor ist für die schweizerische Wirtschaft bedeutsam. 2018 arbeiteten mehr als 46000 Personen in der Schweiz und rund 100000 Personen im Ausland für schweizerische Privatversicherer. Im Bereich der Schadensversicherung (Nichtlebensversicherung) belaufen sich die über Zweigniederlassungen verdienten Bruttoprämien aus der EU auf mehr als eine 1 Mia. CHF (www.eda.admin.ch/dea und Link zu Bilaterale Abkommen bis 1999/Versicherungen).

Das Versicherungsabkommen bezweckt, auf der Grundlage der Gegenseitigkeit die Voraussetzungen zu regeln, um Agenturen und Zweigniederlassungen von Unternehmen, die ihren Sitz im Hoheitsgebiet einer Vertragspartei haben und sich im Hoheitsgebiet der anderen Vertragspartei niederlassen wollen oder dort bereits niedergelassen sind, die Aufnahme oder Ausübung der selbständigen Tätigkeit der Direktversicherung, mit Ausnahme der Lebensversicherung, zu ermöglichen (Art. 1). Das Abkommen gewährt die sekundäre Niederlassungsfreiheit auf der Basis der Gegenseitigkeit; es gewährt kein Recht, grenzüberschreitend Dienstleistungen zu erbringen oder zu empfangen (Dienstleistungsfreiheit). Die Vertragsparteien verpflichten sich, im Geltungsbereich des Abkommens den Grundsatz der Nichtdiskriminierung zu beachten (Art. 5). Das Abkommen harmonisiert gewisse Voraussetzungen, welche erfüllt sein müssen, damit die Errichtung einer Agentur oder Zweigniederlassung im Hoheitsgebiet der anderen Vertragspartei genehmigt wird (z.B. Solvabilität und Mindestgarantiefonds, Art. 10 und Protokoll Nr. 1), und zählt die zulässigen Rechtsformen auf, die ein Unternehmen annehmen kann (Art. 9 und Anhang Nr. 3).

2018 wurde das Abkommen bzw. die einschlägigen Anhänge und Protokolle an die in der EU (Solvency II) und der Schweiz (Schweizer Solvenztest) mittlerweile geltenden Vorgaben betr. Berechnung der Solvabilität angepasst. Auch wurden die im Zug der EUErweiterungsrunden von 2004, 2007 und 2013 neu ebenfalls gültigen Rechtsformen von Versicherungsunternehmen eingefügt und der Umrechnungskurs CHF-EUR aktualisiert (Pressemitteilung des Bundesrates vom 5. Juli 2018, Die Schweiz und die EU passen ihr Versicherungsabkommen an).

185 Das Abkommen ist auf den Bereich der direkten Schadensversicherung anwendbar. Dazu gehören insbesondere die folgenden Versicherungsfälle: Unfall, Krankheit, Landfahrzeug-Kasko und Haftpflicht, Transportgüter, Feuer- und Elementarschäden, sonstige Sachschäden, verschiedene finanzielle Verluste, Rechtsschutz, touristische Beistandsleistungen (Anhang Nr.1). Lebensversicherungen, Rentenversicherungen, Versicherungen im Rahmen eines gesetzlichen Systems der sozialen Sicherheit, Rückversicherungen und 
Ausfuhrkreditversicherungen fallen nicht in den Geltungsbereich des Abkommens (Anhang Nr. 2). Des Weiteren betrifft das Abkommen gewisse namentlich aufgeführte Unternehmen nicht; dazu gehören typischerweise Gebäudeversicherungsanstalten, wie sie in Deutschland und der Schweiz gängig sind.

Das BGer war 2012 aufgerufen, die Zulässigkeit einer unternehmerischen Tätigkeit der Kantonalen Sachversicherung Glarus (Glarnersach) zu beurteilen (BGE 138 I 378). Die Glarnersach ist in einem Monopolbereich tätig (Gebäudeversicherung), kann von Gesetzes wegen im Wettbewerb mit privaten Versicherungsgesellschaften aber auch weitere Gefahren versichern (Sachversicherung). Das BGer kam zum Schluss, dass dies zulässig ist, insbesondere auch im Licht der Wirtschaftsfreiheit (Art. 27 und Art. 94 BV). Weiter setzte es sich mit der Reichweite der Ausnahme der Glarnersach vom Geltungsbereich des VersA auseinander (Anhang Nr. 2 Bst. D. 1. lit. g). Es kam mit Blick auf dessen Zweckartikel (Art. 1) zum Schluss, dass das VersA «einzig die grenzüberschreitende Tätigkeit der Versicherungen regelt; innerstaatliche Sachverhalte sind nur im Hinblick auf den grenzüberschreitenden Aspekt erfasst, soweit nicht der schweizerische Gesetzgeber unabhängig von dieser Zielsetzung parallel analoge Bestimmungen erlässt (...). Der Umstand, dass die in Anhang 2 Bst. D genannten Versicherungen nicht unter das Abkommen fallen, bedeutet demnach, dass diese Monopolversicherungen weiterhin auch im Verhältnis zwischen der Schweiz und der EU zulässig sind und die entsprechenden Versicherungsbereiche mithin den Versicherungsunternehmen aus der jeweils anderen Vertragspartei nicht zugänglich sind. Dadurch wird nicht ausgeschlossen, dass die genannten Versicherungen ihre Tätigkeitsbereiche im Wettbewerb - und damit in Konkurrenz auch zu den Versicherungsunternehmen aus der EU - erweitern. Sie unterstehen den Anforderungen des Abkommens einzig soweit sie in der EU tätig sein wollen.» (kritisch zu diesem Urteil T. CotTIER et al., Rz. 599, weil dieses Businessmodell die Gefahr einer Wettbewerbsverzerrung zugunsten des Monopolisten und zu Lasten der Privatassekuranz birgt).

Institutionell beruht das Abkommen auf dem traditionellen Zweisäulenprinzip (N. 69). Das Bundesgericht bejaht die unmittelbare Anwendbarkeit des Abkommens (BGE 138 I 378, E. 10; N. 185). Das Abkommen sieht vor, zur Lösung einer Streitigkeit über seine Auslegung und Anwendung ein Schiedsgericht einzusetzen, dessen Entscheidung für die Parteien bindend ist (Art. 38, N. 99). Es verpflichtet die Behörden der Vertragsparteien zur Zusammenarbeit. Sie übermitteln einander alle Unterlagen und Auskünfte, die für die Ausübung der Aufsicht zweckdienlich sind; spezielle Bestimmungen gelten für Geschäftsgeheimnisse (Art. 30-33).

Über das VersA hinaus haben die Europäische Aufsichtsbehörde für das Versicherungswesen und die betriebliche Altersversorgung (EIOPA) und die Eidgenössische Finanzmarktaufsicht (FINMA) in einem Memorandum of Understanding von 2012 vereinbart, bei Aufsichtsfragen im Zusammenhang mit Versicherungsgruppen mit internationaler Geschäftstätigkeit im EWR und der Schweiz zusammenzuarbeiten (www.finma.ch und Link $\mathrm{zu}$ News/FINMA und EIOPA unterzeichnen ein Memorandum of Understanding). Weiter hat die Europäische Kommission - ebenfalls unabhängig vom VersA - das in der Schweiz geltende Solvabilitäts- und Aufsichtssystem für Versicherungs- und Rückversicherungsunternehmen mit Sitz in der Schweiz als den Anforderungen gemäss der Richt- 
linie 2009/138/EG (Solvency II) gleichwertig betrachtet (Delegierter Beschluss (EU) 2015/ 1602). Dieser Gleichwertigkeitsbeschluss bildet die Grundlage für den grenzüberschreitenden Marktzugang schweizerischer Versicherungsunternehmen in der EU in den vom Beschluss betroffenen Geschäftsfeldern.

\section{Kurzzeitige Dienstleistungen}

Das Freizügigkeitsabkommen liberalisiert die Erbringung von kurzzeitigen Dienstleistungen im Hoheitsgebiet der Vertragsparteien (Art. 1 lit.b). Es umfasst sowohl die aktive Dienstleistungsfreiheit als auch die passive Dienstleistungsfreiheit (Art. 5 FZA, Art. 17-23 Anhang I FZA; s. für das FZA im Allg. N. 152-177):

- Dienstleistungserbringerinnen und -erbringern einschliesslich Gesellschaften in der EU und der Schweiz wird das Recht eingeräumt, Dienstleistungen im Hoheitsgebiet der anderen Vertragspartei zu erbringen, deren tatsächliche Dauer 90 Arbeitstage pro Kalenderjahr nicht überschreitet. Natürliche und juristische Personen können sich auf die aktive Dienstleistungsfreiheit berufen. Dieses Recht besteht unbeschadet besonderer Abkommen über die Erbringung von Dienstleistungen zwischen den Vertragsparteien (einschliesslich des Abkommens über das öffentliche Beschaffungswesen, sofern es die Erbringung von Dienstleistungen umfasst).

- Natürlichen Personen, die Staatsangehörige eines EU-Mitgliedstaates oder der Schweiz sind und sich nur als Empfänger einer Dienstleistung in das Hoheitsgebiet einer Vertragspartei begeben, wird das Einreise- und Aufenthaltsrecht eingeräumt. Die passive Dienstleistungsfreiheit wird nur natürlichen Personen gewährt; juristische Personen können sich nicht darauf berufen.

188 Diverse EU-Rechtsakte, auf die in den Anhängen II und III verwiesen wird, sind auch bei der grenzüberschreitenden Dienstleistungserbringung anwendbar (N. 192). Demgegenüber ist die Richtlinie 2006/123/EG über Dienstleistungen im Binnenmarkt im Verhältnis zwischen der Schweiz und der EU nicht einschlägig.

189 Das Abkommen umschreibt den Begriff der Dienstleistungen nicht. Es ist gestützt auf die Auslegungsregel gemäss Art. 16 FZA (N. 84-86) davon auszugehen, dass die im EU-Recht entwickelten (Abgrenzungs-) Kriterien auch im bilateralen Verhältnis einschlägig sind.

Das Verwaltungsgericht des Kantons Zürich kam 2014 zum Schluss, dass das FZA deutschen und österreichischen Taxifahrerinnen und Taxifahrern das Recht einräumt, während 
90 Tagen pro Jahr Gäste auf der Strecke zum und vom Flughafen Zürich zu transportieren, weil das Abkommen - im Unterschied zum EU-Recht (Art. 58 AEUV) - Verkehrsdienstleistungen nicht speziell regelt und solche Dienstleistungen folglich als Dienstleistungen im Sinn des Abkommens zu qualifizieren sind. Aus diesem Grund erklärte das Verwaltungsgericht den Staatsvertrag zwischen der Schweiz und Deutschland von 1953 (SR 0.741.619.136) und den Staatsvertrag zwischen der Schweiz und Österreich von 1958 (SR 0.741.619.163), welche umfassende gewerbsmässige Beförderungsrechte nur 5 bzw. $10 \mathrm{~km}$ diesseits und jenseits der Grenze vorsehen, als nicht anwendbar (Urteil vom 4. September 2014, VB.2013.00231; s. zu diesen Staatsverträgen auch N. 179; zum Ganzen M. OESCH, Wirtschaft, S. 322-323; vgl. auch Entscheid des Rekursgerichts im Ausländerrecht des Kantons Aargau vom 30. Mai 2008, AGVE 2008, S. 412, zur Abgrenzung der Erbringung einer Dienstleistung von der Auslieferung und Montage einer Ware).

\section{(a) Aktive Dienstleistungsfreiheit}

Die Vertragsparteien räumen den Staatsangehörigen der anderen Vertragspartei einschliesslich Gesellschaften, die nach dem Recht eines Mitgliedstaats der EU oder nach schweizerischem Recht gegründet wurden und ihren satzungsmässigen Sitz, ihre Hauptverwaltung oder ihre Hauptniederlassung im Gebiet einer Vertragspartei haben, das Recht ein, Dienstleistungen zu erbringen, deren tatsächliche Dauer 90 Arbeitstage pro Kalenderjahr nicht überschreitet (Art. 5; s. BGE 131 I 223, E. 1, betr. Anspruchsberechtigung einer juristischen Person mit Sitz in Deutschland, welche im Bereich der Finanzierung von Rechtsprozessen tätig ist). Dieses Recht umfasst das Recht auf Einreise und Aufenthalt der Dienstleistungserbringerin und des Dienstleistungserbringers, um die Tätigkeit auszuführen; dazu gehören auch die Arbeitnehmenden der Dienstleistungserbringerin und des Dienstleistungserbringers - unabhängig von ihrer Staatsangehörigkeit -, welche zwecks Erbringung einer Dienstleistung entsandt werden (Art. 17 Anhang I). Ungeklärt ist, ob nur Dienstleistungen unter persönlicher Anwesenheit der dienstleistenden Person oder entsandter Arbeitnehmenden unter das Abkommen fallen oder auch Dienstleistungen auf dem Korrespondenzweg (wie etwa die juristische Beratung von Mandanten via Telefon oder Email; s. N. DieBOLD, Freizügigkeit, Rz. 263 und 956; A. Epiney/B. Metz/B. Pirker, Parallelität, S. 213; C. Tobler/J. BeGlinger, Grundzüge, Rz. 321).

Die Dienstleistungserbringerin und der Dienstleistungserbringer verfügen über die gleichen Bedingungen, wie sie der betroffene Staat für seine eigenen Staatsangehörigen vorschreibt (Art. 19 Anhang I; s. dazu Urteil des Verwaltungsgerichts des Kantons Zürich vom 4. September 2014, VB.2013.00231, E. 6.4; zum Grundsatz der Nichtdiskriminierung N. 164-165). Das Abkommen verbietet - zumindest gemäss dem Wortlaut von Art. 17 Anhang I - auch darüber hinausgehende Beschränkungen (A. EPINEY/B. METZ/B. PIRKER, Paralleli- 
tät, S. 219; a.M. C. TOBLER/J. BEGLINGER, Grundzüge, Rz. 327). Ungleichbehandlungen und Beschränkungen können aus Gründen der öffentlichen Ordnung, Sicherheit und Gesundheit (Art. 5 Anhang I) und weiterer zwingender Gründe des Allgemeininteresses (Art. 22 Abs. 4) eingeschränkt werden (N. 168-169). Tätigkeiten, die auch nur gelegentlich die Ausübung hoheitlicher Befugnisse umfassen, profitieren nicht vom vertraglich gewährleisteten Marktzugang (Art. 22 Anhang I; s. zu den analogen Bereichsausnahmen im Rahmen der Personenfreizügigkeit N.160). Ausgenommen sind zudem Tätigkeiten von Arbeitsvermittlungs- und -verleihunternehmen sowie genehmigungspflichtige Finanzdienstleistungen (Art. 22 Anhang I).

192 Die von den Vertragsparteien vereinbarten Begleitmassnahmen zur Erleichterung der Ausübung der Freizügigkeit im Bereich der Koordinierung der Systeme der sozialen Sicherheit (Anhang II) und der gegenseitigen Anerkennung beruflicher Qualifikationen (Anhang III) greifen auch bei der Erbringung von kurzzeitigen Dienstleistungen:

- $\quad$ Die Verordnung (EG) Nr. 883/2004 und die Verordnung (EG) Nr. 987/ 2009 sind bei der grenzüberschreitenden Erbringung von Dienstleistungen anwendbar (s. zu diesen Verordnungen N. 171-172). Dienstleistungserbringerinnen und Dienstleistungserbringer sowie entsandte Arbeitnehmende unterliegen weiterhin den Rechtsvorschriften des Heimatstaates (Art. 12 der Verordnung (EG) Nr. 883/2004).

- Die Richtlinie 2005/36/EG sieht ausdrücklich Regeln für die Anerkennung von Berufsqualifikationen für Staatsangehörige eines EU-Mitgliedstaats oder der Schweiz vor, welche sich bloss vorübergehend zur Dienstleistungserbringung in einen anderen Vertragsstaat begeben (s. zu dieser Richtlinie N. 173-175). Solche Personen dürfen ohne Überprüfung ihrer Qualifikationen am Erbringungsort tätig werden (N. DiEBOLD, Freizügigkeit, Rz. 1137). Nur bei Berufen, welche nicht von der automatischen Anerkennung oder der Anerkennung gestützt auf die Berufserfahrung profitieren und bei denen ein Risiko für die öffentliche Gesundheit oder Sicherheit besteht, können die Qualifikationen des Dienstleistungserbringers nachgeprüft werden. Dabei sind strikte Fristen zu beachten. Wenn ein wesentlicher Unterschied zur geforderten Ausbildung besteht, können die Dienstleistungserbringerin und der Dienstleistungserbringer verpflichtet werden, eine Eignungsprüfung zu absolvieren. Sie verwenden die Berufsbezeichnung ihres Herkunftsstaats. In der Schweiz stellen das Bundesgesetz über die Meldepflicht und die Nachprüfung der Berufsqualifikationen von Dienstleistungserbringerinnen und -erbringern in regle- 
mentierten Berufen von 2012 (BGMD, SR 935.01) und die dazugehörige Verordnung (VMD, SR 935.011) die Rechtsgrundlagen für die Meldepflicht und Nachprüfung dar.

Die Richtlinie 2005/36/EG erfasst nur die aktive Dienstleistungsfreiheit. Die Vorgaben, welche bei der passiven Dienstleistungsfreiheit und der Korrespondenzdienstleistung (s. zur ungeklärten Anwendbarkeit des FZA auf Korrespondenzdienstleistungen N. 190) zu beachten sind, richten sich im EU-Recht nach der Richtlinie 2006/123/EG über Dienstleistungen im Binnenmarkt und der Richtlinie 2000/3/EG über den elektronischen Geschäftsverkehr (N. DiEBOLD, Freizügigkeit, Rz. 1136). Diese Richtlinien sind im bilateralen Verhältnis nicht anwendbar.

\section{(b) Flankierende Massnahmen}

Die Vertragsparteien sind berechtigt, Vorschriften über die Arbeits- und Beschäftigungsbedingungen für die im Rahmen der Erbringung von Dienstleistungen entsandten Arbeitnehmerinnen und Arbeitnehmer zu erlassen (Art. 22 Anhang I). Zu diesem Zweck wird auf die Richtlinie 96/71/EG über die Entsendung von Arbeitnehmern im Rahmen der Erbringung von Dienstleistungen verwiesen. Diese Richtlinie bezweckt, sowohl einen fairen Wettbewerb zu garantieren als auch die Wahrung der Rechte der Arbeitnehmenden zu schützen und damit Sozialdumping zu verhindern (Erwägungsgrund 5; s. dazu und zu den FlaM A. Epiney/L. Hehemann, Rz. 3-15, 24-31; K. PÄrLI, Entsendegesetz, passim; J.M. TIEFENTHAL, passim).

In der EU wurde die Entsenderichtlinie 96/71/EG durch die Richtlinie (EU) 2018/957 revidiert. Zudem wurde die Richtlinie 2014/67/EU zur Durchsetzung der Richtlinie 96/71/EG (Durchsetzungsrichtlinie) erlassen. Diese Novellierungen wurden bis anhin nicht in das FZA aufgenommen (s. zur Forderung der EU, dies im Rahmen des geplanten InstA nachzuholen, N. 113).

In der Schweiz wurden flankierende Massnahmen (FlaM) in Kraft gesetzt, um diese Ziele zu erreichen:

- $\quad$ Das Entsendegesetz (EntsG, SR 823.20) regelt die minimalen Arbeits- und Lohnbedingungen für Arbeitnehmerinnen und Arbeitnehmer, die eine Arbeitgeberin oder ein Arbeitgeber mit Wohnsitz oder Sitz im Ausland in die Schweiz entsendet, um dort eine Dienstleistung zu erbringen. Die Arbeitgeberinnen und Arbeitgeber müssen die Arbeits- und Lohnbedingungen einhalten, die in Bundesgesetzen, Verordnungen, allgemein verbindlich erklärten Gesamtarbeitsverträgen und Normalarbeitsverträgen vorgeschrieben sind und die folgenden Bereiche betreffen: minimale Entlöhnung inklusive Zuschläge, Arbeits- und Ruhezeit, Mindestdauer der 
Ferien, Arbeitssicherheit und Gesundheitsschutz am Arbeitsplatz, Schutz von Schwangeren, Wöchnerinnen, Kindern und Jugendlichen, Nichtdiskriminierung, namentlich Gleichbehandlung von Frau und Mann. Die Kontrolle obliegt Behörden, paritätisch zusammengesetzten Organen, tripartiten Kommissionen und Inspektoren. Bei Verstössen drohen Verwaltungssanktionen und Strafen (Wegweisung, Tätigkeitsverbot bis zu fünf Jahren, Busse bis 1 Mio. CHF; s. BGE 140 II 447 = Pra 104 [2015] Nr. 43, E. 4, zur Vereinbarkeit der Beschränkung des Erlasses von Dienstleistungssperren auf ausländische Dienstleistungserbringer mit Art. 2 FZA). Der Erstunternehmer haftet solidarisch für die Nichteinhaltung der Arbeits- und Lohnbedingungen durch die Subunternehmer.

- Das Bundesgesetz über die Allgemeinverbindlicherklärung von Gesamtarbeitsverträgen (SR 221.215.311) ermöglicht den Behörden im Fall von wiederholter missbräuchlicher Unterbietung der orts-, berufs- oder branchenüblichen Löhne und Arbeitszeiten, einen zwischen Verbänden abgeschlossenen Gesamtarbeitsvertrag auf Arbeitgeber und Arbeitnehmende des betroffenen Wirtschaftszweiges oder Berufs auszudehnen.

- Das Obligationenrecht (SR 220) erlaubt den Behörden, einen befristeten Normalarbeitsvertrag zu erlassen, der nach Regionen differenzierte Mindestlöhne vorsieht, sofern innerhalb einer Branche oder einem Beruf die orts-, berufs- oder branchenüblichen Löhne wiederholt in missbräuchlicher Weise unterboten werden und kein Gesamtarbeitsvertrag mit Bestimmungen über Mindestlöhne vorliegt, der allgemein verbindlich erklärt werden kann (Art. 360a-360f).

2019 kontrollierten die schweizerischen Vollzugsorgane die Einhaltung der Lohn- und Arbeitsbedingungen bei rund 41000 Unternehmen und 160000 Personen in der Schweiz (wobei die nachfolgend festgestellten Verstösse nicht repräsentativ für die Gesamtsituation auf dem Schweizer Arbeitsmarkt sein dürften, weil gezielt dort kontrolliert wurde, wo Lohndumping vermutet wird). Auf nationaler Ebene wurden $8 \%$ der Schweizer Arbeitgeberinnen und Arbeitgeber, $29 \%$ der Entsandten und $32 \%$ der selbständigen Dienstleistungserbringerinnen und -erbringer einer Kontrolle unterzogen. Die Quote der Verstösse gegen GAVs im Entsendebereich betrug gemäss den Paritätischen Kommissionen $21 \%$. Die kantonalen tripartiten Kommissionen stellten bei Entsandten Lohnunterbietungen in $15 \%$ der kontrollierten Fälle fest. Bei den Schweizer Arbeitgebern wurden Unterbietungen von Lohnbestimmungen in $11 \%$ der kontrollierten Fälle festgestellt. Bei den selbständigen Dienstleistungserbringerinnen und -erbringern wurde bei $8 \%$ der knapp 6000 durchgeführten Kontrollen eine Scheinselbständigkeit vermutet (SECO, FlaM Bericht 2019, passim).

Die Schweiz ist nicht an der Europäischen Arbeitsbehörde beteiligt, welche mit der Verordnung (EU) 2019/1149 errichtet wurde (wobei im Titel ausdrücklich von einem «Text von Bedeutung für den EWR und die Schweiz» die Rede ist). Diese Behörde unterstützt die Mitgliedstaaten und die Kommission bei der wirksamen Anwendung und Durchsetzung des 
EU-Rechts im Bereich der Arbeitskräftemobilität und der Koordinierung der Systeme der sozialen Sicherheit (Art. 1); dazu gehört auch der Kampf gegen Lohndumping. Eine formelle Beteiligung der Schweiz - welche zurzeit Beobachterstatus geniesst (Tagesanzeiger vom 25. Februar 2020, S. 6) - bedarf eines separaten völkerrechtlichen Vertrags.

Ausländische Dienstleistungserbringerinnen und Dienstleistungserbringer, die sich auf die selbstständige Erwerbstätigkeit berufen, haben diese gegenüber den Kontrollorganen auf Verlangen nachzuweisen (Art. 1a EntsG); auf diese Weise soll Scheinselbständigkeit verhindert werden (s. zum Ganzen J. DimitriJewitsch, passim). Ausländische Dienstleistungserbringerinnen und Dienstleistungserbringer müssen ihren Einsatz zudem acht Tage vor Aufnahme des Einsatzes den Behörden melden (Art. 6 Abs. 3 EntsG). Diese «AchtTage-Regel» gilt für die meisten Branchen; ausgenommen sind Notfälle (Reparaturen, Unfälle, Naturkatastrophen oder andere nicht vorhersehbare Ereignisse; www.sem.admin.ch und Link zu Einreise \& Aufenthalt/Meldeverfahren für kurzfristige Erwerbstätigkeit). Sofern ein allgemein verbindlich erklärter Gesamtarbeitsvertrag die Hinterlegung einer Kaution durch die Arbeitgeberin oder den Arbeitgeber vorsieht, so gelten die entsprechenden Bestimmungen auch für Arbeitgeberinnen und Arbeitgeber, welche Arbeitnehmende in die Schweiz entsenden (Art. 2 Abs. $2^{\text {ter }}$ EntsG). Die Kautionspflicht wurde eingeführt, damit die Kontrollorgane beim Vollzug der flankierenden Massnahmen eine Sicherheit haben.

Die EU stellt sich auf den Standpunkt, wonach die Wartefrist von acht Tagen gegen das FZA verstösst (Entschliessung des Europäischen Parlaments vom 7. September $2010 \mathrm{zu}$ dem Thema EWR-Schweiz, Rz. 12; Schlussfolgerungen des Rates vom 20. Dezember 2012, Rz. 35). Die Schweiz argumentiert, die Voranmeldung sei notwendig, um die Kontrolle der Einhaltung der FlaM vor Ort zu organisieren und sicherzustellen. Bis heute konnte diese Meinungsverschiedenheit im Gemischten Ausschuss nicht gelöst werden. Auch die Rechtmässigkeit der Kautionspflicht ist umstritten (s. zum Ganzen A. EPINEY/L. HeHEMAnN, Rz. 31).

Das geplante InstA wäre auf das Freizügigkeitsabkommen anwendbar. In Bezug auf die flankierenden Massnahmen hat die EU eine spezielle Regelung vorgeschlagen (N. 113). Die Schweiz akzeptiert diesen Vorschlag nicht; sie fordert Klärungen und Präzisierungen (N. 117).

\section{(c) Passive Dienstleistungsfreiheit}

Natürlichen Personen, welche Staatsangehörige eines Mitgliedstaates der EU oder der Schweiz sind und sich als Empfänger einer Dienstleistung - z.B. für touristische Zwecke oder medizinische Behandlungen - in das Hoheitsgebiet einer Vertragspartei begeben, wird das Einreise- und Aufenthaltsrecht einge- 
räumt (Art. 5 Abs. 3). Für Aufenthalte von mehr als drei Monaten erhalten Dienstleistungsempfänger eine Aufenthaltserlaubnis, deren Gültigkeitsdauer der Dauer der Dienstleistung entspricht; ein Anspruch auf Sozialhilfe besteht nicht (Art. 23 Anhang I). Die passive Dienstleistungsfreiheit kann aus Gründen der öffentlichen Ordnung, Sicherheit und Gesundheit (Art. 5 Anhang I) und weiterer zwingender Gründe des Allgemeininteresses (Art. 22 Abs. 4) eingeschränkt werden (N. 168-169).

Der EuGH und das Bundesgericht legen die passive Dienstleistungsfreiheit nach Massgabe des Freizügigkeitsabkommens restriktiv aus. Gemäss höchstrichterlicher Praxis gewährt die passive Dienstleistungsfreiheit - gestützt auf eine wörtliche Auslegung von Art. 5 Abs. 3 - kein umfassend geltendes Recht auf Nichtdiskriminierung. Dies gilt sowohl in Bezug auf die Behandlung, welche Personen im Hoheitsgebiet der anderen Vertragspartei erfahren (Urteil Hengartner und Gasser, C-70/09, EU:C:2010:430, betr. Pacht eines Jagdgebiets), als auch in Bezug auf die Behandlung, welche ihnen in ihrem Heimatstaat zuteil wird, nachdem sie im Hoheitsgebiet der anderen Vertragspartei eine Dienstleistung empfangen haben.

Das BGer bestätigte in einem Urteil von 2007 den Entscheid der Behörden des Kantons Tessin, wonach sich ein von einem Geburtsgebrechen betroffener Minderjähriger nicht auf das FZA berufen kann, um die Übernahme (eines Teils) der Kosten einer in Deutschland erfolgten ambulanten Behandlung zu beanspruchen (BGE $133 \mathrm{~V}$ 624; kritisch dazu V. BollLEt, S. 77-80; A. EPINEY/B. METZ/B. PIRKER, Parallelität, S. 214-215).

Vereinzelt hat die Schweiz mit anderen europäischen Staaten Vereinbarungen abgeschlossen, wonach die Staatsangehörigen der Vertragsstaaten in Bezug auf spezifische Aktivitäten nicht diskriminiert werden dürfen, wenn diese in das Staatsgebiet der anderen Vertragspartei einreisen und eine Dienstleistung konsumieren. Solche Verträge ergänzen die Bestimmungen zur Dienstleistungserbringung im Freizügigkeitsabkommen.

Die Schweiz und Italien vereinbarten 2007, dass Staatsangehörigen der Schweiz und Italiens gleiche Zugangsbedingungen (Eintrittspreis, Ermässigungen) zu Kulturstätten wie Museen, Galerien, Pärke und archäologischen Stätten gewährt werden wie einheimischen Personen (Decreto 19 ottobre 2007: Ingresso gratuito e agevolato nelle sedi espositive dello Stato ai cittadini della Confederazione svizzera; zit. aus C. TOBLER/J. BEGLINGER, Grundzüge, Rz. 352).

\section{Landverkehr}

1992 schlossen die EWG und die Schweiz das Abkommen über den Güterverkehr auf Strasse und Schiene ab (AS 1993 1198). Es bezweckte, die Zusammenarbeit insbesondere beim alpenquerenden Transitverkehr zu verstärken. Die 
Schweiz verpflichtete sich langfristig zum Bau einer Eisenbahntransitstrecke durch die Alpen (Neue Eisenbahn-Alpentransversale, NEAT), inkl. des Gotthard-Basistunnels und des Lötschberg-Basistunnels (Art. 5). Die EWG verpflichtete sich, die Kapazitäten für die Nord- und Südzulaufstrecken zur NEAT zu erhöhen (Art. 6). Dieses Abkommen blieb in Teilen bis 2005 in Kraft. In Ergänzung dazu schloss die Schweiz mit Deutschland (1996) und Italien (1999) Abkommen, mit denen sich die beiden letztgenannten Staaten verpflichteten, ausreichende Kapazitäten auf den Haupt-Zulaufstrecken zur NEAT zur Verfügung zu stellen, um den grenzüberschreitenden Verkehr auf der Schiene in seiner Leistungsfähigkeit zu sichern (SR 0.742.140.313.69; SR 0.742.140.345.43).

1994 nahmen Volk und Stände die Alpenschutzinitiative an (Art. 36 ${ }^{\text {sexies }}$ aBV, Art. 84 BV). Hauptanliegen der Initiative war die Reduktion der transitverkehrsbedingten Umweltbelastung in den Alpen, insbesondere auf der Nord-Süd-Achse durch den Gotthard. Entsprechend gilt folgendes: Der alpenquerende Gütertransitverkehr von Grenze zu Grenze erfolgt auf der Schiene (Abs. 2). Die Transitstrassen-Kapazität im Alpengebiet darf nicht erhöht werden (Abs. 3). Die Annahme der Initiative führte zu Beginn der Verhandlungen über die Bilateralen I zu einer Blockade, welche erst aufgelöst werden konnte, als die Schweiz eine nichtdiskriminierende Umsetzung der Initiative in Aussicht stellte (Botschaft Bilaterale I, S. 6129; s. zum Konfliktpotential mit dem Grundsatz der Nichtdiskriminierung nach Massgabe des LandVA A. EPINEY, Art. 84 Rz. 11-13). Problematisch ist, dass der Verfassungsauftrag zur Verlagerung des Schwerverkehrs auf die Schiene bis heute nicht erfüllt worden ist - was allerdings nicht allein am fehlenden politischen Willen in der Schweiz liegt, sondern auch am fehlenden Engagement der Nachbarländer (unzureichende Anbindung an das Schienennetz der Schweiz) und der EU (unzulängliche Verlagerungspolitik).

1999 unterzeichneten die EG und die Schweiz das Abkommen über den Güterund Personenverkehr auf Schiene und Strasse (Landverkehrsabkommen, LandVA). Es wurde 2002 in Kraft gesetzt und bezweckt, den Zugang der Vertragsparteien zum Güter- und Personenverkehrsmarkt auf der Strasse und auf der Schiene zu liberalisieren, damit eine effizientere Verkehrsabwicklung auf jener Route gewährleistet ist, die technisch, geographisch und wirtschaftlich am besten auf die verschiedenen Verkehrsträger abgestimmt ist; des Weiteren soll es die Bedingungen für eine koordinierte Verkehrspolitik festlegen (Art. 1; s. zum Ganzen K. SOlLBERGER, passim; K. SOlLbERGER/A. EPINEY, passim).

Das Abkommen beruht auf den Grundsätzen der Gegenseitigkeit und der freien Wahl des Verkehrsträgers. Die Schweiz verpflichtet sich zur Anwendung von Vorschriften, welche mit den im Abkommen und seinen Anhängen aufgeführten EU-Rechtsakten gleichwertig sind (Art. 52 Abs. 6, Ingress zu Anhang 1). Diese Rechtsakte betreffen im Wesentlichen den Zugang zum Beruf der Kraftverkehrsunternehmerin und des Kraftverkehrsunternehmers, Sozialvorschriften, technische Vorschriften für den Betrieb von Kraftfahrzeugen, Zugangsund Transitrechte im Eisenbahnverkehr und die Operabilität und Sicherheit 
der Eisenbahnsysteme. Bei der Anwendung des Abkommens ist der Grundsatz der Nichtdiskriminierung zentral (Art. 1); dieser Grundsatz wird an diversen Stellen konkretisiert (s. etwa Art. 32 betr. Massnahmen zur Verwirklichung der koordinierten Verkehrspolitik; zum Ganzen A. EPINEY/N. FreI, Rz. 39-64; A. EPINEY/J. HEUCK/M. KERN, passim). Das Abkommen enthält auch beihilferechtliche Bestimmungen (N. 227).

Das Abkommen liberalisiert den grenzüberschreitenden gewerblichen Strassengüterverkehr und Personenverkehr mit Kraftomnibussen. Dies gilt in Bezug auf den Gütertransport auch für den Verkehr zwischen zwei EU-Mitgliedstaaten (grosse Kabotage). Beförderungen zwischen zwei Orten im Gebiet eines EU-Mitgliedstaats (Güter; kleine oder nationale Kabotage) und zwischen zwei Orten im Gebiet der EU (Personen) sowie Beförderungen zwischen zwei Orten im Gebiet der Schweiz (Güter und Personen) werden vom Abkommen nicht erfasst (Art. 14 und Art. 20). Für den Güterverkehr gilt in der Schweiz ein Nacht- (22.00 Uhr bis 05.00 Uhr) und Sonntagsfahrverbot (Art. 15). Bei Fahrten aus dem Ausland in die Schweiz (und umgekehrt) ist ein Gesamtgewicht von 40 Tonnen zulässig.

Art. 3 definiert den Begriff des «Fahrzeugs» u.a. als «Kraftfahrzeug, das nach seiner Bauart und Ausstattung dazu bestimmt und geeignet ist, mehr als neun Personen, einschliesslich des Fahrers, zu befördern». Aus diesem Grund ist das Abkommen nicht anwendbar auf Taxidienstleistungen mit herkömmlichen Kraftfahrzeugen (s. zum Taxistreit N. 189).

Das Abkommen liberalisiert den grenzüberschreitenden Eisenbahnverkehr für den Gütertransport. Es gewährt den im Gebiet der Vertragsparteien niedergelassenen Eisenbahnunternehmen bestimmte Zugangs- und Transitrechte, um Verkehrsdienstleistungen im grenzüberschreitenden kombinierten Verkehr zu erbringen (Art. 24). Die nationalen Zuweisungsstellen gewährleisten, dass die Fahrwegkapazität (Zugtrasse) gerecht und in nichtdiskriminierender Weise zugewiesen wird und dass das Zuweisungsverfahren eine effiziente und optimale Nutzung des Fahrwegs erlaubt (Art. 27). Das Abkommen enthält keine Vorgaben zum Personenverkehr auf der Schiene.

Die Vertragsparteien sind übereingekommen, eine koordinierte Verkehrspolitik auf dem Gebiet des Güter- und Personenverkehrs zu entwickeln; sie bemühen sich darum, eine weitgehende Vergleichbarkeit der Bedingungen im Verkehrsbereich, einschliesslich betreffend die Steuervorschriften, zu schaffen, insbesondere um Umwegverkehr im Alpenraum zu vermeiden oder dort eine bessere Verkehrsverteilung zu erzielen (Art. 30). Zu diesem Zweck ergreifen die Vertragsparteien Massnahmen, die darauf abzielen, einen lauteren Wettbewerb zwischen den Verkehrsträgern und innerhalb der Verkehrsträger zu 
gewährleisten und den Einsatz umweltverträglicherer Verkehrsmittel zu erleichtern (Art. 31). Solche Massnahmen müssen den Grundsatz der Nichtdiskriminierung und den Grundsatz der Nichteinführung einseitiger mengenmässiger Beschränkungen beachten (Art. 32; A. EPINEY/B. METZ/B. PIRKER, Parallelität, S. 242-248). Die Schweiz wird ermächtigt, eine nichtdiskriminierende Gebührenregelung für Kraftfahrzeuge einzuführen (Art. 38 und Art. 40, Anhang 10). Gestützt auf diese Vorgaben hat die Schweiz die leistungsabhängige Schwerverkehrsabgabe (LSVA) geschaffen. Die LSVA wird auf alle Kraftfahrzeuge und deren Anhänger entrichtet, die ein Gesamtgewicht von mehr als 3,5 Tonnen aufweisen, Güter transportieren, im In- oder Ausland immatrikuliert sind und das öffentliche Strassennetz der Schweiz befahren. Die Höhe der geschuldeten Abgabe ist vom Gesamtgewicht, von der Schadstoffemissionsstufe (Fahrzeugkategorie) und von den gefahrenen Kilometern in der Schweiz abhängig. Das Abkommen deckelt den gewichteten Durchschnitt der Gebühren auf höchstens 325 CHF für ein Fahrzeug, dessen Gesamtgewicht in beladenem Zustand nicht über 40 Tonnen liegt und das eine alpenquerende Strecke von $300 \mathrm{~km}$, also beispielweise die Strecke Basel-Chiasso, zurücklegt. Die Gebühr für die Kategorie mit dem höchsten Verschmutzungsgrad beträgt nicht mehr als 380 CHF. Der Gemischte Ausschuss passt die Erhebungsmodalitäten periodisch an (www.eda.admin.ch/dea und Link zu Bilaterale Abkommen I (1999)/Landverkehr; s. zur Umsetzung von Beschlüssen des Gemischten Ausschusses im schweizerischen Recht BGE 136 II 337, E. 4.1).

Die Nettoeinnahmen, welche die Schweiz aus der LSVA generiert, betragen jährlich durchschnittlich ca. 1,5 Mia. CHF. Rund ein Viertel dieses Betrags stammt von ausländischen Transporteuren. Die Einnahmen werden vorwiegend zur Finanzierung von Infrastrukturvorhaben des öffentlichen Verkehrs verwendet (www.eda.admin.ch/dea und Link zu Bilaterale Abkommen I (1999)/Landverkehr).

Weiterentwicklungen im EU-Recht - wie die Bahnpakete von 2000, 2004, 2007 und 2016 - sind auch für das Landverkehrsabkommen bedeutsam, soweit sie abkommensrelevante Sachbereiche betreffen (s. www.bav.admin.ch und Link zu Bahnreformen in der EU). Diesfalls liegt es am Gemischten Ausschuss, die Anhänge zu aktualisieren oder eine Änderung des Abkommens selbst vorzuschlagen, sofern ein solcher Schritt - v.a. auch mit Blick auf das ordnungsgemässe Funktionieren des Abkommens - als sachgerecht erachtet wird.

In der EU wurde 2006 die Eisenbahnagentur gegründet (Verordnung (EU) 2016/796). Ziel der Agentur ist es, zur weiteren Entwicklung und zum reibungslosen Funktionieren eines einheitlichen europäischen Eisenbahnraums ohne Grenzen beizutragen (Sicherheit, Interoperabilität, Konkurrenzfähigkeit des Eisenbahnsektors, Genehmigungen für das Inverkehrbringen von Eisenbahnfahrzeugen und von Sicherheitsbescheinigungen für Eisenbahnunternehmen). Die Schweiz beteiligt sich nicht an der Eisenbahnagentur. Seit 2015 
existiert ein Mandat zur Aufnahme von Verhandlungen mit der EU (Pressemitteilung des Bundesrates vom 20. Mai 2015). Zurzeit laufen entsprechende Gespräche zwischen der Schweiz und der EU.

207 Das geplante InstA wäre auf das Landverkehrsabkommen anwendbar (s. für den Textentwurf des InstA vom Dezember 2018 N. 105-116).

\section{Luftverkehr}

208 Die EWG hat seit den 1980er-Jahren schrittweise einen europäischen Luftverkehrsbinnenmarkt entwickelt. Luftfahrtunternehmen der EWG/EG/EU sind berechtigt, innergemeinschaftliche Strecken zu nutzen sowie Flugpreise und Frachtraten frei festzusetzen, sofern sie die gemeinsamen Vorgaben an die Sicherheit ihrer Flugzeuge, die fachliche Eignung ihres Personals und die Strukturen ihrer Organisation einhalten. Flankierende Massnahmen wie etwa Vorgaben über Kartelle, den Missbrauch einer marktbeherrschenden Stellung und staatliche Beihilfen schützen den Wettbewerb und die Fluggäste. 2002 wurde die Europäische Agentur für Flugsicherheit gegründet (EASA). Seit 2004 ist der europäische Gesetzgeber zudem bestrebt, einen einheitlichen europäischen Luftraum (Single European Sky, SES) zu schaffen. Damit soll die Effizienz des Flugverkehrsmanagements und der Flugsicherungsdienste erhöht werden.

1999 schlossen die EG und die Schweiz das Abkommen über den Luftverkehr (Luftverkehrsabkommen, LVA) ab. Es wurde 2002 in Kraft gesetzt und ermöglicht der Schweiz die Teilnahme am europäischen Luftverkehrsbinnenmarkt. Das Abkommen wird als «partiellen Integrationsvertrag» bezeichnet (Botschaft Bilaterale I, S. 6156), weil sich die Schweiz damit zur weitreichenden Übernahme des Acquis communautaire verpflichtet hat und die Durchführung des Abkommens und die Gewährung von Rechtsschutz teils durch die Organe der EU wahrgenommen werden (N. 75). Das Abkommen enthält kartell- und beihilferechtliche Bestimmungen (N. 219, N. 221, N. 222, N. 225).

210 Das Abkommen gewährt den Staatsangehörigen eines EU-Mitgliedstaats oder der Schweiz sowie nach dem Recht eines EU-Mitgliedstaats oder der Schweiz gegründeten Gesellschaften, die ihren satzungsmässigen Sitz, ihre Hauptverwaltung oder ihre Hauptniederlassung in der EU oder in der Schweiz haben, die primäre und sekundäre Niederlassungsfreiheit (Art.4). Die Niederlassungsfreiheit umfasst das Recht zur Aufnahme und Ausübung selbstständiger Erwerbstätigkeiten sowie zur Gründung und Leitung von Unternehmungen, Agenturen und Zweigniederlassungen. Vorbehalten bleibt die Verordnung (EWG) Nr. 2407/92 über die Erteilung von Betriebsgenehmigungen an Luftfahrtunternehmen (Art. 4 und Art. 5; gemäss Ingress zum Anhang sind die Ver- 
weise auf die Verordnung (EWG) Nr. 2407/92 mittlerweile als Verweise auf die Verordnung (EG) Nr. 1008/2008 zu verstehen). Der EuGH hat festgestellt, dass das Abkommen keine spezielle Bestimmung enthält, die darauf abzielt, die betroffenen Luftfahrtunternehmen in den Genuss der unionsrechtlichen Vorschriften über die Dienstleistungsfreiheit kommen zu lassen (Urteil Fluglärmstreit, C-547/10 P, EU:C:2013:139, Rn. 81; N. 81 und N. 214).

Das Abkommen gewährleistet Luftfahrtunternehmen der EU und der Schweiz, welche über eine Genehmigung nach Massgabe der Verordnung (EWG) Nr. 2407/92 über die Erteilung von Betriebsgenehmigungen an Luftfahrtunternehmen verfügen, vorbehältlich der Verordnung (EWG) Nr. 2408/92 über den Zugang von Luftfahrtunternehmen der Gemeinschaft zu Strecken des innergemeinschaftlichen Flugverkehrs, Verkehrsrechte (Art. 15; gemäss Ingress zum Anhang sind die Verweise auf die Verordnungen (EWG) Nr. 2407/ 92 und (EWG) Nr. 2408/92 als Verweise auf die Verordnung (EG) Nr. 1008/ 2008 zu verstehen; s. zur Weitergeltung von Luftverkehrsabkommen zwischen EU-Mitgliedstaaten und der Schweiz Art. 16; R. DetTLing-OtT, Rz. 74-76):

- Verkehrsrechte zwischen jedem Punkt in der EU und jedem Punkt in der Schweiz (3. und 4. Freiheit);

- Verkehrsrechte für schweizerische Luftfahrtunternehmen zwischen Punkten in verschiedenen EU-Mitgliedstaaten (7. Freiheit);

- Überflugrechte und Zwischenlandungen zu nichtkommerziellen Zwecken sind im Abkommen nicht erwähnt, werden aber stillschweigend vorausgesetzt (1. und 2. Freiheit; R. DETTLING-OTT, Rz. 73);

- Inlandflüge in einem EU-Mitgliedstaat und in der Schweiz werden nicht erfasst; die Vertragsparteien haben sich zwar darauf geeinigt, der Abschluss steht aber noch aus (8. und 9. Freiheit; www.eda.admin.ch/dea und Link zu Bilaterale Abkommen I (1999)/Luftverkehr).

Die Schweiz ist verpflichtet, eine Vielzahl von EU-Rechtsakten zu beachten, auf welche im Abkommen selbst oder in dessen Anhang verwiesen wird. Prominente Beispiele betreffen die Fluggastrechteverordnung (EG) Nr. 261/2004 (s. zur Auslegung dieser Verordnung N. 87), die Verordnung (EU) 2018/1139 zur Festlegung gemeinsamer Vorschriften für die Zivilluftfahrt und zur Errichtung einer Europäischen Agentur für Flugsicherheit, die Verordnung (EG) Nr. 300/ 2008 über gemeinsame Vorschriften für die Sicherheit in der Zivilluftfahrt und die Verordnung (EG) Nr. 1008/2008 über gemeinsame Vorschriften für die Durchführung von Luftverkehrsdiensten in der Gemeinschaft. Diese Rechtsakte sind auch in der Schweiz unmittelbar anwendbar. 
In einem Urteil von 2012 bejahte das BGer die unmittelbare Anwendbarkeit der Anhänge der Verordnung (EWG) Nr. 3922/91 zur Harmonisierung der technischen Vorschriften und der Verwaltungsverfahren in der Zivilluftfahrt. Es kam zum Schluss, dass diese Anhänge die in casu strittigen Anforderungen, denen ein «Postholder Flight Operations» genügen muss, mit hinreichender Klarheit umschreiben. Dabei hielt das Bundesgericht auch fest, dass diese Vorgaben eine gesetzliche Grundlage für einen Grundrechtseingriff (Art. 36 BV) - in casu die Wirtschaftsfreiheit (Art. 27 BV) - darstellen können (BGE 138 II 42, E. 3.).

In der EU wurden mehrere Vorabentscheidungsersuchen, welche die Auslegung der qua Verweis im LVA bilateral einschlägigen Verordnung (EG) Nr. 261/2004 (Fluggastrechteverordnung) betrafen, wieder zurückgezogen und vom EuGH im Register gestrichen (s. Beschluss Recinto-Pfingsten, C-259/13, EU:C:2014:236; Beschluss Stück, C-3/15, EU:C:2015:595; Beschluss Kammerer, C-172/16, EU:C:2016:625). Die Parteien einigten sich vermutungsweise aussergerichtlich.

213 Bei der Auslegung des Abkommens und der in den Anhängen referenzierten Rechtsakte gilt für die schweizerischen Behörden ein Beachtungsgebot hinsichtlich der Praxis in der EU (N. 84 und N. 87). Die Anhänge müssen bei Weiterentwicklungen des EU-Rechts aufdatiert werden, um das gute Funktionieren des Abkommens nicht zu gefährden, was routinemässig geschieht. Das geplante InstA wäre auf das Luftverkehrsabkommen anwendbar (s. für den Textentwurf des InstA vom Dezember 2018 N. 105-116).

Das Abkommen verbietet jegliche Diskriminierung auf Grund der Staatsangehörigkeit (Art. 3). Ungleichbehandlungen können aus Gründen der öffentlichen Ordnung, Sicherheit oder Gesundheit gerechtfertigt werden (Art. 7). Hinzu kommen die Vorgaben der einschlägigen EU-Rechtsakte (C. TOBLER/ J. BEGLINGER, Grundzüge, Rz. 309).

Im Fluglärmstreit erklärten das EuG und der EuGH die von Deutschland verfügten Beschränkungen von An- und Abflügen zum und vom Flughafen Zürich mit dem Ziel, im deutsch-schweizerischen Grenzgebiet die Lärmbelastung zu verringern, als zulässig. Dabei liessen das EuG und der EuGH die Frage offen, ob die Schweiz überhaupt zur Einreichung einer Nichtigkeitsklage berechtigt war. Generalanwalt Niilo Jääskinen bejahte die Klagebefugnis der Schweiz gestützt auf Art. 263 Abs. 4 AEUV, während er eine Klagebefugnis gestützt auf Art. 263 Abs. 2 AEUV verneinte, weil der Umstand, dass die Schweiz Vertragspartei des LVA ist, nicht ausreiche, um sie einem Mitgliedstaat gleichzustellen (Schlussanträge Fluglärmstreit, C-547/10 P, EU:C:2012:565, Rn. 55-83). Das EuG stellte fest, dass die deutsche Massnahme zu einer indirekten Schlechterbehandlung der Swiss International Air Lines führte; diese Schlechterbehandlung konnte durch objektive Umstände gerechtfertigt werden und stand auch in einem angemessenen Verhältnis zu dem mit der Massnahme verfolgten Ziel des Lärmschutzes. Das Verbot der Diskriminierung von Luftfahrtunternehmen auf Grund der Staatsangehörigkeit gemäss Art. 3 LVA war folglich nicht verletzt. Der EuGH wies die Beschwerde der Schweiz gegen das Urteil des EuG ab. Er kam zum Schluss, dass die unionsrechtlich gewährleistete Dienstleistungsfreiheit (Art. 56 AEUV) nicht unbesehen auf das LVA übertragen werden kann, ohne dass dieses Abkommen ausdrücklich eine entsprechende Bestimmung enthält. Dies war weder im Abkom- 
men selbst noch in der darin referenzierten Verordnung Nr. 2408/92 der Fall. Der EuGH folgerte, dass die Dienstleistungsfreiheit und damit auch die entsprechende Praxis des EuGH in casu nicht einschlägig waren (Urteil Fluglärmstreit, C-547/10 P, EU:C:2013:139, s. dazu auch N. 81; L.M. BAUDENBACHER, Luftverkehrsabkommen, passim; M. OESCH/G. SPECK, S. 269-270; C. TOBLER, Luftverkehrsrecht, passim).

Das Abkommen stellt die Grundlage dar für die Beteiligung der Schweiz an der Europäischen Agentur für Flugsicherheit (EASA; Verordnung (EU) 2018/1139 zur Festlegung gemeinsamer Vorschriften für die Zivilluftfahrt und zur Errichtung einer Europäischen Agentur für Flugsicherheit). Diese Agentur wurde 2002 gegründet; ihr Sitz befindet sich in Köln. Sie ist für die Sicherheit in der europäischen Luftfahrt (Zulassung und Aufsicht im technischen Betrieb, Flugausbildung und Flugbetrieb) zuständig und unterstützt die Europäische Kommission bei der Vorbereitung von Massnahmen (insbesondere zur Durchführung von Verordnungen). Die EASA nimmt die ihr zugewiesenen Aufgaben wie Musterzulassungen von Luftfahrzeugen, Überwachung der Marktteilnehmerinnen und Marktteilnehmer, Qualifikation von Pilotinnen und Piloten und von Fachpersonal - auch gegenüber der Schweiz wahr.

Der Beitrag der Schweiz an die EASA belief sich 2014-2019 auf 9 Mio. CHF (Stellungnahme des Bundesrates vom 20. November 2019 auf die Interpellation 19.4008 «Aktualisierte Zusammenstellung aller Zahlungen und Beiträge der Schweiz an die EU»).

Im Jahr 2005 errichtete die EU das Emissionshandelssystem (EU-EHS). Dabei handelt es sich um ein zentrales Instrument der EU, um die Treibhausgasemissionen der Industrie kostenwirksam zu verringern und den Klimawandel zu bekämpfen. Dieses System hat sich als weltweit grössten Markt für Emissionsrechte etabliert (www.eda.admin.ch/dea und Link zu Bilaterale Abkommen und Kooperationsabkommen ab 2004/Emissionshandel). Die Schweiz kennt seit 2013 ein Emissionshandelssystem (www.bafu.admin.ch und Link zu Klima/ Emissionshandel). 2017 entschieden die EU und die Schweiz, ihre Emissionshandelssysteme für $\mathrm{CO}_{2}$-Emissionsrechte zu verknüpfen, und unterzeichneten zu diesem Zweck das Abkommen zur Verknüpfung der jeweiligen Systeme für den Handel mit Treibhausgasemissionen. Dieses Abkommen trat 2020 in Kraft. Da das Emissionshandelssystem der EU auch den Luftverkehrssektor umfasst, hat sich die Schweiz verpflichtet, den Luftverkehrssektor ebenfalls in das Emissionshandelssystem $\mathrm{zu}$ integrieren. Das Emissionshandelssystem funktioniert nach dem Prinzip von «cap and trade». Am Schweizer Emissionshandelssystem teilnehmende Unternehmen erhalten eine gewisse Anzahl an Emissionsrechten gratis zugeteilt. Wenn ein Unternehmen pro Jahr mehr $\mathrm{CO}_{2}$ ausstösst als es Rechte besitzt, muss es die fehlenden Emissionsrechte auf dem Markt kaufen. Stösst es demgegenüber weniger $\mathrm{CO}_{2}$ aus, kann es die 
überzähligen Emissionsrechte verkaufen («trade»). Es ist vorgesehen, den Gesamtbestand an Emissionsrechten jährlich zu verringern («cap»). Das Abkommen regelt zudem die Anrechnung von Beiträgen zur Emissionsreduktion im Ausland (www.eda.admin.ch/dea und Link zu Bilaterale Abkommen und Kooperationsabkommen ab 2004/Emissionshandel).

\section{E. Wettbewerbsrecht}

217 Die bis anhin vorgestellten Abkommen thematisieren vorwiegend Marktzugangsfragen in einem klassischen Sinne. Sie bezwecken, staatliche Marktzugangshürden für den Waren- und Dienstleistungshandel abzubauen (z.B. mengenmässige Beschränkungen, Zölle, technische Handelshemmnisse, interne Vorschriften) und die Personenfreizügigkeit zu fördern (z.B. Dienstleistungsund Niederlassungsfreiheit, Familiennachzug, Anerkennung von Berufsqualifikationen). In Ergänzung dazu besteht eine Vielzahl von weiteren staatlichen und privaten Praktiken, welche den Handel zwischen der EU und der Schweiz mutmasslich behindern. Dazu gehören Verhaltensweisen privater Unternehmen, staatliche Beihilfen und das öffentliche Beschaffungswesen. Massnahmen, welche verpönte Verhaltensweisen privater Unternehmen und protektionistische Praktiken des Gemeinwesens disziplinieren, werden hinlänglich unter dem Titel des Wettbewerbsrechts zusammengefasst. Auch der bilaterale Acquis Schweiz-EU enthält punktuell wettbewerbsrechtliche Vorgaben (s. zum Ganzen C. TOBLER, Wirtschaftsrecht, passim). Einzelne Abkommen enthalten Bestimmungen zum Verhalten privater Unternehmen und zu staatlichen Beihilfen. Das Abkommen über bestimmte Aspekte des öffentlichen Beschaffungswesens und das Abkommen über die Zusammenarbeit bei der Anwendung des Wettbewerbsrechts der EU und der Schweiz machen Vorgaben zu den titelgebenden Sachbereichen.

218 Diese bilateralen Regeln werden selektiv durch multilaterale Regelwerke ergänzt, welche auch für die EU und die Schweiz verbindlich sind. Im Vordergrund steht das WTO-Recht. In Bezug auf staatliche Beihilfen disziplinieren Art. II:8(b) und Art. XVI GATT sowie das WTO-Übereinkommen über Subventionen und Ausgleichsmassnahmen die Gewährung von Beihilfen durch die EU und die Schweiz; diese Vorgaben verbieten gewisse Kategorien staatlicher Beihilfen (z.B. Exportsubventionen); sie ermächtigen WTO-Mitglieder zum Erlass von Ausgleichsmassnahmen gegen Beihilfen, sofern dadurch die einheimische Industrie geschädigt wird (s. zum Ganzen M. OEsCH, Subventionen, S. 258-262). Das Abkommen über bestimmte Aspekte des öffentlichen Beschaffungswesens erweitert den Anwendungsbereich des WTO-Übereinkommens 
über das öffentliche Beschaffungswesen im Verhältnis zwischen der EU und der Schweiz; dieses Übereinkommen sieht für Beschaffungen durch die öffentliche Hand über einem gewissen Schwellenwert eine öffentliche Vergabe vor (Transparenz- und Nichtdiskriminierungsgebot, Verfahrensgarantien). Demgegenüber enthält das WTO-Recht keine Regeln über die Zulässigkeit von Kartellen und anderen Wettbewerbsbeschränkungen durch Private; das Vorhaben, im Rahmen der WTO umfassende Wettbewerbsregeln einzuführen, wurde - trotz einer gegenteiligen Absichtserklärung anlässlich der WTOMinisterkonferenz in Singapur 1996 (WT/MIN/96/DEC, para. 20) - bis heute nicht weiterverfolgt.

\section{Kartellrechtliche Bestimmungen}

Das Freihandelsabkommen (Art. 23 Abs. 1 lit. i und ii) und das Luftverkehrsabkommen (Art. 8 und Art. 9) enthalten kartellrechtliche Bestimmungen, die sich an private Unternehmen richten. Demnach sind wettbewerbsverhindernde, -einschränkende oder -verfälschende Vereinbarungen und aufeinander abgestimmte Verhaltensweisen sowie die missbräuchliche Ausnutzung einer marktbeherrschenden Stellung mit dem guten Funktionieren der Abkommen unvereinbar, soweit sie geeignet sind, den Handel zwischen der EU und der Schweiz zu beeinträchtigen. Diese Bestimmungen ähneln dem Wortlaut der kartellrechtlichen Bestimmungen im EWGV (Art. 85, Art. 86 und Art. 90) bzw. AEUV (Art. 101, Art. 102 und Art. 106); die EWG erklärte beim Abschluss des Freihandelsabkommens, sie würde die kartellrechtlichen Bestimmungen des Freihandelsabkommens auf der Grundlage der Kriterien beurteilen, welche sich aus der Anwendung der Bestimmungen des EWGV ergeben (N. 89). Im Geltungsbereich des Luftverkehrsabkommens sind qua Verweis im Anhang (2. Wettbewerbsregeln) zudem eine Vielzahl von EU-Rechtsakten anwendbar; dazu gehören auch die Verordnung (EG) Nr. 487/2009 (Freistellung bestimmter Vereinbarungen im Luftverkehr) und die Verordnung (EG) Nr. 139/2004 (Fusionskontrollverordnung).

Unternehmen in der Schweiz bzw. EU sind zudem verpflichtet, das unionale bzw. schweizerische Wettbewerbsrecht zu beachten, sofern sich ihre Praktiken in der EU bzw. Schweiz auswirken, auch wenn sie im Ausland veranlasst wurden. Das Auswirkungsprinzip wurde in der EU wesentlich durch den EuGH entwickelt (s. zum räumlichen Geltungsbereich des unionalen Kartellrechts F. SCHUHMACHER, Art. 101 Rz. 31-39). In der Schweiz ist es mittlerweile ausdrücklich in Art. 2 Abs. 2 KG verankert (vgl. dazu BGE 127 III 219, E. 3; T. CoTTIER et al., Rz. 692-697).

Die kartellrechtlichen Bestimmungen des Freihandelsabkommens sind in der

Praxis bis heute selten getestet worden. Ein Grund dafür ist zumindest mit 
Blick auf die Schweiz, dass das Bundesgericht diese Bestimmungen als nicht unmittelbar anwendbar qualifiziert (BGE 104 IV 175, E. 2c; s. zur Kritik dazu B. MERKT, S. 289). Sofern eine Vertragspartei der Meinung ist, eine bestimmte Praktik sei mit Art. 23 Abs. 1 FHA nicht vereinbar, kann sie den Gemischten Ausschuss befassen und - sofern keine gütliche Einigung erzielt wird Schutzmassnahmen ergreifen (Art. 27 FHA).

Die Europäische Kommission und die (damalige) schweizerische Kartellkommission untersuchten in der zweiten Hälfte der 1990er-Jahre kartellrechtlich problematische Vereinbarungen der Volkswagen AG mit ihren Händlern in Italien zur Verhinderung von Direkt- und Parallelimporten von Autos der Marke VW und Audi in andere europäische Staaten (s. für die Haltung der Kommission, wonach Volkswagen AG Zuwiderhandlungen gegen Art. 85 EGV beging, Entscheid vom 28. Januar 1998, 1998/L 124/60). Die Schweiz stellte sich im Gemischten Ausschuss auf den Standpunkt, dass das Verhalten der Volkswagen AG auch gegen Art. 23 Abs. 1 lit. i FHA verstiess. Der Gemischte Ausschuss verzichtete allerdings darauf, eine Entscheidung zu fällen (C. TOBLER/J. BEGLINGER, Grundzüge, Rz. 380; B. MERKT, S. 281). bis anhin einige Male im Zusammenhang mit Handlungen der Lufthansa AG/ Swiss International Air Lines AG angewendet. Abweichend vom Grundsatz, wonach die Vertragsparteien für die Durchführung der Abkommen zuständig sind, obliegt die Durchführung der kartellrechtlichen Bestimmungen des Luftverkehrsabkommens auch vis-à-vis der Schweiz der Europäischen Kommission (Art. 11); der Gerichtshof der EU ist für die Gewährung des Rechtsschutzes zuständig (Art. 20).

2005 genehmigte die Europäische Kommission die Übernahme der Swiss International Air Lines AG durch die Deutsche Lufthansa AG auf der Grundlage der Fusionskontrollverordnung und Art. 11 LVA (Entscheidung vom 4. Juli 2005, COMP/M.3770). 2010 stellte die Europäische Kommission Zuwiderhandlungen diverser Flugunternehmen - u.a. der Swiss International Air Lines AG und der Deutschen Lufthansa AG - gegen Art. 101 AEUV und Art. 8 LVA fest wegen unrechtmässiger Absprachen über das Preisverhalten bei Luftfrachtflügen (Beschluss vom 9. November 2010, 2014/C 371/09).

\section{Beihilferechtliche Bestimmungen}

Das Freihandelsabkommen (Art. 23 Abs. 1 lit. iii) und das Luftverkehrsabkommen (Art. 13) enthalten Bestimmungen zu staatlichen Beihilfen (s. zum Ganzen J.-F. MAYORAZ, passim). Demnach sind mit dem guten Funktionieren der Abkommen staatliche Beihilfen, die den Wettbewerb durch Begünstigung bestimmter Unternehmen oder Produktionszweige verfälschen oder zu verfälschen drohen, unvereinbar, soweit sie geeignet sind, den Handel zwischen der EU und der Schweiz zu beeinträchtigen. Das Luftverkehrsabkommen enthält 
zudem eine Liste von Beihilfen, welche mit dem Abkommen vereinbar sind. Diese Bestimmungen sind praktisch gleich formuliert wie die beihilferechtlichen Bestimmungen im EWGV (Art. 92) bzw. AEUV (Art. 107 AEUV); die EWG erklärte beim Abschluss des Freihandelsabkommens, sie würde die beihilferechtlichen Bestimmungen des Freihandelsabkommens auf der Grundlage der Kriterien beurteilen, welche sich aus der Anwendung der Bestimmungen des EWGV ergeben (N. 89).

Art. 23 Abs. 1 lit. iii FHA enthält keine Ausnahmetatbestände. Das dürfte auf ein Missverständnis zurückzuführen sein und nicht auf einem bewussten Entscheid, im bilateralen Verhältnis Beihilfen ausnahmslos zu verbieten. Die im EU-Recht einschlägigen Ausnahmen dürften mutatis mutandis auch bilateral greifen, wobei die Schweiz seit jeher darauf beharrt, die Beihilferegelung im FHA autonom auszulegen und nicht nach Massgabe der Praxis in der EU. Dies gilt auch mit Blick auf die Zulässigkeit der präzedenzlos weitreichenden Massnahmen zur Stützung und Revitalisierung der Wirtschaft im Zug der durch das Coronavirus (COVID-19) ausgelösten Krise (wenngleich sich dabei selbstredend auch ein Seitenblick auf das unionale Recht anbietet; s. etwa Mitteilung der Kommission, Befristeter Rahmen für staatliche Beihilfen zur Stützung der Wirtschaft angesichts des derzeitigen Ausbruchs von COVID-19, C(2020) 1863 final).

Die beihilferechtlichen Bestimmungen des Freihandelsabkommens haben in der Praxis nur vereinzelt eine Rolle gespielt. Sie richten sich primär an die Behörden der Schweiz und der EU und ihrer Mitgliedstaaten. Ungeklärt ist, ob und, sofern dies der Fall ist, unter welchen (restriktiven) Voraussetzungen Einzelne sich unmittelbar darauf berufen können.

Der bekannteste Fall, in dem Art. 23 Abs. 1 lit. iii FHA zur Debatte stand, war der Steuerstreit zwischen der EU und der Schweiz. Gestützt auf eine betont binnenmarktrechtlich orientierte Auslegung dieser Bestimmung kam die Europäische Kommission 2007 zum Schluss, dass kantonale Steuerprivilegien für im Ausland tätige Unternehmen mit Sitz in der Schweiz mit dem bilateralen Beihilfeverbot nicht vereinbar waren (Entscheidung vom 13. Februar 2007, C (2007) 411). 2014 unterzeichneten die EU und die Schweiz ein Memorandum of Understanding, um diese Auseinandersetzung gütlich beizulegen. Der Bundesrat erklärte sich bereit, sich für die Abschaffung von Steuerregimes, welche eine unterschiedliche Behandlung in- und ausländischer Erträge vorsehen, einzusetzen. Die EU-Mitgliedstaaten bekräftigten ihre Absicht, gegen die Schweiz ergriffene Gegenmassnahmen aufzuheben. Die Auseinandersetzung fand mit der Annahme des Bundesgesetzes über die Steuerreform und die AHV-Finanzierung (STAF) durch die Stimmbürgerinnen und Stimmbürger im Mai 2019 zumindest auf Bundesebene ihren Abschluss (s. zur rechtlichen Einschätzung der unterschiedlichen Positionen der Schweiz und der EU S. ARIOLI, Tragweite, passim; T. Cottier et al., Rz. 737-742; T. CotTier/R. MAtTeOtTI, passim; A. EPiney, Steuern, passim).

Die Eidgenössische Rekurskommission für Infrastruktur und Umwelt qualifizierte in einem Entscheid von 2005 den Vorzugstarif für die Beförderung von Presseerzeugnissen, welche im Inland gedruckt wurden, von denen im Ausland gedruckte Presseerzeugnisse aber nicht profitieren konnten, als staatliche Beihilfe gemäss Art. 23 FHA und bestätigte gestützt auf 
eine völkerrechtskonforme Auslegung von Art. 15 des Postgesetzes (in der damaligen Fassung) die Unrechtmässigkeit der unterschiedlichen Behandlung (Entscheid vom 20. Oktober 2005, H-2004-174, E. 13.6-13.8).

In Frankreich qualifizierte das Tribunal de Commerce de Lyon in einem Entscheid von 2016 finanzielle Unterstützungsleistungen der Schweizerischen Post AG an die CarPostal France SAS (eine Tochtergesellschaft der Schweizerischen Post AG) und eine Tochtergesellschaft der CarPostal France SAS als staatliche Beihilfen i.S.v. Art. 23 FHA. Mithilfe dieser Beihilfen waren diese Gesellschaften in der Lage, künstlich tiefe Angebote für öffentliche Aufträge auf dem Markt des öffentlichen Verkehrs einzureichen, und verursachten eine unlautere Wettbewerbsverzerrung. Das Gericht verurteilte die CarPostal France SAS und deren Tochtergesellschaft wegen des Verstosses gegen das FHA und weiterer Verstösse gegen wettbewerbsrechtliche Vorgaben im französischen Recht zur Bezahlung von Schadenersatz an ihre Konkurrenzunternehmen in der Höhe von 10,6 Mio. EUR (Jugement du 23 septembre 2016, Rôle $n^{\circ}$ 2014J00357). Die CarPostal France SAS und deren Tochtergesellschaft legten in der Folge Beschwerde ein. Vor dem Entscheid des Appellationsgerichts einigten sich die Parteien aussergerichtlich (Niederer Kraft Frey Client News vom 22. April 2020, www.nkf.ch).

224 Das geplante Institutionelle Abkommen enthält eine Gemeinsame Erklärung, worin die Vertragsparteien ihre Absicht kundtun, Verhandlungen über die Modernisierung des Freihandelsabkommens einzuleiten; darin werden auch die Regeln betreffend die staatlichen Beihilfen erwähnt. Weiter enthält ein dem InstA beigefügter Entwurf für einen Beschluss des Gemischten Ausschusses nach Art. 29 FHA in der Präambel eine - stark auslegungsbedürftige Anleitung zur Auslegung von Art. 23 Abs. 1 lit. iii FHA (N. 107).

Das Luftverkehrsabkommen verpflichtet die Vertragsparteien, die in den EUMitgliedstaaten und der Schweiz bestehenden und geplanten Beihilferegelungen fortlaufend zu prüfen (Art.14). In der EU übernimmt die Europäische Kommission diese Aufgabe. In der Schweiz wurde diese Aufgabe der Wettbewerbskommission (WEKO) übertragen. Sie überprüft, ob geplante Beihilfen durch den Bundesrat, die Kantone, Gemeinden oder andere öffentlich-rechtliche Körperschaften und Anstalten mit dem Luftverkehrsabkommen vereinbar sind; die für die Beihilfengewährung zuständigen Behörden berücksichtigen das Ergebnis der Prüfung der WEKO, sind aber nicht daran gebunden (Art. 103 LFG).

Die WEKO war bis anhin zwei Mal aufgerufen, als Beihilfeaufsichtsbehörde nach Massgabe von Art. 14 LVA i.V.m. Art. 103 LFG zu amten. 2004 kam sie zum Schluss, dass bei einem staatlich unterstützten Projekt zur Wiederinbetriebnahme des früheren Terminals T2 des Flughafens Genf keine staatliche Beihilfe i.S.v. Art. 13 LVA vorlag (RPW 2004/4, S. 1300). 2018 prüfte die WEKO einen Entlastungsmechanismus zugunsten der SWISS im Zusammenhang mit dem Erlass eines neuen Lärmgebührenmodells für Luftfahrzeuge am Flughafen Zürich; sie qualifizierte diesen Mechanismus nicht als staatliche Beihilfe gemäss Art. 13 LVA (RPW 2019/3b, S. 1040; Verfügung des BAZL vom 5. Juni 2019, Rz. 112-115). 
Das geplante Institutionelle Abkommen sieht vor, dass die darin formulierten beihilferechtlichen Grundsätze und Verfahren auf das Luftverkehrsabkommen anwendbar sind. Ein Anhang listet zudem unionale Rechtsakte und Mitteilungen auf, welche bei der Auslegung des Begriffs der Beihilfe im Luftfahrtsektor zu beachten sind (N. 112).

Das Landverkehrsabkommen enthält zwei Bestimmungen zu staatlichen Beihilfen. Die Vertragsparteien können zur Schaffung eines angepassten Angebots im Schienen- und kombinierten Verkehr Investitionen in die Eisenbahninfrastruktur, die festen und beweglichen Umschlageinrichtungen zwischen Landverkehrsträgern, das im kombinierten Verkehr eingesetzte Material und die Betriebskosten der kombinierten Verkehrsdienste im Transit durch die Schweiz finanziell unterstützen, sofern diese Massnahmen die Qualität und die Wettbewerbsfähigkeit des Preisangebots im Schienen- und kombinierten Verkehr verbessern und keine unverhältnismässigen Wettbewerbsverzerrungen zwischen den Betreibern verursachen (Art. 35 Abs. 2). Die Vertragsparteien gewähren Unternehmen, insbesondere Verkehrsunternehmen, keine direkten oder indirekten staatlichen Beihilfen mit dem Ziel, die Auswirkungen zu mildern, die sich für die Unternehmen dadurch ergeben, dass die Kosten, welche die in diesem Abkommen vorgesehenen Gebühren verursachen, der Beförderung angelastet werden (Art. 38 Abs. 6).

\section{3. Öffentliches Beschaffungswesen}

Die EG und die Schweiz einigten sich im Nachgang zur Uruguay Runde (1986-1994) darauf, bilateral über eine Ausweitung des Geltungsbereichs des WTO-Übereinkommens über das öffentliche Beschaffungswesen (GPA) zu verhandeln. Diese Verhandlungen führten 1999 zur Unterzeichnung des Abkommens über bestimmte Aspekte des öffentlichen Beschaffungswesens. Es trat 2002 in Kraft.

Gemäss Schätzungen der Europäischen Kommission weist der Beschaffungsmarkt der öffentlichen Hand in der EU jährlich ein Volumen von rund 2400 Mia. EUR auf. Zu diesem Markt haben nach Massgabe des WTO-Übereinkommens und des bilateralen Abkommens auch schweizerische Unternehmen Zugang (www.eda.admin.ch/dea und Link zu Bilaterale Abkommen I (1999)/Öffentliches Beschaffungswesen).

Dieses Abkommen bewirkt eine mit dem EWR-Abkommen vergleichbare gegenseitige Marktöffnung zwischen den EU-Mitgliedstaaten und der Schweiz. Es dehnt den Anwendungsbereich des WTO-Übereinkommens auf die schweizerischen Gemeinden und Bezirke aus, lässt einzelne im Rahmen des WTO-Übereinkommens angebrachte Vorbehalte der EU gegenüber der 
Schweiz fallen und unterstellt gewisse öffentliche und private Vergabestellen in den Sektoren Telekommunikation, Wasser-, Energie- und Verkehrsversorgung der Ausschreibungspflicht. Eine Ausklinkklausel stellt sicher, dass solche Vergabestellen nicht mehr der Ausschreibungspflicht unterstehen, sofern in dem Sektor, in dem sie tätig sind, nachweislich Wettbewerb herrscht (Art. 3 Abs. 5).

Zurzeit sind die Sektoren Telekommunikation und Güterverkehr auf Normalspur vom Geltungsbereich des Abkommens ausgenommen (www.eda.admin.ch/dea und Link zu Bilaterale Abkommen I (1999)/Öffentliches Beschaffungswesen).

230 Die einschlägigen Schwellenwerte werden im WTO-Übereinkommen und - für die erwähnten öffentlichen und privaten Vergabestellen, die ebenfalls ausschreibungspflichtig sind - in Art. 3 Abs. 4 umschrieben.

231 Das Abkommen verpflichtet die Vertragsparteien, darauf zu achten, dass die von ihren Vergabestellen angewandten Verfahren und Praktiken der Auftragsvergabe den Grundsätzen der Nichtdiskriminierung, der Transparenz und der Gleichbehandlung entsprechen (Art. 4 und Art. 6). Das für die Auftragsvergabe massgebende Kriterium ist das wirtschaftlich günstigste Angebot unter Berücksichtigung besonderer Wertungskriterien (wie Lieferfrist, Ausführungsdauer, Preis-Leistungs-Verhältnis, Qualität, technischer Wert, Kundendienst und Verpflichtungen hinsichtlich Ersatzteile) oder ausschliesslich der niedrigste Preis (Art. 4 Abs. 1 lit. e). Die Vertragsparteien sind verpflichtet, nichtdiskriminierende, rasch greifende, transparente und wirksame Verfahren einzurichten, damit Lieferantinnen und Lieferanten oder Dienstleistungserbringerinnen und Dienstleistungserbringer gegen angebliche Verletzungen des Abkommens bei Beschaffungen, an denen sie ein Interesse haben oder hatten, Beschwerde erheben können (Art. 5, Anhang V). Das Prinzip der Nichtdiskriminierung gilt auch in Verfahren zur Vergabe von Aufträgen, deren Auftragswert unter den einschlägigen Schwellenwerten liegt (Art. 6 Abs. 3); diesfalls sieht das Abkommen allerdings keine Möglichkeit vor, die Vergabe an einen Konkurrenten anzufechten.

232 Uneinigkeiten zwischen den Vertragsparteien werden im Gemischten Ausschuss erledigt (N. 90). Die Durchführung des Abkommens wird in jeder Vertragspartei zudem von einer unabhängigen Überwachungsbehörde kontrolliert (Art. 8). In der Schweiz wurde zu diesem Zweck die Kommission für das Beschaffungswesen Bund-Kantone (KBBK) geschaffen (Art. 68a-68d der Verordnung über das öffentliche Beschaffungswesen vom 11. Dezember 1995, SR 172.056.11). In der EU übernimmt die Europäische Kommission diese Aufgabe. 
Die Europäische Kommission forderte Italien 2011 auf, die Verpflichtungen gegenüber der Schweiz im Bereich des öffentlichen Beschaffungswesens zu respektieren, nachdem bekannt geworden war, dass Schweizer Firmen bei der Vergabe von öffentlichen Aufträgen wiederholt benachteiligt wurden. Im Anschluss daran beschloss der italienische Wirtschafts- und Finanzminister die Aufhebung der umstrittenen Praktiken gegen Schweizer Unternehmen (Stellungnahme des Bundesrates vom 18. Mai 2011 zur Motion 11.3157 «Beziehungen zwischen der Schweiz und Italien. Wogen glätten»).

Das Abkommen lässt die Rechte und Pflichten gemäss WTO-Übereinkommen unberührt, womit bei Streitigkeiten im Anwendungsbereich des Kapitels I (Erweiterung des Geltungsbereichs des WTO-Übereinkommens) das WTOStreitbeilegungsverfahren zur Verfügung steht. Das Abkommen sieht ausdrücklich vor, dass die Rechte und Pflichten, die sich für die Vertragsparteien aus den im Rahmen der WTO geschlossenen Übereinkommen ergeben, vom Abkommen unberührt bleiben (Art. 15).

Das Abkommen geht von der Gleichwertigkeit der Rechtssysteme der EU und der Schweiz aus (Botschaft Bilaterale I, S. 6147). Es verweist nicht auf EURechtsakte, womit keine Notwendigkeit besteht, das Abkommen periodisch an das sich weiterentwickelnde EU-Recht anzupassen.

\section{Zusammenarbeit der Wettbewerbsbehörden}

Die Zusammenarbeit zwischen der Schweiz einerseits und der EU und ihren 235 Mitgliedstaaten andererseits bei der Durchsetzung ihres Wettbewerbsrechts bei Abreden von Unternehmen, bei Missbräuchen marktbeherrschender Stellungen und bei Unternehmenszusammenschlüssen ist seit jeher von grosser Bedeutung. Bis vor einigen Jahren war eine solche Zusammenarbeit - mit Ausnahme der Zusammenarbeit gestützt auf das Luftverkehrsabkommen (Art. 19 LVA, Art. $42 a \mathrm{KG}$ ) - allein informell möglich. Sie erfolgte aufgrund von nichtverbindlichen OECD-Empfehlungen über die Zusammenarbeit im Wettbewerbsbereich (Recommendation of the OECD Council Concerning Co-operation between Member Countries on Anticompetitive Practices Affecting International Trade, 1986/1995; Recommendation on Effective Action against Hard-Core Cartels, 1998) sowie im Rahmen des International Competition Network (ICN) und des OECD-Wettbewerbsausschusses (T. COTTIER et al., Rz. 714).

Die EU und die Schweiz schlossen 2013 das Abkommen über die Zusammenarbeit bei der Anwendung ihres Wettbewerbsrechts (Wettbewerbsabkommen, WettbA), um diese Lücke zu schliessen. Es trat 2014 in Kraft und bezweckt, durch Zusammenarbeit und Koordinierung einschliesslich des Informationsaustauschs zwischen den Wettbewerbsbehörden der Vertragsparteien zur wirksamen Durchsetzung des Wettbewerbsrechts der Vertragsparteien beizu- 
tragen und die Möglichkeit von Konflikten zwischen den Vertragsparteien in allen Angelegenheiten, die die Anwendung ihres Wettbewerbsrechts betreffen, auszuschliessen oder zu verringern (Art. 1). Die vom Abkommen erfassten Wettbewerbsbehörden sind die Europäische Kommission (EU) und die Wettbewerbskommission (WEKO) einschliesslich ihres Sekretariats (Schweiz). Die Wettbewerbsbehörden der EU-Mitgliedstaaten wie auch die Gerichte der EU und der Schweiz gehören nicht dazu.

237 Die Wettbewerbsbehörden koordinieren Durchsetzungsmassnahmen, wenn sie solche Massnahmen in Bezug auf miteinander verbundene Vorgänge durchführen (Art. 4). Die Wettbewerbsbehörde einer Vertragspartei trägt den wichtigen Interessen der anderen Vertragspartei in allen Phasen von Durchsetzungsmassnahmen Rechnung; Konflikte sind zu vermeiden (Art. 5, negative comity). Ist die Wettbewerbsbehörde einer Vertragspartei der Auffassung, dass wettbewerbswidrige Verhaltensweisen im Hoheitsgebiet der anderen Vertragspartei wichtige Interessen ihrer Vertragspartei beeinträchtigen können, so kann sie unter Berücksichtigung der Bedeutung der Vermeidung von Zuständigkeitskonflikten und dessen, dass die Wettbewerbsbehörde der anderen Vertragspartei möglicherweise wirksamer gegen die betreffenden wettbewerbswidrigen Verhaltensweisen vorgehen kann, die Wettbewerbsbehörde der anderen Vertragspartei ersuchen, geeignete Durchsetzungsmassnahmen einzuleiten oder auszuweiten (Art. 6, positive comity). Das Abkommen regelt den Austausch von Informationen und ihre Verwendung; vertrauliche Informationen unterliegen besonders strikten Anforderungen (Art. 7-9).

Das Abkommen beruht auf dem Prinzip der Gleichwertigkeit der Wettbewerbsrechte der EU und der Schweiz. Es verweist nicht auf EU-Rechtsakte, womit keine Notwendigkeit besteht, das Abkommen periodisch an das sich weiterentwickelnde EU-Recht anzupassen.

\section{F. Justiz und Inneres}

239 Mit dem Vertrag von Maastricht von 1992 wurde die EU gegründet. Sie fungierte als Dach über die weiter geltenden (Gründungs-) Verträge und als Grundlage für eine stärkere innen- und aussenpolitische Zusammenarbeit über die wirtschaftliche Integration hinaus. Die EG, die EGKS und die EAG bildeten die erste Säule der EU. Die zweite Säule der EU, die Gemeinsame Aussen- und Sicherheitspolitik (GASP), und die dritte Säule der EU, die Zusammenarbeit in den Bereichen Justiz und Inneres (ZBJI), nutzten zwar die Institutionen der EG, folgten aber weiterhin zwischenstaatlichen Kooperationsformen. Bei der ZBJI ging es um die Zusammenarbeit in den Bereichen 
Grenzkontrollen, Asyl und Einwanderung sowie um die Zusammenarbeit der Gerichte in Zivil- und Strafsachen und der Polizeibehörden. Die Europäische Kommission, das Europäische Parlament und der EuGH verfügten in diesen Bereichen über keine nennenswerten Befugnisse; im Rat dominierte das Einstimmigkeitsprinzip. Erst mit dem Vertrag von Amsterdam von 1997 wurden ausgewählte Politiken wie Grenzkontrollen, Asyl und Einwanderung in die erste Säule (EG) integriert und vergemeinschaftet. Als Folge davon wurde 1999 die dritte Säule in Polizeiliche und Justizielle Zusammenarbeit in Strafsachen (PJZS) umbenannt; auch wurde der bis anhin völkerrechtlich geregelte Schengen-Besitzstand unter das Dach der EU gestellt (N. 242). Mit dem Vertrag von Lissabon wurde die PJZS - also Teile des Strafrechts und damit einen klassischen Bereich staatlicher Souveränität betreffend (power of the sword) - vollständig in den AEUV integriert. Titel V des AEUV (Art. 67-89; Raum der Freiheit, der Sicherheit und des Rechts) enthält die allgemeinen Grundsätze (Kap. 1) sowie die Vorgaben zu den Politiken in den Bereichen Grenzkontrollen, Asyl und Einwanderung (Kap. 2), Justizielle Zusammenarbeit in Zivilsachen (Kap. 3), Justizielle Zusammenarbeit in Strafsachen (Kap. 4) und Polizeiliche Zusammenarbeit (Kap. 5). Gestützt auf die Kompetenzbestimmungen in diesem Titel hat die EU eine Vielzahl von Massnahmen erlassen, welche ein eindrückliches Mass an Kooperation und Integration reflektieren und auf den Grundsätzen des gegenseitigen Vertrauens und der gegenseitigen Anerkennung beruhen (vgl. Urteil Minister for Justice and Equality, C-216/18 PPU, EU:C:2018:586, Rn. 35-36; zum Ganzen T. Oppermann/C.D. Classen/M. NetTesheim, § 33 Rz. 66-77).

Die Schweiz war seit den 1990er-Jahren bestrebt, mit der EU in ausgewählten Bereichen der ZBJI zusammenzuarbeiten. Die EU und ihre Mitgliedstaaten zeigten sich anfänglich wenig begeistert über eine formalisierte Zusammenarbeit (T. CоTTIER et al., Rz. 793). Eine beidseits zufriedenstellende Lösung gelang erst 2004 mit dem Abschluss der Bilateralen II. Der Schweiz war es ein Anliegen, in den Bereichen Grenzkontrollen, Visa und Asyl mit der EU und ihren Mitgliedstaaten zu kooperieren und an den Schengen/Dublin-Regimes teilzuhaben. Die EU war primär daran interessiert, in den Bereichen Betrugsbekämpfung und Zinsbesteuerung Abkommen abzuschliessen. Weitere Abkommen existieren mittlerweile auch für die Zusammenarbeit bei der Strafverfolgung und Kriminalitätsbekämpfung (Europol, Eurojust). Diese Abkommen werden in diesem Kapitel vorgestellt (s. zum Abkommen über die Zinsbesteuerung N. 317-319).

Ein wesentliches Merkmal der unionalen Politikgestaltung in den Bereichen 241 Grenzkontrollen, Asyl und Einwanderung ist der zunehmende Einsatz von 
computergestützten Datenbanken. In den letzten 20 Jahren errichtete die EU eine Vielzahl von unionsweit operierenden automatisierten IT-Systemen. Im Vordergrund stehen das Schengener Informationssystem (SIS II, N. 261), das Visa-Informationssystem (VIS, N. 253), Eurodac (N. 289), das Einreise-/Ausreisesystem (EES, N. 251) und das Europäische Reiseinformations- und -genehmigungssystem (ETIAS, N. 255). Die Agentur der EU für das Betriebsmanagement von IT-Grosssystemen im Raum der Freiheit, der Sicherheit und des Rechts (eu-LISA) ist für das Betriebsmanagement dieser Datenbanken zuständig (Verordnung (EU) 2018/1726). 2019 schuf die EU die Grundlagen, um die Interoperabilität zwischen den verschiedenen Informationssystemen sicherzustellen (Verordnung (EU) 2019/817 und Verordnung (EU) 2019/818, wobei die Übernahme dieser Verordnungen durch die Schweiz vom Parlament noch genehmigt werden muss). Die automatisiert generierte Erhebung und Speicherung von Daten und der erleichterte Austausch der Daten vereinfachen die tägliche Arbeit der unionalen und nationalen Stellen; sie tragen zur Sicherheit im Schengen-Raum bei. Gleichzeitig stehen die Behörden vor der Herausforderung, die Grundrechte der Betroffenen, darunter insbesondere das Recht auf Achtung der Privatsphäre und das Recht auf Schutz der personenbezogenen Daten (vgl. Art. 8 EMRK, Art. 7 und Art. 8 GRC, Art. 13 BV), zu respektieren. Die bilateralrechtlichen Vorgaben zum Datenschutz werden am Schluss dieses Kapitels behandelt.

Die Schweiz ist an den genannten Datenbanken nach Massgabe der Schengen/DublinAssoziierung beteiligt. Eine separate Vereinbarung zwischen der EU einerseits und Norwegen, Island, der Schweiz und dem Fürstentum Liechtenstein andererseits regelt die Beteiligung dieser Staaten an der Agentur der EU für das Betriebsmanagement von ITGrosssystemen im Raum der Freiheit, der Sicherheit und des Rechts (eu-LISA; SR 0.362.315; s. zum Ganzen C. TOBLER, IT-Agentur, passim).

\section{Schengen}

Belgien, Deutschland, Frankreich, Luxemburg und die Niederlande vereinbarten mit dem Schengener Übereinkommen (SÜ) von 1985 und dem Schengener Durchführungsübereinkommen (SDÜ) von 1990, die Grenzkontrollen an ihren gemeinsamen Grenzen («Binnengrenzen») vollständig abzubauen, gemeinsame Vorschriften für die Kontrolle der Grenzen zu den Nicht-Schengenstaaten («Aussengrenzen») einzuführen und als Ausgleich zur Abschaffung der Binnengrenzkontrollen flankierende Massnahmen betr. die Polizeizusammenarbeit, die Bekämpfung des Drogen- und Waffenschmuggels, die justizielle Zusammenarbeit in Strafsachen sowie die Regelung der Zuständigkeit für die Behandlung von Asylbegehren einzuführen (wobei die Einführung von Regeln 
betr. Asylbegehren sodann ausserhalb des Schengen-Besitzstands erfolgte, N. 275). Diese differenzierte Integration stützte sich ursprünglich auf klassisch völkerrechtliche Verträge zwischen den beteiligten Staaten und erfolgte ausserhalb des institutionellen Rahmens der EWG/EG. Der Schengen-Besitzstand wurde erst 1999 in die EU integriert (Protokoll Nr. 19 zum EUV). Bis heute beteiligen sich das Vereinigte Königreich (bis zu seinem Austritt aus der EU) und Irland nur partiell an den einschlägigen Regeln des Raums der Freiheit, der Sicherheit und des Rechts; sie geniessen primärrechtlich einen Sonderstatus (Protokoll Nr. 19 und Nr. 21 zum EUV). Dasselbe gilt in eingeschränkterem Mass auch für Dänemark (Protokoll Nr. 19 und Nr. 22 zum EUV). Bulgarien, Rumänien, Kroatien und Zypern sind Schengen-Kandidatenländer. Die EWR-EFTA-Mitgliedstaaten Island, Liechtenstein und Norwegen sowie die Schweiz sind durch völkerrechtliche Abkommen an den Schengen-Besitzstand gebunden. Die räumliche Ausdehnung der aktuell 26 EU- und EFTA-Mitgliedstaaten, welche sich zur Beachtung des Schengen-Besitzstands verpflichtet haben, wird als Schengen-Raum bezeichnet.

Mittlerweile stellen Art. 67-76 AEUV (allgemeine Bestimmungen) und Art. 77 AEUV die primärrechtlichen Grundlagen für die Grenzschutzpolitik der EU und damit auch für den Schengen-Besitzstand dar. Demnach entwickelt die EU eine Politik, mit der a) sichergestellt wird, dass Personen beim Überschreiten der Binnengrenzen nicht kontrolliert werden, b) die Personenkontrolle und die wirksame Überwachung des Grenzübertritts an den Aussengrenzen sichergestellt werden soll und c) schrittweise ein integriertes Grenzschutzsystem an den Aussengrenzen eingeführt werden soll (Art. 77 Abs. 1 AEUV). Dabei gilt der Grundsatz der Solidarität (Art. 80 AEUV). Ergänzend sind auch die Vorgaben zur justiziellen Zusammenarbeit in Strafsachen und zur polizeilichen Zusammenarbeit einschlägig (Art. 82-89 AEUV). Gestützt auf die entsprechenden Kompetenzbestimmungen hat die EU eine Vielzahl von Rechtsakten erlassen. Im Zentrum steht dabei - in Ergänzung zum Schengener Übereinkommen (SÜ) von 1985 und zum Schengener Durchführungsübereinkommen (SDÜ) von 1990 - die 2016 neu gefasste Verordnung (EU) 2016/399 über einen Gemeinschaftskodex für das Überschreiten der Grenzen durch Personen (Schengener Grenzkodex, SGK). Diese und weitere zentrale Erlasse werden im Folgenden vorgestellt.

Die EU entwickelt gestützt auf Art. 79 AEUV eine gemeinsame Einwanderungspolitik. Dabei verfügt die EU auch über eine Zuständigkeit zum Erlass von Regeln für die Erteilung von Visa und Aufenthaltstiteln für einen langfristigen Aufenthalt (d.h. für Visa, welche für einen längeren Aufenthalt als 90 Tage berechtigen, s. zum Schengen-Visum N. 252-255). Die Richtlinie 2009/50/EG regelt die Bedingungen für die Einreise und den Aufenthalt von Drittstaatsangehörigen zur Ausübung einer hochqualifizierten Beschäftigung; unter die- 
sem Titel können an Drittstaatsangehörige und ihre Familienangehörigen «Blaue Karten EU» vergeben werden. Die Schweiz beteiligt sich nicht an der gemeinsamen Einwanderungspolitik der EU.

\section{(a) Schengen-Assoziierungsabkommen: Im Allgemeinen}

Die Schweiz und die EG unterzeichneten 2004 das Abkommen zwischen der Schweiz, der EU und der EG über die Assoziierung der Schweiz bei der Umsetzung, Anwendung und Entwicklung des Schengen-Besitzstands (SAA). Es trat 2008 in Kraft und ermöglicht der Schweiz, mitgliedstaatsähnlich am Schengen-Besitzstand und an seiner weiteren Entwicklung teilzunehmen. Die Schweiz wird bei der Tätigkeit der EU in den vom Abkommen erfassten Bereichen assoziiert (Art. 1). Sie ist verpflichtet, die in den Anhängen A und B aufgeführten Vorgaben des Schengen-Besitzstands, welche für die EU-Mitgliedstaaten gelten, umzusetzen und anzuwenden (Art. 2).

Der Bundesrat veröffentlichte 2018 als Antwort auf das Postulat 15.3896 «Wirtschaftliche Vorteile dank Schengen-Partnerschaft» einen Bericht zu den volkswirtschaftlichen und finanziellen Auswirkungen der Schengen/Dublin-Assoziierung der Schweiz. Er kam zum Schluss, dass die Zusammenarbeit in diesem Bereich bedeutsame volkswirtschaftliche und finanzielle Vorteile mit sich bringt. Über den volkswirtschaftlichen und finanziellen Aspekt hinaus stellt Schengen ein grundlegendes Instrument im Bereich der inneren Sicherheit dar. Hinzu kommen weitere messbare Effekte wie die Attraktivität des Wirtschaftsstandorts Schweiz, von welchem touristische Orte wie auch die grenznahen Regionen profitieren (Bericht vom 21. Februar 2018).

Der Gesamtbeitrag der Schweiz an die EU im Rahmen der Schengen/Dublin-Assoziierung belief sich 2014-2019 auf 224 Mio. CHF (inkl. Beiträge für EES, ETIAS, eu-LISA, Eurodac, Frontex, ISF, SIS und VIS), wobei in diesem Zeitraum 23 Mio. CHF in die Schweiz zurückgeflossen sind (Stellungnahme des Bundesrates vom 20. November 2019 auf die Interpellation 19.4008 «Aktualisierte Zusammenstellung aller Zahlungen und Beiträge der Schweiz an die EU»).

Es ist für das ordnungsgemässe Funktionieren des Schengen-Besitzstands zentral, dass neue Schengen-relevante Rechtsakte der EU zeitnah und unkompliziert in das Schengen-Abkommen inkorporiert werden. Zu diesem Zweck enthält das Abkommen einen Mechanismus, neue Rechtsakte dynamisch in die Anhänge zu übernehmen (N. 93). Aus dem gleichen Grund legen die schweizerischen Behörden den Schengen-Besitzstand im Licht der einschlägigen Praxis des EuGH aus (N. 83). Die rechtlichen Schicksale der Schengen- und Dublin-Assoziierungsabkommen sind miteinander verknüpft: Das eine Abkommen wird nur angewendet, wenn auch das andere angewendet wird (sog. «kleine» Guillotine [im Gegensatz zur «grossen» Guillotine, welche die Bilateralen I aneinander kettet]; Art. 15 Abs. 4 SAA, Art. 14 Abs. 2 DAA). 
Zwischen den Schengen/Dublin-Abkommen und dem FZA besteht keine rechtliche Verbindung: Das SAA regelt den Grenzschutz und Belange von Drittstaatsangehörigen. Das FZA regelt den Zugang zum Arbeitsmarkt sowie die Einreise und den längerfristigen Aufenthalt von nicht erwerbstätigen Staatsangehörigen der Vertragsparteien ohne Bezug zum Grenzschutz (s. zur Abgrenzung bei Drittstaatsangehörigen, welche - z.B. unter dem Titel des Familiennachzugs - freizügigkeitsberechtigt sind, T. COTTIER et al., Rz. 841-842). Gleichwohl stellte die Personenfreizügigkeit bei den Verhandlungen zur Schengen/Dublin-Assoziierung der Schweiz aus Sicht der EU eine Grundlage für diese Assoziierung dar (Botschaft Begrenzungsinitiative, S. 5055). Im Nachgang zur Annahme der Masseneinwanderungsinitiative 2014 (N. 63) verlautete die EU, dass eine nicht FZA-konforme Umsetzung dieser Initiative «Zweifel an der Assoziierung der Schweiz mit dem Schengen-Besitzstand und dem Besitzstand des Dubliner Übereinkommens (...) aufkommen lässt» (Schlussfolgerungen des Rates vom 16. Dezember 2014, Rz. 45). Nach Ansicht der EU besteht folglich zumindest ein politischer Konnex.

Die Präambel des Schengen-Abkommens weist darauf hin, dass die Zusammenarbeit der Schweiz und der EU in diesem Bereich «auf den Grundsätzen der Freiheit, der Demokratie, der Rechtsstaatlichkeit und der Achtung der Menschenrechte, wie sie insbesondere in der Europäischen Konvention zum Schutze der Menschenrechte und Grundfreiheiten vom 4. November 1950 gewährleistet sind, beruht». Unionale Rechtsakte des Schengen-Besitzstands verweisen mitunter zudem - wie die folgenden Beispiele zeigen - auf internationale Grundrechtsinstrumente und unionale Grundrechte (wobei die Übernahme einzelner dieser Rechtsakte durch die Schweiz noch hängig ist): Art. 4 SGK verpflichtet die EU-Mitgliedstaaten, bei der Anwendung des Schengener Grenzkodexes «unter umfassender Einhaltung der einschlägigen Rechtsvorschriften der Union, einschliesslich der Charta der Grundrechte der Europäischen Union (...), und des einschlägigen Völkerrechts» zu handeln. Art. 1 der Rückführungsrichtlinie 2008/115/EG betont die Verpflichtung, die Normen und Verfahren inkl. allfälliger Zwangsmassnahmen «im Einklang mit den Grundrechten als allgemeinen Grundsätzen des Gemeinschafts- und des Völkerrechts» anzuwenden. Gemäss Art. 1 Abs. 2 der Verordnung (EU) 2019/1896 handelt die Europäische Grenz- und Küstenwache (inkl. Frontex) «unter uneingeschränkter Wahrung der Grundrechte». Art. 5 der Verordnung (EU) 2019/817 zur Errichtung eines Rahmens für die Interoperabilität zwischen EUInformationssystemen verpflichtet die Behörden bei der Verarbeitung personenbezogener Daten zur Beachtung der Menschenwürde und der Grundrechte der Betroffenen. Es ist selbstverständlich, dass nicht nur die EU und ihre Mitgliedstaaten, sondern auch die Schweiz bei der Durchführung dieser Rechtsakte die Grund- und Menschenrechte des Landes- und Völkerrechts zu beachten hat. Fraglich ist, ob und - sofern dies der Fall ist - inwieweit diese Kaskadenverweisungen auf die allgemeinen Rechtsgrundsätze des EU-Rechts und die Grundrechtecharta der EU dazu führen, dass das grundrechtliche 
Schutzsystem, wie es sich in der EU entwickelt hat, auch in der Schweiz direkt wirkt, oder ob hierzulande weiterhin zuvörderst die Grundrechte der Verfassung und der EMRK und die dazugehörige Praxis der jeweiligen Höchstgerichte gelten (s. dazu auch N. 78).

Art. 8 Abs. 2 SAA ermächtigt die Schweiz, im Rahmen von Vorabentscheidungsverfahren vor dem EuGH, in denen die Auslegung des Schengen-Besitzstands zur Debatte steht, Schriftsätze einzureichen oder schriftliche Erklärungen abzugeben. Die Schweiz macht von diesem Recht vereinzelt Gebrauch. Sie betont dabei regelmässig, dass sie sich zur Auslegung der Grundrechtecharta nicht äussert, da diese «für die Schweiz (...) nicht bindend» ist (s. für eine Intervention in einem Verfahren zur Auslegung des Dublin-Besitzstands N. 282).

\section{(b) Grenzkontrollen}

247 Das Schengen-Regime beruht auf der Maxime, dass die Binnengrenzen an jeder Stelle ohne Personenkontrollen überschritten werden dürfen (Art. 2 SDÜ; Art. 1 und Art. 22 SGK). Dieser Anspruch steht allen im Einreisestaat aufenthaltsberechtigten Personen unabhängig ihrer Staatsangehörigkeit zu. An den Binnengrenzen müssen zudem sämtliche Verkehrshindernisse entfernt werden (s. aber zu den Vorkehren für die Kontrolle von Waren an den Schweizer Grenzen N. 248). Sofern die öffentliche Ordnung oder die innere Sicherheit in einem Schengen-Staat ernsthaft bedroht ist, ist dieser Staat berechtigt, wieder Kontrollen an seinen Binnengrenzen für einen begrenzten Zeitraum einzuführen. Diesfalls ist die Verhältnismässigkeit zwischen der Massnahme und der Bedrohung darzulegen (Art. 26 SGK). Strikte Verfahrensvorgaben insbesondere die Verpflichtung zur vorgängigen Erörterung der geplanten Wiedereinführung von Grenzkontrollen mit der Europäischen Kommission und den anderen Schengenstaaten - stellen sicher, dass ein solcher aussergewöhnlicher Schritt nicht leichthin getan wird (Art. 27 SGK). Eine Zustimmung der Kommission oder der anderen Mitgliedstaaten ist aber nicht notwendig.

Typische - und wiederkehrende - Auslöser für die temporäre Wiedereinführung von Kontrollen an den Binnengrenzen sind das World Economic Forum (WEF) in Davos und Sportgrossveranstaltungen wie Fussballeuropameisterschaften. In den letzten Jahren führten zudem Frankreich, Österreich, Deutschland, Dänemark, Schweden und Norwegen temporär Grenzkontrollen ein, um auf die Defizite des Grenzschutzes in Griechenland und die damit einhergehende unrechtmässige Einreise von Flüchtlingen vorwiegend aus dem Nahen Osten und aus Nordafrika in den Schengen-Raum zu reagieren (N. 277).

Alle EU-Mitgliedstaaten führten im Zuge der Bekämpfung der durch das Coronavirus (COVID-19) ausgelösten epidemiologischen Krise im Frühjahr 2020 Kontrollen an den Binnengrenzen ein. Dies geschah weitgehend unkoordiniert, worauf die Kommission - trotz der beschränkten Kompetenzen im Schengen-Bereich und in der Gesundheitspolitik (vgl. Art. 168 AEUV) - das Heft in die Hand nahm, die gesundheitliche Krise auch als Gefahr 
für das gute Funktionieren des Binnenmarktes begriff und Leitlinien für gesundheitsbezogene Grenzmanagementmassnahmen veröffentlichte (C(2020) 1753; s. auch C(2020) 1897; C(2020) 2051; Gemeinsame Erklärung der Mitglieder des Europäischen Rates vom 26. März 2020, Rz. 3). Sie forderte die Mitgliedstaaten auf, die Durchreise von Unionsbürgerinnen und Unionsbürgern zu ermöglichen, welche an ihren Wohnsitz oder in ihren Heimatstaat zurückkehren wollten, die Ein- und Ausreise von in systemrelevanten Berufen tätigen Grenzgängerinnen und Grenzgängern zu ermöglichen und die Einreise der in der Landwirtschaft unverzichtbaren Saisonarbeiterinnen und Saisonarbeitern zuzulassen. Sie wies auf die für die Versorgung der Bevölkerung wichtige Aufrechterhaltung des freien Warenverkehrs hin und empfahl, an den Grenzübergängen «grüne Korridore» einzurichten. In Bezug auf die Aussengrenzen initiierte die Kommission eine Einigung des Europäischen Rates über die Beschränkung nicht essentieller Einreisen von Drittstaatsangehörigen in die EU (COM(2020) 115 final). Schliesslich legte die Kommission einen gemeinsamen europäischen Fahrplan vor für eine möglichst koordinierte Aufhebung der Grenzkontrollen (Bericht vom 15. April 2020).

Auch die Schweiz führte im Frühjahr 2020 Einreisebeschränkungen für Personen aus allen Ländern ein. Ausgenommen davon waren u.a. Personen aus dem Fürstentum Liechtenstein, Personen, welche über einen schweizerischen Aufenthaltstitel verfügten, Grenzgängerinnen und Grenzgänger, Personen, welche im Gesundheitsbereich tätig waren, sowie Personen, welche sich in einer «Situation der äussersten Notwendigkeit» befanden (Art. 3 der COVID-19-Verordnung 2; Weisung des SEM vom 24. März 2020). Dabei wurde v.a. die letztgenannte Kategorie restriktiv gehandhabt: Einreisen von Personen zwecks Besuchen bei Paar-, Liebesbeziehungen und Bekanntschaften von nicht verheirateten oder nicht registrierten Partnerschaften oder von Paaren ohne gemeinsame Kinder waren nicht möglich, was grundrechtlich problematisch war (s. zur asylrechtlichen Problematik N. 281). Die Schweiz hob die Einreisebeschränkungen gegenüber allen EU/EFTA-Staaten und dem Vereinigten Königreich per 15. Juni 2020 wieder auf. Dieser Schritt erfolgte im Anschluss an eine informelle Videokonferenz der Innenminister der Schengen-Staaten (Pressemitteilung des Bundesrates vom 5. Juni 2010, Coronavirus: Die Schweiz öffnet die Grenze zu allen EU/EFTA-Staaten).

An den Grenzübergängen der Schweiz zu ihren Nachbarstaaten ist es - im Gegensatz zu den Grenzübergängen zwischen EU-Mitgliedstaaten - weiterhin zulässig und üblich, Zollabfertigungen und stichprobenweise Kontrollen durchzuführen, etwa um den Ursprung der Waren festzustellen. Folglich darf die Schweiz weiterhin Grenzeinrichtungen unterhalten und Geschwindigkeitsbegrenzungen vorsehen (Bericht der Kommission vom 13. Oktober 2010, COM(2010) 554 final, S. 7 Fn. 8). Die Schweiz ist nicht Mitglied der Zollunion der EU und handelt damit auch gegenüber der EU handelspolitisch autonom. Im Zug einer solchen Warenverkehrskontrolle ist es auch zulässig, in beschränktem Umfang akzessorisch Personen zu überprüfen (etwa auf das Mitführen von Waren; vgl. T. COTTIER et al., Rz. 818, betr. die Verwertbarkeit von dabei entdeckten fahndungstechnischen Zufallstreffern).

Der Schengen-Besitzstand erlaubt den Schengen-Staaten, Personenkontrollen im Inland (inkl. in den Grenzgebieten) durchzuführen, sofern die Ausübung 
solcher Befugnisse nicht die gleiche Wirkung wie Grenzübertrittskontrollen hat und sich eindeutig von systematischen Personenkontrollen an den Aussengrenzen unterscheidet (Art. 23 SGK). Problematisch sind insbesondere mobile Kontrollen im grenznahen Raum, welche von manchen Staaten als Korrelativ zur Abschaffung der Kontrollen an den Binnengrenzen vermehrt durchgeführt werden (sog. «Schleierfahndung»).

Die geltende Fassung des Schengener Grenzkodexes beruht wesentlich auf der Praxis des EuGH zur Zulässigkeit von Personenkontrollen im Inland. Der EuGH fordert, dass solche Kontrollen faktisch nicht Grenzkontrollen gleichkommen dürfen; sie können aber gleichwohl verdachtsunabhängig und stichprobenmässig erfolgen (vgl. etwa Urteil A, C-9/16, EU:C:2017:483, kritisch zu einer Regelung, welche den Behörden erlaubt, innerhalb eines Gebiets von $30 \mathrm{~km}$ ab der Landgrenze Kontrollen unabhängig vom Verhalten der Person und vom Vorliegen besonderer Umstände zu kontrollieren; Urteil Adil, C-278/12 PPU, EU:C:2012:508, wonach eine Regelung zulässig ist, die es den Behörden erlaubt, in einem räumlichen Gebiet von $20 \mathrm{~km}$ ab der Landgrenze Kontrollen durchzuführen, um zu überprüfen, ob die kontrollierten Personen die Voraussetzungen für einen rechtmässigen Aufenthalt erfüllen, sofern die Durchführung dieser Kontrollen Beschränkungen hinsichtlich ihrer Intensität und Häufigkeit unterliegt; Urteil Touring Tours und Travel, C-412/ 17, EU:C:2018:1005, wonach eine Regelung nicht zulässig ist, welche Beförderungsunternehmer, die im Schengen-Raum einen grenzüberschreitenden Linienbusverkehr betreiben, verpflichtet, den Pass und den Aufenthaltstitel der Passagiere zu kontrollieren, um zu verhindern, dass Drittstaatsangehörige, die nicht im Besitz dieser Reisedokumente sind, in das Hoheitsgebiet dieses Mitgliedstaats befördert werden.

Die Regeln über die Kontrolle der Einreise von Drittstaatsangehörigen an den Schengen-Aussengrenzen wurden vereinheitlicht. Die Aussengrenzen dürfen nur an den Grenzübergangsstellen und während der festgesetzten Verkehrsstunden überschritten werden; die Mitgliedstaaten bezeichnen die Grenzübergangsstellen (Art. 3 SDÜ; Art. 5 SGK). Drittstaatsangehörige müssen für einen geplanten Aufenthalt im Hoheitsgebiet der Schengenstaaten von bis zu 90 Tagen im Besitz eines gültigen Reisedokuments und eines gültigen Visums oder eines gültigen Aufenthaltstitels sein, über ausreichende Mittel zur Bestreitung des Lebensunterhalts für die Dauer des beabsichtigten Aufenthalts verfügen und dürfen nicht im Schengener Informationssystem (SIS II, N. 261) zur Einreiseverweigerung ausgeschrieben sein; sie dürfen zudem keine Gefahr für die öffentliche Ordnung, die innere Sicherheit, die öffentliche Gesundheit oder die internationalen Beziehungen eines Mitgliedstaats darstellen und insbesondere nicht in den nationalen Datenbanken zur Einreiseverweigerung aus denselben Gründen ausgeschrieben worden sein (Art. 6 SGK). Die Schweiz verfügt an den internationalen Flughäfen über Aussengrenzen: Hier können Drittstaatsangehörige direkt in den Schengen-Raum einreisen (s. zur Pflicht von Fluggesellschaften, Angaben über die beförderten Personen vorab an die nationalen Behörden zu übermitteln, Richtlinie 2004/82/ 
EG; zur Pflicht, sich zu vergewissern, dass Drittstaatenangehörige über die für die Einreise erforderlichen Reisedokumente verfügen, Art. 26 SDÜ und Richtlinie 2001/51/EG; Art. 92, Art. 104 und Art. 122a-122c AIG).

Die EG erliess 2004 Normen für Sicherheitsmerkmale und biometrische Daten in von den Mitgliedstaaten ausgestellten Pässen und Reisedokumenten (Verordnung (EG) Nr. 2252/ 2004). Damit wurde u.a. bezweckt, die Verfahren, die von den Mitgliedstaaten bei der Durchführung der Personenkontrollen an den Aussengrenzen einzuhalten sind, zu vereinheitlichen und zu vereinfachen. In der Schweiz wurde gegen die Übernahme dieser auch für die assoziierten Staaten bedeutsamen Weiterentwicklung des Schengen-Besitzstands und die dazugehörige Umsetzungsgesetzgebung das Referendum ergriffen. 2009 stimmten 50.1 \% der abstimmenden Stimmbürgerinnen und Stimmbürger zu (wobei das BGer in der Folge diverse Abstimmungsbeschwerden u.a. mit der Begründung abwies, dass das Resultat zwar relativ knapp ausgegangen sei, aber jedenfalls nicht derart knapp, dass die verlangte Nachzählung vorgenommen werden müsse; Urteile vom 1. Oktober 2009, 1C 241/ 2009, 1C 3/2009, 1C 275/2009). Bei einer Ablehnung - es fehlten nur rund 5600 Stimmen - wäre die Weiterführung der Schengen/Dublin-Abkommen ernsthaft gefährdet gewesen (N. 93). Hauptgrund für die Opposition gegen die Vorlage war gemäss der Vox-Analyse die zentrale Passdatenbank, welche mit der Umsetzungsgesetzgebung eingeführt wurde, ohne dass die EU-Verordnung dies verlangt hätte (NZZ vom 12. April 2019, S. 15).

Zurzeit laufen die Vorarbeiten für die Errichtung eines einheitlichen Einreise/Ausreisesystems (EES) zur Erfassung der Ein- und Ausreisedaten von Drittstaatsangehörigen (Verordnung (EU) 2017/2226). Damit wird bezweckt, den Zeitpunkt und den Ort der Ein- und Ausreise von Drittstaatsangehörigen zu erfassen und zu speichern, die Dauer des zulässigen Aufenthalts solcher Drittstaatsangehörigen zu berechnen, Warnmeldungen für die Mitgliedstaaten zu erstellen, wenn der zulässige Aufenthalt abgelaufen ist, und den Zeitpunkt und den Ort der Einreiseverweigerung für Drittstaatsangehörige, denen die Einreise für einen Kurzaufenthalt verweigert wurde, sowie die Gründe dafür zu erfassen und zu speichern; zum Zweck der Verhütung, Aufdeckung und Untersuchung terroristischer oder sonstiger schwerer Straftaten werden zudem die Bedingungen festgelegt, unter denen die Behörden der Mitgliedstaaten und Europol für Datenabfragen Zugang zum EES erhalten können (Art.1). Die Agentur der EU für das Betriebsmanagement von IT-Grosssystemen im Raum der Freiheit, der Sicherheit und des Rechts (eu-LISA) ist für das Betriebsmanagement des Zentralsystems des EES zuständig (N. 241).

\section{(c) Schengen-Visum}

Ein zentraler Bestandteil des Schengen-Besitzstands ist die gemeinsame

Visumpolitik für die Ausstellung von Visa für einen Zeitraum von maximal 90 Tagen. Die Verordnung (EU) 2018/1806 (Visums-Verordnung) listet die Drittländer auf, deren Staatsangehörige beim Überschreiten der Aussengren- 
zen im Besitz eines Visums sein müssen, sowie die Drittländer, deren Staatsangehörige von dieser Visumpflicht befreit sind. Ein einheitliches SchengenVisum ist für den ganzen Schengen-Raum gültig. Im Gegensatz zu einem einheitlichen Schengen-Visum berechtigt ein Schengen-Visum mit räumlich beschränkter Gültigkeit nur zum Aufenthalt im Hoheitsgebiet eines oder mehrerer Mitgliedstaaten; solche Visa werden in Ausnahmefällen erteilt, wenn der betreffende Mitgliedstaat eine Beschränkung aus humanitären Gründen, aus Gründen des nationalen Interesses oder aufgrund internationaler Verpflichtungen für erforderlich hält (Art. 25 der Verordnung (EG) Nr. 810/2009, Visakodex).

Der Visumsbefreiung nach Massgabe der Verordnung (EU) 2018/1806 (Visums-Verordnung) geht häufig der Abschluss eines völkerrechtlichen Abkommens zwischen der EU und dem betreffenden Drittstaat im Rahmen eines sog. Visums-Dialogs voraus. Visumsbefreiungen werden dabei an spezifische Bedingungen in anderen Politikbereichen geknüpft. Die Schweiz ist zwar nicht Vertragspartei solcher Abkommen, durch die anschliessende Aufnahme eines Drittstaats in die Visums-Verordnung (EU) 2018/1806 wird eine Visumbefreiung jedoch auch für die Schweiz verbindlich. Faktisch verliert sie damit die Möglichkeit, eine autonome Visumpolitik zu betreiben (T. COTTIER et al., Rz. 846). operationalisieren die gemeinsame Visumpolitik: Erstere legt die Verfahren und Voraussetzungen für die Erteilung von Schengen-Visa fest (Visakodex); letztere erleichtert die Bedingungen und Verfahren für den Datenaustausch zwischen den Mitgliedstaaten über Anträge auf Erteilung eines SchengenVisums und legt die Funktionen und Zuständigkeiten in Bezug auf das VisaInformationssystem (VIS) fest. Das Visa-Informationssystem ist eine schengenweit verfügbare Plattform, auf die nationale Behörden zugreifen, um Daten zu sammeln und zu speichern und automatisiert Visa auszustellen. Die Agentur der EU für das Betriebsmanagement von IT-Grosssystemen im Raum der Freiheit, der Sicherheit und des Rechts (eu-LISA) ist für das Betriebsmanagement des Visa-Informationssystems (VIS) zuständig (N. 241).

254 Ein Schengen-Visum ermöglicht einem Drittstaatsangehörigen, in den Schengen-Raum einzureisen und sich für einen Zeitraum von maximal 90 Tagen in einem Gesamtzeitraum von 180 Tagen frei zwischen den Schengen-Staaten zu bewegen. Voraussetzung dafür ist, dass es sich beim Drittstaatsangehörigen nicht um eine im Schengener Informationssystem (SIS II, N. 261) zur Einreiseverweigerung ausgeschriebene Person handelt und er keine Gefahr für die öffentliche Ordnung, die innere Sicherheit, die öffentliche Gesundheit oder die internationalen Beziehungen eines Mitgliedstaats darstellt (Art. 6 SGK; T. CotTIER et al., Rz. 851; s. Urteil Kommission/Spanien, C-503/03, 
EU:C:2006:74, zur Prüfung, ob die Anwesenheit von Personen «eine tatsächliche, gegenwärtige und hinreichend schwere Gefährdung eines Grundinteresses der Gesellschaft» darstellt).

Die gemeinsame Visumpolitik ist insbesondere für den Tourismus in der Schweiz bedeutsam. Touristinnen und Touristen aus anderen Weltgegenden, welche in Europa herumreisen und über ein Schengen-Visum verfügen, können einen Abstecher in die Schweiz machen, ohne ein Visum spezifisch für die Schweiz beantragen und die Unkosten dafür tragen zu müssen (s. dazu Bericht des Bundesrates zu den volkswirtschaftlichen und finanziellen Auswirkungen der Schengen/Dublin-Assoziierung der Schweiz von 2018, N. 244).

Die Schweiz stellte bis 2018 für Personen, welche im Herkunftsstaat ernsthaft an Leib und Leben gefährdet waren und ein Asylgesuch auf einer Schweizer Vertretung im Ausland einreichten, humanitäre Visa als Schengen-Visa mit räumlich beschränkter Gültigkeit aus (Art. 25 der Verordnung (EG) Nr. 810/2009, Visakodex). Im Nachgang zu einem Urteil des EuGH - wonach der Visakodex die Mitgliedstaaten nicht verpflichtet, Personen, die sich in ihr Hoheitsgebiet begeben möchten, um dort Asyl zu beantragen, ein humanitäres Visum zu erteilen; es steht ihnen weiterhin frei, dies nach Massgabe ihres nationalen Rechts zu tun (Urteil X, C-638/16 PPU, EU:C:2017:173) - revidierte die Schweiz die Verordnung über die Einreise und die Visumserteilung (VEV, SR 142.204); neu werden solche Visa als nationale Visa D ausgestellt (Art. 4 Abs. 2 VEV). Bei dieser Novellierung handelte es sich um ein seltenes Beispiel einer Anpassung einer schweizerischen Verordnung an die Praxis in der EU direkt gestützt auf ein Präjudiz des EuGH.

Neu müssen Reisewillige aus einem Drittstaat, deren Staatsangehörige von der Visumpflicht befreit sind, für die Einreise in den Schengen-Raum vorab eine Reisegenehmigung nach Massgabe der Verordnung (EU) 2018/1240 über die Einrichtung eines Europäischen Reiseinformations- und -genehmigungssystems (ETIAS) beantragen (wobei die Übernahme dieser Verordnung durch die Schweiz vom Parlament noch genehmigt werden muss). Die Angaben werden automatisch mit den unionalen Fahndungs- und Migrationsdatenbanken, u.a. SIS II, abgeglichen. Bei triftigen Gründen wird die Einreise verweigert.

\section{(d) Rückführung von Drittstaatsangehörigen}

Der für die Schweiz relevante Schengen-Besitzstand enthält keine Vorgaben zum Einwanderungsrecht in einem klassischen Sinn. Der Schweiz steht es frei, den Aufenthalt und die Niederlassung von Ausländerinnen und Ausländern zu regeln; dazu gehört auch die Regelung, unter welchen Voraussetzungen Aufenthaltsrechte widerrufen und Ausländerinnen und Ausländer aus der Schweiz ausgewiesen werden (vgl. Art. 121 und Art. 121a BV). Eine Ausnahme besteht einzig in Bezug auf Visa, welche gestützt auf die gemeinsame Visumpolitik der Schengen-Staaten ausgestellt werden: Ein einheitliches Schengen-Visum 
ermöglicht Drittstaatsangehörigen, in das Hoheitsgebiet der Schengen-Staaten einzureisen und sich für einen Zeitraum von maximal 90 Tagen im gesamten Schengen-Raum aufzuhalten (N. 254).

Darüber hinaus sind selbstredend weitere völker- und bilateralrechtliche Vorgaben zu beachten. Dazu gehören insbesondere das Non Refoulement-Gebot als Teil des völkerrechtlichen ius cogens, das Genfer Abkommen über die Rechtsstellung der Flüchtlinge von 1951 und das dazugehörige New Yorker Protokoll von 1967 sowie das Freizügigkeitsabkommen, welches den Staatsangehörigen der EU-Mitgliedstaaten und ihren Familienangehörigen Ansprüche auf Einreise und Aufenthalt gewährt (N. 157-163). Problematisch war vor diesem Hintergrund das Einreiseverbot für ausländische Staatsangehörige, welches der Bundesrat zur Bekämpfung der durch das Coronavirus (COVID-19) ausgelösten epidemiologischen Krise erliess (Art. 3 der COVID-19-Verordnung 2, SR 818.101.24; N. 247 und N. 281).

Die Richtlinie 2008/115/EG über gemeinsame Normen und Verfahren in den Mitgliedstaaten zur Rückführung illegal aufhältiger Drittstaatsangehöriger (Rückführungsrichtlinie) gehört demgegenüber zum Schengen-Besitzstand, zu deren Beachtung sich auch die Schweiz verpflichtet hat (s. für weitere verfahrensrechtliche Bestimmungen bei der Einreiseverweigerung Art. 14 und Anhang V Teil A SGK; zur Einführung eines europäischen Reisedokuments bei Rückführungen Verordnung (EU) 2016/1953; zur Umsetzung der Rückführungsrichtlinie in der Schweiz Art. 64-82 AIG). Diese Richtlinie ist anwendbar auf Personen, welche sich im Schengen-Raum ohne Aufenthaltsberechtigung aufhalten und kein Gesuch auf internationalen Schutz gestellt haben. Sie sieht ein mehrstufiges Vorgehen vor (Rückführung, Abschiebung bzw. - in der schweizerischen Terminologie - Wegweisung, Ausschaffung; s. zum Ganzen T. COTTIER et al., Rz. 855-860). Die Richtlinie verpflichtet zum Einsatz von Zwangsmassnahmen, sofern keine anderen ausreichenden, jedoch weniger intensiven Zwangsmassnahmen wirksam angewandt werden können; dazu gehört auch die Inhaftnahme, wobei die Gesamthaftdauer eineinhalb Jahre nicht überschreiten darf (Art. 15 Abs. 5 und Abs. 6).

Der EuGH hat sich wiederholt zur Auslegung der Rückführungsrichtlinie 2008/115/EG und zu den rechtsstaatlich gebotenen Anforderungen an die bei einer Rückführung zu beachtenden Modalitäten geäussert (s. etwa Urteil Kadzoev, C-357/09 PPU, EU:C:2009:741, betr. Haftdauer bei hinreichender Aussicht auf Abschiebung; Urteil Pham, C-474/13, EU:C:2014:2096, zur Inhaftierung in einer gewöhnlichen Haftanstalt; Urteil El Dridi, C-61/11 PPU, EU:C:2011:268, und Urteil Achughbabian, C-329/11, EU:C:2011:807, beide zum Verhältnis der Rückführung zu strafrechtlichen Massnahmen; Urteil Országos Idegenrendeszeti Fơigazgatóság Dél-alföldi Regionális Igazgatóság, C-924/19 PPU, EU:C:2020:367, zu den Voraussetzungen für die Inhaftnahme und ihre Höchstdauer sowie zum Rechtsschutz). 
Mit der Verordnung (EU) 2018/1860 wurde die Grundlage für die Nutzung des Schengener Informationssystems (SIS II, N. 261) für die Rückkehr illegal aufhältiger Drittstaatsangehöriger geschaffen (wobei die Übernahme dieser Verordnung durch die Schweiz vom Parlament noch genehmigt werden muss).

(e) Polizei, Amts- und Rechtshilfe, Sicherheit

Die Zusammenarbeit zwischen der Schweiz und anderen europäischen Staaten im Bereich der Strafverfolgung - d.h. im Bereich der Verhinderung oder Verfolgung von Straftaten (polizeiliche Zusammenarbeit, Amtshilfe) und der justiziellen Beurteilung von Straftaten (Rechtshilfe) - erfolgt traditionell im Rahmen von internationalen Organisationen, von Konventionen unter den Auspizien des Europarates und von bilateralen Verträgen. Dazu gehören zuvörderst Interpol (Statuten der Internationalen Kriminalpolizeilichen Organisation im Anhang zur Interpol-Verordnung, SR 351.21), das Europäische Übereinkommen über die Rechtshilfe in Strafsachen von 1959 (SR 0.351.1), das Europäische Auslieferungsübereinkommen von 1957 (SR 0.353.1) und das Übereinkommen über die Überstellung verurteilter Personen von 1983 (SR 0.343). Die Schweiz hat mit allen Nachbarstaaten (Vertrag zwischen der Schweiz und Deutschland über die grenzüberschreitende polizeiliche und justitielle Zusammenarbeit von 1999, SR 0.360.136.1; Abkommen zwischen der Schweiz und Italien über die Zusammenarbeit der Polizei- und Zollbehörden von 2013, SR 0.360.454.1; Abkommen zwischen der Schweiz und Frankreich über die grenzüberschreitende Zusammenarbeit in Justiz-, Polizei- und Zollsachen von 2007, SR 0.360.349.1; Vertrag zwischen der Schweiz, Österreich und dem Fürstentum Liechtenstein über die grenzüberschreitende polizeiliche Zusammenarbeit von 2012, SR 0.360.163.1) wie auch mit weiteren Staaten vor allem Süd- und Osteuropas Abkommen abgeschlossen.

Der Schengen-Besitzstand hat die polizeiliche Zusammenarbeit und die Rechtshilfe in Strafsachen für sämtliche Schengen-Staaten auf eine einheitliche Basis gestellt, wobei die entsprechenden Instrumente einen Mindeststandard darstellen. Die Verträge der Schweiz mit einzelnen europäischen Staaten gehen - abgesehen vom Schengener Informationssystem (SIS II, N. 261) - über diesen Mindeststandard hinaus, was gemäss Art. 12 Abs. 2 SAA zulässig ist, soweit keine Unvereinbarkeit besteht (s. zum Ganzen T. COTTIER et al., Rz. 901-929). Das Schengener Durchführungsübereinkommen (SDÜ) von 1990 enthält die folgenden Vorgaben in Bezug auf die polizeiliche Zusammenarbeit (Art. 39-47) und die Rechtshilfe in Strafsachen (Art. 48-69): 
- $\quad$ Das SDÜ regelt den polizeilichen Informationsaustausch zur Kriminalitätsbekämpfung und Gefahrenabwehr auf Ersuchen oder spontan; massgeblich ist das nationale Recht (Art. 39 und Art. 46). Es regelt lediglich den Übermittlungsweg (Austausch via nationale Zentralstellen). Auf Ersuchen und unter Vorbehalt der Rechtshilfebestimmungen können auch Informationen beschafft werden, soweit dies das nationale Recht zulässt. Sind Zwangsmassnahmen erforderlich, ist der Rechtshilfeweg zu beschreiten (vgl. Art. 39 Abs. 1 SDÜ). Der Erleichterung des Amtshilfeverkehrs dient auch die Entsendung von Polizeiverbindungsleuten, d.h. die Stationierung einer Beamtin oder eines Beamten bei den ausländischen Behörden (Art. 47 SDÜ). Weiter regelt das SDÜ die grenzüberschreitende Observation (Art. 40 SDÜ) und die Nacheile (Verfolgung von auf frischer Tat ertappter Täterinnen und Täter, Art. 41 SDÜ). Eine grenzüberschreitende Observation ist nur gestützt auf ein Rechtshilfeersuchen und die Zustimmung der anderen Vertragspartei zulässig; bei besonderer Dringlichkeit gelten besondere Voraussetzungen. Die Nacheile kann ohne Ersuchen erfolgen, da dort naturgemäss Dringlichkeit besteht. Sie darf innerhalb eines Gebiets und für einen Zeitraum erfolgen, welche jeder SchengenStaat mittels Erklärung einseitig festgelegt hat (vgl. Art. 41 Abs. 3 SDÜ). Die auf dem Gebiet eines anderen Schengen-Staates agierenden Beamtinnen und Beamten unterstehen sowohl ihrem eigenen Recht als auch dem Recht des Einsatzstaates (vgl. Art. 41-43 SDÜ). Die Vertragsparteien ermöglichen im Rahmen ihrer Verfassung und ihrer Rechtsordnung, dass die kontrollierte Lieferung beim unerlaubten Handel mit Betäubungsmitteln angewandt werden kann (Abfangen von kriminellen Lieferungen und allenfalls Ersatz der Kuriere durch Polizeibeamtinnen und Polizeibeamte; Art. 73 SDÜ).

- Das Schengener Durchführungsübereinkommen erlaubt eine direkte postalische Zustellung gerichtlicher Urkunden (inkl. Ordnungsbussen, Art. 52 SDÜ), den direkten Behördenverkehr (Art. 53 SDÜ), die vereinfachte Auslieferung (Art. 66 SDÜ) und eine stellvertretende Strafvollstreckung auch gegen den Willen des Betroffenen (Art. 69 SDÜ). Es sieht in Bezug auf Fiskaldelikte lediglich eine Rechtshilfeverpflichtung im Bereich der indirekten Steuern (Verbrauchs- und Mehrwertsteuern, Zölle), jedoch nicht im Bereich der direkten Steuern vor (vgl. Art. 50 Abs. 1 SDÜ). Die Schweiz hat ein Opt-out für den Fall, dass eine Weiterentwicklung des Schengen-Besitzstands die Voraussetzung der doppelten Strafbarkeit für die Erledigung von Rechtshilfeersuchen um Durchsuchung und Beschlag- 
nahme abschwächt (Art. 51 SDÜ). Auf diese Weise wird das Bankkundengeheimnis bzw. die Steuerhinterziehung vor Strafverfolgung geschützt (Art. 7 Abs. 5 SAA).

Die vertraglichen Beziehungen zwischen der Schweiz und der EU folgen im Bereich der justiziellen Strafverfolgung weiterhin klassischen rechtshilferechtlichen Ansätzen. Folgerichtig wurde die Einführung des Europäischen Haftbefehls (Rahmenbeschluss 2002/584/ JI) für die Schweiz als nicht Schengen-relevant betrachtet. Eine weitergehende Assoziierung der Schweiz an den Rechtshilfeinstrumenten der EU, welche auf dem Grundsatz der gegenseitigen Anerkennung basieren, ist aber nicht ausgeschlossen. So hat die EU mit Norwegen und Island ein Abkommen abgeschlossen, welches die Grundsätze des Rahmenbeschlusses über den europäischen Haftbefehl auf diese Länder ausdehnt (Übereinkommen über das Übergabeverfahren zwischen den Mitgliedstaaten der EU und Island und Norwegen vom 28. Juni 2006, ABl 2006 L 292, 2). Bisher haben weder die Schweiz noch die EU ein Interesse an entsprechenden Verhandlungen bekundet (T. COTTIER et al., Rz. 920).

Ein zentrales Element der polizeilichen Zusammenarbeit ist das Schengener 261 Informationssystem (SIS), mit dem ein gemeinsames europaweites System zur Fahndung von Personen (gegen die ein Haftbefehl vorliegt, die vermisst werden, die für die Teilnahme an einem Gerichtsverfahren gesucht werden, gegen die verdeckt ermittelt wird oder gegen die ein Einreiseverbot vorliegt) sowie von Gegenständen (die verdeckt kontrolliert werden sollen oder die zur Sicherstellung oder Beweissicherung gesucht werden) eingerichtet wurde; neu ist auch die präventive Ausschreibung von Kindern, die von Entführung durch einen Elternteil bedroht sind, möglich. Das aktuelle System der zweiten Generation (SIS II) beruht auf der Verordnung (EU) 2018/1861 über die Einrichtung, den Betrieb und die Nutzung des Schengener Informationssystems (SIS) im Bereich der Grenzkontrollen und der Verordnung (EU) 2018/1862 über die Einrichtung, den Betrieb und die Nutzung des Schengener Informationssystems (SIS) im Bereich der polizeilichen Zusammenarbeit und der justiziellen Zusammenarbeit in Strafsachen (wobei die Übernahme dieser Verordnungen durch die Schweiz vom Parlament noch genehmigt werden muss). Es besteht aus einem zentralen System (C-SIS), in dem die Ausschreibungen der Schengen-Staaten gespeichert werden, und den nationalen Subsystemen (N-SIS), welche die Schnittstelle zu den nationalen Behörden und Datenbanken bilden. Dabei erfüllen die nationalen SIRENE-Büros eine wichtige Aufgabe (supplementary information request at the national entry): Sie nehmen Ausschreibungen vor und tauschen im Fall eines Treffers weitere Informationen aus. Die Agentur der EU für das Betriebsmanagement von IT-Grosssystemen im Raum der Freiheit, der Sicherheit und des Rechts (eu-LISA) ist für das Betriebsmanagement des Schengener Informationssystems zuständig (N. 241). 
Die Schweiz hat die Vorgaben zum Schengener Informationssystem im Bundesgesetz über die polizeilichen Informationssysteme des Bundes (BPI, SR 361) und in der Verordnung über den nationalen Teil des Schengener Informationssystems (N-SIS) und das SIRENEBüro (N-SIS-Verordnung, SR 362.0) umgesetzt. Der Zugang der Schweiz zum SIS - welches über 75 Mio. Einträge umfasst, wobei die Schweiz 2017 über das SIS 17000 Fahndungstreffer im In- und Ausland erzielte, welche der Verhinderung irregulärer Migration, der Aufdeckung von Straftaten und der Auffindung von Vermissten dienten (www.eda.admin.ch/ dea und Link zu Bilaterale Abkommen II (2004)/Schengen/Dublin) - ist für die Schweiz im Bereich der inneren Sicherheit essentiell (s. Bericht des Bundesrates zu den volkswirtschaftlichen und finanziellen Auswirkungen der Schengen-Assoziierung der Schweiz vom 21. Februar 2018, N. 244).

Die Abschaffung der Personen- und Sicherheitskontrollen an den Binnengrenzen setzt voraus, dass die Schengen-Staaten bei sicherheitsrelevanten Aspekten vergleichbare (Mindest-) Standards einhalten. Dazu gehört auch eine Angleichung des Waffenrechts. Es muss sichergestellt werden, dass in den Schengen-Staaten der Erwerb und der Besitz von Feuerwaffen und ihr Verbringen in einen anderen Staat wirksam kontrolliert werden können. Folgerichtig verpflichtet das Schengener Durchführungsübereinkommen (SDÜ) in «Titel III Polizei und Sicherheit» die Schengen-Staaten, ihre nationalen Gesetze über den Erwerb, den Besitz, den Vertrieb und das Überlassen von Feuerwaffen und Munition zu harmonisieren (Art. 77-91 SDÜ). Auch die Richtlinie 91/477/EWG (Waffenrichtlinie) regelt die Kontrolle des Erwerbs und des Besitzes von Waffen, wobei diese Richtlinie auf Art. 100a EWGV (Art. 114 AEUV) gestützt wurde, aber gleichwohl zum für die Schweiz bedeutsamen SchengenBesitzstand gehört. Der Erwerb einer Waffe durch Private erfordert einen Waffenerwerbsschein und muss in einem kantonalen Waffenregister eingetragen werden; dieses ist elektronisch zu führen. Es gilt eine Markierungspflicht für Feuerwaffen. Die Tätigkeit der Waffenhändlerin und des Waffenhändlers ist von einer Zulassung abhängig zu machen. Detaillierte Vorgaben regeln den Informationsaustausch zwischen den Schengen-Staaten.

Die Waffenrichtlinie 91/477/EWG wird periodisch angepasst, um auf technologische Entwicklungen und neue Bedrohungslagen zu reagieren. Solche Weiterentwicklungen gehören zum für die Schweiz bedeutsamen Schengen-Besitzstand. Ein Beispiel betraf die Übernahme der Richtlinie (EU) 2017/853 zur Änderung der Waffenrichtlinie 91/477/EWG. Mit dieser Neufassung - welche durch die Europäische Sicherheitsagenda von April 2015 vorgespurt wurde und nach den Terroranschlägen in Paris von November 2015 noch an Dringlichkeit gewann - wurde bezweckt, das Waffenrecht zu verschärfen. Dabei war es der Schweiz gelungen, den Inhalt der neuen Richtlinie aktiv mitzugestalten. Sie überzeugte die Vertreter der anderen Mitgliedstaaten, eine spezifisch auf die Verhältnisse in der Schweiz zugeschnittene - wenngleich allgemein formulierte - Bestimmung aufzunehmen und damit gleichsam einen Swiss Finish zu installieren. Gemäss Art. 6 Abs. 6 der Richtlinie können «Mitgliedstaaten, in denen allgemeine Wehrpflicht herrscht und in denen seit über 50 Jahren ein System der Weitergabe militärischer Feuerwaffen an Personen besteht, die 
die Armee nach Erfüllung ihrer Wehrpflicht verlassen, an diese Personen in ihrer Eigenschaft als Sportschützen eine Genehmigung erteilen, eine während des Wehrdienstes benutzte Feuerwaffe zu behalten». Die zuständige Behörde überprüft in regelmässigen Abständen, ob diese Personen keine Gefahr für die öffentliche Sicherheit darstellen. Diese Personen müssen zudem Mitglieder eines Schützenvereins sein und aktiv am Schiesssport teilnehmen. Der EuGH beurteilte diesen Swiss Finish im Rahmen einer gegen die Richtlinie gerichteten Nichtigkeitsklage als nicht diskriminierend, weil diese Bestimmung «sowohl der Kultur als auch den Traditionen der Schweizerischen Eidgenossenschaft sowie dem Umstand Rechnung [trägt], dass dieser Staat aufgrund dieser Traditionen über die Erfahrung und die Fähigkeit verfügt, die betreffenden Personen und Waffen nachzuverfolgen und zu überwachen, eine Erfahrung und eine Fähigkeit, die vermuten lassen, dass die von der angefochtenen Richtlinie verfolgten Ziele der öffentlichen Sicherheit trotz dieser Ausnahme erreicht werden» (Urteil Tschechische Republik/Parlament und Rat, C-482/17, EU:C:2019:1035, Rn. 166); eine bemerkenswerte Begründung. In der Schweiz wurde gegen die Übernahme der Richtlinie das Referendum ergriffen. $63.7 \%$ der Stimmberechtigten votierten für die Übernahme und die damit einhergehenden Änderungen des Waffengesetzes (SR 514.54; s. zu dieser Abstimmung auch N. 93).

Die Zusammenarbeit der Polizeibehörden in der EU im Bereich des automatisierten Informationsaustauschs von DNA-Profilen, Fingerabdruckdaten und Daten zu Fahrzeugen und deren Haltern beruht im Wesentlichen auf den Beschlüssen 2008/615/JI und 2008/616/JI (Prümer Zusammenarbeit). Die Prümer Zusammenarbeit gilt nicht als Weiterentwicklung des Schengen-Besitzstands; der Übernahmeprozess des Schengen-Assoziierungsabkommens wurde hier nicht aktiviert. Die EU und die assoziierten Schengen-Staaten haben stattdessen entschieden, die Beteiligung dieser Staaten an der Prümer Zusammenarbeit gestützt auf ein separates Abkommen zu regeln. Auf diese Weise erhält auch die Schweiz Zugang zu solchen Daten. Das Abkommen wurde am 27. Juni 2019 unterzeichnet. Zurzeit befindet es sich zusammen mit der Umsetzungsgesetzgebung im Genehmigungsprozess im Parlament (s. Erläuternder Bericht zur Genehmigung des Abkommens zur Vertiefung der grenzüberschreitenden Zusammenarbeit (Prümer Zusammenarbeit) und des EurodacProtokolls zwischen der Schweiz und der EU (...) vom 13. Dezember 2019). Die Teilnahme an der Prümer Zusammenarbeit ist auch eine Voraussetzung dafür, dass die Strafverfolgungsbehörden in der Schweiz Zugriff auf die in der Eurodac-Datenbank gespeicherten Daten erhalten (www.eda.admin.ch/dea und Link zu Bilaterale Abkommen und Kooperationen ab 2004/Polizeizusammenarbeit; N. 291).

\section{(f) Frontex, ISF - Grenzen und Visa}

Die Schengen-Staaten sind verpflichtet, die zur wirksamen Sicherung der Aussengrenzen und zur Gewährleistung effizienter Grenzkontrollen notwendigen Vorkehren zu treffen. Dazu gehört, geeignete Kräfte in ausreichender 
Zahl und angemessene Mittel in ausreichendem Umfang zur Verfügung zu stellen (Art. 15 SGK). Dabei unterstützen die Staaten einander und pflegen eine enge und ständige Zusammenarbeit. Die EU hat zum Zweck der effektiven Koordination und operativen Zusammenarbeit diverse Instrumente entwickelt. Dazu gehören Frontex und der Fonds für die innere Sicherheit. Die Schweiz beteiligt sich im Rahmen der Schengen-Assoziierung an diesen Einrichtungen.

Frontex wurde 2004 mit dem Ziel gegründet, die Schengen-Staaten beim Schutz der Aussengrenzen zu unterstützen. Sie hat ihren Sitz in Warschau. 2016 wurde Frontex in Europäische Agentur für die Grenz- und Küstenwache umbenannt. Aktuell finden sich die rechtlichen Vorgaben in der Verordnung (EU) 2019/1896 (wobei die Übernahme dieser Verordnung durch die Schweiz vom Parlament noch genehmigt werden muss; aktuell beteiligt sich die Schweiz gestützt auf die in der EU nicht mehr einschlägigen Verordnungen (EU) Nr. 1052/2013 und (EU) 2016/1624; vgl. Botschaft Frontex, passim). Das Mandat und die finanziellen Ressourcen von Frontex wurden über die Jahre sukzessive erweitert. Sie unterstützt die Mitgliedstaaten bei der Kontrolle und Überwachung der Aussengrenzen mit operativen Massnahmen, mit dem Sammeln und Verarbeiten von Informationen, mit der Förderung von Ausbildungsmassnahmen und mit der Erleichterung des Erfahrungsaustauschs. Auf Ersuchen eines Schengen-Staates koordiniert Frontex gemeinsame Operationen der Schengen-Staaten. Darüber hinaus kann der Rat die Agentur in Fällen, in denen die Wirksamkeit der Aussengrenzkontrollen so weit reduziert ist, dass das Funktionieren des Schengen-Raums gefährdet ist, zum Handeln anweisen (Art. 42 der Verordnung (EU) 2019/1896). Sofern nötig wird die neu eingerichtete ständige Reserve - geplant im Umfang von bis zu 10000 Expertinnen und Experten - im Rahmen von Grenzverwaltungsteams, Teams zur Unterstützung der Migrationsverwaltung und Rückkehrteams (sog. «Teams») für gemeinsame Aktionen sowie für Soforteinsätze zu Grenzsicherungszwecken, Rückkehraktionen und Rückkehreinsätzen eingesetzt. Das Mandat umfasst mittlerweile auch die Bekämpfung von grenzüberschreitender Kriminalität sowie Suchund Rettungseinsätze für Menschen in Seenot, wenn derartige Aufgaben im Zusammenhang mit der Überwachung der Seegrenzen erforderlich werden. EUROSUR stellt einen integrierten Rahmen für den Informationsaustausch und die operative Zusammenarbeit innerhalb der Europäischen Grenz- und Küstenwache dar. Die Modalitäten der Beteiligung der Schweiz an Frontex wird in einer speziellen Vereinbarung geregelt (SR 362.313), wobei sich die Schweiz nicht nur finanziell, sondern auch personell an Einsätzen unter der Ägide von Frontex beteiligt. Diese Vereinbarung listet auch die Sachbereiche 
auf, bei denen die Schweiz - als Ausnahme zur Regel, wonach Drittstaaten aus Gründen der Autonomie des EU-Rechts und der im EUV/AEUV geregelten Zuständigkeiten der Organe der EU und ihrer weiteren Einrichtungen beim decision making nicht beteiligt sind - im Verwaltungsrat stimmberechtigt ist (v.a. operative Fragen).

Frontex wurde seit der Aufnahme ihrer operativen Tätigkeit wiederholt vorgeworfen, bei der Unterstützung und Durchführung von Operationen die grundrechtlichen Mindeststandards nicht zu respektieren. Ein Beispiel betrifft die verheerende Praxis, Flüchtlingsboote daran zu hindern, Häfen von Schengen-Staaten anzulaufen und die Boote stattdessen ins offene Meer zurückzustossen (push backs; dazu Human Rights Watch, passim). Ähnliche Vorwürfe gibt es auch in Bezug auf Rückweisungen an den Landgrenzen (NZZ vom 27. Juni 2020, S. 5). Vor diesem Hintergrund war der EU-Gesetzgeber auch bei der Neufassung der Frontex-Verordnung (EU) 2019/1896 darauf bedacht zu unterstreichen, dass die Europäische Grenz- und Küstenwache - d.h. die zuständigen nationalen Behörden und Frontex - ihre Aufgaben «unter uneingeschränkter Wahrung der Grundrechte» erfüllt (Art. 1); Frontex wird ausdrücklich zur «Überwachung der Einhaltung der Grundrechte bei allen ihren Tätigkeiten an den Aussengrenzen und bei Rückkehraktionen» verpflichtet (Art. 10 Abs. 1 lit. e). 2014 hat die EU mit der Verordnung (EU) Nr. 656/2014 zudem die Rahmenbedingungen für die Überwachung der Seeaussengrenzen im Rahmen der von Frontex an den Aussengrenzen koordinierten operativen Zusammenarbeit konkretisiert. Es ist allerdings nicht immer offenkundig, welche grundrechtlichen Gewährleistungen - die Genfer Abkommen über die Rechtsstellung der Flüchtlinge von 1951 und das dazugehörige New Yorker Protokoll von 1967, die EMRK, weitere völkerrechtliche Instrumente, die Grundrechtecharta und/oder nationale Grundrechtskataloge - in einem konkreten Fall greifen. Auch bei staatlichen Verantwortlichkeiten tritt die unionale Dimension dazu, wenn nationale Behörden «bei der Durchführung des Rechts der Union» handeln (Art. 51 GRC; s. zur Frage der Anwendbarkeit der GRC auch in der Schweiz N. 246). Für die Verpflichtung zur Beachtung zumindest des Flüchtlingsrechts ist die Ausübung von Hoheitsbefugnissen entscheidend, nicht die Anknüpfung an ein Territorium (s. zum Ganzen J. STERN/T. TOHIDIPUR, Rz. 58-62; S. Neumann, passim; J. SeEHASE, passim; EU Fundamental Rights Agency, Fundamental rights at Europe's southern sea borders, Luxembourg 2013).

Die EU richtete 2007 den Aussengrenzenfonds ein (Entscheidung Nr. 574/ 2007/EG). Damit wurde ein Instrument zur Unterstützung der mit den Aussengrenzkontrollen verknüpften Aktivitäten der Schengen-Staaten geschaffen. Die Schweiz beteiligte sich an diesem Fonds durch die Übernahme der Rechtsgrundlagen und den Abschluss einer Zusatzvereinbarung (SR 0.362.312). Der Aussengrenzenfonds lief Ende 2013 aus. Als Nachfolgeinstrument schuf die EU den Fonds für die innere Sicherheit - Grenzen und Visa (ISF - Grenzen und Visa, Verordnung (EU) Nr. 515/2014). Seine Laufzeit beträgt 2014-2020. Damit wird wie bereits unter dem Aussengrenzenfonds bezweckt, SchengenStaaten, welche aufgrund ausgedehnter Land- oder Seegrenzen sowie bedeutender internationaler Flughäfen hohe Kosten für den Schutz der SchengenAussengrenzen tragen, mit projektgebundenen Mitteln zu unterstützen. Die 
Schweiz hat sich auch beim ISF - Grenzen und Visa beteiligt (Abkommen über zusätzliche Regeln in Bezug auf das Instrument für die finanzielle Unterstützung für Aussengrenzen und Visa im Rahmen des Fonds für die innere Sicherheit für den Zeitraum von 2014 bis 2020 von 2018, SR 0.362.314).

Die Finanzausstattung für die Durchführung des IFS - Grenzen und Visa beträgt insgesamt 2.76 Mia. EUR (Art. 5 der Verordnung (EU) Nr. 515/2014). Die Beiträge der assoziierten Staaten erhöhten diesen Betrag noch leicht: Die Schweiz - als Land mit Aussengrenzen nur an den internationalen Flughäfen offenkundig eine Nettozahlerin - rechnete mit Kosten von rund 20 Mio. CHF jährlich (Botschaft Fonds für die innere Sicherheit, S. 5085).

\section{Betrugsbekämpfung, Europol, Eurojust}

Die Schweiz und die EU haben ergänzend zum Schengen-Assoziierungsabkommen und zu weiteren Verträgen über die polizeiliche Zusammenarbeit und die Rechtshilfe in Strafsachen zwischen europäischen Staaten (N. 259-263) die folgenden Abkommen zur Betrugsbekämpfung und Zusammenarbeit in Strafsachen abgeschlossen.

\section{(a) Betrugsbekämpfung}

Die EU und ihre Mitgliedstaaten sind seit der Inkraftsetzung des Vertrags von Maastricht 1993 verpflichtet, Betrügereien und sonstige gegen die finanziellen Interessen der Union gerichtete rechtswidrige Handlungen mit abschreckenden Massnahmen zu bekämpfen (Art. 209a EGV, Art. 325 AEUV). 1999 wurde zu diesem Zweck das Europäische Amt für Betrugsbekämpfung (OLAF) gegründet (Beschluss der Kommission 1999/352/EG, Beschluss der Kommission 2013/ 478/EU, Verordnung (EU, Euratom) Nr. 883/2013; s. zur Errichtung der Europäischen Staatsanwaltschaft N. 273). Zur Bekämpfung von Betrügereien, die sich gegen die finanziellen Interessen der Union richten, ergreifen die Mitgliedstaaten die gleichen Massnahmen, die sie auch zur Bekämpfung von Betrügereien ergreifen, die sich gegen ihre eigenen finanziellen Interessen richten (Art. 325 Abs. 2 AEUV). 2017 erliess die EU mit der Richtlinie (EU) 2017/ 1371 über die strafrechtliche Bekämpfung von gegen die finanziellen Interessen der Union gerichtetem Betrug zudem Mindestvorschriften für die Definition von Straftatbeständen und Strafen zur Bekämpfung von Betrug und sonstigen gegen die finanziellen Interessen der Union gerichteten rechtswidrigen Handlungen.

Die Europäische Kommission veröffentlicht periodisch eine Betrugsbekämpfungsstrategie. Dies geschah letztmals 2019 (COM(2019) 196 final). Dieses Strategiepapier ist für die Dienststellen der Kommission und die Exekutivagenturen bei der Bekämpfung von Betrug und Korruption zum Nachteil der finanziellen Interessen der EU massgeblich. 
1997 vereinbarten die Schweiz und die EG in einem Zusatzprotokoll zum Freihandelsabkommen von 1972, sich einander Amtshilfe bei der Sicherstellung der ordnungsgemässen Anwendung des Zollrechts, insbesondere bei der Verhütung und der Aufdeckung von Zuwiderhandlungen gegen das Zollrecht und bei Ermittlungen im Zollbereich, zu leisten (SR 0.632.401.02). Der EG war es in der Folge ein Anliegen, die Zusammenarbeit bei der Betrugsbekämpfung noch weiter zu verstärken und auszubauen. Sie hatte insbesondere Betrügereien im Zusammenhang mit dem Zigarettenschmuggel im Blick. Dadurch entgingen der EU beträchtliche Mehrwertsteuereinnahmen. Die fehlende Zusammenarbeit der Schweiz im Bereich der Fiskaldelikte erlaubte es den Drahtziehern, ihre Operationen aus der Schweiz zu leiten und Erträge aus den kriminellen Aktionen zumindest vorübergehend auf Konten von Schweizer Banken zu lagern (T. COTTIER et al., Rz. 930).

Die Schweiz und die EG unterzeichneten 2004 das Abkommen über die Zusammenarbeit zur Bekämpfung von Betrug und sonstigen rechtswidrigen Handlungen, die ihre finanziellen Interessen beeinträchtigen (Betrugsbekämpfungsabkommen, SR 0.351.926.81). Es handelt sich um ein gemischtes Abkommen. Die Schweiz und die EG haben das Abkommen ratifiziert. Auf Seiten der EU-Mitgliedstaaten fehlt noch die Ratifizierung von Irland. Die Schweiz wendet das Abkommen seit 2009 provisorisch gegenüber denjenigen EU-Mitgliedstaaten an, welche das Abkommen ratifiziert und erklärt erhaben, dass sie es gegenüber der Schweiz ebenfalls provisorisch anwenden. Das Betrugsbekämpfungsabkommen bezweckt, die Amts- und Rechtshilfe in Strafsachen zwischen den Vertragsparteien auszudehnen, um die im Abkommen genannten rechtswidrigen Handlungen zu bekämpfen (Art. 1). Zu diesen Handlungen gehören Delikte im Bereich der Zölle, der indirekten Steuern (Mehrwertsteuern, besondere Verbrauchssteuern auf Alkohol, Tabak, Mineralöl etc.), der Subventionen, der öffentlichen Ausschreibungen und der mit diesen Delikten verknüpften Geldwäschereitatbeständen; die direkten Steuern (z.B. Einkommens-, Vermögens- und Gewinnsteuern) sind vom Anwendungsbereich des Abkommens ausgeschlossen (Art. 2; www.eda.admin.ch/dea und Link zu Bilaterale Abkommen II (2004)/Betrugsbekämpfung). In Bezug auf die zu leistende Amts- und Rechtshilfe baut das Abkommen auf vorbestehenden Übereinkommen zwischen den Vertragsparteien auf, namentlich auf dem Zusatzprotokoll über die gegenseitige Amtshilfe im Zollbereich von 1997 (N. 269), dem Europäischen Übereinkommen über die Rechtshilfe in Strafsachen von 1959 (N. 259) und dem Übereinkommen über Geldwäsche sowie Ermittlung, Beschlagnahme und Einziehung von Erträgen aus Straftaten von 1990 (Art. 7 und Art. 25). Der Amts- und Rechtshilfeverkehr ist nicht nur mit 
den Verwaltungs- und Justizbehörden der EU-Mitgliedstaaten, sondern auch mit den zuständigen Einrichtungen und Stellen der EU möglich. Für den Umfang der zu leistenden Hilfe ist in erster Linie das Recht der Vertragsparteien massgebend, wobei die Behörden so verfahren, als ob sie in Erfüllung eigener Aufgaben oder auf Ersuchen einer anderen Behörde derselben Vertragspartei handeln würden (Art. 9 und Art.15). Rechtshilfeersuchen um Durchsuchung und Beschlagnahme sind zulässig, wenn die dem Ersuchen zugrunde liegende Tat nach dem Recht beider Vertragsparteien strafbar ist (doppelte Strafbarkeit, Art. 31). Das Betrugsbekämpfungsabkommen ermöglicht den direkten Behördenverkehr (Art.27) und die direkte postalische Zustellung von Verfahrensunterlagen an Personen (Art. 28). Ersuchen um Bank- und Finanzauskünfte sind besonders geregelt (Art. 32 Abs. 2). Das Betrugsbekämpfungsabkommen ermöglicht kontrollierte Lieferungen (Art. 33) und die Übergabe von Sachen, insbesondere Vermögenswerten, zum Zweck der Einziehung oder Rückgabe an die Berechtigten (Art. 34).

\section{(b) Europol}

Die EU-Mitgliedstaaten gründeten 1995 das Europäische Polizeiamt (Europol). Anfänglich stand Europol ausserhalb des institutionellen Rahmens der EU. Erst mit dem Vertrag von Lissabon wurde Europol 2009 formell in die EU integriert (Art. 88 AEUV, Beschluss 2009/371/JI). Sitz von Europol ist Den Haag. Seit 2016 stellt die Verordnung (EU) 2016/794 über die Agentur der Europäischen Union für die Zusammenarbeit auf dem Gebiet der Strafverfolgung (Europol) die Grundlage dar; im Zug der Neufassung der Rechtsgrundlagen wurde Europol auch als eigenständige Agentur konstituiert. Europol fördert die Zusammenarbeit der Strafverfolgungsbehörden in der EU bei der Bekämpfung von schwerer grenzüberschreitender Kriminalität. Im Vordergrund steht die Erhebung, die Speicherung, die Verarbeitung und der Austausch von Informationen. Europol-Personal kann an den Tätigkeiten von gemeinsamen Ermittlungsgruppen mitwirken (Art. 5 der Verordnung (EU) 2016/794). Europol besitzt keine eigenen Ermittlungsbefugnisse.

2722004 schlossen die Schweiz und Europol ein Abkommen (SR 0.362.2). Es trat 2006 in Kraft und bezweckt die verstärkte Zusammenarbeit der EU-Mitgliedstaaten durch Europol mit der Schweiz bei der Bekämpfung schwerwiegender Formen der internationalen Kriminalität, insbesondere durch den Austausch von strategischen und operativen Informationen sowie regelmässige Kontakte zwischen Europol und der Schweiz auf allen Ebenen (Art. 2). Die Zusammenarbeit bezieht sich auf den illegalen Drogenhandel, den illegalen Handel mit 
nuklearen und radioaktiven Substanzen, den Menschenschmuggel (Schlepperwesen), den Menschenhandel, die Motorfahrzeugkriminalität, Straftaten, die im Rahmen von terroristischen Handlungen begangen werden, die Geldfälschung sowie die mit diesen Kriminalitätsformen verbundene Geldwäscherei (Art. 3). 2008 und 2018 wurden - parallel zur Weiterentwicklung der Zusammenarbeit in der EU - weitere Formen der Kriminalität aufgenommen; dazu gehören Tötungen, illegaler Organhandel, Entführungen und Geiselnahmen, Produktpiraterie, illegaler Waffenhandel, Korruption, Völkermord, Verbrechen gegen die Menschlichkeit, Kriegsverbrechen sowie gewisse Formen der Wirtschaftskriminalität (SR 0.362.21, SR 0.362.22). Das Abkommen ermöglicht der Schweiz und Europol, Expertenwissen auszutauschen, an Ausbildungsaktivitäten teilzunehmen und sich bei Ermittlungen gegenseitig zu beraten und zu unterstützen. Zur Koordination und Erleichterung der Zusammenarbeit betreibt die Schweiz in Den Haag ein Verbindungsbüro mit zwei Polizeiattachés und einer Verbindungsoffizierin oder einem Verbindungsoffizier des Grenzwachtkorps (www.eda.admin.ch/dea und Link zu Bilaterale Abkommen und Kooperationen ab 2004/Europol).

Die Polizeikooperation im Rahmen von Europol ergänzt die Zusammenarbeit der Schweiz mit den Nachbarstaaten und diejenige im Rahmen von Interpol (N. 259). Der Informationsaustausch belief sich 2018 auf 16391 operative Meldungen vor allem in den Bereichen des Menschenhandels und -schmuggels, des illegalen Drogenhandels, der Cyberkriminalität, des Betrugs sowie anderer Formen schwerer Kriminalität und Terrorismus (www.eda.admin.ch/dea und Link zu Bilaterale Abkommen und Kooperationen ab 2004/ Europol).

\section{(c) Eurojust}

Die EU errichtete 2002 Eurojust als Einheit für justizielle Zusammenarbeit zur Verstärkung der Bekämpfung der schweren Kriminalität (Beschluss des Rates 2002/187/JI). Mit dem Vertrag von Lissabon wurde Eurojust primärrechtlich verankert (Art. 85 und Art. 86 AEUV). 2018 wurde Eurojust neu als Agentur für justizielle Zusammenarbeit in Strafsachen konstituiert (Verordnung (EU) 2018/1727). Eurojust unterstützt und verstärkt die Koordinierung und Zusammenarbeit zwischen den nationalen Behörden, welche für die Ermittlung und Verfolgung von schwerer Kriminalität zuständig sind, von der zwei oder mehr Mitgliedstaaten betroffen sind oder die eine Verfolgung auf gemeinsamer Grundlage erfordert; Eurojust stützt sich dabei auf die von den Behörden der Mitgliedstaaten, von Europol (N. 271), von der Europäischen Staatsanwaltschaft (s. sogleich) und von OLAF (N. 268) durchgeführten Operationen und gelieferten Informationen (Art. 2 der Verordnung (EU) 2018/1727). Eurojust verfügt über jeweils ein nationales Mitglied pro Mitgliedstaat mit dem Status 
einer Staatsanwältin oder eines Staatsanwalts oder einer Richterin oder eines Richters, das von dem betreffenden Mitgliedstaat entsandt wird. Der Sitz von Eurojust ist Den Haag. Das 1998 eingerichtete Europäische Justizielle Netz justizieller Kontaktstellen zwischen den Mitgliedstaaten unterstützt Eurojust (Beschluss 2008/976/JI).

2017 einigten sich 20 Mitgliedstaaten im Rahmen einer verstärkten Zusammenarbeit auf die Errichtung der Europäischen Staatsanwaltschaft (EUStA, Art. 86 AEUV, Verordnung (EU) 2017/1939). Mittlerweile sind 22 Mitgliedstaaten beteiligt. Die EUStA ist zuständig für die strafrechtliche Untersuchung und Verfolgung sowie die Anklageerhebung in Bezug auf Personen, die Straftaten zum Nachteil der finanziellen Interessen der Union gemäss Richtlinie (EU) 2017/1371 (N. 268) begangen haben. Hierzu führt die EUStA Ermittlungen, ergreift Strafverfolgungsmassnahmen und nimmt vor den Gerichten der Mitgliedstaaten die Aufgaben der Staatsanwaltschaft wahr (Art. 4 der Verordnung (EU) 2017/1939).

2008 schlossen die Schweiz und Eurojust ein Abkommen (SR 0.351.6). Es trat 2011 in Kraft und bezweckt die Verstärkung der Zusammenarbeit zwischen der Schweiz und Eurojust im Kampf gegen schwere Formen der internationalen Kriminalität (Art. 2). Die vom Abkommen erfassten Zuständigkeitsbereiche betreffen die Kriminalitätsformen und Straftaten, welche in die Zuständigkeit von Europol fallen, sowie die Computerkriminalität, den Betrug und die Korruption sowie alle Straftaten, welche die finanziellen Interessen der EU berühren, das Waschen von Erträgen aus Straftaten, die Umweltkriminalität sowie die Beteiligung an einer kriminellen Vereinigung gemäss der Gemeinsamen Massnahme 98/733/JI. Das Abkommen erleichtert die internationale Rechtshilfe, die Erledigung von Auslieferungsgesuchen und die Koordination mit den anderen nationalen Behörden. Die Schweiz arbeitet mit Eurojust über eine nationale Kontaktstelle zusammen; das Bundesamt für Justiz (BJ) nimmt diese Aufgabe wahr (Art. 7). Das Abkommen ermöglicht es der Schweiz, eine Verbindungsstaatsanwältin oder einen Verbindungsstaatsanwalt zu Eurojust zu entsenden; die Schweiz macht von diesem Recht seit 2015 Gebrauch (Art. 6; www.eda.admin.ch/dea und Link zu Bilaterale Abkommen und Kooperationen ab 2004/Eurojust).

2018 wurden Eurojust 3317 Fälle gemeldet (www.eda.admin.ch/dea und Link zu Bilaterale Abkommen und Kooperationen ab 2004/Eurojust). Die Schweiz hat nach Massgabe des Eurojust-Abkommens Zugang zu diesen Informationen. Demgegenüber gibt es keine Bestrebungen, auch mit der Europäischen Staatsanwaltschaft (N. 273) zusammenzuarbeiten. 


\section{Dublin}

Die EWG-Mitgliedstaaten unterzeichneten 1990 das Übereinkommen über die Bestimmung des zuständigen Staates für die Prüfung eines in einem Mitgliedstaat der EG gestellten Asylantrags (Dubliner Übereinkommen). Dieser völkerrechtliche Vertrag, welcher formell ohne Bezug zum Gemeinschaftsrecht abgeschlossen wurde, trat 1997 in Kraft. Zentrales Anliegen war es zu verhindern, dass asylsuchende Personen, die keinen Anspruch auf Asyl haben, über Jahre hinweg untertauchen oder von einem Dublin-Staat in einen anderen gelangen und wiederholt Asylgesuche stellen können.

Mit dem Vertrag von Amsterdam, welcher 1999 in Kraft trat, wurde eine Kompetenzgrundlage der EG zum Erlass von Massnahmen im Asylbereich geschaffen. Heute findet sich die primärrechtliche Grundlage in Art. 78 AEUV; demnach entwickelt die EU eine gemeinsame Politik im Bereich Asyl, subsidiärer Schutz und vorübergehender Schutz, mit der jedem Drittstaatsangehörigen, der internationalen Schutz benötigt, ein angemessener Status angeboten und die Einhaltung des Non Refoulement-Gebots gewährleistet werden soll, inkl. der ausdrücklichen Verpflichtung, das Genfer Abkommen von 1951 und das dazugehörige New Yorker Protokoll von 1967 über die Rechtsstellung der Flüchtlinge sowie die anderen relevanten völkerrechtlichen Verträge zu respektieren. In den Jahren 2000 und 2003 wurden die Verordnung (EG) Nr. 2725/2000 über die Einrichtung von «Eurodac» für den Vergleich von Fingerabdrücken zum Zwecke der effektiven Anwendung des Dubliner Übereinkommens und die Verordnung (EG) Nr. 343/2003 zur Festlegung der Kriterien und Verfahren zur Bestimmung des Mitgliedstaats, der für die Prüfung eines von einem Drittstaatsangehörigen in einem Mitgliedstaat gestellten Asylantrags zuständig ist (Dublin II-Verordnung), in Kraft gesetzt. Damit wurden die Grundlagen geschaffen für das geltende Dublin-System. Die beiden genannten Verordnungen wurden in der Zwischenzeit neugefasst und durch die Verordnung (EU) Nr. 603/2013 (Eurodac-Verordnung) und die Verordnung (EU) Nr. 604/2013 (Dublin III-Verordnung) abgelöst. Heute sind alle EU-Mitgliedstaaten sowie die EFTA-Staaten Island, Liechtenstein, Norwegen und die Schweiz - letztere als assoziierte Staaten - Teil des Dublin-Systems.

Die EU verfolgt - über die Festlegung der Kriterien und Verfahren zur Bestimmung der Zuständigkeit zur Prüfung von Asylanträgen (Dublin-System) hinaus - das Ziel, ein Gemeinsames Europäisches Asylsystem (GEAS) zu errichten. Zu diesem Zweck wurden insbesondere die Richtlinie 2013/32/EU zu gemeinsamen Verfahren für die Zuerkennung und Aberkennung des internationalen Schutzes (Asylverfahrensrichtlinie), die Richtlinie 2013/ 33/EU zur Festlegung von Normen für die Aufnahme von Personen, die internationalen Schutz beantragen (Asylaufnahmerichtlinie), und die Richtlinie 2011/95/EU über Nor- 
men für die Anerkennung von Drittstaatsangehörigen oder Staatenlosen als Personen mit Anspruch auf internationalen Schutz, für einen einheitlichen Status für Flüchtlinge oder für Personen mit Anrecht auf subsidiären Schutz und für den Inhalt des zu gewährenden Schutzes (Qualifikationsrichtlinie) erlassen. Diese Richtlinien sind für die Schweiz nicht einschlägig; sie gehören nicht zum Dublin-Besitzstand, der von den assoziierten Staaten übernommen werden musste. Dasselbe gilt für den mit der Verordnung (EU) Nr. 516/2014 eingerichteten Asyl-, Migrations- und Integrationsfonds; damit wird bezweckt, einen Beitrag zur effizienten Steuerung der Migrationsströme und zur Durchführung und Weiterentwicklung der GEAS zu leisten.

277 Das Dublin-System ist in den letzten Jahren stark unter Druck geraten. Im Herbst 2015 wurde es angesichts der präzedenzlos grossen Flüchtlingsströme nach Griechenland und Italien faktisch ausser Kraft gesetzt, als Deutschland Flüchtlinge vor allem aus dem Nahen Osten (Syrien) nicht mehr in den gemäss der Dublin III-Verordnung für die Prüfung eines Asylantrags zuständigen Ersteinreisestaat zurückschickte, sondern solche Anträge selbst prüfte. Bestrebungen, das System im Geist der Solidarität zu reformieren und eine gerechte Verteilung der Lasten und Verantwortlichkeiten vorzunehmen (vgl. Art. 80 AEUV), sind bis heute gescheitert (s. für eine Auslegeordnung Mitteilung der Kommission vom 6. April 2016, COM(2016) 197 final). Selbst der moderate Beschluss des Rates, 120000 Antragstellende, welche einen Antrag auf internationalen Schutz in Italien oder Griechenland stellten, in andere Mitgliedstaaten umzusiedeln, stiess mancherorts auf Widerstand und wurde nicht konsequent umgesetzt (Beschluss (EU) 2015/1523, Beschluss (EU) 2015/ 1601; s. zur Rechtmässigkeit Urteil Slowakei und Ungarn/Rat, C-643/15 und C-647/15, EU:C:2017:631; Urteil Kommission/Polen, Tschechische Republik und Ungarn, C-715/17, C-718/17 und C-719/17, EU:C:2020:257; zum Ganzen S. Progin-Theuerkauf/V. Zufferey, passim). Stattdessen führten Frankreich, Österreich, Deutschland, Dänemark, Schweden und Norwegen an den Binnengrenzen temporär Grenzkontrollen ein, um auf die Defizite des Grenzschutzes in Griechenland und die damit einhergehende unrechtmässige Einreise von Personen in den Schengen-Raum zu reagieren (N. 247). Ein signifikanter Rückgang der Flüchtlingsströme wurde erst durch einen Deal der EU-Mitgliedstaaten mit der Türkei zur Beendigung der irregulären Migration in die EU ermöglicht (Erklärung EU-Türkei vom 18. März 2016; s. zur Qualifikation dieser Erklärung als Handlung, welche nicht dem Europäischen Rat zugerechnet werden konnte, womit eine dagegen gerichtete Nichtigkeitsklage unzulässig war, Urteil NF/Europäischer Rat, T-192/16, EU:C:2017:762).

Die erwähnten Beschlüsse (EU) 2015/1523 und (EU) 2015/1601 wurden ausserhalb des für die Schweiz relevanten Dublin-Besitzstands erlassen (Art. 78 Abs. 3 AEUV). Aus diesem Grund war die Schweiz nicht verpflichtet, sie nach Massgabe des Dublin-Assoziierungs- 
abkommens zu übernehmen. Die Schweiz erklärte gleichwohl, sich freiwillig an gewissen Massnahmen zu beteiligen (Pressemitteilung des EJPD vom 22. September 2015, Justizund Innenministertreffen der EU: Schweiz begrüsst Entscheid zu Umverteilung).

Die Europäische Kommission hat angekündigt, demnächst einen Vorschlag für eine grundlegende Revision des Dublin-Systems zu unterbreiten. Eine allfällige Novellierung der Dublin III-Verordnung müsste nach Massgabe des Dublin-Assoziierungsabkommens auch von der Schweiz übernommen werden. Als Korrelativ zu dieser Verpflichtung besteht für die Schweiz die Möglichkeit, sich bei der Ausarbeitung des Vorschlags durch die Kommission aktiv einzubringen (vgl. Art. 2 DAA; N. 94) - womit eine (Mit-) Verantwortung der Schweiz für die Gestaltung der Flüchtlingspolitik im paneuropäischen Kontext einhergeht.

\section{(a) Dublin-Assoziierungsabkommen: Im Allgemeinen}

Die Schweiz und die EG unterzeichneten 2004 das Abkommen über die Kriterien und Verfahren zur Bestimmung des zuständigen Staates für die Prüfung eines in einem Mitgliedstaat oder in der Schweiz gestellten Asylantrags. Es trat 2008 in Kraft und ermöglicht der Schweiz, mitgliedstaatsähnlich am DublinBesitzstand und an seiner weiteren Entwicklung teilzunehmen (s. zum finanziellen Beitrag der Schweiz an die EU im Rahmen der Schengen/Dublin-Assoziierung N. 244). Die Schweiz ist verpflichtet, die Verordnungen des DublinBesitzstands umzusetzen und im Rahmen ihrer Beziehungen zu den EUMitgliedstaaten anzuwenden; letztere wenden die genannten Verordnungen ihrerseits in ihren Beziehungen zur Schweiz an (Art. 1). Dabei handelt es sich um die Verordnung (EU) Nr. 604/2013 (Dublin III-Verordnung), die Verordnung (EU) Nr.603/2013 (Eurodac-Verordnung) sowie die dazugehörigen Durchführungsverordnungen.

Es ist für das ordnungsgemässe Funktionieren des Dublin-Besitzstands zentral, dass neue Dublin-relevante Rechtsakte der EU zeitnah und unkompliziert in das Dublin-Abkommen inkorporiert werden. Zu diesem Zweck enthält das Abkommen einen Mechanismus, neue Rechtsakte dynamisch in die Anhänge zu übernehmen (N. 93). Aus dem gleichen Grund legen die schweizerischen Behörden den Dublin-Besitzstand im Licht der einschlägigen Praxis des EuGH aus (N. 83). Die rechtlichen Schicksale der Schengen- und Dublin-Assoziierungsabkommen sind miteinander verknüpft: Das eine Abkommen wird nur angewendet, wenn auch das andere angewendet wird (sog. «kleine» Guillo- 
tine-Klausel [im Gegensatz zur «grossen» Guillotine, welche die Bilateralen I aneinander kettet]; Art. 15 Abs. 4 SAA, Art. 14 Abs. 2 DAA; s. zur - wie von der EU betont - faktischen Verknüpfung mit dem FZA N. 245). Staaten die Grundrechte respektieren. Entsprechend weist auch die Präambel des Dublin-Abkommens darauf hin, dass die Zusammenarbeit der Schweiz und der EU in diesem Bereich «auf den Grundsätzen der Freiheit, der Demokratie, der Rechtsstaatlichkeit und der Achtung der Menschenrechte, wie sie insbesondere in der Europäischen Konvention zum Schutze der Menschenrechte und Grundfreiheiten vom 4. November 1950 gewährleistet sind, beruht». Die Erwägungsgründe der Dublin III-Verordnung betonen die Bedeutung der Grundrechtecharta bei der Auslegung der Verordnung (vgl. v.a. Ziff. 39). Art. 3 Abs. 2 der Dublin III-Verordnung verpflichtet die Dublin-Staaten, einen Antragsteller nicht an den zunächst als zuständig bestimmten Staat zu überstellen, sofern das Asylverfahren und die Aufnahmebedingungen in diesem Staat systemische Schwachstellen aufweisen, die eine Gefahr einer unmenschlichen oder entwürdigenden Behandlung gemäss Art. 4 GRC mit sich bringen; diese Bestimmung kodifiziert im Wesentlichen die Praxis des EuGH (Urteil N.S. u.a., C-411/10 und C-493/10, EU:C:2011:865, Rn. 88, unter Bezugnahme auf das Urteil des EGMR vom 21. Januar 2011, M.S.S. gegen Belgien und Griechenland, Nr. 30696/09). Auch wenn keine systemischen Schwachstellen vorliegen, darf eine Überstellung an einen anderen Dublin-Staat nur vorgenommen werden, wenn der Person dadurch keine unmenschliche oder erniedrigende Behandlung droht (Urteil C.K. u.a./Slowenien, C-578/16, EU:C:2017:127). Es ist selbstverständlich, dass nicht nur die EU und ihre Mitgliedstaaten (N. 78), sondern auch die Schweiz bei der Durchführung der Dublin III-Verordnung die Grund- und Menschenrechte des Landes- und Völkerrechts zu beachten hat.

Problematisch war das Einreiseverbot für ausländische Staatsangehörige, welches der Bundesrat zur Bekämpfung der durch das Coronavirus (COVID-19) ausgelösten epidemiologischen Krise erliess (Art. 3 der COVID-19-Verordnung 2, SR 818.101.24; N. 247). Dabei wurde auch Personen, welche im Zug der Kontrolle an der Schweizer Landesgrenze ein Asylgesuch stellen wollten, die Einreise verweigert; ein humanitäres Visum (Visum D, vgl. N. 254) wurde nur erteilt, «wenn sich eine Person in äusserster Not befindet, die ein behördliches Handeln zur Rettung ihres Lebens und ihrer Existenz zwingend notwendig macht und ein unverzügliches Handeln gefordert ist» (Weisung des SEM vom 16. Mai 2020 zur Umsetzung der COVID-19-Verordnung 2, S. 10). Eine derart restriktive Praxis verstösst unter Umständen gegen das Non Refoulement-Gebot als Teil des völkerrechtlichen ius cogens und das Dublin-Regime. Diese Vorgaben gelten auch in Krisenzeiten. Es ist auch in ausserordentlichen Situationen sicherzustellen, dass Asylsuchende Zugang zu einem Verfahren haben, in dem Asylgesuche individuell und einzelfallgerecht geprüft werden. 
Fraglich ist, ob und - sofern dies der Fall ist - inwieweit die soeben erwähnten Kaskadenverweisungen auf die Grundrechtecharta der EU dazu führen, dass das grundrechtliche Schutzsystem, wie es sich in der EU entwickelt hat, auch in der Schweiz direkt wirkt, oder ob hierzulande weiterhin zuvörderst die Grundrechte der Verfassung und der EMRK und die dazugehörige Praxis der jeweiligen Höchstgerichte einschlägig sind (vgl. Urteil des EGMR vom 4. November 2014, Tarakhel gegen die Schweiz, Nr. 29217/12; zum Ganzen auch N. 78). Interessanterweise geht das Bundesverwaltungsgericht ohne weiteres davon aus, dass Art. 4 GRC bei der Prüfung, ob eine Überstellung eines Antragstellers an einen anderen Staat zumutbar ist, anwendbar ist. In einem Urteil von 2015 führte es gestützt auf die Erwägungsgründe der Dublin III-Verordnung aus: «Die Dublin-III-VO ist im Lichte der EMRK und für die Schweiz als Nicht-EU-Mitglied etwas systemfremd der EU-Grundrechtecharta auszulegen» (BVGE 2015/41, E. 5.3.3; s. auch Urteil vom 29. September 2015, D-5756/ 2015).

Immerhin dürfte der Verweis in Art. 3 Abs. 2 der Dublin III-Verordnung aus Sicht der Schweiz, welche sich zwar zur Beachtung dieses Rechtsaktes verpflichtet hat, institutionell aber den Status eines Drittstaats innehat und in der EU keine mitgliedstaatsähnlichen Partizipationsrechte besitzt, statischer Natur sein. Neue Präjudizien des EuGH zu Art. 4 GRC - etwa ein Leitentscheid darüber, ob ein Dublin-Staat die menschenrechtlichen Minimalstandards beachtet oder nicht, oder eine Konkretisierung des sachlichen Schutzbereichs von Art. 4 GRC - sind für die Schweiz nicht bindend. Die Schweiz behält das Letztentscheidungsrecht. Gleichwohl entfalten solche Präjudizien faktische Wirkmacht: Im Rahmen der europakompatiblen (parallelen) Auslegung des DAA werden die schweizerischen Behörden auch das künftige Fallrecht des EuGH nur dann nicht übernehmen, wenn sachliche Gründe eine autonome Lesart nahelegen - was nicht leichthin der Fall sein dürfte (N. 83).

Art. 5 Abs. 2 DAA ermächtigt die Schweiz, im Rahmen von Vorabentscheidungsverfahren vor dem EuGH, welche die Auslegung des Dublin-Acquis zum Gegenstand haben, Schriftsätze einzureichen oder schriftliche Erklärungen abzugeben. Die Schweiz macht von diesem Recht vereinzelt Gebrauch. Dabei betont sie regelmässig, dass sie sich zur Auslegung der Grundrechtecharta nicht äussert, da diese «für die Schweiz (...) nicht bindend» ist (s. etwa Schriftliche Erklärung der Schweiz betr. verb. Rs. C-411/10 und C-493/10, Bundesamt für Justiz, 3. Februar 2011, Rz. 4).

Die Behörden in der Schweiz wenden die Verordnungen des Dublin-Besitzstands unmittelbar an (Botschaft Bilaterale II, S. 6300). Eine Umsetzung ins Landesrecht erfolgte nur ganz punktuell.

\section{(b) Für die Bearbeitung eines Asylantrags verantwortlicher Staat}

Die Dublin-Staaten sind verpflichtet, jeden Antrag auf internationalen Schutz, den ein Drittstaatsangehöriger oder Staatenloser im Hoheitsgebiet eines Dublin-Staates einschliesslich an der Grenze oder in den Transitzonen stellt, zu 
prüfen; der Antrag wird von einem einzigen Mitgliedstaat geprüft, der nach den Kriterien des Kapitels III der Dublin III-Verordnung als zuständiger Staat bestimmt wird (Art. 3). Auf diese Weise sollen Mehrfachverfahren innerhalb des Dublin-Raums verhindert werden (One chance only-Prinzip). Sofern sich anhand dieser Kriterien der zuständige Staat nicht bestimmen lässt, ist der erste Dublin-Staat, in dem der Antrag auf internationalen Schutz gestellt wurde, für dessen Prüfung zuständig.

Die Kriterien zur Bestimmung des zuständigen Dublin-Staates und die Rangfolge ihrer Anwendung sind in Art. 7-15 der Dublin III-Verordnung festgelegt. Massgebend für die Anwendung der Kriterien ist der Zeitpunkt, in dem erstmals ein Antrag auf internationalen Schutz in einem Mitgliedstaat gestellt wurde (Art. 7 Abs. 2). Für die Ermittlung des zuständigen Dublin-Staates sind in erster Linie familiäre Verbindungen und Verwandtschaftsverhältnisse zu Personen, welche sich bereits in einem Dublin-Staat aufhalten, massgebend (Art. 8-11 und Art. 16). Den Interessen Minderjähriger ist besonders Rechnung zu tragen (Art. 8). Sie müssen jedoch nicht in jedem Fall mit Familienangehörigen, Geschwistern oder anderen Verwandten zusammengeführt werden. Entscheidendes Kriterium ist immer das Wohl des Kindes. In der Praxis kommt jedoch am häufigsten die Zuständigkeit desjenigen Dublin-Staates zum Tragen, in dessen Hoheitsgebiet eine asylsuchende Person nachweislich illegal in den Dublin-Raum eingereist ist (Art. 13), oder, subsidiär, die Zuständigkeit des Dublin-Staates, in dem erstmals ein Antrag auf internationalen Schutz gestellt wurde (Art. 3 Abs. 2).

Jeder Dublin-Staat kann freiwillig, d.h. ohne dass er gemäss den genannten Kriterien zuständig wäre, die Zuständigkeit zur Prüfung eines Asylantrags an sich ziehen (Selbsteintritts- oder Souveränitätsklausel, Art. 17 Abs. 1). Weiter kann eine Zuständigkeit auch nach Massgabe der humanitären Klausel begründet werden (Art. 17 Abs. 2). Diesfalls ersucht der zuständige Dublin-Staat einen anderen Dublin-Staat um die Aufnahme einer asylsuchenden Person, wenn sich im ersuchten Dublin-Staat bereits Personen aufhalten, welche zur antragstellenden Person in einer verwandtschaftlichen Beziehung stehen. Die humanitäre Klausel kann etwa zur Anwendung kommen, wenn nach dem Zeitpunkt der Einreichung des Erstgesuchs Familienangehörige in den DublinRaum eingereist sind und ein Asylgesuch gestellt haben (T. COTTIER et al., Rz. 883).

287 Der zuständige Dublin-Staat ist verpflichtet, die Schutz suchende Person zwecks Durchführung oder Abschluss eines Verfahrens auf internationalen Schutz aufzunehmen oder wieder aufzunehmen, sollte diese in der Zwischen- 
zeit den zuständigen Staat verlassen haben (Art. 18 Abs. 1; s. zur Notwendigkeit der ausdrücklichen oder stillschweigenden Zustimmung zur Überstellung an den zuständigen Staat Urteil Hassan, C-647/16, EU:C:2018:368). Die Verpflichtung zur Aufnahme, respektive zur Wiederaufnahme, erlischt, wenn die internationalen Schutz suchende Person den zuständigen Staat nachweislich für eine Zeitdauer von drei Monaten verlassen hat (Art. 19 und Art. 20). Um eine internationalen Schutz suchende Person in den zuständigen Dublin-Staat überstellen zu können, muss ein Dublin-Staat diesen Staat um Aufnahme oder Wiederaufnahme ersuchen. Dieses Ersuchen muss in der Regel innerhalb von zwei Monaten, nachdem mittels Eurodac-Abfrage festgestellt wurde, dass ein anderer Dublin-Staat zuständig sein könnte, erfolgen (s. zu Eurodac N. 289). Bei einem Aufnahmegesuch hat der ersuchte Dublin-Staat zwei Monate Zeit, das Ersuchen zu prüfen. Sowohl im Fall einer Aufnahme als auch im Fall einer Wiederaufnahme muss der überstellende Dublin-Staat einen förmlichen Entscheid erlassen, gegen den ein Rechtsmittel zur Verfügung stehen muss (Art. 26 und Art. 27; s. zum Rechtsbehelf Urteil Shiri, C-201/16, EU:C:2017:805; zum Rechtsmittelverfahren in der Schweiz T. COTTIER et al., Rz. 888).

Die Dublin III-Verordnung gewährt den Betroffenen diverse individuelle Rechte (Berücksichtigung der familiären Zusammengehörigkeit, Kindeswohl und besondere Schutzbedürftigkeit Minderjähriger, Anspruch auf Information, Anspruch auf ein persönliches Gespräch, Verfahrens- und Rechtsschutzgarantien, Minimalvorschriften bei der Inhaftnahme zum Zweck der Überstellung).

Die Dublin III-Verordnung enthält keine Vorschriften hinsichtlich der Haftbedingungen. Sie verweist in Art. 28 Abs. 4 auf die Richtlinie 2013/33/EU (Asylaufnahmerichtlinie, N. 276). Da die Schweiz diese Richtlinie im Rahmen der Dublin-Assoziierung nicht übernommen hat, gelten die entsprechenden Bestimmungen im bilateralen Verhältnis Schweiz-EU grundsätzlich nicht. Die Botschaft des Bundesrates führte dazu sybillinisch aus: «Diese Richtlinie ist für die Schweiz nicht verbindlich; es gilt aber dennoch, dem Inhalt der Richtlinie Rechnung zu tragen und ähnliche Standards in Bezug auf die Haft bei Dublin-Verfahren anzuwenden» (Botschaft Dublin 2014, S. 2707). Das BGer kam unter Bezugnahme auf die Botschaft zu einem gegenteiligen Schluss; es erklärte die einschlägigen Bestimmungen der Asylaufnahmerichtlinie 2013/33/EU qua Kaskadenverweisung auch für die Schweiz als verbindlich: «Durch den in Art. 28 Abs. 4 Dublin-Verordnung enthaltenen Verweis wird die Regelung, auf welche verwiesen wird (Art. 9, Art. 10, Art. 11 der Richtlinie 2013/33/EU), durch Inkorporation Teil der Dublin-Verordnung und ist als solche als Teil des acquis auch im Verhältnis zur Schweiz anwendbar (...; ausdrücklich auch Botschaft Dublin 2014, BBl 2014 2707).» (BGE 143 II 361, E. 3.3); eine etwas gar saloppe Argumentation des BGer. 


\section{(c) Eurodac}

Ein zentraler Pfeiler des Dublin-Systems ist die Datenbank Eurodac, welche 2000 eingerichtet wurde und zurzeit auf der Verordnung (EU) Nr. 603/2013 (Eurodac-Verordnung) beruht. Aufgabe von Eurodac ist es, die Bestimmung des Mitgliedstaats, welcher gemäss der Verordnung (EU) Nr. 604/2013 (Dublin III-Verordnung) für die Prüfung eines von einem Drittstaatsangehörigen oder Staatenlosen in einem Mitgliedstaat gestellten Antrags auf internationalen Schutz zuständig ist, zu unterstützen und allgemein die Anwendung der Dublin III-Verordnung unter den in dieser Verordnung festgelegten Bedingungen zu erleichtern (Art. 1 Abs. 1). Weiter werden in der Eurodac-Verordnung die Bedingungen festgelegt, unter denen die Behörden der Mitgliedstaaten und Europol den Abgleich von Fingerabdruckdaten mit den im Zentralsystem gespeicherten Daten zum Zweck der Gefahrenabwehr und Strafverfolgung beantragen können (Art. 1 Abs. 2). In Eurodac werden die Fingerabdrücke und persönlichen Daten von sämtlichen Personen erfasst, welche im Dublin-Raum ein Asylgesuch stellen oder illegal in diesen Raum eingereist sind und mindestens 14 Jahre alt sind. Damit wird der Reiseverkehr asylsuchender Personen nachvollziehbar und die für die Anwendung des Dublin-Systems notwendige Transparenz geschaffen.

290 Eurodac besteht aus einer rechnergestützten zentralen Fingerabdruck-Datenbank mit einer Zentraleinheit inkl. Notfallplan und Notfallsystem («Zentralsystem») und aus einer Kommunikationsinfrastruktur zwischen dem Zentralsystem und den Mitgliedstaaten, die ein verschlüsseltes virtuelles Netz für Eurodac-Daten zur Verfügung stellt («Kommunikationsinfrastruktur»; Art. 3). Jeder Mitgliedstaat hat eine einzige nationale Zugangsstelle. Die Agentur der EU für das Betriebsmanagement von IT-Grosssystemen im Raum der Freiheit, der Sicherheit und des Rechts (eu-LISA) ist für das Betriebsmanagement von Eurodac zuständig (N. 241).

Die Eurodac-Verordnung regelt auch die Voraussetzungen für den Zugriff der Strafverfolgungsbehörden auf die Daten in der Eurodac-Datenbank ohne Bezug zu asylrechtlichen Fragen (N. 263). Diese Bestimmungen gelten - im Gegensatz zum restlichen (asylbezogenen) Teil der Verordnung - nicht als Weiterentwicklung des Dublin-Besitzstands; der Übernahmeprozess nach Massgabe des Dublin-Assoziierungsabkommens wurde für diese Bestimmungen nicht aktiviert. Die EU und die assoziierten Dublin-Staaten haben stattdessen entschieden, diese Bestimmungen gestützt auf ein separates Abkommen zu übernehmen. Auf diese Weise erhält auch die Schweiz Zugang zu Eurodac für Zwecke der Strafverfolgung und damit der Verbesserung der 
Wahrung der inneren Sicherheit. Das Abkommen wurde am 27. Juni 2019 unterzeichnet. Zurzeit befindet es sich zusammen mit der Umsetzungsgesetzgebung im Genehmigungsprozess im Parlament (s. Erläuternder Bericht zur Genehmigung des Abkommens zur Vertiefung der grenzüberschreitenden Zusammenarbeit (Prümer Zusammenarbeit) und des Eurodac-Protokolls zwischen der Schweiz und der EU (...) vom 13. Dezember 2019).

\section{(d) Europäisches Unterstützungsbüro für Asylfragen}

Das Europäische Unterstützungsbüro für Asylfragen (EASO) wurde 2010 eingerichtet (Verordnung (EU) Nr. 439/2010). Die Aufgaben des Büros bestehen darin, zu einer besseren Umsetzung des Gemeinsamen Europäischen Asylsystems (GEAS, N. 276) beizutragen, die praktische Zusammenarbeit zwischen den Mitgliedstaaten im Asylbereich zu stärken und die Mitgliedstaaten, deren Asyl- und Aufnahmesysteme besonderem Druck ausgesetzt sind, mit operativen Massnahmen zu unterstützen oder eine solche Unterstützung zu koordinieren (Art. 1). Das Büro ist Bestandteil des GEAS und arbeitet mit den Einrichtungen der Union zusammen, die mit seinem Tätigkeitsbereich in $\mathrm{Zu}$ sammenhang stehende Tätigkeiten ausüben, insbesondere mit Frontex (N. 264-265) und der Agentur der EU für Grundrechte, sowie mit internationalen Organisationen, die in den unter diese Verordnung fallenden Bereichen tätig sind, z.B. mit dem UNO-Hochkommissariat für Flüchtlinge (vgl. Art. 52). Sitz des EASO ist Valletta auf Malta.

Die Beteiligung des EASO stellt keine Weiterentwicklung des Dublin-Besitzstands dar. Die assoziierten Dublin-Staaten beteiligen sich gestützt auf eine separate Abmachung an den Arbeiten des Büros. Die Modalitäten der Beteiligung der Schweiz werden in einer Vereinbarung von 2014 festgelegt (SR 142.392.681). Diese Vereinbarung trat 2016 in Kraft. Sie sieht vor, dass die Schweiz an allen Aktivitäten des EASO beteiligt wird. Sie nimmt im Verwaltungsrat des EASO als Beobachterin Einsitz und beteiligt sich finanziell an den Ausgaben des EASO.

Der Beitrag der Schweiz an das EASO belief sich 2016-2019 auf 11,9 Mio. CHF (Stellungnahme des Bundesrates vom 20. November 2019 auf die Interpellation 19.4008 «Aktualisierte Zusammenstellung aller Zahlungen und Beiträge der Schweiz an die EU»).

\section{Datenschutz}

Bei der Anwendung der unionalen Vorgaben in den Bereichen Grenzkontrollen, Asyl und Einwanderung wird eine Unmenge von persönlichen Daten erhoben, ausgewertet und gespeichert. Anfänglich geschah dies primär im Rahmen 
traditioneller Formen der Verwaltungszusammenarbeit. Mittlerweile werden Daten in computergestützten Systemen automatisiert verarbeitet, gespeichert und den unionalen Einrichtungen und nationalen Behörden europaweit zur Verfügung gestellt (s. zu den auch in der Schweiz zugänglichen Datenbanken und Systemen N.241). Es ist rechtsstaatlich fundamental, dass dabei die Grundrechte der Betroffenen respektiert werden. Im Vordergrund stehen das Recht auf Achtung der Privatsphäre und das Recht auf Schutz der personenbezogenen Daten (vgl. Art. 8 EMRK, Art. 7 und Art. 8 GRC, Art. 13 BV).

295 In den Anfängen der Schengener- und Dubliner-Zusammenarbeit war das Datenschutzrecht der EWG/EG/EU wenig entwickelt. Die älteren Rechtsakte verwiesen auf die gemeinsamen Rechtsgrundlagen des Europarats, insbesondere auf das Übereinkommen über den Schutz des Menschen bei der automatischen Verarbeitung personenbezogener Daten von 1981 (Europaratskonvention Nr. 108; SR 0.235.1) und die Empfehlung R (87) 15 des Ministerausschusses des Europarates über die Nutzung personenbezogener Daten im Polizeibereich von 1987 (vgl. noch heute Art. 117 und Art. 126 SDÜ; T. CoTTIER et al., Rz. 940). In den 1990er-Jahren begann die EU, ein eigenes Datenschutzrecht zu entwickeln. Mit dem Vertrag von Lissabon wurde eine allgemein geltende Bestimmung zum Datenschutz - prominent platziert im ersten Teil des AEUV - geschaffen (Art. 16 AEUV); auch wurde der grundrechtliche Charakter des Schutzes personenbezogener Daten gestärkt (Art. 8 GRC). Mittlerweile existiert in der EU ein hohes Schutzniveau, wozu der EuGH einen wesentlichen Beitrag geleistet hat (s. zum «Recht auf Vergessenwerden» Urteil Google, C-131/12, EU:C:2014:317; zur Vorratsdatenspeicherung Urteil Digital Rights Ireland und Seitlinger, C-293/12 und C-594/12, EU:C:2014:238; zur Übermittlung von Daten in ein Drittland Urteil Schrems, C-362/14, EU:C:2015:650; Urteil C-311/18, Facebook Ireland und Schrems, EU:C:2020:559; dazu auch N. 365). Gleichzeitig ist das unionale Datenschutzrecht weiterhin zersplittert. Eine Vielzahl von Rechtsakten enthält spezifische, teilweise von den allgemeinen Vorgaben abweichende datenschutzrechtliche Bestimmungen. Immerhin wurde dieser Wildwuchs mit dem Erlass der Verordnung (EU) 2016/679 (Datenschutz-Grundverordnung) und der Richtlinie (EU) 2016/680, welche den Datenschutz im Bereich der Strafverfolgung und -vollstreckung regelt, etwas gelichtet.

296 In den Bereichen Grenzkontrollen, Asyl und Einwanderung sind die folgenden datenschutzrechtlichen Rechtsakte zu beachten. Punktuell sind sie auch bei der Anwendung des bilateralen Acquis Schweiz-EU einschlägig (Botschaft 
Bilaterale II, S. 6107-6110, 6120-6121; Botschaft Totalrevision Datenschutzgesetz， S. 6963-6964; A. EPINEY/Y. SCHLEISS，§ 4 Rz. 272-305; R.J. SCHWEIZER, Datenschutzrechte, passim):

- Die EG erliess 1995 die Richtlinie 95/46/EG zum Schutz natürlicher Personen bei der Verarbeitung personenbezogener Daten und zum freien Datenverkehr (allgemeine Datenschutzrichtlinie). Diese Richtlinie verpflichtete die Mitgliedstaaten, den Schutz der Grundrechte und Grundfreiheiten und insbesondere den Schutz der Privatsphäre natürlicher Personen bei der Verarbeitung personenbezogener Daten zu gewährleisten; gleichzeitig verbot sie die Beschränkung des freien Verkehrs personenbezogener Daten (Art. 1). Diese Richtlinie wurde ergänzt durch den Rahmenbeschluss 2008/977/JI über den Schutz personenbezogener Daten, die im Rahmen der polizeilichen und justiziellen Zusammenarbeit in Strafsachen verarbeitet werden. 2018 wurde die allgemeine Datenschutzrichtlinie durch die Verordnung (EU) 2016/679 (Datenschutz-Grundverordnung) abgelöst, welche in der EU seither den datenschutzrechtlichen Grunderlass darstellt. Gleichzeitig wurde der Rahmenbeschluss 2008/ 977/JI durch die Richtlinie (EU) 2016/680 zum Schutz natürlicher Personen bei der Verarbeitung personenbezogener Daten durch die zuständigen Behörden zum Zwecke der Verhütung, Ermittlung, Aufdeckung oder Verfolgung von Straftaten oder der Strafvollstreckung sowie zum freien Datenverkehr ersetzt; gleichsam die «kleine Schwester» der Datenschutz-Grundverordnung (R.J. SchweIzER, Datenschutzrechte, Rz. 7). Diese unionalen Rechtsakte gehören in Bezug auf die Schweiz nur teils zum einschlägigen Schengen-Besitzstand. Dies gilt seit jeher für die Richtlinie 95/46/EG, welche qua Verweis im Schengen-Assoziierungsabkommen bei der Anwendung dieses Abkommens für die Schweiz bis heute verbindlich ist. Auch der Rahmenbeschluss 2008/977/JI war Teil des für die Schweiz relevanten Schengen-Besitzstands. 2019 wurde dessen Nachfolgerin, die Richtlinie (EU) 2016/680, in den Anhang des Schengen-Assoziierungsabkommens übernommen; seither ist diese Richtlinie bei der Anwendung des Schengen-Besitzstands für die Schweiz einschlägig (s. zur Umsetzung dieser Richtlinie in der Schweiz das SchengenDatenschutzgesetz, SDSG).

Die allgemeine Datenschutzrichtlinie 95/46/EG ist im Anwendungsbereich des SAA und DAA weiterhin einschlägig, auch wenn diese Richtlinie im EU-Recht längst aufgehoben wurde; eine unschöne und rechtlich komplizierte Konstellation (s. zu den Gründen, weshalb die Datenschutz-Grundverordnung (EU) 2016/679 für die Assoziierung der Schweiz als nicht relevant erachtet wurde, und zur Frage, ob diese Verordnung - etwa qua Art. 94 
Abs. 2 der neuen Verordnung - indirekt für die Schweiz gleichwohl Wirkung entfaltet, G. BAUR, Datenschutz, passim; C. TOBLER, Homogenität, S. 218-225; zur Bedeutung dieser Verordnung im Rahmen des autonomen Nachvollzugs N. 364-368).

- Weitere unionale Rechtsakte, welche spezifische Aspekte in den Bereichen Grenzkontrollen, Asyl und Einwanderung regeln, enthalten datenschutzrechtliche Vorgaben. Dazu gehören u.a. das Schengener Durchführungsübereinkommen (s. v.a. Art. 126-130, N. 242), die SIS II-Verordnung (EU) 2018/1862 (s. v.a. Art. 66-71, N. 261), die VIS-Verordnung (EG) Nr. 767/2008 (N. 253), die Verordnung (EU) 2019/1896 zu Frontex (s. v.a. Art. 86-92, N. 265), die Eurodac-Verordnung (EU) Nr. 603/2013 (s. v.a. Art. 23-37, N. 289), die Verordnung (EU) 2017/2226 zur Errichtung eines einheitlichen Einreise-/Ausreisesystems (EES, N. 251) und die Vorlagen zu Informationsabläufen in den Anhängen der Durchführungsverordnung (EU) Nr. 118/2014 zur Dublin III-Verordnung. Diese Rechtsakte gehören zu dem auch für die Schweiz relevanten Schengen/Dublin-Besitzstand. Entsprechend sind auch diese datenschutzrechtlichen Vorgaben bei der Anwendung der beiden Assoziierungsabkommen für die Schweiz verbindlich.

Diverse Rechtsakte verweisen dabei auf die Verordnung (EU) 2018/1725 zum Schutz natürlicher Personen bei der Verarbeitung personenbezogener Daten durch die Organe, Einrichtungen und sonstigen Stellen der Union. Diese Verordnung gehört nicht zum für die Schweiz bedeutsamen Schengen/Dublin-Besitzstand. zipien des Datenschutzrechts, welches bei der Anwendung der Schengen/ Dublin-Assoziierungsabkommen einschlägig ist, weitgehend deckungsgleich (T. CотTIER et al., Rz. 944-946). Zentral sind die Grundsätze der Rechtmässigkeit, Verarbeitung nach Treu und Glauben, Transparenz, Zweckbindung, Datenminimierung, Richtigkeit, Speicherbegrenzung, Integrität und Vertraulichkeit sowie Rechenschaftspflicht. Betroffene Personen haben das Recht auf Berichtigung unrichtiger Daten und das Recht auf Löschung («Recht auf Vergessenwerden»), wenn die personenbezogenen Daten für die Zwecke, für die sie erhoben wurden, nicht mehr notwendig sind. Die Notwendigkeit der Speicherung von personenbezogenen Daten muss regelmässig überprüft werden. Die Übermittlung von Daten in ein Drittland ist besonderen Voraussetzungen unterworfen (N. 364). Bei einer Schädigung infolge einer rechtswidrigen Verarbeitung besteht Anspruch auf Schadenersatz. Die Schengen/Dublin-Staaten sind verpflichtet, unabhängige nationale Kontrollstellen einzurichten, welche beratende Aufgaben wahrnehmen und eine Aufsichtsfunktion innehaben. Sie müssen über wirksame Untersuchungsbefugnisse sowie Klagerechte oder Anzeigebefugnisse bei Verstössen verfügen. 
Das Datenschutzniveau der Schweiz entsprach beim Inkrafttreten des SAA/DAA 2008 zwar bereits weitgehend den datenschutzrechtlichen Vorgaben der EU. Punktuell musste das Datenschutzgesetz (DSG) aber gleichwohl angepasst werden. Insbesondere musste die Unabhängigkeit des Eidgenössischen Datenschutzbeauftragten gestärkt werden, «indem die Bestimmungen über seine Wahl und Amtsenthebung expliziter gestaltet werden, ihm grössere Autonomie in Budget- und Personalfragen eingeräumt wird und sichergestellt wird, dass keine Behörde bestimmen kann, wie der Beauftragte bei der Ausübung seiner Befugnisse und Kompetenzen im Einzelfall handeln und entscheiden soll» (Botschaft Rahmenbeschluss Personendaten, S. 6756). Bei der Umsetzung des Rahmenbeschlusses 2008/ 977/JI wurde die Wahl der Beauftragten oder des Beauftragten durch den Bundesrat neu der Genehmigung des Parlaments unterstellt und die Amtsdauer explizit auf vier Jahre festgelegt (Art. 26 Abs. 1 DSG). Auch die Unabhängigkeitsgarantie, die (Nicht-) Wiederwahl, die Amtsenthebung und die Anwendbarkeit des Bundespersonalgesetzes auf die Beauftragte oder den Beauftragten wurden aus diesem Anlass präzisiert (Art. 26 und 26a DSG). Im Zug der Übernahme und Umsetzung der Richtlinie (EU) 2016/680 wurden diverse Bestimmungen des DSG in Bezug auf die Beauftragte oder den Beauftragten nochmals geändert (vgl. BBl 2018 6003).

Ergänzend zu den genannten Rechtsakten, welche qua Verweis in den Schengen/Dublin-Assoziierungsabkommen bilateralrechtlich anwendbar sind, enthalten auch weitere bilaterale Abkommen im Bereich Justiz und Inneres datenschutzrechtliche Bestimmungen. Dies gilt für das Betrugsbekämpfungsabkommen, das Abkommen zwischen der Schweiz und Europol, das Abkommen zwischen der Schweiz und Eurojust sowie das Abkommen zur Vertiefung der grenzüberschreitenden Zusammenarbeit (Prümer Zusammenarbeit), welches sich zurzeit im Zustimmungsprozess in der EU und der Schweiz befindet.

\section{G. Forschung, Kultur, Bildung}

Die Schweiz und die EU teilen im Bereich der Forschung, der Kultur und der Bildung gemeinsame Grundwerte und verfolgen ähnliche Ziele. Es ist nahe liegend, dass eine enge Kooperation angestrebt wird. Wesentliche vertragliche Grundlagen wurden im Rahmen der Bilateralen I (Forschung) und der Bilateralen II (Kultur, Bildung) gelegt. Die Beteiligung der Schweiz an den Programmen der EU steht allerdings auf wackligen Füssen. Sie wurde zwischenzeitlich unterbrochen (Forschung) oder gar gänzlich abgebrochen (Kultur, Bildung). Zurzeit laufen die Vorbereitungsarbeiten für eine Beteiligung der Schweiz an den Programmen der EU für den Zeitraum 2021-2027, wobei die EU die Beteiligung der Schweiz von Fortschritten beim Institutionellen Abkommen abhängig macht. 


\section{Forschung}

Ein Ziel der EU ist es, den wissenschaftlichen und technischen Fortschritt zu fördern und einen europäischen Raum der Forschung zu schaffen (Art. 3 EUV, Art. 179 AEUV). Zu diesem Zweck erlassen das Europäische Parlament und der Rat ein mehrjähriges Rahmenprogramm, in dem alle diesbezüglichen Aktionen der EU zusammengefasst werden (Art. 182 AEUV). Bei der Durchführung des Rahmenprogramms kann die EU eine Zusammenarbeit auf dem Gebiet der Forschung, technologischen Entwicklung und Demonstration der EU mit dritten Ländern oder internationalen Organisationen vorsehen; die Einzelheiten können Gegenstand von Abkommen der EU mit den betreffenden dritten Parteien sein (Art. 186 AEUV).

Die Schweiz und die EWG schlossen 1986 das Rahmenabkommen über wissenschaftlich-technische Zusammenarbeit ab. Dieses Abkommen trat 1987 in Kraft (SR 0.420.518). Es sieht ausdrücklich vor, dass die mit dem Abkommen angestrebte Zusammenarbeit durch geeignete Vereinbarungen durchgeführt wird (Art. 6). 1999 unterzeichneten die Schweiz und die EG das Abkommen über die wissenschaftliche und technologische Zusammenarbeit als Teil der Bilateralen I (Forschungsabkommen, AS 2002 1998). Dieses Abkommen bezog sich auf die Teilnahme der Schweiz am fünften Rahmenprogramm der EG im Bereich der Forschung, technologischen Entwicklung und Demonstration und am Fünften Rahmenprogramm der Europäischen Atomgemeinschaft (Euratom) für Massnahmen im Bereich der Forschung und Ausbildung (1998-2002); Forschungsinstitute in der Schweiz beteiligten sich vorderhand allerdings nur projektweise. Weitere Verhandlungen führten zur vollen Assoziierung der Schweiz am sechsten Rahmenprogramm (2003-2006) und am Siebten Rahmenprogramm (2007-2013). Nach der Annahme der Masseneinwanderungsinitiative 2014 wurden die Verhandlungen zur vollen Assoziierung der Schweiz am achten Rahmenprogramm Horizon 2020 durch die EU zwischenzeitlich auf Eis gelegt; die Schweiz konnte sich vorläufig nur an ausgewählten Projekten beteiligen (SR 0.424.11). 2017 wurde die Schweiz wiederum voll an Horizon 2020 assoziiert (SR 0.424.11), nachdem die Schweiz das Protokoll III zum FZA zur Ausdehnung der Personenfreizügigkeit auf Kroatien ratifiziert hatte (N. 28). Eine Vollassoziierung ermöglicht den Forscherinnen und Forschern in der Schweiz (Forschungsinstitute, Unternehmen, Einzelpersonen), sich gleichberechtigt wie die Forscherinnen und Forscher in der EU an Ausschreibungen für Fördermittel zu beteiligen (www.eda.admin.ch/dea und Link zu Bilaterale Abkommen I (1999)/Forschung). 
In der EU werden zurzeit die Grundlagen gelegt für das Rahmenprogramm Horizon Europe für den Zeitraum 2021-2027. Auch dieses Programm deckt von der Grundlagenforschung über angewandte Forschung, technologische Entwicklung, Innovation und Demonstrationsprojekte bis zum Markteintritt (namentlich von KMUs) die gesamte Wertschöpfungskette ab. Es umfasst die Stipendien des Europäischen Forschungsrats (European Research Council, ERC), Mobilitätsstipendien (Marie-Skłodowska-Curie-Massnahmen) und transnationale Verbundprojekte. Neu ist die systematische Ausrichtung des gesamten Programms auf die Umsetzung der UNO-Ziele für nachhaltige Entwicklung (Sustainable Development Goals, SDG). In der Schweiz laufen die Vorbereitungen für die Beteiligung an Horizon Europe. Das Parlament muss über die Bereitstellung der finanziellen Mittel entscheiden, worauf mit der EU über die Erneuerung des Forschungsabkommens und die Modalitäten der Schweizer Assoziierung verhandelt werden kann. Der Bundesrat geht davon aus, dass für die Beteiligung am Horizon-Paket, am Euratom-Programm und an weiteren EU-Programmen inkl. nationalen Begleitmassnahmen insgesamt 6,1 Mia. CHF notwendig sind (Botschaft Horizon-Paket, passim).

Die Beteiligung der Schweiz an den Forschungsrahmenprogrammen der EU ist für den Wirtschafts- und Wissenschaftsstandort Schweiz essentiell. Der internationale Wettbewerb und die Vernetzung wirken sich positiv auf die Exzellenz und Leistungsfähigkeit des Forschungs- und Innovationsstandortes Schweiz aus. Die Forschungsrahmenprogramme der EU sind nach dem SNF die zweitwichtigste öffentliche Förderquelle für Forschung und Innovation in der Schweiz. Seit der erstmaligen Vollassoziierung der Schweiz 2004 verzeichnet die Schweiz zudem einen positiven finanziellen Rückfluss: Die von Schweizer Forscherinnen und Forscher eingeworbenen Fördermittel übersteigen den Beitrag, den die Schweiz an die EU leistet (Botschaft Horizon-Paket, S. 4847).

Angesichts des unklaren Schicksals des Institutionellen Abkommens bleibt die Beteiligung der Schweiz an Horizon Europe ungewiss (Aussenpolitischer Bericht 2019, S. 1583).

\section{Kultur}

Die EG/EU unterstützt die audiovisuelle Industrie seit den 1990er-Jahren. Dabei geht es zuvörderst um die Unterstützung der Ausbildung und Weiterbildung von Werktätigen, die Förderung der Entwicklung einzelner Werke und ihre Verbreitung, die Stärkung der Wettbewerbsfähigkeit der Kultur- und Kreativbranche sowie die grenzüberschreitende Zusammenarbeit. Zu diesem Zweck wurden die MEDIA-Programme (1991-1995, 1996-2000, 2001-2006, 2007-2013) eingerichtet. 2014 wurde das Programm Kreatives Europa in Kraft gesetzt. Dieses Programm bezweckt die Wahrung, Entwicklung und Förderung 
der kulturellen und sprachlichen Vielfalt Europas und die Förderung des kulturellen Erbes Europas sowie die Stärkung der Wettbewerbsfähigkeit des europäischen Kultur- und Kreativsektors, insbesondere des audiovisuellen Sektors, um intelligentes, nachhaltiges und integratives Wachstum zu fördern (Art. 3 der Verordnung (EU) Nr.1295/2013). Diverse Programme, welche bereits im Rahmen der MEDIA-Programme initiiert wurden, laufen auch unter dem Programm Kreatives Europa weiter. Die Einrichtung von Kreatives Europa basiert auf den unionalen Kompetenzen in den Bereichen Bildung, Jugend und Sport (Art. 166 Abs. 4 AEUV), Kultur (Art. 167 Abs. 5 AEUV) und Industrie (Art. 173 Abs. 3 AEUV). Die Exekutivagentur Bildung, Audiovisuelles und Kultur (EACEA) ist zuständig zur Verwaltung der Förderprogramme.

Die Schweiz und die EG schlossen 2004 das Abkommen im Bereich audiovisuelle Medien über die Festlegung der Voraussetzungen und Bedingungen für die Beteiligung der Schweiz an den Gemeinschaftsprogrammen MEDIA Plus und MEDIA-Fortbildung (MEDIA-Abkommen; AS 2006 1039); dieses Abkommen bezog sich auf die Teilnahme der Schweiz am MEDIA-Programm 2001-2006. Die Teilnahme der Schweiz am Nachfolgeprogramm (2007-2013) wurde in einem Abkommen von 2007 geregelt (s. für Beispiele der Unterstützung Schweizer Filmschaffender durch MEDIA-Fördermittel www.eda.admin.ch/ dea und Link zu Bilaterale Abkommen II (2004)/MEDIA). Nach der Annahme der Masseneinwanderungsinitiative 2014 wurden die Verhandlungen zur Teilnahme der Schweiz am Programm Kreatives Europa vorerst unterbrochen und sodann abgebrochen. Die Schweiz und die EG konnten sich nicht auf eine Teilnahme der Schweiz einigen.

Das Eidgenössische Departement des Innern (EDI) erliess 2016 die Verordnung über die Förderung der internationalen Präsenz des Schweizer Filmschaffens und die MEDIAErsatz-Massnahmen (SR 443.122), um die Nachteile der Nichtteilnahme der Schweiz am Programm Kreatives Europa zumindest teilweise auszugleichen. Sie ist als Übergangslösung konzipiert und regelt die Voraussetzungen für die Gewährung von Finanzhilfen zur Förderung der internationalen Präsenz des schweizerischen Filmschaffens und für die Gewährung von Finanzhilfen als Ersatz für die Nichtteilnahme am MEDIA-Programm der EU im Hinblick auf den Wiedereinstieg der Schweiz in dieses Programm (Art. 1).

306 In der EU werden zurzeit die Grundlagen gelegt für die Fortführung des Programms Kreatives Europa für den Zeitraum 2021-2027. Die Schweiz prüft eine Teilnahme an diesem Programm und führt mit der EU Gespräche. Die EU macht die Teilnahme der Schweiz überdies vom Abschluss des Institutionellen Abkommens abhängig (www.eda.admin.ch/dea und Link zu Bilaterale Abkommen II (2004)/MEDIA). 


\section{Bildung, Berufsbildung, Jugend}

Die EWG/EG/EU hat seit den 1970er-Jahren eine Vielzahl von Aktionen und Programmen eingerichtet, um zur Entwicklung einer qualitativ hochstehenden Bildung beizutragen. Diese Aktionen und Programme unterstützen und ergänzen die Massnahmen der Mitgliedstaaten und ihre Zusammenarbeit. Seit dem Inkrafttreten des Vertrags von Lissabon enthält Titel XII des AEUV (Allgemeine und berufliche Bildung, Jugend, Sport) die primärrechtlichen Vorgaben (Art. 165-166 AEUV). Für den Zeitraum 2014-2020 gilt das Programm Erasmus+ (Verordnung (EU) Nr. 1288/2013). Es erstreckt sich auf die Bereiche i) allgemeine und berufliche Bildung auf allen Ebenen im Sinne des lebenslangen Lernens, einschliesslich Schulbildung (Comenius), Hochschulbildung (Erasmus), internationale Hochschulbildung (Erasmus Mundus), berufliche Aus- und Weiterbildung (Leonardo da Vinci) und Erwachsenenbildung (Grundtvig), ii) Jugend (Jugend in Aktion), und iii) Sport. Erasmus+ verfügt über Mittel im Umfang von knapp 15 Mia. EUR und ermöglicht Unionsbürgerinnen und Unionsbürgern, im Ausland zu studieren, sich weiterzubilden, Berufserfahrung zu sammeln oder Freiwilligenarbeit zu leisten. Das 1975 gegründete Europäische Zentrum für die Förderung der Berufsbildung (Cedefop) unterstützt die Entwicklung von Strategien im Berufsbildungsbereich in der EU.

Die Schweiz beteiligte sich seit 1992 an verschiedenen Programmen der EG/ $\mathrm{EU}$ in den Bereichen Bildung, Berufsbildung und Jugend. Zeitweise erfolgten diese Beteiligungen projektweise und ohne formelle staatsvertragliche Vereinbarung. Zeitweise war die schweizerische Beteiligung staatsvertraglich abgesichert; dies war der Fall bei der Teilnahme am Erasmus-Programm zwischen 1992 und 1994 (vgl. SR 0.414.91). In den Verhandlungen, welche zu den Bilateralen II von 2004 führten, einigten sich die Schweiz und die EU auf eine politische Erklärung, die Beteiligung der Schweiz an den Programmen Lebenslanges Lernen (u.a. Comenius, Erasmus, Leonardo da Vinci und Grundtvig) und Jugend in Aktion zu formalisieren. 2010 unterzeichneten die Schweiz und die EG ein entsprechendes Abkommen (SR 0.402.268.1); es trat 2011 in Kraft. Nach der Annahme der Masseneinwanderungsinitiative 2014 wurden die Verhandlungen zur Teilnahme der Schweiz am Programm Erasmus+ suspendiert und schliesslich abgebrochen. Die Schweiz und die EG konnten sich nicht auf eine Teilnahme der Schweiz als Programmland einigen. Seither gilt die Schweiz als Partnerland, was bedeutet, dass Schweizer Institutionen nicht an den Mobilitätsaktivitäten und nur unter erschwerten Bedingungen an Kooperationsakti- 
vitäten von Erasmus+ teilnehmen können; auch verfügt die Schweiz über keine Mitsprache in strategischen Bildungsgremien der EU (Botschaft Mobilität in der Bildung, S. 3894).

Der Bundesrat erliess 2015 die Verordnung über die internationale Zusammenarbeit im Bereich der Bildung, der Berufsbildung, der Jugend und der Mobilitätsförderung (SR 414.513). Mit der damit einhergehenden Bereitstellung von Fördermitteln soll sichergestellt werden, dass Schweizer Bildungsinstitutionen weiterhin an Kooperations- und Mobilitätsaktivitäten mit den Programmländern von Erasmus+ teilnehmen können. Von diesem als Übergangslösung konzipierten Swiss-European Mobility Programme (SEMP) profitieren Studierende, welche einen Austausch im europäischen Ausland absolvieren (Outgoings), wie auch ausländische Studierende, welche einen Aufenthalt in der Schweiz absolvieren (Incomings; s. zum Ganzen www.movetia.ch). 2017 bewilligte die Bundesversammlung für dieses Programm für den Zeitraum 2018-2020 einen Gesamtkredit von 114,5 Mio. CHF (Bundesbeschluss über die Förderung der internationalen Mobilität in der Bildung in den Jahren 2018-2020, BBl 2018 27). Gemäss Bundesrat überwogen die Vorteile einer autonomen Schweizer Lösung die Nachteile des schweizerischen Abseitsstehens; der Bundesrat erachtete vor allem auch die Höhe des von der EU geforderten Beitrags der Schweiz an Erasmus+ als zu hoch, um sich doch noch als Programmland zu beteiligen (Botschaft Mobilität in der Bildung, passim).

In der EU werden zurzeit die Grundlagen für die Fortführung des Programms Erasmus+ für den Zeitraum 2021-2027 gelegt. Die Schweiz prüft eine Assoziierung als Programmland und führt mit der EU Gespräche (www.eda.admin.ch/ dea und Link zu Bilaterale Abkommen II (2004)/Bildung, Berufsbildung, Jugend), wobei eine Beteiligung der Schweiz angesichts des unklaren Schicksals des Institutionellen Abkommens ungewiss bleibt (Aussenpolitischer Bericht 2019, S. 1583).

Es bleibt zu hoffen, dass sich die Teilnahme der Schweiz an Erasmus+ wieder realisieren lässt. Es trifft zwar zu, dass der Alleingang seit 2015 für die Schweiz finanziell vorteilhaft war. Auch blieb die Zahl der Studierenden an Schweizer Bildungseinrichtungen, welche einen Austausch im europäischen Ausland absolvierten, stabil (Outgoings). Gleichzeitig müssen die hiesigen Bildungseinrichtungen einen enormen Aufwand betreiben, damit ausländische Studierende weiterhin in die Schweiz kommen (Incomings). Weiter haben sie mitunter Mühe, mit renommierten Universitäten im europäischen Ausland Austauschprogramme zu vereinbaren. Schliesslich verfügen Vertreterinnen und Vertreter der Schweiz über keinen Zugang zu den relevanten EU-Netzwerken, in denen über die Bildungspolitik in Europa diskutiert wird. Hier bleibt die Schweiz aussen vor. Swissuniversities, die Dachorganisation der Schweizer Hochschulen, bezeichnet das derzeitige Regime als «Scheinlösung» (NZZ vom 17. September 2020, S. 12).

\section{H. Diverses} und Kooperationen vereinbart. Die Abkommen in den Bereichen Statistik, 
Umwelt, Ruhegehälter und Zinsbesteuerung gehören zum Paket der Bilateralen II (wobei das Zinsbesteuerungsabkommen 2017 durch das Abkommen über den automatisierten Informationsaustausch abgelöst wurde). Kooperationen im Bereich der Verteidigung und der Satellitennavigation existieren seit 2012 und 2015. 2020 trat zudem das Abkommen zwischen der Schweiz und der EU zur Verknüpfung ihrer jeweiligen Systeme für den Handel mit Treibhausgasemissionen in Kraft (s. dazu N. 216).

\section{Statistik}

Eurostat ist das statistische Amt der EU. Es wurde 1953 im Rahmen der EGKS eingerichtet. 1958 wurde es als Dienststelle (Generaldirektion) in die Kommission der EG integriert. Sein Sitz ist Luxemburg. Eurostat ist für die Entwicklung, Erstellung und Verbreitung europäischer Statistiken zuständig (Beschluss der Kommission 2012/504/EU über Eurostat). Zu diesem Zweck führt Eurostat folgende Massnahmen durch: a) Sammlung und Zusammenstellung der für die Erstellung europäischer Statistiken erforderlichen statistischen Daten; b) Entwicklung und Förderung statistischer Normen, Methoden und Verfahren; c) Lenkung des Europäischen Statistischen Systems (EES), Stärkung der Zusammenarbeit zwischen dessen Partnern und Sicherstellung der führenden Rolle dieses Systems in der amtlichen Statistik weltweit; d) Zusammenarbeit mit internationalen Organisationen und Drittländern zur Vereinfachung der Vergleichbarkeit europäischer Statistiken mit Statistiken, die von anderen statistischen Systemen erstellt werden und gegebenenfalls Unterstützung von Drittländern bei der Verbesserung ihrer statistischen Systeme; Eurostat stellt sicher, dass europäische Statistiken allen Nutzern gemäss statistischen Grundsätzen, insbesondere denen der fachlichen Unabhängigkeit, der Unparteilichkeit und der statistischen Geheimhaltung, zugänglich gemacht werden (Art. 6 des Beschlusses 2012/504/EU). Ergänzend zu Eurostat wurde das Europäische Statistische System (ESS) gegründet. Das ESS ist eine Partnerschaft zwischen Eurostat, den nationalen statistischen Ämtern (NSÄ) und anderen einzelstaatlichen Stellen, welche in den Mitgliedstaaten für die Entwicklung, Erstellung und Verbreitung europäischer Statistiken zuständig sind (Verordnung (EG) Nr. 223/2009 über europäische Statistiken). Die Aktivitäten von Eurostat werden durch das mehrjährige Europäische Statistische Programm finanziert (ESP; vgl. für den Zeitraum 2013-2020 Verordnung (EU) Nr. 99/2013).

Die Schweiz und die EG unterzeichneten 2004 das Abkommen über die Zusammenarbeit im Bereich der Statistik. Es trat 2007 in Kraft. Sein Ziel 
besteht darin, für die Erstellung und Verbreitung von kohärenten und vergleichbaren Statistiken für die Beschreibung und Überwachung aller für die bilaterale Zusammenarbeit relevanten Wirtschafts-, Sozial- und Umweltpolitiken zu sorgen; zu diesem Zweck entwickeln und benutzen die Vertragsparteien harmonisierte Methoden, Definitionen und Klassifikationen sowie gemeinsame Programme und Verfahren, in denen die statistischen Arbeiten bei den Verwaltungen im Bereich der Statistik organisiert werden (Art. 1). Die Hauptbereiche und statistischen Themen des Europäischen Statistischen Programms (ESP) sind auch für die statistische Zusammenarbeit zwischen der EU und der Schweiz relevant und stehen der Schweiz zur uneingeschränkten Teilnahme offen. Eurostat veröffentlicht «europakompatible» Daten aus der Schweiz, womit die internationale Sicht- und Vergleichbarkeit erhöht wird (www.eda.admin.ch/dea und Link zu Bilaterale Abkommen II (2004)/Statistik). In der Schweiz niedergelassene Einrichtungen haben das Recht, mit denselben vertraglichen Rechten und Pflichten wie Einrichtungen mit Sitz in der EU an von Eurostat verwalteten Programmen der EU teilzunehmen. Die Schweiz beteiligt sich auch an den Aktivitäten des Europäischen Statistischen Systems (ESS). Der finanzielle Beitrag der Schweiz beträgt rund 4,5 Mio. EUR jährlich (www.eda.admin.ch/dea und Link zu Bilaterale Abkommen II (2004)/Statistik). Das Abkommen verweist in Anhang A auf eine Reihe von Rechtsakten der EU, welche für die Vertragsparteien verbindlich erklärt werden.

Der Gemischte Ausschuss hat das Abkommen bis anhin zweimal aufdatiert und den Anhang A entsprechend revidiert $(2013,2019)$. Auf diese Weise wird gewährleistet, dass die Kohärenz und Vergleichbarkeit der Statistikerfassung in der EU und der Schweiz bei Weiterentwicklungen der unionalen Gesetzgebung sichergestellt ist (www.eda.admin.ch/dea und Link zu Bilaterale Abkommen II (2004)/Statistik).

\section{Umwelt}

Die EG errichtete 1993 die Europäische Umweltagentur (EUA). Gleichzeitig führte sie das Europäische Umweltinformations- und Umweltbeobachtungsnetz (European Environment Information and Observation Network, Eionet) ein. Heute regelt die auf Art. 192 AEUV (Umwelt) gestützte Verordnung (EG) Nr. 401/2009 über die Europäische Umweltagentur und das Europäische Umweltinformations- und Umweltbeobachtungsnetz die Einzelheiten. Die Agentur und Eionet sind damit beauftragt, zur Erreichung der im AEUV und in den unionalen Umweltaktionsprogrammen gesetzten Ziele zum Schutz und zur Verbesserung der Umwelt und zur Verwirklichung der nachhaltigen Entwicklung beizutragen. Zu diesem Zweck werden der EU und den Mitgliedstaaten a) objektive, zuverlässige und auf europäischer Ebene vergleichbare Infor- 
mationen zur Verfügung gestellt, anhand derer sie die notwendigen Umweltschutzmassnahmen ergreifen, die Ergebnisse dieser Massnahmen bewerten und eine sachgerechte Unterrichtung der Öffentlichkeit über den Zustand der Umwelt sicherstellen können, und hierfür b) die nötige technische und wissenschaftliche Unterstützung gegeben (Art. 1 der Verordnung (EG) Nr. 401/ 2009). Weiter unterstützt die Agentur die Europäische Kommission bei der Erarbeitung von Vorschlägen für neue Rechtsakte im Bereich des Umweltschutzes. Sie hat ihren Sitz in Kopenhagen.

Die Schweiz und die EG schlossen 2004 das Abkommen über die Beteiligung der Schweiz an der Europäischen Umweltagentur und dem Europäischen Umweltinformations- und Umweltbeobachtungsnetz (Eionet) ab. Es trat 2006 in Kraft und ermöglicht der Schweiz, sich in vollem Umfang an der Agentur und am Eionet zu beteiligen (Art. 1). Die Schweiz erhält Zugang zu den Daten und Informationen in der EU, welche über das Eionet erhoben und verbreitet werden. Sie wirkt in vollem Umfang, jedoch ohne Stimmrecht, im Verwaltungsrat der Agentur mit und wird an der Arbeit des wissenschaftlichen Beirats der Agentur beteiligt (Art. 3). Der finanzielle Beitrag der Schweiz beträgt rund 1,5 Mio. CHF jährlich (www.eda.admin.ch/dea und Link zu Bilaterale Abkommen II (2004)/Umweltschutz). Die Schweiz wendet die in Anhang I aufgeführten Rechtsakte an. Darüber hinaus verpflichtet das Abkommen die Schweiz aber nicht, unionales Sekundärrecht im Bereich des Umweltschutzes zu beachten.

\section{Ruhegehälter}

Die Beamtinnen und Beamten und sonstigen Bediensteten der EU sind in Bezug auf Gehälter, Löhne und andere Bezüge in der EU steuerpflichtig (Protokoll Nr. 7 über die Vorrechte und Befreiungen der EU, Verordnung (EWG, Euratom, EGKS) Nr. 260/68). Dazu gehören auch Ruhegehälter wegen Dienstunfähigkeit und Alters- und Hinterbliebenenversorgungsbezüge (Art. 2 der Verordnung). Die Steuer wird im Wege des Steuerabzugs erhoben (Art. 8 der Verordnung); dies gilt unabhängig davon, ob eine Beamtin oder ein Beamter oder eine sonstige Bedienstete oder ein sonstiger Bediensteter in einem EUMitgliedstaat oder in einem Drittstaat wohnt.

Der Schweizerische Bundesrat und die Europäische Kommission schlossen 2004 das Abkommen zur Vermeidung der Doppelbesteuerung von in der Schweiz ansässigen ehemaligen Beamten der Organe und Agenturen der EG ab. Es trat 2005 in Kraft. Das Abkommen findet auf Pensionen Anwendung, die von der Kommission oder aus einem von ihr errichteten Sondervermögen an 
natürliche Personen, die nach schweizerischem Steuerrecht ihren Wohnsitz oder Aufenthalt in der Schweiz haben, für die einem Organ oder einer Agentur der EU geleisteten Dienste gezahlt werden (Art. 1). Sofern die Kommission von den genannten Pensionen eine Steuer an der Quelle erhebt (was in der Praxis regelmässig der Fall ist), befreit die Schweiz diese Pensionen gemäss ihren innerstaatlichen Rechtsgrundsätzen von den Bundes-, Kantons- und Gemeindesteuern (Art. 2). Auf diese Weise wird eine ungerechtfertigte Doppelbesteuerung vermieden. Die steuerbefreiten Renten können allerdings zur Festsetzung des Steuersatzes herangezogen werden, der für andere steuerpflichtige Einkünfte gilt (www.eda.admin.ch/dea und Link zu Bilaterale Abkommen II (2004)/Ruhegehälter).

\begin{abstract}
Die Schweiz hat - bereits vor der Aushandlung des Abkommens mit der EG - mit mehreren EU-Mitgliedstaaten Doppelbesteuerungsabkommen abgeschlossen, welche ähnliche (reziproke) Vorgaben für die Vermeidung der Doppelbesteuerung enthalten. Diese Doppelbesteuerungsabkommen sind auf ehemalige Beamte und sonstige Bedienstete der EU allerdings nicht anwendbar. Aus diesem Grund war der Abschluss eines separaten Abkommens mit der EG notwendig (www.eda.admin.ch/dea und Link zu Bilaterale Abkommen II (2004)/Ruhegehälter).
\end{abstract}

\title{
4. Zinsbesteuerung / Automatischer Informationsaustausch
}

317 Der Rat der EU verabschiedete 2003 die Richtlinie 2003/48/EG im Bereich der Besteuerung von Zinserträgen. Das Ziel dieser Richtlinie bestand darin, dass Erträge, die in einem Mitgliedstaat im Wege von Zinszahlungen an wirtschaftliche Eigentümer, die natürliche Personen sind und die in einem anderen Mitgliedstaat steuerlich ansässig sind, erzielt werden, nach den Rechtsvorschriften dieses letzteren Mitgliedstaats effektiv besteuert werden. $\mathrm{Zu}$ diesem Zweck wurde der automatische Informationsaustausch eingeführt (wobei Belgien, Luxemburg und Österreich vorbehalten blieb, gleichwertige Massnahmen zu treffen). Vor dem Erlass dieser Richtlinie waren in einem Mitgliedstaat Ansässige häufig in der Lage, die Besteuerung von in einem anderen Mitgliedstaat vereinnahmten Zinsen im Mitgliedstaat ihres Wohnsitzes zu vermeiden; dieser Zustand führte zu mit dem Binnenmarkt nicht zu vereinbarenden Verzerrungen des Kapitalverkehrs zwischen den Mitgliedstaaten.

Die EU war sich bewusst, dass in der EU ansässige Personen ihre Gelder weiterhin Finanzinstituten in Drittländern anvertrauen konnten und eine solche Kapitalflucht die Erreichung der Ziele der Richtlinie gefährden würde. Aus diesem Grund verpflichtete die Richtlinie die EU-Mitgliedstaaten nur dann zur Anwendung der Richtlinie, sofern gewisse Drittländer - unter ihnen auch die Schweiz - Massnahmen anwenden, die den in der Richtlinie vorgesehenen 
Massnahmen gleichwertig sind; es war vorgesehen, mit den Drittländern zu diesem Zweck Abkommen zu schliessen (Art. 17). 2004 unterzeichneten die Schweiz und die EG das Abkommen über Regelungen, die den in der Richtlinie 2003/48/EG im Bereich der Besteuerung von Zinserträgen festgelegten Regelungen gleichwertig sind. Dieses Abkommen trat 2005 in Kraft und bezweckte, die grenzüberschreitende Steuerhinterziehung zu bekämpfen (Zinsbesteuerungsabkommen, ZinsbestA). Schweizer Banken waren verpflichtet, auf in der Schweiz anfallende Zinserträge von natürlichen Personen mit steuerlichem Wohnsitz in der EU einen Steuerrückbehalt zu erheben. Der Satz des Steuerrückbehalts betrug in den ersten drei Jahren der Anwendung des Abkommens $15 \%$, in den darauffolgenden drei Jahren $20 \%$ und danach $35 \%$ (Art. 1). Die Schweiz behielt $25 \%$ der Einnahmen aus dem Steuerrückbehalt als Entgelt für ihre Kooperationsbereitschaft und für die pauschale Abgeltung des Erhebungsaufwands und leitete $75 \%$ der Einnahmen an den Mitgliedstaat weiter, in dem der Nutzungsberechtigte ansässig war (Art. 8). Das Abkommen sah ein Verfahren vor, das es einem Nutzungsberechtigten ermöglichte, den Steuerrückbehalt zu vermeiden, indem er seine Zahlstelle in der Schweiz ausdrücklich ermächtigte, die Zinszahlungen an die zuständige Behörde dieses Staates zu melden (freiwillige Offenlegung, Art. 2). Die Schweiz und die einzelnen Mitgliedstaaten tauschten für die unter das Abkommen fallenden Erträge Informationen über Handlungen aus, die nach den Rechtsvorschriften des ersuchten Staates als Steuerbetrug galten oder ein ähnliches Delikt darstellten (Informationsaustausch, Art. 10).

2014 verabschiedete der Rat der EU die Richtlinie 2014/107/EU zur Änderung der Richtlinie 2011/16/EU bezüglich der Verpflichtung zum automatischen Austausch von Informationen im Bereich der Besteuerung. Damit wurde zuvörderst bezweckt, den globalen Standard für den automatischen Informationsaustausch über Finanzkonten in Steuersachen der OECD in der EU umzusetzen. Im Nachgang dazu unterzeichneten die Schweiz und die EU 2015 das Abkommen über den automatischen Informationsaustausch über Finanzkonten zur Förderung der Steuerehrlichkeit bei internationalen Sachverhalten. Es trat 2017 in Kraft und löste - formell als Änderungsprotokoll ausgestaltet - das Zinsbesteuerungsabkommen von 2004 ab. Die zuständige Behörde der Schweiz und jede zuständige Behörde der Mitgliedstaaten sind verpflichtet, die steuerrelevanten Informationen meldepflichtiger Konti (Name, Anschrift, Kontonummer, Finanzinstitut, Saldo etc.) jährlich untereinander automatisch auszutauschen (Art. 2). Zu diesem Zweck melden die Finanzinstitute die Informationen ihren Behörden, welche sie automatisch weiterleiten. Ein Hauptziel des Abkommens besteht analog zur unionalen Richtlinie darin, den 
globalen Standard für den automatischen Informationsaustausch der OECD auch im bilateralen Verhältnis umzusetzen. Weiter regelt das Abkommen den Informationsaustausch auf Ersuchen neu; dieser beruht nun auf dem Standard für Steuertransparenz und steuerlichen Informationsaustausch der OECD (Art. 26 des OECD-Musterabkommens zur Vermeidung der Doppelbesteuerung; www.eda.admin.ch/dea und Link zu Bilaterale Abkommen und Kooperationen ab 2004/Automatischer Informationsaustausch). Seit 2018 findet der Informationsaustausch jährlich statt. Damit leisten die Schweiz und die EU einen Beitrag zur Verhinderung von Steuerdelikten. Gleichzeitig besiegelte das Abkommen mit der EU, ausgehend von den Entwicklungen in der OECD, das Ende des Bankkundengeheimnisses im grenzüberschreitenden Bankgeschäft.

\section{Verteidigung}

320 Die EU-Mitgliedstaaten verpflichten sich, ihre militärischen Fähigkeiten schrittweise zu verbessern (Art. 42 Abs. 3 EUV). Dies gilt auch für ihre Verteidigungsfähigkeiten. Zur Unterstützung der Mitgliedstaaten und zur besseren Koordinierung von Massnahmen in den Bereichen Forschung, Beschaffung und Rüstung wurde 2004 die Europäische Verteidigungsagentur (EVA) mit Sitz in Brüssel gegründet (Gemeinsame Aktion 2004/551/GASP). Alle EU-Mitgliedstaaten beteiligen sich an der Agentur; einzig Dänemark hat sich gegen eine Teilnahme entschieden. Seit Lissabon findet die Agentur ihre primärrechtliche Verankerung in Art. 42 Abs. 3 und Art. 45 EUV. Der Beschluss (GASP) 2015/1835 über die Rechtsstellung, den Sitz und die Funktionsweise der Europäischen Verteidigungsagentur regelt die organisationsrechtlichen Einzelheiten. Die Europäische Verteidigungsagentur nimmt gemeinsam mit dem Europäischen Auswärtigen Dienst (EAD) auch die Sekretariatsaufgaben für die Ständige Strukturierte Zusammenarbeit (Permanent Structured Cooperation, PESCO) wahr, in deren Rahmen 25 Mitgliedstaaten eine vertiefte Verteidigungszusammenarbeit vereinbart haben (Beschluss (GASP) 2017/2315).

321 Die Agentur kann zur Erfüllung ihrer Aufgaben Verwaltungsvereinbarungen mit dritten Staaten, Organisationen und Einrichtungen schliessen, um gemeinsam einzelne Projekte und Programme durchzuführen (Art. 26 des Beschlusses (GASP) 2015/1835). Eine derartige Vereinbarung besteht auch mit der Schweiz (Framework for Cooperation, s. für den Text www.eda.admin.ch/ dea und Link zu Bilaterale Abkommen und Kooperationen ab 2004/Zusammenarbeit EVA). Die rechtlich nicht bindende Rahmenvereinbarung von 2012 ermöglicht es der Schweiz, über rüstungspolitische Entwicklungen informiert zu werden und punktuell Zugang zur multilateralen Rüstungskooperation zu 
erhalten. Es findet ein regelmässiger Austausch statt. Dabei entscheidet die Schweiz selbst, welche Informationen sie austauschen und an welchen Projekten und Programmen sie teilnehmen will (www.eda.admin.ch/dea und Link zu Bilaterale Abkommen und Kooperationen ab 2004/Zusammenarbeit EVA). Die Vereinbarung mit der Europäischen Verteidigungsagentur ergänzt das Netz bestehender bilateraler Rüstungsabkommen, welche die Schweiz mit zahlreichen europäischen Ländern abgeschlossen hat.

2017 entschied die Schweiz, zum ersten Mal an einem Zusammenarbeitsprojekt im Rahmen der EVA teilzunehmen. Es handelt sich um eine internationale Studie zum Schutz autonomer Systeme vor feindlichen Zugriffen (Protection of Autonomous Systems against Enemy Interference, PASEI). Auf Seiten der Schweiz sind der Bund (armasuisse) und der Industriepartner RUAG beteiligt (Pressemitteilung des Bundesrates vom 5. April 2017, Zusammenarbeit mit der EVA - erstes Zusammenarbeitsprojekt mit Schweizer Beteiligung).

\section{Satellitennavigation}

Die EG/EU hat seit den späten 1980er-Jahren diverse Initiativen und Massnahmen im Bereich der Satellitenkommunikation und -navigation ergriffen. Seit dem Inkrafttreten des Vertrags von Lissabon finden sich die Vorgaben zu den transeuropäischen Netzen und zur europäischen Raumfahrtpolitik in Art. 170 und Art. 189 AEUV. Beim Auf- und Ausbau transeuropäischer Netze im Bereich der Satellitennavigation und bei der Ausarbeitung einer europäischen Raumfahrtpolitik unterhält die EG/EU traditionell enge Bande mit der Europäischen Weltraumorganisation (European Space Agency, ESA, www.esa.int); diese internationale Organisation wurde 1975 ausserhalb der institutionellen Struktur der EWG/EG/EU gegründet (vgl. nun Art. 189 Abs. 3 AEUV zur Zusammenarbeit). Sie umfasst 22 Mitgliedstaaten - inkl. der Schweiz (SR 0.425.09) - und bezweckt, den Weltraum friedlich zu erforschen und zu nutzen und die wissenschaftlichen und wirtschaftlichen Interessen Europas im All zu fördern.

2004 gründete die EG die Agentur für das Europäische GNSS (European Global Navigation Satellite System) mit Sitz in Prag (Verordnung (EG) Nr. 1321/2004, heute: Verordnung (EU) Nr. 912/2010). Die Agentur verwaltet die Entwicklung und den Unterhalt der beiden zentralen Dienste der europäischen Satellitennavigationspolitik, das Programm Galileo und den Geostationären Navigations-Ergänzungsdienst für Europa (European Geostationary Navigation Overlay System, EGNOS; Verordnung (EU) Nr. 1285/2013): 
- das im Rahmen des Programms Galileo errichtete zivile System stellt die Infrastruktur des autonomen weltweiten Satellitennavigationssystems (GNSS) dar, die eine Satellitenkonstellation und ein weltweites Netz von Bodenstationen umfasst;

- $\quad$ EGNOS ist eine regionale Infrastruktur, die der Überwachung und Korrektur von offenen Signalen dient, die von bestehenden globalen Satellitennavigationssytemen gesendet werden, sowie von den Signalen des offenen Dienstes, der von dem im Rahmen des Programms Galileo geschaffenen System angeboten wird; sie umfasst Bodenstationen und mehrere auf geostationären Satelliten installierte Transponder.

Galileo ist das erste, unter ziviler Kontrolle stehende, weltweite Satellitennavigations- und Ortungssystem, das der internationalen Zusammenarbeit offensteht und kommerziell betrieben wird. Die ersten Satelliten wurden 2011 ins Weltall befördert. Galileo bezweckt, einen weltumspannenden und krisenfesten Kommunikationsdienst zu betreiben. Dabei soll die Unabhängigkeit Europas von den beiden militärisch kontrollierten Systemen GPS (Vereinigte Staaten) und GLONASS (Russland) gewährleistet werden. Gleichwohl konkurrenziert Galileo die beiden Systeme nicht, sondern ergänzt sie; es basiert auf derselben Grundtechnologie wie GPS und ermöglicht im Tandem mit GPS eine höhere Genauigkeit und Zuverlässigkeit (www.esa.int; vgl. Beschluss 2011/ 901/EU betr. Abkommen mit den Vereinigten Staaten über die Förderung, Bereitstellung und Nutzung von Galileo- und GPS-Satellitennavigationssystemen). In Ergänzung zu ihrer geopolitischen Bedeutung verfolgen Galileo und EGNOS auch soziökonomische Nutzeffekte (Art. 2 der Verordnung (EU) Nr. 1285/2013). Das Budget für den Unterhalt und die weitere Entwicklung der europäischen Satellitennavigationssysteme beläuft sich im Zeitraum 2014-2020 auf über 7 Mia. EUR.

325 Die Schweiz einerseits und die EU und ihre Mitgliedstaaten andererseits unterzeichneten 2013 das Kooperationsabkommen über europäische Satellitennavigationsprogramme. Die Schweiz ratifizierte das Abkommen 2015. Unionsrechtlich stellt es ein gemischtes Abkommen dar, womit es nicht nur von der EU, sondern auch von den EU-Mitgliedstaaten ratifiziert werden muss; einzelne Ratifizierungen stehen noch aus, weshalb das Abkommen vorerst provisorisch angewendet wird (www.eda.admin.ch/dea und Link zu Bilaterale Abkommen und Kooperationen ab 2004/Satellitennavigation). Ziel des Abkommens ist es, die langfristige Zusammenarbeit zwischen den Vertragsparteien im Bereich der Satellitennavigation unter ziviler Kontrolle, insbesondere durch die Teilnahme der Schweiz an den europäischen GNSS-Program- 
men, zu fördern, zu erleichtern und zu vertiefen (Art. 1). Die Schweiz trägt zur Finanzierung der europäischen GNSS-Programme bei: Ihr Beitrag belief sich 2014-2019 auf 252 Mio. CHF (Stellungnahme des Bundesrates vom 20. November 2019 auf die Interpellation 19.4008 «Aktualisierte Zusammenstellung aller Zahlungen und Beiträge der Schweiz an die EU»). Die Vertreterinnen und Vertreter der Schweiz können als Beobachterinnen und Beobachter an den Ausschüssen, die für die Verwaltung, Entwicklung und Durchführung der Tätigkeiten im Rahmen der europäischen GNSS-Programme eingerichtet wurden, ohne Stimmrecht teilnehmen (Art. 17).

Das Kooperationsabkommen enthält keine Bestimmungen über die Teilnahme der Schweiz an der Agentur für das Europäische GNSS. Eine solche Teilnahme ist in einem separaten Abkommen zwischen der EU und der Schweiz festzulegen (Art. 16). Die Paraphierung des fertig ausgehandelten Abkommens über die Teilnahme der Schweiz an dieser Agentur wurde von der EU angesichts des unklaren Schicksals des Institutionellen Abkommens auf Eis gelegt (Aussenpolitischer Bericht 2019, S. 1582). 



\section{Exkurs: Kohäsion}

Ein Ziel der EU ist es, den wirtschaftlichen, sozialen und territorialen Zusammenhalt und die Solidarität zwischen den Mitgliedstaaten zu fördern und die Unterschiede im Entwicklungsstand der verschiedenen Regionen und den Rückstand der am stärksten benachteiligten Gebiete zu verringern (Präambel zum EUV und AEUV, Art. 3 EUV, Art. 174 AEUV, Protokoll Nr. 28 zum EUV). Das EU-Recht reflektiert einen Solidaritätsgedanken, der zumindest im Ansatz an Finanzausgleichsysteme bundesstaatlicher Prägung erinnert. Gleichzeitig ist die Sicherstellung eines Mindestmasses an sozialer und wirtschaftlicher Kohärenz für die Konsolidierung und Weiterentwicklung des Integrationsprozesses unerlässlich. Folgerichtig enthält das unionale Haushaltsrecht diverse distributive Elemente:

- $\quad$ Auf der Einnahmenseite bestimmt sich der von den einzelnen Mitgliedstaaten zu entrichtende Anteil am Haushalt der EU wesentlich mit Blick auf ihre wirtschaftliche Leistungsfähigkeit. Dies gilt für die Berechnung der Bruttonationaleinkommen-Eigenmittelquote. Die Grundlage für die Bemessung der Mehrwertsteuer-Eigenmittel wurde zudem auf höchstens $50 \%$ des Bruttonationaleinkommens eines Mitgliedstaates festgesetzt (Art. 311 AEUV; Eigenmittelbeschluss 2014/335/EU; Verordnung (EU, Euratom) Nr. 608/2014; M. OESCH, Europarecht, Rz. 147-167).

- $\quad$ Auf der Ausgabenseite stehen die Mittel im Vordergrund, welche unter dem Budgetposten «Wirtschaftlicher, sozialer und territorialer Zusammenhalt» zugunsten strukturschwächerer und volkswirtschaftlich weniger prosperierender Regionen bereitstehen und einen Umverteilungseffekt zur Folge haben. Für den Zeitraum 2014-2020 sind dafür rund 325 Mia. EUR vorgesehen. Damit betreibt die EU Struktur- und Regionalpolitik bzw. - in der unionalen Terminologie - Kohäsionspolitik. Die rechtlichen Grundlagen für kohäsionspolitische Massnahmen finden sich zuvörderst in Art. 174-178 AEUV. Darüber hinaus sind weitere Vorgaben wie Art. 43 und Art. 162-164 AEUV einschlägig. Gestützt auf diese Vorgaben hat die EU eine Reihe von Struktur- und Investitionsfonds errichtet, deren Hauptzweck darin besteht, die unionale Regional- und Strukturpolitik durchzuführen, weitere Ziele wie die Förderung der Wettbewerbsfähigkeit von Unternehmen in der EU im Allgemeinen zu verfolgen und die im Haushaltsplan dafür vorgesehenen Mittel zweckmässig einzusetzen (Europäischer Fonds für regionale Entwicklung, Europäischer Sozialfonds, Kohäsionsfonds, Europäischer Landwirtschaftsfonds für die Ent- 
wicklung des ländlichen Raums, Europäischer Garantiefonds für die Landwirtschaft, Europäischer Meeres- und Fischereifonds, Europäischer Investitionsfonds, Europäischer Fonds für strategische Investitionen).

Die Kommission veröffentlicht periodisch Lagebeurteilungen. Dabei identifiziert sie Schlüsselbereiche, welche prioritär gefördert werden sollen, und bereitet den Boden für die künftige Ausrichtung der Kohäsionspolitik (vgl. Siebter Bericht über den wirtschaftlichen, sozialen und territorialen Zusammenhalt von 2017).

327 Die EU erwartet auch von Nicht-EU-Mitgliedstaaten, dass diese als Ausgleich zur Teilnahme am Binnenmarkt einen Beitrag zur Verringerung der wirtschaftlichen, sozialen und territorialen Ungleichheiten in der EU leisten. Dabei handelt es sich nicht nur um «das Eintrittsticket zum EU-Binnenmarkt», sondern auch um «ein Zeichen der Solidarität» (Botschafter Michael Matthiessen, NZZ vom 15. August 2020). Für die Beteiligung der EWR-EFTA-Mitgliedstaaten Island, Liechtenstein und Norwegen an der Kohäsionspolitik der EU wurde der EWR-Finanzierungsmechanismus eingerichtet (Art.115-117 und Protokoll Nr. 38 des EWR-Abkommens). Zwischen 2014 und 2021 bezahlen die EWREFTA-Mitgliedstaaten rund 2,8 Mia. EUR, wobei Norwegen mit $97 \%$ den weitaus grössten Teil stemmt und in der laufenden Periode jährlich rund drei Mal mehr als die Schweiz bezahlt (www.eda.admin.ch/erweiterungsbeitrag).

Die Schweiz bekräftigte 2006 in einem Memorandum of Understanding ihre Bereitschaft, sich finanziell an der Kohäsionspolitik der EU zu beteiligen und Projekte in den neuen Mitgliedstaaten in Mittel- und Osteuropa zu unterstützen. Gemäss diesem Memorandum handelt «[d]er Bundesrat (...) mit den [2004 der EU beigetretenen] Mitgliedstaaten Abkommen über die Modalitäten eines schweizerischen Beitrags in Höhe von einer Milliarde Schweizer Franken aus, der für einen Zeitraum von fünf Jahren ab der Genehmigung der entsprechenden Mittel durch das Schweizerische Parlament bereitgestellt wird»; «[d]er Bundesrat wählt die Projekte und Programme im Einvernehmen mit den Empfängerstaaten aus und berücksichtigt dabei ihre Wünsche, ihren Bedarf und ihre tatsächliche Aufnahmefähigkeit.» (www.eda.admin.ch/dea und Link zu Offene Themen/Zweiter Schweizer Beitrag). 2008 und 2014 wurden Zusätze in Bezug auf den schweizerischen Erweiterungsbeitrag zugunsten von Bulgarien, Rumänien und Kroatien unterzeichnet. Im Anschluss an die Unterzeichnung des Memorandums von 2006 wurde in der Schweiz das Bundesgesetz über die Zusammenarbeit mit den Staaten Osteuropas geschaffen (Osthilfegesetz, SR 974.1). Gegen dieses Gesetz wurde das Referendum ergriffen; in der Volksabstimmung sprachen sich $53 \%$ der abgegebenen Stimmen für die Annahme des Gesetzes und die damit verbundene Beitragsleistung an die EU 
- umgangssprachlich als «Kohäsionsmilliarde» betitelt - aus. In der Folge beteiligte sich die Schweiz an knapp 250 Projekten im Umfang von gesamthaft 1,3 Mia. CHF (www.eda.admin.ch/erweiterungsbeitrag). Die Empfängerstaaten waren die 2004, 2007 und 2013 der EU beigetretenen mittel- und osteuropäischen Staaten. Dieser Erweiterungsbeitrag stellte eine autonome Massnahme der Schweiz dar. Er war nicht Teil der Kohäsionspolitik der EU. Die Rahmenbedingungen für konkrete Projektförderungen wurden bilateral mit den Partnerländern vereinbart. Das Osthilfegesetz war auf zehn Jahre befristet und lief 2017 aus.

Eine unabhängige Expertengruppe evaluierte 2015 die Verwendung des Erweiterungsbeitrags durch die schweizerischen Behörden. Sie stellte hinsichtlich Relevanz, Wirksamkeit, Effizienz und Nachhaltigkeit der durchgeführten Projekte ein gutes Zeugnis aus. Bei der grossen Mehrheit der Projekte wurden die Ziele erreicht oder gar übertroffen. Verbesserungsbedarf wurden vor allem bei der Effizienz ausgemacht (Evaluationsbericht zum Erweiterungsbeitrag 2015, www.eda.admin.ch/erweiterungsbeitrag). Ähnlich positiv war die Bilanz zum Abschluss der Länderprogramme in den EU-12 Staaten, welche die DEZA und das SECO 2020 veröffentlichten; dabei wurde auch darauf hingewiesen, dass $10 \%$ des Erweiterungsbeitrags den in den Projekten involvierten Schweizer Unternehmen, Hochschulen und anderen Schweizer Institutionen zugute kamen (www.eda.admin.ch/erweiterungsbeitrag).

Die EU forderte die Schweiz wiederholt auf, ihr Engagement zu verlängern (vgl. zuletzt Schlussfolgerungen des Rates vom 19. Februar 2019, Rz. 11), weil es sich dabei gemäss der Europäischen Kommission um eine «natürliche Konsequenz» des Zugangs der Schweiz zum Binnenmarkt handelt (NZZ vom 17. Juli 2020, S. 9). Der Bundesrat erledigte die Vorbereitungsarbeiten, wobei er vorschlug, den Fokus nicht nur auf die Verringerung wirtschaftlicher und sozialer Ungleichheiten zu legen, sondern auch auf die Unterstützung von Massnahmen im Bereich der Migration (Botschaft zweiter Schweizer Beitrag, passim). Die Bundesversammlung verlängerte und revidierte 2018 das Bundesgesetz über die Zusammenarbeit mit den Staaten Osteuropas und legte damit den Grundstein für die Leistung eines weiteren Erweiterungsbeitrags im Bereich der Kohäsion (vgl. Art. 10; demgegenüber stützt sich der neue Rahmenkredit für Projekte im Bereich der Migration auf das AsylG). Gegen diese Gesetzesrevision wurde kein Referendum ergriffen. Ende 2019 bewilligte die Bundesversammlung den zweiten Erweiterungsbeitrag der Schweiz in Form zweier Rahmenkredite für Kohäsion und Migration. Es ist vorgesehen, dafür insgesamt wiederum rund 1,3 Mia. CHF aufzuwenden. Dabei folgte die Bundesversammlung der Empfehlung des Bundesrates, die Leistung des Beitrags mit Fortschritten in anderen europapolitischen Dossiers zu verknüpfen (Botschaft zweiter Schweizer Beitrag, S. 6667). Gemäss Bundesbeschluss werden keine 
Verpflichtungen auf der Grundlage dieser Rahmenkredite eingegangen (d.h. es werden keine Vereinbarungen mit den Partnerländern zur Durchführung konkreter Projekte unterzeichnet), «wenn und solange die EU diskriminierende Massnahmen gegen die Schweiz erlässt» (Bundesbeschlüsse vom 3. Dezember 2019, BBl 2020 757, BBl 2020 759). Damit zielt die Bundesversammlung auf die zumindest nach ihrer Einschätzung - diskriminierende Nichtverlängerung der Börsenäquivalenz durch die EU (N. 369).

Mit Blick auf die enormen Finanzmittel, welche die EU zur Revitalisierung und Stärkung der europäischen Wirtschaft im Nachgang zu den einschneidenden Massnahmen zur Bekämpfung der durch das Coronavirus (COVID-19) ausgelösten epidemiologischen Krise aufzubringen gewillt ist, ist es durchaus denkbar, dass die EU die Schweiz auffordern wird, einen höheren Beitrag als die bis anhin vorgesehenen 1,3 Mia. CHF aufzuwenden (vgl. Tagesanzeiger vom 19. Juni 2020, S. 6).

Die Schweiz und die EU verleihen in einer Erklärung zur Kohäsion, welche dem geplanten InstA beigefügt ist, ihrer Überzeugung Ausdruck, «dass es wichtig ist, die koordinierte Zusammenarbeit bei Massnahmen zur Verringerung der wirtschaftlichen und sozialen Ungleichheiten zwischen ihren Regionen aufrechtzuerhalten, um eine kontinuierliche und ausgewogene Stärkung der wirtschaftlichen und sozialen Beziehungen untereinander zu fördern.» Es wird festgehalten, dass die Schweiz die Beiträge weiterhin autonom und abhängig vom Zugang zum Binnenmarkt entrichtet. Eine Verpflichtung wird nicht begründet (s. auch Präambel des InstA; Erläuterungen des EDA, S. 16). Es wird vereinbart, dass die Durchführungsbestimmungen für den autonomen Beitrag der Schweiz weiterhin Gegenstand eines spezifischen «politischen Abkommens» in der Form eines Memorandum of Understanding zwischen der EU und der Schweiz sind. Diese Erklärung würde beim Abschluss des InstA ebenfalls in Kraft gesetzt. 


\section{Dritter Teil}

\section{Autonomer Nachvollzug}





\section{Politik der Europaverträglichkeit}

\section{A. Begriff und Zweck}

Das schweizerische Recht wird seit jeher durch das europäische Recht beeinflusst, auch ohne dass staatsvertragliche Verpflichtungen Rezeptionen und Anpassungen auslösen.

Ein anschauliches - und in seiner Aussagekraft unübertroffenes - Beispiel des autonomen Nachvollzugs betrifft die Sommerzeit. Im Sommer 1980 stellten die damaligen EWG-Mitgliedstaaten (sowie weitere europäische Staaten) zum ersten Mal gemeinsam die Uhren eine Stunde nach vorne. Um die unterschiedlichen einzelstaatlichen Regelungen für die Folgejahre zu harmonisieren, setzte die EWG mit der Richtlinie 80/737/EWG ein gemeinsames Datum für den Beginn der Sommerzeit 1981 fest. Die Schweiz verpasste es nach einer negativen Referendumsabstimmung, die Sommerzeit 1980 ebenfalls einzuführen, und mutierte zur «Zeitinsel» inmitten Europas und zum «Sonderfall» im wahrsten Sinn des Wortes (G. BiAGGinI, BV-Kommentar, Art. 125 Rz. 5). Erst 1981 wurde die Sommerzeit auch in der Schweiz eingeführt; die Wiederaufnahme der gesetzlichen Einführung der Sommerzeit wurde nicht mehr mit dem Referendum bekämpft. Eine 1982 lancierte Volksinitiative zur Abschaffung der Sommerzeit scheiterte zudem im Stadium der Unterschriftensammlung (BBl 1982 II 968, BBl 1984 I 619). Seither ticken die Uhren in der Schweiz im Einklang mit denjenigen in der EU (Richtlinie 2000/84/EG; Art. 15 MessG; Sommerzeitverordnung, SR 941.299.1).

Ein Beispiel für die Auseinandersetzung mit dem gemeinschaftlichen Grundrechtsschutz qua Rechtsvergleichung betrifft die Gleichstellung von Mann und Frau. 1981 nahmen Volk und Stände den neuen «Gleichberechtigungsartikel» an (Art. 4 Abs. 2 BV 1874, heute Art. 8 Abs. 3 BV). In der bundesrätlichen Botschaft wurde die rechtliche Stellung von Mann und Frau in mehreren europäischen Staaten und in den Vereinigten Staaten analysiert; dabei wurde auch die grundrechtsähnliche Gewährleistung des gleichen Entgelts für Männer und Frauen bei gleicher oder gleichwertiger Arbeit im EG-Recht thematisiert (Art. 119 EWGV, heute Art. 157 AEUV; Botschaft Gleiche Rechte für Mann und Frau, S. 106-107). Auch beim Erlass des Gleichstellungsgesetzes von 1995 (GlG) wurde das einschlägige EG-Recht rechtsvergleichend aufgearbeitet (Botschaft Gleichstellungsgesetz, S. 1286-1291).

Ende der 1980er-Jahre wurde die Politik der Anpassung an das europäische Recht in der Schweiz formalisiert und systematisiert. Seither dominiert die Politik des autonomen Nachvollzugs bzw. - synonym verstanden - der Europaverträglichkeit die hiesige Gesetzgebungstätigkeit. Der «Brussels effect» (A. BRADFORD, passim) wirkt auch in der Schweiz - und dies in ganz erheblichem Ausmass. Auslöserin dieser Entwicklung war die spektakuläre Revitalisierung des europäischen Integrationsprozesses im Rahmen der EWG in den 1980er-Jahren. Mit dem Weissbuch der Kommission von 1985, der Einheitlichen Europäischen Akte (EEA) von 1986 und dem Cecchini-Bericht von 1988 wurden die rechtlichen und programmatischen Grundlagen für die Vollendung 
des Binnenmarkts gelegt. Ebenso konkretisierten sich die Pläne für die Errichtung einer Wirtschafts- und Währungsunion sowie für die Schaffung einer politischen Union. Vor diesem Hintergrund entschied der Bundesrat, aktiv darauf hinzuarbeiten, die Nachteile, die sich aus dem Abseitsstehen der Schweiz ergeben, zu minimieren. 1988 veröffentlichte er seinen ersten Integrationsbericht. Als einen zentralen Pfeiler seiner Europapolitik führte er dabei die Politik der Europaverträglichkeit ein: Neue Gesetze und Verordnungen wie auch die Änderung von bestehenden Erlassen sollen im Rahmen des verwaltungsinternen Vorbereitungsverfahrens systematisch auf ihre Europakompatibilität überprüft werden. Das schweizerische Recht soll auch ohne staatsvertragliche Verpflichtung an das europäische angeglichen werden (Integrationsbericht 1988, S. 380):

«Unser Ziel muss sein, in Bereichen von grenzüberschreitender Bedeutung (und nur dort)
eine grösstmögliche Vereinbarkeit unserer Rechtsvorschriften mit denjenigen unserer euro-
päischen Partner zu sichern. (...) Es geht bei diesem Streben nach Parallelität nicht darum,
das europäische Recht automatisch nachzuvollziehen, wohl aber darum, zu verhindern,
dass ungewollt und unnötigerweise neue Rechtsunterschiede geschaffen werden, welche
die grundsätzlich angestrebte gegenseitige Anerkennung der Rechtsvorschriften auf euro-
päischer Ebene behindern.»

Nach der Ablehnung des Beitritts zum EWR 1992 bekräftigte der Bundesrat, dass es «in unserem eigenen Interesse [liegt], die systematische Prüfung der Europaverträglichkeit des aktuellen und künftigen schweizerischen Rechts weiterzuführen» (Botschaft Folgeprogramm 1993, S. 821). Damit wurde die Politik des autonomen Nachvollzugs zu einer grundlegenden Rechtsetzungsmaxime erhoben, welche die schweizerische Rechtsordnung als Leitmotiv in ihrer ganzen Breite durchdringt. Abweichungen von europarechtlichen Vorbilderregelungen bleiben zwar selbstredend möglich. Eine verbindliche Nachvollzugsverpflichtung besteht nicht. Es handelt sich allein um eine Prüfungsobliegenheit. Helvetische Sonderlösungen bedürfen in Kenntnis der Sachlage aber einer sachlichen Begründung. Sie sollen dort bewusst gewählt werden, wo eine Abweichung der eigenen Interessenlage dient und auch unter integrationspolitischen Gesichtspunkten vertretbar scheint.

Die Politik des autonomen Nachvollzugs verfolgt einen doppelten Zweck:

- Der autonome Nachvollzug beruht auf wirtschaftlichen Gründen. Durch eine konsequente Angleichung des schweizerischen Rechts sollen die wirtschaftlichen Nachteile, welche sich aus der Nichtmitgliedschaft der Schweiz in der EU bzw. im EWR ergeben, minimiert und die Wettbewerbsfähigkeit der Wirtschaft gestärkt werden. 
- Die möglichst europakompatible Ausgestaltung des schweizerischen Rechts bildet die Grundlage, um für alle integrationspolitischen Optionen gewappnet zu sein, d.h. - wie der Bundesrat 1993 ausführte - für «einen möglichen (späteren) EWR- oder EG-Beitritt ohne unüberwindbare Hürden oder allenfalls eine auf bilaterale Abkommen beschränkte Alternative» (Botschaft Folgeprogramm 1993, S. 810).

Als Grundlage für die Prüfung der Europaverträglichkeit von neuen bzw. geänderten Bundesgesetzen dienen die Botschaften des Bundesrates. Art. 141 Abs. 1 ParlG schreibt vor, dass der Bundesrat dem Parlament seine Erlassentwürfe zusammen mit einer erläuternden Botschaft unterbreitet. Darin informiert er über die Ziele, welche mit dem vorgeschlagenen Erlass verfolgt werden, und begründet die gewählte Lösung. Gemäss Art. 141 Abs. 2 lit. a ParlG erläutert der Bundesrat - «soweit substantielle Angaben dazu möglich sind» - auch «das Verhältnis [der Vorlage] zum europäischen Recht»; gemäss Art. 141 Abs. 2 lit. a ${ }^{\text {bis }}$ ParlG orientiert er über «die Nutzung des Handlungsspielraums der Schweiz». Diese Ausführungen finden sich im sog. «Europakapitel». Der Botschaftsleitfaden äussert sich dazu wie folgt (Fassung von 2019, S. 25, www.bk.admin.ch und Link zu Dokumentation/Sprachen/Hilfsmittel):

\footnotetext{
«Es ist darzulegen, ob die vorgesehenen Regelungen mit dem geltenden oder in Ausarbeitung stehenden EU-Recht, das die Schweiz nicht bindet, sowie mit einschlägigen Empfehlungen im Bereich des Menschenrechtsschutzes (Europarat, UNO) kompatibel sind. Wenn eine Vorlage Angleichungen schweizerischer Normen an europäische Regelungen enthält, sind Umfang und Reichweite dieser Angleichungen darzustellen. Zeigen Sie auf, wie bei der Ausgestaltung der neuen Regelung der Handlungsspielraum der Schweiz bei einer autonomen Übernahme ausgenutzt wurde (...). Begründen Sie gegebenenfalls, weshalb die schweizerische Regelung von der europäischen abweicht. Halten Sie ausserdem fest, ob mit Synergien oder Reibungsverlusten zu rechnen ist.»
}

In der Praxis beschränkt sich der autonome Nachvollzug keineswegs mehr wie ursprünglich gefordert und bis heute zeitweise missverständlich kolportiert - allein auf grenzüberschreitende Tatbestände. Er reicht seit jeher über das klassische Aussenwirtschaftsrecht hinaus und erfasst im Gleichschritt mit dem sich ebenfalls ausdehnenden Unionsrecht eine immer grössere Vielzahl von Regelungsbereichen (Spillover-Effekt). Mittlerweile ist der autonome Nachvollzug der Regelfall der Gesetz- und Verordnungsgebungspraxis im Bund. Das Bundesrecht wird dem europäischen Recht gleichsam systematisch nachgebildet. 1998 hielt der Bundesrat fest, dass mit der Einführung der Europaverträglichkeitsprüfung ein «Europareflex» geschaffen wurde; neue schweizerische Regeln «sind im allgemeinen eurokompatibel, ausnahmsweise nicht» (Antwort vom 13. Mai 1998 auf die Einfache Anfrage 98.1032 «Swisslex II»). Im 
Europabericht von 2006 bestätigte der Bundesrat die Politik des autonomen Nachvollzugs. Gleichzeitig erinnerte er daran, dass dieser Grundsatz nur gilt, «insofern er den Interessen der Schweiz nützt» (Europabericht 2006, S. 6832). Ähnlich stellte der Bundesrat im Europabericht von 2010 fest: Der autonome Nachvollzug soll «nur dort angestrebt werden, wo wirtschaftliche Interessen dies erfordern oder rechtfertigen» (Europabericht 2010, S. 7288).

\section{B. Verhältnis zur Rechtsvergleichung}

337 Die Politik des autonomen Nachvollzugs bewirkt eine fortlaufende Europäisierung des schweizerischen Rechts. Dieser Anpassungsmechanismus unterscheidet sich strukturell und methodisch von früheren Rezeptionen und Nachahmungen ausländischer Rechtsinstitute, wie sie auch für die schweizerische Rechtsordnung unter dem Titel der Rechtsvergleichung seit jeher prägend sind.

Bereits die Bundesverfassung von 1848 wurde wesentlich von angloamerikanischen Konzepten und Idealen der Französischen Revolution beeinflusst (A. KLEY, S. 248). Auch die kantonalen Kodifikationen des Zivil- und Strafrechts im 19. Jahrhundert sowie die nationalen Rechtsvereinheitlichungen im 19. und 20. Jahrhundert gründeten erheblich und wechselseitig auf dem Vergleich mit anderen Kodifikationen aus dem In- und Ausland (R. PAHUD DE MORTANGES, S. 236). Der Blick über die Grenze und die Methode der Rechtsvergleichung gehören spätestens seit Mitte des letzten Jahrhunderts zum Standardrepertoire des schweizerischen Gesetzgebers bzw. der Dienststellen, welche im Gesetzgebungsverfahren die grundlegenden Vorarbeiten leisten. In bundesrätlichen Botschaften finden sich regelmässig Ausführungen zur rechtlichen Situation vor allem in unseren Nachbarländern sowie - im Wirtschaftsrecht und in Bezug auf Grundrechte - in den Vereinigten Staaten. Legislatorische Lösungen, welche andere Rechtsordnungen für ein gleichgelagertes Problem gewählt haben, werden zusammengetragen, verglichen und bewertet. Einen entscheidenden Beitrag dazu leistet das 1982 gegründete Schweizerische Institut für Rechtsvergleichung (SIR) in Lausanne, welches für Bundesstellen unentgeltlich rechtsvergleichende Studien erstellt.

339 Ein wesentliches Element der Rechtsvergleichung als Methode besteht darin, dass sie grundsätzlich zweckfrei ist (P.V. KunZ, Rechtsvergleichung, S. 34; A. Rusch, Rz. 5). Sie folgt keinem vorgegebenen Ziel im Sinn etwa einer Präferenz für eine bestimmte Rechtsordnung, deren Lösung aus rechtsetzungstechnischen Erwägungen als überlegen gilt oder aus politischen oder anderen Gründen $a b$ initio favorisiert wird. In diesem Punkt unterscheidet sich die 
Methode der Rechtsvergleichung grundlegend von der Politik des autonomen Nachvollzugs. Der autonome Nachvollzug beruht auf einem bewussten politischen Entscheid, das schweizerische Recht europakompatibel auszugestalten und unterschiedliche legislatorische Lösungen möglichst zu vermeiden. Die Politik hat sich ausdrücklich dafür ausgesprochen, das schweizerische Recht dem Unionsrecht anzupassen und nachzubilden - als «materieller Legislativfaktor von neuartiger Gestalt» (D. THÜRER, Europaverträglichkeit, S. 580) und grundsätzlich unabhängig davon, ob in einem konkreten Fall die europäische Lösung mit Blick auf den zu normierenden Sachverhalt in der Tat die überzeugendste darstellt oder nicht. Abweichungen vom EU-Recht sollen nur dort weiterverfolgt werden, wo das Interesse an einer Sonderlösung - etwa zur Förderung von Standort- und Wettbewerbsvorteilen - höher zu gewichten ist als die integrationspolitisch und volkswirtschaftlich begründete Vorabentscheidung zugunsten einer europaverträglichen Ausgestaltung. Das EU-Recht entfaltet seine Relevanz im schweizerischen Gesetzgebungsverfahren nicht als eine von mehreren gleichrangigen Inspirationsquellen, sondern beansprucht als «Leitrechtsordnung» systembedingt eine Vormachtstellung. Der Prozess des vorurteilslosen Vergleichens und Abwägens von legislatorischen Lösungen, welche ausländische Rechtsordnungen für ein gleichgelagertes Problem gewählt haben, verliert in der Schweiz an Bedeutung. Für das EU-Recht findet dieser Prozess vorgängig in Brüssel und Strassburg statt, wo die Rechtskulturen der 27 Mitgliedstaaten in den Gesetzgebungsprozess einfliessen und ihren Niederschlag in den gewählten legislatorischen Lösungen wiederfinden mögen. Zu diesem Gesetzgebungsprozess hat die Schweiz keinen Zugang (s. zu den Mitwirkungsrechten bei der Erarbeitung von unionalen Rechtsakten im Anwendungsbereich von bilateralen Abkommen N. 94).

Darüber hinaus beeinflusst das EU-Recht das schweizerische Recht weiterhin auch unter dem Titel der Rechtsvergleichung, ohne dass seine Berücksichtigung als autonomer Nachvollzug zu qualifizieren wäre. Diesfalls dient das Unionsrecht gleichberechtigt mit anderen Rechtsordnungen als Inspirationsquelle. Für diese Form der Europäisierung des schweizerischen Rechts ist typisch, dass ein unionales Rechtsinstitut meist nicht tel quel Eingang in das schweizerische Recht findet, sondern in abgeänderter Form und adaptiert an die schweizerische Problemlage, welche sich von derjenigen im Unionsrecht unterscheiden mag. Im Rahmen der Rechtsanwendung geht es dabei - im Unterschied zur europarechtsverträglichen Auslegung (N. 354-362) - um eine «europarechtsorientierte Auslegung» (S. EMMENEGGER/A. TsCHENTSCHER, Art. 1 Rz. 303). 
Ein Beispiel stellt die Kodifikation des Herkunftsortsprinzips im Binnenmarktgesetz (BGBM) von 1995 dar. Dieses Prinzip wird im Grundsatz dem europarechtlichen Cassis de Dijon-Prinzip nachgeformt, unterscheidet sich bezüglich Anwendungsbereich, Inhalt und Wirkungsweise davon aber in wesentlichen Punkten (vgl. Art. 3 BGBM; Botschaft BGBM, S. 1282). Es fand unter dem Titel der Rechtsvergleichung - das Gemeinschaftsrecht diente als «Vorbild» (BGE 125 I 276, E. 4e) - Eingang ins schweizerische Recht; «a classic EC solution for a purely Swiss problem» (F. MAIANI, Europeanization, S. 114). Demgegenüber erfolgte die einseitige Einführung des Cassis de Dijon-Prinzips vis-à-vis Produkten aus dem EWR unter dem Titel des autonomen Nachvollzugs (N. 374-376).

Bundesrätliche Botschaften verweisen auch bei grundrechtsrelevanten Erlassentwürfen rechtsvergleichend auf die Rechtslage in der EU (vgl. etwa Botschaft Gleichstellungsgesetz, S. 1286-1291; Botschaft Strafprozessrecht, S. 1121-1125; s. für Beispiele von Anpassungen in grundrechtssensiblen Bereichen unter dem Titel des autonomen Nachvollzugs N. 346). Auch das BGer und das BVerwG richten ihr Augenmerk bei der Auslegung von Grundrechten rechtsvergleichend auf den Grundrechtsschutz in der EU und die Rechtsprechung des EuGH (vgl. BGE 123 I 152; BGE 125 I 21; BGE 133 II 136; BGE 139 I 72; BVGE 2011/51; Urteil des BVerwG E-1422/2014 vom 31. Juli 2014). Die schweizerischen Gerichte tragen auf diese Weise zur Kohärenz der grundrechtlichen Praxis in Europa bei.

341 Die Schweiz profitiert bei der Übernahme von EU-Recht von den Vorleistungen der EU. Sie lässt sich rechtsvergleichend von Lösungen inspirieren, welche die EU für ähnlich gelagerte Probleme und Sachverhalte entwickelt hat, und übernimmt unionale Lösungen im Rahmen des autonomen Nachvollzugs, um die wirtschaftlichen Nachteile des institutionellen Abseitsstehens zu minimieren. Häufig geben solche Anpassungen keinen Anlass zu Kontroversen. Sie erfolgen routinemässig und segeln unterhalb des Radars einer breiteren Öffentlichkeit. Dies ist paradigmatisch bei der Verabschiedung von technischen Vorschriften - Ursprung und hauptsächlicher Anwendungsbereich der Politik des autonomen Nachvollzugs - der Fall. Hier handelt es sich um eine Materie, deren Regelung getrost den Spezialistinnen und Spezialisten in der EU überlassen werden kann, solange es um technische Details geht und keine politischen Grundsatzentscheidungen zu klären sind. Die EU gibt den Takt an; die Schweiz folgt hintenan. In dieser Konstellation ist der «Europareflex» (N. 336) des Gesetz- und Verordnungsgebers demokratietheoretisch unproblematisch.

Heikel wird es, wenn die Auseinandersetzung mit den Vor- und Nachteilen einer unionalen Lösung nurmehr pro forma erfolgt und die Angleichung an das EU-Recht quasi automatisch stattfindet, obwohl in einem konkreten Fall politisch umstrittene Fragen aufgeworfen werden, die der Gesetzgeber debattieren und beantworten muss. Die Politik des autonomen Nachvollzugs darf nicht dazu führen, dass die grundlegenden politischen Weichenstellungen für die Schweiz in Brüssel und Strassburg erfolgen. Die Behörden sind gut beraten, 
die Rezeption und Übernahme von EU-Recht transparent auszuweisen, die Vor- und Nachteile einer Anpassung zu benennen und Alternativen aufzuzeigen. Der Gesetzgeber steht sodann in der Verantwortung, die verwaltungsinternen Vorarbeiten kritisch zu reflektieren. Dazu gehört, autonome Lösungen zu wählen, wenn sich eine Gleichschaltung im Licht verfügbarer Alternativen nicht als sachgerecht erweist und auch integrationspolitisch vertretbar ist. 



\section{Beispiele}

Die Politik des autonomen Nachvollzugs gilt als Rechtsetzungsmaxime umfassend für die Legislativtätigkeit auf Stufe Bund. Die nachfolgenden Beispiele legen Zeugnis darüber $\mathrm{ab}$, in welch erheblichem Ausmass das schweizerische Recht vom Recht der EU beeinflusst wird.

In den Kantonen gibt es grundsätzlich keine ausdrücklichen Vorgaben, das kantonale Recht systematisch an das EU-Recht anzupassen. Eine Verpflichtung, das EU-Recht im Gesetzgebungsprozess zu beachten, findet sich soweit ersichtlich einzig im Kanton Freiburg: Gemäss Art. 197 Abs. 2 lit. f des Grossratsgesetzes von 2006 (GRG) informiert die Botschaft zu einem Gesetzesvorhaben «insbesondere über die Verfassungsmässigkeit, die Übereinstimmung mit dem Bundesrecht und die Europaverträglichkeit des Entwurfs.» In den anderen Kantonen fliesst das EU-Recht fallweise und punktuell ein, wenn Schnittstellen bestehen, ohne dass eine ausdrückliche Prüfungsobliegenheit existiert.

\section{A. Gesetzesrecht}

Die erste umfassende Anpassung des schweizerischen (Wirtschafts-) Rechts an das europäische Recht erfolgte im Rahmen der Swisslex-Vorlage von 1993. Ursprünglich handelte es sich um eine geplante Anpassung von 61 Bundesgesetzen, zu der die Schweiz aufgrund des EWR-Beitritts verpflichtet gewesen wäre. Zur Beratung dieser damals Eurolex genannten Vorlage wurden 1992 zwei Sondersessionen abgehalten. Die Erlasse wurden nicht dem Referendum unterstellt; ihr Inkrafttreten wurde von der Annahme des EWR-Abkommens abhängig gemacht. Nach der Ablehnung des EWR-Beitritts fielen die geplanten Anpassungen des schweizerischen Rechts zwar dahin. Gleichwohl nahmen Bundesrat und Parlament einen Teil dieser Reformvorhaben wieder auf. Diese wurden in der Folge in Swisslex-Vorlage umgetauft. Unter diesem Titel wurden 27 Gesetzesrevisionen, welche im Wesentlichen eine Übernahme des Gemeinschaftsrechts bezweckten, verabschiedet (Botschaft Folgeprogramm 1993, S. 805).

Prominente neue Gesetze und Änderungen von bestehenden Gesetzen der Swisslex-Vorlage betrafen Art. 40a-g und Art. 333 des Obligationenrechts (OR, s. zur Auslegung von Art. 333 OR N. 361), das Produktehaftpflichtgesetz (PrHG), das Pauschalreisegesetz und das Konsumkreditgesetz (KKG). In Ergänzung zum Swisslex-Paket wurden Mitte der 1990erJahre auch das Binnenmarktgesetz (BGBM), das Bundesgesetz über die technischen Handelshemmnisse (THG) und das Bundesgesetz über Kartelle und andere Wettbewerbsbeschränkungen (KG) erlassen bzw. - im Fall des letzteren - totalrevidiert. Auch diese Gesetze beruhen wesentlich auf den Vorbilderregelungen in der EG bzw. EU. 

che unter dem Titel des autonomen Nachvollzugs eine europakompatible Ausgestaltung erfahren haben.

Dazu gehören etwa das Anwaltsgesetz (BGFA), das Heilmittelgesetz (HMG), das Luftfahrtgesetz (LFG), das Fernmeldegesetz (FMG), das Nationalbankgesetz (NBG), das Fusionsgesetz (FusG), das Mehrwertsteuergesetz (MWSTG), das Produktsicherheitsgesetz (PrSG) und das Kollektivanlagengesetz (KAG). Auch die Bestimmungen zu Freisetzungsversuchen und zum Inverkehrbringen von gentechnisch veränderten Organismen (Gentechnikgesetz, GTG), das Patentgesetz (PatG) und das Urheberrechtsgesetz (URG) beruhen mittlerweile auf EU-Recht. Selbst im Lebensmittelrecht nähert sich die Schweiz in grossen Schritten dem EU-Recht an; die 2014 abgeschlossene Totalrevision des Lebensmittelgesetzes (LMG) stand ganz im Zeichen der Anpassung an das EU-Recht. Ähnliches gilt für das Finanzmarktinfrastrukturgesetz (FinfraG) sowie - in Teilen - für das Finanzdienstleistungsgesetz (FIDLEG) und das Finanzinstitutsgesetz (FINIG). Die laufende Revision des Datenschutzgesetzes (DSG) steht unter dem Stern des autonomen Nachvollzugs. Im Finanzmarkt- und Datenschutzrecht ist die Schweiz faktisch gar gezwungen, das EU-Recht zum Nennwert zu nehmen, weil die Wirtschaft darauf angewiesen ist, dass die Europäische Kommission die schweizerische Gesetzgebung als gleichwertig mit derjenigen der EU qualifiziert ( $\mathrm{N}$. 364-371).

Eine laufende Revision des erwähnten KAG offenbart, wie auch im Rahmen des autonomen Nachvollzugs Spielräume bestehen und wie die Schweiz diese nutzt. Aktuell soll eine neue Fondskategorie für qualifizierte Anlegerinnen und Anleger geschaffen werden, die von der Bewilligungspflicht befreit ist, der sog. Limited Qualified Investor Fund (L-QIF). Die Richtlinie 2011/61/EU über die Verwalter alternativer Investmentfonds setzt unionsrechtlich die Leitplanken; die Schweiz hat diese Richtlinie autonom übernommen. Gleichzeitig belässt die Richtlinie in mehrerlei Hinsicht einen Gestaltungsspielraum. Folgerichtig schlägt der Bundesrat eine Lösung vor, welche sich auch mit Blick auf die Rechtslage in den Mitgliedstaaten als sachlich stimmig und wirtschaftlich vorteilhaft erweist. Er orientiert sich in der Botschaft an denjenigen Mitgliedstaaten, «die ein dem L-QIF sehr ähnliches Produkt kennen oder aus denen die meisten Fonds (gemessen am Anlagevolumen) stammen, die an Anlegerinnen und Anleger in der Schweiz verkauft werden»; dazu gehören Luxemburg, Malta, Irland und Frankreich (Botschaft zur Änderung des Kollektivanlagengesetzes, S. 12, noch nicht im BBl veröffentlicht; vgl. Pressemitteilung des Bundesrates vom 19. August 2020, Bundesrat verabschiedet Botschaft zur Änderung des Kollektivanlagengesetzes). Auf diese Weise soll das primäre Ziel der Revision erreicht werden: «Der Schweizer Fondsplatz schliesst mit dem L-QIF (...) zu seinen europäischen Peers auf.» (ibid., S. 14).

Diese keineswegs abschliessende Auflistung veranschaulicht, dass sich der autonome Nachvollzug längst nicht mehr nur auf den traditionellen Bereich des Warenverkehrs und auf Regelungsbereiche mit grenzüberschreitender Wirkung beschränkt. Das EU-Recht durchdringt das schweizerische Recht in seiner ganzen Breite und Tiefe (s. zum Spillover-Effekt auch oben N. 336). Dabei übernimmt die Schweiz mitunter auch unionales Sekundärrecht, welches aufgrund der Regelungsmaterie einer besonders eingehenden grundrechtlichen Überprüfung bedarf. 
Ein Beispiel ist die partielle Übernahme der Richtlinie 98/44/EG über den rechtlichen Schutz biotechnologischer Erfindungen in das schweizerische Patentgesetz 2007. Damit wurde die «Eurokompatibilität des schweizerischen Patentschutzes für Erfindungen, die biologisches Material zum Gegenstand haben, (...) sichergestellt» (Botschaft Änderung Patentgesetz, S. 29). Diese Richtlinie war in der EU selbst umstritten. Im Anschluss an ihre Inkraftsetzung wurde der EuGH mit der Überprüfung der Richtlinie befasst; er bejahte ihre Vereinbarkeit mit den Verträgen und den Grundrechten, namentlich der Menschenwürde und der Unversehrtheit der Person (Urteil Niederlande/Parlament und Rat, C-377/ 98, EU:C:2001:523, Rn. 69-81). Darauf wurde in der bundesrätlichen Botschaft hingewiesen (Botschaft Änderung Patentgesetz, S. 28). In solchen Fällen wirken die unionalen Grundrechte erheblich, wenngleich indirekt, auf das schweizerische Recht ein. Ähnliches war beim Erlass des Bundesgesetzes über genetische Untersuchungen beim Menschen von $2004 \mathrm{zu}$ beobachten. Die Botschaft äusserte sich eingehend zu den einschlägigen Richtlinien in der EG; auch nahm sie auf die «im Bereich der Medizin und Biologie zu beachtende[n] Grundsätze» gemäss Art. 3 Abs. 2 der - damals noch nicht verbindlichen - Grundrechtecharta Bezug (Botschaft genetische Untersuchungen, S. 7471-7472).

Von offizieller Seite fehlen Untersuchungen zur Frage, in welchem Ausmass sich das schweizerische Recht in quantitativer Hinsicht (d.h. prozentual zur gesamten Rechtsetzungstätigkeit) oder in qualitativer Hinsicht (d.h. in Bezug auf die Rechtsetzung im Bereich von «Schlüsselgesetzen») an das europäische Recht angleicht und unter dem Titel des autonomen Nachvollzugs europäisiert wird. Der Bundesrat lehnt es ab, Bundesgesetze und Verordnungen, welche im Zug des autonomen Nachvollzugs erlassen werden, zu kennzeichnen oder über den prozentualen Anteil solcher Erlasse im Verhältnis zur gesamten Gesetzgebungstätigkeit Bericht zu erstatten: «Die Anzahl Seiten an EURechtsvorschriften, welche die Schweiz in den letzten fünf Jahren übernommen hat, ist keine relevante Grösse. Wesentlich sind vielmehr Inhalt und Tragweite von übernommenem EU-Recht.» (Antwort vom 3. Dezember 2010 auf die Interpellation 10.3810 «Gesetzesflut und Souveränitätsverlust»; vgl. auch Antwort vom 14. Februar 2007 auf das Postulat 06.3839 «Autonomer Nachvollzug und Kennzeichnung des Schweizer EU-Rechtes»). Wissenschaftliche Studien schätzen, dass im vorletzten Jahrzehnt zwischen $40 \%$ und $60 \%$ der (damals) neueren oder revidierten Bundesgesetze vom EU-Recht beeinflusst wurden (Antwort des Bundesrates vom 3. Dezember 2010 auf die Interpellation 10.3810 «Gesetzesflut und Souveränitätsverlust», m.V.a. A. ARBIA, passim; E. KOHLER, Influence, passim; vgl. auch R. GAVA/P. SCIARINI/F. VARONE, passim; S. JENNI, Differentiated, passim). Teils wird das EU-Recht dabei ausdrücklich und vollständig übernommen; teils spielt es indirekt in den Gesetzgebungsprozess hinein. 


\section{B. Verordnungsrecht}

Der autonome Nachvollzug entfaltet seine Wirkung nicht nur auf Gesetzesstufe, sondern auch im Verordnungsrecht des Bundesrates und der ihm unterstellten Dienststellen. Sofern der Gesetzgeber bewusst europakompatibel legiferiert, enthält die Rechtsetzungsdelegation implizit ein Gebot der europakompatiblen Verordnungsgebung. In diversen Bereichen wird der Bundesrat zudem explizit verpflichtet, das EU-Recht zu berücksichtigen (ohne spezifisch auf namentlich bezeichnete EU-Rechtsakte Bezug zu nehmen); diesfalls handelt es sich um indirekte Verweise auf EU-Recht.

Solche Vorschriften existieren etwa für die Ausgestaltung von technischen Normen und Vorschriften, welche «auf die technischen Vorschriften der wichtigsten Handelspartner der Schweiz abgestimmt» werden (Art. 4 Abs. 2 THG), und für den Anlegerschutz und die Gewährleistung der Transparenz und Funktionsfähigkeit des Marktes für kollektive Kapitalanlagen, wo «[d]er Bundesrat und die FINMA (...) beim Erlass von Verordnungsrecht die massgebenden Anforderungen des Rechts der Europäischen Gemeinschaften» beachten (Art. 152 Abs. 2 KAG).

349 Vereinzelt wird EU-Recht qua Verweis ausdrücklich als anwendbar erklärt. Diesfalls handelt es sich um direkte Verweise auf EU-Rechtsakte, welche tel quel - allenfalls unter Beachtung ausdrücklich normierter Anpassungen und Ausnahmen - auch in der Schweiz verbindlich sind. Dabei wird standardmässig statisch auf eine im Zeitpunkt des Inkrafttretens der schweizerischen Verordnung in der EU geltende Fassung verwiesen (unter Angabe der Fundstelle im Amtsblatt der EU). Ausnahmsweise finden sich auch Verweise, welche dynamisch auf einen EU-Rechtsakt verweisen; diesfalls gilt die unionale Regelung in der jeweilig geltenden Fassung.

Ein Beispiel für einen statischen Verweis auf unionale Rechtsakte findet sich in Art. 2 Abs. 3 der Lebensmittel- und Gebrauchsgegenständeverordnung (LGV; s. für weitere Beispiele Gesetzgebungsleitfaden, 2019, Rz. 756). Ein Beispiel für einen dynamischen Verweis findet sich in Art. 133 Abs. 2 der Binnenschifffahrtsverordnung (BSV), wonach auf Schiffen Radargeräte verwendet werden dürfen, welche über eine Konformitätserklärung der Herstellerin oder des Herstellers gemäss der Richtlinie 2014/90/EU «in ihrer jeweils in der EU geltenden Fassung» verfügen (s. L. MADER/C. KROPF, S. 89, welche anmerken, dass es sich hierbei um Produkte handelt, welche auch nach Massgabe von Art. 16a THG in der Schweiz in Verkehr gebracht werden dürfen). Im Rahmen der Revision des Heilmittelgesetzes (HMG) von 2019 ging der Gesetzgeber noch einen Schritt weiter: Art. 82 Abs. 3 HMG ermächtigt den Bundesrat zur dynamischen Rechtsübernahme, soweit bestimmte delegierte Rechtsakte und Durchführungsrechtsakte der Europäischen Kommission im Bereich der Medizinprodukte technische oder administrative Einzelheiten betreffen, deren Regelung fortlaufend und in der Regel kurzfristig angepasst wird; diese Bestimmung war in den Beratungen der eidgenössischen Räte entsprechend umstritten (vgl. C. KROPF, S. 99-100). 
Beim Erlass von Verordnungsrecht gilt die Obliegenheit zur Prüfung der Europakompatibilität analog wie beim Erlass von Gesetzesrecht (N. 335). Die Richtlinien für Bundesratsgeschäfte (sog. «Roter Ordner») halten fest, dass im Antrag an den Bundesrat «ein Vergleich der vorgesehenen Regelung mit ausländischem Recht, insbesondere jenem der EU, angestellt werden muss» (www.bk.admin.ch und Link zu Rechtsetzungsbegleitung/Übernahme von EU-Recht). Unbefriedigend ist, dass solche Anträge wie auch die Erläuterungen dazu im Bundesblatt nicht veröffentlicht werden und der Öffentlichkeit nicht ohne Weiteres zugänglich sind.

\section{Rechtsetzungstechnik}

Bei der Anpassung des schweizerischen Rechts an dasjenige der EU stellen sich regelmässig komplexe Fragen in Bezug auf die Rechtsetzungstechnik: Ist es zielführend, das EU-Recht wörtlich (d.h. mittels copy- und paste-Tasten) oder sinngemäss (d.h. mittels eigener Formulierung) zu übernehmen (Inkorporation)? Oder ist es sachgerechter, eine unionale Verordnung oder Richtlinie durch einen Verweis tel quel anwendbar zu erklären (Verweisung)? Im Unterschied zu den EU-Mitgliedstaaten, welche unionsrechtlich verpflichtet sind, Verordnungen unmittelbar anzuwenden und Richtlinien umzusetzen, steht es der Schweiz frei, Verordnungen ins schweizerische Recht zu überführen und Richtlinien unmittelbar anzuwenden, sofern sie dies als sachgerecht erachtet.

Die Bundeskanzlei hat zur Übernahme von EU-Recht «Faustregeln» entwickelt. Dabei wird auch auf die Gesetzestechnischen Richtlinien des Bundes (GTR) und den Gesetzgebungsleitfaden des Bundesamtes für Justiz verwiesen (www.bk.admin.ch und Link zu Rechtsetzungsbegleitung/Übernahme von EU-Recht: Formale Aspekte; s. zum Ganzen auch B. GRÜTER/M. NUSSBAUMER, passim; C. KROPF, passim; zur Rechtsetzungstechnik in der Schweiz im Allg. S. HÖFler/M. Nussbaumer/F. Uhlmann, passim; G. MÜller/F. Uhlmann, passim). - Die Publikation von EU-Rechtsakten, auf die in einem schweizerischen Erlass verwiesen wird, ist speziell geregelt: Sie werden in der Amtlichen Sammlung (AS) nur mit dem Titel und der Fundstelle im Amtsblatt der EU (www.eur-lex.europa.eu) aufgenommen (Art. 5 Abs. 2 PublG; Botschaft Änderung Publikationsgesetz, S. 7078).

Der Gesetz- und Verordnungsgeber muss bei der Beantwortung dieser Fragen mitunter kollidierende Anliegen im Auge behalten: Er muss sich darum bemühen, die unionale Vorbilderregelung möglichst harmonisch ins normative System des schweizerischen Rechts einzupassen. Er muss die Grundsätze zur Bestimmung der Normstufe beachten, wonach alle wichtigen rechtsetzenden Bestimmungen in der Form des Bundesgesetzes zu erlassen sind (Art. 164 BV); zeitweise mag die Notwendigkeit, das schweizerische Recht flexibel im Gleichschritt mit dem sich rasant ändernden EU-Recht weiterzuentwickeln, 
allerdings dazu führen, intuitiv Verordnungsrecht zu präferieren und die Gesetzesform nur für offenkundig wichtige Bestimmungen zu wählen (und dabei nach Möglichkeit auf Verweisungen zu verzichten, B. GRÜTER/M. Nussbaumer, S. 116). Der Gesetz- und Verordnungsgeber muss sich darum bemühen, autonom nachvollzogenes EU-Recht in die Systematik des schweizerischen Rechts einzupassen; zeitweise mag der Übernahmeprozess allerdings dazu führen, die Systematik innerhalb eines Erlasses sowie auch das Verhältnis zwischen Erlassen neu zu justieren und allenfalls gänzlich neue Erlasse zu schaffen (s. zur «Dekodifikation» des Obligationenrechts als Folge des autonomen Nachvollzugs T. COTTIER et al., Rz. 262). Schliesslich besteht in der Schweiz eine sorgsam gepflegte Kultur der einfachen und verständlichen Gesetzessprache, welche auch bei der Übernahme von oftmals umständlich formulierten EU-Rechtsakten mit ihren ausschweifenden und materiell-rechtlich aufgeladenen Legaldefinitionen, ihren mehrfach verschachtelten komplizierten Satzkonstruktionen und ihren langfädigen Anhängen nicht aufgegeben werden soll (s. zur «oft sehr schwer verständlich[en]», «umfangreichen und komplizierten Regelungssprache» des EU-Rechts auch Botschaft Bereinigung Bundesrecht, S. 6128-6129). Der Gesetz- und Verordnungsgeber hat dabei idealiter auch die primären Adressatinnen und Adressaten der Regelung im Auge: Je breiter und heterogener der Kreis der Adressatinnen und Adressaten ist, desto eher drängt sich eine Reformulierung auf; je enger und fachspezifischer der Kreis ist, desto eher rechtfertigt es sich, mittels Verweisungen zu arbeiten (B. GRÜTER/M. NUSSBAUMER, S. 117).

Die Gesetzesredaktion in der Schweiz ist darauf ausgelegt, auch beim autonomen Nachvollzug von EU-Recht Begriffe in der in der Schweiz üblichen Terminologie zu verwenden. So ist hierzulande etwa von der «Konzession» die Rede und nicht von der «Lizenz», von der «Bewilligung» und nicht von der «Genehmigung» und vom «Motorfahrzeug» und nicht vom «Kraftfahrzeug» (www.bk.admin.ch und Link zu Übernahme von EU-Recht: Formale Aspekte/Terminologie). Gleichwohl haben sich über die Jahre in der schweizerischen Rechtspraxis Begriffe eingenistet, welche hierzulande früher nicht gebräuchlich waren. Dazu gehören die «Marktüberwachung» ebenso wie das «Inverkehrbringen» und die «Verbraucherinnen» und «Verbraucher»; allesamt Begriffe, welche von Brüssel und Strassburg aus das schweizerische Recht infiltriert haben. Immerhin ist die Schweiz mit den Tücken der Mehrsprachigkeit vertraut, wobei in der EU zurzeit 24 gleichermassen verbindliche Amtssprachen gelten (Art. 342 AEUV i.V.m. Art. 1 der Verordnung Nr. 1 zur Regelung der Sprachenfrage für die EWG von 1958); nicht zu Unrecht werden die Übersetzungsdienste als eigene Gewalt qualifiziert, welche den Inhalt von Erlassen in den Kapillaren beeinflussen (T. COTTIER et al., Rz. 259). Idealiter werden die verschiedenen Sprachfassungen beim autonomen Nachvollzug folglich berücksichtigt. Vorbilderregelung die Gefahr, dass sich die schweizerische Umsetzungsge- 
setzgebung allzu weit vom ursprünglichen, in der EU avisierten Normgehalt entfernt und damit zweckentfremdet wird. Es mag allein mit Blick auf den Wortlaut einer dem EU-Recht nachgebildeten Norm und ihre systematische Einpassung in der SR mitunter nicht mehr erkennbar sein, dass die Bestimmung ihren Ursprung im EU-Recht hat und europarechtskonform ausgelegt werden soll (s. zur europarechtskonformen Auslegung N. 354-362). Umso wichtiger ist in solchen Fällen das sorgfältige Studium der Materialien, was vor allem bei Verordnungsrecht allerdings nicht leichthin möglich ist (N. 350).

Der Gesetz- und Verordnungsgeber entscheidet sich zeitweise bewusst für eine Übernahme, welche sich terminologisch und systematisch eng an der unionalen Vorbilderregelung orientiert (d.h. mittels Verweisung oder copy/paste-Tasten), weil auf diese Weise der Nachweis gegenüber der EU, die Schweiz hätte eine analoge Regelung getroffen, einfacher erscheint (S. HÖFLER, S. 92). Dies mag nicht nur bei der Umsetzung der bilateralen Abkommen bedeutsam sein, sondern auch beim autonomen Nachvollzug in denjenigen Bereichen, in denen die Schweiz auf einen Gleichwertigkeitsbeschluss der EU angewiesen ist (N. 363-373). 



\section{Europaverträgliche Auslegung}

Die Politik des autonomen Nachvollzugs beschränkt sich nicht nur auf die Rechtsetzung. Ihr Zweck - die Minimierung der wirtschaftlichen Nachteile, welche sich systembedingt aus der Nichtmitgliedschaft der Schweiz in der EU bzw. im EWR ergeben - wird nur dann erreicht, wenn die Behörden autonom nachvollzogenes Recht tatsächlich europarechtsverträglich bzw. europarechtskonform auslegen. Der gesetzgeberische Wille, sich an der Rechtslage in der EU zu orientieren, muss seinen Niederschlag zwangläufig auch in der Rechtsanwendung finden, welche einen integralen Teil der Rechtsschöpfung darstellt und generell-abstrakte Erlasse zur Anwendungsreife führt (s. zum Ganzen E. KOHLER, rôle, passim).

In aller Regel bereitet das Postulat der europarechtskonformen Auslegung keine Probleme. Dies gilt dann, wenn die Anwendung der in der Schweiz traditionell im Vordergrund stehenden Auslegungselemente - grammatikalische, teleologische, systematische, historische und geltungszeitliche Auslegung zum Schluss führt, dass eine fragliche Bestimmung europarechtsverträglich auszulegen ist, d.h. wenn sich mit Blick auf ihren Wortlaut (welcher entweder identisch ist mit der EU-Vorbilderregelung oder zumindest eine «helvetisierte» Übersetzung in die Rechtsterminologie der Schweiz darstellt; vgl. N. 352), die ratio legis und die Materialien ergibt, dass eine parallele Rechtslage angestrebt wird. Das Bundesgericht umschrieb die Verpflichtung zur europarechtskonformen Auslegung in einem leading case von 2003 wie folgt (BGE 129 III 335, E. 6, wobei autonom nachvollzogenes Recht recte nicht nur «im Zweifel» europarechtskonform auszulegen ist, sondern ganz grundsätzlich; s. zu diesem Urteil auch N. 361):

«Nachvollzogenes Binnenrecht ist im Zweifel europakonform auszulegen. (...) Wird (...) die schweizerische Ordnung einer ausländischen - hier der europäischen - angeglichen, ist die Harmonisierung nicht nur in der Rechtssetzung, sondern namentlich auch in der Auslegung und Anwendung des Rechts anzustreben, soweit die binnenstaatlich zu beachtende Methodologie eine solche Angleichung zulässt. (...) Die Angleichung in der Rechtsanwendung darf sich dabei nicht bloss an der europäischen Rechtslage orientieren, die im Zeitpunkt der Anpassung des Binnenrechts durch den Gesetzgeber galt. Vielmehr hat sie auch die Weiterentwicklung des Rechts, mit dem eine Harmonisierung angestrebt wurde, im Auge zu behalten.»

Zentral ist das bundesgerichtliche Diktum, wonach eine europarechtskonforme Auslegung nur möglich ist, soweit die Auslegung im Lichte der in der Schweiz einschlägigen Auslegungselemente eine europaverträgliche Lesart 
erlaubt. Sofern die Auslegung zu einem Ergebnis führt, welches von der EUrechtlichen Regelung abweicht, bleibt für eine «Umdeutung pro Europa» kein Raum. Diesfalls bedarf eine Anpassung an das EU-Recht zwingend einer gesetzgeberischen Intervention.

Auch das Bundesverwaltungsgericht betont regelmässig, dass eine Norm nur insoweit europarechtskonform auszulegen ist, als dass «die binnenstaatliche Methodologie dies zulässt» (Urteil des BVerwG B-3064/2008 vom 13. September 2010, E. 3):

«Ist eine schweizerische Rechtsnorm in Erfüllung völkervertraglicher Pflichten, etwa der bilateralen Verträge, dem europäischen Recht nachgebildet, ist auch die entsprechende gemeinschaftsrechtliche Regelung und gegebenenfalls hierzu bestehende Rechtsprechung der Gemeinschaftsgerichte oder mitgliedstaatlicher Gerichte als Auslegungshilfe heranzuziehen (...). Gleiches muss gelten, wenn feststeht, dass der Gesetzgeber, wie im vorliegenden Fall geschehen (...), unabhängig von völkervertraglichen Pflichten, nach europäischem Vorbild legiferieren wollte (BGE 129 III 335 E. 6, ...). Die europarechtskonforme Interpretation ist in der Schweiz auf das Gebot der teleologischen Interpretation, gleichzeitig aber auch auf das historische Auslegungselement zurückzuführen. Der autonome Nachvollzug bezweckt die Angleichung an die europäischen Vorgaben (...). Im Zweifel ist die in Frage stehende Norm, soweit die binnenstaatliche Methodologie dies zulässt, europarechtskonform auszulegen und wie in der Rechtssetzung auch in der Auslegung und Anwendung des Rechts eine Harmonisierung anzustreben (BGE 129 III 335 E. 6 S. 350, ...). Dies kann auch bedeuten, dass selbst ein klarer, aber die europarechtliche Zielsetzung fehlerhaft, entgegen der eigenen Absicht, planwidrig lückenhaft nachvollziehender Wortlaut im Sinne des erreichten Ergebnisses teleologisch und europarechtskonform zu reduzieren ist ('Ausnahmelücke'; ...).»

358 Gewisse Konstellationen, in denen sich unter Umständen eine europarechtskonforme Auslegung anbietet, stellen Verwaltungsbehörden und Gerichte vor komplexe Herausforderungen. Es kommt häufig vor, dass sich der Gesetzgeber bei einzelnen Bestimmungen eines Erlasses bewusst um eine Angleichung an das europäische Recht bemüht, bei anderen Bestimmungen desselben Erlasses aber ebenso bewusst eine eigenständige Lösung wählt; er setzt die unionsrechtliche Vorbilderregelung gleichsam à la carte (E. KRAMER, S. 354) um, womit ein «Europarecht sui generis» (T. COTTIER et al., Rz. 214) entsteht. Diesfalls gilt das Gebot der europarechtskonformen Auslegung selbstredend nur für erstere Regelungsbereiche - sofern die rechtsanwendende Behörde die Harmonisierungsabsicht erkennt, weil etwa die Materialien darüber Aufschluss geben.

Anschauliche Beispiele dafür sind das Kartellgesetz (KG; s. BGE 124 III 495, E. 2a; BGE 137 II 199, E. 4.3; BGE 143 II 297; zum Ganzen M. STURNy, passim) und das Mehrwertsteuergesetz (MWSTG; s. BGE 124 II 193, E. 6a; zum Ganzen R. IMSTEPF, passim). Bei beiden Erlassen 
strebte der Gesetzgeber in relevanten Teilen eine Annäherung an das EU-Recht an. Gleichzeitig wich er punktuell davon ab, soweit ihm dies sachlich sinnvoll erschien, und verzichtete folglich bewusst auf eine vollständige Übernahme.

Das Postulat der europarechtskonformen Auslegung darf nicht zu einer sklavischen Orientierung am EU-Recht führen, auch wenn bei der Auslegung eines schweizerischen Erlasses feststeht, dass der Gesetzgeber damit eine Angleichung an die Rechtslage in der EU bezweckte. Entscheidend bleibt der gesetzgeberische Wille, sich am EU-Recht zu orientieren, um damit die wirtschaftlichen Nachteile des schweizerischen Abseitsstehens soweit als möglich zu kompensieren. Unter Umständen deckt sich dieser Wille aber nicht in allen Teilen mit dem telos der unionalen Vorbilderregelung, womit eine unreflektierte Übernahme der EU-Praxis zu ungewollten Resultaten führen würde (T. CotTIER/D. DzAmKo/E. Evtimov, S. 368).

Ein Beispiel dafür ist das Markenschutzgesetz (MSchG) von 1992, welches die einschlägigen Bestimmungen der ersten Richtlinie 89/104/EWG zur Harmonisierung des Markenrechts von 1988 autonom übernommen hat. Das BGer legte die Bestimmungen über die Zulässigkeit von Parallelimporten sodann aber nicht im Sinne der EuGH-Rechtsprechung aus (wonach innerhalb des Binnenmarktes die regionale bzw. gemeinschaftsweite Erschöpfung gilt), sondern sprach sich davon abweichend für die internationale Erschöpfung aus (EuGH, Urteil Silhouette International Schmied GmbH \& Co. KG, C-355/96, EU:C:1998:374; BGE 122 III 469).

Der Harmonisierungszweck des autonomen Nachvollzugs wirkt pro futuro. Damit rückt nicht nur die Rechtsprechung des EuGH in den Fokus, sondern auch die Praxis der mitgliedstaatlichen Verwaltungsbehörden und Gerichte (vgl. BGE 130 III 182, E. 5.5.1). Die Auslegung von autonom nachvollzogenem Recht soll sich grundsätzlich an der Praxis orientieren, wie sie sich nach der Übernahme durch die Schweiz in der EU weiterentwickelt (BGE 129 III 335, E. 6; N. 361). Dieses Prinzip beruht auf dem Vertrauen in die Vernünftigkeit der Rechtsfortbildung in der EU (F. NYFFELER, S. 50). Gleichzeitig befriedigt es das Bedürfnis nach Rechtssicherheit. Nur eine möglichst parallele Rechtsentwicklung garantiert die Verwirklichung der ratio legis, im fraglichen Rechtsbereich das schweizerische Recht mit dem europäischen zu harmonisieren. Dies gilt selbst dann, wenn die rechtsanwendenden Behörden in der Schweiz eine von der EU-Praxis abweichende Auslegung des EU-Rechtsaktes und damit reflexartig auch des schweizerischen «Umsetzungserlasses» bevorzugen würden (E. KRAMER, S. 352). Sofern sich das EU-Recht allerdings in einem Masse weiterentwickelt und verändert, dass eine parallele Rechtslage nicht mehr allein durch eine schöpferische Auslegung des schweizerischen Rechts erreicht werden kann, ist der Gesetzgeber aufgerufen, das schweizerische Recht formell anzupassen. 

dierte Kenntnisse des EU-Rechts voraus. Es geht zuvörderst darum zu realisieren, dass ein schweizerischer Erlass ein Produkt des autonomen Nachvollzugs darstellt; das mag insbesondere dann herausfordernd sein, wenn weder Wortlaut noch Materialien darüber hinreichend Auskunft geben (s. zum abgelehnten Vorschlag, autonom nachvollzogenes Recht zu kennzeichnen, N. 347). Sodann geht es darum, den Normgehalt des dem schweizerischen Erlass zugrundeliegenden EU-Rechtsakts zu eruieren. Dazu gehört das Studium der Praxis der unionalen und mitgliedstaatlichen Behörden; sofern die Faktenlage dazu dürftig ist, geht es darum, den EU-Rechtsakt im Lichte der spezifisch unionsrechtlichen Auslegungselemente selbst auszulegen (s. zu diesen Auslegungselementen T. OpPERMANN/C.D. ClaSSEN/M. NETTESHEIM, § 9 Rz. 165-188; M. OESCH, Europarecht, Rz. 460-476). In der Schweiz bestehen im fachkundigen Umgang mit dem EU-Recht erhebliche Unterschiede: Zeitweise setzen sich die Behörden sorgfältig mit dem Recht und der Praxis in der EU auseinander; zeitweise verzichten die Behörden gänzlich auf eine Analyse des EURechts.

In einer Streitsache von 2003 war das BGer aufgerufen, Art. 333 OR, welcher im Rahmen des Swisslex-Pakets an das EU-Recht angepasst wurde (N. 344), auszulegen und auf das Gebot der europakompatiblen Auslegung zurückzugreifen (BGE 129 III 335). Es ging um die Frage, ob Art. 333 OR auch im Konkurs eine solidarische Haftung des neuen Betriebsinhabers für Forderungen der Arbeitnehmer bestimmt. Das BGer stellte fest, dass «sich weder der Wortlaut von Art. 333 Abs. 3 OR noch die Materialien explizit darüber [äussern], ob der Erwerber für vor der Betriebsübernahme fällig gewordene Lohnforderungen haftet, wenn die Übernahme aus einer Konkursmasse erfolgt» (E. 5). Weiter kam es zum Schluss, dass «eine Solidarhaft des Erwerbers eines Betriebes aus der Konkursmasse im Sinne von Art. 333 Abs. 3 OR in Auslegung contra verba legis auszuschliessen» ist (E. 5.8). Sodann wandte es sich der europakompatiblen Auslegung zu. Im Zentrum stand die Richtlinie 77/ 187/EWG, deren Nachvollzug Art. 333 OR bezweckte. Das BGer analysierte ein Urteil des EuGH zur Auslegung dieser Richtlinie, die Umsetzung und Praxis in ausgewählten EGMitgliedstaaten, die Revision der Richtlinie 77/187/EWG durch die Richtlinie 98/50/EG und den Erlass der Richtlinie 2001/23/EG, mit der die Richtlinie 77/187/EWG aufgehoben wurde. Es kam zum Schluss: «Da der Schweizer Gesetzgeber für das Konkursverfahren 'nichts anderes' im Sinne von Art. 5 Abs. 1 der Richtlinie vorgesehen hat, bedeutet diese Regelung auf das schweizerische Recht übertragen, dass Art. 333 Abs. 3 OR, der eine autonome Satzung im Sinne von Art. 3 Abs. 1 Satz 2 der Richtlinie enthält, bei Übernahme eines Betriebes aus einer Konkursmasse nicht anwendbar ist. Auch eine europarechtskonforme Auslegung von Art. 333 Abs. 3 OR führt also zu dessen Nichtanwendbarkeit im Konkursfall (...).» (E. 6). Es ist vorbildhaft, mit welcher Akribie das BGer in casu die Rechtsentwicklung in der EWG/EG analysierte. 
Fundierte Kenntnisse des EU-Rechts sind auch deshalb wichtig, weil es für die

Gerichte in der Schweiz nicht möglich ist, dem EuGH eine Frage über die Auslegung des EU-Rechts zur Vorabentscheidung vorzulegen. Dieses Recht ist den Gerichten der Mitgliedstaaten vorbehalten (Art. 267 AEUV).

Das BVerwG sistierte 2008/2009 ein Beschwerdeverfahren während rund zehn Monaten bis zur rechtskräftigen Erledigung eines vor dem EuGH hängigen Verfahrens. Der Rechtsstreit vor dem EuGH drehte sich um die Auslegung von gemeinschaftsrechtlichen Bestimmungen über die Schaffung von ergänzenden Schutzzertifikaten im Patentrecht, welche autonom in das schweizerische Patentgesetz (PatG) übernommen worden waren; neckischerweise war die AHP Manufacturing BV sowohl im Verfahren vor dem BVerwG als auch im Verfahren vor dem niederländischen Gericht, welches den EuGH um Vorabentscheidung ersuchte, als klägerische Partei beteiligt. Nach Verkündigung des Urteils des EuGH (AHP Manufacturing, C-482/07, EU:C:2009:501) nahm das BVerwG das Beschwerdeverfahren wieder auf und entschied den Rechtsstreit auch unter Berücksichtigung der Erwägungen des EuGH (Urteil des BVerwG B-3064/2008 vom 13. September 2010; s. dazu auch N. 357). Dieses Vorgehen ist typisch für den Pragmatismus, den die schweizerischen Gerichte beim Umgang mit EU-Recht mitunter an den Tag legen. 



\section{Spezialfälle}

\section{A. Faktische Alternativlosigkeit}

Mitunter ist die Schweiz faktisch gezwungen, das eigene Recht an das unionale anzupassen. Damit sind Konstellationen gemeint, bei denen völkerrechtlich zwar keine Verpflichtung besteht, in Tat und Wahrheit aber auch keine wirkliche Entscheidungsautonomie vorhanden ist. Methodisch finden solche Anpassungen unter dem Titel des autonomen Nachvollzugs statt. Gleichwohl erreicht die Angleichung an das EU-Recht eine neue Qualität. Namentlich aber nicht ausschliesslich - wirtschaftspolitische Erwägungen lassen eine Angleichung gleichsam als alternativlos erscheinen. Die folgenden Beispiele illustrieren diese Sachzwänge.

\section{Datenschutzrecht}

Gemäss Art. 45 der Datenschutz-Grundverordnung (EU) 2016/679 (DSGVO) muss bei der Übermittlung personenbezogener Daten in ein Drittland sichergestellt sein, dass das durch diese Verordnung und die Grundrechtecharta garantierte Schutzniveau für natürliche Personen nicht untergraben wird. Auf der Basis von Art. 45 der Datenschutz-Grundverordnung kann die Europäische Kommission beschliessen, dass ein Drittland ein angemessenes Schutzniveau gewährleistet (Angemessenheitsbeschluss). Datenübermittlungen in ein Land, für das ein solcher Angemessenheitsbeschluss besteht, bedürfen keiner weiteren Genehmigung. Die Europäische Kommission entschied 2000, dass dies in der Schweiz der Fall ist (C(2000) 2304). Dieser Beschluss ist für die schweizerische Wirtschaft von enormer Bedeutung. Andernfalls würde der Datenaustausch mit Unternehmen und Kunden in der EU erschwert. Wenig überraschend herrscht in der Schweiz ein Grundkonsens, bei der laufenden Revision des Datenschutzgesetzes (DSG) das unionale Datenschutzrecht - inkl. der durch die Organe der EU erfolgten Austarierung der mitunter kollidierenden Grundrechtsgewährleistungen (Schutz der Privatsphäre, Schutz personenbezogener Daten, Meinungs- und Informationsfreiheit, Wirtschaftsfreiheit) zum Nennwert zu nehmen. Abweichungen sollen nur insoweit ins Auge gefasst werden, als damit die Angemessenheit des Schutzniveaus nicht gefährdet wird (Bundesamt für Justiz, Normkonzept zur Revision des Datenschutzgesetzes: Bericht der Begleitgruppe Revision DSG, 29. Oktober 2014, S. 5-6; vgl. auch Botschaft Totalrevision Datenschutzgesetz, S. 6965): 
«Der Datenverkehr steht diesbezüglich [gegenüber Drittstaaten] grundsätzlich unter der Voraussetzung, dass die EU das Datenschutzniveau der Schweiz als angemessen anerkennt (Angemessenheitsentscheidung), was gegenwärtig der Fall ist. Will die Schweiz diesen Status beibehalten, hat sie somit alles Interesse daran, ihre Datenschutzvorschriften zu stärken und der europäischen Gesetzgebung anzugleichen, auch wenn nicht eine wörtliche Anpassung an den Entwurf zur EU-Datenschutz-Grundverordnung erforderlich ist.»

Vor diesem Hintergrund kommt den Urteilen Schrems I und Schrems II des EuGH von 2015 und 2020 eine besondere Bedeutung zu (Urteil Schrems, C-362/14, EU:C:2015:650; Urteil C-311/18, Facebook Ireland und Schrems, EU:C:2020:559). Im Urteil Schrems I von 2015 erklärte der EuGH die Entscheidung der Europäischen Kommission, wonach die Vereinigten Staaten im Rahmen des Safe Harbor-Programms ein angemessenes Schutzniveau für die Übermittlung personenbezogener Daten gewährleisteten, ungültig. In der Folge einigten sich die Kommission und die Vereinigten Staaten auf einen neuen Rahmen für die transatlantische Übermittlung von Daten, den EU-USPrivacy Shield (s. für den Angemessenheitsbeschluss der Kommission C(2016) 4176 final). Nach Ansicht der Kommission wurden mit dem neuen Privacy Shield die Defizite ausgemerzt, welche der EuGH im Urteil Schrems I beim Safe Harbor-Programm identifiziert hatte. Dieser Ansicht widersprach der EuGH im Urteil Schrems II von 2020; er erklärte den Beschluss der Europäischen Kommission, wonach die Vereinigten Staaten im Rahmen des Privacy Shield ein angemessenes Schutzniveau gewährleisteten, für ungültig.

Die Schweiz schloss mit den Vereinigten Staaten 2008 ein Safe Harbor-Abkommen ab (SR 0.235.233.6). Dieses Abkommen war mit dem Safe Harbor-Programm, welches dem Angemessenheitsbeschluss der Europäischen Kommission von 2000 zugrunde lag, inhaltlich weitgehend identisch. Das Schrems I-Urteil des EuGH führte allerdings auch in der Schweiz zu einem Umdenken. Nach der Veröffentlichung dieses Urteils erklärte der Eidgenössische Datenschutz- und Öffentlichkeitsbeauftragte (EDÖB), dass er das Safe Harbor-Abkommen zwischen der Schweiz und den Vereinigten Staaten nicht mehr als genügende Rechtsgrundlage für die Übermittlung von Personendaten in die Vereinigten Staaten betrachte, weil auch dieses Abkommen «unter dem Aspekt der Verhältnismässigkeit von Grundrechtseingriffen und der verfassungsmässigen Verfahrensgarantien aus Schweizer Sicht problematisch» ist; mit Blick auf das bilaterale Verhältnis zur EU kam der EDÖB zu folgendem Schluss (Bericht und Empfehlungen an den Bundesrat vom 14. Oktober 2015 (...) betr. U.S.-Swiss Safe Harbor Framework, S. 3):

«Auch wenn die Ungültigerklärung des Kommissionsentscheids betreffend EU Safe Harbor Framework durch [den] EuGH das Schweizer Abkommen nicht direkt betrifft, wird es nicht möglich sein, dass die Schweiz in der Sache eine andere Haltung vertritt und am U.S.-Swiss 
Safe Harbor Framework unverändert festhält. Nebst den (...) Risiken für die Grundrechte betroffener Personen aus der Schweiz könnte ein solches Vorgehen auch zur Konsequenz haben, dass die EU ihre Angemessenheitserklärung zum Datenschutzniveau in der Schweiz aufhebt. (...) Eine Aufhebung der Angemessenheitserklärung hätte schwerwiegende Konsequenzen für den freien Austausch von Personendaten mit EU-Staaten und könnte zur Isolation der Schweiz führen.»

Im Nachgang zu den Verhandlungen zwischen der EU und den Vereinigten Staaten, welche zum Abschluss des Privacy Shield führten, erklärten auch die Schweiz und die Vereinigten Staaten den Privacy Shield im Verhältnis zwischen diesen beiden Staaten für anwendbar (vgl. Pressemitteilung des EDÖB vom 11. Januar 2017, Swiss-US Privacy Shield: neuer Rahmen für Datenübermittlungen in die USA). Der EuGH hatte hierfür den Boden gelegt. Die Schweiz profitierte vom Urteil Schrems I, dessen präjudizielle Wirkung weit über die EU hinausstrahlte. Ohne entsprechende Vorleistung des EuGH wäre die Schweiz demgegenüber kaum in der Lage gewesen, die Vereinigten Staaten zu einer Privacy Shield-ähnlichen Lösung zu motivieren. Auch wäre ein solcher autonomer «Vorvollzug» zumindest aus wirtschaftlicher Sicht kaum ratsam gewesen. Nach dem Urteil des EuGH von 2015 blieb der Schweiz faktisch aber keine andere Wahl, als die Regelung der Datenübermittlung gegenüber den Vereinigten Staaten europakompatibel auszugestalten. Im Nachgang zum Schrems II-Urteil des EuGH dürfte eine ähnliche Anpassung zu erwarten sein. Ein Festhalten am Swiss-US Privacy Shield wäre mit dem Angemessenheitsbeschluss der Europäischen Kommission in Bezug auf das Schutzniveau in der Schweiz nicht zu vereinbaren. Wenig überraschend kam der EDÖB im September 2020 zum Schluss, dass «das Privacy Shield Regime trotz der Gewährung von besonderen Schutzrechten für Betroffene in der Schweiz kein adäquates Schutzniveau für Datenbekanntgaben von der Schweiz an die USA gemäss Bundesgesetz über den Datenschutz (DSG) bietet» (Pressemitteilung des EDÖB vom 8. September 2020, Privacy Shield CH-USA bietet nach Auffassung des EDÖB kein adäquates Datenschutzniveau; s. auch Stellungnahme des EDÖB zur Übermittlung von Personendaten in die USA und weitere Staaten ohne angemessenes Datenschutzniveau i.S.v. Art. 6 Abs. 1 DSG vom 8. September 2020).

Die Europäische Kommission ist verpflichtet, die Entwicklungen in Drittländern fortlaufend zu überwachen und bei Bedarf Angemessenheitsbeschlüsse zu widerrufen (Art. 45 Abs. 4 und Abs. 5 der Datenschutz-Grundverordnung). Nach allgemein vorherrschender Ansicht besteht unionsrechtlich kein Anspruch auf eine Verlängerung, auch wenn das Drittland überzeugt ist, über ein angemessenes Schutzniveau zu verfügen. Zurzeit steht die Überprüfung des Angemessenheitsbeschlusses in Bezug auf die Schweiz an. 


\section{Finanzmarktrecht}

Ein ähnliches Schema zeigt sich im Finanzmarktrecht. Der EU-Gesetzgeber erliess in den letzten Jahren mehrere neue Rechtsakte zur Regelung der Märkte für Finanzinstrumente und der Marktinfrastruktur. Dazu gehören die Verordnung (EU) 648/2012 (EMIR), die Verordnung (EU) 600/2014 (MiFIR) und die Richtlinie 2014/65/EU (MiFID II). Diese Rechtsakte bezwecken, die Transparenz zu erhöhen, den Anlegerschutz zu verbessern und den Wettbewerb zu stärken. Sie ermächtigen die Europäische Kommission, Gleichwertigkeitsbeschlüsse in Bezug auf die Rechtslage in Drittstaaten zu erlassen, um Marktteilnehmerinnen und Marktteilnehmern aus diesen Drittstaaten selektiv Marktzugang zu gewähren (s. zum Ganzen Mitteilung der Kommission, Gleichwertigkeit im Bereich der Finanzdienstleistungen, COM(2019) 349 final):

- Wertpapierfirmen in der EU müssen sicherstellen, dass ihre Handelsgeschäfte mit Aktien, die zum Handel an einem geregelten Markt zugelassen sind oder an Handelsplätzen gehandelt werden, an einem geregelten Markt oder im Rahmen eines multilateralen Handelssystems (MTF), eines systematischen Internalisierers oder an einem Drittlandhandelsplatz, der von der Europäischen Kommission als gleichwertig eingestuft wird, getätigt werden (vgl. Art. 23 MiFIR, Art. 25 Abs. 4 MiFID II). Im letzteren Fall prüft die Kommission, ob die Anforderungen an die Tätigkeit von Handelsplätzen und die Beaufsichtigung und Durchsetzung in einem Drittland mit den unionsrechtlichen Vorgaben gleichwertig sind. Die Kommission bejahte die Gleichwertigkeit des Rechts- und Aufsichtsrahmens, der für die beiden Börsen in der Schweiz, die SIX Swiss Exchange AG und die BX Swiss AG gilt, für 2018 (Durchführungsbeschluss (EU) 2017/2441). Ende 2018 bestätigte die Kommission diesen Beschluss, befristete die Verlängerung bis zum 30. Juni 2019 und kündigte an, die weitere Verlängerung vom Gang der Verhandlungen über ein institutionelles Abkommen abhängig zu machen (Durchführungsbeschluss (EU) 2018/2047):

«Dieser Beschluss trägt auch den Schlussfolgerungen des Rates vom 28. Februar 2017 Rechnung, wonach eine Voraussetzung für die weitere Entwicklung des sektorbezogenen Ansatzes mit der Schweiz nach wie vor die Schaffung eines gemeinsamen institutionellen Rahmens für bestehende und künftige Abkommen über die Beteiligung der Schweiz am Binnenmarkt der EU ist. Bei der Entscheidung über eine etwaige Verlängerung dieses Beschlusses hat die Kommission dem Stand der Fortschritte Rechnung getragen, die mit Blick auf ein Abkommen zur Schaffung eines solchen gemeinsamen institutionellen Rahmens erzielt wurden. Die Verhandlungsführer der EU und der Schweiz haben sich auf einen vollständigen Entwurf des Abkommens geeinigt. Der Schweizer Bundesrat hat dieses Abkommen zur Kenntnis genommen und beschlossen, einen Konsultationsprozess einzuleiten, der bis zum Frühjahr 2019 andauern wird.» 
Im Juni 2019 entschied die Kommission, den Gleichwertigkeitsbeschluss nicht mehr zu verlängern. Aktien von börsenkotierten Unternehmen aus der EU können seither nicht mehr an den Börsen in der Schweiz gehandelt werden. Die Schweiz erliess die Verordnung über die Anerkennung ausländischer Handelsplätze für den Handel mit Beteiligungspapieren von Gesellschaften mit Sitz in der Schweiz (SR 958.2); demnach ist es Handelsplätzen mit Sitz in der EU untersagt, den Handel mit Aktien von Gesellschaften mit Sitz in der Schweiz anzubieten oder diesen Handel zu ermöglichen. Diese Massnahme der Schweiz mag kurzfristig sinnvoll sein; «der grosse Knall ist ausgeblieben» (NZZ vom 9. August 2019). Längerfristig hat die Schweiz aber kaum ein Interesse daran, den Börsenhandel gleichsam künstlich auf die EU und die Schweiz aufzuteilen und zu regionalisieren; dasselbe gilt wohl auch für die EU.

- Die Erbringung grenzüberschreitender Dienstleistungen gegenüber geeigneten Gegenparteien und professionellen Kunden durch Drittlandunternehmen ohne Zweigniederlassung ist in der EU nur möglich, sofern die Europäische Kommission zum Schluss kommt, dass die Finanzmarktregulierung des Drittstaats aufsichtsrechtlich äquivalent ist (Art. 46-47 MiFIR). Wenig überraschend orientieren sich folglich das Finanzmarktinfrastrukturgesetz (FinfraG) sowie - in Teilen - das Finanzdienstleistungsgesetz (FIDLEG) und das Finanzinstitutsgesetz (FINIG) an den europäischen Vorbilderregelungen. In der Botschaft zum FIDLEG und FINIG äusserte sich der Bundesrat wie folgt (Botschaft FIDLEG und FINIG, S. 8939-8940; vgl. auch Botschaft FinfraG, S. 7509-7512):

«Die beschriebenen Abweichungen der vorgelegten Gesetzesentwürfe gegenüber den europäischen Normen zur Finanzmarktregulierung lassen sich auf eine Berücksichtigung der besonderen Eigenschaften des Schweizer Finanzmarkts zurückführen. Die vorgeschlagenen Vorschriften enthalten jedoch grundsätzlich keine Vorschriften, die über die Anforderungen der europäischen Regelwerke hinausgehen. Sie stellen kein Hindernis für eine allfällige Äquivalenzanerkennung der Schweiz unter der Verordnung MiFIR dar.»

Das Parlament hat die Entwürfe in der Folge zwar in mehrfacher Weise abgeändert und dabei vereinzelt auch Elemente eingeführt, welche nicht gleichwertig mit den unionalen Vorgaben sind (vgl. T. JUTZI/K. WESS/ D. SIERADZK, passim; R. SETHE, passim). Gleichwohl bleibt zu hoffen, dass die Anerkennung der Äquivalenz durch die Kommission nicht gefährdet ist. Der damit einhergehende Marktzugang ist schlicht zu bedeutsam (auch wenn sich die Zulässigkeit der Erbringung von - wirtschaftlich noch bedeutsameren - Dienstleistungen gegenüber Kleinanlegerinnen und Kleinanlegern weiterhin nach dem Recht der Mitgliedstaaten 
bestimmt, s. Art. 39 MiFID II). Die Anpassungen an die Rechtsakte der EU erweisen sich vor diesem Hintergrund faktisch als alternativlos. Eine eigene vorurteilslose Auseinandersetzung mit den zentralen Rechtsfragen findet nurmehr innerhalb der Leitplanken statt, welche vom EURecht vorgegeben werden.

Analog zum Datenschutzrecht geht die herrschende Meinung auch im Bereich des Finanzmarktrechts davon aus, dass die Europäische Kommission unionsrechtlich nicht verpflichtet ist, einen Gleichwertigkeitsbeschluss zu erlassen, wenn ein Drittland über eine äquivalente Regulierung verfügt (Mitteilung der Kommission, Gleichwertigkeit im Bereich der Finanzdienstleistungen, COM(2019) 349 final). Bei diesem Beschluss mögen auch - prima vista sachfremde - politische Gründe eine Rolle spielen, wie die Nichtverlängerung des Beschlusses über die Börsenäquivalenz illustriert (s. für weitere Äquivalenzverfahren, welche die Schweiz betreffen, S. EMMENEGGER/A. BIGLER, S. 173-174).

Unter Umständen setzt das GATS dem Spielraum der Europäischen Kommission beim Erlass von Gleichwertigkeitsbeschlüssen gewisse Schranken (Art. II und Art. VII GATS, Anhang über Finanzdienstleistungen). Sofern die Kommission den regulatorischen Rahmen eines WTO-Mitglieds als gleichwertig akzeptiert, mögen andere WTO-Mitglieder eine nicht weniger vorteilhafte Behandlung einfordern, wenn sie darlegen können, dass sie die Voraussetzungen der Gleichwertigkeit ebenfalls erfüllen.

2019 legte die Europäische Kommission - nicht zuletzt vor dem Hintergrund der Verhandlungen über die zukünftigen Beziehungen der EU zum Vereinigten Königreich - eine Neubeurteilung ihres Ansatzes zur Gleichwertigkeit von Regulierungssystemen im Bereich der Finanzdienstleistungen vor (COM(2019) 349 final). Sie weist ausdrücklich darauf hin, dass sie bei der Prüfung der Gleichwertigkeit auch den «allgemeinen politischen Kontext» zu einem Drittstaat berücksichtigt. Damit mutiert das Instrument des Gleichwertigkeitsbeschlusses zu einem Instrument, mit dem gegenüber Drittstaaten auch weitere politische Interessen verfolgt werden, welche mit finanzdienstleistungsrechtlichen Belangen nicht oder nur am Rand zusammenhängen. Die trostlose Saga der verweigerten Börsenäquivalenz könnte sich in anderen Bereichen des Finanzdienstleistungsrechts durchaus wiederholen, womit der Gestaltungsspielraum der Schweiz noch stärker als bis anhin auch in anderen Politikbereichen eingeschränkt werden würde. 


\section{COVID-19: Ausfuhr persönlicher Schutzausrüstung}

Die Europäische Kommission unterstellte bei der Bekämpfung der durch das

Coronavirus (COVID-19) ausgelösten epidemiologischen Krise die Ausfuhr von bestimmten Gegenständen im Bereich der medizinischen Schutzausrüstung einer speziellen Ausfuhrgenehmigung (Durchführungsverordnung (EU) 2020/ 402). Dabei handelte es sich um Produkte, welche notwendig waren, «um eine weitere Ausbreitung der Krankheit zu verhindern und die Gesundheit des medizinischen Personals zu schützen, das infizierte Patienten behandelt» (Erwägungsgrund 2). Von der Genehmigungspflicht ausgenommen wurden wenngleich erst eine Woche später - Ausfuhren in die EWR-EFTA-Mitgliedstaaten, in die in Anhang II AEUV aufgeführten überseeischen Länder und Hoheitsgebiete, in die Färöer, nach Andorra und San Marino, in die Vatikanstadt sowie in die Schweiz (Durchführungsverordnung (EU) 2020/426). Im Gegenzug forderte die Kommission diese Länder und Gebiete auf, die Ausfuhr dieser Gegenstände ebenfalls zu kontrollieren, und führte ein - nicht weiter spezifiziertes - Kriterium der Angemessenheit der Kontrolltätigkeit ein (Erwägungsgrund 4):

«Die Behörden der ausgenommenen Länder und Gebiete sollten angemessene Garantien dafür bieten, dass sie ihre eigenen Ausfuhren der betreffenden Produkte kontrollieren, damit das mit der Durchführungsverordnung (EU) 2020/402 verfolgte Ziel nicht untergraben wird. Die Kommission sollte diesen Aspekt aufmerksam verfolgen.»

Die Schweiz führte in der Folge ebenfalls eine Ausfuhrbewilligung für die von der EU-Verordnung erfassten Produkte ein; davon ausgenommen wurden Ausfuhren in die EU und die weiteren Länder und Gebiete, welche die EU von der Genehmigungspflicht ausgenommen hatte, «soweit die Reziprozität gewährleistet ist» (Art. 10d der COVID-19-Verordnung 2, SR 818.101.24). Der Bundesrat verfolgte mit der Einführung dieser Ausfuhrbewilligung ein doppeltes Ziel: Zum einen ging es darum sicherzustellen, dass das eigene medizinische Personal und weitere Personen in der Schweiz weiterhin Zugang zu solchen Schutzausrüstungen hatten. Zum anderen ging es darum, den Handel mit diesen Produkten zwischen der EU und der Schweiz nicht zu erschweren; im Vordergrund standen diesfalls - im Unterschied zum Datenschutzrecht (N. 364-368) und zum Finanzmarktrecht (N. 369-371) - nicht primär wirtschaftliche Gründe, sondern Anliegen des Gesundheitsschutzes und der Krisenbekämpfung. 


\section{B. Cassis de Dijon-Prinzip}

Die Politik des autonomen Nachvollzugs wurde 2010 um eine neue Dimension erweitert. Im Rahmen der Revision des Bundesgesetzes über die technischen Handelshemmnisse (THG) führte die Schweiz das Herkunftsortsprinzip (Cassis de Dijon-Prinzip) für Waren aus dem EWR ein: Produkte, welche nach den Vorschriften des EU-Rechts (im harmonisierten Bereich) oder des EU- bzw. EWR/ EFTA-Herkunftslandes (im nicht-harmonisierten Bereich) hergestellt und dort rechtmässig in Verkehr gebracht worden sind, können gemäss Art. 16a THG auch in der Schweiz ohne weitere Prüfung eingeführt und verkauft werden. Das ist praktisch bedeutsam, wenn der schweizerische Gesetz- oder Verordnungsgeber technische Vorschriften der EU nicht autonom ins schweizerische Recht übernommen hat und damit nicht ohnehin eine parallele Rechtslage besteht. Vorbehalten bleiben die Anforderungen an die Produktinformation; diese muss grundsätzlich in mindestens einer schweizerischen Amtssprache abgefasst sein (Art. $4 a$ und Art. 16e THG). Art. 16a Abs. 2 THG sieht Ausnahmefälle vor (N. 375). Für Lebensmittel gilt eine Sonderregelung: Lebensmittel, welche im EWR rechtmässig in Verkehr gesetzt worden sind, die Bestimmungen der schweizerischen Lebensmittelgesetzgebung aber nicht erfüllen, bedürfen einer speziellen Bewilligung für den Vertrieb in der Schweiz (Art. 16c und Art. 16d THG). Diese Bewilligung wird in Form einer Allgemeinverfügung erteilt.

Auf diese Weise können etwa Schinken aus Italien (Prosciutto cotto) mit einem höheren Wassergehalt als in der Schweiz erlaubt und verdünnter Obstsaft aus Dänemark (Cider) mit einem geringeren Fruchtsaftanteil als in der Schweiz vorgeschrieben auch hierzulande rechtmässig vertrieben und verkauft werden (s. für eine Übersicht über die erteilten Allgemeinverfügungen www.blv.admin.ch und Link zu Cassis de Dijon). Es ist geplant, das Zulassungsverfahren für Lebensmittel zu vereinfachen und neu ein Meldeverfahren vorzusehen (Medienmitteilung der DEA vom 23. Juli 2019: Cassis-de-Dijon-Prinzip für Lebensmittel wird verstärkt). Gleichzeitig führte die Revision der Lebensmittelgesetzgebung von 2017 und die damit einhergehende weitere Anpassung des schweizerischen Rechts an dasjenige der EU dazu, dass das Cassis de Dijon-Prinzip nach Massgabe des THG an Bedeutung verliert. Ähnliches wird der Fall sein, wenn sich die Schweiz und die EU im Rahmen der - zurzeit allerdings sistierten - Verhandlungen über den Agrarfreihandel über die Gleichwertigkeit und die gegenseitige Anerkennung weiterer lebensmittelrechtlicher Vorschriften einigen (s. zu diesen Verhandlungen N. 141).

Die schweizerische Lösung unterscheidet sich mehrfach vom Cassis de Dijon-Prinzip, wie es im europäischen Binnenmarkt entwickelt wurde. Es handelt sich um systembedingte Abweichungen, welche sich aus dem Umstand ergeben, dass die Schweiz institutionell nicht in die EU bzw. in den EWR eingebunden ist. Die Anpassung an die hiesigen Bedürfnisse widerspiegelt «hel- 
vetischen Eigensinn und Pragmatismus» - Cassis de Dijon wird zu «Cassis de Berne» (T. COTTIER/D. HERREn, S. 275). So führt die autonome Einführung dazu, dass der erleichterte Marktzugang nur einseitig, d.h. zugunsten von Produkten, welche aus dem EWR eingeführt werden, wirkt. Schweizerische Produkte und ihre Hersteller profitieren nicht von einem erleichterten Marktzugang im EWR. Die Schweiz hat diesen Nachteil - unter Verzicht auf die handelspolitische Maxime der Reziprozität - mit Blick auf die erhofften Vorteile bewusst in Kauf genommen (vgl. zu einer ersten - positiven - Einschätzung der Auswirkungen des Cassis de Dijon-Prinzips in der Schweiz Bericht des SECO vom April 2013, www.seco.admin.ch und Link zu Aussenwirtschaft/Technische Handelshemmnisse). Weiter erfasst die schweizerische Variante des Cassis de Dijon-Prinzips sowohl Produkte, welche gestützt auf unionsrechtliche Produktevorschriften rechtmässig in Verkehr gesetzt worden sind, als auch Produkte, welche - bei unvollständiger oder fehlender Harmonisierung - den technischen Vorschriften eines EU- oder EWR-EFTA-Mitgliedstaats entsprechen. Schliesslich hat die Tatsache, dass die Schweiz das Cassis de Dijon-Prinzip autonom eingeführt hat, zur Folge, dass sie ebenso autonom über die Ausnahmen entscheidet. Das Cassis de Dijon-Prinzip gilt gemäss Art. 16a Abs. 2 THG nicht für i) Produkte, die einer Zulassungs- oder Anmeldepflicht unterliegen (z.B. Arzneimittel); ii) Produkte, die einer vorgängigen Einfuhrbewilligung bedürfen oder einem Einfuhrverbot unterliegen (z.B. Kriegsmaterial oder Güter aus Ländern, gegen welche die Schweiz ein Embargo erlassen hat); iii) Produkte, für welche der Bundesrat aus überwiegenden öffentlichen Interessen eine Ausnahme beschliesst (s. Verordnung über das Inverkehrbringen von nach ausländischen technischen Vorschriften hergestellten Produkten und über deren Überwachung auf dem Markt, VIPaV, SR 946.513.8).

$\mathrm{Zu}$ den Produkten, welche nach Massgabe von Art. 2 VIPaV bei der Einfuhr in die Schweiz nicht vom Cassis de Dijon-Prinzip profitieren, gehören etwa Alcopops, Reinigungsmittel, welche Phosphat enthalten, und Eier aus in der Schweiz nicht zugelassener Käfighaltung von Hühnern ohne entsprechende Deklaration. Die schweizerischen Gerichte haben sich in einer Vielzahl von Fällen zur Auslegung des helvetisierten Cassis de Dijon-Prinzips geäussert, insbesondere zur Konkurrentenklage gegen Allgemeinverfügungen bei Lebensmitteln und zu den Massnahmen der Marktüberwachung (s. zum Ganzen N. DiebolD/ M. LUdin, passim; J.-A. STADELHOFER/B. ZIRLICK, passim).

Methodisch und inhaltlich geht die einseitige Einführung des Cassis de Dijon-Prinzips über die bisherige Politik des autonomen Nachvollzugs hinaus. Sie hat eine neue Epoche dynamischer Anpassung eingeläutet. Das Cassis de Dijon-Prinzip beinhaltet auch in seiner helvetisierten Form naturgemäss eine prospektive Komponente: Es erfasst auch künftige Änderungen von Produktevorschriften. Dabei gilt die unbesehene Anerkennung neuen Rechts nicht nur 
in Bezug auf die EU. Sie umfasst im nicht-harmonisierten Bereich auch die Entwicklungen in den EU- und EWR-EFTA-Mitgliedstaaten. Damit wird ein hohes Mass an Vertrauen in diese Rechtsordnungen zum Ausdruck gebracht. Im praktischen Ergebnis bricht die Einführung des Cassis de Dijon-Prinzips mit dem souveränitätspolitisch begründeten Kardinalsprinzip der schweizerischen Integrationspolitik, wonach europäisches Recht nur statisch, d.h. gestützt auf die Rechtslage an einem Stichtag, Eingang in das schweizerische Recht findet. Im Anwendungsbereich des Cassis de Dijon-Prinzips besteht zumindest aus der Perspektive der Schweiz auch ohne fortlaufende Gesetzesund Verordnungsanpassungen eine parallele Rechtslage. Die Schweiz hat im Warenverkehr damit einen Integrationsgrad erreicht, welcher über tradierte Vorstellungen hinausreicht.

Durch die konsequente Anwendung des Cassis de Dijon-Prinzips zugunsten von Produkten aus dem EWR besteht mitunter die Gefahr einer Inländerdiskriminierung (discrimination à rebours). Aus diesem Grund lässt das THG schweizerischen Produzentinnen und Produzenten die Wahl, ob sie ihre Produkte gestützt auf das schweizerische Recht oder gestützt auf das EU-Recht bzw. das Recht eines EU- oder EWR-EFTA-Mitgliedstaats herstellen möchten (vgl. Art. 16a THG [für Produzentinnen und Produzenten, welche auch für den Export in die EU produzieren] und Art. 16b THG [für Produzentinnen und Produzenten, welche nur für den inländischen Markt produzieren]), sofern das schweizerische Recht nicht ohnehin bereits autonom an dasjenige der EU angepasst worden ist. 
Vierter Teil

Epilog 

Die Doppelstrategie der völkerrechtlichen Anbindung qua sektorieller Abkommen und der selbstgewählten Anpassung im Zuge des autonomen Nachvollzugs hat sich für die Schweiz bewährt. Sie wird vielerorts als schweizerischer Königsweg betrachtet, welcher politisch von Volk und Ständen getragen wird und inhaltlich die gewünschten Resultate liefert:

- Der bilaterale Weg wurde von den Stimmbürgerinnen und Stimmbürgern in Referendumsabstimmungen diverse Male bestätigt. Verfassungsrechtlich wurde die Frage des Verhältnisses der Schweiz zu Europa explizit bei der Volksinitiative «EU-Beitrittsverhandlungen vors Volk!» von 1997, der Volksinitiative «Ja zu Europa!» von 2001 und der Volksinitiative «Für eine massvolle Zuwanderung (Begrenzungsinitiative)» von 2020 thematisiert; Volk und Stände lehnten diese Initiativen ab und bestätigten den eingeschlagenen Kurs (s. zum Ganzen und auch zu den europapolitisch problematischen Initiativen für die Ausschaffung krimineller Ausländer und gegen Masseneinwanderung N. 60-64).

- Der bilaterale Weg hat dazu beigetragen, die Nachteile, welche im Nachgang zur Ablehnung der schweizerischen Beteiligung im EWR befürchtet wurden, zu minimieren und schweizerischen Unternehmen einen in mancherlei Hinsicht vergleichbaren Zugang zum europäischen Markt zu ermöglichen. In Ergänzung zu den Marktzugangsabkommen profitiert die Schweiz von weiteren Abkommen, welche die Integration in den europäischen Rechtsraum - etwa in den Bereichen Justiz und Inneres, Visa und Asyl - und die Teilnahme an Agenturen, weiteren Einrichtungen und Programmen der EU ermöglichen.

Der Bundesrat hat wiederholt bekräftigt, dass der bilaterale Weg die bevorzugte europapolitische Option darstellt, nachdem er sich 2006 vom 1992 erklärten Ziel des Beitritts zur EU verabschiedet hatte und den Beitritt seither nurmehr «als längerfristige Option» betrachtet (Europabericht 2006, S. 6982; wobei das Beitrittsgesuch erst 2016 formell zurückgezogen wurde, N. 26). Seither gilt die Maxime, dass die Weiterführung des bilateralen Wegs «das am besten geeignete Instrument zur Wahrung der schweizerischen Interessen in Europa» darstellt (Europabericht 2010, S. 7246, 7341). Gemäss Bundesrat bilden die bilateralen Abkommen «einen massgeschneiderten rechtlichen Rahmen, welcher den engen wirtschaftlichen und politischen Beziehungen zwischen der Schweiz und der EU sowie der geografischen Lage der Schweiz im Zentrum Europas gerecht wird» und den Interessen der Schweiz weiterhin am besten dient (Bericht Freihandelsabkommen statt bilaterale Abkommen, S. 75). Gleichzeitig ist sich der Bundesrat bewusst, dass sich die erfolgreiche Weiter- 
führung des bilateralen Wegs als zunehmend komplizierter und ungewisser erweist, und «[g]erade angesichts der zunehmenden Schwierigkeiten eine vergleichende Prüfung der Vor- und Nachteile anderer europapolitischer Instrumente für die Schweiz umso wichtiger» ist (Europabericht 2010, S. 7285).

Die Rahmenbedingungen für die erfolgreiche Weiterführung des bilateralen Wegs haben sich in den letzten fünfzehn Jahren in der Tat verschlechtert. Die folgenden fünf Aspekte stehen im Vordergrund:

- $\quad$ Die EU verlangt mittlerweile konsequent, bei der völkerrechtlichen Einbindung der Schweiz auf den Acquis communautaire abzustellen. Die Schweiz beteiligt sich an einem «multilateralen Projekt» (Schlussfolgerungen des Rates vom 16. Dezember 2014, Rz. 44). Entsprechend geht die EU bei der Schweiz davon aus, dass die Regeln des Clubs akzeptiert werden oder auf eine Beteiligung verzichtet wird; tertium non datur.

- Das Verständnis für den europapolitischen Sonderweg der Schweiz hat mit der Osterweiterung 2004/2007/2013 und der damit praktisch einhergehenden Verdoppelung der Anzahl der Mitgliedstaaten der EU weiter abgenommen. Die schweizerischen Unterhändlerinnen und Unterhändler verhandeln nicht mehr nur mit den Vertreterinnen und Vertretern der EU-15, sondern auch mit den Vertreterinnen und Vertretern mittel- und osteuropäischer Staaten, mit denen traditionell weniger enge Bande bestehen und die bei den Verhandlungen über den EU-Beitritt ihrerseits nur ganz beschränkt von Ausnahmen und Sonderlösungen profitieren konnten.

- $\quad$ Die Schweiz hat sich mit dem Entscheid des Bundesrates von 2006, den EU-Beitritt nicht mehr als Ziel der schweizerischen Europapolitik zu verstehen, sondern nurmehr als eine Option, gleichsam aus eigenem Antrieb in die Kategorie «normaler» Drittstaaten degradiert. Damit mutierte der pragmatische Bilateralismus stillschweigend von einer Übergangslösung zu einem provisoire qui dure, und die Bereitschaft der EU, Hand zu bieten für massgeschneiderte Sonderlösungen, verringerte sich weiter.

- Die EU verlangt seit 2008 die Schaffung eines institutionellen Rahmens, der für alle bestehenden und zukünftigen Marktzugangsabkommen gilt, welche auf EU-Recht beruhen. Sie hat die Botschaft ausgesendet, wonach sie den Status quo nicht mehr als valable Option betrachtet. Ein institutionelles Abkommen gehört gleichsam zum Preis, den die Schweiz dafür bezahlt, sektoriell weiterhin in mitgliedstaatsähnlicher Weise in den Binnenmarkt eingebunden zu sein, ohne die Regeln des Clubs vollumfänglich akzeptieren zu müssen. 
- $\quad$ Die komplizierten Verhandlungen über das künftige Verhältnis der EU zum Vereinigten Königreich führen dazu, dass die EU gegenüber europäischen Drittstaaten noch konsequenter auftritt und ihnen nur privilegierten Marktzugang gewährt, wenn der von der EU formulierte Grundsatz der Unteilbarkeit der Grundfreiheiten (Schlussfolgerungen des Rates vom 19. Februar 2019, Rz. 10; Leitlinien des Europäischen Rates (Artikel 50) für die Brexit-Verhandlungen, 29. April 2017, Rz. 1; Empfehlung des Europäischen Parlaments vom 18. Juni 2020 für die Verhandlungen über eine neue Partnerschaft mit dem Vereinigten Königreich Grossbritannien und Nordirland, 2020/2023(INI), Rz. 8) und das institutionelle Credo des form follows function respektiert werden.

Damit steht die Schweiz europapolitisch vor wegweisenden Entscheiden. Kurzfristig geht es darum, die Voraussetzungen für die Weiterführung des bilateralen Wegs in seiner aktuellen Form zu schaffen. Die enge Anbindung an die EU qua bilateraler Abkommen hat sich bewährt. Personen und Unternehmen in der Schweiz und der EU profitieren von präferentiellen Marktzugangsrechten. Diverse Abkommen erlauben der Schweiz, als assoziierter Drittstaat in mitgliedstaatsähnlicher Weise in den unionalen Rechtsraum eingebunden zu sein und in paneuropäisch operierenden Agenturen, weiteren Einrichtungen und Programmen teilzunehmen. Über den unmittelbaren volkswirtschaftlichen Nutzen hinaus drückt das dichte Vertragsnetz eine Geisteshaltung aus, wonach sich die Schweiz als Teil Europas versteht und ebenfalls einen Beitrag zum Wohlergehen und zur gemeinsamen Problemlösung auf dem europäischen Kontinent zu leisten bereit ist. Die Weiterführung des schweizerischen Modells der europäischen Integration bedingt, dass die Schweiz Hand bietet für die von der EU geforderte Schaffung eines neuen institutionellen Rahmens. Das auf dem Tisch liegende Institutionelle Abkommen stellt die Beziehungen der Schweiz mit der EU auf eine solide Grundlage, trägt zur weiteren Verrechtlichung des bilateralen Acquis bei und stellt ein belastbares Fundament für weitere pragmatische Integrationsschritte der Schweiz dar. Andernfalls besteht die Gefahr, dass der bilaterale Weg schleichend erodiert; das wäre keine verlockende Perspektive - weder für die Schweiz noch für die EU.

Das gute Einvernehmen und die Einsicht, zur Lösung aktueller Herausforderungen eng und konstruktiv zusammenzuarbeiten, manifestierte sich eindrücklich bei der Bekämpfung der durch das Coronavirus (COVID-19) ausgelösten epidemiologischen Krise im Frühjahr 2020. Die Schweiz wurde von der EU zu Gesprächskreisen und Koordinationsgruppen eingeladen - wie etwa dem Frühwarn- und Reaktionssystem (EWRS, Beschluss Nr. 1082/2013/EU) des Europäischen Zentrums für die Prävention und die Kontrolle von Krankheiten (Verordnung (EG) Nr. 851/2004) -, zu denen die Schweiz zuvor keinen Zugang hatte (s. zu den Verhandlungen über ein Gesundheitsabkommen N. 35). Die Schweiz konnte an online 
durchgeführten Treffen der ständigen Botschafterinnen und Botschafter der EU-Mitgliedstaaten in Brüssel, der Innenministerinnen und Innenminister und der Landwirtschaftsministerinnen und Landwirtschaftsminister teilnehmen. Die Schweiz beteiligte sich an einer von der EU betriebenen Informationsplattform zur Repatriierung von eigenen Staatsangehörigen. Als Folge davon wurden Schweizer Bürgerinnen und Bürger mit Flugzeugen von Gesellschaften aus anderen europäischen Staaten repatriiert; umgekehrt brachte die Swiss International Air Lines im Ausland gestrandete Unionsbürgerinnen und Unionsbürger nach Europa zurück. Schweizer Grenzkantone nahmen an Covid-19 erkrankte Patientinnen und Patienten aus den Nachbarstaaten in ihren Spitälern auf. Entsprechend positiv fiel eine erste Bilanz der Zusammenarbeit gemäss einem Sprecher der Europäischen Kommission aus: «Wir können uns nur zu der engen Zusammenarbeit zwischen der Schweiz und der Union beglückwünschen, die in dieser beispiellosen Krise in vielen Sektoren vorherrschte»; es sei stärker denn je messbar gewesen, dass man eine «Schicksalsgemeinschaft» sei (Tagesanzeiger vom 6. April 2020, S. 4; NZZ vom 23. Mai 2020, S. 13). Gleichzeitig belasteten einzelne Aktionen der EU das bilaterale Verhältnis. So erliess die Kommission kurzzeitig ein faktisches Ausfuhrverbot von Gegenständen im Bereich der medizinischen Schutzausrüstung auch gegenüber der Schweiz (N. 128). Die Einführung von Personenkontrollen an den Grenzen durch alle EU-Mitgliedstaaten wie auch die Schweiz - alles Schengen-Staaten - erfolgte weitgehend unkoordiniert (N. 247). Aktuell bleibt die Schweiz aussen vor bei der Zusammenarbeit im Bereich von Tracing Apps. Aufgrund der fehlenden rechtlichen Grundlage ist es nicht möglich, Daten der SwissCovid App mit Daten von Apps anderer europäischer Staaten zu synchronisieren. Voraussetzung dafür ist - zumindest aus Sicht der EU - der Abschluss eines Gesundheitsabkommens (Tagesschau SRF vom 20. Juli 2020, www.srf.ch). Diese Situation ist vor allem auch mit Blick auf die 320000 Grenzgängerinnen und Grenzgänger aus der EU, welche in der Schweiz arbeiten, ärgerlich. EU grundsätzlich zu überdenken. Der bilaterale Ansatz bleibt störungsanfällig. Negativ fällt ins Gewicht, dass das bilaterale Vertragswerk unübersichtlich und wenig systematisch aufgebaut ist. Konkrete Bedürfnisse und windows of opportunities bestimmen den Sachbereich und Zeitpunkt der staatsvertraglichen Annäherung. Das Vorgehen folgt einem punktuell-pragmatischen induktiven Ansatz. Die fehlende konzeptionelle Kohärenz und Koordination springen insbesondere bei einem Vergleich mit dem EWR-Recht ins Auge. Das EWR-Abkommen dehnt die vier Grundfreiheiten in ihrer Gesamtheit auf die EWR-EFTA-Mitgliedstaaten aus. Die bilateralen Abkommen umfassen nur punktuell Aspekte der binnenmarktrechtlich gewährleisteten Warenverkehrsfreiheit, Personenfreizügigkeit (Arbeitnehmerfreizügigkeit und Niederlassungsfreiheit) und Dienstleistungsfreiheit; die Kapitalverkehrsfreiheit wird im bilateralen Acquis gar nicht geregelt. Das EWR-Abkommen übernimmt das Binnenmarktrecht zu den horizontalen (flankierenden) Politiken, welches das gute Funktionieren der Grundfreiheiten sicherstellt und zu diesem Zweck Regelungen im Bereich Forschung und Entwicklung, Umwelt, Bildung, Konsumentenschutz, Gesellschaftsrecht, Wettbewerbsrecht und Sozialpolitik enthält. Die bilateralen Abkommen regeln solche Politikbereiche nur punktuell; 
Beispiele sind kartell- und beihilferechtliche Vorgaben in ausgewählten Abkommen. Das EWR-Abkommen enthält ein ausgeklügeltes Set an institutionellen Regeln und Einrichtungen mit faktisch-supranationalem Charakter. Die bilateralen Abkommen kennen keinen allgemeingültigen institutionellen Rahmen; immerhin wird das Institutionelle Abkommen hier Verbesserungen bringen. Damit offenbaren die bilateralen Abkommen ein janusköpfiges Naturell: Partiell ist die Schweiz mitgliedstaatsähnlich in den unionalen Binnenmarkt integriert; das einschlägige EU-Recht wird auf das Verhältnis zur Schweiz ausgedehnt. Partiell verbleibt die Schweiz in der bunt zusammengewürfelten Kategorie «normaler» Drittstaaten; die gegenseitigen Rechte und Pflichten bestimmen sich nach klassisch völkerrechtlichen Mustern. Auch erfahrene Juristinnen und Juristen empfinden solche Subtilitäten mitunter - und zu Recht - als verwirrend und haben Mühe, sich in diesem rechtlichen Dickicht zurechtzufinden. Das ist der Rechtssicherheit abträglich.

In der Schweiz wurde vereinzelt vorgeschlagen, einen nochmaligen Anlauf für einen EWRBeitritt zu wagen (vgl. etwa D. FreIBURGHAUS/G. KreIS, passim). Die EWR-Option scheint für die Schweiz - ungeachtet der offenkundig vorhandenen Vorteile insbesondere in Bezug auf die umfassende Binnenmarktteilhabe - aus drei Gründen kaum erstrebenswert. Erstens ist der EWR weiterhin durch institutionelle Besonderheiten charakterisiert, welche bereits 1992 umstritten waren und zur Ablehnung beitrugen (v.a. fehlende Mitentscheidungsrechte beim Erlass neuer EU-Rechtsakte, welche «quasi obligatorisch» bzw. «quasi automatisch» übernommen werden müssen, Europabericht 2010, S. 7313-7315; supranationaler Charakter). Zweitens dürfte ein Beitritt der Schweiz - wie notabene auch anderer Staaten - das one voice-Prinzip auf Seiten der EWR-EFTA-Staaten arg strapazieren. Es bestünde das Risiko, dass die fein austarierte Balance zwischen den EWR-EFTA-Staaten Island, Liechtenstein und Norwegen wie auch das eingespielte working arrangement zwischen der EU und den EWR-EFTA-Staaten unnötig aufs Spiel gesetzt würden. Drittens stösst nicht nur der bilaterale Acquis Schweiz-EU, sondern auch der EWR systembedingt an Grenzen. Stirnrunzeln bereiten etwa die zeitweise unklare Binnenmarkt- und damit EWR-Relevanz von EU-Rechtsakten und die Verzögerung ihrer Übernahme in das EWRA (backlogs), das komplizierte Verhältnis der EWR-EFTA-Staaten zu den unionalen Agenturen und die unklare Rechtslage in Bezug auf den Grundrechtsschutz im EWR-EFTA-Pfeiler (s. zum Ganzen die Beiträge in EuR Beiheft 1, 2020, 25 Jahre Europäischer Wirtschaftsraum).

Aus demokratietheoretischer Warte fällt die fortlaufende Übernahme von EURecht negativ ins Licht. Die Schweiz hat die Rechtsetzung in durchaus relevanten Bereichen faktisch an die Organe der EU delegiert. Dabei zeigt sich immer deutlicher, dass zwischen der fortlaufenden Rechtsübernahme und der (direkten) Demokratie ein Spannungsverhältnis besteht; die Substanz der demokratischen Rechte wird ausgehöhlt (N. 65-68). Diese Entwicklung wird sich mit der Dynamisierung der Rechtsübernahme unter dem Institutionellen Abkommen noch verstärken. Des Weiteren erweisen sich der Einbezug neuer Sachbereiche in den bilateralen Acquis und die Beteiligung der Schweiz an 
Agenturen und Programmen zunehmend als schwierig; der unionale Verwaltungsverbund ist nur beschränkt in der Lage, Drittstaaten zu akkommodieren. Die Politik, sektoriell am multilateralen Integrationsprojekt der EU teilzunehmen, ohne sich institutionell den gemeinsamen Regeln zu unterwerfen, stösst systembedingt an Grenzen. Schliesslich entwickelt sich die EU selbst weiter. Diverse Massnahmen, welche in der Folge der Finanz-, Wirtschafts- und Staatsschuldenkrise erlassen wurden, weisen ein erhebliches Diskriminierungspotential für Unternehmen in Drittstaaten aus; dies dürfte etwa auf die Errichtung der Bankenunion und weitere regulatorische Verschärfungen im Finanzdienstleistungsbereich zutreffen. Ähnliches gilt in der Aussenwirtschaftspolitik, wo die Vorteile der handelspolitischen Autonomie zunehmend kleiner werden und sich unter Umständen schon bald eine Neubeurteilung der Optionen aufdrängt (T. COTTIER, Strukturwandel, passim; M. OESCH, Autonomie, passim). Beim Abschluss von Freihandelsabkommen mit Partnern auf der ganzen Welt hat die EU die Schweiz bereits mehrfach überholt. Sofern die zurzeit zwar blockierten, aber nicht definitiv abgebrochenen - Verhandlungen über privilegierte Handelsbeziehungen zwischen den Vereinigten Staaten und der EU zu einem erfolgreichen Abschluss führen (TTIP, allenfalls TTIP light), werden schweizerische Unternehmen im Vergleich zu ihren europäischen Konkurrenten weiter benachteiligt.

Vor diesem Hintergrund stellt sich die Frage, ob der bilaterale Weg auch zukünftig den schweizerischen Königsweg darstellt oder ob sich «im Hinblick auf die Wahrung der Interessen der Schweiz» (Europabericht 2010, S. 7284) eine weitergehende Integration aufdrängt - weg von der Politik, als «zugewandter Ort» politisch isoliert alte Privilegien und Nischen zu verteidigen, hin zur konstruktiven Mitwirkung und Übernahme von Verantwortung (T. CотTIER et al., Rz. 955-1002). Letztlich ist das Schicksal der Schweiz wirtschaftlich, politisch, gesellschaftlich, kulturell und wissenschaftlich unweigerlich mit demjenigen ihrer Nachbarn und weiterer Staaten in Europa verbunden. Dies gilt auch in Bezug auf die Wahrung und Förderung der europäischen Werte und Interessen auf dem globalen Parkett und die Positionierung Europas als wirtschaftlich und politisch einflussreicher Akteur etwa gegenüber China und den Vereinigten Staaten; dieser Aspekt rückt mit dem Ende der Pax Americana und den aktuellen geopolitischen Verschiebungen immer mehr in den Vordergrund. Die aktive Mitgestaltung der Zukunft im Verbund mit gleichgesinnten Staaten liegt im ureigenen Interesse der Schweiz. Sie tut gut daran, sich sachlich und vorurteilslos der politischen Gretchenfrage des EU-Beitritts zu stellen. 

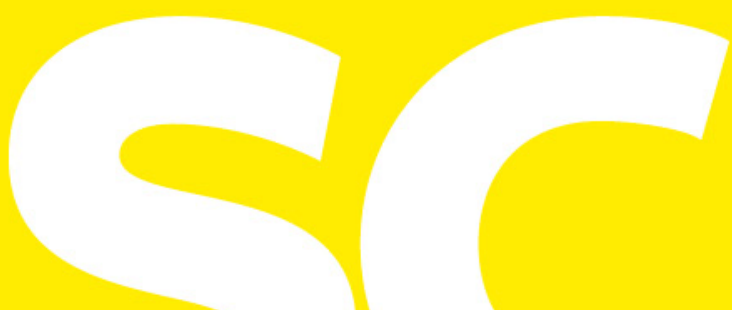

Die schweizerische Europapolitik steht auf dem Prüfstand. Nach der Ablehnung der Volksinitiative «Für eine massvolle Zuwanderung (Begrenzungsinitiative)» rückt das geplante Institutionelle $\mathrm{Ab}$ kommen in den Fokus der Öffentlichkeit. Die EU erwartet von der Schweiz, sich zum vorliegenden Text zu bekennen. Der Bundesrat beurteilt den Entwurf zwar positiv, hat bislang aber darauf verzichtet, ihn zu unterzeichnen. Er verlangt Klärungen und Präzisierungen in Bezug auf den Lohnschutz, die Unionsbürgerrichtlinie und die Beihilferegeln.

Die nächsten Monate werden zeigen, ob der Bundesrat und die Europäische Kommission einen tragfähigen Kompromiss finden und bereit sind, das Abkommen den Vertragsgebern in der Schweiz und der EU zur Genehmigung vorzulegen, oder ob wir auf eine Phase der Unsicherheit und Erosion der bilateralen Beziehungen zusteuern. Letzteres wäre keine verlockende Perspektive - weder für die Schweiz noch für die EU. 